\title{
Ultrasonic welding of thermoplastics
}

This thesis submitted to the University of Sheffield for the degree of Doctor of Philosophy

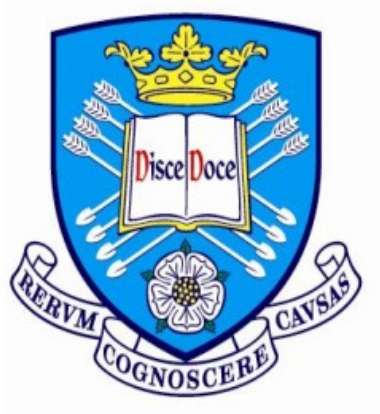

The

University

Of

Sheffield.

Department of Mechanical Engineering

By

Syed Farhan Raza

Supervised by

Dr. Candice Majewski

Dr. Christophe Pinna

August, 2015 


\section{Abstract}

Ultrasonic welding (UW) is not only a well-known industrial process but it has also been an active research area. Materials ranging from metals to non-metals e.g. polymers and from virgin materials to non-virgin materials e.g. composites are easily welded using this welding technique. Some research has already been carried out but more thorough analysis is needed on ultrasonic welding of thermoplastics.

Two thermoplastics selected for this research are commercially known as Acrylo-nitrileButadiene Styrene (ABS) and Polypropylene (PP). ABS belongs to amorphous type of thermoplastic, whereas PP is semi-crystalline thermoplastic. Owing to this dissimilarity in their molecular structure, ultrasonic welding of these two plastics has already been considered to be different. Energy director (ED) is usually protruded on anyone of the samples to be welded. Ultrasonic energy is uniformly driven in the presence of energy director at a localized area between the samples.

In this research, triangular (TRI) and semi-circular (SEMI) energy directors (ED) were protruded on the surface of specimen by designing and manufacturing injection molds. Tensile testing in shear was performed for measuring lap shear strength of joints after being welded ultrasonically at constant strain rate of $3.24 \mathrm{~mm} /$ minute. Maximum LSS (lap shear strength) of $17 \mathrm{MPa}$ and $6 \mathrm{MPa}$ were found for ABS with TRI ED and PP with SEMI ED respectively. In other words, these LSSs (lap shear strengths) were $34 \%$ and $14.63 \%$ of base material strength for ABS and PP respectively.

In this work, a statistical analysis (General Linear Model) was also used to deduce meaningful information from experimentation for both materials. For different factor settings, percent change in LSS was also calculated. Maximum percent change in LSS was found for weld time e.g. 2682 and 76.67 from low to high level was computed for ABS and PP respectively. Similarly, 55 \& 47.2 for amplitude and 41 \& 6 for static force were calculated with ABS \& PP respectively. Percent change in LSS of 409 and 47 was also evaluated from SEMI to TRI and TRI to SEMI EDs for ABS and PP respectively. All the factors appeared to be significant to affect the LSS but weld time was found to be the most significant to achieve the higher bond strength. 
After doing the GLM (General Linear Model) analysis, some interesting results were also highlighted. Experimental techniques were used to investigate the main reasons for these findings. Difference in softening temperatures, viscosities, temperature spreads, ED collapses, heat affected zones (HAZ) and fractured surfaces for both materials were used to determine the reasons for interesting effects. For example, low static force was required for $A B S$ in gaining higher LSS due to its lower softening temperature and viscosity drop at weld zone. Thus this work presented new and deeper understanding of the ultrasonic welding of thermoplastics.

Various hypotheses were also made after having gone through the literature. Experimental tools were also used to test these hypotheses. These technical tools included differential scanning calorimeter (DSC), melt flow index (MFI) tests, finite element analysis (FEA), high speed video camera (HSVC) and microscopy (optical and scanning electron). These tools helped approve or disapprove the hypotheses.

Viscoelastic heating was considered to be crucial mechanism in joining thermoplastics ultrasonically. Viscoelastic heating was depending mainly upon loss modulus, applied frequency and strain amplitude. Weld strength further depended upon the temperature development at weld interface.

Apart from above description, simulation based on FEA was also validated for its accuracy and precision. A good match was found between experimental and simulated results. 


\section{Acknowledgments}

First of all, I am really indebted to continuous support and assistance of my supervisors Dr. Candice Majewski and Dr. Christophe Pinna particularly for their vital technical backing and guidance throughout the course of this research.

I will also like to express my sincere gratitude to my first supervisor (Dr. Candice Majewski) for her planned monthly meetings besides her other commitments. Candice's instructions and guidance gave me a better experience in researching and writing thesis.

I am also grateful to various researchers, technical staff especially Michael Jackson, Chris Grigson, Mike Renison and academic staff of the mechanical engineering department.

Above all, I am really thankful to my creator, my sweet parents and my siblings. Without the best wishes of them, I would not be able to achieve this milestone by fulfilling the requirements of my PhD. 


\section{Table of Contents}

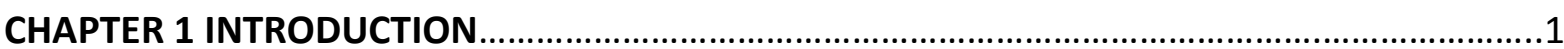

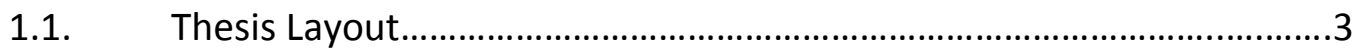

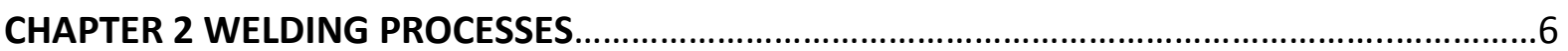

2.1. AIM

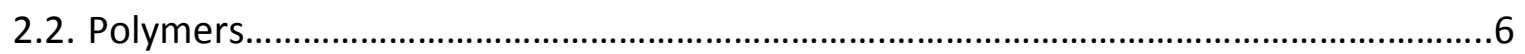

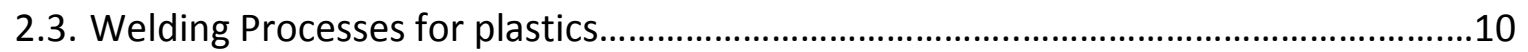

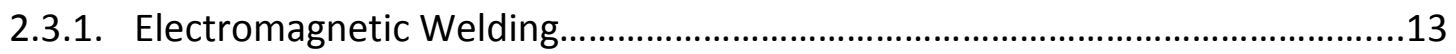

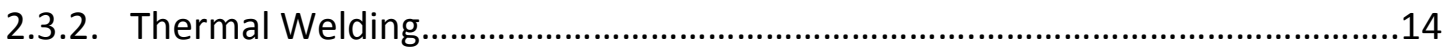

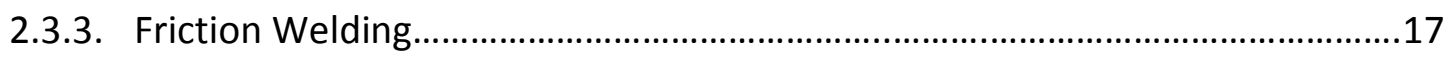

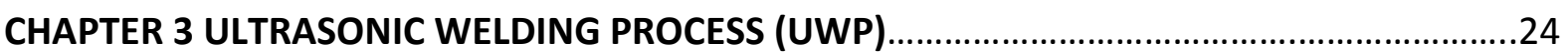

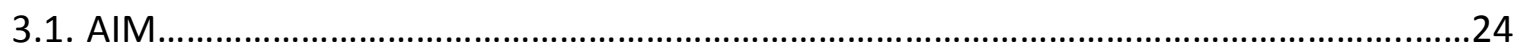

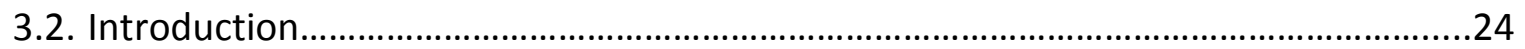

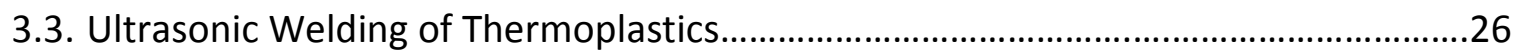

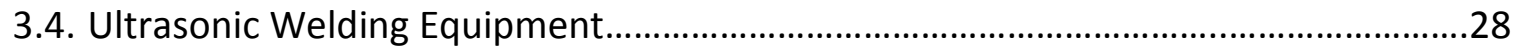

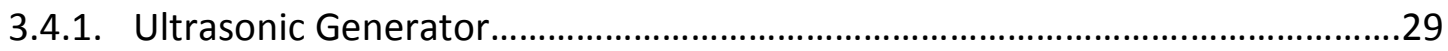

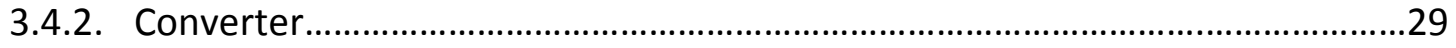

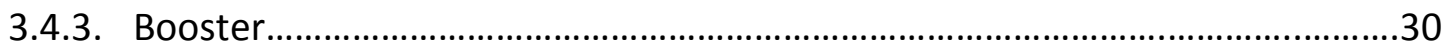

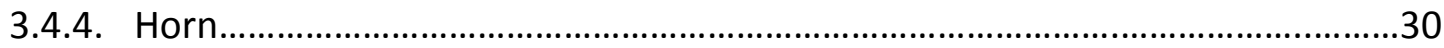

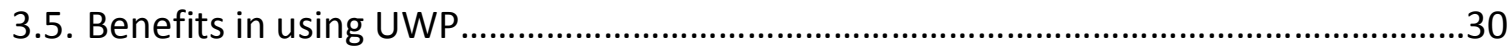

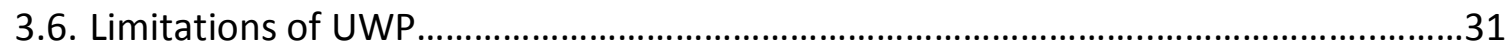

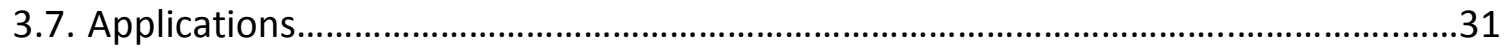




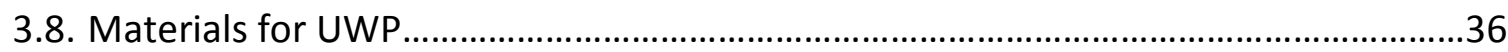

3.8.1. Viscoelastic Behaviour of Polymers..................................................................39

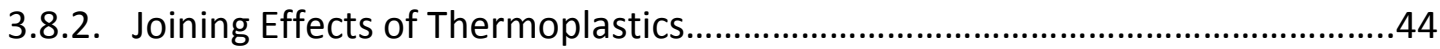

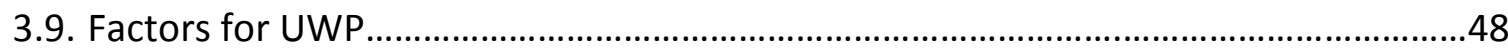

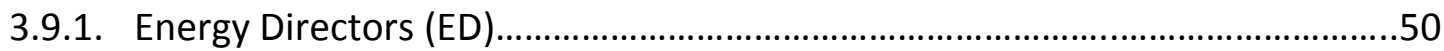

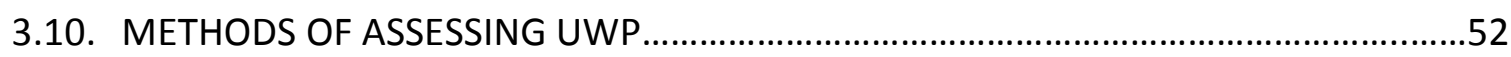

3.10.1. FEA for Ultrasonic Welding Process................................................................52

3.10.2. Role of Differential Scanning Calorimeter (DSC)...........................................62

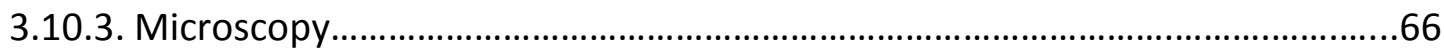

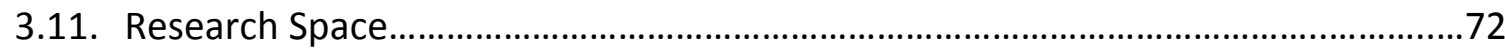

CHAPTER 4 Methodology, Variables and Description of Procedure .......................................75

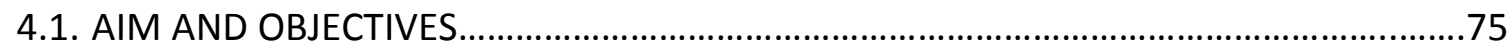

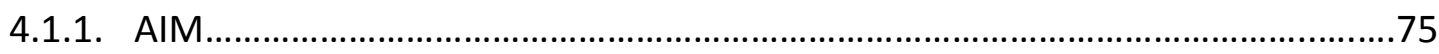

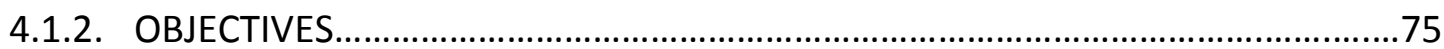

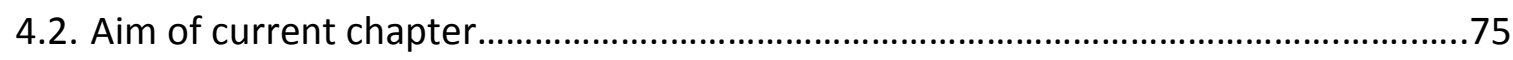

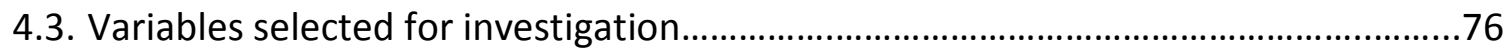

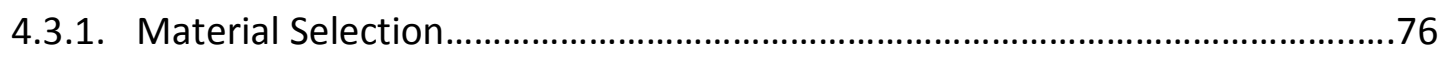

4.3.2. Geometry of Energy Director (ED) ....................................................................

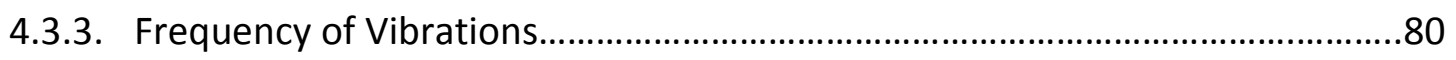

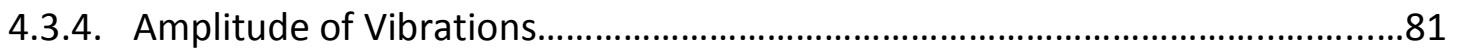

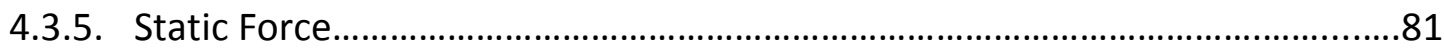

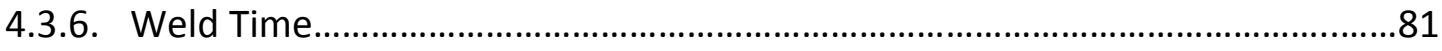

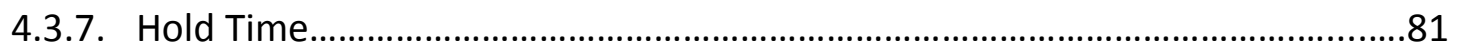




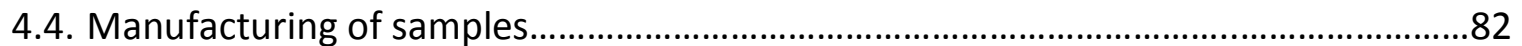

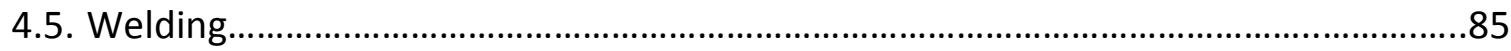

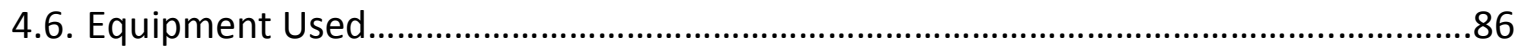

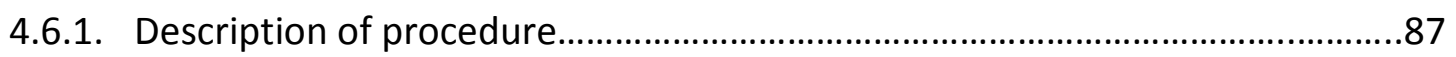

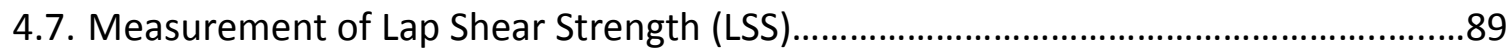

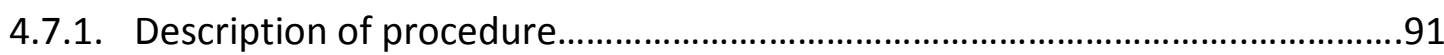

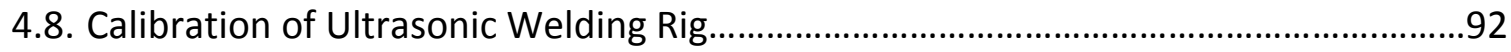

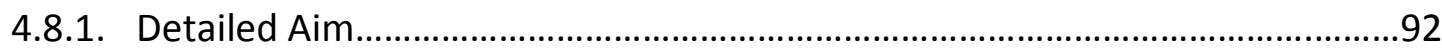

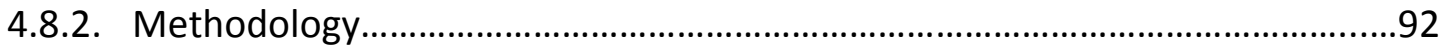

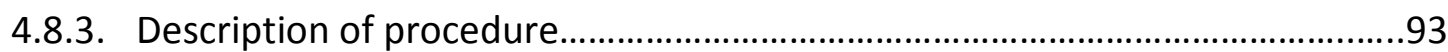

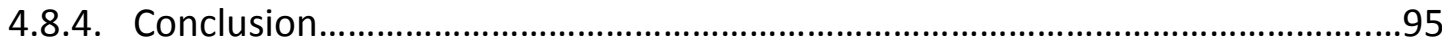

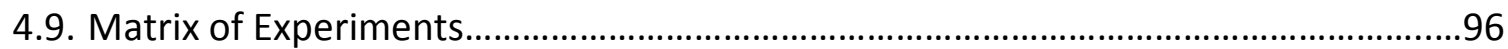

CHAPTER 5 RESULTS

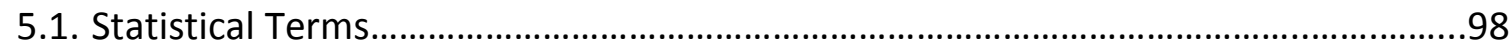

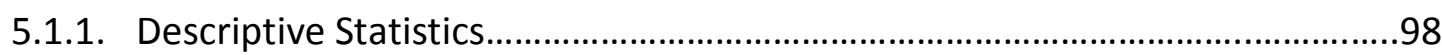

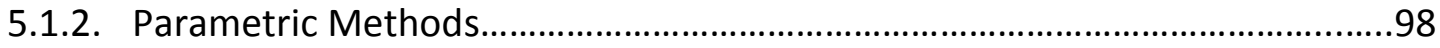

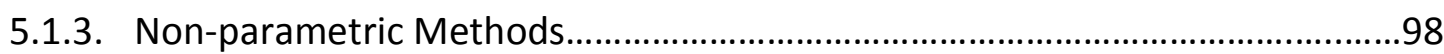

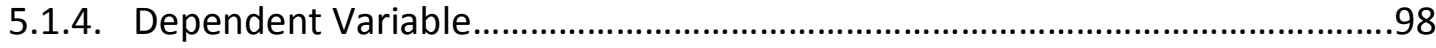

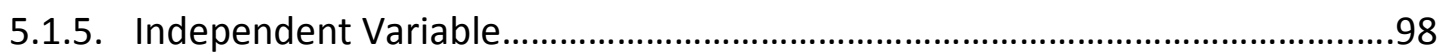

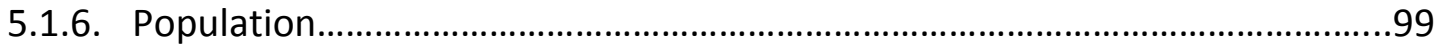

5.1.7. Sample

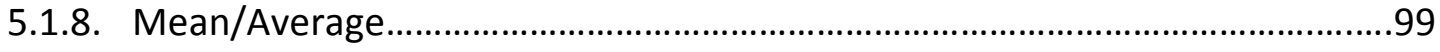

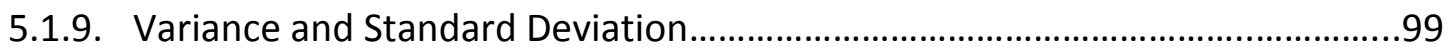




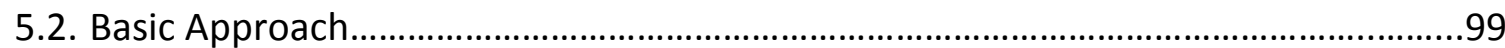

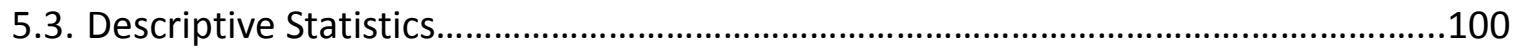

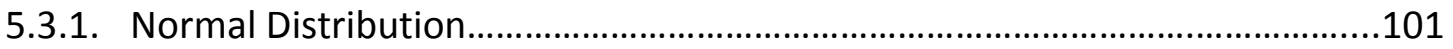

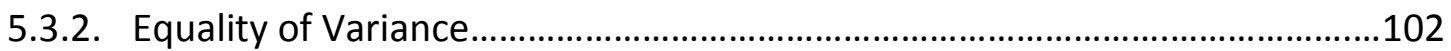

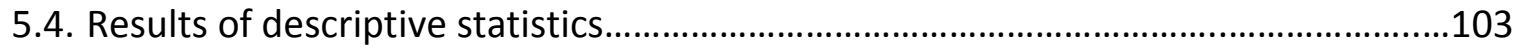

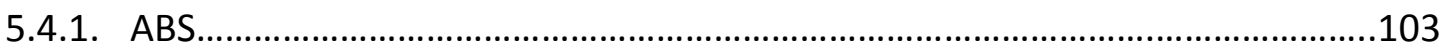

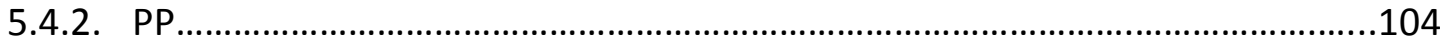

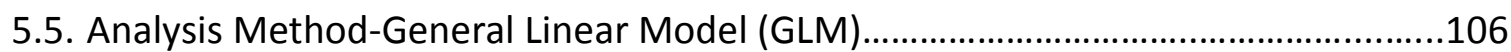

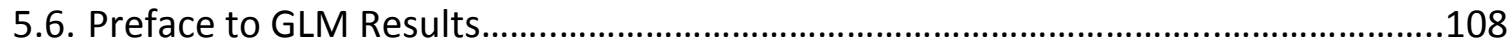

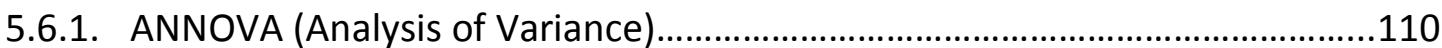

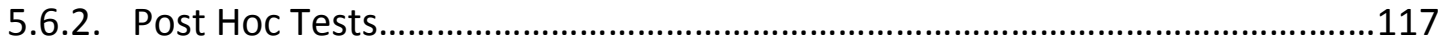

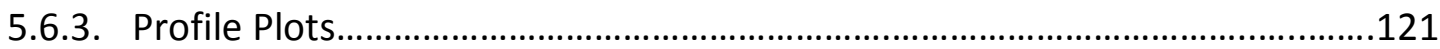

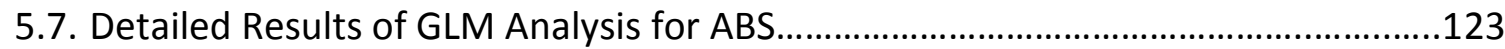

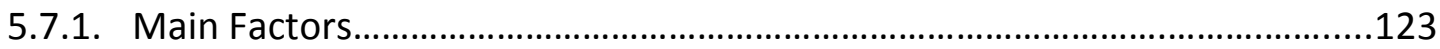

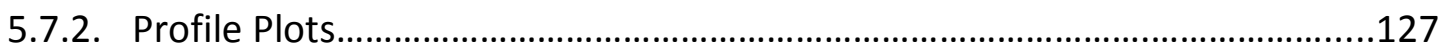

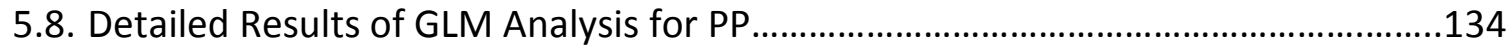

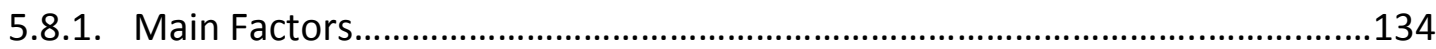

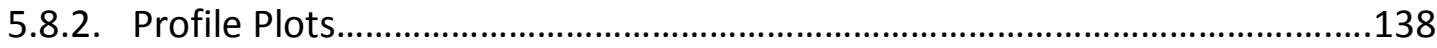

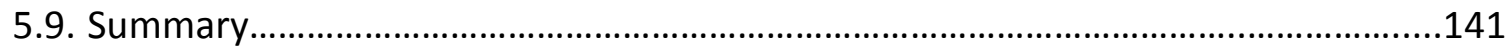

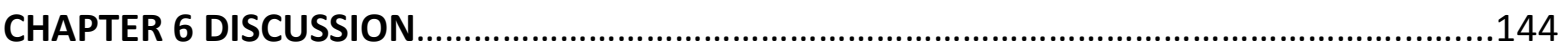

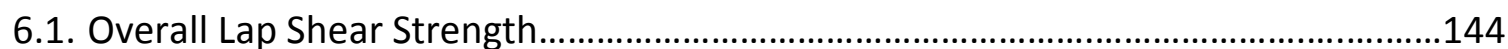

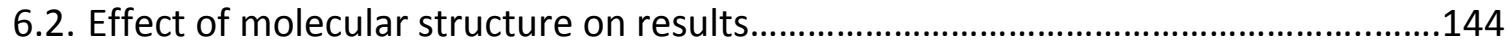

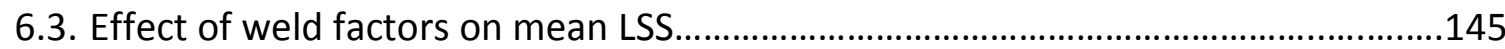




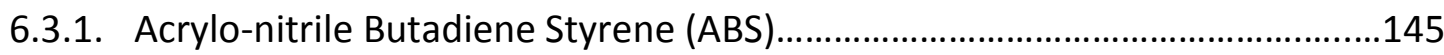

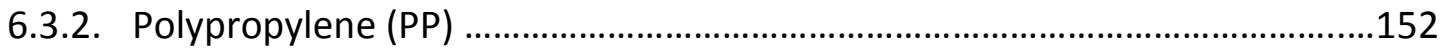

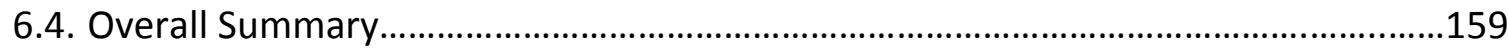

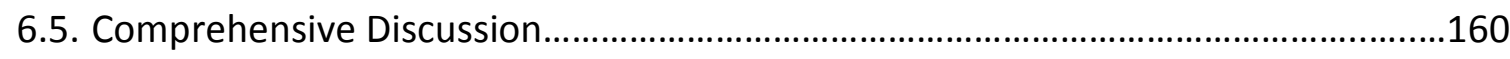

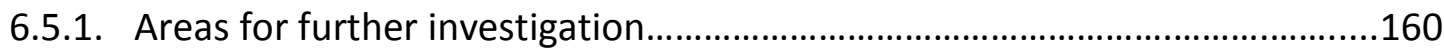

7.1. DSC

7.1.1. AIM

7.1.2. Methodology 167

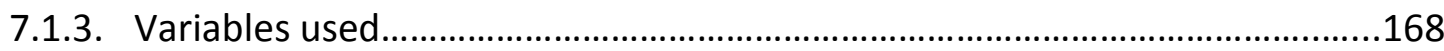

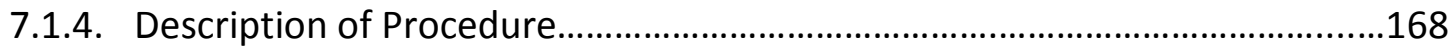

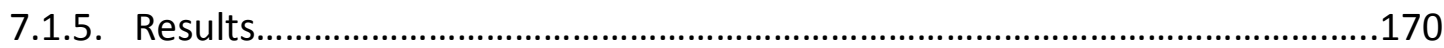

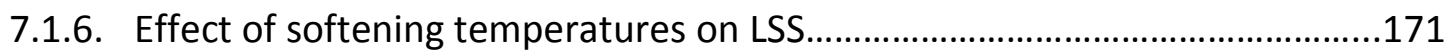

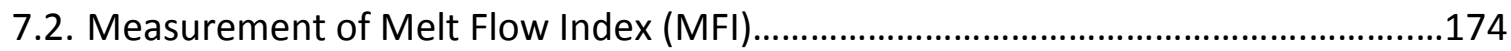

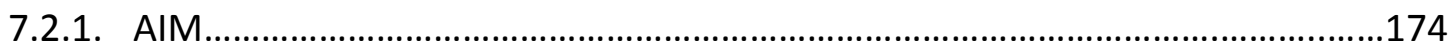

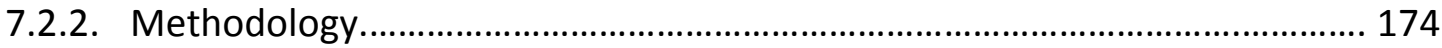

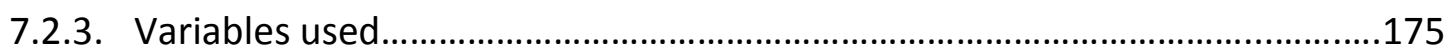

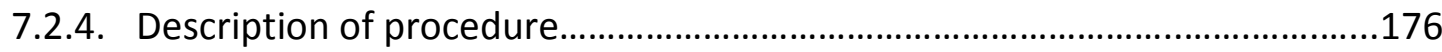

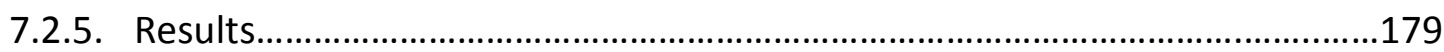

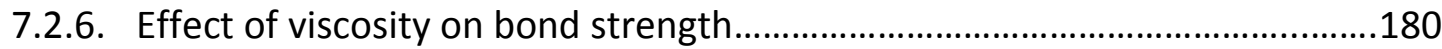

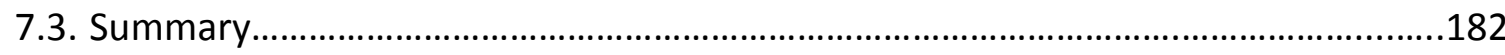

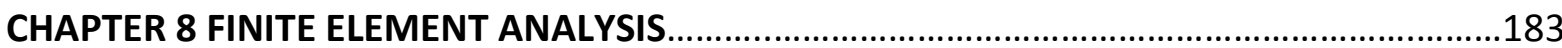


8.1. AIM

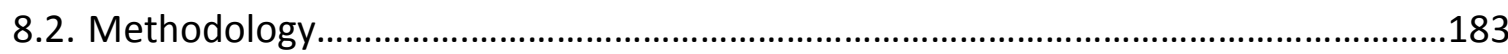

8.3. Variables used

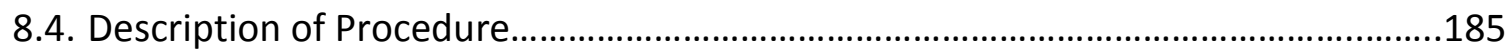

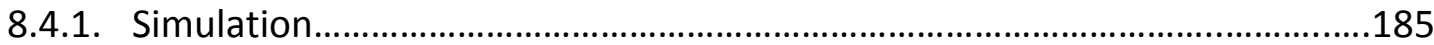

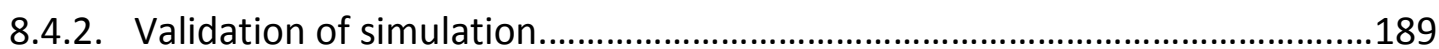

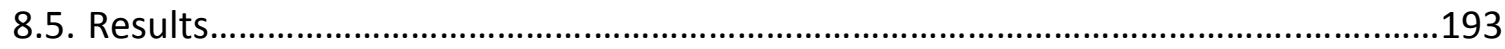

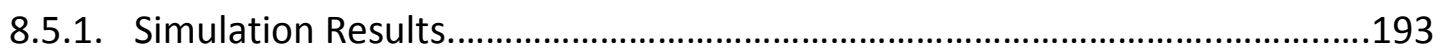

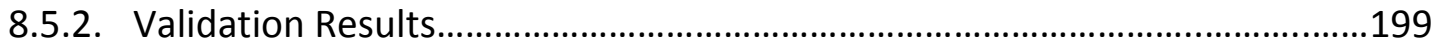

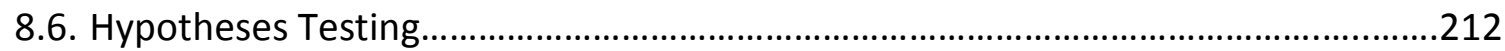

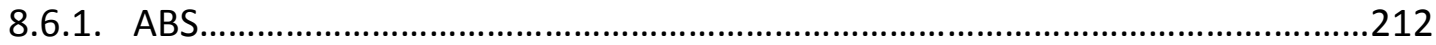

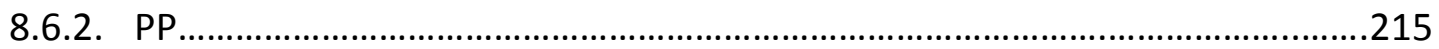

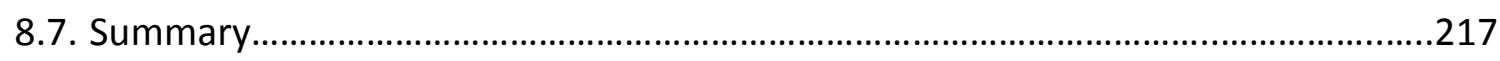

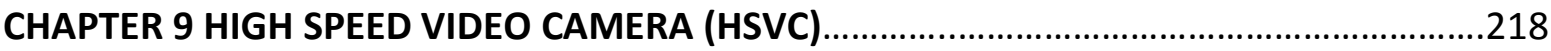

9.1. AIM

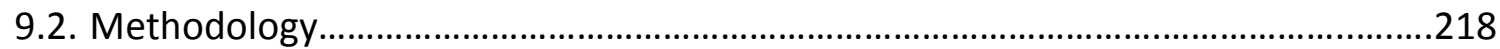

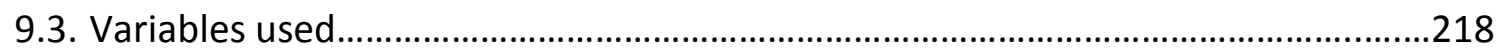

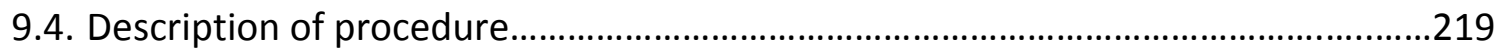

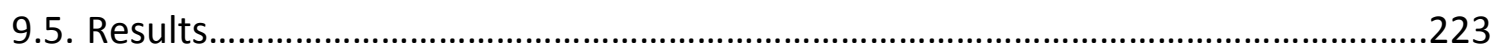

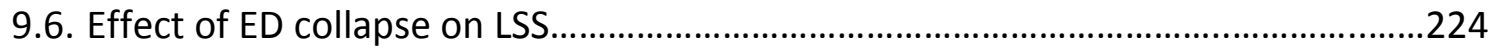

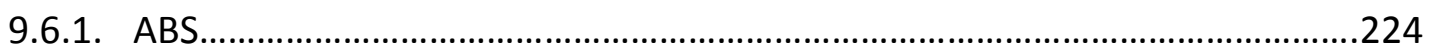

9.6.2. PP

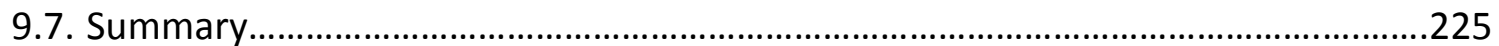




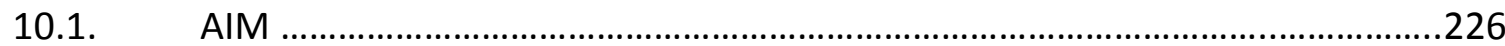

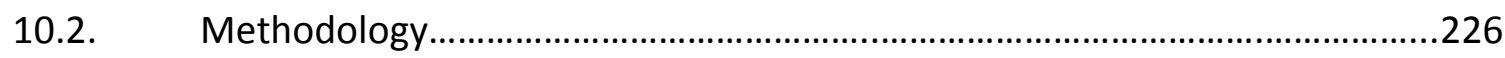

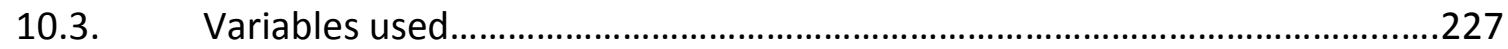

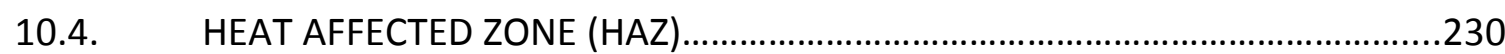

10.4.1. Description of procedure for studying HAZ.................................................230

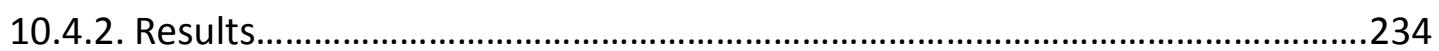

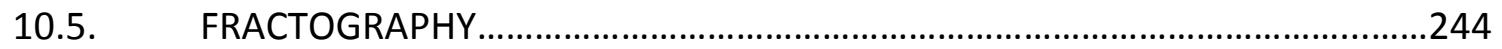

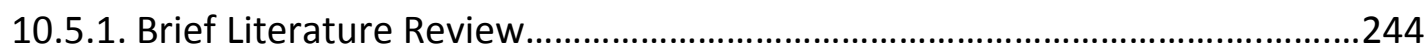

10.5.2. Description of procedure for fractography....................................................245

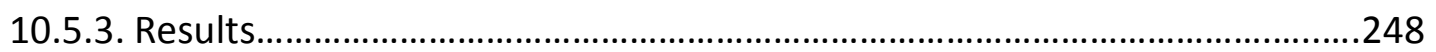

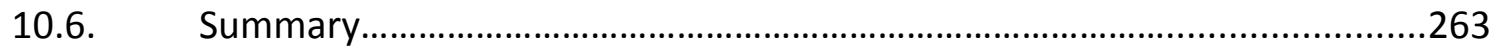

CHAPTER 11 GENERAL SUMMARY OF HYPOTHESES TESTING .......................................264

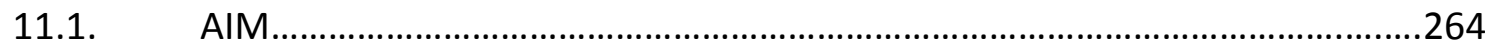

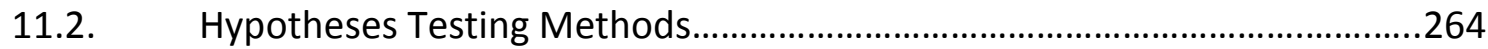

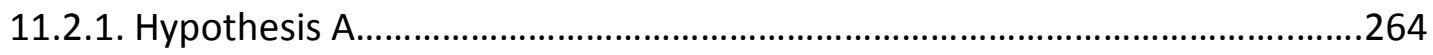

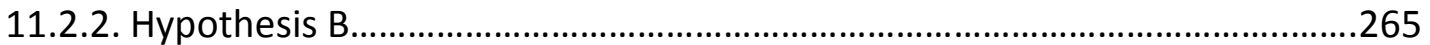

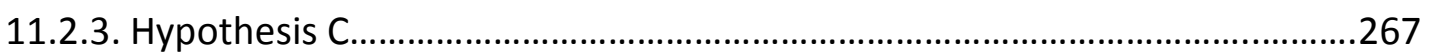

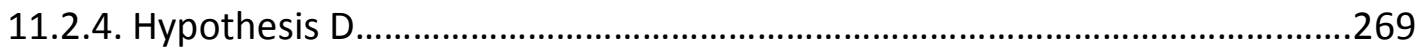

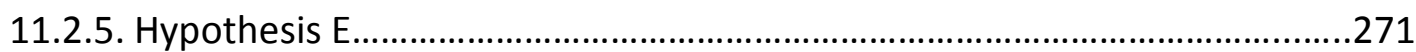

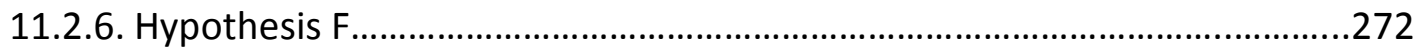

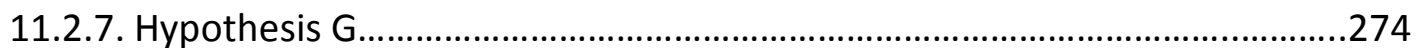


12.1. FUTURE WORK AND RECOMENDATIONS

REFERENCES.

Appendices 292

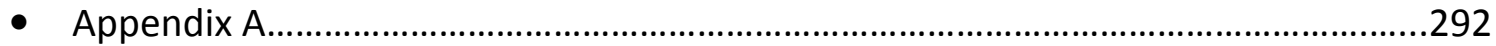

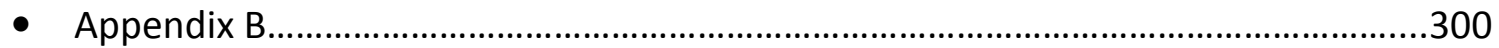

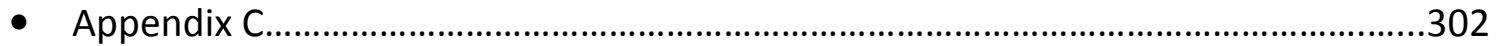

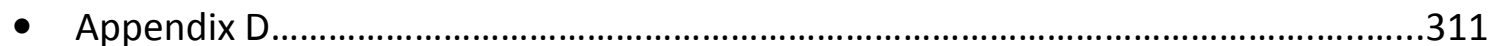

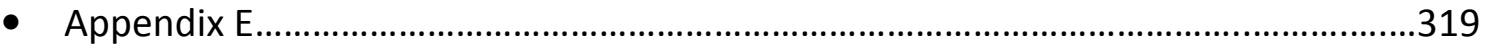




\section{List of Figures}

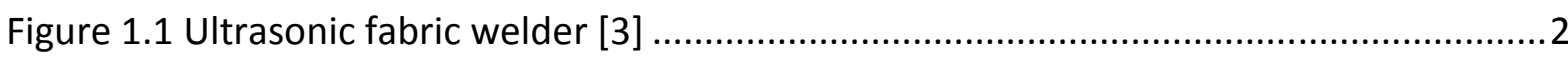

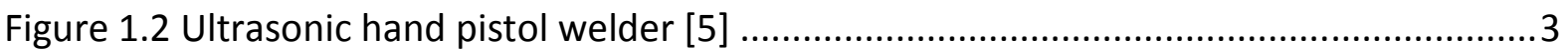

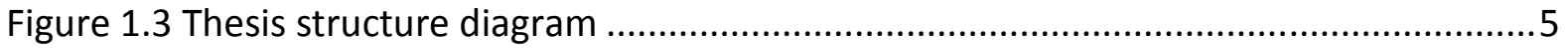

Figure 2.1 Application of plastics in furniture manufacturing [9] .......................................

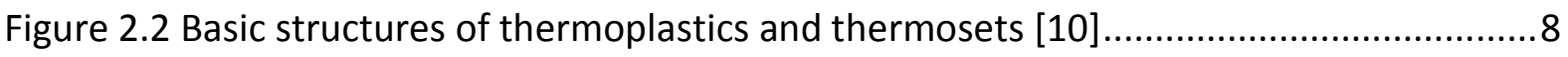

Figure 2.3 Linear, branched and cross-linked plastics [11] ..............................................

Figure 2.4 Basic chain structures of amorphous and semicrystalline thermoplastics [12] .......9

Figure 2.5 Different joining methods for plastics [14] ...................................................11

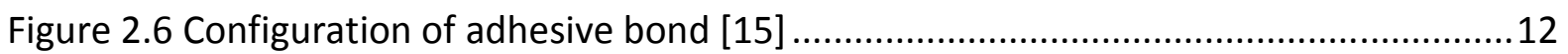

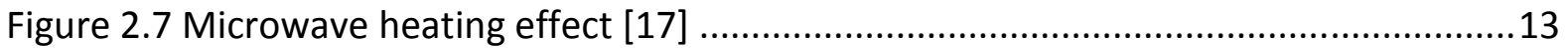

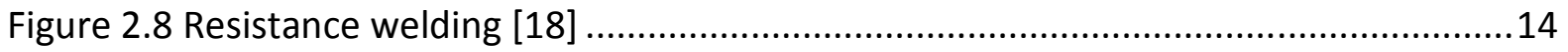

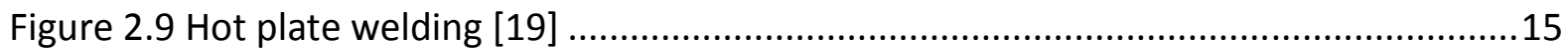

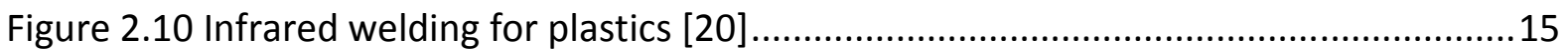

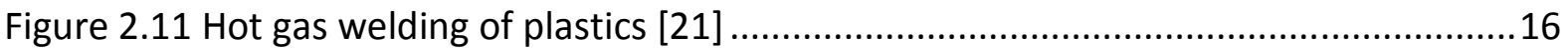

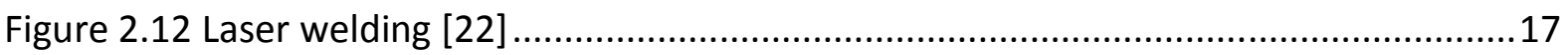

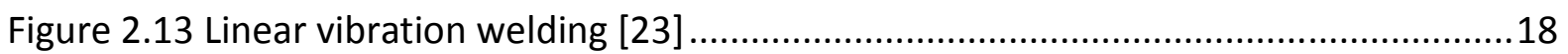

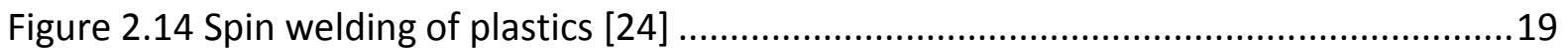

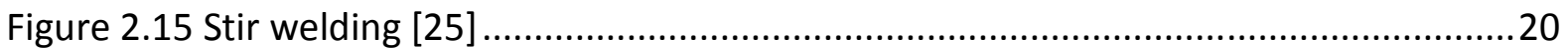

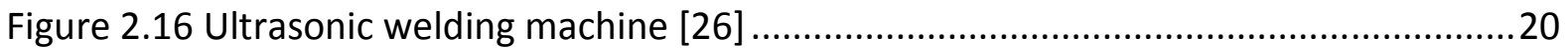

Figure 3.1 Scientific and engineering areas overlapping with ultrasonics [34] $\ldots \ldots \ldots \ldots \ldots \ldots \ldots . . .25$

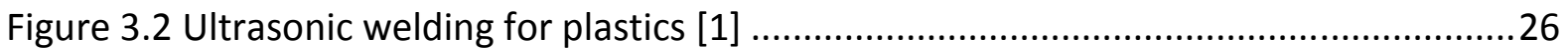


Figure 3.3 Near and far-field ultrasonic welding [36]

Figure $3.4(A \& B)$ TRI and SEMI EDs.

Figure 3.5 Ultrasonic welding equipment [41]

Figure 3.6 Four distinct phases of ultrasonic welding [42]

Figure 3.7 Production line of ultrasonic welding [48] .34

Figure 3.8 Various welded joints [52] 35

Figure 3.9 Vibration welding process [61] .38

Figure 3.10 Polymer viscoelasticity with an elastic spring and a viscous dashpot [67] .40

Figure 3.11 Polypropylene (PP) strapping Tape [77] .42

Figure 3.12 A DMA setup [82]

Figure 3.13 Hot plate welding [89]

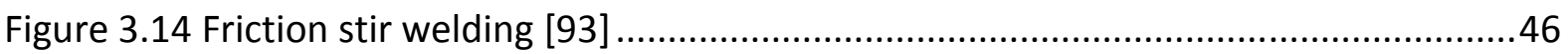

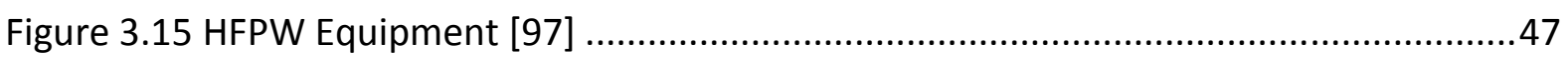

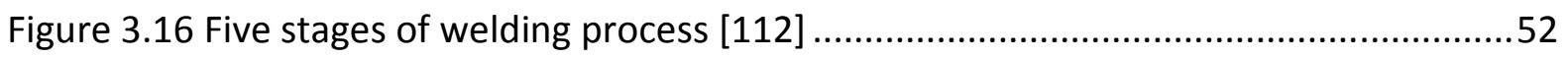

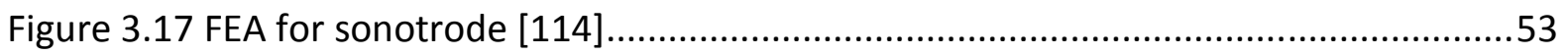

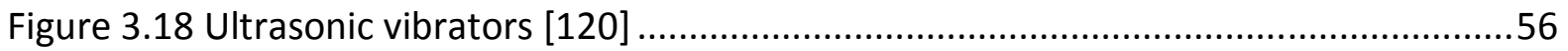

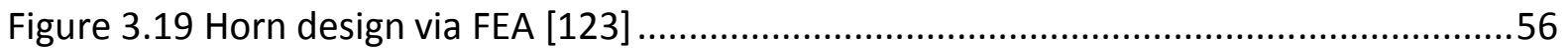

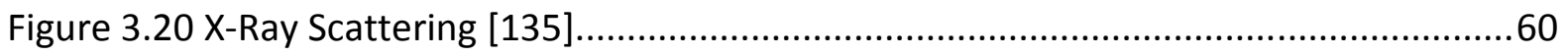

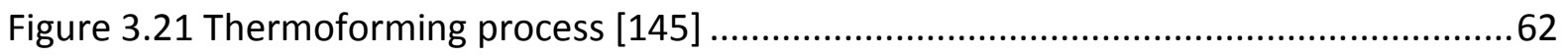

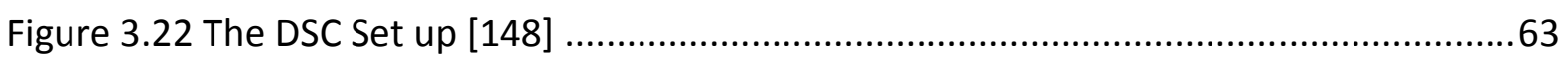

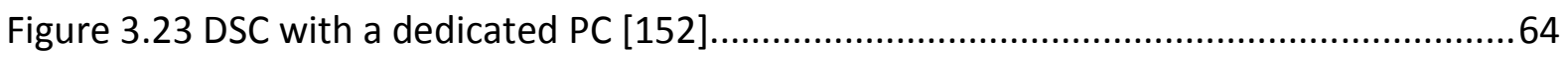

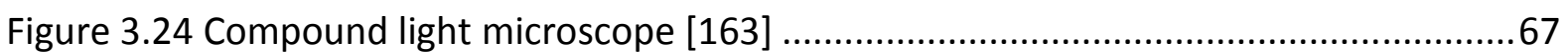


Figure 3.25 Typical engineering microscope [164].

Figure 3.26 A video textile microscope [165]

Figure 4.1 Chemical Formula of Polypropylene [177]

Figure 4.2 Chemical Formula of ABS [178]

Figure 4.3 Geometries of EDs

Figure 4.4 Outer shape and two halves of moulds

Figure 4.5 Cavities with and without a provision of ED......

Figure 4.6 HAAKE* MiniJet Piston Injection Molding System [180]

Figure 4.7 Moulded samples in plastic sealable bags .85

Figure 4.8 Orientation of upper and lower specimens .86

Figure 4.9 Orientation of real samples .86

Figure 4.10 Ultrasonic welding rig with a dedicated computer

Figure 4.11 Real pneumatic circuit .88

Figure 4.12 Compressed and held samples via fixtures, anvil and sonotrode .89

Figure 4.13 Motorized Hounsfield Tensometer.

Figure 4.14 Tensometer applying shear strain at weld interface..... .90

Figure 4.15 Coincidence of line of action of force and centreline of weld zone. .90

Figure 4.16 (A\&B) Fractured sample with welding efficiency 91

Figure 4.17 Calibration of current UW rig from referenced UW rig. .95

Figure 4. 18 Flow chart for weld factors

Figure 5.1 Flow chart for selection of suitable statistical analysis 101

Figure 5.2 Normal distributions [183] .102 
Figure 5.3 An example of spread vs. level plot

Figure 5.4 Normality of data for ABS

.103

Figure 5.5 Spread VS level plot for ABS 104

Figure 5.6 Normality Test. 105

Figure 5.7 Normality Test after square root transformation 105

Figure 5.8 Spread VS level plot for PP 106

Figure 5.9 Profile Plot (ED and Static Force)

Figure 5.10 Effect of static force categories on mean LSS.

Figure 5.11 Effect of amplitude on mean LSS

Figure 5.12 Effect of EDs on mean LSS

Figure 5.13 Effect of weld time categories on mean LSS.

Figure 5.14 Profile Plot (ED and Static Force)

Figure 5.15 Profile Plot (ED and Weld Time)

Figure 5.16 Profile Plot (Amplitude and Weld Time) 130

Figure 5.17 Profile Plot (Amplitude and Static Force)

Figure 5.18 Profile Plot (Amplitude and Static Force) at SEMI ED

Figure 5.19 Profile Plot (Amplitude and Static Force) at TRI ED......

Figure 5.20 Profile Plot (Amplitude and ED).....

Figure 5.21 Profile Plot (Static Force and Weld Time)

Figure 5.22 Effect of static force categories on mean LSS.

Figure 5.23 Effect of amplitude on mean LSS

Figure 5.24 Effect of EDs on Mean LSS 
Figure 5.25 Effect of weld time categories on mean LSS

Figure 5.26 Profile Plot (ED and Weld Time)

Figure 5.27 Profile Plot (Amplitude and Weld Time).....

Figure 5.28 Profile Plot (Amplitude and ED).....

Figure 6.1 Effect of categories of static force

Figure $6.2(A, B, C, \& D)$ The red molten ABS pressed out of the weld zone

Figure 6.3 Effect of categories of static force at SEMI ED

Figure 6.4 Effect of categories of static force at TRI ED

Figure 6.5 Effect of amplitude of ultrasonic vibrations ....... .150

Figure 6.6 Profile Plot (ED and Weld Time)

Figure 6.7 Effect of amplitude of ultrasonic vibrations

Figure 6.8 Voigt model.

Figure 7.1 DSC with a dedicated computer [152]

Figure 7.2 Placing a sample pan in DSC chamber [192]

Figure 7.3 DSC result for ABS

Figure 7.4 DSC result for PP

Figure 7.5 MFI-10 set up

Figure 7.6 Control Console.....

Figure 7.7 Piston and piston support.

Figure 7.8 Simple Diagram showing that how MFI test works[193]

Figure 7.9 Manual Cutting with cut-off knife.

Figure 8.1 Mesh generated after approximating the symmetric geometry. .187 
Figure 8.2 Lab view software screen shot for particular validation case 189

Figure 8.3 Insertion of thermocouple .190

Figure 8.3(A) Thermocouple Position 191

Figure 8.4 Details of USB DAQ .192

Figure 8.5 DAQ in black box as a part of UW rig. .192

Figure 8.6 UWP with USB DAQ

Figure 8.7 Temperature spread Vs Static Force for polypropylene with triangular ED 195

Figure 8.8 Temperature Distribution Vs Static Force for ABS with TRI ED .195

Figure 8.9 Relationship between weld time and Temperature for PP and TRI ED .196

Figure 8.10 Relationship between weld time and Temperature for ABS and TRI ED ...... .196

Figure 8.11 Temperature Distribution for ABS with SEMI ED Vs Static Force

Figure 8.12 Temperature Distribution for PP with SEMI ED Vs Static Force

Figure 8.13 Relationship between weld time and Temperature for ABS and SEMI ED ........198

Figure 8.14 Relationship between weld time and Temperature for PP and SEMI ED..........198

Figure 8.15 Experimental results for 1st attempt. .200

Figure 8.16 Experimental results for 2 nd attempt .201

Figure 8.17 Experimental results for 3rd attempt .202

Figure 8.18 Experimental results for 4th attempt. .203

Figure 8.19 Experimental results for 5th attempt. .204

Figure 8.20 Experimental results for 6 th attempt .205

Figure 8.21 Experimental results for 7 th attempt .206

Figure 8.22 Experimental results for 8th attempt .207 
Figure 8.23 Experimental results for 9th attempt 208

Figure 8.24 Experimental results for 10th attempt .209

Figure 8.25 Experimental results for 11th attempt 210

Figure 8.26 Experimental results for 12 th attempt

Figure 8.27(A\&B) Relationship between simulated temperature and LSS for TRI and SEMI

EDs respectively.

Figure 9.1 Equipment and accessories required for filming

Figure 9.2 Method of calculating ED Collapse

Figure 9.3 Base line of TRI ED with vertices X1 and X2

Figure 9.4 Flow chart for measuring ED Collapse.....

Figure 9.5 Relationship between amplitude and ED Collapse

Figure 9.6 Relationship between ED Collapse and LSS for ABS

Figure 9.7 Relationship between ED Collapse and LSS for PP

Figure 10.1(A) Simple diagram of SEM[197]

Figure 10.1(B) Optical Microscope[198]. .228

Figure 10.2 Variables used for a particular SEM image

Figure 10.3 POLYVAR MET Reichert-Jung optical microscope. 230

Figure 10.4 Cutting procedure of welded joint for ABS .231

Figure 10.4(A) HAZ. .231

Figure 10.4(B) HAZ Thickness.

Figure 10.5 Measurement of HAZ thickness with vernier callipers

Figure 10.6(A, B\&C) Anatomy of Ultrasonic HAZ .234 
Figure 10.7 Relationship between LSS and HAZ thickness for PP (Sr. No. 1 \& 2 of Table 10.2)

Figure 10.8 Relationship between LSS and HAZ thickness for ABS (Sr. No. 3, 4 \& 5)..........236

Figure 10.9 Relationship between LSS and HAZ thickness for ABS (Sr. No. 6 \& 7)..............236

Figure 10.10(A\&B) HAZES viewed from side1 for Serial No.1 …....................................237

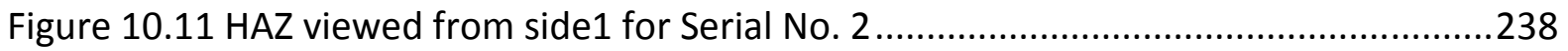

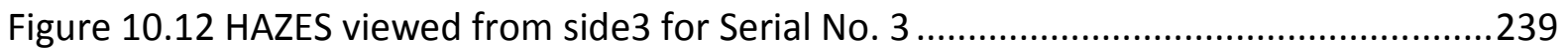

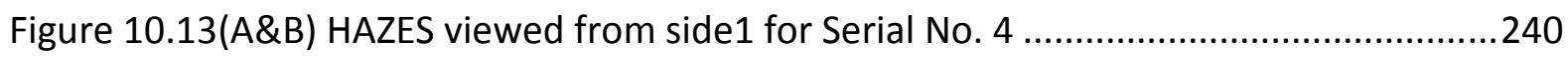

Figure 10.14 HAZ viewed from side4 for Serial No. 5 ...................................................241

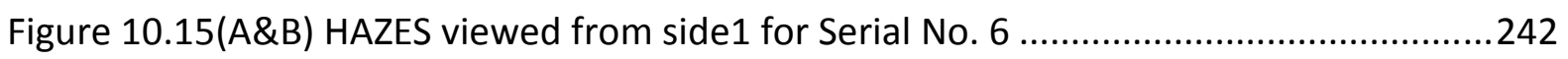

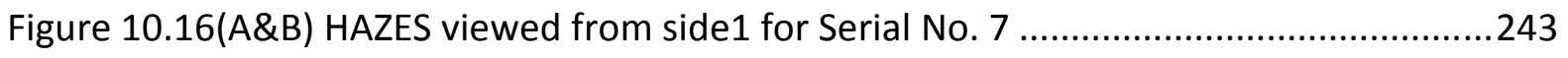

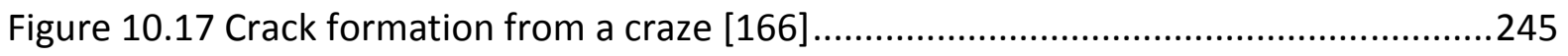

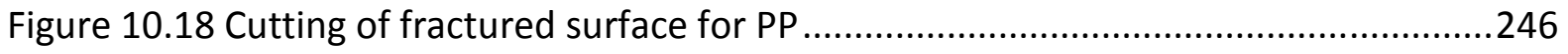

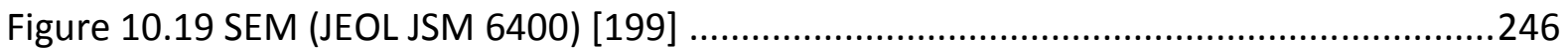

Figure 10.20 Gold Coated and Pasted Samples on SEM Sample Platform ..........................247

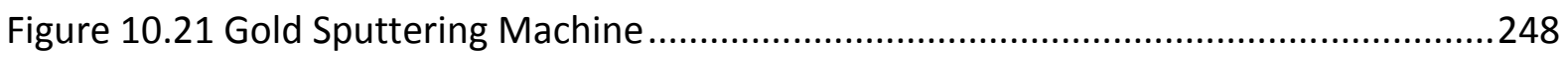

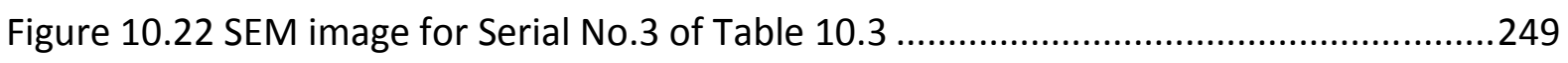

Figure 10.23(A\&B) Microscopic pictures of crazing fibrils for ABS ...................................250

Figure 10.24 Microscopic pictures of crazing fibrils for PP...........................................250

Figure 10.25(A\&B) SEM images showing crazing fibrils for Serial No.3 ..............................251

Figure 10.26 SEM image showing crazing fibrils for Serial No.4.......................................252

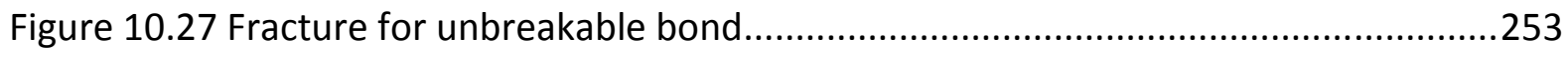


Figure 10.28(A\&B) Two HAZES viewed from side1 for PP ...................................................25

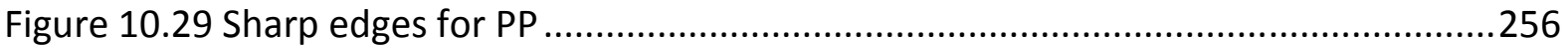

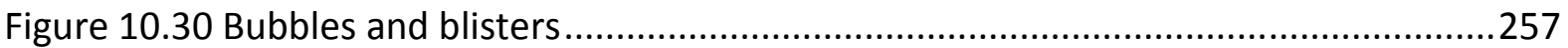

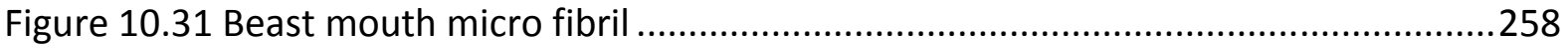

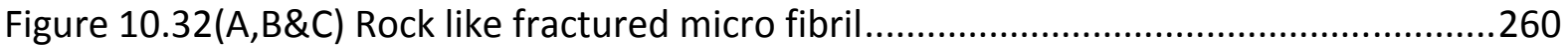

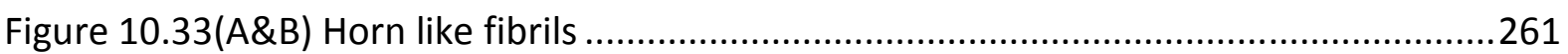

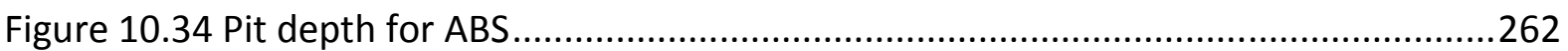

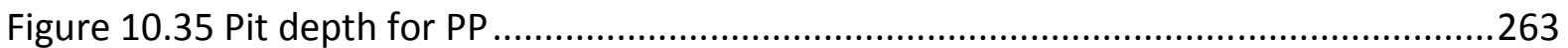




\section{List of Tables}

Table 1.1 Thermoplastic Types

Table 2.1 Difference between semicrystalline and amorphous thermoplastics 10

Table 2.2 Advantages, limitations and applications of plastic welding processes .22

Table 3.1 Research gaps/spaces .73

Table 4.1 Injection moulder's factor settings before preparing samples..... 83

Table 4.2 Room Conditions...... .85

Table 4.3 Near field ultrasonic welding .94

Table 4.4 Extension of table 4.3 .95

Table 4.5 Ultrasonic welding factors

Table 5.1 Average heights of five persons.

Table 5.2 Three 2-levels factors

Table 5.3 Balanced Matrix

Table 5.4 Extension of Table 5.3

Table 5.5 Unbalanced Matrix.

Table 5.6 ANOVA RESULTS.

Table 5.7 Post-Hoc Test

Table 5.8 An extension of Table 5.7.

Table 5.9 Mean LSS in MPa

Table 5.10 Mean LSS in MPa

Table 5.11 Comparison based on Mean LSS.

Table 5.12 Mean LSS in MPa.... 
Table 5.13 Mean Sqrt_LSS

Table 5.14 Mean Sqrt_LSS

Table 5.15 Comparison based on mean Sqrt_LSS

Table 5.16 Mean Sqrt_LSS

Table 5.17 Interaction analysis

Table 6.1 Mean LSS for EDs.

Table 6.2 Mean LSS in MPa

Table 6.3 Mean LSS in MPa

Table 6.4 Mean LSS in MPa

Table 6.5 [189] Complex moduli for both materials.

Table 6.6 Comparison based on mean Sqrt_LSS.

Table 6.7 Mean Sqrt_LSS

Table 6.8 Mean Sqrt_LSS

Table 6.9 Mean Sqrt_LSS

Table 6.10 Factors' level in gaining quality weld for both materials

Table 6.11 Percent change in LSS for both materials

Table 6.12 Heat distortion temperature for both materials

Table 6.13 Hypotheses Development and Testing using Scientific Method

Table 7.1 DSC programme for ABS

Table 7.2 DSC programme for PP

Table 7.3 MFI for ABS 
Table 7.5 Softening Temperatures and MFI for both materials

Table 8.1 Properties for both materials

Table 8.2 Mesh Verification

Table 8.3 First Attempt

Table 8.4 Second Attempt

Table 8.5 Third Attempt

Table 8.6 Fourth Attempt

Table 8.7 Fifth Attempt

Table 8.8 Sixth Attempt .204

Table 8.9 Seventh Attempt 205

Table 8.10 Eighth Attempt.....

Table 8.11 Ninth Attempt

Table 8.12 Tenth Attempt

Table 8.13 Eleventh Attempt

Table 8.14 Twelfth Attempt.

Table 8.15 Results from validation of simulation

Table 9.1 Variables Setting

Table 9.2 Pixels to $\mathrm{mm}$ conversion

Table 9.3 Weld factors which were used for HSVC analysis

Table 10.1 Variables for optical microscope.

Table 10.2 Weld factors and HAZ Thickness

Table 10.3 Weld factors for fractography of welded specimens. 
Abbreviation \& Nomenclature

\begin{tabular}{|c|c|}
\hline ABBREVIATION & NOMENCLATURE \\
\hline UWP & Ultrasonic Welding Process \\
\hline UWE & Ultrasonic Welding Equipment \\
\hline UW & Ultrasonic Welding \\
\hline ABS & Acrylo-Nitrile Butadiene Styrene \\
\hline PP & Polypropylene \\
\hline$P E$ & Polyethylene \\
\hline LSS & Lap Shear Strength \\
\hline LSSs & Lap Shear Strengths \\
\hline Sqrt_LSS & Square root LSS \\
\hline UTS & Ultimate Tensile Strength \\
\hline PEEK & Polyetherether Ketone \\
\hline PEI & Polyether Imide \\
\hline PPS & Polyphenylene Sulfide \\
\hline CF & Carbon Fibres \\
\hline DSC & Differential Scanning Calorimeter \\
\hline $\mathrm{T}_{\mathrm{g}}$ & Glass Transition Temperature \\
\hline$T_{m}$ & Melting Temperature \\
\hline MFI & Melt Flow Index \\
\hline MFR & Melt Flow Rate \\
\hline FEA & Finite Element Analysis \\
\hline HSVC & High Speed Video Camera \\
\hline ED & Energy Director \\
\hline TRI & Triangular \\
\hline SEMI & Semicircular \\
\hline SL Plot & Spread VS Level Plot \\
\hline$E^{\prime \prime}$ & Loss Modulus \\
\hline$E^{\prime}$ & Storage Modulus \\
\hline HDT & Heat Distortion Temperature \\
\hline${ }^{\circ} \mathrm{C}$ & Degree Centigrade, Unit of Temperature \\
\hline $\mathrm{Hz}$ & Hertz, SI unit of frequency \\
\hline $\mathrm{Sec}$ & Second, SI unit of time \\
\hline
\end{tabular}




\begin{tabular}{|c|c|}
\hline ABBREVIATION & NOMENCLATURE \\
\hline $\mathrm{m}$ & Meter, SI unit of length \\
\hline $\mathrm{N}$ & Newton, SI unit of force \\
\hline $\mathrm{Pa}$ & Pascal, SI unit of pressure \\
\hline PZT & Piezoelectric ceramic longitudinal Transducer \\
\hline MW & Molecular Weight \\
\hline CPS & Cycles Per Second \\
\hline 2D & Two Dimensional \\
\hline $3 \mathrm{D}$ & Three Dimensional \\
\hline ANSYS & FEA Software Package \\
\hline OM & Optical Microscope \\
\hline SEM & Scanning Electron Microscope \\
\hline ISO & International Organization for Standardization \\
\hline BS & British Standards \\
\hline ASME & American Society of Mechanical Engineers \\
\hline ASTM & American Society for Testing and Materials \\
\hline Bar & A unit of pressure \\
\hline$\mu$ & Micro, $10^{-6}$ \\
\hline PLC & Programmable Logic Controller \\
\hline PC & Personal Computer \\
\hline GLM & General Linear Model \\
\hline ANOVA & Analysis of variance \\
\hline HSD & Honestly Significant Difference \\
\hline df & Degree of freedom \\
\hline $\mathrm{N}$ & Number of samples \\
\hline$>>$ & Greater than \\
\hline$\geq$ & Greater or equal \\
\hline g & Gram, SI Unit of weight \\
\hline $\mathrm{Kg}$ & Kilogram, SI unit of Weight \\
\hline$\omega$ & Angular velocity \\
\hline$\epsilon_{\mathrm{A}}$ & Strain amplitude \\
\hline Qavg & Viscoelastic heating \\
\hline DAQ & Data acquisition \\
\hline
\end{tabular}




\begin{tabular}{|c|c|}
\hline ABBREVIATION & NOMENCLATURE \\
\hline \hline HAZ & Heat Affected Zone \\
\hline $\mathrm{J}$ & Joule, derived SI Unit of energy \\
\hline $\mathrm{SI}$ & Severity Index \\
\hline $\mathrm{CLC}$ & Condenser Lens Current \\
\hline $\mathrm{Cl}$ & Confidence Interval \\
\hline
\end{tabular}




\section{CHAPTER 1 INTRODUCTION}

Ultrasonic welding has first been used in 1963 after being employed in electronic industry in 1960's for joining wires [1]. Ultrasonic plastic welding consists of imposing ultrasonic vibrations to thermoplastics in the presence of a vertical force/pressure simultaneously for certain time period thereby resulting in a weld. The whole process takes either less than a second or few to finish depending upon material configurations.

Ultrasonic welding has now been recognized as an excellent industrial joining technique. Ultrasonic welding process is equally used for metals as well, with an only difference of solid-state of metals during welding. On contrary, phase change occurs from glassy to rubbery while welding thermoplastics ultrasonically.

Non-ferrous metals and their alloys are easily welded ultrasonically e.g. Aluminium, brass, copper, and nickel alloys etc. Moreover, dissimilar material has easily been welded in industry [2].

From industrial standpoint, joint design is an important aspect of ultrasonic welding. Energy directors (EDs) are usually utilized for welding thermoplastics. These EDs really help in concentrating the ultrasonic energy at localized areas. Furthermore, these EDs are those places where the heat affected zone is developed. Shear joints are proposed for leak-tight joints [2].

Thermoplastics are classified into two major groups;

1. Crystalline or Semicrystalline

2. Amorphous

Industrially acceptable thermoplastics for welding ultrasonically is presented in Table 1.1 [2]: 
Table 1.1 Thermoplastic Types

\begin{tabular}{|c|c|}
\hline \multicolumn{2}{|c|}{ THERMOPLASTICS } \\
\hline \hline AMORPHOUS & SEMICRYSTALLINE \\
\hline Polycarbonate & Polyethylene \\
\hline Polyphenylene Oxide & Polypropylene \\
\hline ABS & Thermoplastic Polyester \\
\hline Polystyrene & Polyphenylene Sulphide \\
\hline Polyetherimide & Acetal \\
\hline Acrylic & Poly-ether-ether-ketone \\
\hline Polysulfone & \\
\hline
\end{tabular}

Despite its application to metals and plastics, ultrasonic welding is found to be harmoniously successful in textile industry. At this point the ultrasonic welding appears to have continuous mode of joining instead of spot welding. Continuous ultrasonic welding needs a roller and horn. Fabric is passed between the roller and horn thereby resulting in perfect continuous seal [2]. Ultrasonic fabric welder is exhibited in Figure 1.1.

Figure 1.1 Ultrasonic fabric welder [3] 
Ultrasonic welders are easily converted into hand welders for special applications e.g. fabric repairs and joining, conveyor belt repair, intricate areas which has difficulty in assessing them, reworking operation, and automotive industry. Ultrasonic hand pistol welder is well suited to those premises where automation is having little importance and production rate is quite small [4]. Ultrasonic hand pistol welder is illustrated in Figure 1.2.

Figure 1.2 Ultrasonic hand pistol welder [5]

Ultrasonic welding process is used flexibly to various applications e.g. apparel, appliance, automotive, electronics, filtration, home furnishings, medical, and packaging [2].

Hence ultrasonic welding has an excellent standing in industry. Although ultrasonic welding has already been accepted as a promising process in industry, it is, still, needed to fully understand this process in terms of weld mechanics, weld morphology, heating mechanism, suitable materials etc.

In this work, a comprehensive study on ultrasonic welding process was endeavoured in order to develop a vast understanding of this welding process.

\subsection{Thesis Layout}

This thesis is structured as follows:

Chapter 2 deals with introduction to polymers and various plastic welding processes including ultrasonic welding of thermoplastics. Thesis structure diagram is shown in Figure 1.3. 
Chapter 3 presents extensive literature review on plastic welding and ultrasonic welding processes. Main topics of literature review include UWP, materials for UWP, viscoelastic behaviour of polymers, factors for UWP, joining effects of thermoplastics, EDs, and methods in assessing UWP. Methods in assessing UWP further consist of FEA, DSC and microscopy.

Chapter 4 describes the methodology to perform ultrasonic welding. It starts with the weld factors selection, material selection, planning for experimentation, manufacturing of moulds \& samples, equipment used and LSS measurement of welded samples.

Chapter 5 presents the GLM statistical analysis results of ABS and PP.. This statistical analysis comprises descriptive statistics to obtain a general idea about data for both ABS and PP, transformation of data for PP in order to meet the condition of normality and GLM (General Linear Model) as an analysis method for both materials.

Chapter 6 discusses the GLM results in detail. Effects of weld factors and their interactions on LSS are determined and quantified for both materials. And reasons for these effects are determined. Most significant levels of factors are tabulated then for their maximum contribution in acquiring higher LSS for both materials. Interesting effects are also deduced. Hypotheses based on literature are made by considering these effects.

Chapter 7 addresses the first experimental technique (DSC) to test some hypotheses. DSC is used to find the softening temperature for both materials. It has been found that softening temperature of ABS is lower than that of PP. This chapter also includes the MFI tests in an effort to understand the fluidity of materials under investigation. Results from MFI tests are also used to test hypotheses.

Chapter 8 defines another experimental tool (HSVC) in testing a hypothesis again. HSVC is utilized to analyse the ED collapse and its effect on LSS for both materials.

Chapter 9 presents the temperature spread at weld interface by using FEA. Simulated results are used to test again certain hypotheses. Numerical results are also validated with the experimental results and a good match has been found.

Chapter 10 addresses the HAZ and fractography by using microscopic tools. Causes of ductile and brittle fractures are explained with help of fractography. Various fractured 
surface features have also been found after testing LSS. Moreover, microscopic results are also employed to test some hypotheses in this chapter.

Chapter 11 is summarising the hypotheses testing which leads to their approval or disapproval. Finally chapter 12 concludes this present work and recommends the future work.

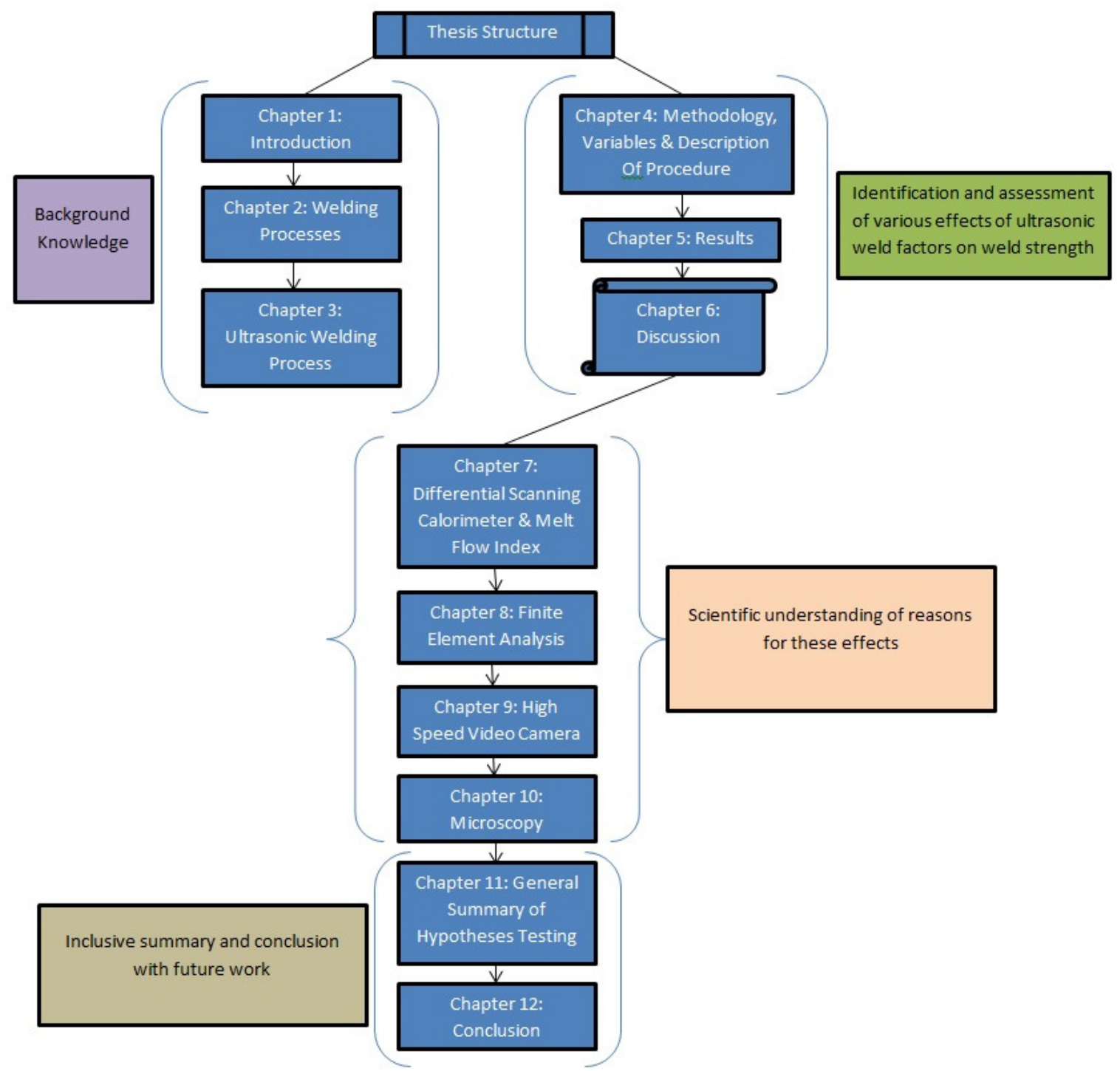

Figure 1.3 Thesis structure diagram 


\section{CHAPTER 2 WELDING PROCESSES}

\subsection{AIM}

Having presented a brief introduction on ultrasonic welding process (UWP), this chapter is intended to present basic definitions of various technical terms and possible plastic welding processes. Advantages, limitations and application of these welding processes have also been provided with a Table 2.2 .

\subsection{Polymers}

Polymer and plastics are synonymously used for one another. Polymer is a Greek word consisting of 'poly' meaning 'many' and 'meros' meaning 'part'. Hence a polymer is a long chain molecule based on small interconnected monomer unit. Many polymers are organic in chemistry and consist of covalent compounds of carbon. Hydrogen, oxygen, fluorine, chlorine, sulphur, etc. are those elements in polymers forming covalent bond with carbon atom. In addition to primary covalent bonding, a polymer molecule has also secondary intermolecular forces comprising dipole forces and hydrogen bonding [6].

The discovery of plastics is among the most amazing accidents in history of science and technology. In 1931, Fawcett and Gibson at Imperial Chemicals Industries (ICI) Australia discovered polyethylene after having observed a waxy solid produced in small amount during their high-pressure gas experiments which used ethylene. It was made properly later as polyethylene and was invented as one of the great commodity polymers. In the same fashion, PTFE (Polytetrafluoroethylene) was discovered by Roy Plunkett at DuPont during an activity that found white residue in the cut cylinder under observation [7].

Since then, various polymers have been made based on the possible combinations of atoms and the way of arranging them in a particular order. This has led to build up many innovative schemes of making polymers [7].

Until now, polymers have captured a huge amount of market share as compared to metals. This is owing to the reason that polymers have high strength to weight ratio, excellent 
toughness, corrosion and abrasion resistance, lower friction and outstanding electrical insulation. These attractive features of polymers are advantageous over other materials [8].

The most important area of application of plastics is in automobile and aerospace industries. Plastics are also being used in medical, agricultural, pharmacy, furniture, electrical appliances, food packaging, printed circuit boards, insulation, golf-ball covers and artificial joints [6]. Application of plastics in furniture manufacturing is illustrated in Figure 2.1.

Figure 2.1 Application of plastics in furniture manufacturing [9] Polymers can broadly be categorized into two major groups by considering their exposure to thermal phenomena [6]:

1. Thermoplastics

2. Thermosets

Thermoplastics are large group of polymers. Thermoplastics are easily heated repeatedly and reversibly resulting in softening and hardening on cooling properly [6]. All 
thermoplastics cannot be listed here owing to their large number except some of them e.g. polypropylene, nylon, polyethylene, polystyrene, polycarbonate etc.

Thermosets are that group of polymers needing to be cured to get the intended function. They do not allow any further change after getting cured once. They have the cross-linked structure so heating and shaping cannot be done repeatedly. If thermosets are heated excessively then they will get decomposed [6]. Basic structures for thermoplastics and thermosets are exhibited in Figure 2.2.

Figure 2.2 Basic structures of thermoplastics and thermosets [10]

Thermoplastics have also been classified into two further types i.e. Linear and Branched. On the other hand, when thermo-sets are heated, a chemical reaction takes place preventing further chemical change in them [6]. Linear, branched and cross-linked plastics respectively are shown in Figure 2.3. 
Figure 2.3 Linear, branched and cross-linked plastics [11]

Thermoplastics can further be divided into two major groups based on molecular structures.

1. Semi-crystalline

2. Amorphous

In case of semi-crystalline thermoplastics, molecules arrange themselves in proper order of crystals. On the other hand, amorphous thermoplastics have no proper order for arranging their molecules or in other words, they have random orientation of their molecules [6]. Difference in these two thermoplastics is shown in Table 2.1. Moreover, basic chain structures for these two types of thermoplastics are illustrated in Figure 2.4.

Figure 2.4 Basic chain structures of amorphous and semicrystalline thermoplastics [12]

In fact, the list of thermoplastics is far from complete, but the aim is to present some examples of thermoplastics. Polypropylene, polyethylene, and nylon are some examples of 
semi-crystalline thermoplastics. And in the same fashion polycarbonate, polystyrene, and ABS are few examples of amorphous thermoplastics.

Thermally, a thermoplastic undergoes majorly two transitions i.e. melting $\left(T_{m}\right)$ and glass transition $\left(T_{g}\right)$. Melting transition is also called as the first order transition, whereas latter is known as the second order transition. Change in polymer properties can easily be observed at $\mathrm{T}_{\mathrm{g}}$ for amorphous and amorphous portions of semi-crystalline thermoplastics including coefficient of thermal expansion, refractive index, electrical properties, heat capacity and mechanical damping. A significant drop in Young's modulus is also observed around the glass transition temperature [6].

Table 2.1 Difference between semicrystalline and amorphous thermoplastics

\begin{tabular}{|c|c|}
\hline \multicolumn{2}{|c|}{ THERMOPLASTICS [13] } \\
\hline \hline SEMICRYSTALLINE & AMORPHOUS \\
\hline Distinctive Properties & Distinctive Properties \\
\hline \hline Sharp Melting Point i.e. $\mathrm{T}_{\mathrm{m}}$ & No Melting Point i.e. $\mathrm{T}_{\mathrm{g}}$ \\
\hline Orderly Molecular Structure & Random Molecular Structure \\
\hline Excellent Chemical Resistance & Tough, Rigid, Good Creep and Chemical \\
\hline Modulus remained until temperature \\
reached the melting point & Modulus reduced gradually near $\mathrm{T}_{\mathrm{g}}$ \\
\hline It gave opaque look & It gave transparent look \\
\hline Resilient & - \\
\hline
\end{tabular}

\subsection{Welding Processes for plastics}

Welding plastics has emerged as an active area of research from increasing development of various kinds of plastics. As lot of research has been done on plastics for their efficient usage in various applications, in the same way, an optimum joining technique is needed to assemble plastics. Various joining techniques for plastics are shown in Figure 2.5. 


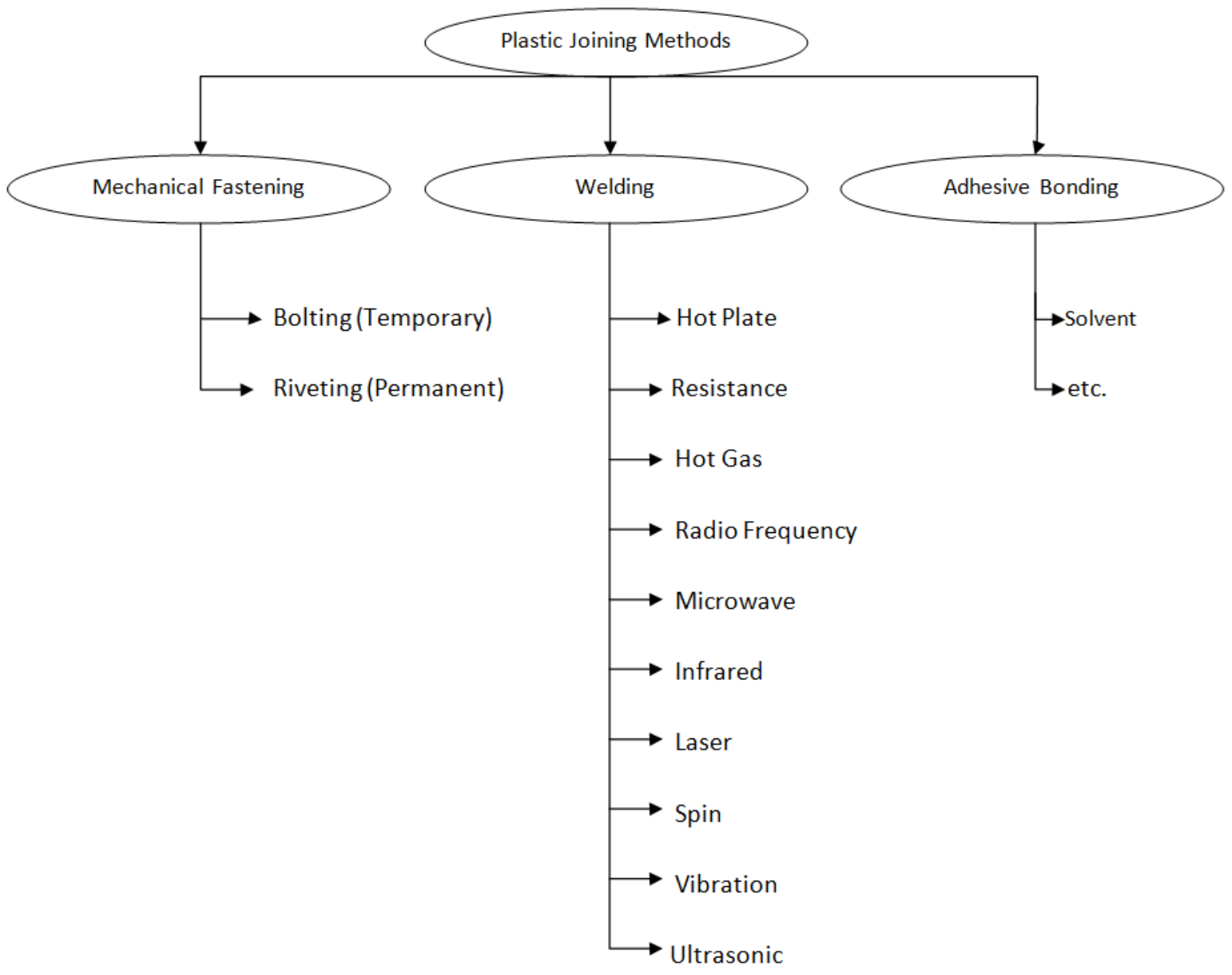

Figure 2.5 Different joining methods for plastics [14]

As a matter of fact, a lot of options are available for joining plastic materials but each option has its own advantages and limitations.

For instance, adhesive bonding is found difficult when it comes to handle and introduce a foreign material in parent materials joining. Configuration of an adhesive bond is exhibited in Figure 2.6. Mechanical fastening has problems regarding increased stress concentration, increased weight of the final assembly, and variation in coefficient of thermal expansion. Above all, these fasteners (temporary or permanent) need holes in parent materials to join them [14]. 
Figure 2.6 Configuration of adhesive bond [15]

Plastic welding replaces above difficulties in a good manner by considering various crucial aspects e.g. time and money etc. These difficulties are [16]:

1. As compared to welding processes, the final strength of adhesive bond cannot be achieved immediately. It takes some time depending further on the type of adhesive. In an effort to reduce this time of proper adhesive joining, chemicals are used to increase the curing time of adhesive bonding.

2. Adhesive bonding is susceptible to various environmental conditions while observing a long curing time. It includes the interaction with moisture, chemicals and ultraviolet light.

3. It involves a time consuming activity of surface preparation that depends on the materials to be bonded and type of adhesives.

4. Adhesives are known as chemical compounds and these need proper safety of operator while bonding. Also wastage is problematic after adhesive bonding due to the requirement for its proper disposal and recycling.

5. In order to achieve a good adhesive bond, it always requires a competent operator for avoiding wrong consumptions of chemicals.

Before discussing ultrasonic welding for thermoplastics, it is imperative to discuss some of welding techniques which are mentioned in Figure 2.5.

Different ways of fusing plastics lay the foundations for further classification of welding processes including [14]:

1. Electromagnetic Welding

2. Thermal Welding 


\section{Friction Welding}

\subsubsection{Electromagnetic Welding}

\section{Microwave Welding}

Microwave welding is deemed be a quick and neat process. All it needs an electromagnetic absorbent material that is placed between the materials to be joined and an application of suitable pressure along with microwave energy in $\mathrm{GHz}$. As a result of this procedure, a temperature develops due to electromagnetic absorbent material at the interface of materials melting the two materials, finally developing the bond or weld. Microwave heat effect is shown in Figure 2.7.

Electromagnetic absorbent materials usually consist of materials and solvents with $\mathrm{OH}, \mathrm{CO}$, $\mathrm{NO}$ and $\mathrm{NH}$. While welding, some of these materials leave interface via evaporation and some other remain in the weld zone [14].

Figure 2.7 Microwave heating effect [17]

\section{Resistance Welding}

Resistance welding is also known as implant resistance welding. Implant is a term used for an electrically resistive material. Resistance welding involves efficient utilization of implant in the form of copper strip or metal mesh between the materials to be joined. Resistance heating is produced due to the application of electric current to the implant. After having developed a suitable temperature for melting plastic, a bond is produced. A typical resistance welding set up is shown in Figure 2.8 .

Several thermoplastics are welded by using this method. Materials to be welded may have larger sizes. In order to control the quality of weld, a proper control on producing resistance 
is required between the two materials by adjusting the input power level during the welding process [14].

Figure 2.8 Resistance welding [18]

\subsubsection{Thermal Welding}

Thermal welding has two prominent stages to consolidate the materials which are: external heating and application of pressure i.e. forging. Let us discuss some of thermal welding processes:

\section{Hot Plate Welding}

During hot plate welding, a hot plate (heated externally) is placed between the materials to be joined for melting the interfaces of two materials. Hot plate is removed after having melted plastic materials. Plastic materials are kept together under the application of pressure until the plastic material again hardens on cooling. Hot plate welding equipment is shown in Figure 2.9.

Thermal conductivity and geometry of the parts decide the time for welding. This welding process is used to weld two different thermoplastics. Exposure of hot plate to materials produces sticking which is a problem in this type of welding. Non sticking materials can be used for eliminating sticking problem but even these materials will not resolve the said issue in extremely high temperature conditions.

Hot plate welding has its special application in automotive industry and it is extensively used in infrastructural applications. Hot plate welding is a slow process, albeit simplicity of equipment is one of its advantages [14]. 
Figure 2.9 Hot plate welding [19]

\section{Infrared welding}

Infrared welding is a thermal welding in which heating is provided to the interfaces of parts to be welded by infrared radiations which come out of infrared heaters. This is a noncontact type of welding in contrast to hot plate welding which is a contact type of thermal welding. Method of giving heat to plastics is also different for both hot plate and infrared welding i.e. former applies heat due to conduction, whereas later transfers heat through radiations. Once the plastic melts or more precisely when temperature of plastic reaches to its optimum processing temperature, the infrared lamps or heaters are withdrawn. Parts are brought together by applying pressure until solidification of molten plastic, producing a good quality and strong weld. An infrared welding set up is shown in Figure 2.10. This process is fast and has the ability of providing the user with an opportunity for automating it [14].

Figure 2.10 Infrared welding for plastics [20] 


\section{Hot Gas Welding}

Hot gas welding is a kind of thermal welding process for joining thermoplastics using convection heat transfer. Hot gas welding is almost similar to gas welding for metals as it uses filler rod of the same materials to be welded with a little difference i.e. instead of using gas flame, hot gas welding utilizes stream of hot gas to heat the interface of materials. After melting the materials, the application of hot gas is turned off and parts are kept together in the presence of pressure to get a weld. Hot gas welding of plastic is illustrated in Figure 2.11 .

Hot gas welding is comparatively slow process. Moreover, operator skills are essential during the process to achieve a quality weld making it difficult to automate. Resultantly the process is not suitable for high production rates [14].

Figure 2.11 Hot gas welding of plastics [21]

\section{Laser Welding}

Laser welding has good capability of welding metals but it can also be used for thermoplastics and even for welding thermoplastic composites materials. In laser welding, parts are pressed together by applying suitable pressure and a laser beam is passed through the interface of two materials to be joined. The material is softened which results in obtaining a weld. Laser welding is shown in Figure 2.12.

A good quality Laser weld depends upon the weld time, laser characteristics and material properties. Laser welding is an expensive process and it requires careful control as laser beams usually have high density to avoid material degradation. This type of welding is avoided when other cheap alternatives are present. It is a fast, neat and non-contact type of 
thermal welding. Polymer laser welding is extensively used in automotive industries and where high concentrated energy is required over small areas [14].

Figure 2.12 Laser welding [22]

\subsubsection{Friction Welding}

Friction Welding is another category of welding in which heat for welding takes place due to friction at the interface of joining parts. Molten materials are held together under the action of pressure followed by solidification and consolidation of parts. Ultrasonic welding, vibration welding, spin welding, and stir welding are examples of friction welding.

\section{Vibration Welding}

Vibration welding is frequently used for joining thermoplastics and it has the ability to weld thermoplastic composites accurately. In vibration welding, two parts to be consolidated are held together with pressure. One part is kept stationary by fixing it on some suitable place and the other part takes the vibrations at a particular frequency \& amplitude and transfers these vibrations at the interface. Vibrations are given parallel to the interface. Owing to friction and viscous dissipation (in case of thermoplastic welding), heat is developed up to a certain value. After that vibrations are stopped and parts are aligned under pressure giving rise to a quality weld on cooling. Linear vibration welding set up is illustrated in Figure 2.13.

This process is easily automated by controlling properly important factors e.g. frequency, amplitude of vibrations, welding time, welding penetration, hold time with pressure etc. This welding process is, still, under development and offers many researches to make it as efficient as possible. 
Vibration welding is used for high production rates especially due to short cycle times, its ability of welding many parts at the same time, welding parts that have size small to medium, and its capability of joining all thermoplastics i.e. amorphous, semi-crystalline and crystalline. This process is preferred in automotive industry for assembling various parts [14].

Figure 2.13 Linear vibration welding [23]

\section{Spin Welding}

Spin welding is another type of friction welding used to weld thermoplastics and its composites. In this welding process, one part is revolved with a certain angular velocity against the other fixed part under the action of pressure. Heat is developed due to friction melting the interface of two parts to be joined together. After melting stage, part is stopped rotating followed by cooling and consolidation of two materials. Spin welding of circular plastic part is shown in Figure 2.14.

Simple machines e.g. drill or lathe can be used to make this process possible. In an effort to get good quality weld, sufficient input energy is ensured at the interface by controlling angular velocity of the rotational part. Also in order to carry out this process successfully, some important factors are properly taken care of i.e. angular velocity, weld time, weld pressure etc.

This process has short cycle time, capability of getting good weld and process simplicity. Circular parts are welded without any trouble but due to uncontrollable distribution of temperature at the interface, there may be chances of having residual stresses. As a result 
of this, hollow circular parts with thin walls are the good customers of this process. This process is used in producing float and aerosol bottles [14].

Figure 2.14 Spin welding of plastics [24]

\section{Stir Welding}

Stir welding is a friction welding process used to weld thermoplastics and even light metal such as aluminium. In this welding, a rotating drill tool is used having a small diameter pin that is protruded out of drill tool face towards the parts. This type of tool rotates along the interface line in such a fashion that the tool pin immerses itself into the interface line. The height of pin is evaluated by considering the thickness of joining parts. Owing to rotation of the tool and application of pressure, friction is produced and parts are joined immediately on cooling after the tool leaves the part. Stir welding has found its lot of applications in composites. This is due to this reason that a lot of room is available for an active research on this process [14]. Stir welding is illustrated in Figure 2.15. 
Figure 2.15 Stir welding [25]

\section{Ultrasonic Welding}

Ultrasonic welding is categorised as the friction welding type used for joining plastics, metals and their respective composites. It utilizes ultrasonic waves at $20 \mathrm{KHz}$ frequency with other vibration characteristics such as amplitude etc. A static force or weld pressure is also introduced at the same time with ultrasonic waves causing the material to join owing to intermolecular friction (for plastics) and surface friction (for metals). A typical ultrasonic welding machine is shown in Figure 2.16.

Figure 2.16 Ultrasonic welding machine [26]

Specimens are usually prepared with energy directors (EDs) via injection moulding. These EDs help the ultrasonic vibrations concentrate at localized area which soften ED at interface thereby resulting in weld on cooling. Ultrasonic welding is efficiently applied to amorphous and semicrystalline type of thermoplastics rather than to crystalline [14].

Of the above discussed plastic welding processes, a perfect welding process must tackle difficulties in welding small \& large bond areas, readily automating it, welding strong, 
reliable \& repeatable bonds, acquiring surface preparation before welding, reducing costs and testing weld strength [14].

Table 2.2 is intended to present some of the advantages, limitations and applications of earlier discussed joining processes. 
Table 2.2 Advantages, limitations and applications of plastic welding processes

\begin{tabular}{|c|c|c|c|c|}
\hline Main Welding Category & Welding Processes & Advantages/Benefits & Disadvantages/Limitations & $\begin{array}{l}\text { Applications } \\
\end{array}$ \\
\hline \multirow[b]{2}{*}{ Electromagnetic } & Microwave Welding & $\begin{array}{c}\text {-Fast and clean [14] } \\
\text {-Shorter Weld Times [27] } \\
\text {-Overall low capital cost [27] } \\
\text {-Suitable for complex geometries [27] } \\
\text {-Disassembly is possible in the presence of } \\
\text { absorbent material [14] }\end{array}$ & $\begin{array}{l}\text {-Specimen Size Limits [14] } \\
\text {-Incomplete removal of absorbent material } \\
\text { after welding [14] } \\
\text {-A controlled environment is required i.e. } \\
\text { shielded container [14] }\end{array}$ & -Automotive Industry [27] \\
\hline & Resistance Welding & $\begin{array}{l}\text {-Fast, simple, cheap and clean [14] } \\
\text {-It is easily made sequential in welding large } \\
\text { structures [14] } \\
\text {-Easily automated [28] } \\
\text {-Suitable for high production rate [28] } \\
\text {-Suitable for flat and curved areas [14] }\end{array}$ & $\begin{array}{l}\text {-Initial equipment cost [28] } \\
\text {-Low tensile and fatigue strengths [28] } \\
\text {-Lap joints adds weight and material [28] }\end{array}$ & $\begin{array}{c}\text {-Aerospace, Medical } \\
\text { Devices, and Automotive } \\
{[\text { [14] }}\end{array}$ \\
\hline \multirow{4}{*}{ Thermal } & Hot Plate Welding & $\begin{array}{l}\text {-Dissimilar thermoplastics are easily welded [14] } \\
\text {-Accurate temperature control at molten interfaces } \\
\qquad \text { [14] } \\
\text {-It handles the complex geometries [14] }\end{array}$ & $\begin{array}{l}\text {-Sticking tendency of molten plastic to hot plate } \\
\qquad \text { [14] } \\
\text {-Application of non-sticking materials generates } \\
\text { the temperature limitations [14] } \\
\text {-Specimen Size Limits [14] }\end{array}$ & $\begin{array}{l}\text {-Automotive, plastic battery } \\
\text { cases, fuel tanks, fuel pipes, } \\
\text { infrastructure applications } \\
\text { e.g. water and gas } \\
\text { distributions, sewage and } \\
\text { effluent disposal pipes [14] }\end{array}$ \\
\hline & Infrared Welding & $\begin{array}{l}\text {-Fast Process [14] } \\
\text {-It joins a combination of flat and curved parts [14] } \\
\text {-It results strong welds [14] } \\
\text {-It is easily automated [14] }\end{array}$ & $\begin{array}{l}\text {-Deconsolidation and warpage of substrate } \\
\text { during heating process [14] }\end{array}$ & $\begin{array}{l}\text {-Instrument Panels [29] } \\
\text {-Door Trims [29] } \\
\text {-Centre Consoles [29] }\end{array}$ \\
\hline & Hot Gas Welding & $\begin{array}{l}\text {-Simple and portable equipment [14] } \\
\text {-It is used for large and complex parts [14] }\end{array}$ & $\begin{array}{l}\text {-Not suitable for lap shear joints [14] } \\
\text {-Slow Process [14] } \\
\text {-Difficult to control [14] } \\
\text {-Not suitable for high production rate [14] }\end{array}$ & $\begin{array}{l}\text {-Welding polyolefin tanks } \\
\text { [14] } \\
\text {-Repairing thermoplastic } \\
\text { containers [14] }\end{array}$ \\
\hline & Laser Welding & $\begin{array}{c}\text {-It is used for thermoplastics [14] } \\
\text {-Fast and clean [14] } \\
\text {-Availability of high concentrated energy at small } \\
\text { localized area [14] }\end{array}$ & $\begin{array}{l}\text {-Limited to metals for its most applications [14] } \\
\text {-High intensity of laser beams can burn the } \\
\text { thermoplastics [14] }\end{array}$ & -Automotive Industry [14] \\
\hline \multirow{4}{*}{ Friction } & Vibration Welding & $\begin{array}{l}\text {-High equipment cost [14] } \\
\text {-It is better than adhesive and riveted joints [14] } \\
\text {-Suitable for high production rates [14] } \\
\text {-Short cycle times [14] } \\
\text {-Ability to join a number of components } \\
\text { simultaneously [14] } \\
\text {-Advantage of welding small to medium parts [14] } \\
\text {-Ease in controlling process [14] } \\
\text {-No requirement of surface preparation [14] }\end{array}$ & -Not suitable for non-flat seamed parts [14] & $\begin{array}{c}\text {-Automotive [14] } \\
\text {-Domestic Appliances [14] }\end{array}$ \\
\hline & Spin Welding & $\begin{array}{l}\text {-Ability to achieve high weld quality [14] } \\
\text {-Simple, Fast and Repeatable [14] } \\
\text {-Suitable for circular parts [14] } \\
\text {-Suitable for hollow circular parts with thin walls } \\
\text { [14] }\end{array}$ & $\begin{array}{c}\text {-Non uniform heat distribution [14] } \\
\text {-Introducing residual stresses at the weld zone } \\
{[14]}\end{array}$ & -Aerosol bottles, floats [14] \\
\hline & Stir Welding & $\begin{array}{l}\text {-Ability to impart superior mechanical } \\
\quad \text { characteristics [30] } \\
\text {-Requirement of low energy [30] } \\
\text {-Lack of generating fumes [30] } \\
\text {-Advantage of joining dissimilar materials [30] }\end{array}$ & $\begin{array}{l}\text {-Suitable for circular parts [31] } \\
\text {-Requirement of proper alignment before } \\
\text { welding [31] } \\
\text {-High initial capital cost [31] }\end{array}$ & $\begin{array}{l}\text {-Automotive [32] } \\
\text {-Drilling pipes [31] }\end{array}$ \\
\hline & Ultrasonic Welding & $\begin{array}{c}\text {-Fast, clean, simple [14] } \\
\text {-Suitable for high production rates [14] } \\
\text {-Ability to be easily automated [14] } \\
\text {-Ability to weld dissimilar materials [33] } \\
\text {-Flash free welds [14] }\end{array}$ & -Surface preparation e.g. EDs [14] & $\begin{array}{l}\text {-Automotive parts [14] } \\
\text {-Medical Devices [14] } \\
\text {-Floppy disks [14] } \\
\text {-Battery housing [14] }\end{array}$ \\
\hline
\end{tabular}


After having highlighted the short summaries of plastic welding processes and their capabilities, our main focus will be on ultrasonic welding throughout the thesis. 


\section{CHAPTER 3 ULTRASONIC WELDING PROCESS (UWP)}

\subsection{AIM}

Having identified ultrasonic welding process (UWP) as a good process, it is desired to conduct more research to gain a deeper understanding of this process. Whilst there has been conducted some research in this area, this work provides with a comprehensive understanding of the link between processing factors, weld features, and joint strength for the first time. The purpose of this chapter is to provide with an extensive background familiarity regarding ultrasonic welding process (UWP). In an attempt to do so, various facets of UWP have been explored. These facets are being discussed hereby.

\subsection{Introduction}

Ultrasonics is a special branch of acoustics dealing with sound waves in gases, liquids and solids at a frequency greater than the upper limit of human hearing, that is, at frequency above $20 \mathrm{KHz}$. Pythagoras and Aristotle were the earliest contributors in the field of ultrasonics. Pythagoras worked on stringed instruments mathematically in sixth century BC, while Aristotle, supposed that sound wave resonated in air due to the air motion in fourth century BC. Nevertheless, when observing in nature, bats are found to be a kind of mammals who produce these ultrasonic waves in an attempt to hunt and travel in the dusk, night, and dawn. In general, this natural navigation system in bats is known as echolocation. Dolphins are also understood for using this principle of echolocation for their navigation, hunting and communication with each other. Hence ultrasonic vibrations are commonly found in nature. Ultrasonic waves are being used in various applications such as medical, imaging, nondestructive testing, accelerating a chemical process, and detecting submarines etc. Scientific and engineering areas overlapping with ultrasonics are illustrated in Figure 3.1. Many applications are strongly dependent on ultrasonic transducer which acts as the electromechanical conversion device. Ultrasonic transducer has emerged to be the heart of various uses of ultrasonics $[34,35]$. 
Figure 3.1 Scientific and engineering areas overlapping with ultrasonics [34]

In ultrasonic welding, parts to be welded are held together on an anvil under a compressive force which is static in nature. Ultrasonic vibrations are applied on these compressed parts. As a result of applying the static force and mechanical vibrations simultaneously, heat is developed. It is deemed a good practice to have man-made energy directors at the mating surface of one of the parts to be welded that give rise to uniform and concentrated heat development. As the temperature approaches and finally reaches the melting point of the material (i.e. thermoplastic) at the weld interface, material starts flowing in the presence of applied pressure, thereby, resulting in a weld. Ultrasonic welding is used for plastics and metals along with their respective composites. In case of metals, under the same processing conditions, material remains in solid-state and a weld is obtained finally. In this research, our focus remained mainly towards thermoplastics as input materials for ultrasonic welding [34]. Ultrasonic welding setup for thermoplastics is shown in Figure 3.2. 
Figure 3.2 Ultrasonic welding for plastics [1]

\subsection{Ultrasonic Welding of Thermoplastics}

Development of plastics and its composites has opened new doors for extensive researches. In fact, welding thermoplastics has been identified to be the most important production process. Ultrasonic welding is believed to be an attractive thermoplastic joining process. A significant number of people uses the ultrasonically welded products every day in the United States [34].

Based on the position of ultrasonic horn from the joining interface of samples to be welded, ultrasonic welding of thermoplastics can be divided into two types [34]:

a. Near-Field Welding

b. Far-Field Welding

Near field welding is that type of ultrasonic welding in which the distance (d), between ultrasonic horn and joining interface of two parts, is less than 6 or $6.35 \mathrm{~mm}$. On the other hand, far field welding is an ultrasonic welding process in which the distance (d) is greater than 6 or $6.35 \mathrm{~mm}$. Near field welding is suggested for soft and porous thermoplastics, whereas far field welding is preferably utilized for rigid and amorphous thermoplastics. Near field and far field welding set-ups are shown respectively in Figure 3.3 [34]. 
Figure 3.3 Near and far-field ultrasonic welding [36]

In ultrasonic welding of thermoplastics, development of heat is taken as an important parameter which is more involved. Viscoelastic heating is a main heating mechanism which does not only depend upon material under study but also on surface asperities (EDs). These energy directors (EDs) are the protruding parts which are moulded on specimens by injection moulding process. Energy directors may consist of semi-circular and triangular shapes. TRI and SEMI EDs are exhibited in Figure 3.4. Energy directors are designed in accordance with overall part geometry, part material and shape of sonotrode etc. In fact, energy director is expected to have maximum energy during the ultrasonic welding of thermoplastics [37]. 


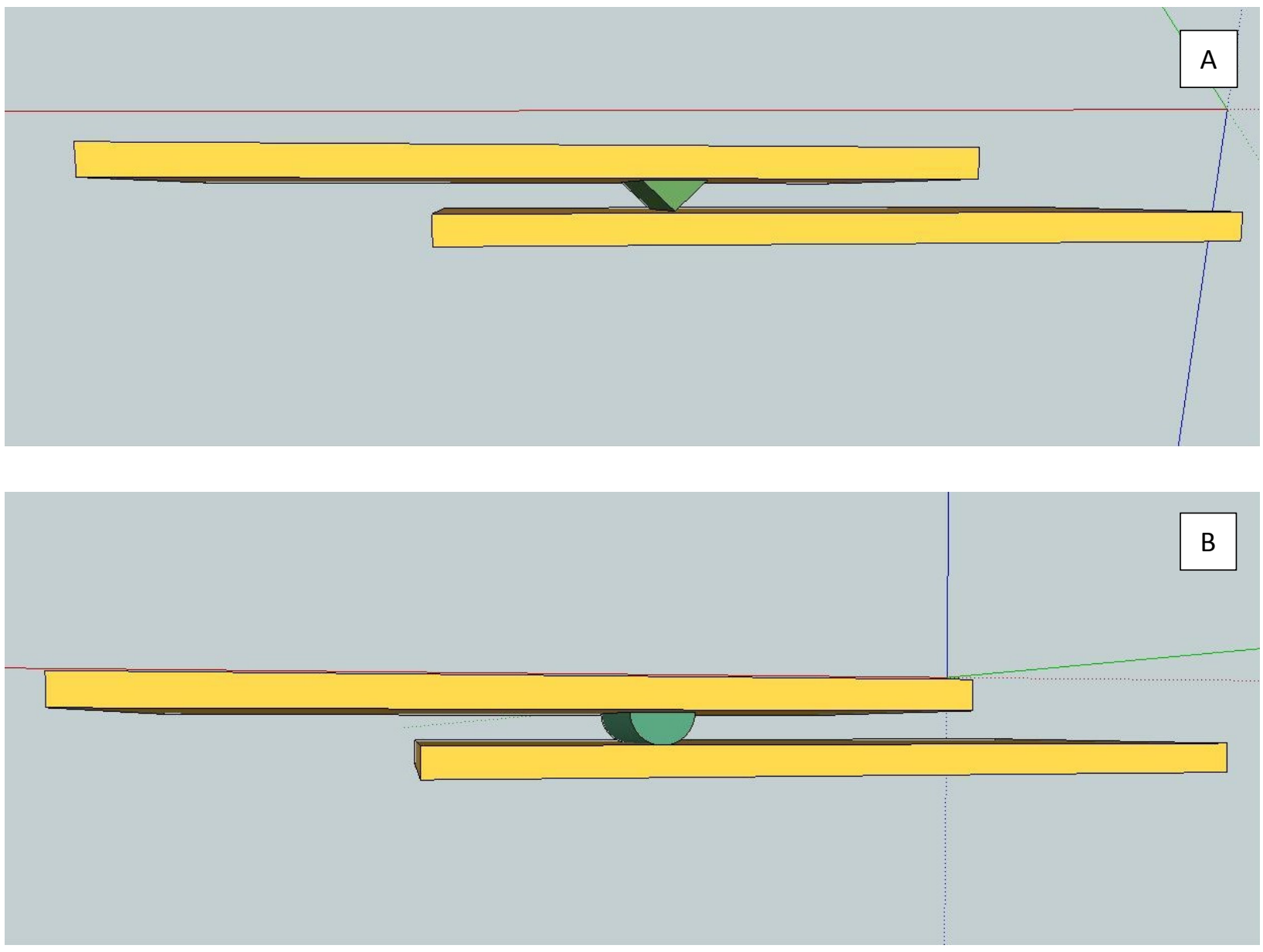

Figure 3.4 (A\&B) TRI and SEMI EDs

Amorphous and semi-crystalline thermoplastics are thought to be better raw materials for ultrasonic welding than that of crystalline thermoplastics. But thermosets are found hard to be welded ultrasonically. Thermoplastics are neither purely elastic nor purely viscous but they show an attitude between elastic and viscous behaviour. So, complex modulus is utilized consisting of two components i.e. storage modulus and loss modulus instead of using young's modulus. Further, storage modulus is called as in-phase component, whereas loss modulus is called as out-of-phase component of complex modulus [14].

\subsection{Ultrasonic Welding Equipment [38-40]}

Ultrasonic welding equipment is used to impart mechanical vibration at high frequency of $20 \mathrm{KHz}$. A static compressive force is also introduced at the same time while holding the parts to be welded with the assistance of suitable fixtures. Owing to simultaneous application of compressive static force and dynamic ultrasonic vibrations, fusion occurs thereby resulting in a weld after cooling. 
An ultrasonic welding equipment (UWE) consists of five components regardless of material (e.g. plastics or metals) to be bonded i.e. power supply or ultrasonic generator, transducer (or converter), booster, horn (or sonotrode) and an anvil on which specimens rest during and after welding. Ultrasonic welding equipment is depicted in Figure 3.5.

Figure 3.5 Ultrasonic welding equipment [41]

\subsubsection{Ultrasonic Generator}

An ultrasonic generator is used to convert a source of electrical power (5000 W (watts)) at $50-60 \mathrm{~Hz}$ into electrical energy at high frequency of 20 to $40 \mathrm{KHz}$. To date, $20 \mathrm{KHz}$ is the most desirable operating frequency in many ultrasonic welding applications. Old power supplies have used open loop control for various weld factors, whereas today's power supplies are equipped with close loop control for frequency and amplitude of vibrations as an input for ultrasonic welding.

\subsubsection{Converter}

Converter or transducer converts high frequency electrical pulses from the generator into mechanical vibrations. Old transducers comprise materials of magnetostrictive 
characteristics but due to many disadvantages of using it, piezo-ceramics are being used in new converters which expand and contract on exposing to alternating voltage.

\subsubsection{Booster}

After having obtained mechanical vibrations from the transducer, vibrations now face a device of particular design and geometry that is called as booster. A booster is used to increase or decrease the amplitude of vibrations by adjusting the geometry of it. For instance, booster having larger entry, attached to converter, than its exit, attached to horn or sonotrode, results in increment in amplitude and vice versa.

\subsubsection{Horn}

Horn has been used in ultrasonic welder whose function is to transfer mechanical oscillations to the parts to be welded. Horns come in different shapes and sizes requiring a lot of designing efforts to deliver right vibrations to the work piece. As horns are available in various shapes so, it is possible to vary amplitude of vibrations by using them in the same fashion as boosters do. Titanium and aluminium are frequently used for making horn. In fact, selection of a particular horn depends upon the particular application under considerations.

In addition to these four components, an UWE requires a number of other components e.g. main frame or structure to hold all components together, fixture, air compressor, and plc (programmable logic controller).

\subsection{Benefits in using UWP [14]}

The ultrasonic welding is preferred to other plastic welding processes due to following advantages:

1. It is a fast process requiring fraction of a second to weld.

2. Ultrasonic welding is a good option to weld neat, clean and aesthetically good welds.

3. It is flash/spark less process.

4. No external heat is required for this type of welding.

5. Parts remain free of any external material after getting welded e.g. adhesives, filler rod etc. 
6. Part strength improves after welding ultrasonically.

7. Dissimilar materials are certainly welded by ultrasonic welding process.

8. It is used for mass production.

9. It is easily automated.

10. Protective equipment is not required during this welding process except in severe case where personal protective equipment, like ear muffs, is needed to attenuate further infrasonic waves.

11. Ultrasonic welding is a fumeless welding process.

12. It is economical with regards to both time and cost.

13. Ultrasonic welding is a pollution free process.

\subsection{Limitations of UWP [14]}

Although UWP for thermoplastics has many benefits, it has also few disadvantages which need to be considered for the samples before being welded ultrasonically;

1. It needs weld surface preparation e.g. energy directors. These EDs are produced by injection molding process. And a considerable time is required to manufacture molds.

2. For welding graphite composites, the fibers sitting away from the bonded area may have heat conduction problems.

\subsection{Applications [14]}

Though UWP has so many applications, some of them are worthy of contemplation:

1. Automotive parts such as door panels, headlamps and engine covers

2. Medical Devices such as sealed bags

3. Floppy disks

4. Battery housing

A substantial amount of research is available for ultrasonic welding of thermoplastics, metals, and their composites. A detailed review of literature is given hereby:

Yousefpour et al. [14] provided a review of various joining techniques for thermoplastics. They have pronounced various fusion bonding techniques along with relevant equipment, 
production methodologies, technical factors' effects on welded joint, and their applications. Although this review presents the description of different welding techniques, this description is short. Hence more work is needed to add further understanding of each welding technique.

Nonhof and Luiten[42] have suggested four steps to perform ultrasonic welding which are based on penetration of specimens. These steps have given rise to various phenomena during ultrasonic welding of plastics. Step I (softening of ED), Step II (Development of weld zone), Step III (Steady state achieved by molten plastic) and Step IV (Turning off the ultrasonic generator) have clearly been stated. These four distinct phases are presented in Figure 3.6. They have also found that sonotrode hammers on the specimens which produce poor repeatability of welding process. This behaviour is also named as chaotic behaviour. A little work has been done to resolve this issue so; more research is required to fix this problem.

Figure 3.6 Four distinct phases of ultrasonic welding [42]

Tsujino et al. [43] reconfirmed the effectiveness of using high frequency welding system for plastics. They have studied the welding properties from $40 \mathrm{KHz}$ ultrasonic welding system with two PZT (Piezoelectric ceramic longitudinal Transducer) and four PZT rings for PP sample sheets. Welding characteristics improve on using such a high frequency welding rig. Effectiveness of frequency has been addressed very well by authors but effect of other weld factors e.g. amplitude, weld time etc. is also needed to be explored for their impact on bond strength.

Khmelev et al. [44] found the basic problem which restricted UW technology from further development. This problem includes the correct choice of optimum way or variant to realize 
the right potential of this technology and manufacturing of new specialized equipment. They have observed that the more is force of initial static pressure on a welded material, the less will be initial current of a welding.

Although a principle of energy batching has been introduced, it is hard to understand. Hence a simplistic way of affecting bond strength needs to be utilized by using different welding conditions.

Villegas \& Bersee[45] studied the effects of orientation and configuration of various energy directors upon carbon fiber/polyetherimide (CF/PEI) by using the near-field ultrasonic welding. Triangular EDs have been made on surface of consolidated composite laminates by utilizing hot platen press. Single lap-shear-welded samples have been joined ultrasonically to determine the effect of orientation and configuration of EDs with respect to load direction.

This work has presented a good effort in finding the effect of triangular ED on weld quality but this is limited to only TRI ED and one material type.

Tsujino et al.[46] studied the welding characteristics from ultrasonic welding systems by using various frequency levels e.g. 20,40 and $67 \mathrm{KHz}$. They have observed that weld characteristics can be improved by utilizing high frequency ultrasonic vibrations which result in considerable vibration loss in plastics. Weld strength achieves its larger value by using 67 $\mathrm{KHz}$ welding equipment differing from those of 20 and $40 \mathrm{KHz}$.

Again, effects of other weld factors have not been considered in this research except effect of different frequency levels is studied on weld strength.

Barsukov et al.[47] recommended the installation of an ultrasonic facility in the production lines for continuously joining and cutting the polymeric materials. Polymeric materials, with thickness from 20 microns to $5 \mathrm{~mm}$, speed from 20 to $200 \mathrm{~mm} / \mathrm{sec}$, and width from 0.5 to $15 \mathrm{~mm}$, have been welded and cut by using continuous, dot or step by step ultrasonic welding and cutting facilities.

Though this work involves a good study on continuous ultrasonic welding and cutting but it is lacking a deeper focus on spot ultrasonic welding type which has a proper industrial prospective. A production line consisting of ultrasonic welding equipment is illustrated in Figure 3.7. 
Figure 3.7 Production line of ultrasonic welding [48]

Tsujino et al.[49] studied various ultrasonic welding techniques for both plastics and metals. Complex ways of similar and dissimilar frequency for ultrasonic vibrations have been identified along with numerous vibration systems. These ultrasonic methods are considered effective in welding plastic and metallic samples which are found difficult to weld by using conventional ultrasonic welding process.

Although this study gives sufficient information on different welding scenarios for plastics, this work fails to present thorough account of effect of weld factors on ultrasonic weld.

Khmelve et al.[50] modelled the energy at the weld zone while welding thermoplastics ultrasonically. This calculation of ultrasonic energy for certain time period is deemed to have great role in managing the overall ultrasonic welding process and imparting the optimized quality to the final welds.

This work presents a better control on welding thermoplastics ultrasonically for attaining quality joint with a different approach of energy estimation. Effect of each weld factor on ultrasonic welding of thermoplastic is also required in order to obtain greater insight into UWP.

Tsujino and Ueoka[51] discussed the ultrasonic butt welding of metals such as aluminum, anticorrosive aluminum and copper with thickness up to $6 \mathrm{~mm}$. They have found that dry specimens give better weld strength than that of wet specimens with grease. Micro-Vickers hardness tester has been used to measure strength. Burrs have also been observed above and below surfaces of the welded specimens. High vibration amplitudes and shorter weld times remove the formation of these burrs. A butt joint is shown in Figure 3.8. 
This work can be considered good for butt type of welded joint for metals. Ultrasonic welding of thermoplastics has not been investigated in this research.

Figure 3.8 Various welded joints [52]

Tsujino [53] discussed the recent developments of ultrasonic welding process (UWP). Different orientation of the basic parts of UWP has been used to welding different materials e.g. metals i.e. aluminum, anticorrosive aluminum, steel, copper, and plastics. To join thick as much as $10 \mathrm{~mm}$ and large metal samples, ultrasonic butt welding has been implemented and suggested with large capacity vibration sources and power amplifiers of 50 and 100kW. Two vibration systems have also been used crossing at right angle for welding medium to large metal specimens. For medium size metal specimens, complex vibration welding tips vibrating in different loci such as elliptical to circular and rectangular to square, have been considered effective.

Though plastic welding has been done in this study by using different ultrasonic welding systems but thickness of specimens seems to be very small i.e. less than $1 \mathrm{~mm}$. Also energy director has not been used for welding thermoplastics. Hence proper considerations of all weld factors are required to have better understanding of UWP for plastics.

Ling et al.[54] worked for acquiring more understanding of the ultrasonic welding of thermoplastics. This work has emphasized on mechanical impedance and force vs. displacement curves used to divide the ultrasonic welding into four distinct phases; 
viscoelastic-plastic phase, phase of melting energy director, completion of melting phase and coupling phase of upper \& lower samples. In an effort to optimize this process, over welding phase should be avoided as much as possible.

Although authors have presented an excellent method to understand the UWP for plastics, a deeper insight in to UWP, still, needs to be attained for welding thermoplastics by using various experimental techniques e.g. FEA etc.

Shoh[55] discussed that ultrasonic welding made a considerable progress over the past years. Complex weld designs have already made the ultrasonic welding, a process that it can easily accommodate intricate weld geometries. Acceptance of ultrasonic welding as an industrial process is the most crucial development in assembling thermoplastics. Ultrasonic welding appears to have new uses in plenty of ways based on original process.

Huang [56]focused on designing aspect of ultrasonic welding process in detail. According to the author, many thermoplastics can be conveniently welded ultrasonically without adding any solvent or adhesives. Various crucial factors need to be implemented for ultrasonic welding including joint design and welding structure with great care.

Author has provided with design guidelines for ultrasonic welding. But when it comes to introducing energy director, this study fails to present design requirements for semicircular energy director.

\subsection{Materials for UWP}

For welding thermoplastics or metals or both ultrasonically, right selection of a particular thermoplastic or metal is essential. Having discussed earlier, thermoplastics can be divided into various types i.e. amorphous, semicrystalline or even crystalline. Ultrasonic welding is best suited to amorphous and semicrystalline types of thermoplastics owing to the fact that ultrasonic bond appears to be developed between glass transitions and melting temperatures. As far as crystalline material is concerned, the bond is hardly achieved before attaining its melting temperature [14].

Metals are also joined by ultrasonic welding. In case of welding metals, ultrasonic welding has already been believed to maintain the solid state of metals while joining [57].

Moreover, composites from metals and polymers have also been welded successfully [14]. 
Wu et al.[58] employed an ultrasonic welding technique for welding plastics with various geometries. A new ultrasonic welding facility has been designed and manufactured. Effect of different welding conditions is found on welding zone and tensile strength of the weld. Shorter welding time has also been proposed with high level of normal pressure in an effort to achieve higher weld strengths.

In this work, a good procedure is developed for welding and testing thermoplastics. However, using ED at joint interface can definitely result in better weld quality.

Shu et al.[59] explained the ultrasonic spin welding of thermoplastics by using various weld factors. Weld strength has been optimized by using the Taguchi method. Analysis of variance shows that hardening time is the most dominant weld factor that affects the welding quality.

This work has only analysed the spin welding considering relevant weld factors. It can also be extended to weld thermoplastics ultrasonically.

Wu et al.[60] used the design of experiments in an effort to get a proper understanding of ultrasonic welding and vibration welding of thermoplastic polyolefin (TPO). Standard samples have been used for both ultrasonic welding as well as vibration welding according to American Welding Society (AWS). For both welding processes, amplitude of vibrations has been observed to be the dominant factor for affecting the weld strength. Maximum weld strength obtained by utilizing ultrasonic welding, is $40 \%$ of the base material strength, whereas $66 \%$ for vibration welding. Standard deviation for vibration weld is significantly lower than that for ultrasonic weld. Vibration welding setup is represented in Figure 3.9.

Although current work presents a good comparison of two welding processes, this work is limited to only one material type. 
Figure 3.9 Vibration welding process [61]

Haddadi [62] has welded the dissimilar metals ultrasonically. Aluminium has been welded with steel. Intermolecular islands can be found to nucleate at interface containing mainly FeAl3 and Fe2Al5 for shorter weld times. For $1.5 \mathrm{sec}$ of weld time and $1.4 \mathrm{KN}$ of axial pressure, $1.0 \mu \mathrm{m}$ of intermetallic layer thickness can be obtained.

Author has developed a better understanding of ultrasonic spot welding for dissimilar metals. But this study is confined to metals. Ultrasonic welding of thermoplastics can be explored for getting more insight into this process.

Shakil et al. [63]studied the ultrasonic spot welding of Aluminium and stainless steel alloy at various weld parameters. Over, under and good welds have been recognized as function of different weld parameters. It is revealed by lap shear tensile test that under weld is achieved at weld energies 75 and $100 \mathrm{~J}$ due to cold worked. Weld obtained with energy values 125 and $150 \mathrm{~J}$ is named as good weld. Over welds are obtained owing to recrystallization at energy values 175 and $200 \mathrm{~J}$. Good weld results in higher strength than that from under and over welds.

Effect of ultrasonic weld parameters has been addressed by authors on dissimilar metals. This effect may also be investigated for thermoplastics leading to identification of different levels of weld quality e.g. good, over and under weld.

Zhang et al. [64]investigated the possibility of welding aluminium and titanium by using high power ultrasonic spot welding. Authors have found that $0.93 \mathrm{~mm}$ thick aluminium can successfully be welded ultrasonically with $1 \mathrm{~mm}$ thick titanium. Two different modes of 
failures have also been found called as interfacial failure and pull out. Lack of formation of brittle intermetallic layer has resulted in better strength of aluminium-titanium joint. The pull out mode transfers to the interfacial failure mode after natural aging.

Although ultrasonic welding of different thermoplastics has not been conducted in this work, the authors established the basic foundation for welding ultrasonically a new metallic combination.

Corre et al. [65]modelled the heating phenomenon in ultrasonic welding of thermoplastic composite (carbon fibre fabric/polyetherimide) with flat energy directors. Neat PEI film has been acted as a flat ED between two samples to be welded. Local heating with progressive adhesion has been produced by flat ED. Heating based on friction is confirmed by simulated results between ED and composite adherends. Viscoelastic heating is developed then after the adhesion has occurred and friction drops. Heating phase has been found to be finished when the interface temperature reaches the glass transition temperature. At this stage, flow of resin has been started. A comparison has been investigated between predicted and experimental electric power. The apparatus is found to deliver a global efficiency of $13 \%$.

An excellent modelling approach for thermoplastic composites has been identified by authors. But this approach is limited to composite materials only. It does not address the virgin materials especially thermoplastics.

Stoehr et al. [66]investigated the ultrasonic welding of neat and plasticized PLA (polylactic acid). Effect of material composition has been examined on processing window and weld quality. It is revealed that plasticizer addition affects greatly the processing window and weld quality. A wider process window is achieved by welding plasticized PLA, as shown by experimental results. Weld parameters are not influenced by multiple processing stages for PLA films. Moreover, weld quality has been improved due to the addition of plasticizer.

Ultrasonic welding of PLA has been done successfully. Ultrasonic welding of different thermoplastics may also be achieved with/without plasticizing the material.

\subsubsection{Viscoelastic Behavior of Polymers}

On imposing the ultrasonic vibrations to thermoplastic samples in the presence of static force, heat is produced. This heat is majorly thought of coming from viscoelastic phenomenon in thermoplastics. Hence viscoselasticity of thermoplastics must be considered properly in an attempt to explore the capacities of ultrasonic welding process. Various 
viscoelastic and viscoplastic models have been presented in order to understand behaviour of polymers. Polymers have also general mechanical properties e.g. tensile strength, stiffness, etc. Various viscoelastic and viscoplastic properties have been addressed in following literature i.e. storage modulus, loss modulus, creep behaviour, stress-strain curve, and relaxation behaviour. Most of these properties are function of time and frequency. In this work, various mechanical and viscoelastic properties of thermoplastics have been considered to attain research oriented objectives. In an effort to understand the viscoelastic behaviour of polymers, a considerable amount of literature has been studied. A basic representation of polymer viscoelasticity in the form of spring and dashpot is exhibited in Figure 3.10.

Figure 3.10 Polymer viscoelasticity with an elastic spring and a viscous dashpot [67]

Lee[68] discussed the problems with stress analysis for linear viscoelastic materials. He has explained three fundamental features of stress analysis; a) quantifying material properties, b) evaluating the operators functions between stress and strain leading to have viscoelastic model and c) employing previous two features for determining the stress distribution. He has concluded that quasi-static analysis is quite general and it is beneficial to evaluate the transient solution over limited time span in order to escape from using Fourier integrals. F. Bueche[69] presented a simplified model of molecule. The model has been employed to understand the viscoelastic behaviour of cross linked polymers under the action of sinusoidal forces. Theoretical data have been seemed to be agreed with experimental data. Dilute solution has also been considered good for the presented method. Improvement in this theory needs to consider two aspects; a) chain segments are neglected due to short time situation and b) friction factor should not be considered as a true constant.

Gates et al.[70] discussed the elastic and viscoelastic response of the advanced thermoplastic polyimide by testing mechanically and having known the molecular weight 
(MW) over a range of temperatures below the glass transition temperature. Tensile strength is found to be a strong function of molecular weight and temperature. But stiffness is observed to be the only function of temperature. Low molecular weight materials fail in a brittle fashion, whereas the materials of high MW have shown ductile failure. On the other hand, low MW materials have high creep compliance and creep compliance rate as compared to those of high MW materials. Elastic properties are measured by monotonic tensile loading i.e. uniaxial tensile loading by using $22.2 \mathrm{KN}$, servo hydraulic testing system, and heated chamber. It has also been found that movement of the polymer chains increases thus creep decreases at high temperature.

Janowski et al.[71] studied the long fibre thermoplastic (LFT) composites experimentally for flexural creep behaviour. It has commonly been observed for thermoplastics and their composites that they lose dimensional accuracy due to the combined effect of average to high stress and temperature conditions during their service life. This viscoelastic behaviour of LFTs brings attentions towards their performance under various working conditions. An empirical model based on four parameters provides a brilliant fit to experimental data for short fibre thermoplastics (SFT). Long term creep strength of neat and reinforced polymers has also been performed with the help of time temperature stress superposition method. Picken et al.[72] studied the linear viscoelastic properties of two olefinic thermoplastic elastomer blends i.e. Blend1 or TPE (Thermoplastic Elastomer) (PP, SEBS (KRATON ${ }^{\circledR}$ G 1651, KRATON Polymers) (elastomer) and OIL) and Blend2 or TPV (Thermoplastic Vulacanisates) (Vulcanized EPDM (ethylene propylene-diene monomer)particles dispersed in PP matrix). Storage modulus has been found to be reduced at low frequencies on increasing the PP content, whereas both storage and loss moduli have been observed to be reduced on increasing the oil content. This is found to be true for both blends. Composition and morphology of blends have the relationship with the model parameters.

Lyons[73] attempted to discuss the effect of stress and material type on long term creep behaviour of six glass reinforced thermoplastics. This long term creep behaviour is important for plastic materials because designers need to select right materials and develop optimum design. In order to investigate the strain for time greater than $1000 \mathrm{~h}$, Maxwell linear viscoelastic model has been used as it consists of one linear elastic spring in series with one linear viscous dashpot. Experimental data have been utilized to acquire design 
properties, isochronous stress strain diagrams, creep modulus charts and material constants.

Lee-Sullivan et al.[74] worked on making a model for estimating creep in random glass mat thermoplastics (GMT) composites. GMT materials of two distinct thicknesses were utilized in this research. Based on short period, a non-linear viscoelastic model has been established by using the creep data. He has also [75] extended the work on short term tensile creep of a 3 $\mathrm{mm}$ continuous long fiber glass mat thermoplastics (GMT). This additional work has been intended to characterize and model longer term creep and recovery in the $3 \mathrm{~mm}$ composite for stresses up to near failure. Data up to stress range of $70 \mathrm{MPa}$ are deemed to be employed for modelling. In order to separate viscoelastic component from the visco-plastic part of non-linear model, an estimation of parameter has been made. A good agreement is found between experimental curves and model estimates.

Govaert et al.[76] predicted the yield and long term failure of PP tape. PP strapping tape is illustrated in Figure 3.11. An anisotropic visco-plastic model has been used to define the short term and long term behaviour. The yield stress of anisotropic PP is found to be depended upon draw ratio, strain rate and loading angle.

Figure 3.11 Polypropylene (PP) strapping Tape [77]

Maffezzoli and Lionetto [78] worked on determining the behaviour of polymers by finding their basic properties with ultrasonic wave propagation. The velocity of sound has been found to have relevance with polymer storage modulus and density, whereas absorption of ultrasonic waves has been found to have relationship with loss modulus. UDMA (Ultrasonic Dynamic Mechanical Analysis) can be used to provide information about the viscoelastic 
behaviour of polymers. The acoustic characteristics have been determined by ultrasonic velocity and ultrasonic attenuation coefficient. Longitudinal waves are preferred to shear waves for more controllable and measurable attenuation conditions. UDMA is able to provide online monitoring of production of polymeric matrix composites and manufacturing operations.

Lu[79] used the small scale dynamic mechanical analysis for evaluating the storage modulus and loss tangent from force amplitude and phase angle. Atactic polystyrene, high density polyethylene and polyisobutylene have been used for evaluating their temperature dependent viscoelastic properties. A power supply was used to supply current for heating thermal chamber and this heating rate was controlled precisely by using the LabView program.

Wang et al.[80] determined the viscoelastic properties of co-polymers by using dynamic mechanical analysis (DMA). A typical DMA setup is portrayed in Figure 3.12. Addition of E51 (an epoxy) in the co-polymers has resulted in improving the toughness of DCPDCE (dicyclopentadiene bisphenol cyanate Esters) resin and reducing the thermal stability. Data from thermo gravimetric analysis (TGA) have been found to be in good agreement with $\mathrm{T}_{\mathrm{g}}$ values from DMA. Mechanical properties of co-polymers were also improved with the addition of E51 in controlled amount. Brostow and Lobland[81] established a connection between the tribological (wear) and mechanical properties of polymer based materials (PBMs). Authors have presented a model which is suitable for ten thermoplastics which have different mechanical properties and chemical structures.

Figure 3.12 A DMA setup [82] 
Gauthier et al.[83] addressed the comparison between the viscoelastic and viscoplastic properties of bulk metallic glass, oxide glasses, and amorphous polymers. Authors have found two main responses from metallic glasses i) linear viscoelastic response with a decrease in storage modulus ii) yield occurrence in stress strain curve preceding the viscoplastic flow. Isono et al.[84] have explained that in linear and nonlinear regions, the viscoelastic properties of well entangled polymers can be denoted by two factors i.e. plateau modulus (i.e. a measure of entanglement structure) and the longest relaxation time (i.e. independent of magnitude of strain). Change in the height of rubbery plateau has been found to be crucial for nonlinear viscoelasticity of well entangled polymers.

Horio et al.[85] explained the vibrating reed method to measure the viscoelastic properties of viscose monofils with different degree of orientation and a range of frequency from 20 to $100 \mathrm{cps}$. Modulus and viscosity coefficient have been found to be frequency and orientation dependent and related to relaxation mechanism. Anomalous dispersion has been expected to be occurred at different low and high frequencies due to chain slip and segmental motion. Measurements have also been stretched to films of synthetic high polymers such as polycaprolactam and polyvinyl chloride. Mocellini et al.[86] worked for relating the low frequency dielectric properties with the viscoelastic properties by applying the electro rheological model to the results from electromechanical study i.e. DMA. EPDM (ethylene propylene-diene monomer) has been used in order to apply the model on it. This rubber has two phases i.e. amorphous and semi-crystalline with polar groups that are rooted in the amorphous phase. In other words, amorphous phase is generally considered as more polar than that of semi-crystalline. An accord has been noted for calculated and measured dielectric spectra. Keunings et al.[87] established and verified a model appropriate for the estimation of linear viscoelasticity for blends of poly-disperse star and linear entangled molecules. The model has been reported to superbly predict if the constraint release solvent is treated consistently by applying the extension of "Graessley Criterion".

\subsubsection{Joining Effects of Thermoplastics}

When thermoplastics are welded with any suitable welding type e.g. ultrasonic welding, the quality of welded joint can never be compromised. Every effort must logically be needed to obtain the intended excellence of weld. Weld quality normally includes the bond strength improvement with the help of appropriate plastic welding technique using different welding 
conditions. A good weld is also obtained not only by changing specimen design but also by using different materials. Numerous studies have been attempted to describe joining effects of thermoplastics, a brief account of which is being presented here.

Stokes[88] studied the joining of three dissimilar thermoplastics by using hot plate welding. Hot plate welding is displayed in Figure 3.13. Polycarbonate (PC), polyetherimide (PEI), and polybutyleneterephthalate (PBT) have been employed in this research. To determine the joining effect, authors have worked on butt welds. Results have also been registered based on joint strengths for different hot tool temperatures and heating times.

Figure 3.13 Hot plate welding [89]

Yarlagadda and Chai [90] worked on a novel welding process for thermoplastics i.e. microwave welding. Three thermoplastics have been utilized in this research i.e. PC, ABS and ultra-high molecular weight polyethylene (UHMWPE). To measure the bond strength, tensile tests have also been conducted. Authors have also carried out microscopic analysis in order to develop more understanding about weld zone. Joining of these three thermoplastics is found to be improved with the addition of primers except the PE due to its lower dielectric loss factor which has resulted in insufficient absorption of microwave energy for fusion to occur.

Though this work gives a good idea of utilizing microwave welding in joining thermoplastics but ultrasonic welding of plastics has been considered to be fast and simple. Hence ultrasonic welding of above thermoplastics may give better quality.

In 2008, Bates and Dai [91] found that weld strength appeared to significantly increase with the degree of glass fibres on using vibration welding. Materials having $40 \%$ long/short glass 
fibres have showed lower bond strength as compared to materials with $30 \%$ glass fibres. On increasing the weld pressure, the strength of short glass fibre reinforced polypropylene compounds has been decreased slightly and vice versa for long fibre reinforced compounds. As a result, different material and processing conditions have affected the weld strength to higher extent. Strand[92] has presented an assessment of various joining techniques for plastic materials. Various factors have comparatively been considered vital including different costs, sample preparation, weld time, welding efficiency, joining proficiency, and repeatability. Friction stir welding has been observed to be a valued contestant among these assembling methods for plastics. Friction stir welding in action is shown in Figure 3.14.

Figure 3.14 Friction stir welding [93]

Kagan [94, 95]observed the likenesses and dissimilarities at local area (knit lines and welds) to analyse the mechanical performance as a result of moulding and welding conditions. These local areas can result in crack origination leading to ultimate failure of specimens. Authors suggest that surfaces subjected to high loads should not have knit and weld lines. Mechanical performance of non-reinforced and reinforced nylon has been noted to be equal to that of matrix material at local areas.

Moulding defects which may affect the subsequent welding process have been discussed. Moulding defects are not limited to just only knit lines. These defects include air bubbles and unfilled cavities. Hence a comprehensive consideration of moulding defects is required to attain the desired mechanical performance. 
Sanchez et al.[96] studied the high frequency plastic (HFPW) welding, welding defects and their detection by using SCADA (supervisory control and data acquisition) System. An equipment of HFPW is depicted in Figure 3.15. HFPW is usually employed to join PVC (polyvinyl chloride) for medical applications. In this research the weld strength of medical bag has been analysed by autoclave and excess air influence.

Figure 3.15 HFPW Equipment [97]

Gillespie et al.[98] studied the resistance welding of large scale thermoplastic composites samples. Two methodologies have been used in this work for resistance welding i.e. single step and multiple step (sequential) welding. A considerable variation in LSS has also been observed for resistance welded CF/PEEK with a co-molded PEI polymer interlayer. Based on various experimental techniques, it has been confirmed that sequential type of resistance welding has resulted in superior bond. Authors have reported higher weld consistency and LSS for multiple step welds instead of single step weld. Lower value of lap shear strengths has indicated either the presence of higher void content or incomplete fusion of the polymer interlayer. 


\subsection{Factors for UWP}

Ultrasonic welding is successfully implemented by manipulating various factors in an attempt to study their importance and effects on the final weld strength. Much of the current literature on welding materials ultrasonically pays particular attention to weld factors and standard sample design. Different sample design and changes in ED geometry are required to find the effect of these changes on bond strength. Following weld factors have been identified for welding various materials ultrasonically [99]:

1. ED Geometry

2. Static force

3. Amplitude

4. Welding Time

5. Hold Time

6. Frequency

Khmelve et al.[100] examined the influence of various ultrasonic weld factors on weld strength. Polypropylene (PP) and Poly-Vinyl Chloride (PVC) have been used in this research. Strength of PVC weld connections is found to be higher than that of PP at same weld factors.

Author has used a fixed combination of ultrasonic weld factors in an attempt to find their effect on LSS. A series of planned experimentation must be utilized with different combinations of welding factors. This enhanced experimentation can help better understand the UWP for various thermoplastics at large scale.

Seven years ago, Khmelve et al. (2008) [101] worked on the distribution of ultrasonic energy in the weld zone that was defined by the time interval, the ultrasonic oscillation energy and the acoustical properties of the materials to be welded. They have observed that the ultrasonic energy to the weld zone softened the materials as the energy gets absorbed by the materials. Due to which, the amplitude, frequency of the UOS (ultrasonic oscillatory system) welding tool and area of the welded polymeric materials have been changed. A decrease in the ultrasonic energy has resulted in incomplete melting of the material at weld zone and poor bond strength. On the other hand, if the ultrasonic energy is intended to increase then an excessive warming up and destruction of thermoplastic materials have 
again been resulted in perceivably poor joint strength. As a whole, ultrasonic welding lasts for a fraction of second so it is necessary to introduce exact amount of energy and various factors in order to get tight control over this welding process. Mathematical models can be used to calculate energy and time required to obtain desired weld strength. Experimental researches have shown that actual time for ultrasonic influence should be more than that of the time obtained by theoretical calculations.

This paper provides with good analysis of weld factors and ultrasonic energy which has great impact on weld strength. However, a simple way of attaining weld strength is required as a function of various weld factors.

Daniels[102] conducted a survey on various factors of ultrasonic metal welding e.g. power, pressure, welding time and their interdependence. According to the author, dissimilar metals can be welded. Surface states i.e. roughness vs. polished have been recommended. A weld can be generated between metals and non-metals e.g. metals to silicon or germanium. Antonevich[103] emphasized on various aspects of ultrasonic welding equipment. According to him, ultrasonic welding equipment should generally have a vibrating device, a method for controlling various times, clamping, and coupling tools. General relationships between weld strength and various weld factors have also been established for welding monel sphere with copper plate.

Tsujino et al.[104] studied new methods of metal welding ultrasonically. Ultrasonic welding has been used for welding small and medium sized samples by using different settings of welding variables. For welding big samples, authors have employed ultrasonic butt welding methods. Mallick[105] worked on vibration welding of the continuous fibre reinforced thermoplastic composites. Various factors have been found to affect the lap shear strength of joined samples from glass and carbon fibres reinforced thermoplastics. Clamping pressure and vibration time are noted to have great impact on LSS, whereas effect of direction of vibrations does not appear substantial. Matrix has been observed to be softened on subjecting to high temperature resulting eventually in considerable reduction of LSS.

Balle and Eifler [106]investigated the joining of Al/CFRP via ultrasonic welding process. A statistical model named "central composite design circumscribed" (CCC) has been used to evaluate the appropriate process parameters. CCC model is best suited for describing the mutual dependence of three weld factors; amplitude, energy and weld force concerning the mechanical properties of joints. Hence CCC model may be used to optimize joints of $\mathrm{Al}$ 
alloys. Microstructural analysis is also attained in an effort to understand in detail the mechanical behaviour of ultrasonically welded hybrid joints.

A good statistical approach has been established for understanding the ultrasonic welding. But this approach is limited to describe mutual dependence of only three weld factors on the joint behaviour. More insight into this welding process can be achieved by adopting a more stable statistical method with due considerations to all possible process factors.

\subsubsection{Energy Directors (ED)}

Energy directors (EDs) are considered essential for ultrasonic welding of thermoplastics successfully. Their main objective is to make sure the availability of consistent and concentrated viscoelastic heating during welding. Energy directors are designed in various shapes e.g. triangular, semicircular, and rectangular. An enormous and growing body of literature has also investigated this aspect of ultrasonic welding:

Chuah [et al][107] studied the improvement in speed and weld strength by using different EDs e.g. triangular, rectangular, and semicircular ED. The most efficient among these three EDs has been found to be semicircular, next rectangular, and then triangular. Liu and Chang[99] worked on optimization of weld strength for ultrasonically welded nylon composites. Optimization essentially depends on trial and error process. Maximization of weld strength has been identified to be the goal of optimization. Maximization of $\mathrm{S} / \mathrm{N}$ ratio has resulted in minimization of any property which is sensitive to noise. In this study, an L18 orthogonal array design has been used based on Taguchi method. EDs having semicircular geometry have been found to give highest weld strength. The weld strength of samples has been found to increase with fibre content in composites and reduce with moisture content within materials.

Liu and Chang[108] studied again optimization of joint strength of ultrasonically welded polypropylene and its composite. Triangular ED has been found to join samples with highest strength for virgin polypropylene and $10 \%$ glass-fibre filled polypropylene composites, whereas a semi-circular energy director has been found to acquire highest strength for $30 \%$ glass-fibre filled composites. Rani et al.[109] studied various ultrasonically welded joints by using semi-crystalline thermoplastics. According to the author, textured joint should be considered as the best to achieve highest strength for welding semi-crystalline thermoplastics. TRI ED has more collapse. Saw tooth joint's volume has been found to be 
proportional to the energy supplied at the weld interface. Textured joint has accommodated higher temperature among all non ED joints. Textured joint has also been observed to utilize low energy in delivering highest strength.

Liu et al. [110] again worked on optimizing the bond strength for thermoplastics. They have found that amorphous plastics need less energy to be successfully welded than those of semicrystalline thermoplastics. SEMI ED has been observed to impart the highest weld strength. Rani et al. [111] implemented fractional factorial design to ultrasonic welding of thermoplastics. They have found that welding time and welding pressure significantly affect the joint strength. Holding time does not contribute a lot to welding results. ABS is easier to weld than that of HDPE (High-density Polyethylene). ABS has been welded by using both ED and shear joint but joining of HDPE is only feasible in near field orientation of samples along with high levels of welding time and pressure. Far field welding of HDPE is found to be possible with shear joint. Joint strengths for $A B S$ have been observed to be higher than those for HDPE with same testing conditions.

Villegas [112]investigated the bond strength development for ultrasonic welding of carbon fibre fabric/polyetherimide (PEI) with flat energy director. Five stages of welding process have been identified, as shown in Figure 3.16, as a function of weld parameters for analysing the weld strength, weld morphology and failure modes. Stage 1 involves heating of ED without any physical change at weld zone. In this stage, power dissipation is increased continuously until a maximum is reached. In stage 2 , ED starts melting locally and nucleation of hot spot is controlled by heating from surface friction. Power decrease is observed at this stage while the sonotrode displacement remains constant. Stage 3 is identified by complete melting of ED. An increase in power and sonotrode displacement is found at this stage. Flow of energy director is obtained in stage 4. Upper layers of composite matrix are also found to start melting at stage 4 . A power drop is again observed at stage 5 due to predominant melting of composite matrix in the adherends. 
Figure 3.16 Five stages of welding process [112]

A brilliant effort has been made for ultrasonic welding of thermoplastic composites with flat energy director. But this research is lacking the focus on welding thermoplastics ultrasonically with different shapes of energy directors.

Villegas et al. [113]presented a comparative study of ultrasonic, induction and resistance welding of carbon fibre reinforced Polyphenylene sulphide samples. TRI ED has been used for ultrasonic welding. For ultrasonic and induction welding, static single lap shear strengths have been found to be alike. Static mechanical properties of resistance welded joints have been reduced by $15 \%$. These three types of welds have shown the similar fatigue performance. The shortest welding time for welding small areas has been observed for ultrasonic welding process.

Although an excellent comparative investigation has been evaluated for ultrasonic, induction and resistance welding processes, an in depth study of ultrasonic welding is still lacking.

\subsection{METHODS OF ASSESSING UWP}

\subsection{1. $\quad$ FEA For Ultrasonic Welding Process}

FEA (finite element analysis) has been identified as an influential computational tool for analysing and predicting the performance of a real product which is subjected to real loads relevant to structural, thermal, fluid dynamics etc. In other words, FEA involves controlling accurately the product development process by investigating the behaviour of a product in 
real world situations. A specific FEA is demonstrated in Figure 3.17. Designing specimens is a crucial part of ultrasonic welding involving time and money to reach a final design. FEA is a powerful tool helping the engineers in reducing the lead time of product to market. To reduce time and money, researchers have undertaken various finite element (FE) analyses to optimize ultrasonic welding process:

Figure 3.17 FEA for sonotrode [114]

In 1972, Aloisio et al. [33] presented a simple thermo-viscoelastic analysis for ultrasonic welding of thermoplastics. According to authors, possibility of ultrasonic bond depends upon the processing temperature of the material to be welded. Dissimilar materials are also deemed to get ultrasonically bonded. In an effort to calculate the heating rate, various mechanical and thermal properties of material are required. Temperature increases at the ED owing to internal dissipation which is calculated by E" (loss modulus). Once melt flow has occurred, this increment in temperature has been observed to decrease in an attempt to avoid thermal degradation. This approach has now motivated the researchers to try various combinations of materials for welding ultrasonically e.g. TEFLON-FEP (fluorinated ethylene propylene) is one successful welding example. Moreover, ultrasonic curing of epoxy cements has also been investigated. Thirty five years later, Prakasan et al. (2007) [37] attempted to simulate the ultrasonic welding of thermoplastics. They have described the 
viscoelastic heating as the main heating mechanism while welding thermoplastics ultrasonically. This viscoelastic heating depends on frequency, square of the amplitude and loss modulus of thermoplastic under investigation. Amorphous thermoplastics have been found to be successfully welded in both near and far field welding scenarios. Simulated results agree with the measured temperatures during ultrasonic welding. TRI ED is found to have high temperature regions and high ultimate tensile strengths (UTS) as compared to other EDs. UTS has been found to increase up to a certain temperature for amorphous thermoplastic and then it decreases for both types of thermoplastics.

Aloisio and Prakasan have provided with excellent approaches in conducting FEA for ultrasonic welding of thermoplastics. But their work is limited to 2D FEA needing to be tested with 3D FEA.

Zhang et al. [115]presented a finite element model via ANSYS to evaluate the temperature profile in glass fibre reinforced polypropylene composites joint during ultrasonic welding. Temperature has been increased by increasing weld time. More heat is dissipated due to interfacial friction and melting temperature of polypropylene may be reached in shorter time by increasing the weld pressure. Interpolation method has been used to acquire the weld map. This weld map derived from FEA results has been found to be in agreement with experimental results.

In this study, authors have found the transient temperature profile for composite materials. Likewise, temperature profiles can be found for virgin thermoplastics.

Rani and Rudramoorthy [116]worked on the computational modelling for vibration performance of five different horns. Five horn profiles used are cylindrical, Gaussian, catenoidal, stepped ad Bezier. Authors have found that stepped and Bezier horns have high amplitude displacement with higher temperature development at weld interface. The displacement amplitude of cylindrical, Gaussian and catenoidal horns is suitable to join ABS in far field region. Bezier horn has produced stronger joints which are 3 and 1.5 times stronger than that of Gaussian and catenoidal horns respectively. The energy utilization of stepped horn is lower than that of Bezier horn. Authors have concluded that cylindrical, Gaussian and catenoidal horns may be used to weld amorphous thermoplastics, whereas stepped and Bezier horn may be utilized for welding semicrystalline thermoplastics and joining samples in far field regions. Simulated results have also been validated in this study. 
In this research, authors have clearly identified the performance of different horn profiles for an ultrasonic welding system. Stepped horn may also be checked for welding different semicrystalline thermoplastics.

Wang et al. [117] worked on exploring ultrasonic welding of continuous carbon fibre reinforced PEEK composites by using finite element modelling. Viscoelastic dissipation theory has also been used in this work. In particular, effects of size and apex angle of ED have been explained.

Nevertheless, authors have presented an excellent approach to model ultrasonic welding of thermoplastic based composite with the help of FEA. But simulated results have not been validated.

Wang et al. [118]studied the new horn design for high displacement amplification. Optimization algorithm and FEA have been used to optimize the displacement amplification of horn. Horn prototypes have also been produced and tested for validating the design procedure. The experimental results agree with the design specifications. The Bezier horn is found to be more suitable than the stepped and catenoidal horns for those applications where higher displacement amplification and lower stress concentration are required.

Although FEA is utilized for optimizing the horn performance, a thorough analysis can also be achieved which encompasses the temperature distribution at weld zone during ultrasonic welding.

Andoh and Kagawa[119] have found that vibratory behaviour of a large sized vibrator is always a problem for researchers. Various ultrasonic vibrators are exhibited in Figure 3.18. Vibration simulation has been applied by using a two dimensional model with negligible or minimum thickness as compared to other dimensions in $X$ and $Y$ axes. Simulated results have also been compared with experimental results. A good agreement has been found between experimental and numerical results. 
Figure 3.18 Ultrasonic vibrators [120]

Prakasan et al.[121] suggested a model for temperature distribution and stress distribution in horn and welded joints during welding. According to authors, with knowledge of forces at weld interface, it is possible to control weld strength and sticking of sonotrode. The FE model is capable of predicting interface temperature, stress distribution and their influences on work piece, sonotrode and anvil. Ming et al.[122] wrote a report on optimizing the horn designs for ultrasonic welding systems. According to them, more accurate predictions are required to achieve good horn performance and efficient energy delivery. An FEA for horn design is shown in Figure 3.19. Working at the resonance frequency, Ultrasonic welding system will significantly result in reducing power delivery if any deviation occurs from resonance frequency. In order to design the horn efficiently, the effects of the work pieces should not be overlooked. Modal \& Harmonic analyses have been performed to optimize the horn design by using ANSYS. The harmonic analysis has given more accurate predictions than the modal analysis in optimization of design.

Figure 3.19 Horn design via FEA [123] 
Zhang and Li[124] addressed a complicated phenomenon of bond formation due to the ultrasonic consolidation for metals. Bond interface in metals has been developed between the two mating surfaces purely due to the friction. Two phenomena are found to be vital i.e. temperature distribution and plastic deformation. The friction has resulted in shearing effect on bonding, whereas bond formation occurs due to plastic deformation. This paper describes what has actually happened during the ultrasonic consolidation process? The roles of heat generation due to friction and heat dissipation due to heat loss via conduction have also been discussed.

The phenomenon of bond formation is crucial in welding both metals and thermoplastics. Both intermolecular and surface frictions remain present on welding thermoplastics. Ultrasonic welding of thermoplastics requires the phase change of thermoplastic material, whereas metals generally maintain their solid state while welding ultrasonically.

Zhao et al.[125] elaborated the advantages of ultrasonic consolidation over traditional autoclave laminated consolidation and repair. According to author, ultrasonic heating is a complex phenomenon requiring various factors to be considered such as horn angle, frequency, young's modulus, density, Poisson's ratio, specific heats, thermal conductivity and thickness of the materials. FEA has given an accurate optimization of the ultrasonic consolidation.

Ando and Kagawa[126] have discussed that bolt clamped langevin type transducers have extensively been used in ultrasonic plastic welding systems due to their high electromechanical conversion efficiency and production of large amplitude vibrations. In this study, finite element formulation comprising two internal heating sources has been used. One is due to mechanical vibration loss in elastic materials which is proportional to the square of the vibration strain and second is owing to dielectric loss in ferroelectric materials (FM) that is proportional to the vibration frequency and square of the impressed electric field i.e. electric potential. It has been established that the numerical (FE) analysis should be taken into account for design consideration of vibrators. Siddiq and Ghassemieh[127] explained the thermomechanical aspect of ultrasonic welding of aluminium alloy. Surface and volume effects have properly been taken into account for doing current FEA. Friction is found to play secondary role in heating weld interface. The numerical results have been verified with the experimental results and a good agreement is 
found. In order to further understand the deformation during ultrasonic welding, micro structure as well as FEA at submicron level needs to be performed in future.

Gao and Doumanidis [57] analysed the ultrasonic welding of thin foils for rapid manufacturing process. A 2D quasi-static/dynamic finite element analysis has been performed and validated. This type of mechanical analysis is considered suitable for multijoint rapid prototyping that has ultrasonic phenomenon. Hong-Jun[128] presented the numerical simulation FVM (finite volume method) study to attain an improvement in polymer flooding by viscoelastic effect. Viscoelasticity of polymer solutions has been found to be the main parameter manipulating sweep efficiency. Higher weissenberg number is crucial in obtaining stable solutions.

Roopa et al.[129] studied the ultrasonic welding of HDPE in far field state. Authors have suggested that selection of amplitude is an important aspect of far field ultrasonic welding of semi-crystalline thermoplastic. Higher weld strengths have been found at the displacement antinodes. Temperature spikes have also been determined for various geometries of specimens by using ANSYS.

Authors have suggested using FEA but the analysis is confined to only semicrystalline type of thermoplastics. A good analysis can be investigated by using amorphous type of thermoplastics as well.

Hubert et al.[130] studied the failure mechanism of resistance welded composite specimens i.e. stringer/skin CF/PEEK composites. Effect of heating element (HE) $\mathrm{TiO}_{2}$ on fatigue performance of resistance welds has also been investigated. In this work two heating elements (HE) were used i.e. stainless steel mesh $\mathrm{HE}$ and $\mathrm{TiO}_{2}$ (titanium oxide) $\mathrm{HE}$. According to authors, FEA can be used as a next step in predicting the fatigue life of resistance welded stringer/skin composites.

Levy et al. [131]studied the time homogenized multiphysical modelling of ED flow at mesoscopic scale. Parametric study of the process is also offered with 2D approach using numerical software. Effect of various parameters has also been studied. The equivalent stiffness analysis, healing degree and risk of porosity entrapment are used for evaluating process efficiency and weld quality. Although current study can be used for ED design at mesoscopic scale, additional work is also required in terms of investigating cooling phase, validating the numerical tool and extending the analysis to three dimensional domains. 
A good effort based on numerical approach has been established for designing ED. But this study does not provide validation of current modelling. Moreover, a good numerical study may also be developed for welding a range of virgin materials ultrasonically.

Levy et al. [132]proposed a model and simulation for polymer flow at the interface. A simulation tool has been developed involving a level set method for handling large geometry change and an iterative solver to deal with multiphysical facets. Code predictions have been qualitatively validated. A tool for automatic mesh refinement is decided to be added in the code.

This work presents a modelling and simulation approach at the weld interface. An FEA with proper validation and 3D capabilities must be used for understanding the temperature development at weld interface.

\section{Selection of Material Model: A Special Case of FEA}

A material model involves deducing the material response to stress and strain for both elastic and plastic regions. Thermoplastics are defined as the viscoelastic materials and their response to stress-strain is different than that of metals. Selection of material model is an important aspect of FEA. For this purpose, there is a large volume of published studies describing the material models for thermoplastics.

Dahoun et al.[133] used the monoclinic polypropylene samples to investigate stress-strain relationships under tension and shear. Viscoelastic analysis, densitometry and X-ray scattering have been used for characterization. An example of X-ray scattering is shown in Figure 3.20. It has been found that stress hardening is approximately equal to zero in shear, whereas it is high in tension. Structural damage is found to take place via plastic deformation in tension but it is observed to be little in shear. A viscoplastic model has then been established taking various slip mechanisms into account. Strain hardening in tension as well as in shear has been reproduced properly by simulations with present model. BrusselleDupend et al.[134] characterized PP in pre-necking area. Viscoelastic and viscoplastic behaviours have been determined by conducting mechanical testing. Relation between crystalline lamellae and amorphous phases has also been considered by dividing the macroscopic stress into back and effective stresses. 
Figure 3.20 X-Ray Scattering [135]

Brusselle-Dupend et al.[136] developed a model for PP in order to forecast the behaviour of PP by using their previous work. Total strain has been divided into viscoelastic and viscoplastic strains for modelling viscoelastoplastic behaviour of PP. Data, based on tests of loading-unloading, loading-relaxation-unloading, and unloading from the punctual reevaluation, have agreed with the prediction of viscoelastoplastic model for PP. Dean and Broughton[137, 138] modelled the creep behaviour of PP under uniaxial tension by employing an exponential function with four factors. Creep behaviour has then been formulated for multiaxial stress state. An assumption relevant to time independent Poisson's ratio has been validated. According to authors, little variations are found between measured and calculated results. It is not clear whether these variations are owing to error in experimentation or due to model limitation. Further work has finally been suggested for multiaxial loading. This type of loading has been considered essential when using current model in FEA for stress analysis of long term deformation behaviour. Drozdov and Christiansen[138] established the constitutive equations for time dependent attitude of semi-crystalline polymers with small strains at isothermal loading. Semi-crystalline network is considered to be inhomogeneous. This network has been modelled as a collection of mesoregions (MRs). MRs are expected to have two types i.e. Passive and Active. Restructuring of strands in active MRs has represented the viscoelastic phenomenon and sliding of junctions has connected with viscoplastic behaviour. Observed data have been found to have good agreement with results from numerical simulation.

Takahashi et al.[139] predicted the fracture for polymers by using a simulation. In this research, the constitutive equation considering craze effects was thermodynamically 
developed. An evolution equation has been derived addressing propagation and growth termination for craze. A new material model is recognized for ductile polymers. This material model has finally been introduced in a 3D FE solver i.e. Radioss with the help of user subroutine. An FE simulation for PP plate has been conducted ultimately having uniaxial tension. Based on craze build-up and fibril strength, failure estimation has also been achieved for validating the material model.

Louche et al.[140] studied the thermomechanical behaviour of a structural component i.e. heel of woman's shoe (made of ABS) during impact testing. Stress-strain relationship considering necking has been developed by tensile and impact testing with kinematic field. High temperature variation ( $\mathrm{a}$ few $\mathrm{K}$ to $25 \mathrm{~K}$ ) has been measured in localized strain field during two testing methods representing the thermal field. Johnson cook material model has been employed owing to its considerations for strain rate, strain and thermal effects. A thermomechanical simulation has been run eventually. Sudduth[141] described the efficiency of yield energy dissipation i.e. " $n$ ". From Scott-Blair's explanation for material viscoelasticity, $\mathrm{n}$ has been supposed to have a range between 0 (solid) and 1 (liquid). In this study, the account of Scott-Blair was established to be stable with generalized model for viscoelasticity which was presented by the current author in his earlier publication. It has also been observed numerically and empirically that $\mathrm{n}$ has found its range between 0 and 0.2 .

Hummel and Nied[142] designed the tensile testing apparatus at raised temperatures. This apparatus has been used to obtain large strain deformation of polymers by using uniaxial and biaxial loading at high temperatures. One of the main objectives of this study was to improve the FEA for thermoforming or blow moulding practices by using a comprehensive material model in Abaqus. A typical thermoforming process is exhibited in Figure 3.21. The results can be used to recuperate the nonlinear response of heated ABS at limited strains.

Sudduth[143] presented a universal viscoelastic model in his another paper. In order to clarify the features of failure principle, the universal viscoelastic model has been authenticated for two ABS materials. The Tresca failure principle has been observed to be better than that of Von-Mises when fitting the pipe burst data for ABS-A. Contradictory to this, Von-Mises failure burst principle has found its better prediction from extrapolated creep failure principle. Based on the findings in this work, universal viscoelastic model has well been employed in FEA for various design uses. Wang et al.[144] employed ABS for 
uniaxial tensile testing. A new model has been developed after considering the strain rate sensitivity, strain hardening, temperature change, and variation in the hardening index. A good agreement has also been found between the results from new model and uniaxial tensile testing.

Figure 3.21 Thermoforming process [145]

Sudduth[146] strived, once again in his another publication, to relate stress relaxation, creep, and constant strain rate for viscoelastic polymeric compounds. This relationship has then resulted in formulating a mathematical model considering all phases of creep. This type of approach has been expected to help design plastic products for short term as well as long term applications. Chen[147] introduced a method of obtaining prony series with the help of loading, unloading and relaxation data. Deformation history is needed to have the time dependent stress and strain for a linear viscoelastic material. Based on time dependent loading by using hereditary integral method, material response has also been analytically represented.

\subsection{2. $\quad$ Role of Differential Scanning Calorimeter (DSC)}

Differential scanning calorimeter (DSC) and other useful tools have also been studied with their applications for thermoplastics. Basic arrangement for DSC is shown in Figure 3.22. In recent years, there has been a growing amount of literature on usefulness of DSC. 
Figure 3.22 The DSC Set up [148]

Blom et al. [149] addressed the correct performance of ABS in medical applications. It is desired that ABS should preserve its properties while processing, fabricating and actually using it. They have studied the mechanical loss in properties of ABS with thermal analysis and mechanical testing. DSC and oxidation onset temperature (OOT) testing have resulted in higher glass transition temperature and lower onset temperature respectively for those specimens that show loss in mechanical properties. DSC has appeared to be a sensitive and beneficial tool for observing the degradation of butadiene phase of ABS. A DSC with dedicated PC (Personal Computer) is presented in Figure 3.23. It has also been found that the degradation of $A B S$ occurs at substantial rate in the presence of oxygen. Relationship between rubber phase and mechanical properties has also been illustrated.

Authors have presented a detailed analysis for ABS performance. Most of the crucial properties of ABS are expected to be changed at $T_{g}$. Hence DSC can be considered to be an adequate tool in determining the performance of $A B S$ at weld interface.

Bandeira et al. [150] described the sol-gel method as a route of coating on the 3D rapid prototyping parts. The coated part has affected the base material properties. Sol-gel metal oxides have been coated on ABS and Nylon substrates which are further characterized with the help of thermal analysis and scanning electron microscope (SEM). On thermally analysing via DSC and TA (thermal analysis), it has been found that this sort of coating has improved effects on substrate's properties e.g. physical, thermal, and chemical. Degradation temperature has also been found to be increased to considerable degrees centigrade. 
Zimmermann et al. [151] worked on combined thermal device (TGA/DSC) for various samples with the aid of soft photo ionization mass spectrometry (EBEL-SPI-MS) (electron beam pumped rare gas excimer lamp- single photon ionisation-mass spectrometry). Decomposition of $\mathrm{ABS}$ has happened between 400 and $450{ }^{\circ} \mathrm{C}$ with greater rate of mass loss at about $420{ }^{\circ} \mathrm{C}$ due to emission of organic compounds. Hence ABS has been found to be highly susceptible to mass degradation at high temperatures.

A good analysis has been done addressing the degradation of ABS at high temperatures. Glass transition temperature is found to be lower than that of degradation for ABS. Hence thermal analysis has provided the first guidelines in setting the right weld factors for welding ABS ultrasonically.

Figure 3.23 DSC with a dedicated PC [152]

Shapi [153] described the biochemical effects of thermal-oxidative fumes for various thermoplastics. Author has concluded that these fumes are toxic. He has also studied the thermal stability of these polymers via DSC and TG (thermogravimetry) in air and nitrogen. Thermal degradation of plastics varies in the presence or absence of oxygen. Results from thermal analyses have shown that no substantial chemical changes have been observed below $200{ }^{\circ} \mathrm{C}$ for materials under investigation. Duh et al. [154] conducted thermal analysis to study the oxidative decomposition of $A B S$ resin powder. Unsteadiness of $A B S$ has been investigated for different polybutadiene (PB) contents using DSC. Exothermic peaks relevant to auto-oxidation, degradation and oxidative decomposition have also been investigated. Unsaturated PB has been found to be possible reactive hazards for ABS powders instead of SAN (polystyrene-acrylonitrile). DSC results have shown that degradation of ABS has 
occurred owing to oxidation of PB component in ABS. Authors have also commented on vitality of process control in preventing thermal hazards of ABS powder plant despite unsafe materials.

Parikh and Zlatkevich [155] studied the chemiluminescence in thermal oxidation of ABS. They have compared the results with those from DSC. According to the authors, DSC has depicted the improvement of $A B S$ stability in the presence of antioxidants but the chemiluminescence is better in showing the differences in durability. Moreover, resistance to oxidation during processing is relevant to DSC thermal oxidative constancy results, whereas results from chemiluminescence are beneficial in addressing the material's lifetime at service temperatures. Tarantili et al. [156] utilized DSC, MFI, and tensile testing for the characterization of pure plastics and their blends. This research has been aimed at providing with waste plastic management from electrical and electronic devices. ABS blend with PC (polycarbonate) has appeared to have plasticizer effect at blending concentration of $20 \%$ due to the distribution of PB phase in blend matrix. A decrease in tensile strength has been noted for both blends ABS/PC and ABS/HIPS (high impact polystyrene). Modulus of elasticity has been improved for both blends.

Kang et al. [157] studied the concentration of $\beta-N A$ (nucleating agent i.e. TMB-5) on the crystallization and polymorphic attitude of two iPP (isotactic polypropylene). These iPP have been affected differently by concentration of $\beta$-NA. Also the polymorphic behaviour and crystallization of iPP (nucleated by TMB-5) have significantly been varied with uniformity of stereo defect distribution.

Liu et al. [158] investigated comparatively the injection moulded iPP macro and micro parts. These parts have been manufactured with same processing conditions for their microstructure and mechanical properties. Authors have used different tools in doing so and DSC is one of them. DSC results have shown that macro parts have lower orientation degree and crystallinity than that of micro parts. This is owing to this reason that the tensile strength and modulus for micro parts are considerably higher. This work has been ended with a better understanding of relationship between processing structure and property.

Authors have presented a relationship between structure and property for iPP. DSC results can also be useful in various processing steps for PP. These processes consist of sampling and welding PP ultrasonically. 
Shashidhara et al. [159] studied the homoploymer (PP) and copolymer (PP-g-GMA) (polypropylene grafted with glycidyl methacrylate) for their melting behaviour and isothermal crystallization kinetics with the aid of DSC. Copolymers have been found to have higher degree of crystallinity and overall crystallization as compared to homoploymer (PP). Moreover, the MFI value of graft copolymers is found to be greater than that of virgin PP showing reduction in molecular weight. The melting temperature of graft copolymer has appeared to be slightly less than that of virgin PP owing to again reduction in molecular weight.

Impact of both DSC and MFI results has been studied in order to better understand the melting behaviour of different types of PP. However, DSC and MFI results can also be used in determining the softening temperature and fluidity of PP respectively. Softening temperature and fluidity of PP has substantial effect on bond strength during ultrasonic welding.

Luijsterburg and Goossens [160] worked on the post-consumer polyolefin recyclates i.e. PP, PE etc. They have used different tools e.g. DSC for assessing the composition of materials prior to reprocess. They have found that the $96 \%$ pure PP is easily obtained from postconsumer PP recyclates. Various steps e.g. hot washing, manual screening, and centrifugation have depicted improvement in mechanical properties and purity of recyclates. Ha [161] performed a thermal analysis for post-consumer PP on recycling it in open loop. He has used DSC and thermogravimetry derivative thermogravimetry (TG-DTG) for the said thermal analysis. He has found that both impact-PP that has $\mathrm{Caco}_{3}$ filler in small amount and mixed impact-PP can be used in refrigerator plastics for its good impact properties without downgrading for open loop recycling.

\subsubsection{Microscopy}

Microscopic studies were often related to biological/medical field years ago without being realized for their numerous applications in engineering and technology. A compound light microscope is illustrated in Figure 3.24. It is worth mentioning here that technical microscopy in various fields of engineering is aimed at providing qualitative as well as quantitative relationships between microscopic images and physical engineering phenomena. A typical engineering microscope is shown in Figure 3.25. For instance, 
metallurgical microscopist has to look at plastics, metals, ceramics, and grease etc. And many types of dirt are observed by textile microscopist [162].

Figure 3.24 Compound light microscope [163]

Understanding of various features on captured images with technical microscopy consists of general and unexpected information about the sample. These unexpected features may have crucial meanings to the microscopist [162].

Figure 3.25 Typical engineering microscope [164] 
Morphology is the organization and form on a size scale above atomic but smaller than the size and shape of the whole sample. Polymer morphology consists of the size, distribution and relationship of structural units within the macrostructure. Structure usually deals with details about atoms and molecules. Moreover, morphology and microstructure are observed to investigate the relationships between structure, property and process using characterization tools [8].

Morphological studies are designed in such a manner that right microscopic tools are used in answering various questions e.g. whether does a relationship between material's structure and material's performance exit? Hence microscopic studies play a vital role in exposing several interesting features [162].

Various phenomena relevant to physical chemistry are well addressed with the assistance of microscopes by directly witnessing the applications of basic principles. Having observed these phenomena, one may have the convincing bases for structural changes which take place in various materials [162].

Engineers from many disciplines are trained for microscopic studies in an effort to reveal material's structure. Pictographic illustrations are of great interest for pictorial minded researchers who do not acquire instant information from tables and curves. Moreover, these illustrative ways of communicating scientific ideas appear to be great recipes for the material engineers. A technical microscopist is thought of preaching various fields of science and engineering at micro level to engineers and scientists solving definitely many technical problems in the physical world [162].

Textile industry has extensively used the light microscopy in exploring many scientific phenomena within textile materials. Textile industries have also focused greatly on research and development by establishing a microscopic section along with production \& control sections. In short owing to general gratitude for the role of microscopy, technical microscopy has resulted in perceiving the nature of various textiles and contributing towards advancement \& manufacturing of various adequate products in textile industry [162]. A video textile microscope is exhibited in Figure 3.26. 
Figure 3.26 A video textile microscope [165]

Fractography is used to explore the welding, loading and environmental conditions which generate fracture by interpreting the visual appearance of fractured parts [166].

Heat affected zone (HAZ) can critically be examined microscopically in an effort to compare bond features for various weld factors which result in high LSS.

\section{Weld Morphology, Heat Affected Zone (HAZ) And Fractography}

Kamal and Chung[167] have explored the HAZ and fractography of polyamide-6 that is welded by vibration welding. Authors have addressed two types of HAZES i.e. deformed and recrystallized. They have found that the thickness of different HAZ surfaces and the size of spherulites in HAZ depend upon weld factors such as weld pressure and amplitude.

Welding factors have great impact on development of HAZ and ultimately on LSS. Thickness of $H A Z$ is an important cause of bond effectiveness needing to be properly considered.

Oliveira et al.[168] have found that microscopy and mechanical tests are essential for analysing polypropylene tensile bars which are prepared by using hot plate welding. The hot plate welding has produced significant changes to the microstructure and shape of the bars at weld area. Weld morphology has been influenced majorly by both thermal history and welding conditions. The key morphological factors affecting the weld quality include oriented textures, coarse spherulites, voids or sharp notches that happen at the beads. As far as quality of weld is concerned, tensile impact test is found to be better in addressing the morphology which affects the fracture behaviour. Authors have also concluded that optimum welds are free of voids and oriented at central zone. 
Balkan et al.[169]examined morphology and mechanical properties of polyvinyl chloride $(\mathrm{PVC})$, polyethylene (PE), and polypropylene (PP) after welding them with hot gas butt welding. Four welding methodologies have been employed i.e. double $\mathrm{V}$ welds, single $\mathrm{V}$ welds, with welding shoe and without welding shoe. Rheological techniques have been followed for evaluating the weld-abilities of base materials. Weld-abilities of PE and PP are found to be superior to that of PVC. Weld quality has been determined by taking welding energy into considerations. Based on the results from various tests, weld strength of PVC sheets has been found to be lower than those of PE and PP sheets. By using welding shoe, weld quality has been improved for three materials owing to sufficient welding pressure and exposure of effective heating. Scanning electron, polarized light, and stereo microscopy have been utilized for investigating the morphology of welded regions. Heat affected zone (HAZ) has been revealed by polarized light microscopic studies comprising welding rod core, deformed spherulitic zone, and molten zone.

Cakmak et al.[170] have found that the weld interface and planes of naphthalene have been observed to be parallel and similar to graphitic structure owing to shearing action. This has imparted a detrimental effect on mechanical properties of specimens which are joined by utilizing amorphous precursors. Traditional HAZ structure has been determined for those parts which are joined by using crystalline precursors. Hubert et al.[171] have found that quality of weld remains good along the weld zone. Presence of voids and porosities at edges of samples has reduced weld quality when observing by ultrasonic C-Scan and optical microscopy. This is due to the fact that pressure has been decreased at the edges during resistance welding.

Carboni [172]investigated the premature failure of two aluminium alloy sonotrodes which are used for welding thermoplastics. Fatigue cracks at stress concentrations are found to be the one reason of premature failure by using NDT (Non-destructive testing) method. Optically dark areas and metallurgical defects are found to be two distinct crack initiation modes, as exhibited from SEM observations. Chemical composition of aluminium is demonstrated by metallurgical analyses. As far as optically dark areas are concerned, segregation defects and oxygen trapped inside may be thought of playing a basic role. 
Author has presented the failure analysis of aluminium alloy sonotrodes by using SEM. SEM may also be used for analysing the failure of ultrasonic bond.

Balle et al. [173]investigated the bonding mechanism of Al/CFRP (Aluminium/Carbon fibre reinforced polymer) joints microscopically. Authors have detected the mechanically deformed areas due to reorientation of crystal structure after welding. It has been found that not only the surface enlargement owing to higher roughness but also the submicroscopic oxide structure generation is crucial to improve the mechanical properties of ultrasonically welded Al/CFRP.

A better microscopic investigation has been achieved for ultrasonically welded joints. Effect of different weld and geometrical factors may also be investigated on mechanical performance of welded samples.

\section{Modes Of Fracture [166]}

A fractured surface is developed just after the fracture when the applied stress at some local region dominates the strength of material. Fractured surface also depends on complex interaction between material type, environmental conditions, loading conditions, and time.

There are two well-known modes of fracture in thermoplastics i.e. ductile and brittle. Microscopic observations can also help us determine the presence of mode of fracture.

\section{Ductile Fracture [166]}

Ductile fractures are found with plastic deformation before fracture. Before failure, ductile fracture encounters with yielding, tearing, plastic deformation, necking, and shear. These all come one after another with certain shape changes and distortions to some extent.

Following are some examples of commercial polymeric systems which reveal ductile behaviour before fracturing;

- Acrylo-nitrile Butadiene Styrene (ABS)

- Polypropylene (PP)

- Polyethylene (PE)

- Polycarbonate (PC) 
- Polyvinyl Chloride(PVC)

\section{Brittle Fracture [166]}

Little or no plastic deformation is found before brittle fracture. This fracture mode happens on macroscopic level with no shape changes and distortions. Polymeric systems showing this type of fracture behaviour are listed below;

- Polymethyl Methacrylate (PMMA)

- Styrene Acrylonitrile(SAN)

- Polystyrene (PS)

- Thermosetting resins e.g. epoxy and polyester

\subsection{Research Space}

Several efforts have been made to join thermoplastics by using other welding processes. For example, vibration, resistance, and microwave welding have been reported as plastic welding techniques. Hence ultrasonic welding may also be used to reveal and understand its various hidden benefits. A comparison between ultrasonic and other welding processes has been presented at the end of chapter 2 .

There is a large amount of published studies which emphasizes on various aspects of ultrasonic welding. Many researchers have delivered state of the art efforts in order to collect sufficient scientific information in this area. Most of the work, still, needs to be addressed due to the presence of room for research on UW of thermoplastics. This section is intended to mention explicitly the overall novelties in the thesis by identifying the research space. 
Research gaps/spaces are being highlighted, as shown in Table 3.1. Ultrasonic welding has extensively been researched by using metals and plastics but thorough research on thermoplastics is necessary for the sake of understanding this process.

Table 3.1 Research gaps/spaces

\begin{tabular}{|c|c|c|c|}
\hline Main References & Topic Area Covered & Research Space/Gap & Current Research to fill gap \\
\hline $\begin{array}{c}43,46,49,106,63 \\
60,45,66,53,58,56 \\
65,112\end{array}$ & $\begin{array}{l}\text { Effect of welding } \\
\text { factors on ultrasonic } \\
\text { welding of } \\
\text { thermoplastics }\end{array}$ & $\begin{array}{l}\text { Effect of few weld factors has frequently been found } \\
\text { on the bond strength in literature without giving due } \\
\text { importance to other ultrasonic welding factors e.g. } \\
\text { amplitude, weld time, ED etc. } \\
\text { Few authors have also attempted to use only one } \\
\text { material type during UW of thermoplastics. A better } \\
\text { insight into this process can also be obtained by } \\
\text { welding experiments for two different thermoplastics. } \\
\text { Ultrasonic welding has also joined thermoplastics } \\
\text { without preparing surface of samples. In fact, one } \\
\text { geometrical shape of energy director (ED) e.g. } \\
\text { triangular (TRI) or semicircular (SEMI) must be utilized } \\
\text { at least to achieve better weld strength. }\end{array}$ & $\begin{array}{l}\text { To fill this gap, all possible weld factors have been } \\
\text { planned and implemented including material type, } \\
\text { weld time, hold time, static force, amplitude of } \\
\text { ultrasonic vibrations and different geometries of } \\
\text { energy directors (EDs). } \\
\text { In this study, ABS and PP were also welded } \\
\text { ultrasonically. } \\
\text { TRI and SEMI EDs were used in this work. } \\
\text { Hence effect of various ED geometries on } \\
\text { ultrasonic bond can also be investigated. }\end{array}$ \\
\hline $\begin{array}{c}33,37,121,174,131 \\
132\end{array}$ & Modelling of UWP & $\begin{array}{l}\text { Previous research has shown the use of 2D model in } \\
\text { FEA for UW. Therefore, a 3D approach has been } \\
\text { desired to optimize FE analysis without compromising } \\
\text { on the computational costs that are associated with } \\
\text { FEA. Moreover, in an analysis of FE (by Wang [174]), } \\
\text { FEA is lacking the validation which is considered the } \\
\text { essential part of computational studies. }\end{array}$ & $\begin{array}{l}\text { To better understand the FEA approach and its } \\
\text { effect on the temperature distribution at weld } \\
\text { zone, different combinations of weld factors were } \\
\text { used for three dimensional finite element } \\
\text { modelling in this research. This simulation has also } \\
\text { been validated. }\end{array}$ \\
\hline 167 to 173 & $\begin{array}{l}\text { Microscopy and } \\
\text { visualization for UWP }\end{array}$ & $\begin{array}{l}\text { Fewest research articles have been found on } \\
\text { investigation of heat affected zone (HAZ) and } \\
\text { fractured surfaces (fractography) which result from } \\
\text { ultrasonic welding. } \\
\text { Collapse of ED can also affect bond strength during } \\
\text { UW of thermoplastics. High speed video camera } \\
\text { (HSVC) has the capability of recording and playing } \\
\text { back various phenomena that occur at high speed. }\end{array}$ & $\begin{array}{l}\text { Hence a broad microscopic analysis was achieved, } \\
\text { in this work, to address sufficiently HAZ and } \\
\text { fractography leading to acquire various hints of } \\
\text { physical phenomena that occur during welding and } \\
\text { testing. HAZ depending on levels of weld factors } \\
\text { has also been investigated to predict the amount } \\
\text { of LSS after welding thermoplastics ultrasonically. } \\
\text { In this study, HSVC was also used to find degree of }\end{array}$ \\
\hline
\end{tabular}




\begin{tabular}{|c|c|c|c|}
\hline Main References & Topic Area Covered & Research Space/Gap & Current Research to fill gap \\
\hline & & $\begin{array}{l}\text { There are no comprehensive studies using HSVC to } \\
\text { find the effect of ED collapse on bond strength. }\end{array}$ & $\begin{array}{l}\text { ED collapse during ultrasonic welding of ABS and } \\
\text { PP. }\end{array}$ \\
\hline $59,99,108,110,111$ & Statistical Analyses & $\begin{array}{l}\text { Some authors have mentioned the experimental } \\
\text { design procedures (e.g. by Taguchi) in determining the } \\
\text { significance of various weld factors. This type of } \\
\text { experimentation is short at its length and breadth for } \\
\text { understanding UW of thermoplastics. An overall } \\
\text { statistical method must be set forth to provide an in- } \\
\text { depth analysis of the effects of each weld factor on } \\
\text { bond strength. }\end{array}$ & $\begin{array}{l}\text { A statistical method called "General Linear Model } \\
\text { (GLM)" was used in this research owing to many } \\
\text { benefits which were associated with this method. }\end{array}$ \\
\hline $149,151,158,159$ & Material analyses & $\begin{array}{l}\text { DSC and MFI tests have rarely been conducted for } \\
\text { gaining thermal and flow characteristics of materials } \\
\text { before sampling and welding. DSC and MFI tools can } \\
\text { be innovatively used to investigate melting behaviour } \\
\text { of thermoplastics, degradation of thermoplastics } \\
\text { under severe heating during sampling and UW, and } \\
\text { viscosity of thermoplastics that may affect the } \\
\text { ultrasonic bond strength. }\end{array}$ & $\begin{array}{l}\text { In this study, DSC and MFI tools were used to know } \\
\text { the thermal and flow characteristics of ABS and PP. }\end{array}$ \\
\hline 50,101 & $\begin{array}{l}\text { Energy calculations } \\
\text { while welding } \\
\text { thermoplastics } \\
\text { ultrasonically }\end{array}$ & $\begin{array}{l}\text { Estimation of ultrasonic energy has also been done } \\
\text { but this appears to be complicated. In other words, a } \\
\text { simple way of calculating this energy needs to } \\
\text { consider for finding out its effect on bond strength. }\end{array}$ & $\begin{array}{l}\text { Welding energy was calculated by using basic } \\
\text { physics. } \\
E=F^{*} f^{*} \lambda^{*} t \\
\text { Where, } E=\text { Energy } \\
F=\text { Force } \\
\lambda=\text { Peak to peak amplitude } \\
t=\text { Weld Time } \\
f=\text { frequency }\end{array}$ \\
\hline
\end{tabular}




\section{CHAPTER 4 Methodology, Variables and Description of Procedure}

It has been clearly demonstrated that there is a lack of understanding of the factors influencing the UWP. This work presents an in-depth study into this area, through the following aim and objectives.

\subsection{AIM AND OBJECTIVES}

\subsubsection{AIM}

Aim of this research is to gain a deeper understanding of factors which influence the UW of thermoplastics in an attempt to improve the weld strength.

\subsubsection{OBJECTIVES}

In undertaking the aim so, following objectives were planned and achieved;

- Determining the bond strength experimentally for a definite range of factors by using both amorphous and semi-crystalline type of thermoplastics

- Identifying a suitable statistical analysis in determining the effect of weld factors on joint strength and the most effective weld factors

- Hypotheses development based on literature review and interesting effects from experimentation.

- Utilizing several experimental techniques in order to identify the reasons for various effects. These experimental techniques include:

1. DSC (Differential Scanning Calorimeter)

2. MFI (Melt Flow Index)

3. FEA (Finite Element Analysis)

4. HSVC (High Speed Video Camera)

5. Microscopy

\subsection{Aim of current chapter}

This chapter presents a detailed introduction to various factors/independent variables and their possible ranges for current ultrasonic welding rig, manufacturing of samples, description of procedures for welding thermoplastics and testing bond strength. At the end, 
experimental procedure in the form of a matrix has also been established after including calibration data of ultrasonic welding rig.

\subsection{Variables selected for investigation}

Having been discussed in literature review, ultrasonic welding is only possible accurately by considering certain process factors e.g. frequency and amplitude of ultrasonic vibrations, weld and hold time, energy director (ED) with certain geometry, material selection and static force. These factors are identified by investigating the available literature on ultrasonic welding process. It can be said with great confidence that ultrasonic welding is totally impossible without imparting frequency and amplitude of vibrations, weld time, and static force. Let us see why we need to introduce above process factors one by one and their threshold values during actual ultrasonic welding process.

According to literature, certain factors appear to be crucial but others affect the weld strength minimally. For example, amplitude, weld time and ED are found to be crucial to obtain optimum weld strength [10]. Based on previous literature, the ultrasonic welding process factors can be listed below:

- Material Selection

- Geometry of Energy Director (ED)

- Frequency of Vibrations

- Amplitude of Vibrations

- Static Force

- Weld Time

- Hold Time

\subsubsection{Material Selection}

The first factor is the selection of thermoplastic materials to be investigated. Holistically explaining, based on difference in their molecular structure, thermoplastics can be divided into two parts i.e. amorphous and semi-crystalline and these are expected to behave differently on welding them ultrasonically [37]. In an effort to understand and explore the ultrasonic welding of thermoplastics, ABS (Acrylo-Nitrile-Butadiene-Styrene) and PP (Polypropylene) have been selected with former as amorphous and later as semi-crystalline 
thermoplastic. These are well known standard manufacturing materials for their numerous applications. Ultrasonic welding of these two selected materials is beneficial $[33,37,43,46$, $111,175]$

\section{Polypropylene (PP)}

PP was manufactured by Natta in Spain for the very first time in 1954 . Since then it has achieved sufficient popularity as a good industrial product. PP is classified as a member of polymer systems having linear hydrocarbon configuration. It is also nominated as polyolefin. The chemical formula of PP is shown in Figure 4.1. Due to its versatility, it easily finds its application in various areas directly. Main properties making it attractive for many manufacturing processes and applications are its semi-crystalline molecular structure, translucence, toughness, chemical resistance, good fatigue, electrical, and heat resistance. As compared to Polyethylene (PE), PP is rigid having higher softening point, higher hardness and lower density [176].

Figure 4.1 Chemical Formula of Polypropylene [177]

Processing compatibility of polypropylene with all thermoplastic-processing methods makes PP even more desirable for manufacturers. Owing to its suitability (because of its low melt viscosity and pseudo plastic nature i.e. fast filling rates) for injection moulding, it is a good selection as a raw material to be welded ultrasonically. Due to high filling rates, surface finish remains better resulting in strong mouldings with lower moulding defects. Thanks to these superb material properties, PP has been selected to make samples from injection moulding [176].

PP is an acceptable commercial commodity having its major use in following areas [176]:

1. Flexible Packaging e.g. Foods, confectionaries, tobacco, and clothing.

2. Rigid Packaging e.g. CD Casings, Reusable, and stackable crates. 
3. Automotive e.g. interiors, PP film cushioning, bumpers, cladding, and paint-less manufacturing.

4. Consumer Products e.g. toys, housewares, furniture, appliances, luggage, and battery case.

\section{Acrylo-Nitrile-Butadiene-Styrene (ABS)}

ABS is a mixture of two polymer phases i.e. SAN (Styrene acrylonitrile) and Polybutadiene (PB). Former gives the necessary rigidity, hardness, and heat resistance but latter is responsible for toughness in ABS. Chemical formula of ABS is presented in Figure 4.2.

Figure 4.2 Chemical Formula of ABS [178]

Many grades of ABS are available in order to meet customer requirements. Modifications in $A B S$ are easily made by controlling the ratio of three monomers i.e. Acrylonitrile, Butadiene, and Styrene. In an attempt to increase the stiffness of ABS, fibre reinforcement is made with additional advantage of having dimensional stability.

Various desired properties are also obtained in ABS by alloying different polymers in controlled amount with ABS e.g. fire retardancy is included within ABS by either blending PVC with ABS or fire retardant additives with ABS.

$A B S$ is naturally found colourless and opaque. However, coloured and transparent $A B S$ grades have also been discovered. ABS is conveniently moulded making it suitable to 
produce samples for ultrasonic welding. Owing to its splendid properties, ABS has a wide range of applications. Following are some of its applications [179]:

1. Domestic Appliances

2. Telephone handsets

3. Office equipment housings

4. Lawn mower covers

5. Safety Helmets

6. Pipes and fittings

7. Automotive exterior and interior trim components.

PP and ABS have been supplied in granules form by SABIC and AZELIS Plastics respectively. PP is homopolymer for injection moulding with its trade name as SABIC ${ }^{\circledR}$ PP 579S, whereas ABS, supplied by AZELIS Plastics, has its trade name as ABS KUMHO $750 \mathrm{SW}$. Please see the datasheets for these materials in appendix B. Moreover, due to difference in molecular structure of both ABS and PP [37], ultrasonic welding may appear to be dissimilar for both materials.

\subsubsection{Geometry of Energy Director/s (ED/EDs)}

Type of ED has a great influence on strength of ultrasonic weld i.e. Lap Shear Strength (LSS). Various ED geometries were employed in the past $[99,107,108]$.

In relation to literature, two geometries of ED were utilized in this research. Two different geometries for EDs are exhibited in Figure 4.3:
i. $\quad$ Triangular (TRI)
ii. Semi-circular (SEMI) 


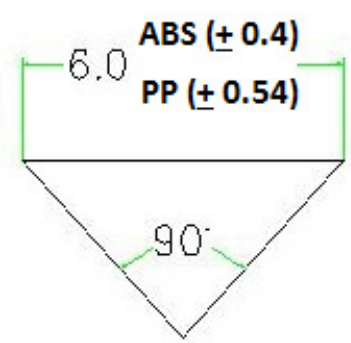

TRI-Angular

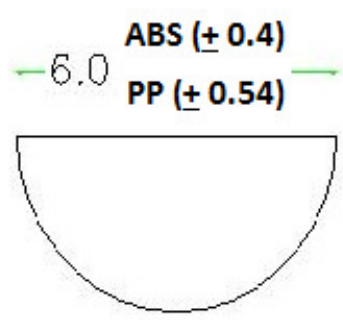

SEMI-Circular

\section{All dimensions are in mm}

Figure 4.3 Geometries of EDs

Effect of two different geometries of ED on bond strength has also been expected to be different. In this work, tolerances were associated with geometries of each ED for both ABS and PP separately according to BS 7010:1988.

In line with the accessible literature, geometrical factors and their dimension for above EDs are found to be small. So, in this study, it was decided to increase the available dimensions to some multiples. For instance, base of triangular ED and diameter of semi-circular ED are found to be equal to $2 \mathrm{~mm}$ in literature but here these have been made equal to $6 \mathrm{~mm}$ i.e. three times greater value $[99,107,108]$. Bigger dimension of ED has been proposed in order to supply more molten material improving weld strength. Also in order to introduce novelty in the sample design, a difference is thought to be necessary between geometries available in literature and the geometries of EDs for this research.

\subsubsection{Frequency of Vibrations}

For ultrasonic welding, frequency of vibrations should remain either equal to $20 \mathrm{KHz}$ or greater than $20 \mathrm{KHz}$ [37]. So, every effort must be made to keep this frequency of vibrations, which is produced by ultrasonic generator, equal to or above $20 \mathrm{KHz}$. In case of current rig, the operating frequency is $20 \mathrm{KHz}$ similar to value of frequency in literature [99, $107,108]$. 


\subsubsection{Amplitude of Vibrations}

Amplitude of vibrations is an important characteristic of vibrations. In an attempt to assess the influence of amplitude, four possible amplitudes of vibrations are present from ultrasonic generator i.e. $21,18.9,16.8$, and $14.7 \mu \mathrm{m}$ for current rig. In addition, these four amplitudes of ultrasonic vibrations are set manually to $100 \%, 90 \%, 80 \%$ and $70 \%$ of $21 \mu \mathrm{m}$.

\subsubsection{Static Force}

A range of static forces was used during present ultrasonic welding: 500, 750, 1000, 1250, 1500 and $1750 \mathrm{~N}$. Static force is applied on perpetual basis during one complete cycle of ultrasonic welding. Once applied, static force remains constant during the whole ultrasonic welding process i.e. during whole weld time and hold time.

\subsubsection{Weld Time}

Weld time is the magnitude of time for which ultrasonic vibrations with certain amplitude are kept ON. Careful selection of this time is necessary in order to get optimal weld strength. In current study, a general range of weld times was utilized for both PP and ABS: $0.4-1.7 \mathrm{sec}$ with an interval of $0.1 \mathrm{sec}$. The selection of this range agrees with the literature [110].

\subsubsection{Hold Time}

Hold time is that time for which welded samples, that are constrained by fixtures, are caused to be held between sonotrode and anvil under the action of static force (which has already been used for welding just a fraction of second ago) after ultrasonic vibrations are turned OFF. It is introduced because it helps crush the ED (softened while welding) and it also makes the ED spread evenly thereby resulting in good bonding [110]. Constant but different values of hold time were used for both PP and ABS in this work i.e. 3 and $0.2 \mathrm{sec}$ respectively. According to literature, hold time for semi-crystalline type of thermoplastic has been found to be higher than that of amorphous thermoplastic [111]. 


\subsection{Manufacturing of samples}

In this work, Injection moulds were designed for making samples with EDs (Energy Directors). Outer shape of moulds was produced and cut into two halves within University of Sheffield's premises. Outer shape and two halves of moulds are shown in Figure 4.4. The cavities with a provision of EDs were planned to be eroded and finished by Cheshire Wire Erosion Limited, UK. Cavities with and without ED are illustrated in Figure 4.5.

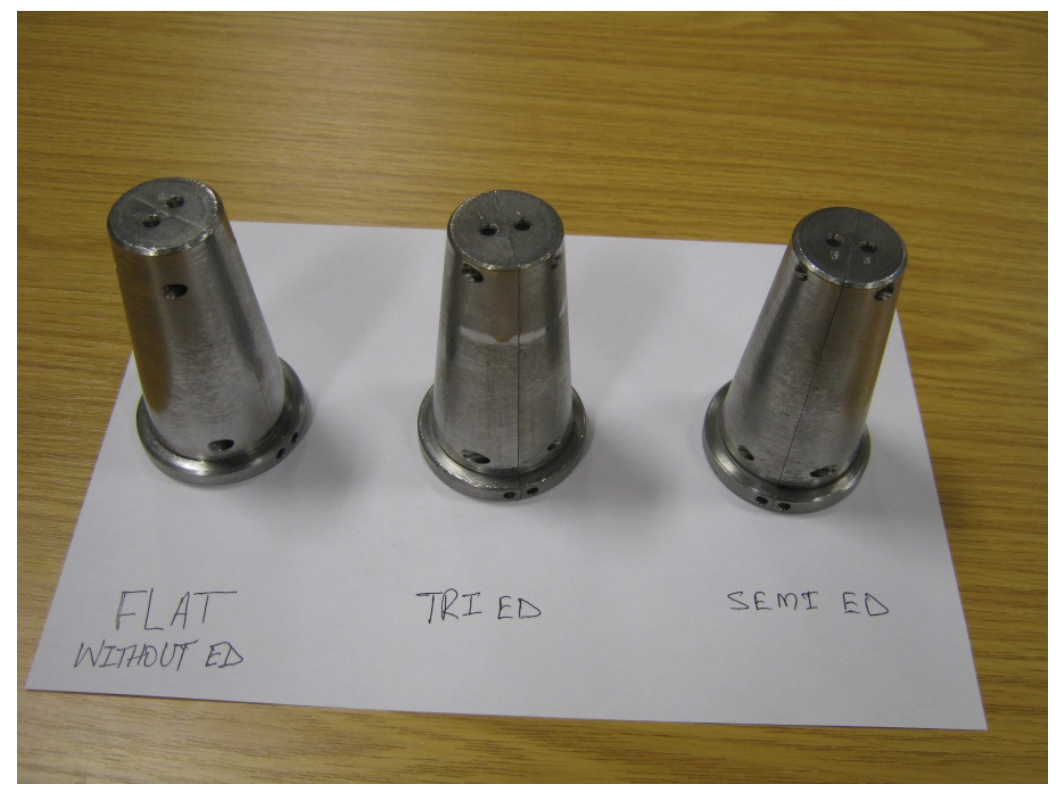

Figure 4.4 Outer shape and two halves of moulds

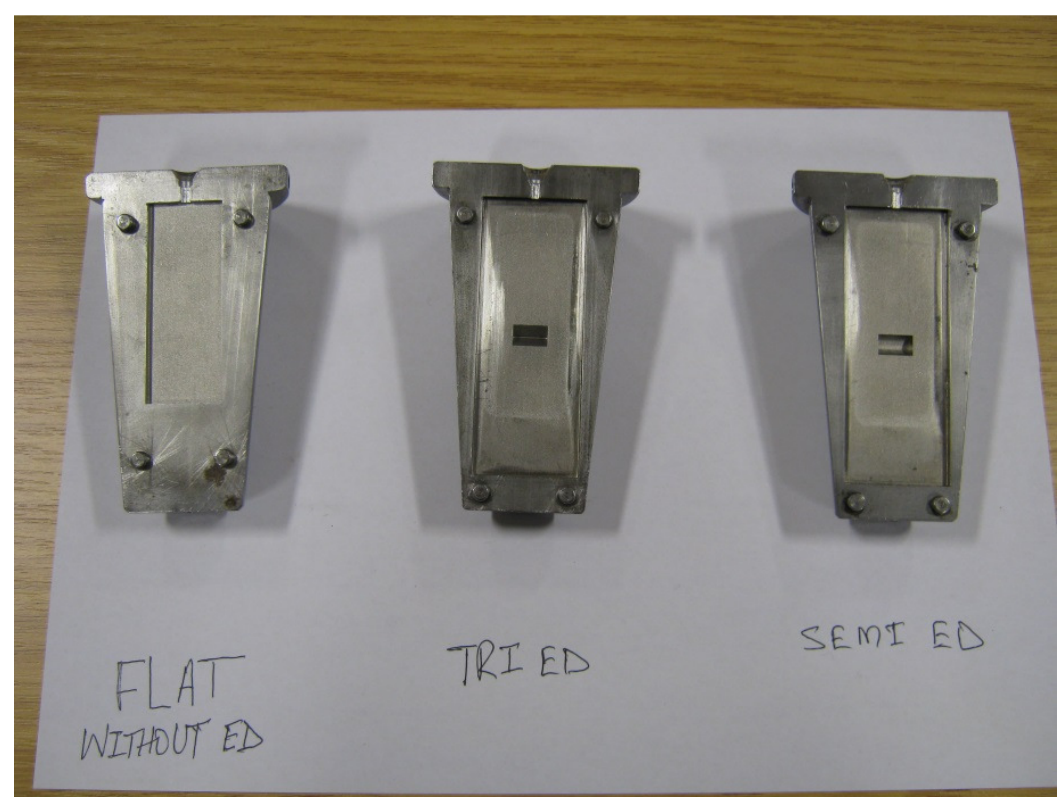

Figure 4.5 Cavities with and without a provision of ED 
HAAKE MiniJet Piston Injection Moulding System was intensively used for production of samples and this is shown in Figure 4.6.

An ASTM Standard i.e. D3641-97(08.02) and a British Standard i.e. EN ISO 294-1:1998 (article 5 Procedure except 5.2.2), which addressed a standard practice for injection moulding test specimens of thermoplastics moulding and extrusion materials, were followed during sample preparation.

Table 4.1 shows the parametric settings for injection moulding system which were observed constantly during sample preparation.

Table 4.1 Injection moulder's factor settings before preparing samples

\begin{tabular}{|c||c|c|c||c||}
\hline \hline Material & $\begin{array}{c}\text { Temperature } \\
\left({ }^{\circ} \mathbf{C}\right)\end{array}$ & $\begin{array}{c}\text { Injection } \\
\text { Pressure (bar) }\end{array}$ & Post Pressure (bar) & $\begin{array}{c}\text { Melting } \\
\text { Time } \\
\text { (minutes) }\end{array}$ \\
\hline \hline ABS & 200 & 660 & 500 & $3-5$ \\
\hline PP & $200-230$ & $\begin{array}{c}200-550(\mathrm{MIN}- \\
\mathrm{MAX})\end{array}$ & $150-500$ (MIN-MAX) & $4-7$ \\
\hline
\end{tabular}


Figure 4.6 HAAKE* MiniJet Piston Injection Molding System [180]

Following cares were adopted to get the desired quality of samples moulded:

- Air bubbles were prevented from being penetrated in the samples without letting the molten thermoplastic expose to air for long time.

- Cleaning of cylinder was done by running PP or ABS molten material through cylinder prior to make parts.

- Good surface finish was obtained by rejecting samples with rough surface through visual inspection.

- Removing useless flashes by a cutter.

Small sandwich plastic sealable bags were repeatedly used to store the samples with clear labels on them to be recognized on welding ultrasonically. Moulded samples in plastic sealable bags are shown in Figure 4.7. Welded samples were written with codes according to the matrix's history and put in a carton. Coded sample in tensile tester is exhibited in 
Figure 4.15. These samples were placed in steel cabinet at room temperature. Table 4.2 shows the room conditions at which samples were stored.

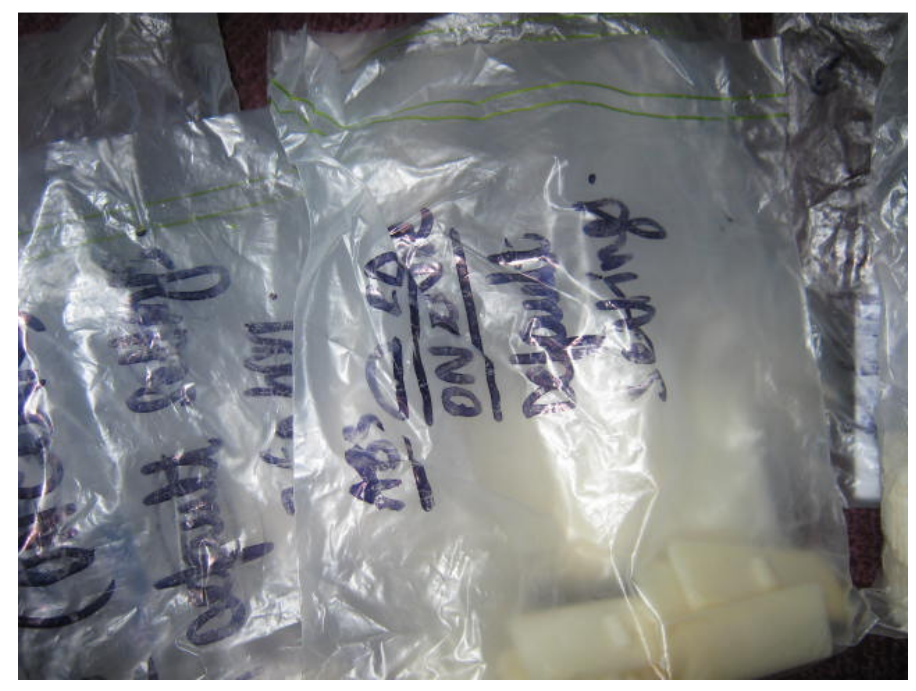

Figure 4.7 Moulded samples in plastic sealable bags

Table 4.2 Room Conditions

\begin{tabular}{|c||c||}
\hline $\begin{array}{c}\text { Room } \\
\text { Temperature }\left({ }^{\circ} \mathrm{C}\right)\end{array}$ & $\begin{array}{c}\text { Room } \\
\text { Humidity (\%) }\end{array}$ \\
\hline \hline 21.03 & 54.63 \\
\hline
\end{tabular}

\subsection{Welding}

After producing the samples, next step was to properly orient them between two fixtures on anvil before welding. Orientation of thermoplastic samples before welding them ultrasonically is shown in Figure 4.8 and Figure 4.9. Drawings of fixtures and anvil are provided with appendix E. 


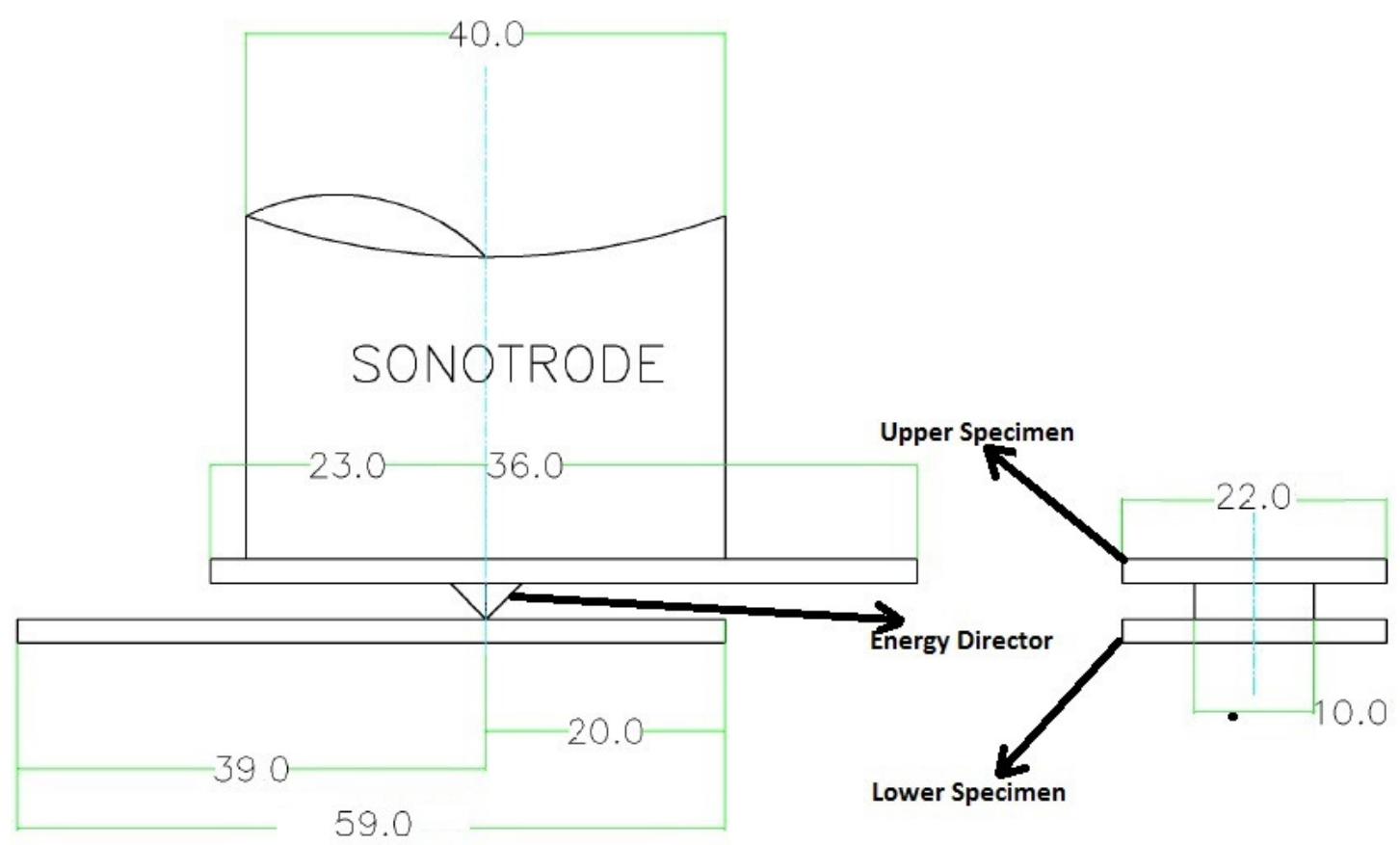

Figure 4.8 Orientation of upper and lower specimens
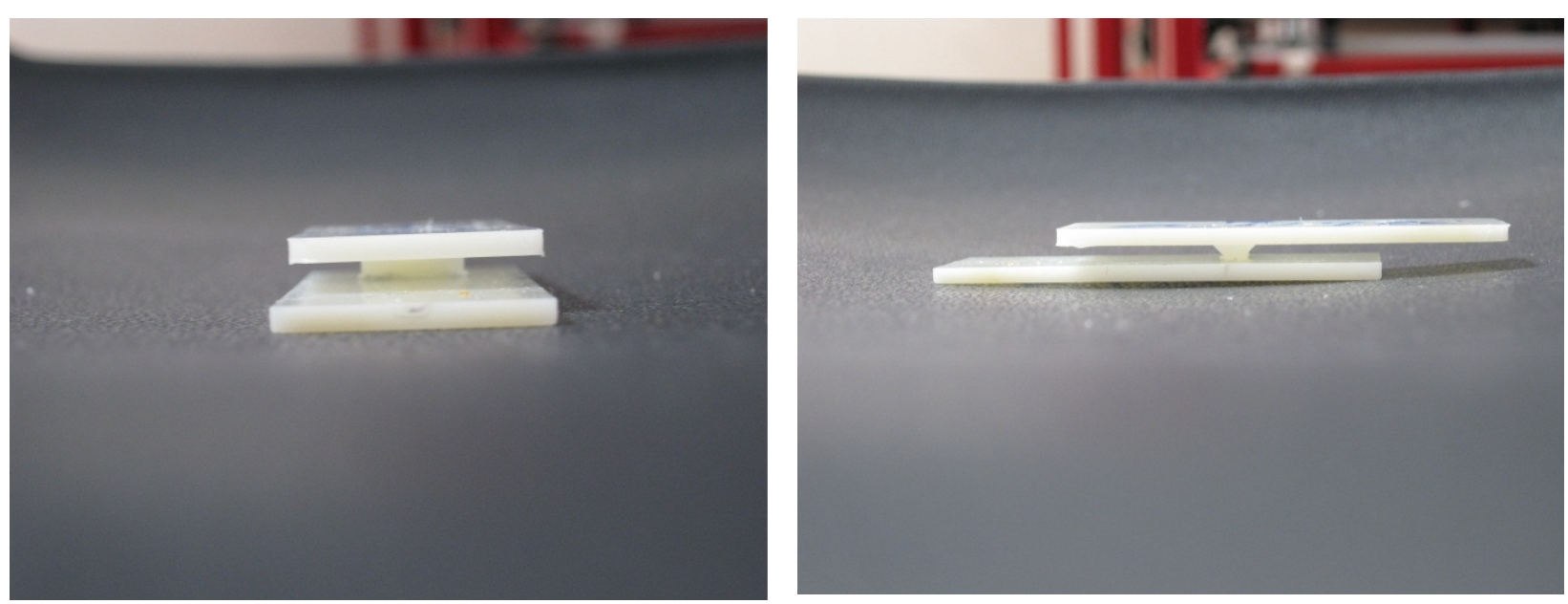

Figure 4.9 Orientation of real samples

After orienting properly upper and lower parts, next step was to identify and set basic factors for welding them ultrasonically by following the matrix of experiments.

\subsection{Equipment Used}

Ultrasonic welding setup needs an ultrasonic generator and sonotrode in delivering ultrasonic vibrations to the specimens. Static force is also required to weld specimens 
ultrasonically. In an ultrasonic welding, both ultrasonic vibrations and static force are applied simultaneously. In this study, ultrasonic welding rig was made by utilizing the Mayes structure in an effort to assemble its necessary parts. For availability of static force in ultrasonic rig, air compressor was used. A pneumatic circuit has been presented in appendix E. Weld time and hold time were set with the help of a PLC and a dedicated computer. Parts of ultrasonic welding rig are shown in Figures 4.10 to 4.12 .

\subsubsection{Description of procedure}

Ultrasonic welding factors were set by utilizing a combination of PLC, pneumatics and a dedicated computer. First of all, weld and hold time were decided and finally set using a PLC programme.

Next stages involved turning the ultrasonic generator on, setting static force by using the air compressor, and setting the proper amplitude of ultrasonic vibrations. In present ultrasonic rig, transducer of sonotrode was used to convert electrical energy at $20 \mathrm{KHz}$ into mechanical energy giving peak to peak amplitude i.e. $14 \mu \mathrm{m}$. This amplitude was amplified by a booster up to a value of $21 \mu \mathrm{m}$ as maximum amplitude.

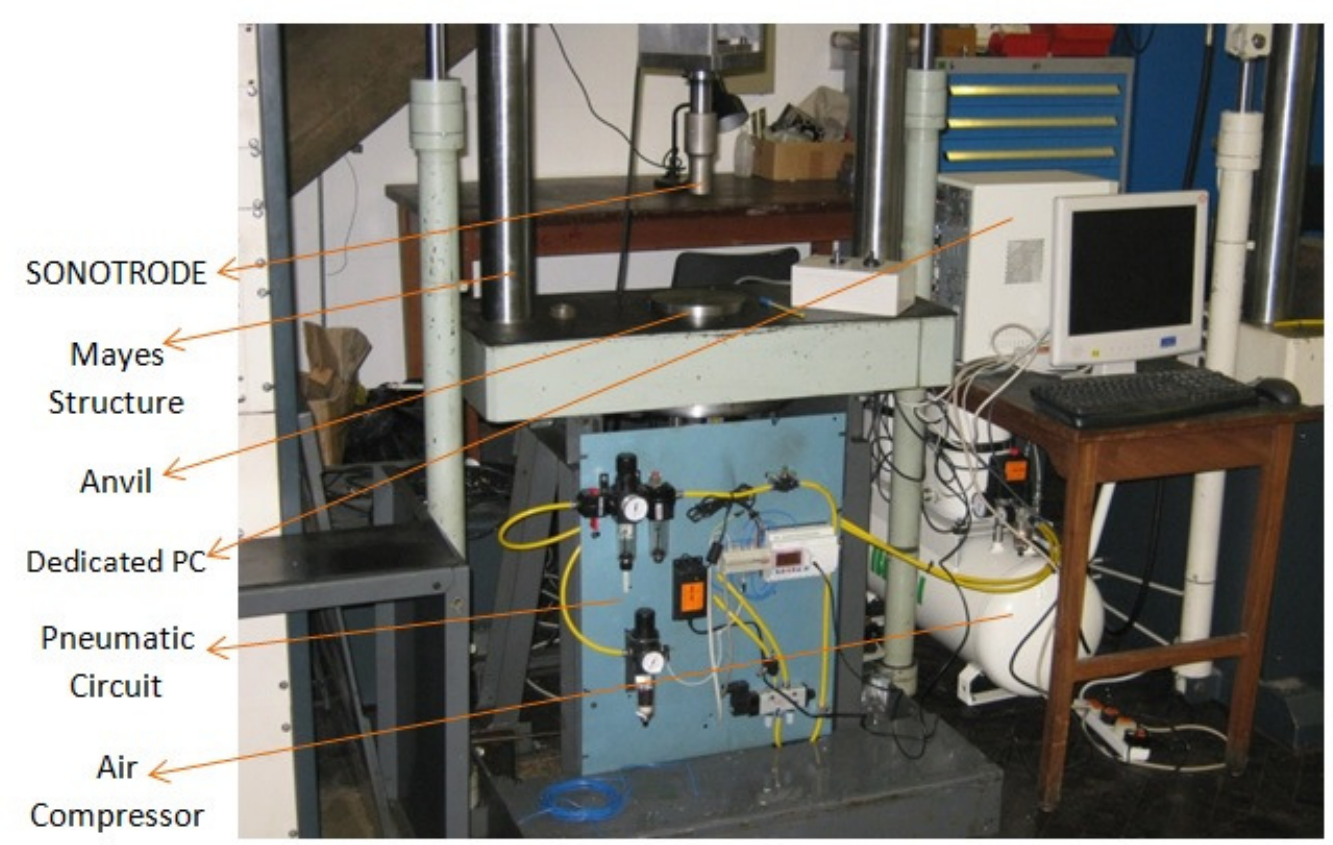

Figure 4.10 Ultrasonic welding rig with a dedicated computer 


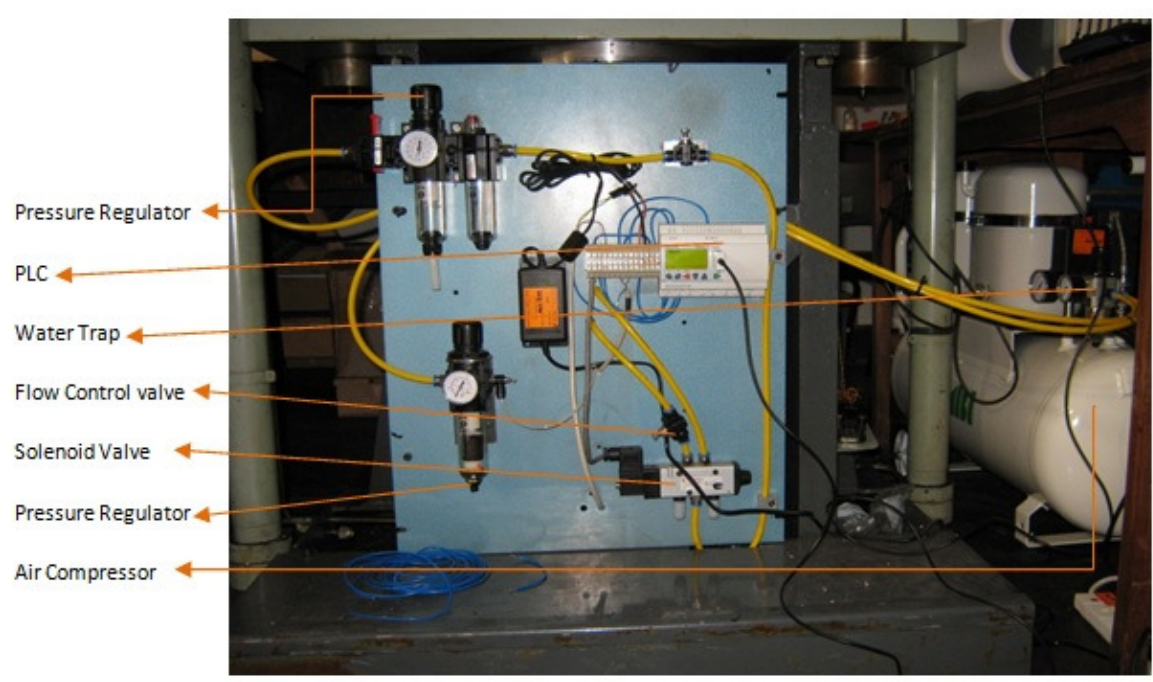

Figure 4.11 Real pneumatic circuit

Ultrasonic welding was initiated with a set static force raising the anvil against sonotrode before locating samples between anvil and sonotrode. The value of static force on the PLC can be seen clearly by using a pressure transducer. Bambi air compressor was used to provide the necessary static forces during welding. Samples to be welded were then loaded and fixed manually between fixtures on an anvil. Ultrasonic welding was finally started by pressing a programmed button on PLC. After ensuring and allowing some time to apply the set static force, ultrasonic vibrations with set characteristics were turned on for certain weld time and turned off for certain hold time as per instructions of PLC programme resulting in welding samples. Anvil was then lowered ultimately by releasing the static force again via PLC programme. Welded samples were lastly removed from the fixtures and anvil. Proper code was written on welded specimens with the permanent marker and brought to Hounsfield Tensometer where it was tested for getting LSS (Lap Shear Strength) measured. 


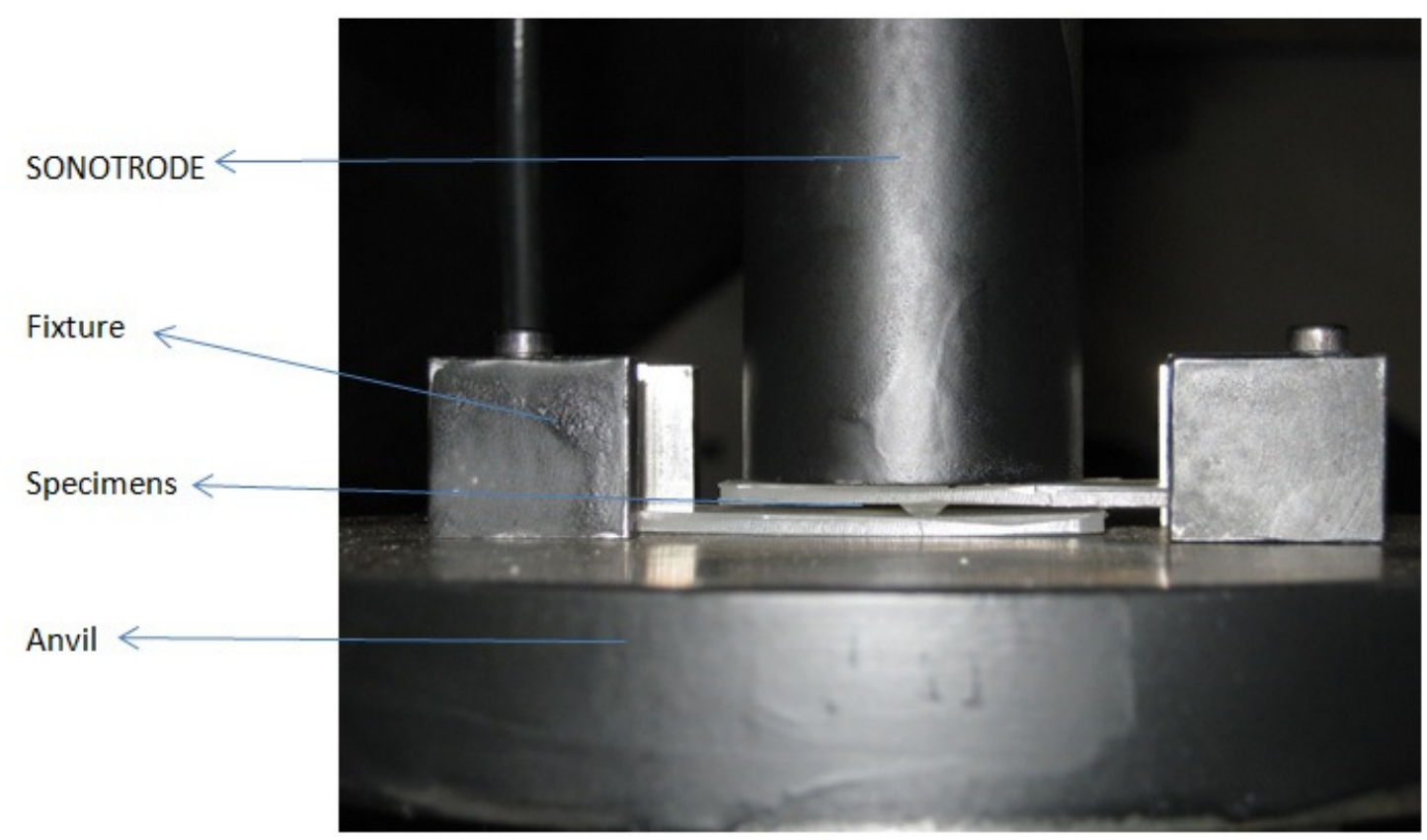

Figure 4.12 Compressed and held samples via fixtures, anvil and sonotrode

\subsection{Measurement of Lap Shear Strength (LSS)}

For various weld factors, LSS of ultrasonic bond was measured by Hounsfield Tensometer and Motorised Hounsfield Tensometer is illustrated in Figure 4.13.

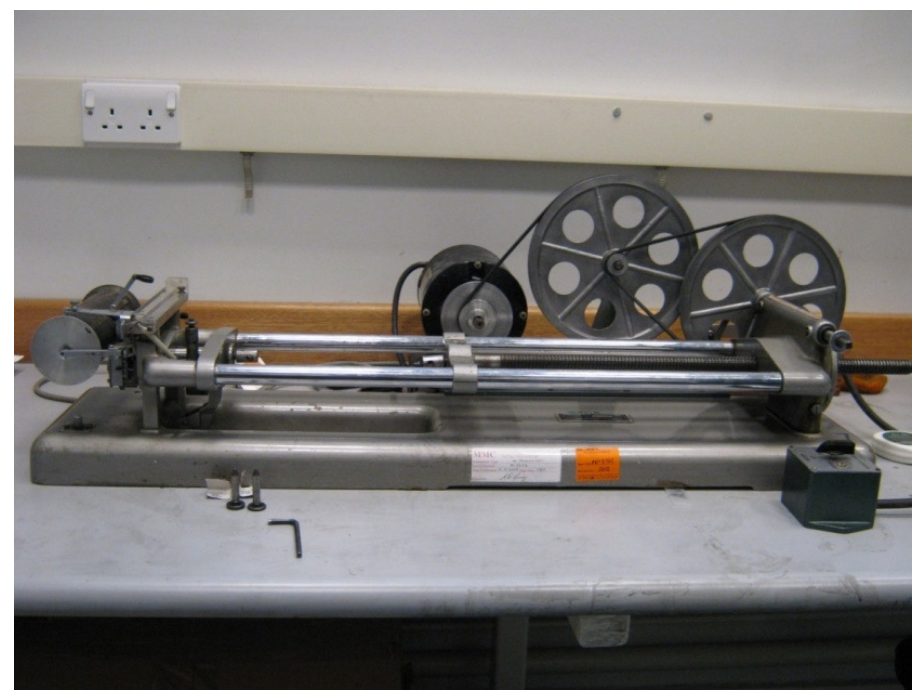

Figure 4.13 Motorized Hounsfield Tensometer

Each welded joint was clamped and sheared by motorized shear tester at a constant shear strain rate of $3.24 \mathrm{~mm} /$ minute throughout. This is due to the reason that different LSS may 
be obtained by different shear strain rates for the same welding conditions. A particular state of welded ABS specimens is exhibited in Figure 4.14 under severe shear force at constant strain rate.

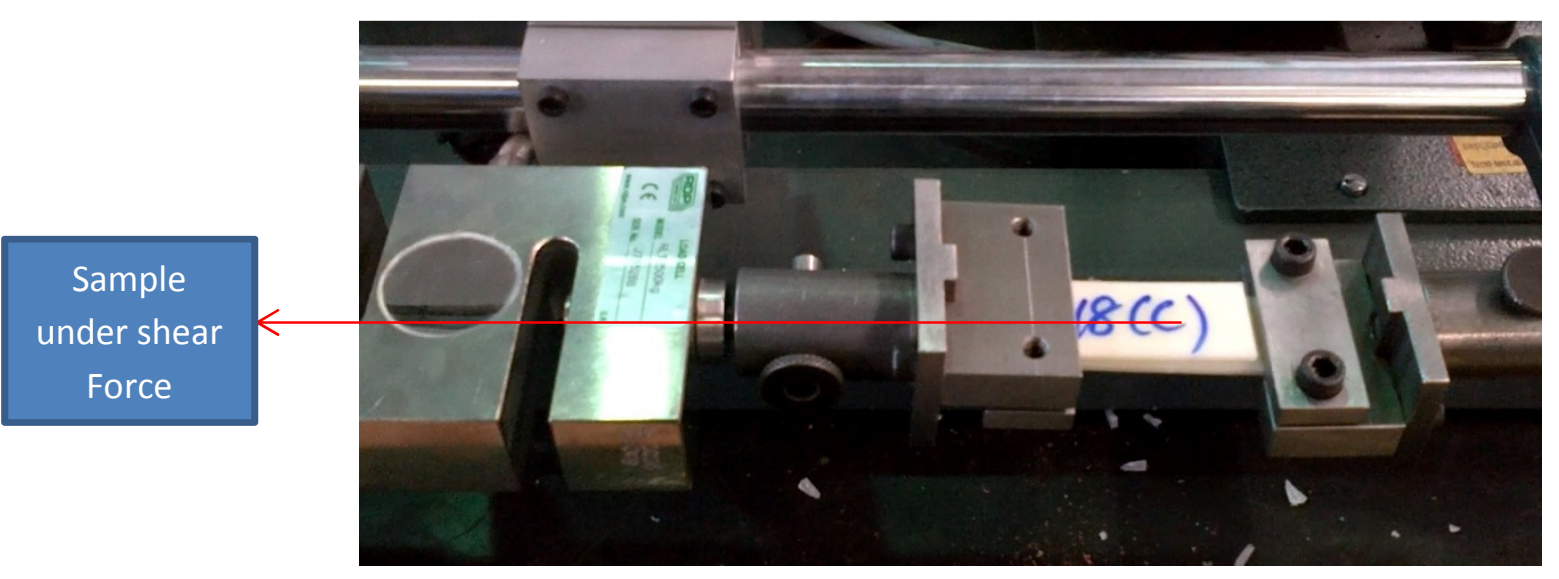

Figure 4.14 Tensometer applying shear strain at weld interface

Moreover, shear clamps were designed considering the configuration of the welded joint and a special care was taken for concurrence of line of action of shear force with centreline of the weld zone. This concurrence of lines is illustrated in Figure 4.15. Moveable and stationary jaws are included by each shear clamp. Moveable jaw can be moved up and down for achieving the coincidence of lines accurately. Furthermore, the right hand side clamp is the inverted image of the left hand side clamp.

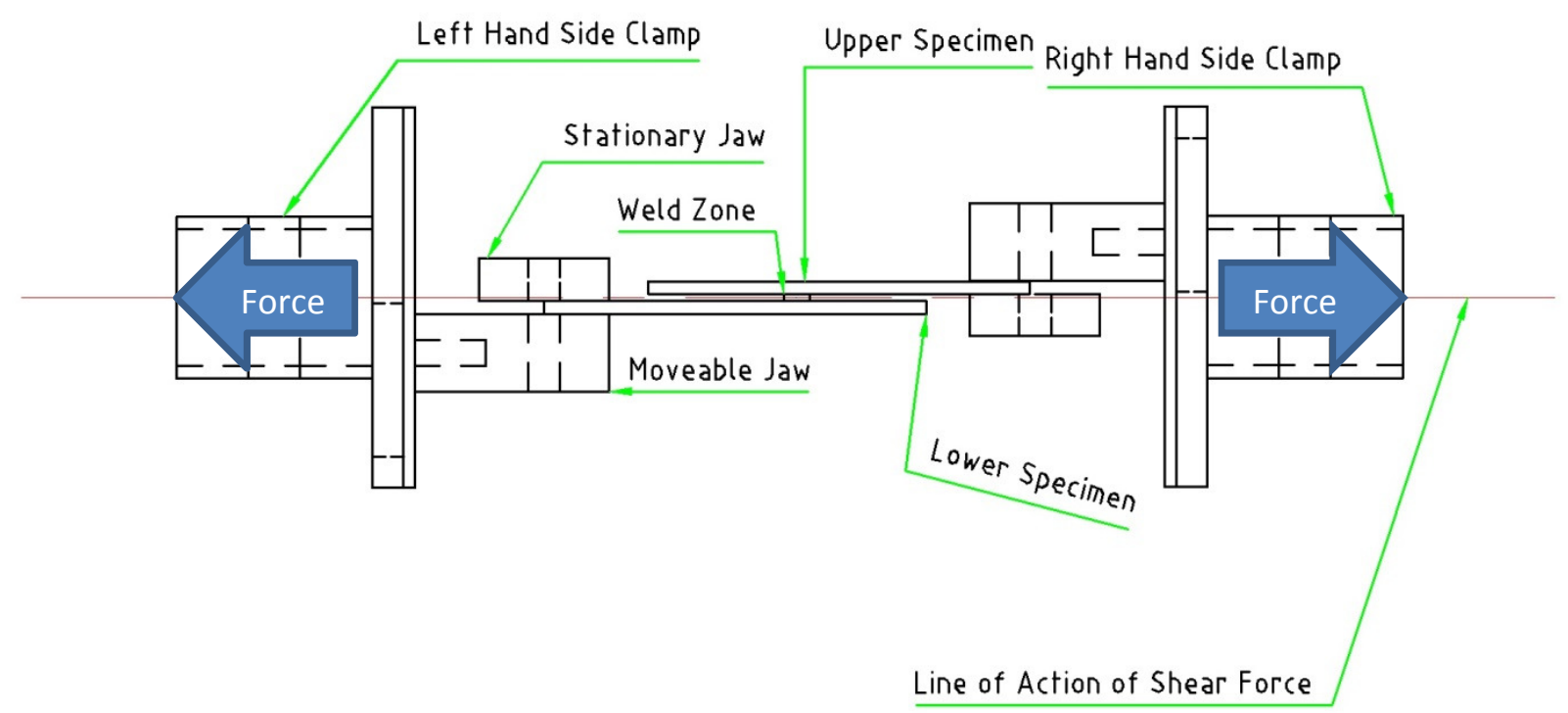

Figure 4.15 Coincidence of line of action of force and centreline of weld zone 


\subsubsection{Description of Procedure}

It consisted of holding a welded sample between two clamps. A pulling force was then applied in order to increase the distance between the jaws resulting in shearing the sample and fracture at the welded zone. Value of force was monitored and noted running on digital force meter for constant strain rate. Fractured area was calculated by means of vernier callipers. Hence a numerical value of lap shear strength was obtained in tensile fashion.

A term defined at this stage was called welding efficiency. Welding efficiency is the ratio of strength of ultrasonic bond and strength of parent material i.e. PP or ABS to be welded. When achieving 'welding efficiency' greater than one, ultrasonic bond between parent materials was practically impossible to measure due to the primary failure of parent component away from the weld zone. This is represented in Figure 4.16 (A\&B).
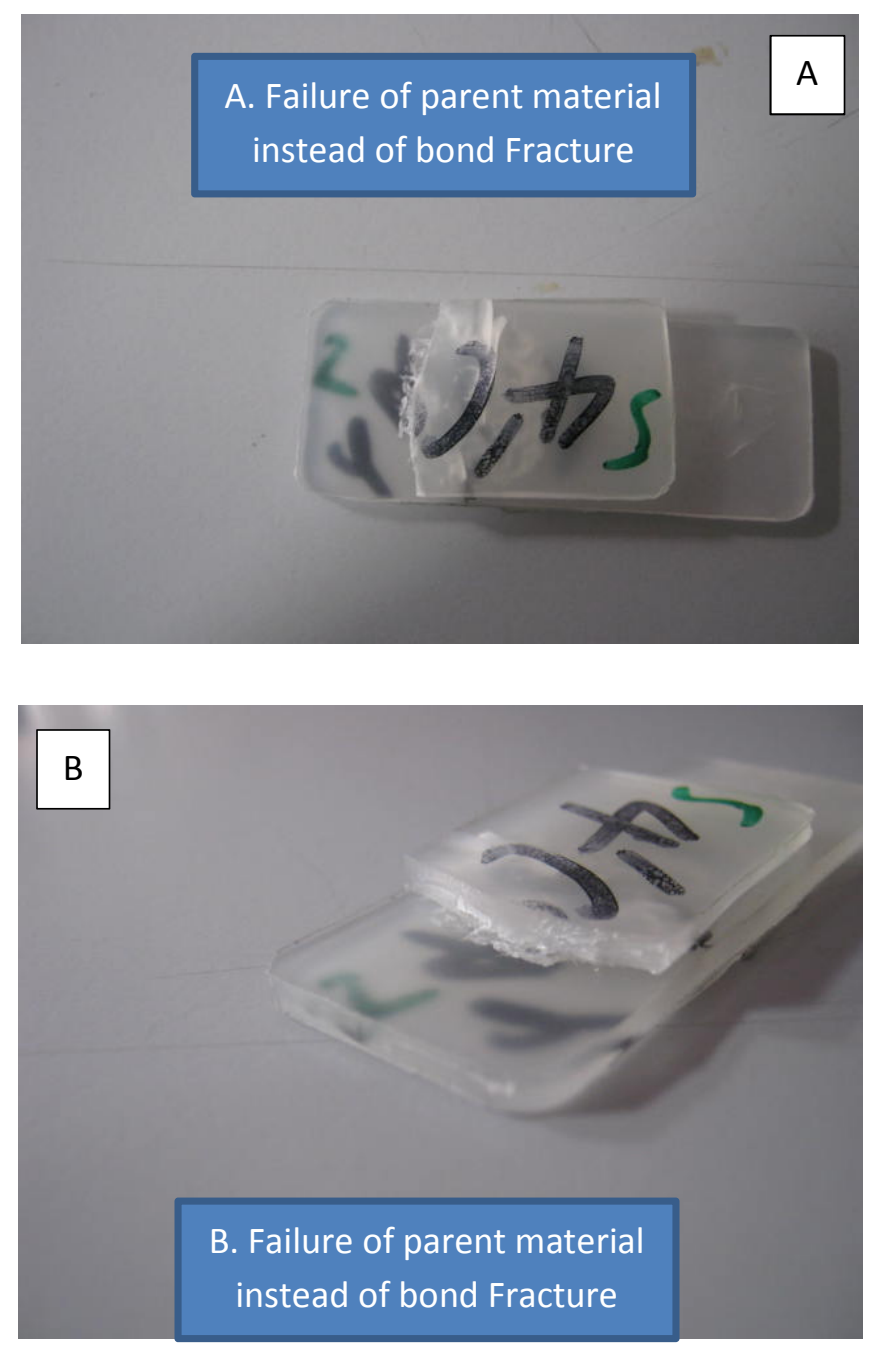

Figure 4.16 (A\&B) Fractured sample with welding efficiency 
Following equation was used to calculate the LSS of each welded sample:

Lap Shear Strength = Force required to break the welded joint /

Calculated resisting area directly from the fractured samples

Force required to break the ultrasonic bond was measured instantaneously from the Hounsfield Tensometer, whereas fractured area was measured by using vernier callipers. This area was approximated to be rectangle or circular depending upon measured values as appropriate.

Drawings of stationary and moveable jaws of clamps can be found in appendix $E$.

\subsection{Calibration of Ultrasonic Welding Rig}

\subsubsection{Detailed Aim}

In this section of chapter 4 , calibration data are presented to provide the reader with an idea of reliability of subsequent results from ultrasonic welding of thermoplastics. Calibration of current ultrasonic welder has been done using a reference ultrasonic welder from literature.

\subsubsection{Methodology}

After designing and manufacturing ultrasonic welding rig, the next stage was to ensure that this rig could deliver the desired results in terms of weld strengths accurately. Calibration covers not only this phase of ensuring accurate strength data but it is also a way of validating the design of new samples and manufacturing of samples.

Hence in context of current research, calibration can be defined as, "It is a method to verify the UW rig for its actual performance with the help of available performance data in literature from an accurate UW rig".

In this work, the actual performance meant how good the assemblage of samples was after welding i.e. bond strength. ABS samples were manufactured for calibration of current UW rig. Therefore, current UW rig and tensometer were used to obtain bond strength and match it with bond strength data of literature by using the same weld factors. 


\subsubsection{Description of procedure}

Ultrasonic welding rig must provide an opportunity to the operator with accurate measurements in the form of weld results. For this purpose, a reference ultrasonic welder (ultrasonic plastic machine) manufactured by $\mathrm{M} / \mathrm{s}$ National Indosonic was selected from literature [37] to calibrate current welding rig. Weld strength data were collected for referenced equipment and present welding rig for same set of weld factors.

\section{Variables used}

Following weld factors were set for ABS with both TRI and SEMI EDs producing maximum LSS. These factors were same for both referenced equipment and present welding rig.

\section{Material Type}

ABS was used for calibrating the ultrasonic welding rig.

Geometry of ED

Two geometries of ED were utilized in this calibration, as can be seen in section 4.2.2.

Frequency of Vibrations

Frequency of ultrasonic vibrations was $20 \mathrm{KHz}$.

Weld Time

For TRI ED, the weld time $(0.4 \mathrm{Sec})$ used for current welder is almost similar to the weld time (0.3 Sec) for referenced welder.

In addition to above factors, there are levels of some weld factors fixed for current welder which may be different from that of the referenced welder. The reason for having different levels of factors is the lack of information on them in published work. For instance, amplitude of vibrations, hold time and static force are not clearly mentioned for the referenced welder. Hence those levels of these three weld factors have been selected contributing to achieve maximum LSS. Therefore, weld time was set to 0.9 sec for SEMI ED. 
Amplitude of Vibrations

Amplitude of vibrations set was equal to $21 \mu \mathrm{m}$.

Hold Time

Hold time was equal to 0.2 sec.

\section{Static Force}

Static force was set to $500 \mathrm{~N}$ and $1500 \mathrm{~N}$ for TRI and SEMI EDs respectively.

These factors are presented in Table 4.3.

Table 4.3 Near field ultrasonic welding

\begin{tabular}{|c||c||c||c|c|c|c||c||}
\hline \hline Sr. & \multirow{2}{*}{ No. } & Materia & Amplitude & $\begin{array}{c}\text { Hold } \\
\text { Time } \\
(\boldsymbol{\mu m})\end{array}$ & $\begin{array}{c}\text { Weld } \\
\text { Time } \\
(\mathbf{s e c})\end{array}$ & $\begin{array}{c}\text { Static } \\
\text { Force } \\
(\mathbf{N})\end{array}$ & $\begin{array}{c}\text { Frequency } \\
(\mathrm{KHz})\end{array}$ \\
\hline 1 & TRI & ABS & 21 & 0.2 & 0.4 & 500 & 20 \\
\hline 2 & SEMI & ABS & 21 & 0.2 & 0.9 & 1500 & 20 \\
\hline
\end{tabular}

Procedure for preparing samples, using ultrasonic welding rig, sample orientation, measuring LSS was same to calibrate the current rig, as can be read in sections 4.3 to 4.6. Ten samples were produced for this calibration. Moreover, ultrasonic welding scenario was near field. There were present commonalities between the results from two welders which guaranteed the soundness of current welding rig.

A. Maximum weld strength was obtained with ABS and TRI ED

B. Maximum bond strength was found within an acceptable margin of error. This is shown in Figure 4.17. 


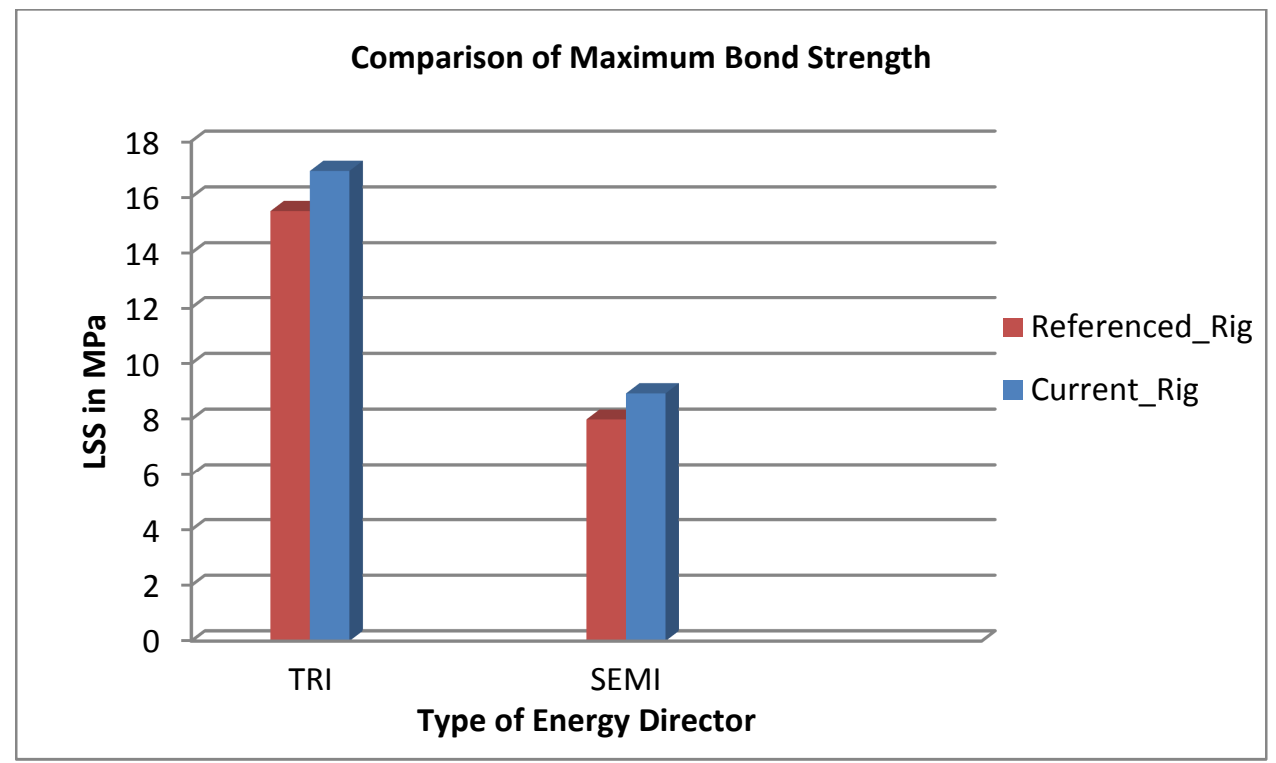

Figure 4.17 Calibration of current UW rig from referenced UW rig

A very little difference in values of bond strengths was noted for ABS with TRI ED and SEMI EDs respectively, as shown in Table 4.4. These minute differences in values of bond strengths show a little drift of current welding rig from giving accurate performance which cannot appear to influence the subsequent results significantly. Hence weld strength data from two welders have been found to be close to each other.

Table 4.4 Extension of table 4.3

\begin{tabular}{|c||c|c||}
\hline \multirow{2}{*}{ Sr.No. } & $\begin{array}{c}\text { Max. Strength (MPa) } \\
\text { from } \\
\text { Referenced_Rig }\end{array}$ & $\begin{array}{c}\text { Max. Strength (MPa) } \\
\text { from Current_Rig }\end{array}$ \\
\hline \hline 1 & 15.5 & 16.94 \\
\hline 2 & 8 & 8.93 \\
\hline
\end{tabular}

\subsubsection{Conclusion}

In this investigation, present ultrasonic welder has been calibrated by an ultrasonic welder from literature in an effort to validate all subsequent results. It can be seen that the weld strength values obtained with the UW equipment which is used in this research are consistent with those from the published literature. Since sampling method before welding and LSS testing method after welding are also main contributors to eventually achieve LSS, 
this agreement between strength data from two welders also delivers satisfactory reliance on sampling and testing methods. Hence this calibration of welding rig provides additional evidence with respect to methods for sampling ABS and testing LSS. This, therefore, provides sufficient confidence that this ultrasonic welder can be utilized to provide the researcher with accurate results throughout the rest of this thesis.

\subsection{Matrix of experiments}

In this research, a matrix was made for various combinations of basic factors welding $A B S$ and PP ultrasonically in order to investigate the effect of weld factors on weld strength. In short, this matrix, having cluster of various ultrasonic welding factors, was established to explore ultrasonic welding of thermoplastics (i.e. ABS and PP).

Let us present the way of structuring the matrix for ABS and PP:

Based on initial welding tests, matrix was arranged for both ABS and PP by keeping EDs, material, hold time, and amplitude constant firstly. Although some ranges of factors were different for each material, the structure of experiments was the same. Now for ABS, weld time was decreased from maximum $(0.9 \mathrm{sec})$ to minimum $(0.4 \mathrm{sec})$ with an interval of 0.1 sec and static force was increased from minimum (500 N) to maximum (1500 N) with an interval of $250 \mathrm{~N}$. Similarly for PP, weld time was decreased from maximum $(1.7 \mathrm{sec})$ to minimum $(0.6 \mathrm{sec})$ with the same interval of $0.1 \mathrm{sec}$ or greater and static force was increased from minimum $(500 \mathrm{~N})$ to maximum $(1750 \mathrm{~N})$ with the same interval of $250 \mathrm{~N}$. For both materials, ED was then changed alternatively i.e. SEMI or TRI after having set weld time and static force. Finally amplitude was set from maximum $(21 \mu \mathrm{m})$ to minimum $(14.7 \mu \mathrm{m})$ with a decreasing interval of $2.1 \mu \mathrm{m}$ again for both materials. A flow chart is representing this way of setting weld factors in Figure 4.18. Intervals and ranges for ultrasonic weld factors are also mentioned in Table 4.5.

In order to make results consistent, reliable and uniform throughout the testing conditions, 10 samples were produced for each test condition. Matrix of experiments for each thermoplastic is available in appendix A. 


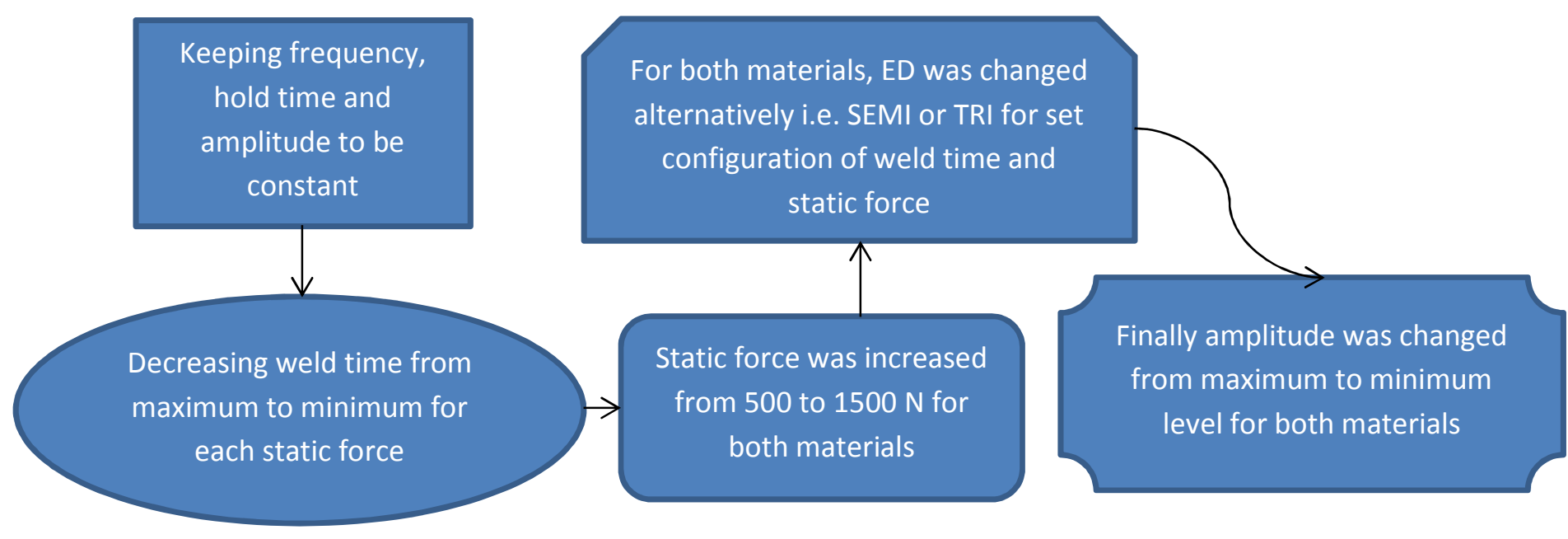

Figure 4. 18 Flow chart for weld factors

Table 4.5 Ultrasonic welding factors

\begin{tabular}{|c||c||c|c|}
\hline \hline Material & Factor & Factor Interval & Range of Factor \\
\hline \hline \multirow{3}{*}{ ABS } & Amplitude & $2.1 \mu \mathrm{m}$ & $14.7-21$ \\
\cline { 2 - 4 } & $\begin{array}{c}\text { Weld } \\
\text { Time }\end{array}$ & $0.1 \mathrm{sec}$ & $0.4-0.9$ \\
\cline { 2 - 4 } & $\begin{array}{c}\text { Static } \\
\text { Force }\end{array}$ & $250 \mathrm{~N}$ & $500-1500$ \\
\hline \multirow{4}{*}{ PP } & Amplitude & $2.1 \mu \mathrm{m}$ & $14.7-21$ \\
\cline { 2 - 4 } & $\begin{array}{c}\text { Weld } \\
\text { Time }\end{array}$ & $0.1 \mathrm{sec}$ & $0.6-1.7$ \\
\cline { 2 - 4 } & $\begin{array}{c}\text { Static } \\
\text { Force }\end{array}$ & $250 \mathrm{~N}$ & $500-1750$ \\
\hline
\end{tabular}

This arrangement of factors covers sufficient set of experiments to achieve the aim of this research. This set of experiments is a representation of holistic paradigm of experiments rather a complete set of experiments. 


\section{CHAPTER 5 RESULTS}

\subsection{Statistical Terms}

To begin with, it is necessary to explain some statistical terms which will be used throughout the following sections:

\subsubsection{Descriptive Statistics}

Descriptive statistics involve using graphs, charts, and tables and calculating several statistical measures to organize and condense the information.

\subsubsection{Parametric Methods}

Those statistical methods wanting the data to first meet the assumptions of normality and equality of variance, as described in sections 5.2.1 and 5.2.2 respectively are called parametric methods.

\subsubsection{Non-parametric Methods}

Statistical methods which do not want the data to meet the assumptions of normality and equality of variance are called non-parametric methods.

\subsubsection{Dependent Variable}

A dependent variable is a variable affected by one or more than one independent variables. In this analysis, lap shear strength (LSS) is a dependent variable.

LSS (Dependent and Quantitative)

\subsubsection{Independent Variable}

An independent variable is that type of variable manipulated by the researcher in an attempt to observe the effect of independent variable on dependent variable. So, collectively five independent variables/factors have affected the LSS excluding constant hold time and constant frequency, as introduced in chapter 4. 


\subsubsection{Population}

A complete collection of items or data under investigation in a statistical study is called a population

\subsubsection{Sample}

A fragment of population selected for analysis is called a sample.

\subsubsection{Mean/Average}

Researchers are usually interested in a typical numerical value to describe a data set. This numerical value is known as mean value or measure of central tendency. Mean/average value $(\bar{X})$ of a data set is obtained when the sum of results or observations $(X)$ is divided by number $(n)$ of observations.

$$
\overline{\mathrm{X}}=\frac{\sum X}{n}
$$

\subsubsection{Variance and Standard Deviation}

Besides the mean value, numerical values are often desired to measure the spread or dispersion of data about the mean of data set. These numerical values are called variance and standard deviation. Variance $\left(\mathrm{S}^{2}\right)$ of a sample size is given by

$$
S^{2}=\frac{\sum\left(X-\bar{X}^{2}\right.}{n-1}
$$

Moreover, square root of the variance is called the standard deviation (S).

$$
S=\sqrt{S^{2}}
$$

\subsection{Basic Approach}

A researcher should adopt two step approach in getting statistically analysed the observed data:

Step I: Using the descriptive statistics to acquire general idea about the data.

Step Il: Adopting the parametric or non-parametric statistical methods in interpreting the data from step $\mathrm{I}$. 


\subsection{Descriptive Statistics}

A univariate analysis was used for obtaining meaningful information from results after welding $A B S$ and PP ultrasonically. In order to perform a univariate analysis, the following conditions must be met by data:

1. Normality

2. Equality of Variance

Test of normality was performed by looking at the distribution of data. If data followed the normal distribution then it would be succeeded in passing the test of normality. Following section discussed briefly the normal distribution.

Equality of variance was tested by spread (standard deviation) vs. level (mean) plot. If the SL (spread vs. level) plot did not show any particular relationship or trend then data would again be succeeded in passing the test for equality of variance.

The procedure used to analysing the weld strength data, is shown in the form of a flow chart in figure 5.1 and has been explained more thoroughly in the following sections. 


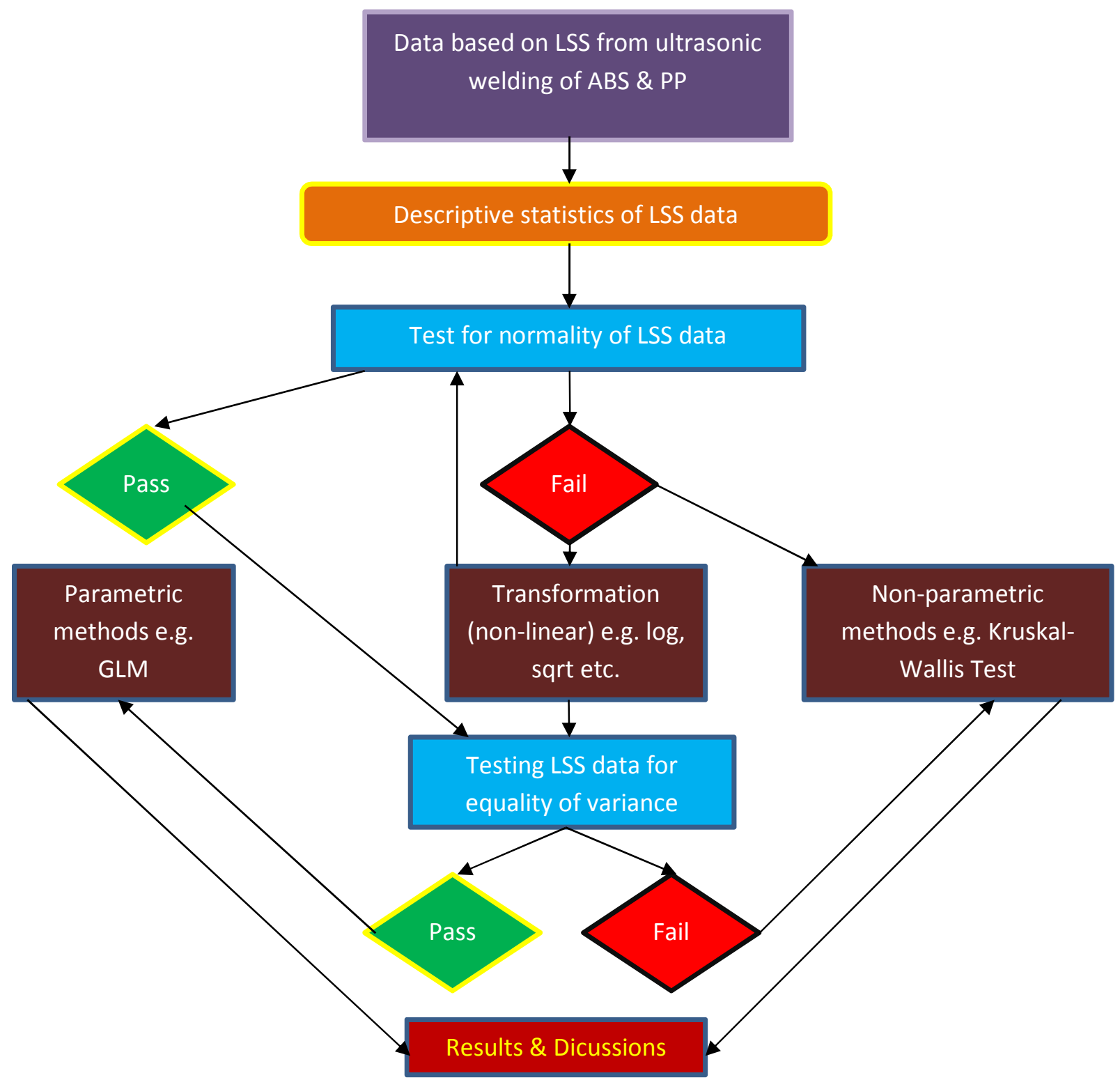

Figure 5.1 Flow chart for selection of suitable statistical analysis

\subsubsection{Normal Distribution}

Normal distribution is extensively used in statistical research analyses. It is also known as bell shape curve or Gaussian distribution. Normal distribution has its hard roots on the mean and the standard deviation. By changing these two parameters, large number of normal distributions comes across, as exhibited in Figure 5.2.

A normal distribution has following properties/characteristics in general;

1. It is found to be symmetric about its mean. 
2. Mean and standard deviation define the Gaussian distribution.

3. Bell curve distribution is found to be thicker in the middle and less thick at the ends.

Another term needed to be briefly explained here is the transformation of data. Data are required to be transformed if it is observed to be non-normal. There are present few options in doing so e.g. square root transformation, logarithmic transformation, square transformation etc. After being transformed, data are again desired to meet above two conditions i.e. normality and equality of variance [181, 182].

Figure 5.2 Normal distributions [183]

\subsubsection{Equality of Variance}

One of the conditions needing to be met by data is to have constant variance. If data do not appear to meet this condition, a researcher will find it hard to interpret the data. In order to check or test for constant variance, spread vs. level plot is used. Spread or variance must not show any relationship with level or mean of data in an attempt to provide sufficient evidence that the data are passing the test for equality of variance. For instance, ultrasonic welding data for $A B S$ result in spread vs. level plot that is illustrated in Figure 5.3 after having met the test for equality of variance. 


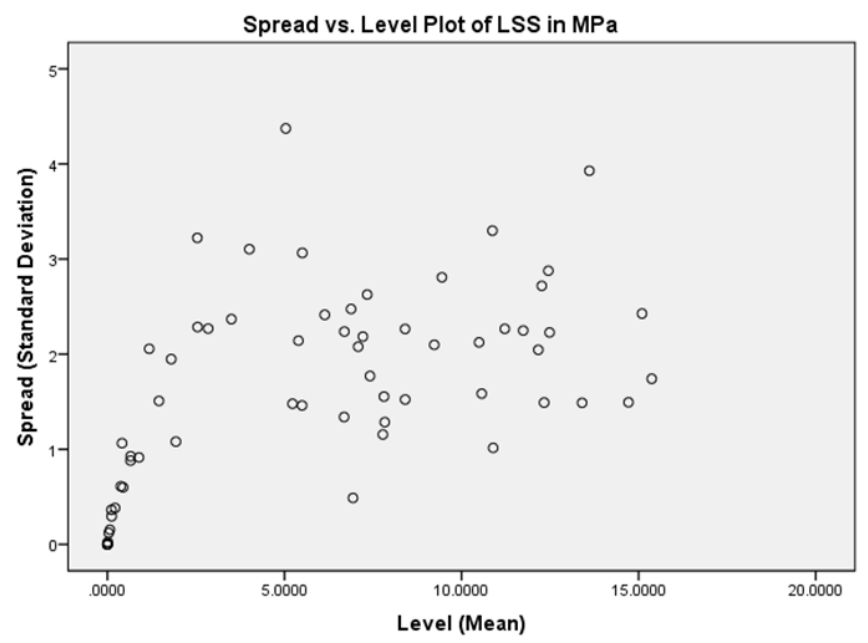

Figure 5.3 An example of spread vs. level plot

\subsection{Results of descriptive statistics}

\subsection{1. $\underline{A B S}$}

\section{Normality Test}

In this research, LSS showed a normal distribution with long histogram at left that represented the number of zero values without having any transformation on data points for ABS. So, the data have already met the condition of normality by ignoring the big column of no weld/zero values of LSS. Normality test of LSS data for ABS is shown in Figure 5.4. This zero value column represented low level of weld conditions (e.g. low level of weld time and amplitude etc.) which resulted in no weld situations.

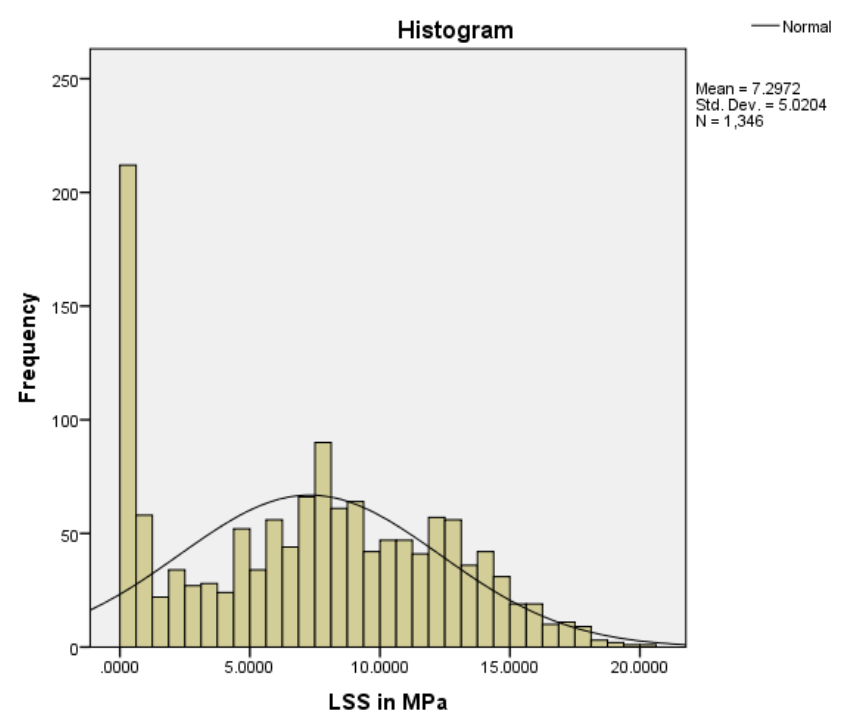

Figure 5.4 Normality of data for ABS 


\section{Spread VS Level (SL) Plot}

Equality of variance was also tested by plotting spread and level of LSS data. In this statistical analysis, a good distribution of data for ABS was found from SL plot without having any relationship between spread and level. So, another condition has also been met by LSS data for ABS during a procedure of selecting right statistical method (parametric/Non-parametric methods). SL plot for ABS data is demonstrated in Figure 5.5. This SL plot was considered good because it did not show any particular trend between standard deviation and mean.

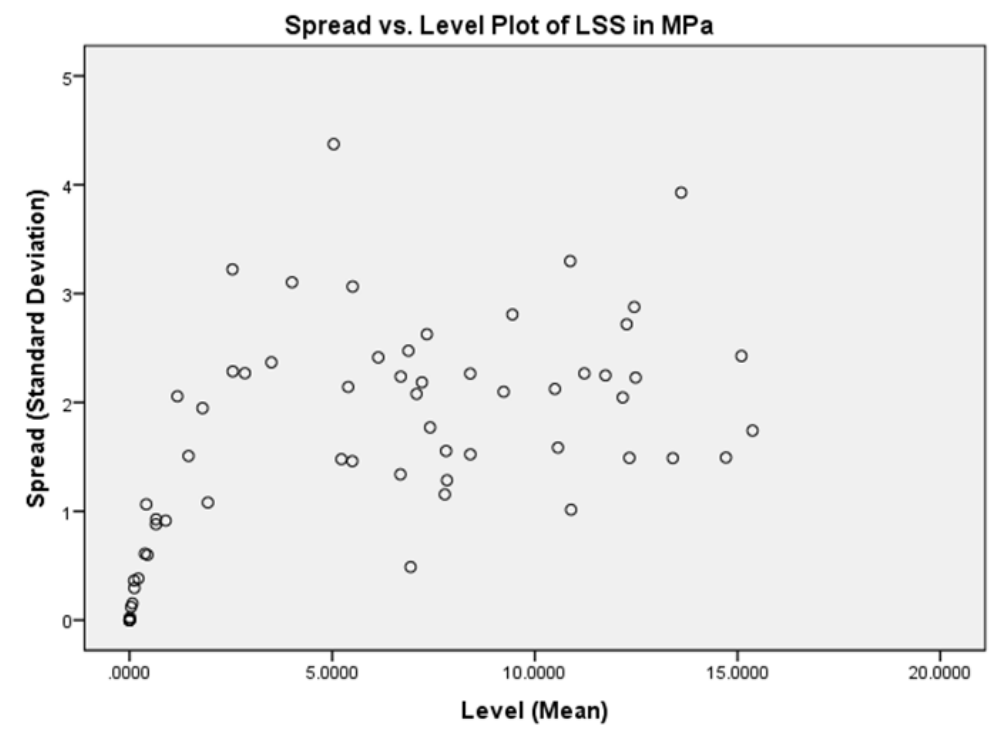

Figure 5.5 Spread VS level plot for ABS

\subsection{2. $\underline{P P}$}

\section{Normality Test}

Without having any transformation on LSS data for PP, a non-normal distribution was obtained, as shown in Figure 5.6, with long histogram at left which represented the number of zero values. So, the data for PP could not meet the condition of normality. 


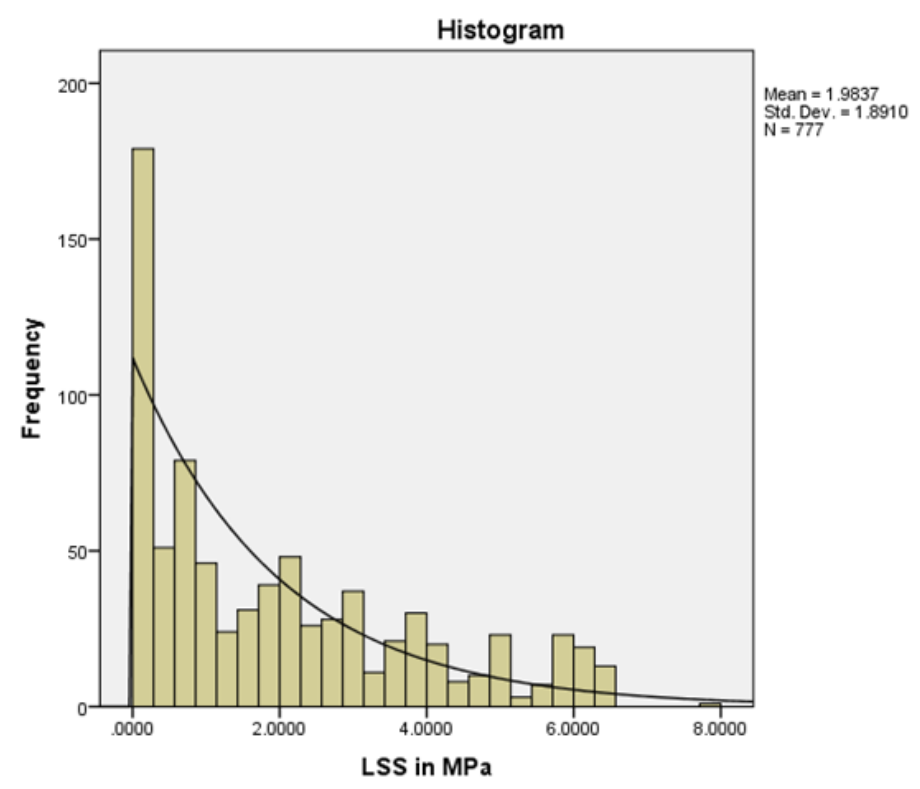

Figure 5.6 Normality Test

After having square root transformation done, a good pattern of normal distribution of data was obtained for PP passing the condition of normality. Transformed data are exhibited in Figure 5.7.

Note: After acquiring the predicted results, the results needed to be squared to properly and correctly interpret them. It has also been accomplished and presented in appendix D.

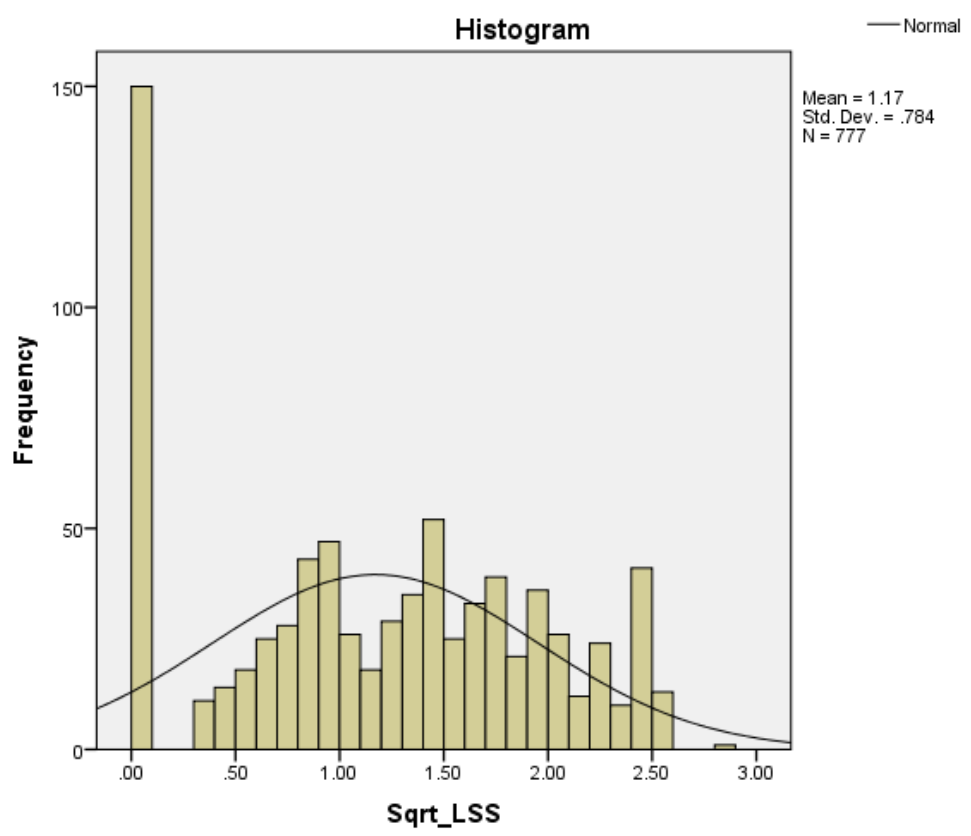

Figure 5.7 Normality Test after square root transformation 


\section{Spread VS Level Plot}

So a good distribution of data for PP was again shown by SL plot passing the second condition that was equality of variance. SL plot for PP data is shown in Figure 5.8.

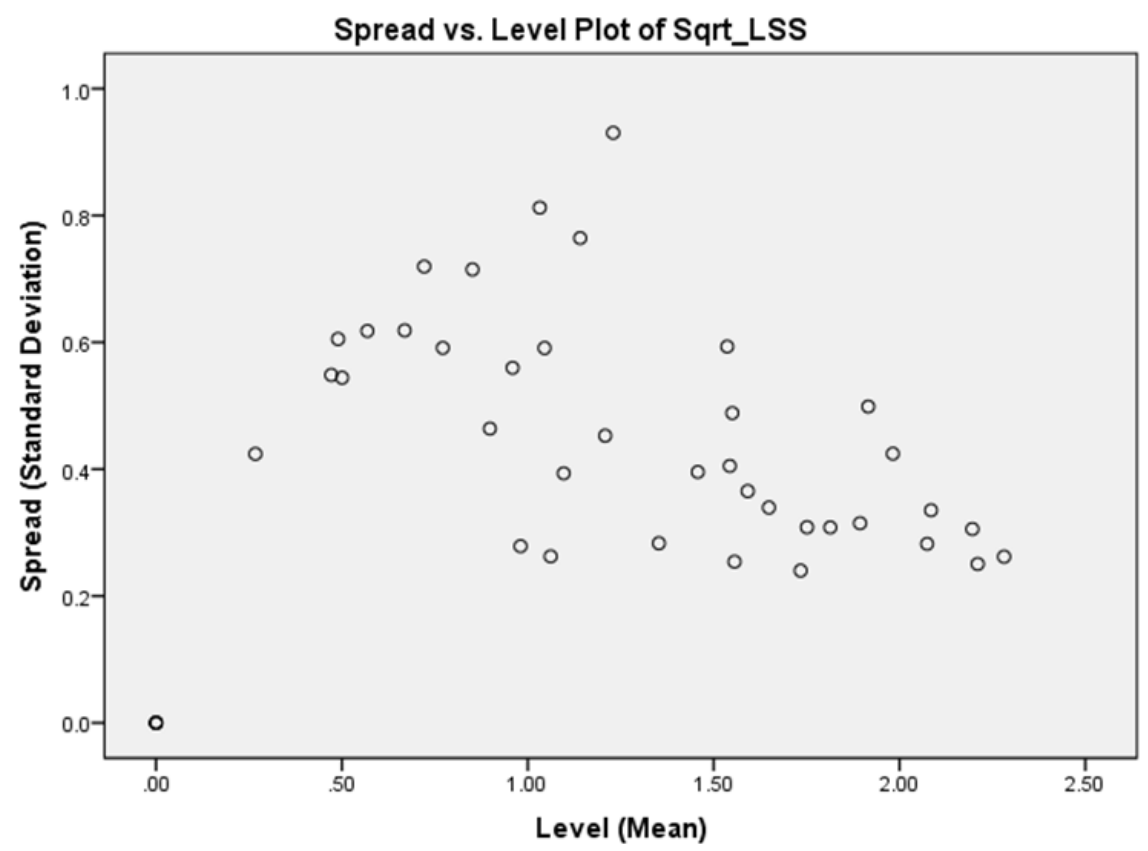

Figure 5.8 Spread VS level plot for PP

Hence LSS data after welding ABS and PP ultrasonically have passed the essential conditions to select a parametric method for subsequent and thorough analysis. Main analysis can be undertaken now.

\subsection{Analysis Method-General Linear Model (GLM)}

To analyse the results, a suitable statistical analysis is required. Analysis based on experimental conditions deals with various terms that are evaluated after experiments. The mathematical and statistical calculations using the empirical results are obtained to support the observations, recommendations and conclusions. Based on complexities in calculating several expressions, an analysis can be divided into two types. An analysis requiring a simple arithmetic calculator (multiplication, subtraction etc.) for getting means/averages of various terms is called a simple analysis. There exists a common problem conducting simple analysis, that is, the averages of two different data sets may result in same values. For example, average heights of five persons are given in Table 5.1. This type of analysis is only 
restricted to determine average of results. On the other hand, analysis of variance (ANOVA) does not only deal with averages but it has also the ability of precisely handling the variances which are associated with many data sets. Likewise, regression analysis provides a best fit line for a given scatterplot using least squares i.e. sum of squared residuals. Various calculations involved in ANOVA and regression analysis are not evaluated conveniently. These statistical derivations involve taking squares and square root of several terms in order to interpret the results in a better way. ANOVA additionally supports the interaction effects, test of significance of a factor and error terms which comprise the effect of those factors which are not included in the experiments.

Table 5.1 Average heights of five persons

\begin{tabular}{|c|c|c|c|c|c|c|}
\hline Data Sets & \multicolumn{4}{|c||}{ Heights of Persons } & Mean of Data Set \\
\hline 1 & 11 & 12 & 8 & 10 & 9 & 10 \\
\hline 2 & 15 & 18 & 6 & 4 & 7 & 10 \\
\hline
\end{tabular}

In this statistical analysis, a statistical technique called General Linear Model (GLM) with Univariate Analysis was used by employing SPSS software. GLM is a parametric method analysing the results which have passed the tests of normality and equality of variance. GLM Univariate Analysis has the ability to perform regression analysis along with analysis of variance (ANOVA) for one dependent variable (e.g. LSS) by considering one or more than one factors or variables [184]. Since lap shear strength (LSS) is the only one dependent variable in this research, current GLM analysis can generally be called a univariate analysis.

Moreover, the word "General" in GLM shows the capability of GLM to accommodate various variables suitable for regression analysis and ANOVA. Similarly, the next two words "Linear Model" simply refer to the regression analysis. Hence GLM also involves predicting a linear model for dependent variable utilizing independent variables. In this research, predicted models have been obtained for results from ultrasonic welding of both ABS and PP and are available in appendix D. 
Based on the common pattern, regression and ANOVA may be explained in a similar way. This conception of common pattern is called GLM. For instance, GLM conception involves accommodating the data in terms of a model and some error.

$$
\text { Data }=\text { Model }+ \text { Error }
$$

Regression analysis endeavours to describe the data (dependent variables) in terms of a model (predictors or independent variables) and error (residuals). Likewise, ANOVA also attempts to explain data (dependent variables) with the help of a model (optimum experimental conditions) and error (the part of data that is not accommodated by the model). Error term for ANOVA indicates that there are some other factors affecting the data. These factors are considered to be unique for each area and consist of anything and everything not controlled in the experiment such as sudden fluctuations in a machine.

Note: Various GLM (regression analysis) results have not been included in the main body of thesis and are presented in appendix D. These include the parameter estimates and prediction of LSS in the form of general linear models by considering all the factors of ultrasonic welding for both ABS and PP. These models must be validated before including them in the current results section. Henceforth, results based on ANOVA will clearly be presented and discussed in this chapter.

\subsection{Preface to GLM Results}

An introduction to GLM results is presented to understand actual meaning of various tabulated terminologies associated with various statistical tests and the exemplary interpretation of results in context of ultrasonic welding of $A B S$ only. Based on this preamble to GLM results, detailed GLM results will be provided with their complete interpretation afterwards, as can be gone through under sections 5.6 and 5.7 for both ABS and $\mathrm{PP}$ respectively.

In this statistical analysis, GLM results consist of following consecutive steps to be performed for fresh LSS data from ultrasonic welding of both ABS and PP:

1. ANOVA (analysis of variance) resulting in detecting the significance of a factor

2. Post Hoc Tests 


\section{Profile Plots}

All univariate statistical procedures are the special cases of multiple regressions (a statistical technique predicting the dependent variable by considering one or more than one independent variables which are known as predictors). In short researchers consider the univariate analysis focusing on a single dependent variable as the first procedure to analyse their data. In other words, effect of each independent variable on LSS will be identified during univariate analysis.

Following are the main reasons for identifying GLM method as an appropriate method for analyses ahead;

1. Statistical analyses using GLM Method are considered suitable for research community with little statistical background e.g. normal distribution, variance, mean, etc.

2. It mainly deals with frequency distributions of dependent variable. So, if a data distribution appears to have non-normal form then a quick transformation is made in an effort to attain normal distribution.

3. A GLM has the possibility of testing null hypothesis (for effects of each variable on the mean of dependent variable) which leads to post hoc tests and profile plots.

4. A long range variable can be categorised in order to make analysis more explicit e.g. categories of static force and weld time in this analysis.

In this analysis, the dichotomisation of weld time and static force was necessary in order to simplify the analysis because higher the number of levels of an independent variable was, more it would make the analysis complex and difficult to understand and interpret. So, the weld time and the static force were categorised/dichotomised into following categories:

Categorisation/Dichotomisation of Weld Time:

For complete data of PP:

$1=0.8$ thru 1.0

$2=1.1$ thru 1.3 
$3=1.4$ thru 1.7

For complete data of ABS:

$1=0.4$ thru 0.5

$2=0.6$ thru 0.7

$3=0.8$ thru 0.9

Categorisation/Dichotomisation of Static Force:

For both $A B S$ and $P P$,

$1=500$ thru 750

$2=1000$ thru 1250

$3=1500$ thru 1750

Therefore, weld time and static force have three levels (categories) which have different meaning from the meaning of level (mean) in SL plot, as discussed earlier in Figure 5.3.

\subsubsection{ANOVA (Analysis of Variance)}

ANOVA is desired in those circumstances which intend to determine the influence of a factor or different factors on results. ANOVA is also overviewed as a picture of process/product performance similar to an X-Ray of a person's body which is taken to acquire an understanding of his health's condition. When visualizing the normal distribution of a population, the spread or width of the distribution is directly linked with the total variation in the data. In order to determine the deviational effect of each factor on this total variation, ANOVA is thought of being the best option [185].

ANOVA is deemed to assume that probability distribution of dependent variable must have Normality and Equality Of Variance i.e. variance of data in groups should be same. In this research, idea of performing ANOVA was actually to assess the variation due to each individual factor which was associated with total variation within the results. In other words, significance of weld factors was determined after performing ANOVA. 
Effects of factors on LSS were intended to be analysed separately for ABS and PP in this analysis by using:

1. Complete LSS Data with ABS

2. Complete LSS Data with PP

In an effort to understand the GLM procedure for current analysis, few ANOVA results from ultrasonic welding of ABS are presented in Table 5.6.

Table 5.6 is a demonstration of main terms in ANOVA table (Complete table is presented in Appendix C); this table is quite revealing in several ways. In fact, ANOVA utilizes various terms which are column headings of Table 5.6. The objective of presenting Table 5.6 is to deliver an ease for interpreting the ANOVA results.

\section{First Column: Source}

First column shows the effects of main factors and their possible interactions on LSS. Main effects simply refer to the mean effects of weld factors on results e.g. mean effects of ED, amplitude, static force and weld time on LSS. Each weld factor is expected to have some influence on results. Moreover, a factor is an independent variable. A plot of these main effects may have parallel lines if these main effects are fully independent of each other.

In order to arrive at good analysis of experimental results, it is a good idea to expect an interaction between various factors in terms of their effects on results. Interaction technically implies interdependence. It is frequently noted that factors behave in a different manner in the presence of some other factors such as the effect of a factor changes when levels of other factors are altered. This interdependence of effects of two factors is known as interaction effect. The interaction between the factors affects the way the factors influence the results. If an interaction is present, main effects interacting will be interpreted carefully. Hence interaction is more an effect than a result. Since Interaction has an effect on results, it is not a factor that can be controlled. In other words, an interaction cannot be controlled making us more vigilant when results are interpreted.

Since four weld factors have been used in this analysis, six two way/two factors interactions (i.e. interaction between two factors at a time) are studied, as can be calculated by a 
formula $N^{*}(N-1) / 2$. $N$ represents the number of factors. When two factors are known as $Y$ and $Z$, the interaction is identified as $Y^{*} Z$ interaction. Hence interaction of factors refers to ED*Amplitude_of_Vibrations, ED*Weld_categories etc., as mentioned in Table 5.6. Interaction effects of main factors imply that the effects of main factors on LSS are not independent of each other. For instance, effect of ED is not independently different from the effect of static force on LSS and these both factors may have some interdependence. Unlike interpretation of effects of a factor levels on results, levels of an interaction effect don't have any physical interpretation. Finally, analysis of two way interaction is included in present ANOVA because it can give vital clues for different levels of factors in achieving the optimal and desired results.

\section{Second Column: Type III sum of squares}

Second column named as type III sum of squares has been set as default before running univariate analysis and this is the most useful for ANOVA as well. Type III sum of squares is used for unbalanced data. Data will be unbalanced if data have been acquired by setting unequal number of levels of a factor within experimental matrix or array for each experiment and opposite is true for balanced data. In other words, if columns of experimental matrix are not balanced with equal number of levels for each factor, the experimental matrix will be known as unbalanced. In this analysis, an unbalanced experimental matrix was utilized. For example, three 2-levels factors are presented here in Table 5.2. Balanced and unbalanced experimental matrices are shown in Tables (5.3/5.4) and (5.5) respectively. From Table 5.2, factors (A, B and C) are represented by first column and factor levels are mentioned in next two columns. From Table 5.3, four experiments are represented by Runs column with experimental conditions of factors ( $A, B$ and $C$ ) in rows. For instance, first experimental run consists of A1B1C1 conditions. Likewise, factors with their original names and actual levels are shown in Table 5.4.

Table 5.2 Three 2-levels factors

\begin{tabular}{|c||c|c|}
\hline \hline Factors & Level 1 & Level 2 \\
\hline \hline A. Weld Time $(\mathrm{sec})$ & 1 & 0.8 \\
\hline B. Amplitude $(\mu \mathrm{m})$ & 21 & 18.9 \\
\hline C. Static Force $(\mathrm{N})$ & 1500 & 1250 \\
\hline
\end{tabular}


Table 5.3 Balanced Matrix

\begin{tabular}{|c|c||c|c|}
\hline Runs & A & B & C \\
\hline \hline 1 & 1 & 1 & 1 \\
\hline 2 & 1 & 2 & 2 \\
\hline 3 & 2 & 1 & 2 \\
\hline 4 & 2 & 2 & 1 \\
\hline
\end{tabular}

Table 5.4 Extension of Table 5.3

\begin{tabular}{||c||c||c|c|}
\hline Runs & Weld Time $(\mathbf{s e c})$ & Amplitude $(\boldsymbol{\mu m})$ & Static Force $(\mathbf{N})$ \\
\hline \hline 1 & 1 & 21 & 1500 \\
\hline 2 & 1 & 18.9 & 1250 \\
\hline 3 & 0.8 & 21 & 1250 \\
\hline 4 & 0.8 & 18.9 & 1500 \\
\hline
\end{tabular}

Table 5.5 Unbalanced Matrix

\begin{tabular}{|c|c|c|c|}
\hline Runs & A & B & C \\
\hline \hline 1 & 1 & 1 & 1 \\
\hline 2 & 1 & 2 & 2 \\
\hline 3 & 2 & 1 & 2 \\
\hline 4 & 2 & 2 & 1 \\
\hline 5 & 1 & 2 & 2 \\
\hline
\end{tabular}

Sum of squares is calculated for each factor and two way interactions between factors compelling any negative values to be positive. The sum of squares for ANOVA may have comprehensive formulae containing sample size, sample mean, sample variance, total sample size and overall mean for all samples in it. ANOVA uses this sum of squares as a basis for calculating other terms in ANOVA table. In fact, there exists an interrelationship among the various ANOVA terms. Hence sum of squares for each factor and interaction between factors was finally evaluated by using average values of LSS with regard to each level of factors.

In the context of linear regression, the residuals are squared to convert all negative values into positive values because average of both positive and negative residuals is zero. The squared values are then added to find out the sum of squares. In order to determine the 
best fit line for a scatterplot, the sum of squared residuals must be smaller i.e. least squares line.

\section{Third Column: Degree of freedom (df)}

Next column is the ' $d f^{\prime}$ ' i.e. degree of freedom that is calculated by subtracting the number of levels of a factor from one. As far as the interaction of factors is concerned, degree of freedom is calculated by multiplying the ' $d f$ ' of individual factors. Hence ' $d f$ ' is a crucial number for scientific data analyses. ' $d f$ ' is commonly used in engineering applications. For instance, in vibration analysis, a mass spring system with two degrees of freedom has two vibration modes which can be investigated under suitable excitation. But in case of experimental data analyses, the interpretation of ' $d f$ ' is slightly different. For statistical analyses of experimental data, ' $d f$ ' represents the amount of information enclosed in a data set. In order to further explain it, an example of four people can be taken. For these four people, three comparisons need to be evaluated in an effort to determine who is the tallest.

\section{Fourth Column: Mean square}

Fourth column illustrates the mean square which is evaluated when the sum of squares of a particular factor is divided by its 'df'. Moreover, mean square is also called the variance. In other words, sums of squares can be changed into variances when they are divided by their 'df'. There can be two sources of variations in a data set; between samples variation and within samples variation. Between samples variations are tackled by mean squares, whereas within samples variations may be quantified by mean square error, as can be seen at the end of fourth column. Hence a significant amount of variations can easily be accommodated by ANOVA.

\section{Fifth Column: F-Ratio/F-test statistic/F-Value}

Fifth column represents the F-Ratio that is determined when the mean square or variance of each factor or its interaction is divided by mean square error or variance for the error term. The values of F-Ratio are also used to find the degree of significance for a particular weld factor. Based on F-Value, significance of weld time categories/levels has been found to be the maximum, as can be seen in Table 5.6. In fact, ANOVA utilizes a statistical F Distribution 
in order to identify the statistical significance of a particular effect at a predefined significance level (e.g. 0.05 or 0.01) using F Tables [186, 187].

\title{
Sixth Column: P-Value/Significance Tester
}

Finally, the sixth column denotes the P-Value giving the significance of F-Ratios for individual as well as interaction of factors. The $p$-value is defined as the possibility of getting a test statistic at least as extreme as the F-test statistic if the null hypothesis is true. It is also the possibility of rejecting the null hypothesis. This null hypothesis asks whether mean LSS for a particular welding factor is different for each category of the same welding factor. In the same way, rejecting the null hypothesis means the presence of difference of mean LSS between two categories that belong to same welding factor. Likewise, null hypothesis for an interaction effect indicates that mean LSS value for the category of one factor does not depend upon the mean LSS value for other factor category.

\section{An example for understanding the role of P-value}

"Two types of engine oil are supplied to a random sample of four owners of different automobiles. Type $S$ is the brand of manufacturer who is interested in conducting this statistical research. Type $\mathrm{H}$ is the competitor's brand. The manufacturer intends to detect any preference for his engine oil if it exists.

The null hypothesis emphasizing on 'no difference', is $\mathrm{HO}: \pi=1 / 2$. Proportion of owners, in the general population who prefer type $S$, is denoted by $\pi$. The second statistical hypothesis, which is often called the alternative hypothesis, states that there is a preference for engine oil S, Ha: $\pi>1 / 2$.

Suppose four owners are asked to choose between the engine oils. Following 16 outcomes are possible with equal probability if there is no preference:

\author{
SSSS, SSSH, SSHH, SHHH \\ HSSH, HSHS. SHSH, SHHS \\ HHSS, SHSS, HSSS, SSHS \\ HHHS, HHSH, HSHH, $\mathrm{HHHH}$
}


Only one outcome out of 16 is felt very different from what has been expected to occur under random sampling if the null hypothesis is true. Since the unlikely case has appeared once if the null hypothesis is true, the significance level (P-value) is set equal to $1 / 16<$ 0.0625 .

In other words, if the outcome is the four choices of type $S$, then $P=P(S S S S)=1 / 16$, and the manufacturer can say that the results are significant, and the null hypothesis is rejected. However, if the outcome is three choices of type S, then P = P (SSSH, SHSS, SSSS, HSSS, SSHS) $=5 / 16>1 / 16$, and null hypothesis is not rejected".

Moreover, any effect of factor is judged to be significant if it has p-value less than 0.05 . If all the effects appear to be significant, profile plots will be made to get clearer visualization and understanding of effect of weld factors on LSS, as will be discussed later in section 5.5.3. Table 5.6 also shows that each source element/effect is significant for LSS data of ABS with P-value less than 0.05 i.e. significance level. Hence effects of main factors and their interactions are significant at $\mathrm{P}=0.05$ level. A significance level of 0.05 implies a $5 \%$ risk of concluding that an effect exists when there is no effect in reality. This indicates that profile plots are essential to be looked at. Moreover, significance of main and interaction effects based on ANOVA calculations is exhibited in the last column of Table 5.6.

Table 5.6 ANOVA RESULTS

Dependent Variable: LSS in MPa
\begin{tabular}{|c|c||c||c||c|c|c||}
\hline \hline Source & $\begin{array}{c}\text { Type III } \\
\text { Sum of } \\
\text { Squares }\end{array}$ & df & $\begin{array}{c}\text { Mean } \\
\text { Square }\end{array}$ & F & P-Value & Significant \\
\hline \hline ED & 2970.80 & 1 & 2970.80 & 477.72 & 0 & Yes \\
\hline Static_Force_Categories & 679.23 & 2 & 339.62 & 54.61 & 0 & Yes \\
\hline Amplitude_of_Vibrations & 3064.81 & 3 & 1021.60 & 164.28 & 0 & Yes \\
\hline Weld_Categories & 7768.73 & 2 & 3884.37 & 624.62 & 0 & Yes \\
\hline Amplitude_of_Vibrations & 417.11 & 3 & 139.04 & 22.36 & 0 & Yes \\
\hline $\begin{array}{c}\text { Static_Force_Categories } \\
\text { E* }\end{array}$ & 190.07 & 6 & 31.68 & 5.09 & 0 & Yes \\
Amplitude_of_Vibrations & 1138.76 & 6 & 189.79 & 30.52 & 0 & Yes \\
\hline $\begin{array}{c}\text { Amplitude_of_Vibrations } \\
* \text { Weld_Categories }\end{array}$ & 1625.74 & 2 & 812.87 & 130.71 & 0 & Yes \\
\hline $\begin{array}{c}\text { ED * } \\
\text { Static_Force_Categories }\end{array}$ & & & & & \\
\hline
\end{tabular}


Dependent Variable: LSS in MPa

\begin{tabular}{|c|c|c|c|c|c|c|}
\hline Source & $\begin{array}{l}\text { Type III } \\
\text { Sum of } \\
\text { Squares }\end{array}$ & df & $\begin{array}{l}\text { Mean } \\
\text { Square }\end{array}$ & $\mathbf{F}$ & P-Value & Significant \\
\hline ED * Weld_Categories & 148.76 & 2 & 74.38 & 11.96 & 0 & Yes \\
\hline $\begin{array}{l}\text { Static_Force_Categories } \\
{ }^{*} \text { Weld_Categories }\end{array}$ & 492.75 & 4 & 123.19 & 19.81 & 0 & Yes \\
\hline Mean Square Error & & & 6.22 & & & \\
\hline
\end{tabular}

Therefore, ANOVA attempts to investigate the significance of main and interaction effects using several conceptual, statistical and mathematical calculations for various terms present in first six columns of Table 5.6.

Hence ANOVA results show that each main and interaction effects of factors are statistically significant. However, further investigations are also needed to identify the importance of each weld factors to the LSS.

\subsubsection{Post Hoc Tests}

Post hoc tests are conducted after finding the significant weld factors via ANOVA. In other words, it needs to be isolated exactly where significant differences lie. Post hoc tests are necessary in those situations that have dire need to make comparison among various means of dependent variable (e.g. LSS) in an effort to justify significance of factors in detail. If a factor has only two levels then the significant F-Ratio is sufficient to explain that two levels are significantly different from each other for affecting the dependent variable. Hence post hoc tests have not been performed for two levels of EDs i.e. SEMI and TRI in this research. However, when there are more than two levels for a factor (e.g. weld time, static force and amplitude), post hoc tests will be needed to tell the significant difference among the levels in addition to significant F-Ratio. Post hoc test will also be considered essential for a significant interaction.

In other words, p-value is used to identifying whether significant difference between at least one pair of means exists or not for a particular factor. But it does not, still, suggest us which means are significantly different from which other means specifically for the same weld 
factor. Owing to this reason, post hoc tests are employed. Although there are present various kinds of post hoc tests, Tukey's HSD or Tukey's Honestly Significant Difference has been used in this analysis, as accepted post hoc test for many analyses. Tukey's HSD is extensively used and easily calculated test permitting the researcher to obtain more understanding of ANOVA. Furthermore, post hoc test must not be used if ANOVA finds any non-significant effects [188].

Note: In this study, post hoc tests were conducted for each weld factor except for ED because ED included fewer than three levels.

Table 5.7 shows the results after conducting the post hoc tests for amplitude of vibrations.

\section{First Column: (I) Amplitude}

First column (I) shows the each value/category/level of amplitude which has been used during ultrasonic welding. Furthermore, each level of amplitude or any other weld factor along with other necessary factor levels utilized for welding ABS and PP can result in some value of LSS. Therefore, LSS value for a particular level of a weld factor will frequently be identified in subsequent analysis.

\section{Second Column: (J) Amplitude}

Second column (J) exhibits all other amplitudes (three amplitudes) to be compared with the single amplitude value present in first column.

\section{Third Column: Mean LSS Difference for (I-J)}

Third column (I-J) represents the mathematical difference between mean LSS for amplitudes in first and second column respectively. Negative value of mean difference presents a larger mean LSS for any amplitude in ' $\mathrm{J}$ ' column than that in ' $\mathrm{l}$ ' column and opposite is true for the positive value. Furthermore, a very little difference of 0.28 has been found between mean LSS for amplitudes 21 and $18.9 \mu \mathrm{m}$. This little difference implies that mean LSS values for amplitudes 21 and $18.9 \mu \mathrm{m}$ are closer to each other. Hence these comparisons deliver firsthand information about numeric difference among mean LSS which corresponds to more than two different categories of weld factors. 


\section{Fourth Column: Confidence Interval}

Last column, having two sub-divided columns that are named lower and upper bounds, illustrates that there is $95 \%$ confidence that difference of means between two amplitudes is expected to be in between lower and upper bounds. Moreover, interval estimation is calculated when a researcher wishes to be impartially sure that a true value of mean is bounded within the estimated interval. If a probability is attached to an estimated interval, a confidence interval $(\mathrm{Cl})$ is acquired. $\mathrm{Cl}$ may also be evaluated by subtracting the P-value or significance level from one. For instance, a P-value of 0.05 means a confidence interval of 95\%. In other words, when a $\mathrm{Cl}$ of $95 \%$ is set, there is a $95 \%$ certainty that a population mean will be covered by the interval. Consequently, there is also a $5 \%$ chance of being wrong for a $\mathrm{Cl}$ of $95 \%$. Lower and upper bounds for $\mathrm{Cl}$ can be calculated by

$$
\frac{\bar{x} \pm Z_{\alpha} \times \sigma / \sqrt{n}}{\overline{2}}
$$

Where $\overline{\mathbf{x}}$ is the sample mean, $\boldsymbol{\alpha}$ is the significance level i.e. 0.05. $\boldsymbol{\sigma}$ is the standard deviation and $\mathbf{n}$ is the sample size. The value of $\mathbf{Z}$ at $\boldsymbol{\alpha} / 2$ can be found from standard normal distribution table. Hence $\mathbf{Z}$ at 0.025 is 1.96 . Therefore, the $\mathrm{Cl}$ can now be calculated conveniently [187].

Table 5.8 is an addition to Table 5.7 showing the actual values of mean LSS that are associated with all amplitudes.

\section{First Column: Amplitude}

First column of Table 5.8 is similar to that of Table 5.7 showing a set of four possible amplitudes for welding thermoplastics ultrasonically.

\section{Second Column: Number (No.) of Samples}

Second column represents the number of samples which have been welded by setting four amplitudes one by one.

\section{Third Column: Subset}


Third column shows the mean LSS values for each of four amplitudes in the form of three subsets. The two values of mean LSS in the third subset imply that these two values are almost equal.

Moreover, from Table 5.7, small difference of 0.28 between mean LSS noted for amplitudes 21 and $18.9 \mu \mathrm{m}$ is due to the similar values of mean LSS (8.04 \& 8.32) for both amplitudes, as shown in Table 5.8 .

Table 5.7 Post-Hoc Test

\begin{tabular}{||c|c|c||c|c|}
\hline \multirow{3}{*}{ (I) Amplitude } & \multirow{2}{*}{ (J) Amplitude } & \multirow{2}{*}{ Mean Difference (I-J) } & \multicolumn{2}{|c|}{ 95\% Confidence Interval } \\
\cline { 4 - 5 } & & & Lower Bound & $\begin{array}{c}\text { Upper } \\
\text { Bound }\end{array}$ \\
\hline \hline \multirow{3}{*}{14.7} & 16.8 & -1.15 & -1.74 & -0.55 \\
\cline { 2 - 5 } & 18.9 & -3.13 & -3.72 & -2.53 \\
\cline { 2 - 5 } & 21 & -2.85 & -3.44 & -2.26 \\
\hline \multirow{3}{*}{16.8} & 14.7 & 1.15 & 0.55 & 1.74 \\
\cline { 2 - 5 } & 18.9 & -1.98 & -2.44 & -1.52 \\
\cline { 2 - 5 } & 21 & -1.70 & -2.16 & -1.25 \\
\hline \multirow{3}{*}{18.9} & 14.7 & 3.13 & 2.53 & 3.72 \\
\cline { 2 - 5 } & 16.8 & 1.98 & 1.52 & 2.44 \\
\cline { 2 - 5 } & 21 & 0.28 & -0.18 & 0.73 \\
\hline \multirow{3}{*}{21} & 14.7 & 2.85 & 2.26 & 3.44 \\
\cline { 2 - 5 } & 16.8 & 1.70 & 1.25 & 2.16 \\
\cline { 2 - 5 } & 18.9 & -0.28 & -0.73 & 0.18 \\
\hline
\end{tabular}

Table 5.8 An extension of Table 5.7

\begin{tabular}{||c||c|c|c|c||}
\hline \multirow{2}{*}{ Amplitude } & \multirow{2}{*}{ No. of Samples (N) } & \multicolumn{3}{|c|}{ Subset } \\
\cline { 3 - 5 } & & $\mathbf{1}$ & $\mathbf{2}$ & $\mathbf{3}$ \\
\hline \hline 14.7 & 166 & 5.19 & & \\
\hline 16.8 & 376 & & 6.34 & \\
\hline 21 & 413 & & & 8.04 \\
\hline 18.9 & 391 & & & 8.32 \\
\hline
\end{tabular}

Hence ANOVA results suggest the significance of a weld factor but they don't give any information regarding the significance of different categories of that factor. Therefore, post hoc tests are performed to investigate profoundly the ANOVA results. 
In general, post hoc tests help determine deeply the significance of levels of a factor e.g. identifying the levels of amplitude which result drastically in optimal values of the dependent variable i.e. LSS. For instance, mean LSS resulted from amplitudes 21 and 18.9 $\mu \mathrm{m}$ indicates that both amplitudes can better contribute in achieving optimal weld strengths. Similar to amplitude, these post hoc tests have been performed for remaining main factors e.g. weld time, static force etc., as can be seen in sections $5.6 .1 \&$ 5.7.1 for both $A B S$ and $P P$ respectively.

Analogous to amplitude, results have been found for the effect of other factors on LSS in the form of tables, as presented in appendix $C$. And the way of understanding these tabulated results is similar to what has been discussed for Tables $5.7 \& 5.8$.

\subsubsection{Profile Plots}

Profile plots can also be obtained during GLM analysis to compare graphically means of LSS as a function of main factors. Profile plot is a multiline plot indicating a relationship between mean of dependent variable and levels of more than one weld factors. Profile plots may be used to find the presence/absence of an interaction between various factors when all possible interaction effects seem to be statistically significant. From these line plots, a researcher also predicts whether there is an increasing or decreasing trend of mean LSS.

A graph is the most efficient way of interpreting and understanding an interaction. To understand the method of graphing these profile plots, an example of Figure 5.9 has been presented here. A profile plot between three categories of static force and two EDs is shown in Figure 5.9 to find the presence or absence of an interaction. To create this profile plot, three categories of static force have been plotted along $x$-axis exhibiting three separate locations on x-axis. Effect of each category of static force on mean LSS may now be plotted for fixed levels of EDs i.e. TRI and SEMI. A profile plot of this kind may be composed of two straight line segments. To decide whether or not an interaction exists in Figure 5.9, two lines plotted will be investigated for being parallel or nonparallel. If these two lines are found to be parallel then there will be no interaction between categories of static force and EDs and reverse is true for presence of interaction. In other words, interaction exists if two lines are nonparallel, as can be seen in Figure 5.9. 


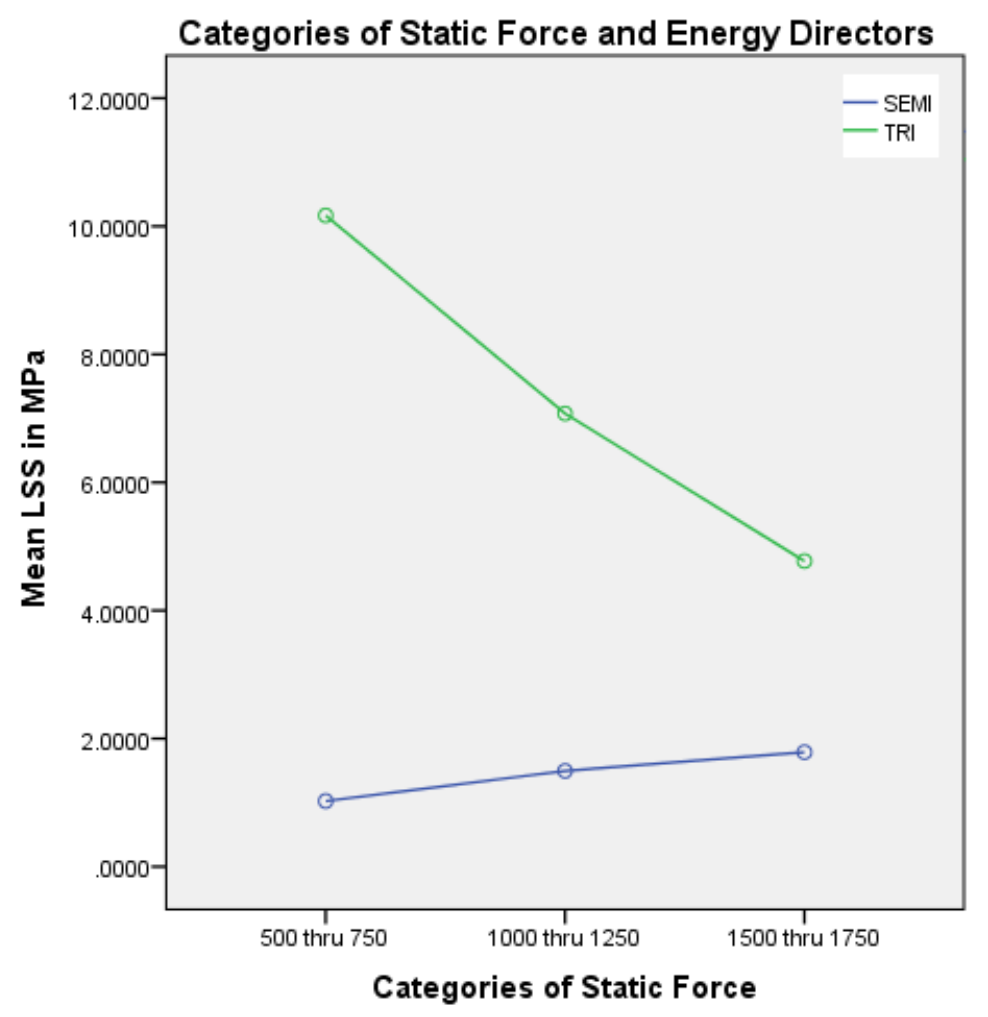

Figure 5.9 Profile Plot (ED and Static Force)

Even though each interaction effect can appear to be significant, two questions must be answered before proceeding ahead with analysis. First, are interactions actually present there? Second, which interactions are more severe or significant than the others? A profile plot can intensely help us answer these two questions.

The judgement about presence of an interaction is achieved by rigorously comparing the slopes of lines in the interaction plot. The magnitude of angle is usually used to compare the slope of lines ranging from 0 to 90 degrees. The term severity is used to express the degree of presence of an interaction. Severity Index (SI) is deemed to be $0 \%$ and $100 \%$ if the angle between lines becomes equal to 0 and 90 degrees. In this analysis, interaction effects will be neglected if $\mathrm{SI}$ is found to be less than $11.67 \%$ because the lines are almost similar to parallel lines. For example, SI for two lines in Figure 5.9 is $53.33 \%$ identifying a severe interaction between static force and EDs.

If an interaction between factors is found to be statistically and graphically (after investigating a profile plot) significant, levels of factors interacted must thoroughly be investigated to acquire their physical interpretation and their effect on the ultimate results. 
For instance, for Figure 5.9, combined effect of both static force and EDs must be discussed in detail to attain optimal results, as will be discussed in discussion chapter.

If all the factors and interactions between factors are found to be statistically significant, levels of interacting factors must be analysed by profile plot in an attempt to find optimum levels or conditions of interacting factors. In fact, analysis of interaction between factors is mainly a thorough study of factors leading to determine the most appropriate levels/conditions of factors if the interaction is not only significant but also present.

In this analysis, profile plots were also gathered after having analysed the post hoc results. These plots provide more insight into the relationships between ultrasonic weld factors and mean LSS graphically. Moreover, some profile plots show negative value for mean LSS at $Y$ axis as a graphical requirement. The SPSS software covers all levels of weld factors in a graph between mean LSS and weld factors even by extending the trend line beyond the zero value of LSS. Hence a negative value of mean LSS represents zero value of LSS. Profile plots will be presented and discussed in sections 5.6.2 \& 5.7.2 for both ABS and PP respectively.

\subsection{Detailed Results of GLM Analysis for ABS}

A simple statistical analysis known as GLM-UNIVARIATE was used to analyse LSS data for ABS. After running the GLM-UNIVARIATE Analysis for ABS, effects of main factors and their interactions were found to be significant, as shown in Table 5.6. This uniformity of significance guided us to consider both post hoc analysis and profile plots.

All the tabulated results are present in appendix $C$.

\subsubsection{Main Factors}

Post hoc analysis revealed the effect of each level of weld factors on mean LSS. Therefore, a comprehensive study is presented in this section to understand deeply the effect of ultrasonic welding factors on mean LSS.

\section{Static Force}

Effect of each category of static force on mean LSS is shown in Table 5.9 in the form of three subsets/means. And ' $N$ ' represents the number of welded samples. 
Table 5.9 Mean LSS in MPa

Tukey HSD

\begin{tabular}{|c|c||c|c|c||}
\hline \hline \multirow{2}{*}{$\begin{array}{c}\text { Categories } \\
\text { of Static } \\
\text { Force }\end{array}$} & \multirow{2}{*}{$\mathbf{N}$} & \multicolumn{3}{|c||}{ Subset } \\
\cline { 3 - 5 } & & $\mathbf{1}$ & $\mathbf{2}$ & $\mathbf{3}$ \\
\hline $\begin{array}{c}1500 \text { thru } \\
1750\end{array}$ & 281 & 5.84 & & \\
\hline $\begin{array}{c}1000 \text { thru } \\
1250\end{array}$ & 536 & & 7.14 & \\
\hline $\begin{array}{c}500 \text { thru } \\
750\end{array}$ & 529 & & & 8.23 \\
\hline
\end{tabular}

Table 5.9 shows that means of LSS are placed in three columns separately and different to each other. Mean LSS for category of static force 500 thru 750 is the highest one and 1000 thru 1250 is having the second highest mean LSS. LSS goes up by $41 \%$ with a decrease in static force of $71.42 \%$. Mean LSS values in Table 5.9 are plotted in Figure 5.10. Figure 5.10 shows the decreasing trend of mean LSS on increasing the static force. This trend will be elaborated in discussion section later on.

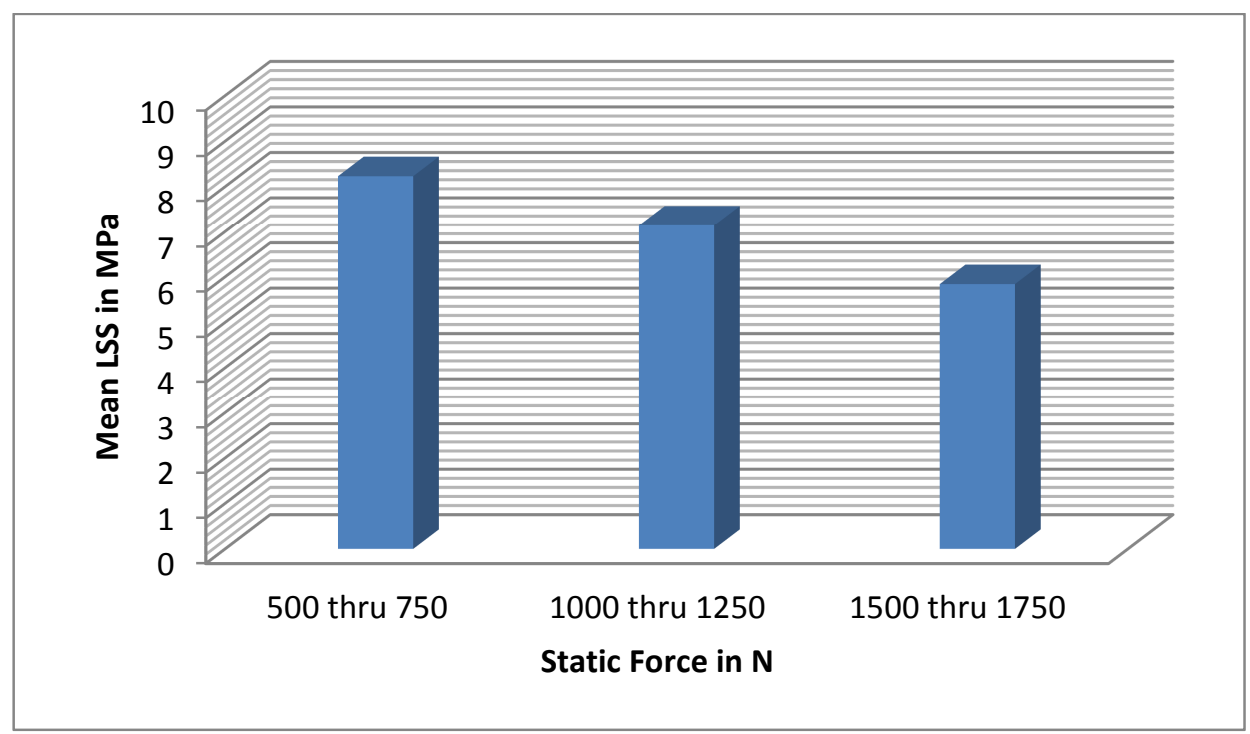

Figure 5.10 Effect of static force categories on mean LSS

\section{Amplitude}

Means of LSS related to 21 and 18.9 amplitudes are almost similar as can be seen from Table 5.10. But 14.7 and 16.8 result in different mean LSS. Mean LSS for amplitude 18.9 is the highest and then $21,16.8$ \& 14.7 respectively. LSS is found to be increased by $60.3 \%$ on increasing the amplitude from 14.7 to 18.9 and it decreases by $3.5 \%$ with an increase of amplitude from 18.9 to 21 . Mean LSS values in Table 5.10 have also been plotted in Figure 
5.11. Graph in Figure 5.11 shows an increasing tendency of mean LSS by increasing amplitude of ultrasonic vibrations.

Table 5.10 Mean LSS in MPa

\begin{tabular}{|c|c|c|c|c|}
\hline \multirow{2}{*}{ Amplitude } & \multirow{2}{*}{$\mathbf{N}$} & \multicolumn{3}{|c|}{ Subset } \\
\hline & & 1 & 2 & 3 \\
\hline 14.7 & 166 & 5.19 & & \\
\hline 16.8 & 376 & & 6.34 & \\
\hline 21 & 413 & & & 8.04 \\
\hline 18.9 & 391 & & & 8.32 \\
\hline
\end{tabular}

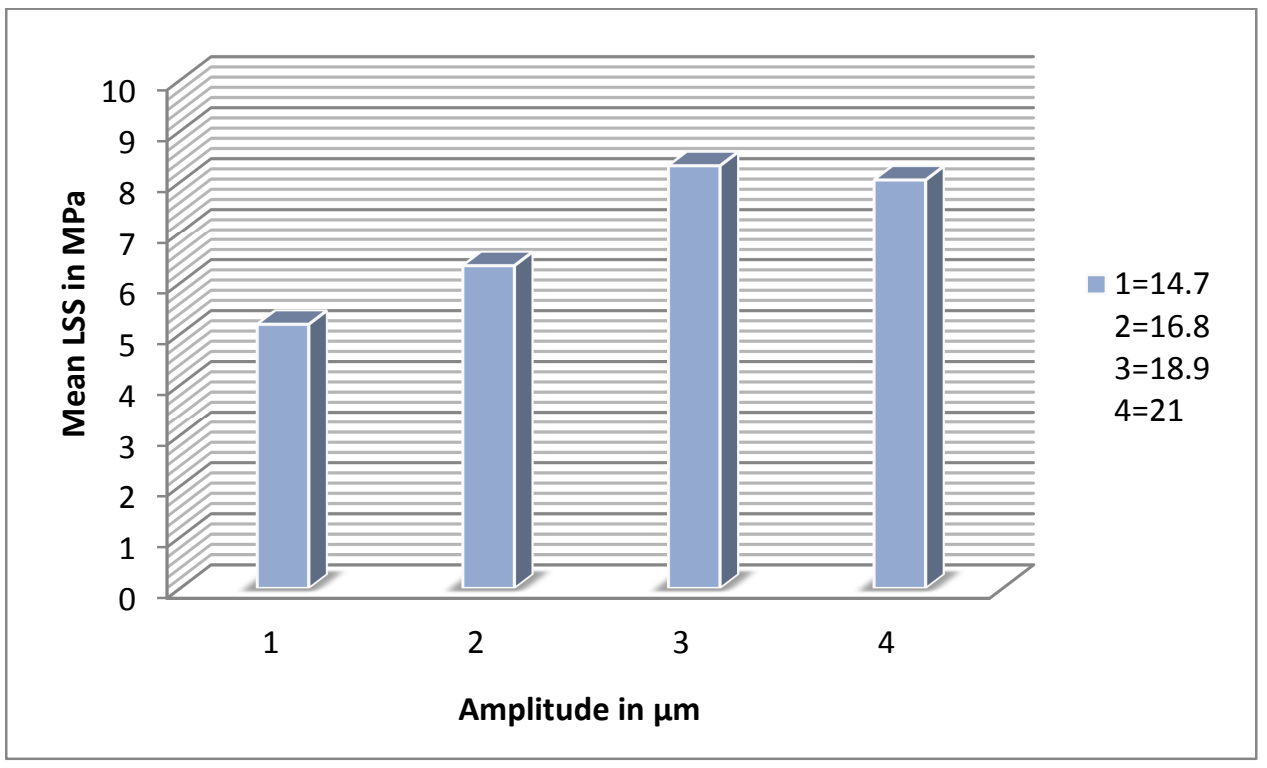

Figure 5.11 Effect of amplitude on mean LSS

$\underline{E D}$

In this analysis, post hoc tests were not performed for energy directors because ED consisted of two levels i.e. TRI and SEMI (less than three levels/categories). It is apparent from the Table 5.11 that mean LSS for TRI ED is much higher than that of SEMI ED. A bar graph based on Table 5.11 is illustrated in Figure 5.12.

Table 5.11 Comparison based on Mean LSS

\begin{tabular}{||c||c||c|c||}
\hline \hline \multirow{2}{*}{$\begin{array}{c}\text { Energy } \\
\text { Director }\end{array}$} & \multirow{2}{*}{ Mean } & \multicolumn{2}{|c|}{ 95\% Confidence Interval } \\
\cline { 3 - 4 } & & $\begin{array}{c}\text { Lower } \\
\text { Bound }\end{array}$ & $\begin{array}{c}\text { Upper } \\
\text { Bound }\end{array}$ \\
\hline \hline SEMI & 1.44 & 0.93 & 1.94 \\
\hline TRI & 7.34 & 7.13 & 7.55 \\
\hline
\end{tabular}




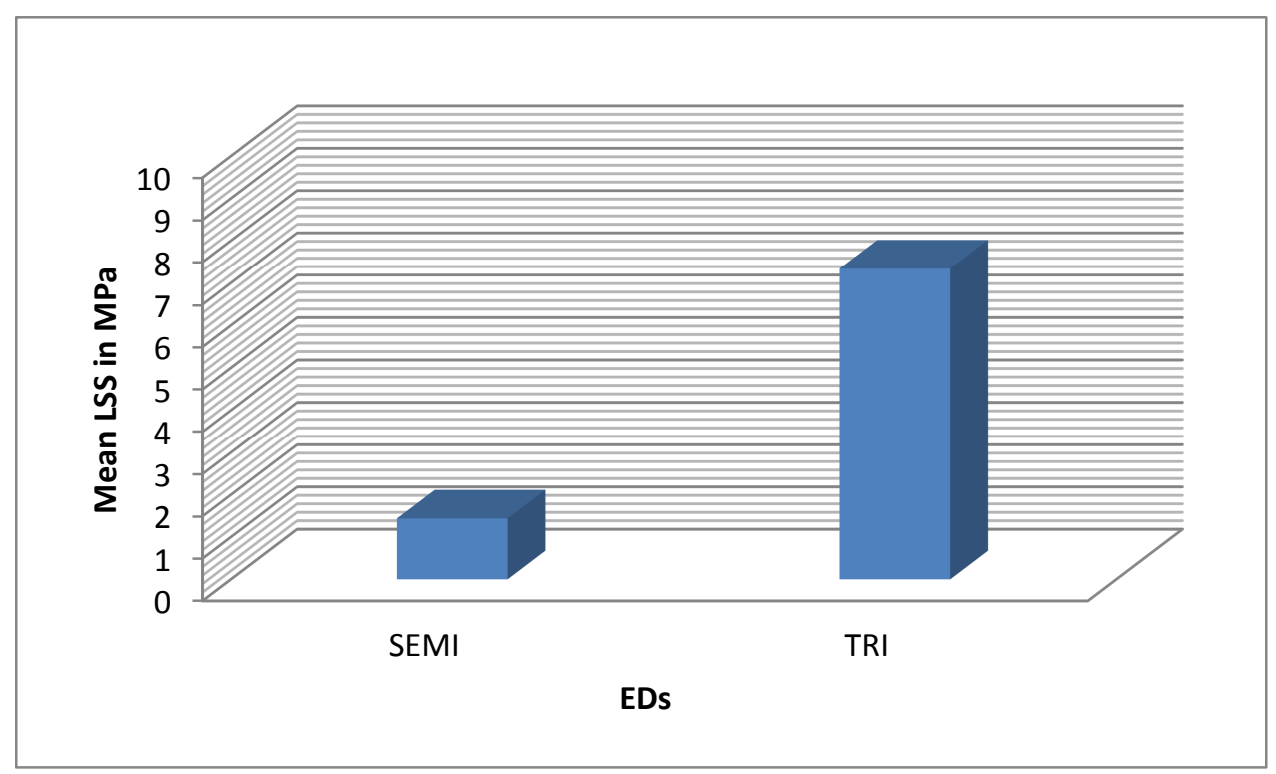

Figure 5.12 Effect of EDs on mean LSS

\section{$\underline{\text { Weld Time }}$}

Mean LSS for category of weld time 0.8 thru 0.9 is the highest and then for 0.6 thru 0.7 is the second highest. And the lowest mean LSS is found for category 0.4 thru 0.5 . Mean LSS values are presented in Table 5.12 for three categories of weld time. From Table 5.12, on increasing the weld time by $125 \%$, LSS increases by $226 \%$. Mean LSS values in Table 5.12 are represented in Figure 5.13 for categories of weld time. Bar graph in Figure 5.13 shows an overall increasing trend for mean LSS by increasing weld time.

Tukey HSD

\begin{tabular}{|c|c|c|c|c|}
\hline \multirow{2}{*}{ Weld Time } & \multirow{2}{*}{$\mathbf{N}$} & \multicolumn{3}{|c|}{ Subset } \\
\hline & & 1 & 2 & 3 \\
\hline 0.4 thru 0.5 & 274 & 2.82 & & \\
\hline 0.6 thru 0.7 & 360 & & 6.98 & \\
\hline 0.8 thru 0.9 & 712 & & & 9.18 \\
\hline
\end{tabular}




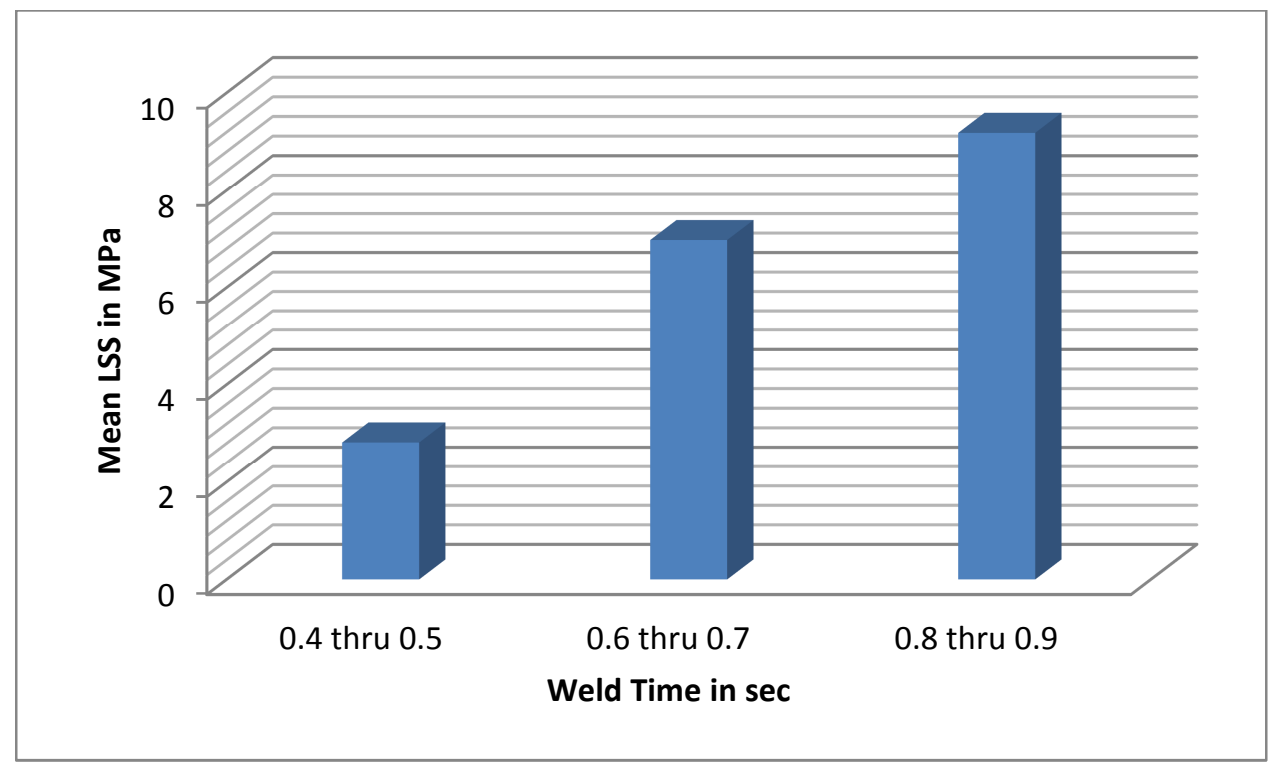

Figure 5.13 Effect of weld time categories on mean LSS

\subsubsection{Profile Plots}

\section{ED and Static Force}

Profile plot in Figure 5.14 shows that there is a $920 \%$ increase of mean LSS from SEMI ED to TRI ED at lowest static force and $150 \%$ increase at highest static force. Obviously, maximum increment in LSS is found at lowest static force. But later, the distance between values of average LSS reduces on increasing static force as shown in Figure 5.14. In other words, on increasing static force, means for LSS increase for SEMI ED and decrease drastically for TRI ED. Mean LSS for TRI ED is found to be higher than that for SEMI ED. Trend of mean LSS for TRI ED appears to decrease and vice versa in case of SEMI ED.

As exemplified earlier in section 5.5 .3 , the severity index (SI) for this profile plot is $53.33 \%$. Therefore, what is the most interesting in this profile plot is that, there exists a severe interaction between high level of static force and EDs. Hence this profile plot will thoroughly be discussed in discussion chapter later. 


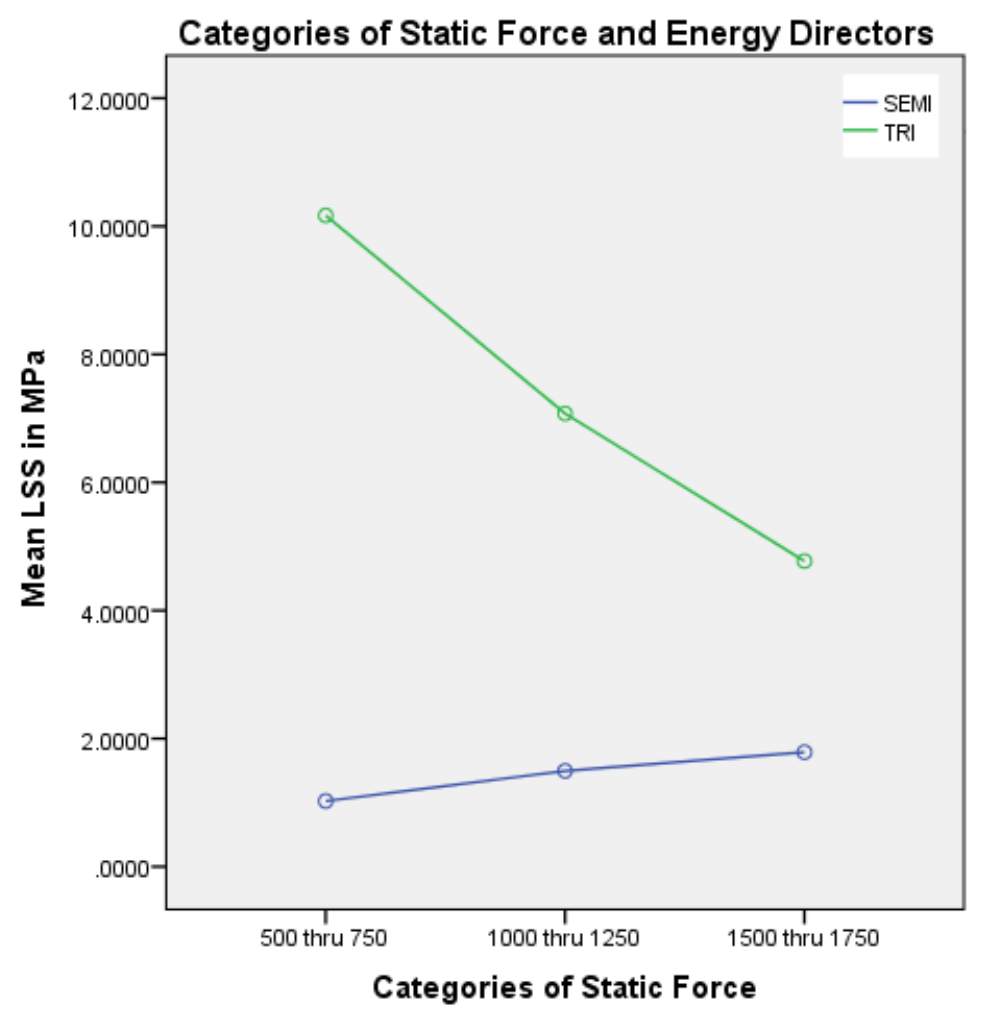

Figure 5.14 Profile Plot (ED and Static Force)

\section{ED and Weld Time}

Profile plot of Figure 5.15 explores that there is no particular difference between values of average LSS for weld time categories with SEMI and TRI ED. Also mean LSS for TRI ED remains greater than that for SEMI ED. Percentage increase of $111 \%$ at highest weld time is noted from SEMI to TRI ED. Overall there are increasing trends for both EDs with no notable differences

Moreover; both lines in current profile plot are parallel. Therefore, there are almost $0 \%$ chances of interaction between ED and weld time owing to $\mathrm{SI}=0 \%$. In this situation, effects of ED and weld time may independently be interpreted because there is no interaction between these two factors. 


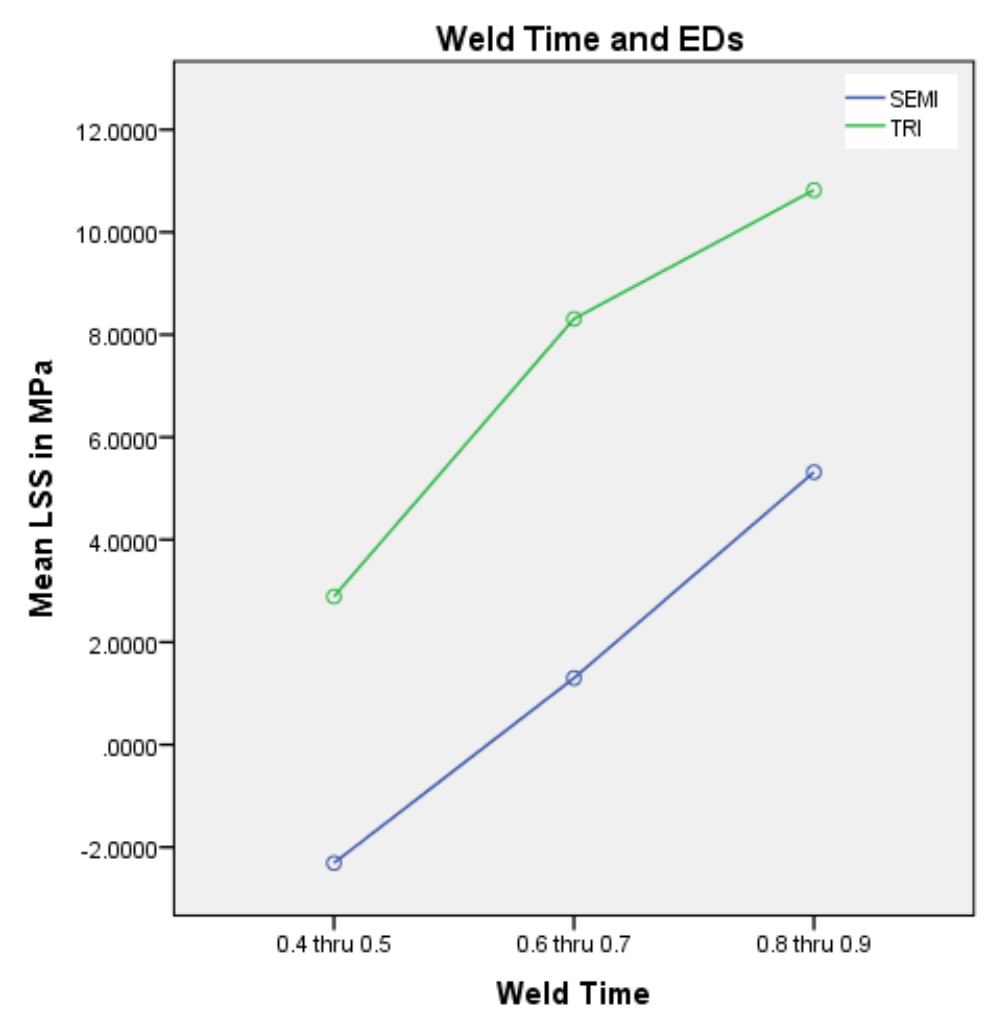

Figure 5.15 Profile Plot (ED and Weld Time)

\section{Amplitude and Weld Time}

Profile plot of Figure 5.16 shows relationship between weld time categories and means of LSS at various levels of amplitudes of vibrations. For weld time category 0.4 to 0.5 , the difference of mean LSS is higher at all levels of amplitude of vibrations. This difference of mean LSS increases for weld time category 0.6 to 0.7 and finally the differences of mean LSS seem to be merged for weld time category 0.8 to 0.9 at all levels of amplitude of vibrations. Overall, these relationships between weld time and mean LSS are showing increasing trends for all levels of amplitude of vibrations.

Severity Index (SI) for current profile plot is only negligibly present i.e. $8 \%$ for amplitudes 21 and 18.9 between weld time categories 0.6 thru 0.7 and 0.8 thru 0.9. Hence interaction between amplitude and weld time will be concluded to be negligible. 


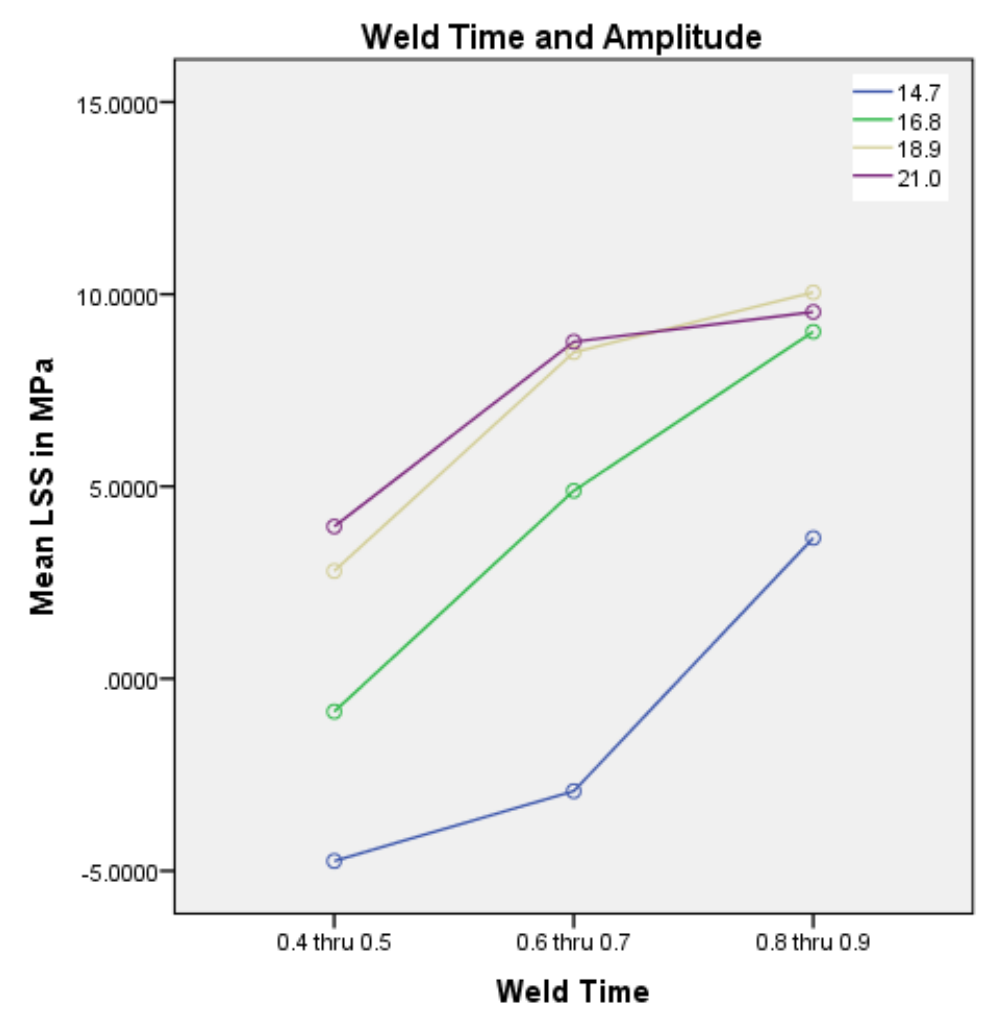

Figure 5.16 Profile Plot (Amplitude and Weld Time)

\section{Amplitude and Static Force}

Profile plot of Figure 5.17 shows the means of LSS for various categories of static force at four levels of amplitude of vibrations. Higher values of mean LSS are noted for amplitude 21 that decrease gradually on increasing the static force. Largest difference between values of mean LSS is found for static force category 1500 to $1750 \mathrm{~N}$ at all amplitudes except for 18.9 and 21 and smallest difference is found for static force category 1000 to 1250 at 18.9 and 21 amplitudes. In fact, little difference of mean LSS is observed for amplitudes 18.9 and 21 overall. At each amplitude, trend is more or less the same.

From Figure 5.17, negligible interaction has been found between amplitude and static force because of lower value of SI. The same interaction is even insignificant for SEMI and TRI EDs, as can be seen in Figure 5.18 and Figure 5.19 respectively. 


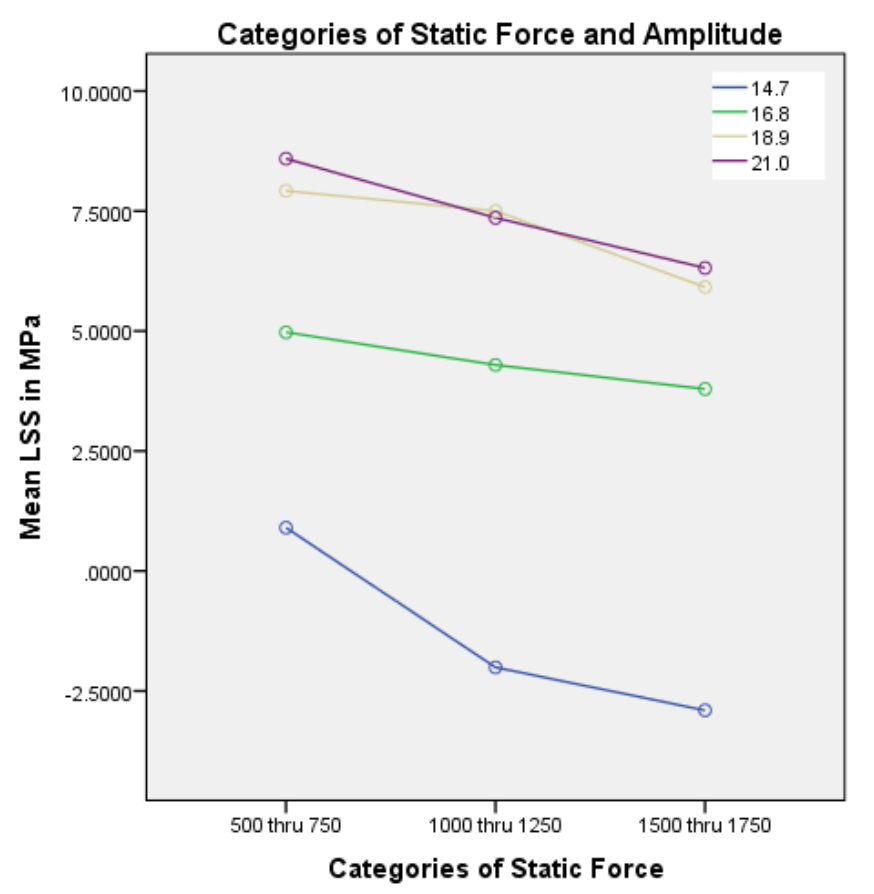

Figure 5.17 Profile Plot (Amplitude and Static Force)

Profile plot in Figure 5.18 shows relationship between different categories of static force and mean LSS for SEMI ED. It gives largest difference of mean LSS for categories of static force 1500 to 1750 at all levels of amplitude of vibrations. At amplitudes 16.8 and 21, the mean LSS increases for all categories of static forces and trends of means LSS are varying at all other amplitudes.

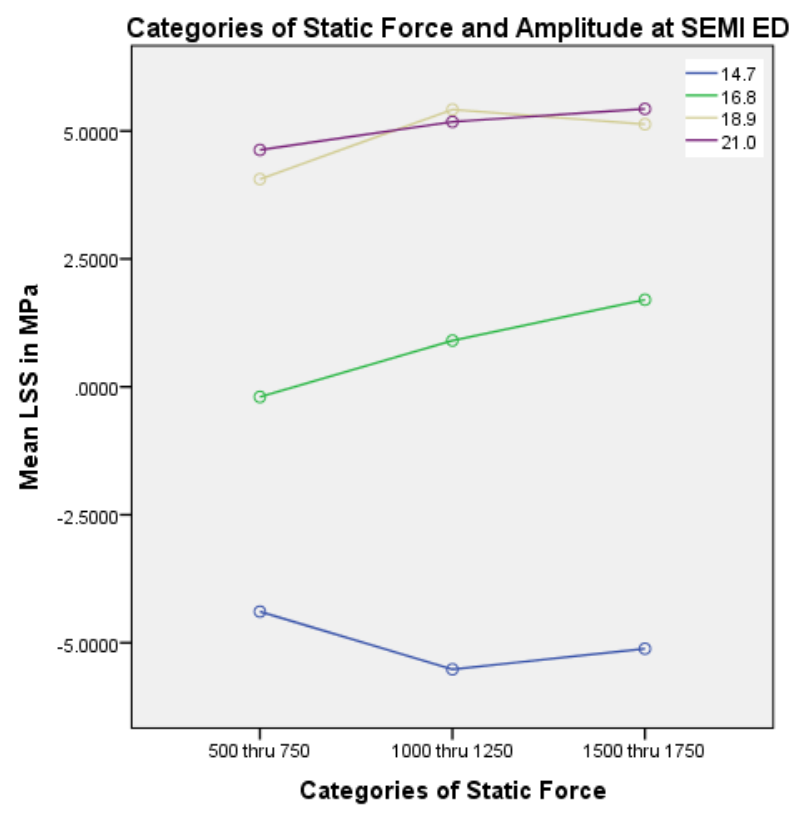

Figure 5.18 Profile Plot (Amplitude and Static Force) at SEMI ED 
Profile plot in Figure 5.19 for TRI ED predicts the relationship between categories of static force and means of LSS at various levels of amplitude again. All the trends are decreasing with large difference of mean LSS between amplitude 14.7 and all other amplitudes of vibrations.

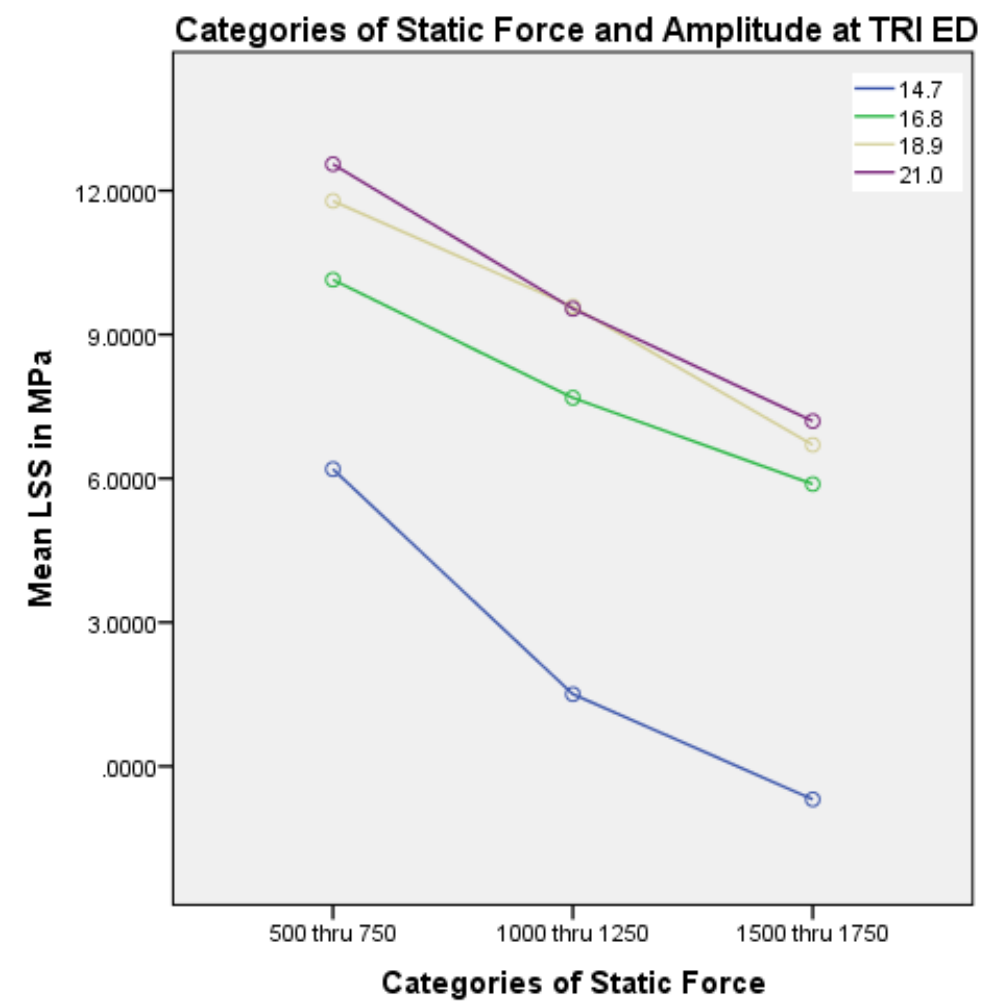

Figure 5.19 Profile Plot (Amplitude and Static Force) at TRI ED

\section{Amplitude and ED}

Profile plot in Figure 5.20 presents a relationship between amplitude of vibrations and means of LSS for SEMI and TRI EDs. Again these relationships show overall increasing trends for both EDs with values of means greater for TRI ED. Also from Figure 5.20, for amplitudes 21 and $18.9 \mu \mathrm{m}$, mean LSS achieves its highest value for both EDs because of straightening of trends after having gradually increased. This is another interesting finding and will be discussed later.

$\mathrm{SI}$ is found to be equal to approximately 0\% for Figure 5.20. Hence the interaction between amplitude and ED is insignificant after investigating the corresponding profile plot. 


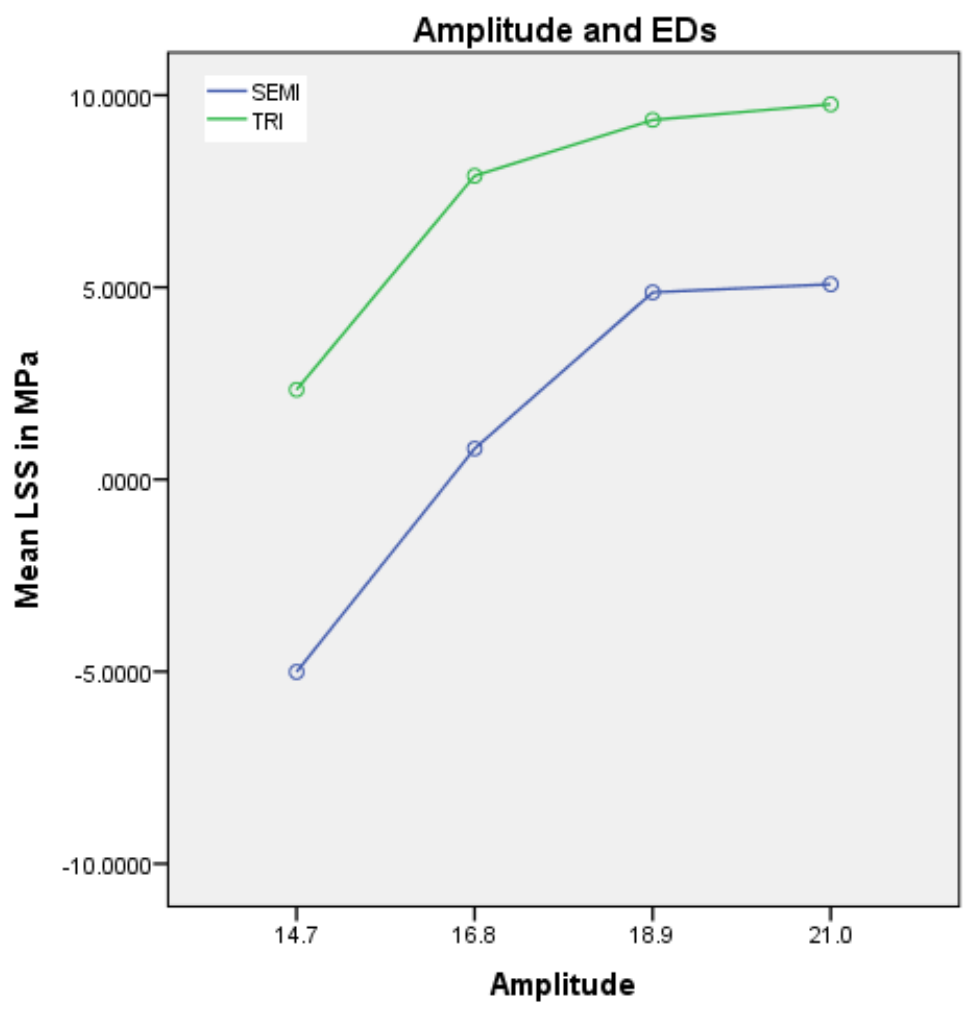

Figure 5.20 Profile Plot (Amplitude and ED)

\section{Static Force and Weld Time}

Profile plot in Figure 5.21 presents the relationship between categories of static force and means of LSS at three weld time categories. Highest LSS is found to be at weld time category 0.8 thru $0.9 \mathrm{sec}$ i.e. maximum weld time which has been used for ABS. LSS decreases on increasing the weld time at all categories of static forces except for weld time category 0.8 thru 0.9 , LSS rises slightly up to $1250 \mathrm{~N}$. Similarly, mean LSS is found to be the highest for static force category of 500 thru $750 \mathrm{~N}$ at all categories of weld time. At category of static force 500 thru $750 \mathrm{~N}$, the gap between mean LSS for all categories of weld time is smaller but this gap increases gradually on increasing the static force which results in maximum gap at 1500 thru $1750 \mathrm{~N}$.

Moreover, interaction between static force and weld time is absent due to the presence of nearly parallel lines in Figure 5.21. 


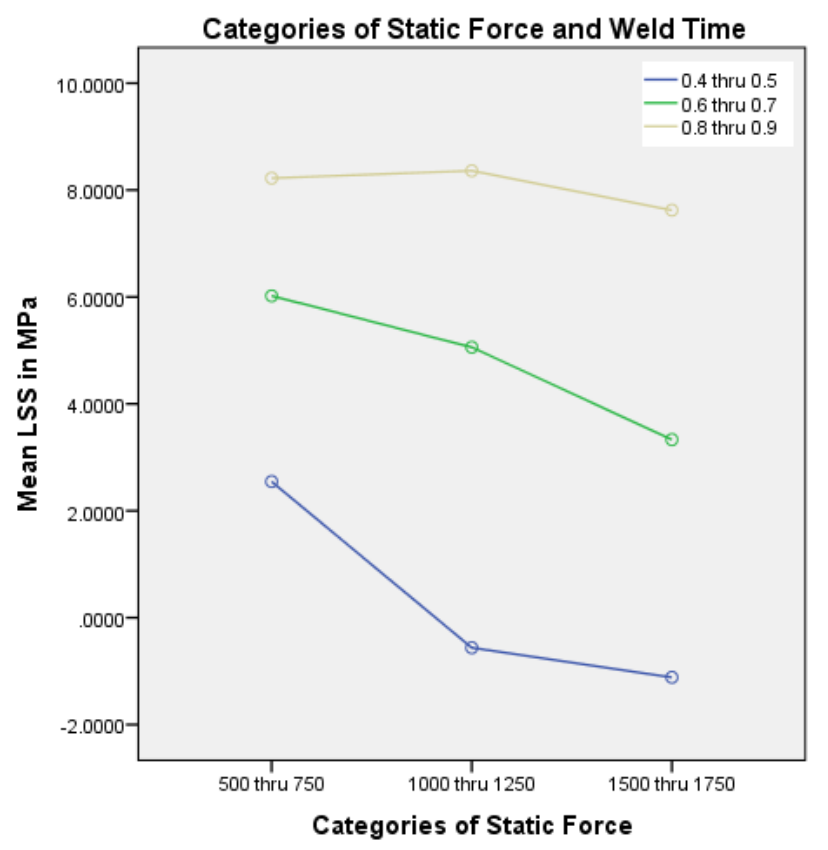

Figure 5.21 Profile Plot (Static Force and Weld Time)

\subsection{Detailed Results of GLM Analysis for PP}

As discussed in section 5.3.2 under the heading "Normality Test", LSS data for PP have been transformed using square root (Sqrt) transformation before running GLM-Univariate Analysis. Therefore, the mean/average LSS (if it appears in the lines) implies the mean of square root of LSS (Sqrt_LSS) throughout this analysis for PP.

After running the GLM-Univariate Analysis for PP, all the factors and their interactions were again found to be significant. Consistency of significance for effects of main factors and their interactions has directed us to investigate again both post hoc analysis and profile plots.

Owing to variability in PP-Data, it has been tough to assess the effect of static force on mean LSS and hence this effect cannot be interpreted confidently for PP.

All the tabulated results are present in appendix $C$.

\subsubsection{Main Factors}

\section{Static Force}

Post hoc analysis shows that means of LSS, as exhibited in Table 5.13, for categories 500 thru $750 \& 1500$ thru 1750 are close to each other and higher than that for remaining 
category i.e. 0.97 . Means of LSS for categories of static force can be ordered as 1500 thru $1750 \gg>500$ thru $750 \gg>1000$ thru 1250 . LSS is increased by $36.08 \%$ with a decrease of static force by $60 \%$ and increased by $6 \%$ with an increase of static force by $250 \%$. Means of LSS from Table 5.13 are plotted in Figure 5.22. Declining and then growing trend of mean LSS is shown in Figure 5.22 on increasing the static force. Reason of this type of trend will be revealed in discussion section.

Table 5.13 Mean Sqrt_LSS

\begin{tabular}{|c|c|c|c|}
\hline \multirow{2}{*}{$\begin{array}{l}\text { Categories } \\
\text { of Static } \\
\text { Force }\end{array}$} & \multirow{2}{*}{$\mathbf{N}$} & \multicolumn{2}{|c|}{ Subset } \\
\hline & & 1 & 2 \\
\hline $\begin{array}{c}1000 \text { thru } \\
1250\end{array}$ & 385 & 0.97 & \\
\hline $\begin{array}{c}500 \text { thru } \\
750\end{array}$ & 152 & & 1.32 \\
\hline $\begin{array}{c}1500 \text { thru } \\
1750\end{array}$ & 240 & & 1.40 \\
\hline
\end{tabular}

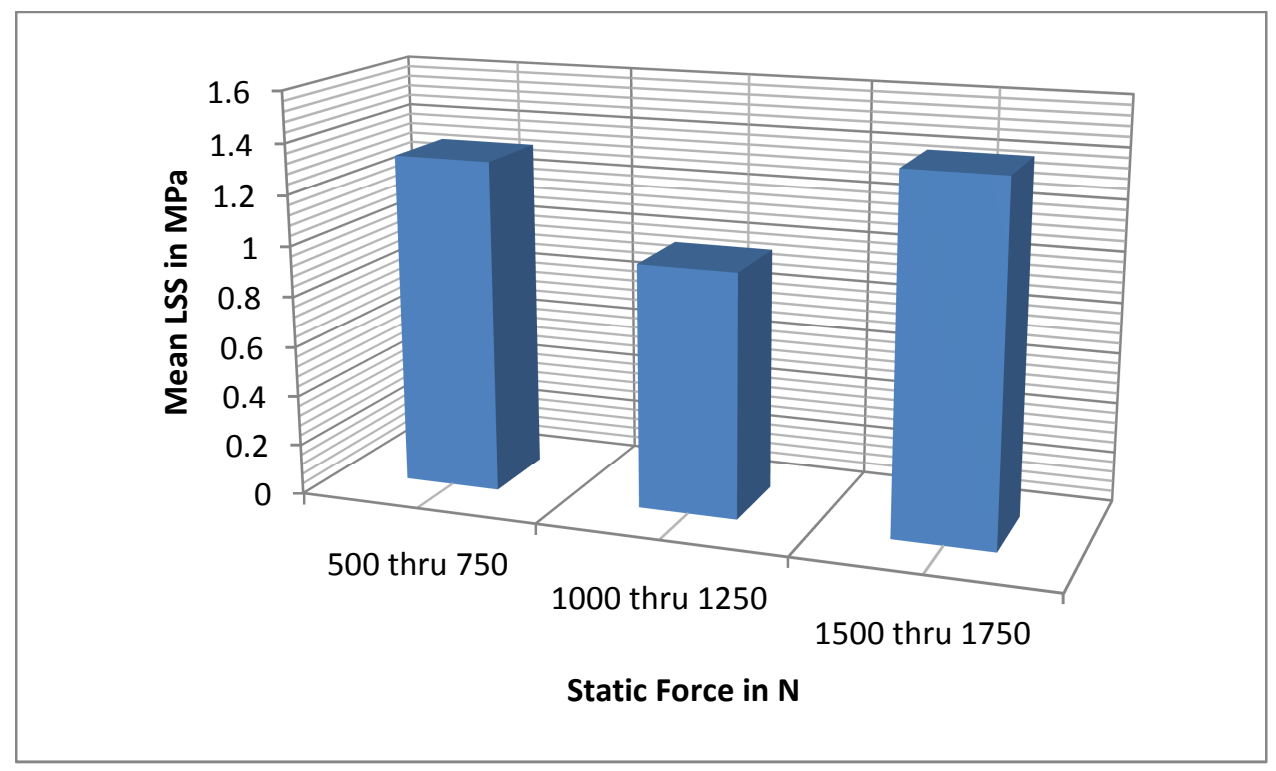

Figure 5.22 Effect of static force categories on mean LSS

\section{Amplitude}

It can be seen from the data in Table 5.14 that means of LSS for amplitudes 21 and 18.9 are close to each other and higher than that for 16.8. Means of LSS for amplitudes are ordered as $21>>18.9 \gg>16.8$. On increasing the amplitude by $25 \%$, LSS is observed to be amplified by 47.2\%. Mean LSS from Table 5.14 is shown graphically in Figure 5.23. The graph in Figure 
5.23 shows an increasing tendency of mean LSS on increasing the amplitude of ultrasonic vibrations until it flattens out.

Table 5.14 Mean Sqrt_LSS

Tukey HSD

\begin{tabular}{||c||c||c|c||}
\hline \multirow{2}{*}{ Amplitude } & \multirow{2}{*}{$\mathbf{N}$} & \multicolumn{2}{|c|}{ Subset } \\
\cline { 3 - 4 } & & $\mathbf{1}$ & $\mathbf{2}$ \\
\hline \hline 16.8 & 240 & 0.89 & \\
\hline 18.9 & 275 & & 1.28 \\
\hline 21 & 262 & & 1.31 \\
\hline
\end{tabular}

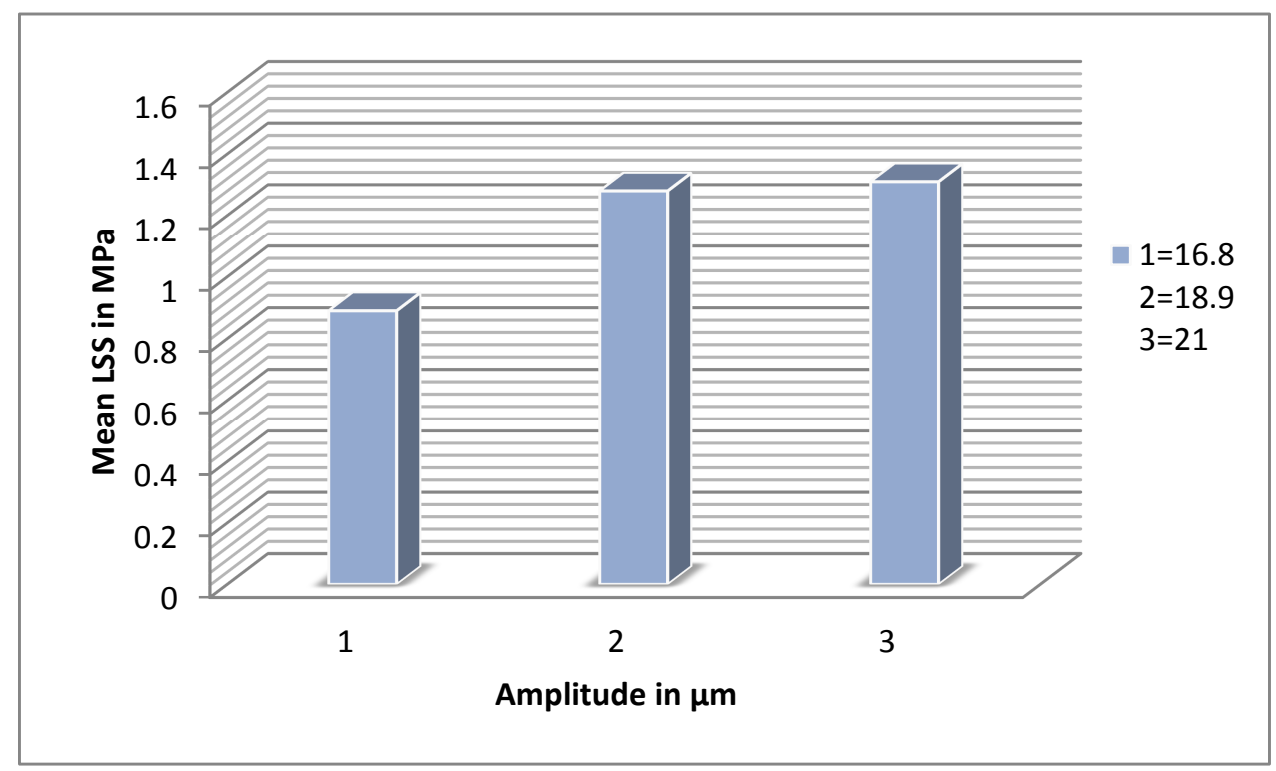

Figure 5.23 Effect of amplitude on mean LSS

$\underline{E D}$

It is apparent from the Table 5.15 that means of LSS for both EDs can be ordered as SEMI > TRI. A graph based on Table 5.15 is illustrated in Figure 5.24. The bar graph of Figure 5.24 shows that mean LSS is higher for SEMI ED than that for TRI ED.

Table 5.15 Comparison based on mean Sqrt_LSS

\begin{tabular}{||c||c||c|c||}
\hline \hline \multirow{2}{*}{$\begin{array}{c}\text { Energy } \\
\text { Director }\end{array}$} & \multirow{2}{*}{ Mean } & \multicolumn{2}{|c|}{ 95\% Confidence Interval } \\
\cline { 3 - 4 } & & $\begin{array}{c}\text { Lower } \\
\text { Bound }\end{array}$ & $\begin{array}{c}\text { Upper } \\
\text { Bound }\end{array}$ \\
\hline \hline SEMI & 1.22 & 1.15 & 1.28 \\
\hline TRI & 0.83 & 0.64 & 1.01 \\
\hline
\end{tabular}




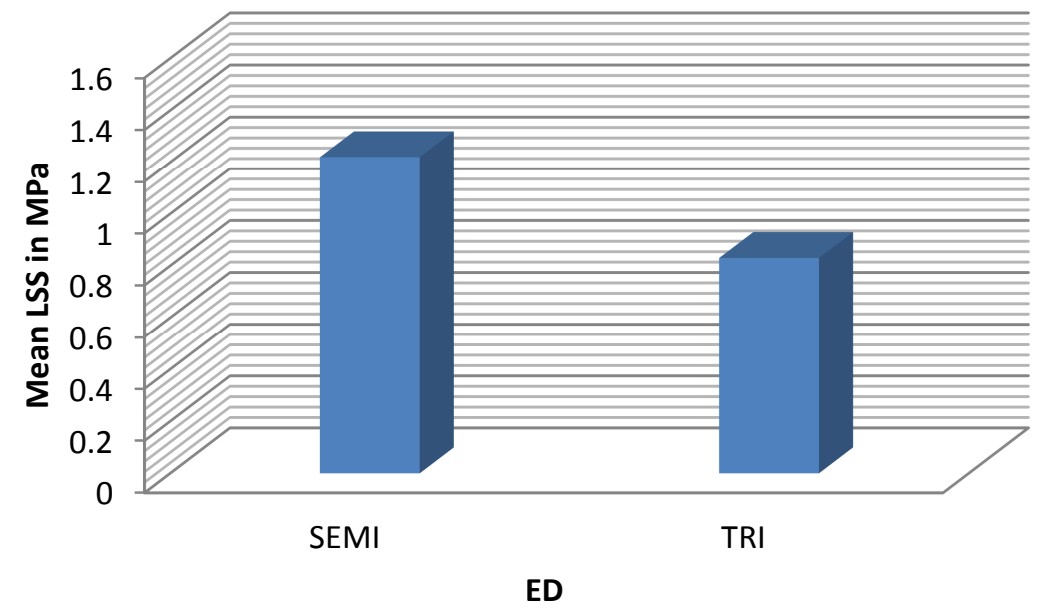

Figure 5.24 Effect of EDs on Mean LSS

\section{$\underline{\text { Weld Time }}$}

The Table 5.16 illustrates that means of LSS for categories of weld time i.e. 1.1 thru $1.3 \& 0.8$ thru 1.0 are smaller and closer to each other as compared to that for weld category 1.4 thru 1.7. Means of LSS for categories of weld time are ordered as 1.4 thru $1.7>>0.8$ thru $1.0>>1.1$ thru 1.3. LSS increases by $76.67 \%$ with an increase of weld time by $112.5 \%$. Mean LSS from Table 5.16 has been represented in Figure 5.25. An increasing trend of mean LSS is observed in Figure 5.25 when weld time is increased.

Table 5.16 Mean Sqrt_LSS

\begin{tabular}{|c|c|c|c|}
\hline \multirow{2}{*}{ Weld Time } & \multirow{2}{*}{$\mathbf{N}$} & \multicolumn{2}{|c|}{ Subset } \\
\hline & & 1 & 2 \\
\hline $\begin{array}{c}1.1 \text { thru } \\
1.3 \\
\end{array}$ & 269 & 0.84 & \\
\hline $\begin{array}{c}0.8 \text { thru } \\
1.0 \\
\end{array}$ & 180 & 0.90 & \\
\hline $\begin{array}{c}1.4 \text { thru } \\
1.7\end{array}$ & 328 & & 1.59 \\
\hline
\end{tabular}




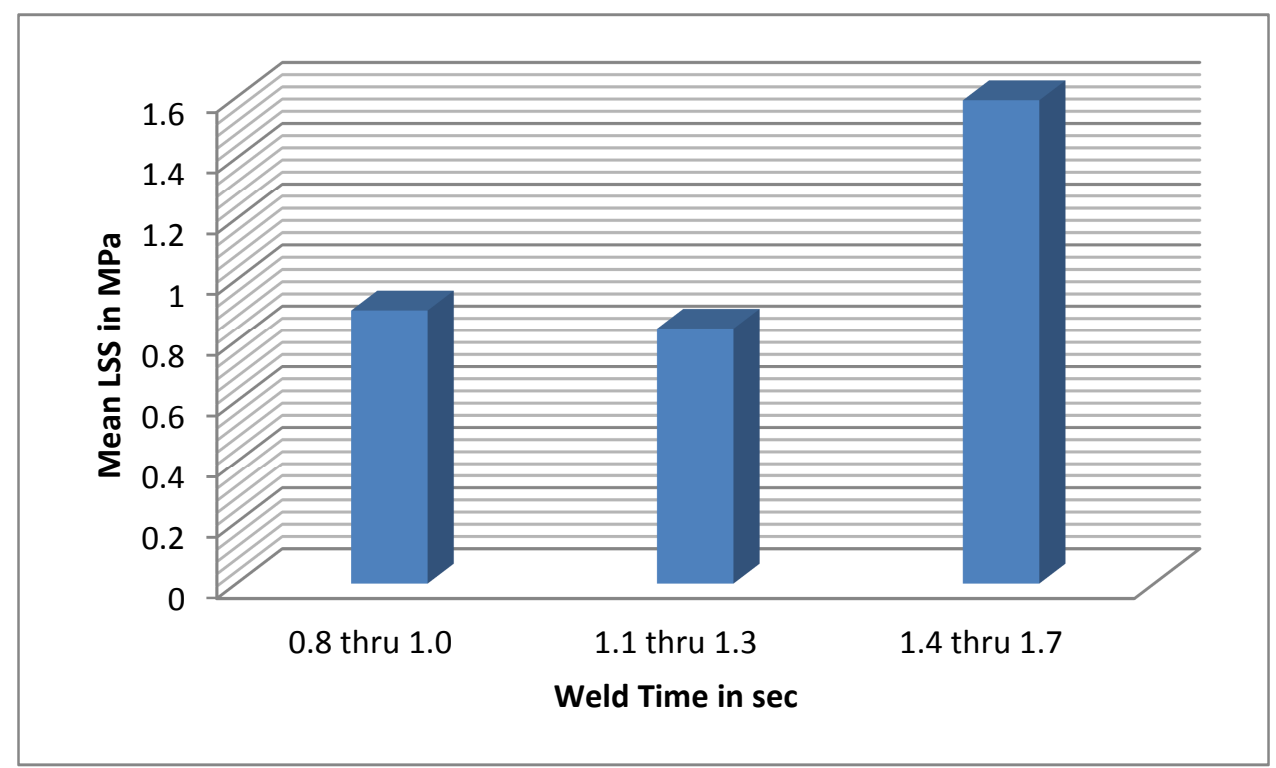

Figure 5.25 Effect of weld time categories on mean LSS

\subsubsection{Profile Plots}

\section{$\underline{\text { ED and Weld Time }}$}

To assess the relationships between mean LSS and various weld factors, profile plots have been acquired from the preliminary analysis. Profile plot in Figure 5.26 shows relationships between weld time categories and means of LSS at two EDs. Again for SEMI and TRI EDs, these relationships increase with increase in welding time. Largest values of mean LSS are found for SEMI ED. Trend for PP\&SEMI ED is similar to what has been observed for ABS\&SEMI ED. Percent increase in mean LSS from TRI to SEMI ED at highest weld time is $12.5 \%$.

SI for two lines in Figure 5.26 is $13.33 \%$. Hence there is a probability of having an interaction between ED and weld time. This is also an interesting interaction effect and will sufficiently be interpreted in discussion chapter. 


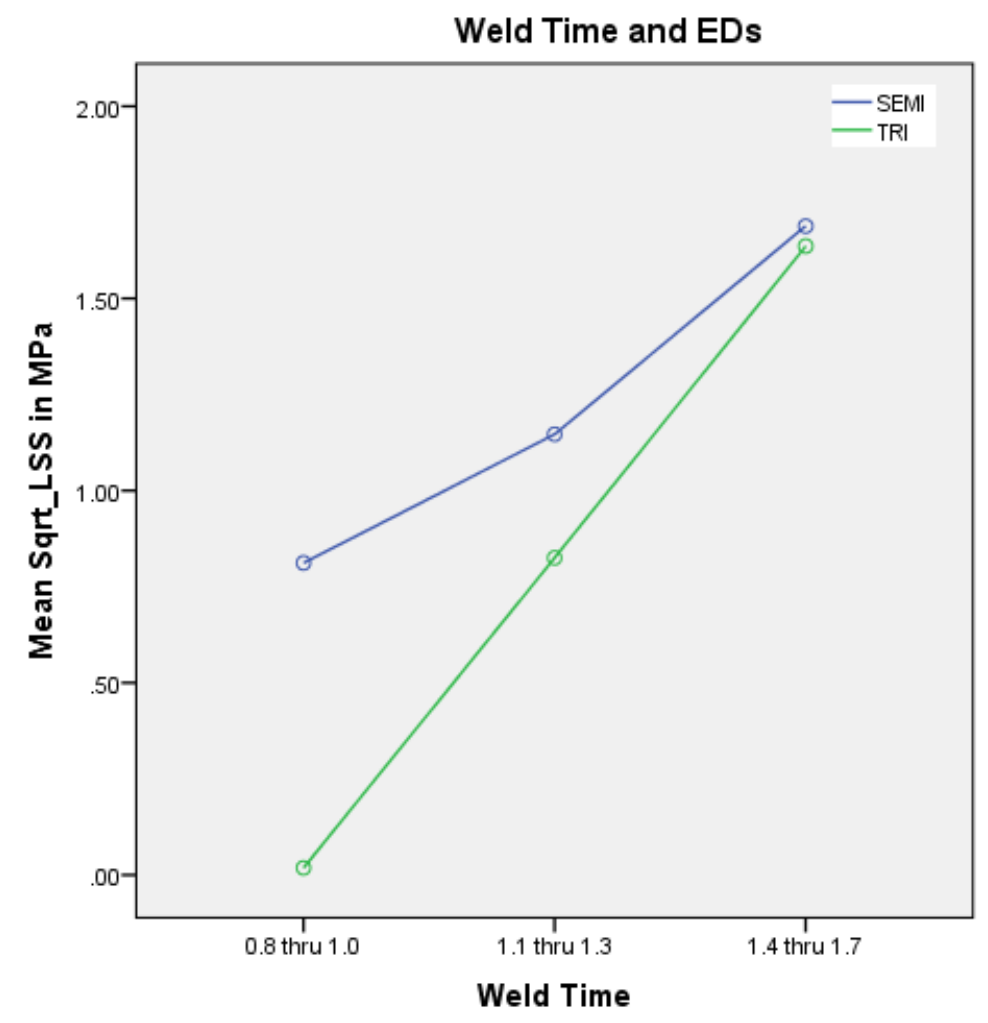

Figure 5.26 Profile Plot (ED and Weld Time)

\section{Amplitude and Weld Time}

Profile plot in Figure 5.27 illustrates the relationship between weld time categories and means of LSS at three amplitudes of ultrasonic vibrations. At weld categories 0.8 thru 1.0, mean LSS for all amplitudes is noted to be far away from each other. At 1.1 thru 1.3, means are observed to be closer to each other. This relationship for PP is also similar to that for ABS. Means of LSS are interestingly observed to achieve their maximum and same contribution at highest weld time for amplitudes $18.9 \& 21$. Hence percent increase from 16.8 to 18.9 or 21 at highest weld time is $25 \%$.

In addition, an interaction between amplitudes and weld time categories cannot be expected due to the presence of parallel lines. 


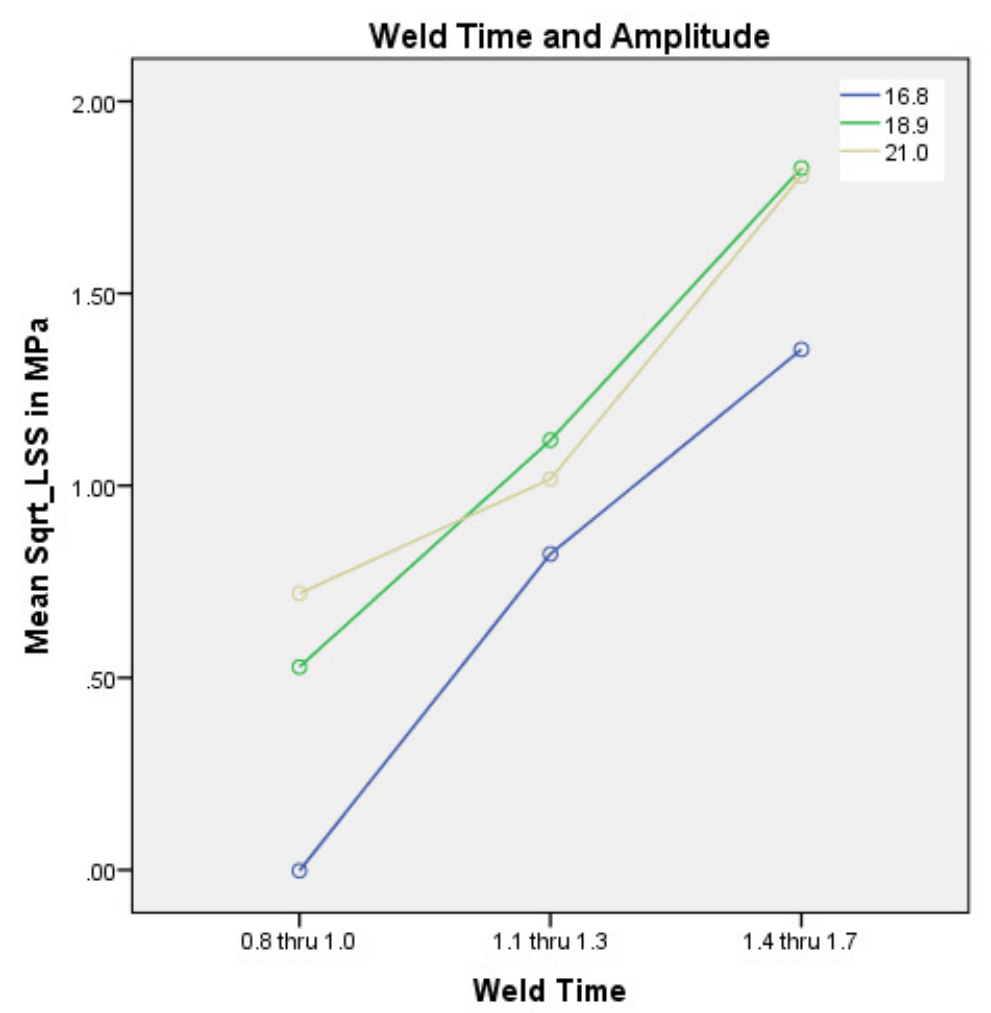

Figure 5.27 Profile Plot (Amplitude and Weld Time)

\section{Amplitude and ED}

Profile plot in Figure 5.28 explains the relationship between amplitude of ultrasonic vibrations and means of LSS at two EDs. Relationships of SEMI and TRI EDs both have trend of increasing LSS consistently. At 21 amplitude, means of LSS have huge difference in values for SEMI and TRI EDs. But at 18.9, considerable difference in values of means exists for SEMI and TRI EDs and small difference of means at 16.8. This trend for PP is also analogous to that for ABS. From Figure 5.28, the percent increase is also calculated to be $33.33 \%$ and $55.8 \%$ from TRI to SEMI ED at lowest and highest amplitudes respectively.

Profile plot in Figure 5.28 shows almost parallel lines as per two intervals of amplitudes. Therefore, interaction between amplitude and ED is absent here. 


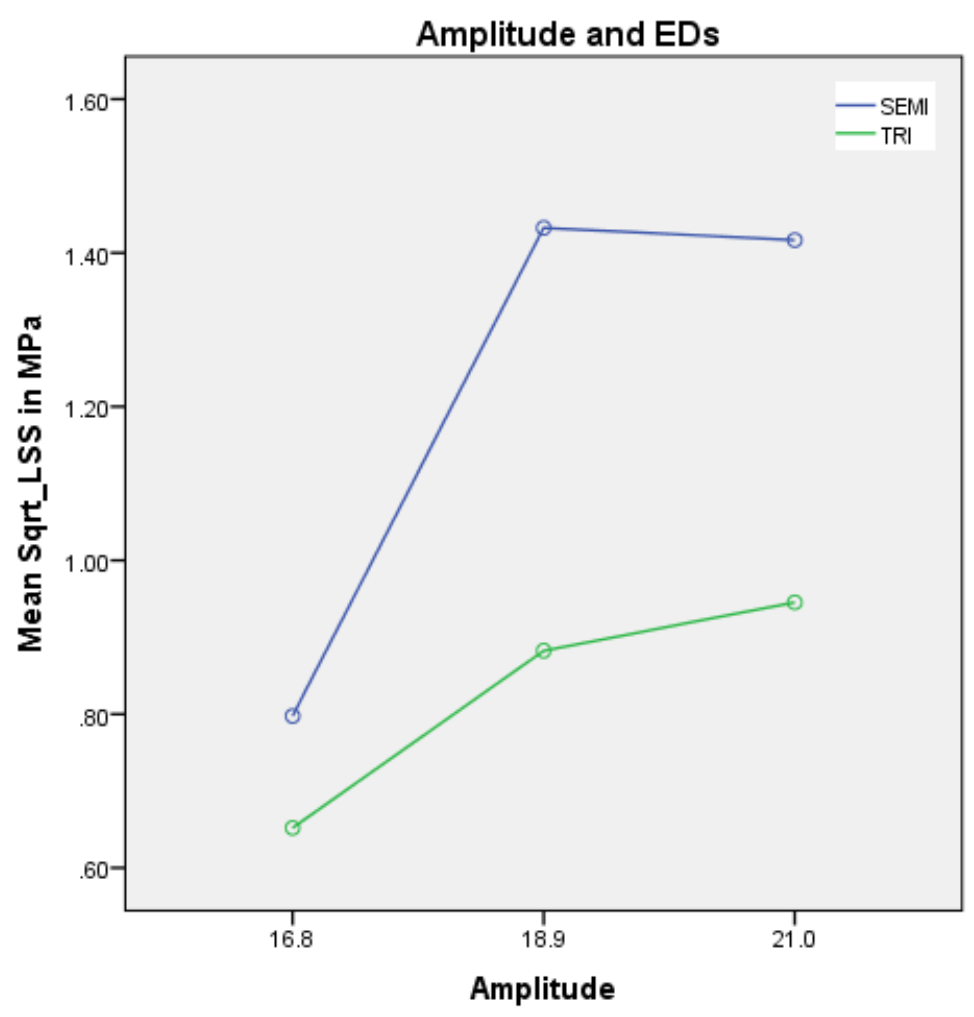

Figure 5.28 Profile Plot (Amplitude and ED)

\subsection{Summary}

A statistical method called GLM has been performed for ultrasonic weld strength data. Significance of main and interaction effects has been tested by ANOVA results. All factors and interactions between factors have been found to be significant for both ABS and PP.

Also statistical significance of a particular factor does not mean for its practical significance.Hence post hoc tests and profile plots have been employed to provide with better insight into significant effects particularly for those factors that have more than two levels/categories.

In current interaction analysis, static forces and EDs were found to have interdependence for ABS, whereas EDs, weld times and amplitudes were also found to have interdependence for PP, as shown in Table 5.17. Therefore, combined effect of interacting factors on LSS must be interpreted. 
Table 5.17 Interaction analysis

\begin{tabular}{||c||c||c||}
\hline Materials & Factors Interacting & $\begin{array}{c}\text { Severity } \\
\text { Index } \\
\text { (\%) }\end{array}$ \\
\hline \hline ABS & ED*Static Force & 53.33 \\
\hline \multirow{2}{*}{ PP } & $\begin{array}{c}\text { Amplitude*Weld } \\
\text { Time }\end{array}$ & 21.11 \\
\cline { 2 - 3 } & ED*Weld Time & 13.33 \\
\hline
\end{tabular}

Moreover, weld time based on F-values has been found to be the most significant weld factor for both ABS and PP, as can be seen from ANOVA Tables in Appendix C.

For ABS, profile plots (Figure 5.14 to 5.21 ) have shown some common tendencies which need to be addressed seriously in discussion section later on. Some of the findings are intended to note down here. For example, means of LSS have been found greater for TRI ED than those for SEMI ED in all profile plots. Higher values of mean LSS are observed for amplitudes 21 and 18.9 in all profile plots. Profile plot between categories of static force and ED is interestingly crucial because of decreasing trend of mean LSS for TRI ED and increasing trend of mean LSS for SEMI ED. An overall increasing trend of mean LSS has been found for relationship between weld time and ED or amplitude and ED. On plotting profiles separately for EDs, plot between categories of static force and amplitude shows the increasing and fluctuating trends of mean LSS for TRI ED and SEMI ED respectively.

For PP, the weld factors and their interactions have also been found to be significant. There are present some common and the most important tendencies in all profile plots for PP. Means of LSS are always found greater for SEMI ED than those for TRI ED. In the same fashion, mean LSS has been found higher for amplitudes 21 and 18.9. Profile plots between welding time and ED for mean LSS are found to be continuously increasing. Similarly, means of LSS increase uninterruptedly for profile plots between amplitude and ED. Profile plots have been observed between categories of static forces and ED or Amplitude for sudden rise and fall of mean LSS. These trends will be explained critically in discussion section in terms of their effects on LSS.

The most important commonality among profile plots for ABS and PP is the highest values of mean LSS for amplitudes 21 and 18.9 which have resulted in flattening of trends. The 
biggest difference among profile plots for $A B S$ and PP is again the highest values of mean LSS that are associated with TRI ED and SEMI ED respectively. Likewise, means of LSS increase continuously for profile plots between weld time and ED for both materials. For both materials, profile plots between categories of static force and amplitude or ED are largely inconsistent in terms of sudden drops and increments of means. These brief summaries of trends for both materials at this stage have made the basic foundations for the discussion section.

Most of the trends have been explained initially but these trends need to be elaborated later on in discussion section in order to make them clearer.

Prediction of GLM Univariate Model is available for ABS and PP in appendix D. 


\section{CHAPTER 6 DISCUSSION}

As mentioned in the literature, weld strength can be affected by various ultrasonic weld factors. Therefore, this study set out with the aim to gain a deeper understanding of factors which influence the UW of thermoplastics in an attempt to improve the weld strength. The current study was also designed to determine the effect of weld factors on ultrasonic bond strength.

\subsection{Overall Lap Shear Strength}

In this study, highest LSSs of $17 \mathrm{MPa}$ and $6 \mathrm{MPa}$ were measured for ABS and PP respectively. According to datasheet of $A B S$, the tensile strength of $A B S$ is $50 \mathrm{MPa}$. Hence the highest LSS was found to be $34 \%$ of original material strength for ABS. In the same fashion, the tensile strength for PP is $41 \mathrm{MPa}$ from its datasheet. So, the highest LSS was $14.63 \%$ of material strength for PP. Above percentages of LSS suggest us that the resulting weld from ABS seems to be stronger than that from PP. Weld strength may further be improved if following options are used:

1. Specimen design including geometry of ED must be improved to attain more weld strength.

2. Current sonotrode has a circular tip contacting with the upper specimen. Rectangular tip of sonotrode can progress the weld strength which is beyond the scope of this research.

3. On using different materials from two major groups of thermoplastics i.e. amorphous e.g. PVC, PEI, PS and semi-crystalline e.g. PEEK, PPS, High or Low density PE etc.

4. With the hindsight, bond strength can furthest be improved by crossing the current limits of weld factors.

5. Heat affected zone and its spread must be controlled firmly to improve the overall joint strength. This will be discussed later in microscopy section (chapter 10).

\subsection{Effect of molecular structure on results}

In this research, ABS and PP were welded ultrasonically. There exists a clear difference between the molecular structure of $\mathrm{ABS}$ and PP. ABS has a randomly oriented molecular 
structure, whereas PP has a definite order for its molecular structure. Former one is known as amorphous thermoplastic and later as semi-crystalline thermoplastic. This will be explored further in the DSC (Differential Scanning Calorimeter) section (7.1). Owing to this difference in molecular structure, ultrasonic welding of ABS and PP was found to be different from every aspect e.g. bond formation for these polymers was different, LSS was different, statistical analysis was different, factor settings were different during sampling and welding as well.

\subsection{Effect of weld factors on mean LSS}

Effect of weld factors on mean LSS needs to be discussed here in detail after identifying the results from GLM-Univariate analysis.

\subsubsection{Acrylo-nitrile Butadiene Styrene (ABS)}

\section{Effect of Categories of Static Force}

Figure 6.1 illustrates that the static force has a different effect on mean LSS for both EDs. Surprisingly; difference of means is larger between TRI and SEMI EDs for minimum value of static force i.e. 500 or $750 \mathrm{~N}$ and the same mean gap is reduced to a greater extent on increasing the static force. In fact, there exists a severe interaction between high level of static force and EDs with a severity index (SI) of 53.33\%.

The practical implications of these findings from Figure 6.1 suggest that it is not beneficial to utilize SEMI ED instead of TRI ED if highest LSS is desired. This is owing to the minimum value of mean LSS that is found at all categories of static force for SEMI ED. Decreasing trend of TRI ED on increasing static force approves of using 500 thru $750 \mathrm{~N}$ static force range in order to have maximum value of mean LSS. This finding may help select the optimal level of static force and type of ED in achieving better weld. 


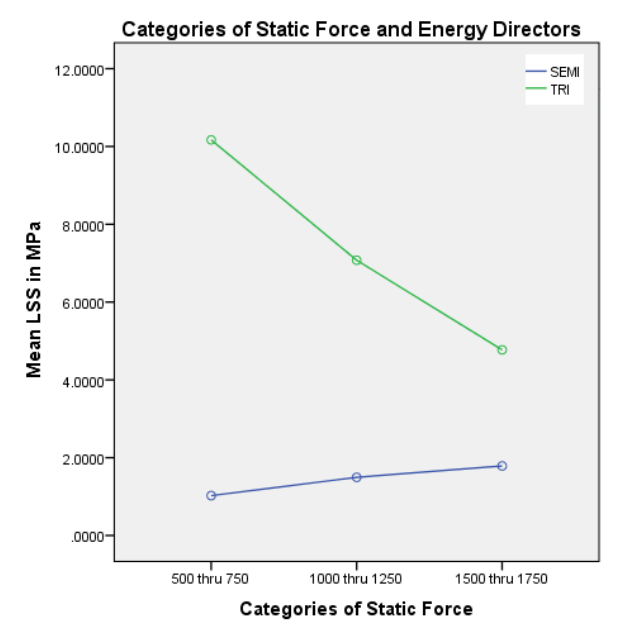

Figure 6.1 Effect of categories of static force

A possible reason for using low level of static force is the smallest volume of TRI ED as compared to volume of SEMI ED which implies that larger concentration of ultrasonic energy in case of TRI ED. Due to this reason, larger portion of a TRI ED has been melted quickly as compared to SEMI ED. As ABS is an amorphous thermoplastic so, it is having glass transition temperature $\left(103{ }^{\circ} \mathrm{C}\right)$. As temperature goes above its glass transition temperature, the viscosity of the ABS drops down and molten ABS starts flowing. It is that point where the role of static force comes in. On increasing the static force to its high level for TRI ED, molten material has been pressed severely out of the expected bond area resulting in low LSS. This is why; high static force is thought of a major reason for observing the decreasing trend of LSS in case of TRI ED for ABS. Crushing and spreading of the molten TRI ED are shown in Figure 6.2 when high level of static force is used. This will further be addressed later in chapters on HSVC (High Speed Video Camera) (chapter 9), FEA (chapter 8) and MFI (Melt Flow Index) (section 7.2).

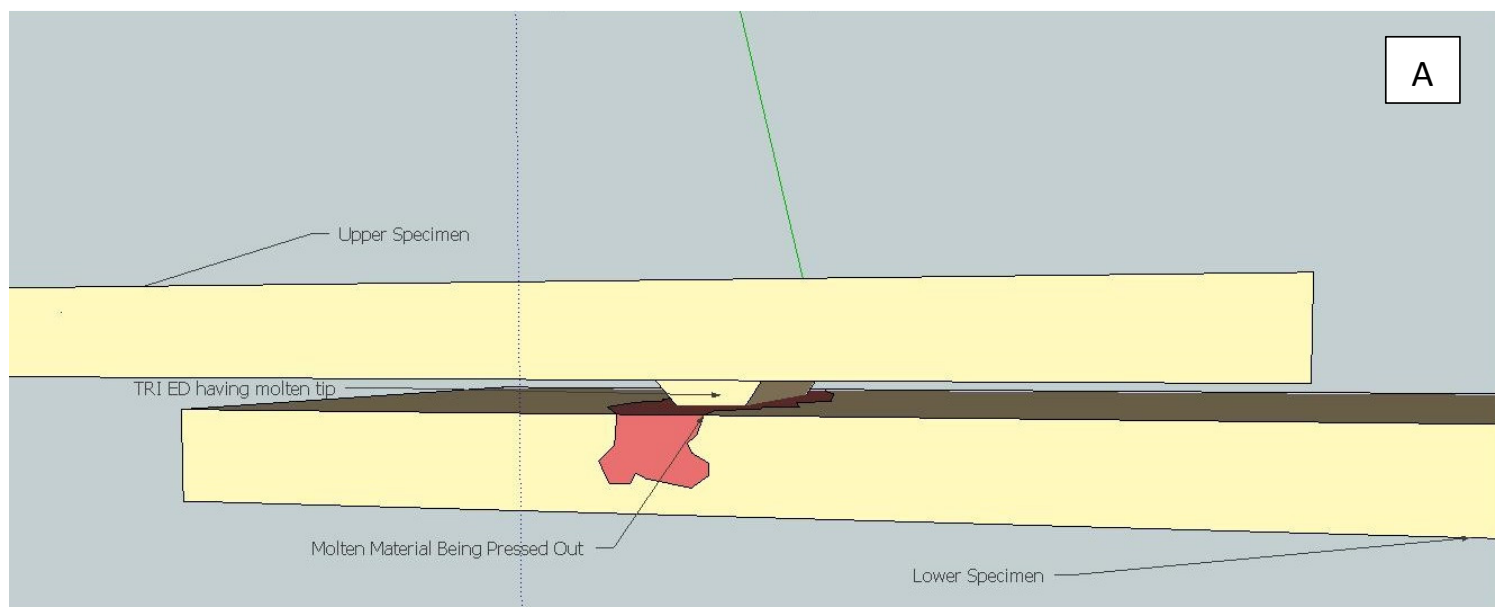



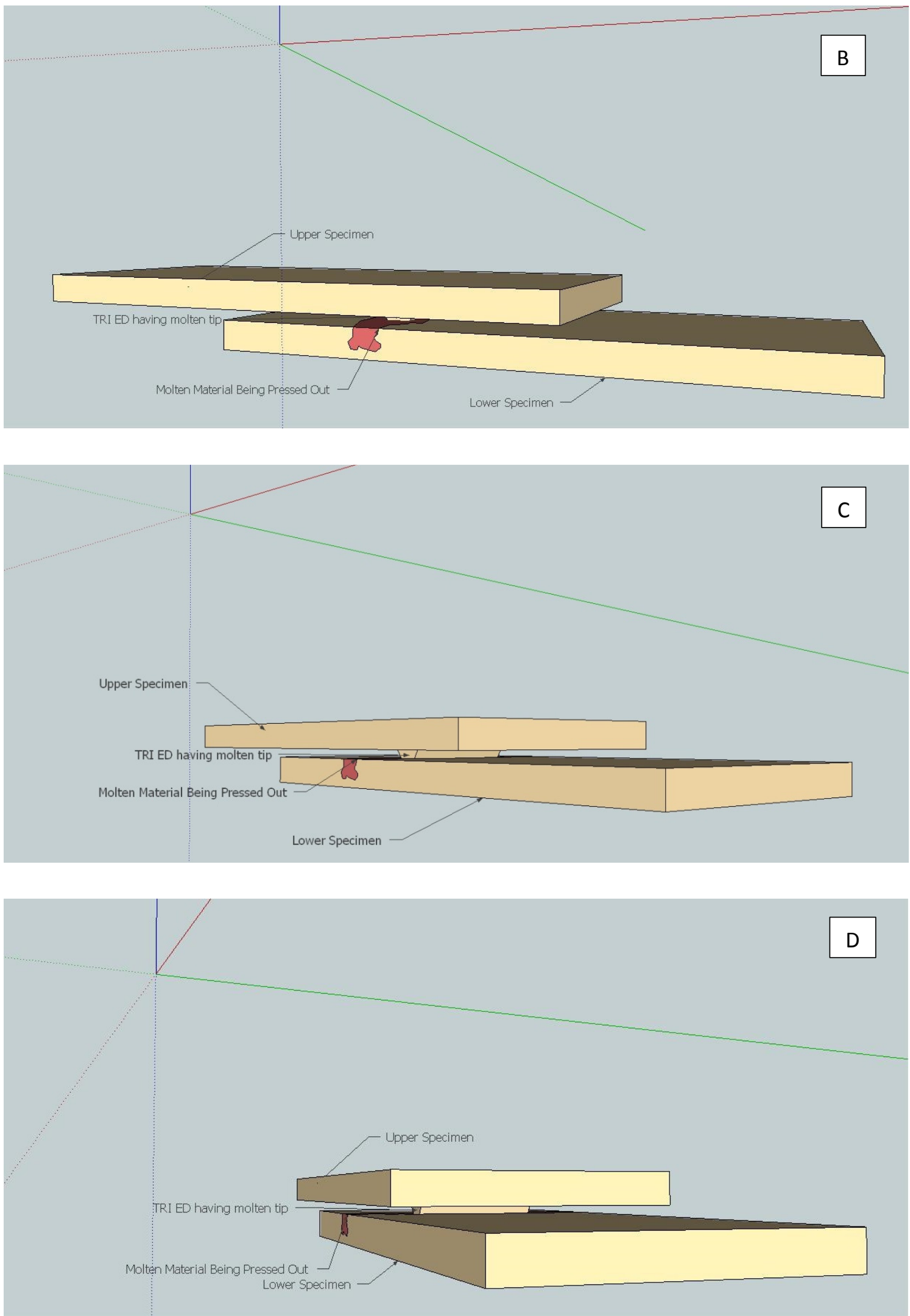

Figure 6.2 (A, B, C, \&D) The red molten ABS pressed out of the weld zone 
As far as SEMI ED is concerned, the situation has been found to be opposite of TRI ED from Figure 6.1. This result can be explained by the fact that complete crushing of SEMI ED has not been found even for a single case. And molten portion of SEMI ED distributes itself really well at weld zone by increasing the static force contributing ultimately in achieving higher LSS. This is also the possible reason that a continuous increase of LSS is observed on increasing the static force for SEMI ED.

These findings from Figure 6.1 are in agreement with Figure 6.3 and Figure 6.4. Moreover, minimum values of mean LSS have been found for SEMI ED for all static forces which do not suggest us to utilize SEMI ED that is illustrated in Figure 6.1 \& 6.3. On the other hand, higher values of mean LSS have been found from Figure $6.1 \& 6.4$ at low level of static force (500 thru 750) which strongly recommends us to use TRI ED for ultrasonic welding of ABS.

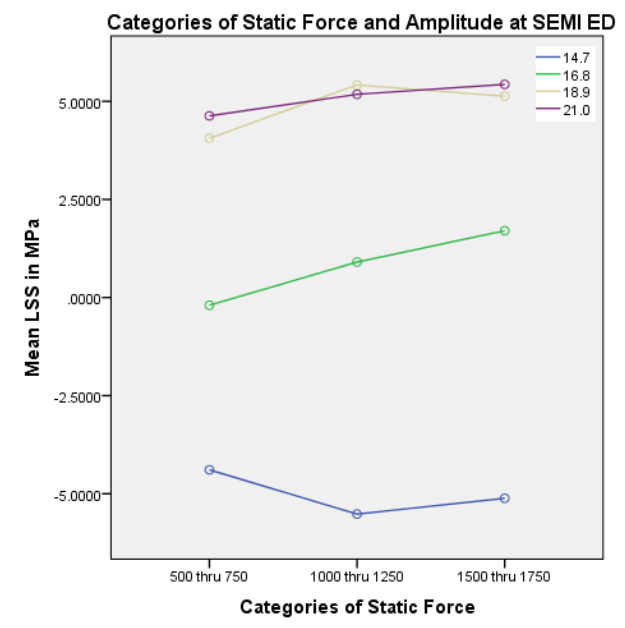

Figure 6.3 Effect of categories of static force at SEMI ED

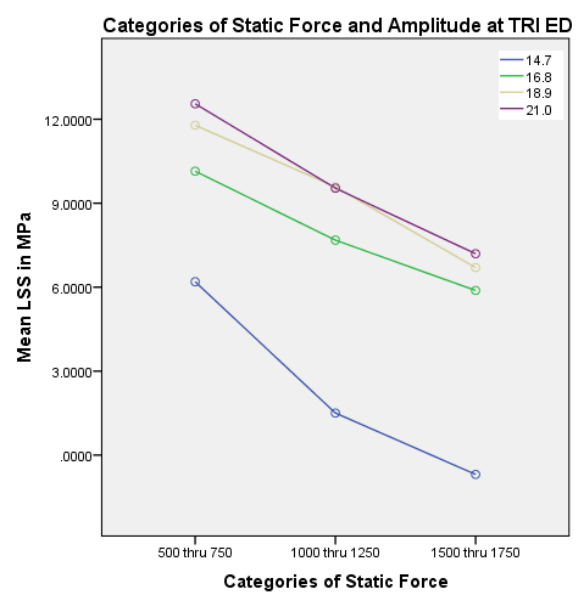

Figure 6.4 Effect of categories of static force at TRI ED 
Utilization of TRI ED is also confirmed from Table 6.1 because mean LSS for TRI ED is higher than that for SEMI ED.

Table 6.1 Mean LSS for EDs

\begin{tabular}{||c||c||c|c||}
\hline \hline \multirow{2}{*}{$\begin{array}{c}\text { Energy } \\
\text { Director }\end{array}$} & \multirow{2}{*}{ Mean } & \multicolumn{2}{|c||}{ 95\% Confidence Interval } \\
\cline { 3 - 4 } & & $\begin{array}{c}\text { Lower } \\
\text { Bound }\end{array}$ & $\begin{array}{c}\text { Upper } \\
\text { Bound }\end{array}$ \\
\hline \hline SEMI & 1.44 & 0.93 & 1.94 \\
\hline TRI & 7.34 & 7.13 & 7.55 \\
\hline
\end{tabular}

On increasing the welding pressure, the welding efficiency tends to decrease for $A B S$. Although increasing welding pressure up to 4 bars, this efficiency is observed to increase for $\mathrm{PE}$. By further increasing the welding pressure beyond 4 bars, welding efficiency for even PE has been decreased [107].

According to literature on statistical analyses of ultrasonic welding, weld pressure does not appear to be effective in attaining optimal weld. This explains why overall increasing trend for both EDs throughout all categories of static force has not been obtained [110].

According to Eswaran, on increasing, weld pressure does not appear to improve weld strength for both types of thermoplastics [189].

Note; welding pressure from previous studies refers to static force for present work.

\section{Effect of Amplitude of Ultrasonic Vibrations}

The relationship between amplitude of vibrations and means of LSS in Figure 6.5 shows an overall increasing trend for both EDs. Values of means shown in Figure 6.5 are greater for TRI ED than those for SEMI ED.

It is also noted that maximum value of LSS has been again obtained for TRI ED. Also from Figure 6.5, for amplitudes 21 and $18.9 \mu \mathrm{m}$, mean LSS seems to achieve its highest value because of straightening of line after gradually increasing. It seems possible that this result is due to the stable effect of high amplitudes on bond formation. 


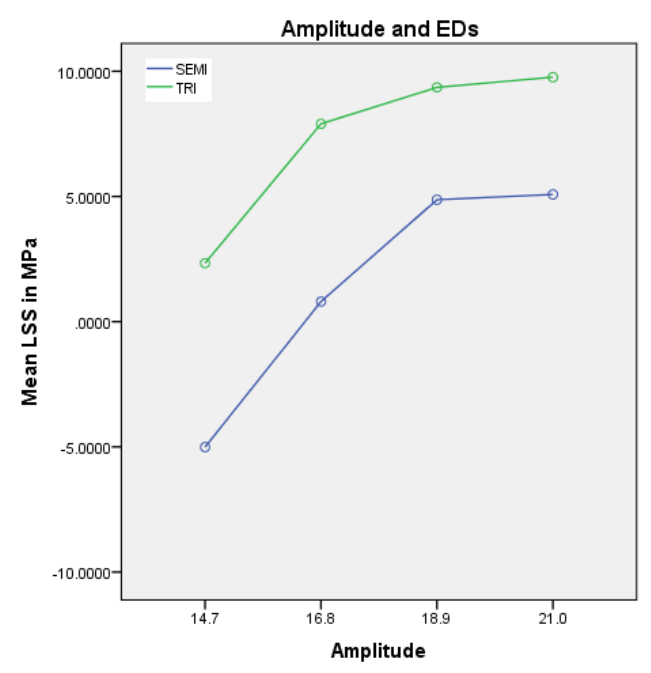

Figure 6.5 Effect of amplitude of ultrasonic vibrations

A likely explanation is that an ultrasonic vibration consists of frequency, amplitude and time period. It is known very well for an ultrasonic vibration that frequency and time period remain constant ( $20 \mathrm{KHz}$ and $50 \mu \mathrm{sec}$ respectively). In this work, it was then only amplitude that was varied at constant intervals of $2.1 \mu \mathrm{m}$. For an optimized value of LSS, a proper and stable supply of molten material is required at expected interface of weld. This is owing to the presence of high level of amplitudes that a sufficient supply of molten material is originated by utilizing the collapse of ED. This stable or uniform availability of molten material is further spread evenly under the action of static force hence resulting in proper HAZ and optimized LSS. Therefore, a tight control over the supply of molten material (ABS or PP) from both EDs (SEMI or TRI) can be ensured by employing high levels of amplitudes. This is because of this reason, a highest value of LSS is observed in Figure 6.5 which results in levelling of trend eventually at higher amplitudes. At higher amplitude, a portion of bonded material contributes unvaryingly to provide resistance to bond failure on shear testing in tension.

This will also be explained later in FEA (Finite Element Analysis) (chapter 8) and HSVC (chapter 9).

According to literature on statistical analyses of ultrasonic welding for thermoplastics, amplitude of ultrasonic vibrations is also believed to be significant to give optimized ultrasonic weld [110]. 
Similarly, increasing both weld time and amplitude contribute significantly to achieve the final joint strength for both types of thermoplastics [189].

\section{Percent Variations in LSS after welding ABS ultrasonically}

Percent variations in mean LSS resulted from weld factors were also analysed in this research. TRI ED with its specific geometry has been preferred for securing better LSS. It can be seen from Table 6.1 that a higher value of mean LSS i.e. 7.34 MPa has been noted through GLM analysis for TRI ED as compared to that for SEMI ED i.e. 1.44 MPa. A percent increase from SEMI to TRI ED is found, therefore, to be almost 409\%.

In this analysis, static force was divided into three categories. 500 thru $750 \mathrm{~N}$ has been found to be the best range of static forces for ABS in achieving better weld strength. As Table 6.2 shows mean LSS from these static forces is found to be $8.23 \mathrm{MPa}$ and similarly $7.14,5.84$ from categories of static forces 1000 thru $1250 \mathrm{~N}, 1500$ thru $1750 \mathrm{~N}$ respectively. Percent change from 500 thru 750 to 1500 thru 1750 reaches to its maximum value of $41 \%$.

Table 6.2 Mean LSS in MPa

Tukey HSD
\begin{tabular}{|c|c||c|c|c||}
\hline $\begin{array}{c}\text { Categories } \\
\text { of Static } \\
\text { Force }\end{array}$ & \multirow{N}{*}{$\mathbf{N}$} & $\mathbf{1}$ & $\mathbf{2}$ & $\mathbf{3}$ \\
\cline { 2 - 5 } & & 5.84 & & \\
\hline $\begin{array}{c}1500 \text { thru } \\
1750\end{array}$ & 281 & & 7.14 & \\
\hline $\begin{array}{c}1000 \text { thru } \\
1250\end{array}$ & 536 & & & 8.23 \\
\hline $\begin{array}{c}500 \text { thru } \\
750\end{array}$ & 529 & & & \\
\hline
\end{tabular}

Four types of amplitudes were chosen in this work. The Table 6.3 shows that means of LSS are 8.04 and $8.32 \mathrm{MPa}$ for 21 and $18.9 \mu \mathrm{m}$ respectively. A little percent increase is observed from 21 to $18.9 \mu \mathrm{m}$ but the percent increase becomes considerable from 14.7 to $21 \mu \mathrm{m}$ or 14.7 to $18.9 \mu \mathrm{m}$ guiding us to preferably use 21 and $18.9 \mu \mathrm{m}$ as one of the welding factors.

Table 6.3 Mean LSS in MPa

Tukey HSD
\begin{tabular}{|c|c||c|c|c||}
\hline \multirow{2}{*}{ Amplitude } & \multirow{2}{*}{ N } & \multicolumn{3}{|c|}{ Subset } \\
\hline \hline 14.7 & 166 & 5.19 & & $\mathbf{2}$ \\
\hline 16.8 & 376 & & 6.34 & \\
\hline 21 & 413 & & & 8.04 \\
\hline 18.9 & 391 & & & 8.32 \\
\hline
\end{tabular}


Weld time was divided into three categories in this study. From the Table 6.4; 9.18, 6.98 and 2.82 MPa are the mean LSS values from categories of weld time 0.8 thru $0.9,0.6$ thru 0.7 and 0.4 thru $0.5 \mathrm{sec}$ respectively. Percent increase in mean LSS from category 0.6 thru 0.7 to 0.8 thru 0.9 is detected to be almost $31.5 \%$. Similarly, a significant increase in percentage from category 0.4 thru 0.5 to 0.6 thru 0.7 has been emerged to be almost 148 and almost 226, from 0.4 thru 0.5 to 0.8 thru 0.9. Percent increase related to all three categories of weld time suggests recommending categories 0.8 thru 0.9 and 0.6 thru $0.7 \mathrm{sec}$ in obtaining optimum results from ultrasonic welding.

Table 6.4 Mean LSS in MPa

Tukey HSD

\begin{tabular}{|c|c|c|c|c|}
\hline \multirow{2}{*}{ Weld Time } & \multirow{2}{*}{ N } & \multicolumn{3}{|c|}{ Subset } \\
\hline & & 1 & 2 & 3 \\
\hline 0.4 thru 0.5 & 274 & 2.82 & & \\
\hline 0.6 thru 0.7 & 360 & & 6.98 & \\
\hline 0.8 thru 0.9 & 712 & & & 9.18 \\
\hline
\end{tabular}

\section{Additional Interesting Effects}

In addition, some interesting effects have also been observed after analysing the results. These effects are listed below.

1. $A B S$ is easier to weld (chapter $7 \&$ chapter 8 )

2. Lower collapse of ED is found for ABS than that for PP (chapter 9)

3. Both fracture modes i.e. ductile and brittle are observed on testing weld strength (section 10.5)

\subsubsection{Polypropylene (PP)}

\section{Effect of welding time on LSS}

Combined effect of ED and weld time can properly be interpreted by acquiring the profile plots from the preliminary analysis. Profile plot in Figure 6.6 shows relationships between weld time categories and means of LSS at two EDs. For both SEMI and TRI EDs, these relationships increase with increase in welding time. Largest values of mean LSS are found for SEMI ED. Trend for PP\&SEMI ED is similar to what has been observed for ABS\&SEMI ED. 
Severity Index for two lines in Figure 6.6 is $13.33 \%$. Hence there is a high probability of having an interaction between ED and weld time. This is essentially an interesting interaction effect and needs to be interpreted in this chapter.

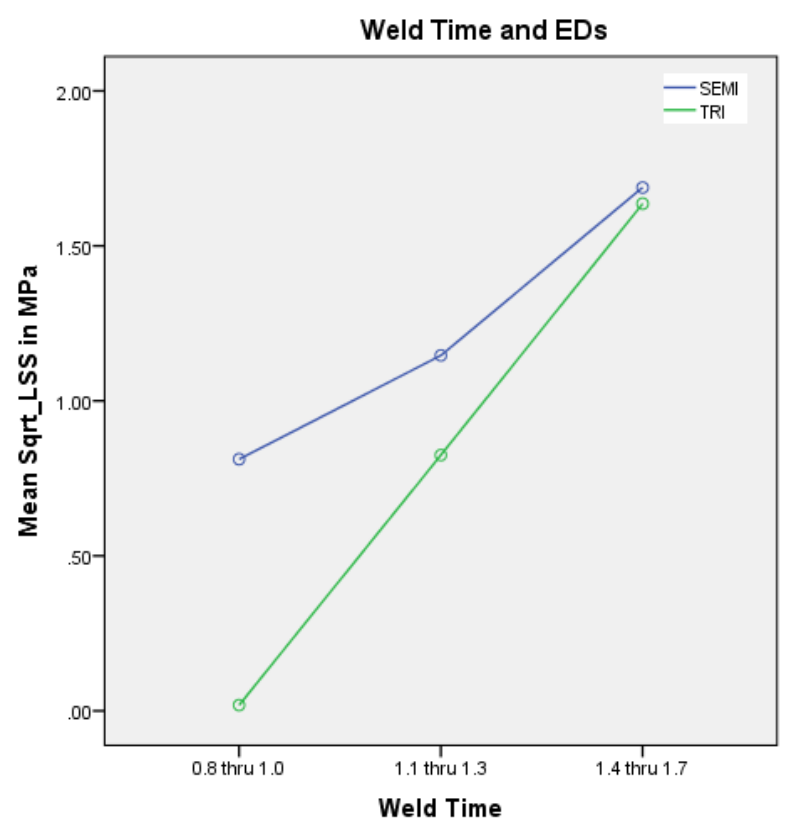

Figure 6.6 Profile Plot (ED and Weld Time)

Weld time is that factor attaining the highest significance with F-Value (103.27) from ANOVA results for PP, as mentioned in Appendix C. One second of weld time means the supply of 20,000 cycles of ultrasonic vibrations to the samples. Slight variation in LSS may be observed if low level of weld time is used because of using two different geometries of ED. SEMI ED has been completely collapsed and melted to a greater extent at low level of weld time as compared to TRI ED owing to its higher volume $\left(140.13 \mathrm{~mm}^{3}\right)$ than that of TRI ED $\left(90 \mathrm{~mm}^{3}\right)$. Bond spread and bond formation are also found better using semicircular geometry of ED at low level of weld time because bigger heat affected zone is acquired by higher volume of semicircular energy directors.

Moreover, higher weld time implies that more cycles of vibrations will be imposed on the samples to be welded. Furthermore, PP has lower loss modulus and heat distortion temperature which mean that ED will severely be crushed if high level of weld time will be set before welding. Loss modulus is related to the energy dissipated as heat when ultrasonic vibrations are applied to the specimens. Loss modulus is also called as out-of-phase component of complex modulus. Moreover, heat distortion temperature (HDT) is defined as 
the distortion rate of a material at a particular temperature. This is also a likely reason for obtaining same resisting weld area after welding and same weld strength after testing the ultrasonic weld. Hence at high level of welding time, a complete collapse of both EDs is expected which may result in almost similar LSS. These are additional and plausible reasons for achieving approximately equal mean LSS at higher weld times. Hence optimal LSS can absolutely be attained when high level of welding time is set for welding PP. In general, role of any ED may be neglected if high level of weld time is utilized to weld PP.

\section{Effect of Amplitude of Ultrasonic Vibrations}

The relationship between amplitude of ultrasonic vibrations and means of LSS is exhibited in Figure 6.7 for two EDs. These relationships of SEMI and TRI EDs are found to increase consistently. At $21 \mu \mathrm{m}$ amplitude, means of LSS have huge difference in values for SEMI and TRI EDs. But at amplitude $18.9 \mu \mathrm{m}$, considerable difference in values of means exists for SEMI and TRI EDs and the lowest difference of means is found for $16.8 \mu \mathrm{m}$. The highest value for mean LSS is noted at amplitudes 18.9 and $21 \mu \mathrm{m}$ for both TRI and SEMI EDs. On increasing the amplitude from 18.9 to $21 \mu \mathrm{m}$, LSS appears to attain its maximum value after being gradually increased. Reasons for achieving this LSS are similar to those for ABS but the maximum value of mean LSS for PP here is less than that for ABS.

One of the likely reasons that mean LSS is comparable for both EDs at low level of amplitude is, amplitude is attenuated or decreased when an ultrasonic vibration is propagated through PP. Hence lower LSS is obtained with PP as compared to ABS. For PP, the attenuation rate of vibrations is higher with TRI ED rather with SEMI ED because higher concentration of ultrasonic energy is attained with TRI ED. Therefore, higher LSS is achieved with SEMI ED after welding PP. Moreover, amplitude and decay rate of ultrasonic oscillations are also affected by the ratio of energy stored to energy dissipated which is higher for PP i.e. 12.09, as shown in Table 6.5. 
Table 6.5 [189] Complex moduli for both materials

\begin{tabular}{||c||c||c||c||}
\hline \hline Complex Moduli (GPa) & Frequency (KHz) & ABS & PP \\
\hline \hline Storage Modulus (GPa) & 20 & 2.75 & 3.87 \\
\hline Loss Modulus (GPa) & 20 & 0.42 & 0.32 \\
\hline $\begin{array}{c}\text { Storage Modulus /Loss } \\
\text { Modulus }\end{array}$ & 20 & 6.55 & 12.09 \\
\hline
\end{tabular}

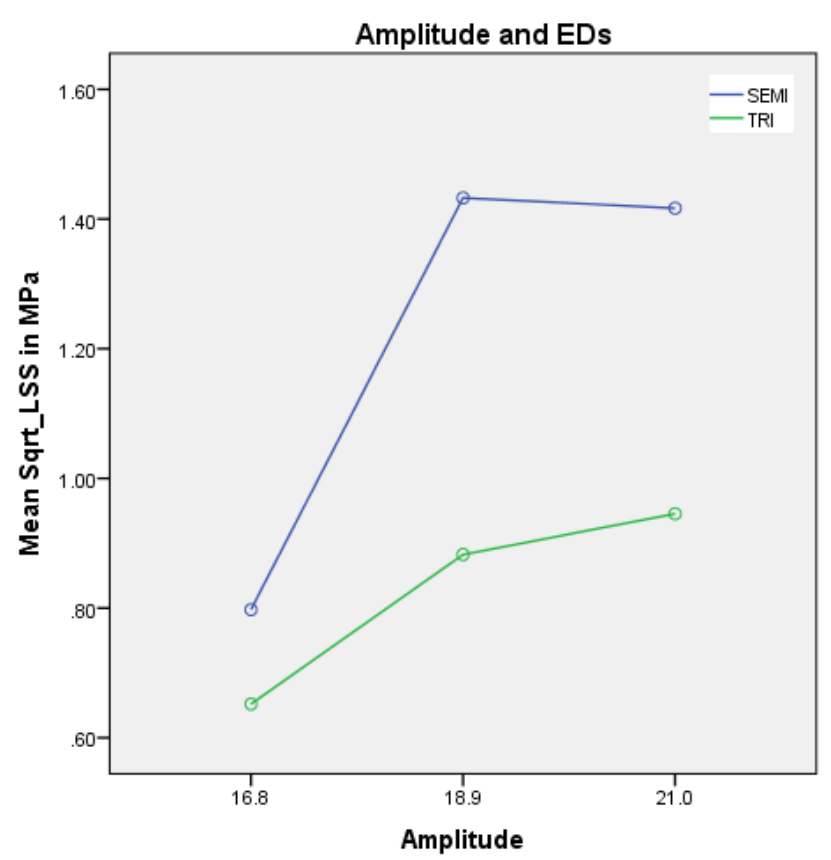

Figure 6.7 Effect of amplitude of ultrasonic vibrations

Following account of basic concepts and experimental observations are intended to help explain the above reasons in order to justify the selection of high levels of weld factors especially amplitude for PP.

Behaviour of thermoplastic has been modelled with the help of a dashpot and a spring. A basic model known as Voigt model is shown in Figure 6.8 in an attempt to gain basic understanding of thermoplastic materials. Moreover, this model must not be thought of implementing it practically. This model is considered as a guide for qualitative thinking about thermoplastics. 


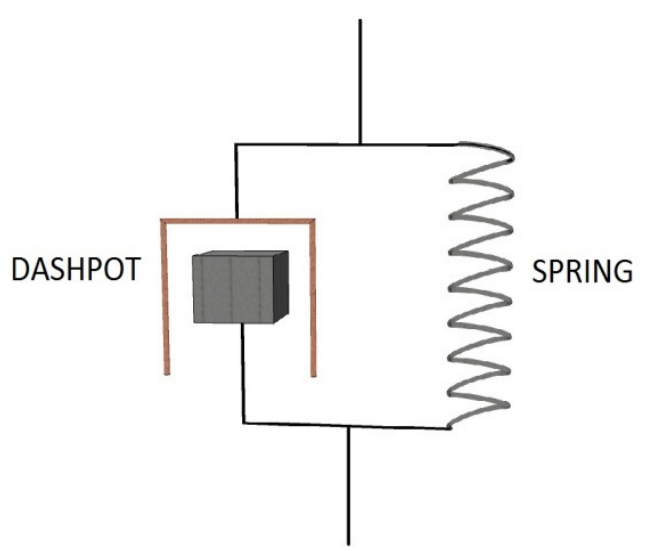

Figure 6.8 Voigt model

For instance, according to literature and Voigt model, PP is found to be more elastic like a spring than that of ABS. Due to this fact, it is expected that a portion of supplied ultrasonic energy is recovered after being stored properly on welding PP that is problematic [189].

Polypropylene (PP) is a semi-crystalline thermoplastic and it has definitely sharp melting point of $170.55{ }^{\circ} \mathrm{C}$. In order to melt ED during ultrasonic welding, sufficient amount of ultrasonic energy needs to be supplied to PP specimens. A specific portion of ultrasonic energy always returns back owing to the higher spring content i.e. storage modulus for PP. And high ultrasonic energy is only possible through utilizing high levels of weld factors. This is also deduced on later pages showing percent variation in LSS for PP. This energy is also necessary due to the sharp melting point of PP. Melting point of PP has been tested in DSC section of chapter 7 .

\section{Area of Cross Section of bond vs bond strength: Experimental Observations}

In this research, the area of cross section obtained after getting the ultrasonic bond fractured was supposed to be circular in most of the cases because of using high level of ultrasonic welding factors. Fewer cases were also observed with rectangular cross sections of the bond resulting from either low weld time or low amplitude or in general low level of ultrasonic welding factors. For acquiring greater LSS or higher welding efficiency with PP, production of circular bond cross section or considerably large rectangular cross section was necessary after welding. This finding is somewhat surprising and suggests us to use high 
level of ultrasonic welding factors. Also the volume of SEMI ED is higher than that of TRI ED meaning the availability of sufficient molten material on welding PP ultrasonically. The Table 6.10 suggests using the high level of weld factors to acquire strong bond for PP.

Similarly, increasing weld time and amplitude contribute significantly to achieve the final joint strength. But the joint strength has been levelled off (i.e. no further improvement in bond strength) by increasing the weld time up to greater than $1.5 \mathrm{sec}$ for PP [189].

In conclusion, higher amplitude, higher weld time and SEMI ED are preferred to other amplitudes, weld times and EDs respectively for welding PP ultrasonically. Suitable considerations will also be paid to other weld factors for their selection.

\section{Percent Variations in LSS after welding PP ultrasonically}

In this research, SEMI ED was selected as the best welding factor for polypropylene after scrutinizing the results. From Table 6.6, mean LSS from SEMI ED is determined to be 1.22MPa, whereas from TRI ED, it is $0.83 \mathrm{MPa}$. The percent increase from TRI to SEMI ED is witnessed to be almost $47 \%$ approving SEMI ED to achieve better welds.

Table 6.6 Comparison based on mean Sqrt_LSS

\begin{tabular}{||c||c||c|c||}
\hline \multirow{2}{*}{$\begin{array}{c}\text { Energy } \\
\text { Director }\end{array}$} & \multirow{2}{*}{ Mean } & \multicolumn{2}{|c|}{ 95\% Confidence Interval } \\
\cline { 3 - 4 } & & $\begin{array}{c}\text { Lower } \\
\text { Bound }\end{array}$ & $\begin{array}{c}\text { Upper } \\
\text { Bound }\end{array}$ \\
\hline \hline SEMI & 1.22 & 1.15 & 1.28 \\
\hline TRI & 0.83 & 0.64 & 1.01 \\
\hline
\end{tabular}

In this study, static force was divided into three categories. From Table 6.7; 1.40, 1.32 and 0.97MPa are noted to be the mean LSS values from categories of static force 1500 thru 1750,500 thru 750 and 1000 thru $1250 \mathrm{~N}$ respectively. The percent increase from 1000 thru $1250 \mathrm{~N}$ to 1500 thru 1750 and from 1000 thru $1250 \mathrm{~N}$ to 500 thru750 is found to be almost 44.33 and 36.08 respectively recommending 1500 thru $1750 \mathrm{~N}$ for getting better bond strength. 
Table 6.7 Mean Sqrt_LSS

\begin{tabular}{|c|c|c|c|}
\hline \multirow{2}{*}{$\begin{array}{c}\text { Categories } \\
\text { of Static } \\
\text { Force }\end{array}$} & \multirow{2}{*}{$\mathbf{N}$} & \multicolumn{2}{|c|}{ Subset } \\
\hline & & 1 & 2 \\
\hline $\begin{array}{c}1000 \text { thru } \\
1250\end{array}$ & 385 & 0.97 & \\
\hline $\begin{array}{c}500 \text { thru } \\
750\end{array}$ & 152 & & 1.32 \\
\hline $\begin{array}{c}1500 \text { thru } \\
1750\end{array}$ & 240 & & 1.40 \\
\hline
\end{tabular}

For welding PP, three amplitudes were considered i.e. $21,18.9$ and $16.8 \mu \mathrm{m}$ with the fact that $14.7 \mu \mathrm{m}$ was that value of amplitude of ultrasonic vibrations which resulted in no weld situations. Means of LSS from three amplitudes are 1.31, 1.28 and 0.89 respectively, as can be seen from Table 6.8. The percent increase from 18.9 to $21 \mu \mathrm{m}$ is the lowest i.e. almost $2 \%$, from 16.8 to 21 and $18.9 \mu \mathrm{m}$ is closer to each other i.e. round about 47.2 and 44 respectively. This recommends the utilization of 21 or $18.9 \mu \mathrm{m}$ as a crucial amplitude level in welding PP ultrasonically.

Table 6.8 Mean Sqrt_LSS

\begin{tabular}{|c|c|c|c|}
\hline \multirow{2}{*}{ Amplitude } & \multirow{2}{*}{$\mathbf{N}$} & \multicolumn{2}{|c|}{ Subset } \\
\hline & & 1 & 2 \\
\hline 16.8 & 240 & 0.89 & \\
\hline 18.9 & 275 & & 1.28 \\
\hline 21 & 262 & & 1.31 \\
\hline
\end{tabular}

Weld time for PP was divided into three categories i.e. 0.8 thru 1.0, 1.1 thru 1.3 and 1.4 thru $1.7 \mathrm{sec}$ in this analysis. From Table 6.9, weld time category 1.4 thru $1.7 \mathrm{sec}$ is the best with the highest mean LSS i.e. 1.59MPa.

Table 6.9 Mean Sqrt_LSS

\begin{tabular}{|c|c|c|c|}
\hline \multirow{2}{*}{ Weld Time } & \multirow{2}{*}{$\mathrm{N}$} & \multicolumn{2}{|c|}{ Subset } \\
\hline & & 1 & 2 \\
\hline $\begin{array}{c}1.1 \text { thru } \\
1.3\end{array}$ & 269 & 0.84 & \\
\hline $\begin{array}{c}0.8 \text { thru } \\
1.0\end{array}$ & 180 & 0.90 & \\
\hline $\begin{array}{c}1.4 \text { thru } \\
1.7\end{array}$ & 328 & & 1.59 \\
\hline
\end{tabular}




\section{Further Interesting Effects}

In addition to earlier discussed effects, some effects are also worth mentioning here which will be discussed in detail later. These effects are mentioned below.

1. PP is difficult to weld (chapter 8)

2. Higher collapse of ED is found for PP than that for ABS (chapter 9)

3. Both fracture modes i.e. ductile and brittle are observed on testing weld strength (section 10.5)

\subsection{Overall Summary}

From Table 6.10, it has already been known that levels of main weld factors are quite similar for both thermoplastics except choice of ED. Another exception found here is the difference in setting the levels of static forces. This is one of the unanticipated finding examined in this research. Low level of static force for ABS is logically necessary in order to stabilize the flow of molten ABS into flat molten form with ED collapse. But as far as PP is concerned, another aspect of static force needs to be considered here that is to counterbalance the return of some ultrasonic energy during welding. As high ultrasonic energy is required to break orderly structure of PP hence high level of static force needs also to be ensured before setting high levels of other welding factors.

Table 6.10 Factors' level in gaining quality weld for both materials

\begin{tabular}{|c||c||c||c||c|}
\hline Material & Static Force $(\mathbf{N})$ & $\begin{array}{c}\text { Weld Time } \\
(\mathbf{s e c})\end{array}$ & $\begin{array}{c}\text { Amplitude of Vibration } \\
(\boldsymbol{\mu m})\end{array}$ & ED \\
\hline ABS & Low & High & High & TRI \\
\hline PP & High & High & High & SEMI \\
\hline
\end{tabular}

Table 6.11 is representing a comparison of percent changes in LSS between both ABS and PP. Overall, percent changes are the highest for ABS as far as all ranges of factors are concerned [37]. Welding time, static force, and ED have higher percent change in mean LSS for $A B S$ than that for PP. Additionally; percent change from amplitude range for $A B S$ is 
almost similar to that for PP. This finding confirms that the stability of bond strength is associated with amplitudes for both thermoplastics.

Table 6.11 Percent change in LSS for both materials

\begin{tabular}{||c|c|c|c|c|}
\hline \multicolumn{1}{|c||}{ Factors } & \multicolumn{2}{c||}{ Range } & \multicolumn{2}{c|}{ \% change in LSS } \\
\hline \hline Material & ABS & PP & ABS & PP \\
\hline $\begin{array}{c}\text { Energy } \\
\text { Director }\end{array}$ & $\begin{array}{c}\text { SEMI to } \\
\text { TRI }\end{array}$ & $\begin{array}{c}\text { TRI to } \\
\text { SEMI }\end{array}$ & 409 & 47 \\
\hline Weld Time & min-max & min-max & 226 & 76.67 \\
\hline Static Force & min-max & min-max & 41 & 6 \\
\hline Amplitude & min-max & min-max & 55 & 47.2 \\
\hline
\end{tabular}

\subsection{Comprehensive Discussion}

\subsubsection{Areas for further investigation}

Carey [190] defines the aim of science in his book "A Beginner's Guide to Scientific Method" that Science is that activity which aims to further our understanding of why things happen as they do in the natural world. It accomplishes this goal by applications of scientific methodprocess of observing, proposing explanations and testing these explanations. Hence scientists investigate that why things happen as they do in the natural world. And the method by which a scientist studies these things is called the scientific method. This very scientific method is fully acquainted with the predefined domain of research field.

Therefore, scientific method is generally a way of acquiring knowledge with three basic steps after having defined the research aim i.e. observation, speculation and testing [190].

\section{Observation/Identifying interesting effects}

Before exploring something scientifically, it must be made sure that proper sense of the facts surrounding the phenomenon has been investigated at first place. In fact, an explicit research aim needs to be stated even before actually starting this observation. At very basic level, this research aim can be exemplified as "Why do we not see the moon every night on the sky?" or "What is the natural phenomenon behind sunrise and sunset every day?" etc. Background knowledge of phenomenon/process under investigation is necessary in order to get more understanding of the process because it may lay basic foundations for correct and accurate implementation of next stages of scientific method. 
In this research, the aim of current work was mentioned clearly at the end of chapter 3. Background knowledge was achieved by going through literature. Research spaces were identified in an effort to establish a need for acquiring new perspective of UWP. Facts about UWP were also extensively obtained by primary but considerable experimentation. Hence in present context, the observation for scientific method consists of literature review and findings from initial experimentation using ultrasonic welding.

Whilst some of the results obtained were as expected, there were several effects identified warranting further investigation. This observation step of scientific method was also devoted to briefly describing these interesting effects, as mentioned in Table 6.13.

First interesting effect indicates that weld strength in terms of LSS is mostly found to be higher for ABS than that for PP. This reveals that dissimilar bond strengths may be produced by welding different thermoplastics ultrasonically. Therefore, difference in molecular structure and material strength of both $A B S$ and PP is thought to be a reason for first interesting effect.

Second effect is related to ED geometry. Two ED geometries were used in this research named as TRI and SEMI. Effect of ED geometries was found to be alternatively effective when both $A B S$ and PP were welded ultrasonically. Hence the likely reason for this interesting effect is the difference in the volumes of TRI and SEMI EDs. In other words, volume of TRI ED is lower than that of SEMI ED.

Levels of weld factors must be carefully selected for welding both ABS and PP, as mentioned in third interesting effect. In this work, some weld factors were set at their low levels in acquiring better weld strength for ABS. The plausible reason for setting low levels of weld factors is the higher value of loss modulus that is associated with ABS.

Effect of ED collapse is stated by fourth interesting effect in Table 6.13. When ultrasonic vibrations are imposed on samples with $E D$, distortion of ED is significantly expected while the samples are welded. Degree of LSS is also associated with this ED collapse for both ABS and PP. The expected reason for this interesting effect is the difference in thermal (Heat Distortion Temperature), as shown in Table 6.12, and dynamic properties (Loss Modulus) of both $A B S$ and PP. 
Table 6.12 Heat distortion temperature for both materials

\begin{tabular}{|c|c|}
\hline Material Type & HDT $\left({ }^{\circ} \mathbf{C}\right)$ \\
\hline \hline ABS & 85 \\
\hline PP & 56 \\
\hline
\end{tabular}

In this study, weld strength (LSS) was tested and measured by tensometer after welding both ABS and PP. Bond fracture was obtained by testing LSS. Both types of fracture modes (ductile or brittle) were observed during LSS measurement, as can be seen in $5^{\text {th }}$ interesting effect. The probable reason for the bond fracture is crazing, as will be discussed in section 10.5 of chapter 10 . However, fracture modes must not be affected by crazing.

Heedful selection of static force was also needed to weld both ABS and PP, as mentioned in $6^{\text {th }}$ interesting effect. As observed from experimental results, low level of this force was required for efficiently welding $A B S$ but this was not true for welding PP. The feasible reason for this interesting effect is the higher viscosity and lower glass transition temperature of ABS.

In this research, LSS was evaluated after welding ABS and PP. For last interesting effect, LSS was found to be higher and uniform at higher amplitudes of ultrasonic vibrations. This was not only observed for ABS but also for PP. The anticipated reason for this effect is the possibility of attaining the similar resisting area at weld zone after welding both ABS and PP.

\section{Speculation/Hypothesis Development}

A number of explanatory factors is introduced in getting fully explained the answer of the research question that why does this phenomenon occur in that way. For instance, why does the sun appear to rise and set every day? The answer of this question is the way earth rotates about its own axis in twenty four hours of the full sunny day. If the answer of a scientific question is not fully understood then the explanation usually results in implicit implications of apparent description. Hence in order for a process/question to get sufficiently understood and challenge the best explanations of the day, some conjectures/hypotheses are associated with the problem which, if true, can help better explain answer of question. 
Having reviewed and looked back the literature on UWP after experimentation, reasons of various interesting results were pondered for their accuracy and precision. In fact, various descriptive statements were hypothesized in order to answer a question that involves why do rudimentary experimental results appear like this way from UW of thermoplastics by setting fair factors in a matrix? Table 6.13 is a complete representation of interesting results/effects of certain causes leading to proposed explanations/hypotheses which have the potential of either supporting existing explanations or disproving the existing prevailing views about existing reasons. Hence these hypotheses were finally tested with appropriate testing methods in this work.

For example, from Table 6.13, hypothesis A presents a reason for first interesting effect that higher LSS for ABS may be related to its different molecular structure and higher base material strength. On the other hand, PP is a semicrystalline type of thermoplastics with lower base material strength. Hence hypothesis A is defined as "ABS is an amorphous thermoplastic with higher tensile strength than that of PP". Various testing methods can be employed in order to check empirically whether hypotheses predicted are right. If the proposed hypothesis is right then a good reason will be there to accept the assumed description for its correctness. If experimental results fail to prove the tentative speculation then hunch/educated guess/hypothesis will either be rejected or modified. In this research, DSC, MFI and FEA were used to test hypothesis A, as stated in Table 6.13. In general, Table 6.13 intends to make a comprehensive assessment of effects of weld factors on weld strength by introducing various interesting effects along with their observational findings. Each hypothesis deduced will be tested with the help of certain testing methods which are linked with different sections of thesis. In fact, this Table 6.13 is quite revealing and involved in many ways unlike other tables in this thesis.

Hypothesis $B$ has also been deduced to find out the probable reason for $2^{\text {nd }}$ interesting effect. Proposed reason states that volume of TRI ED is actually lower than that of SEMI ED. And low volume of any ED may result in higher concentration of ultrasonic energy during welding ABS. But for welding PP, higher ED volume will be needed due to the fact that sufficient molten material at weld interface may help attain a good bond. Therefore, hypothesis B is stated as "Volume of TRI ED is less than that of SEMI ED resulting in high 
concentration of ultrasonic energy". To test this hypothesis, MFI and FEA were utilized in this study.

Hypothesis $\mathrm{C}$ attempts to describe the possible reason for setting low level of weld factors for $A B S$. The likely reason declares that welding temperature may easily be obtained for $A B S$ owing to its higher loss modulus as compared to PP. Hence hypothesis $C$ is suggested that "Welding temperature is more conveniently attainable for ABS owing to its higher loss modulus as compared to PP". In order to assess the hypothesis C, DSC, MFI and FEA were again employed in this work.

Hypothesis $D$ has been deduced to introduce the likely explanation for the $4^{\text {th }}$ interesting effect. Higher ED collapse leading to higher LSS is detected due to higher heat distortion temperature and higher loss modulus for ABS and opposite is true for PP. Moreover, the heat distortion temperature (HDT) of $A B S$ is also higher than that of PP implying that distortion rate of ED under ultrasonic vibration is not higher for ABS than that for PP. Henceforth hypothesis D is recommended as "EDs made of ABS have higher heat distortion temperature (HDT) \& higher loss modulus than those for PP" In this study, HSVC, microscopy and FEA were used to check hypothesis D for its approval/disapproval.

Hypothesis $E$ addresses the likely reason for $5^{\text {th }}$ interesting effect. When the ultrasonic bond is tested for measuring LSS, both ductile and brittle fracture modes are found. Crazing is expected to affect generally the bond fracture without having any effect on fracture mode. Application of load on lap shear joint results in formation of crazes. Crazes are crack predecessors. A detailed explanation of crazing is presented in section 10.5.1. Hence hypothesis E is hypothesized as "Crazing affects bond fracture without influencing fracture mode for ABS and PP" In this research, fractography or fracture analysis was used to test the hypothesis $E$.

Hypothesis F suggests setting the static force at its lower and higher level for ABS and PP respectively. Observational results hint that higher force will cause the molten material at weld zone to leave the weld zone. To control supply of molten material at weld zone, static force is required to be lower for ABS and vice versa for PP. Hence the possible explanation for $6^{\text {th }}$ interesting effect is relevant to setting differently the static force for both ABS and PP. Therefore, hypothesis F has been defined as "Role of static force is different for ABS due 
to its higher viscosity and lower glass transition temperature". In this work, this Hypothesis was tested by DSC, MFI and FEA.

Similarly, the stable effect of setting higher amplitude on LSS is obtained for both materials. The plausible reason for $7^{\text {th }}$ interesting effect is the formation of similar resisting area during ultrasonic welding of both ABS and PP at higher amplitudes. Hence hypothesis $\mathrm{G}$ is formed by a statement "Almost same resisting area is expected to be produced at weld interface by using higher amplitudes". To investigate hypothesis G, DSC, MFI and FEA were used once more in this study. 
Table 6.13 Hypotheses Development and Testing using Scientific Method

\begin{tabular}{|c|c|c|c|c|}
\hline Material & Interesting Effects & Hypotheses & Testing Method & Thesis Section \\
\hline \multirow{7}{*}{$A B S \& P P$} & $\begin{array}{l}\text { 1. Higher LSS for ABS as } \\
\text { compared to LSS for PP } \\
\text { at same welding } \\
\text { conditions }\end{array}$ & $\begin{array}{l}\text { A. ABS is an amorphous } \\
\text { thermoplastic with higher } \\
\text { tensile strength than that } \\
\text { of PP }\end{array}$ & DSC, FEA \& MFI & 7.1 , chapter 8 \& 7.2 \\
\hline & $\begin{array}{l}\text { 2. TRI ED is better in } \\
\text { getting higher LSS with } \\
\text { ABS as contrary to SEMI } \\
\text { ED with PP }\end{array}$ & $\begin{array}{l}\text { B. Volume of TRI ED is } \\
\text { less than that of SEMI ED } \\
\text { resulting in high } \\
\text { concentration of } \\
\text { ultrasonic energy }\end{array}$ & MFI \& FEA & 7.2 \& Chapter 8 \\
\hline & $\begin{array}{l}\text { 3. ABS requires lower } \\
\text { level of weld factors } \\
\text { than that for PP to } \\
\text { attain higher LSS }\end{array}$ & $\begin{array}{l}\text { C. Welding temperature } \\
\text { is more conveniently } \\
\text { attainable for ABS owing } \\
\text { to its higher loss modulus } \\
\text { as compared to PP }\end{array}$ & MFI, FEA \& DSC & 7.2, Chapter 8 \& 7.1 \\
\hline & $\begin{array}{l}\text { 4. Higher ED collapse } \\
\text { leads to higher LSS for } \\
\text { ABS but opposite for PP } \\
\text { at similar weld factors }\end{array}$ & $\begin{array}{l}\text { D. EDs made of ABS have } \\
\text { higher heat distortion } \\
\text { temperature (HDT) \& } \\
\text { higher loss modulus than } \\
\text { those for PP }\end{array}$ & $\begin{array}{c}\text { HSVC, Microscopy \& } \\
\text { FEA }\end{array}$ & $\begin{array}{c}\text { Chapter } 9 \text {, chapter } \\
10 \& \text { chapter } 8\end{array}$ \\
\hline & $\begin{array}{l}\text { 5. Brittle and ductile } \\
\text { fractures affecting LSS } \\
\text { for both ABS and PP }\end{array}$ & $\begin{array}{l}\text { E. Crazing affects bond } \\
\text { fracture without } \\
\text { influencing fracture mode } \\
\text { for ABS and PP }\end{array}$ & Fractography & 10.5 \\
\hline & $\begin{array}{l}\text { 6. Lower static force is } \\
\text { required to achieve } \\
\text { higher LSS for ABS }\end{array}$ & $\begin{array}{l}\text { F. Role of static force is } \\
\text { different for ABS due to } \\
\text { its higher viscosity and } \\
\text { lower glass transition } \\
\text { temperature }\end{array}$ & MFI \& FEA & $7.2 \&$ chapter 8 \\
\hline & $\begin{array}{l}\text { 7. LSS is found to be } \\
\text { stable at higher } \\
\text { amplitudes for both } \\
\text { ABS and PP }\end{array}$ & $\begin{array}{l}\text { G. Almost same resisting } \\
\text { area is expected to be } \\
\text { produced at weld } \\
\text { interface by using higher } \\
\text { amplitudes }\end{array}$ & DSC, FEA \& MFI & 7.1 , chapter $8 \& 7.2$ \\
\hline
\end{tabular}




\section{CHAPTER 7 Differential Scanning Calorimeter (DSC) and Melt Flow Index (MFI)}

\subsection{DSC}

7.1.1. AIM

In this section of chapter 7, thermal properties of ABS and PP were measured with the help of DSC. For both materials, a considerable difference of softening temperature was expected. Hypotheses deduced were tested with the assistance of DSC results. Hypotheses $A, C \& G$ were checked for both $A B S$ and PP. These hypotheses were pointed out; (A) ABS is an amorphous thermoplastic with higher tensile strength than that of PP, (C) Welding temperature is more conveniently attainable for ABS owing to its higher loss modulus as compared to PP and (G) Almost same resisting area is expected to be produced at weld interface by using higher amplitudes.

\subsubsection{Methodology}

Differential scanning calorimeter is an effective facility to deliver reliable thermal examination. DSC is widely available and has been used in many investigational studies. It is one of the most practical ways of measuring the softening temperatures for thermoplastics. Many industries have used it and accepted its power of analysis. These industries include pharmaceutical, food, polymers etc. DSC produces an accurate and precise set of thermal information about a material under investigation in order to understand crystallinity and amorphous content in a material. Moreover, DSC can also be used for determining specific heat, reaction kinetics, percent crystallinity and purity. These results are really helpful in obtaining both qualitative and quantitative information about different types of changes in a material involving endothermic and exothermic processes. DSC 8500 supplied by PerkinElmer was utilized in this research in an effort to identify the glass transition temperature for ABS and melting point of PP. A distinctive DSC with a dedicated computer is illustrated in Figure 7.1. 
Figure 7.1 DSC with a dedicated computer [152]

Ultrasonic welding involves phase change (solid to liquid) of thermoplastic samples to be welded. In this research, the bond strength was greatly affected by thermal properties particularly softening temperature of thermoplastics. Weld factors were varied as an input in order to achieve sufficient melting of samples for formation of desired bond.

\subsubsection{Variables used}

In a DSC analysis, a set of variables is used, as shown in Table 7.1, comprising sample weight, heating range, cooling rate, heating rate and hold time at a temperature. Sample weight is the weight of sample measured during sample preparation. Heating range involves the range of set temperatures during one complete cycle of DSC programme at a specific heating or cooling rate. Heating range can be seen at the baseline of Figure 7.3. Heating rate is the quantity of heat absorbed (endothermic) per unit time. Cooling rate is the quantity of heat released (exothermic) per unit time. Hold time is the time for which the sample is held at a temperature. In this DSC analysis, dependent variable measured was softening temperature of $A B S$ and $P P$.

\subsubsection{Description of Procedure}

Softening temperatures for both ABS and PP were intended to be measured hereby using a DSC. These temperatures were then used for approving or disapproving some of derived hypotheses.

DSC 8500 set up mainly consists of provisions for sample pan, nitrogen cylinder and a chiller. In this work, polymers under investigation were cut and placed in the aluminium pan after 
weighing them. A thin aluminium cover was then placed carefully on aluminium pan and finally pressed gently by using a small hand press. This sample pan was then placed in the DSC chamber. The insertion of a sample pan in DSC is shown in Figure 7.2. A DSC program was written by using Pyris software including heating range, heating and cooling rates, sample weight, sampling rate and holding time at certain temperature. For example, Table 7.1 and Table 7.2 show the same programs to run thermal analyses for ABS and PP respectively except the different lower limits of heating range. Lower limit of heating range for PP starts with negative temperature $\left(-50^{\circ} \mathrm{C}\right)$ to observe the glass transition temperature of PP which is difficult to capture at a starting temperature of $20{ }^{\circ} \mathrm{C}[191]$. The glass transition temperature of PP is the best indicator for its material properties. Physical properties e.g. coefficient of thermal expansion, heat capacity etc. are modified by changing the glass transition temperature owing to different degree of polymerization. Moreover, $\mathrm{T}_{\mathrm{g}}$ for $A B S$ can easily be seen by setting the lower limit of heating range at $20{ }^{\circ} \mathrm{C}$. Upper limit of heating range is $200^{\circ} \mathrm{C}$, same for both $A B S$ and $P P$, in an attempt to achieve the melting point of PP which is expected to be higher. A sample weight of $15 \mathrm{mg}$ is chosen, in accordance with standard laboratory protocols.

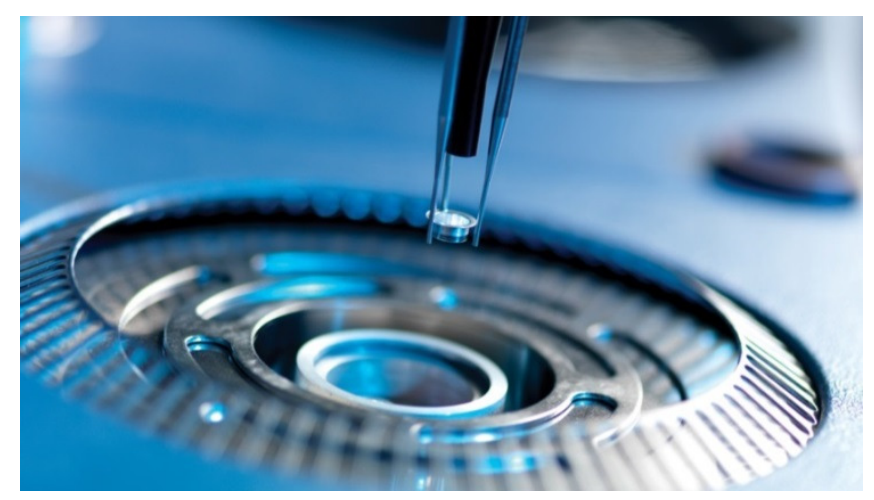

Figure 7.2 Placing a sample pan in DSC chamber [192]

Table 7.1 DSC programme for ABS

\begin{tabular}{|c||c|}
\hline Crucial Factors & $\begin{array}{c}\text { Set } \\
\text { Values }\end{array}$ \\
\hline \hline Sample Weight $(\mathrm{mg})$ & 15 \\
\hline Heating Range & 20 to \\
$200^{\circ} \mathrm{C}$ \\
\hline Cooling rate $\left({ }^{\circ} \mathrm{C} / \mathrm{min}\right)$ & 10 \\
\hline Heating rate $\left({ }^{\circ} \mathrm{C} / \mathrm{min}\right)$ & 10 \\
\hline Hold Time at $200^{\circ} \mathrm{C}(\mathrm{min})$ & 1 \\
\hline
\end{tabular}


Table 7.2 DSC programme for PP

\begin{tabular}{|c||c||}
\hline Crucial Factors & $\begin{array}{c}\text { Set } \\
\text { Values }\end{array}$ \\
\hline \hline Sample Weight $(\mathrm{mg})$ & 15 \\
\hline Heating Range & $\begin{array}{c}-50 \text { to } 200 \\
{ }^{\circ} \mathrm{C}\end{array}$ \\
\hline Cooling rate $\left({ }^{\circ} \mathrm{C} / \mathrm{min}\right)$ & 10 \\
\hline Heating rate $\left({ }^{\circ} \mathrm{C} / \mathrm{min}\right)$ & 10 \\
\hline Hold Time at $200{ }^{\circ} \mathrm{C}(\mathrm{min})$ & 1 \\
\hline
\end{tabular}

\subsubsection{Results}

For $A B S$, the glass transition or softening temperature was found to be $103{ }^{\circ} \mathrm{C}$ from DSC results, as shown in Figure 7.3. There was a proper signal of phase change at $103{ }^{\circ} \mathrm{C}$ during endothermic processes. Moreover, endothermic processes consist of endothermic events of material where the energy is absorbed by the material i.e. melting, whereas exothermic events of material imply that energy is released by the material.

Hypotheses A, C\&G were tested later by using this DSC result for ABS.

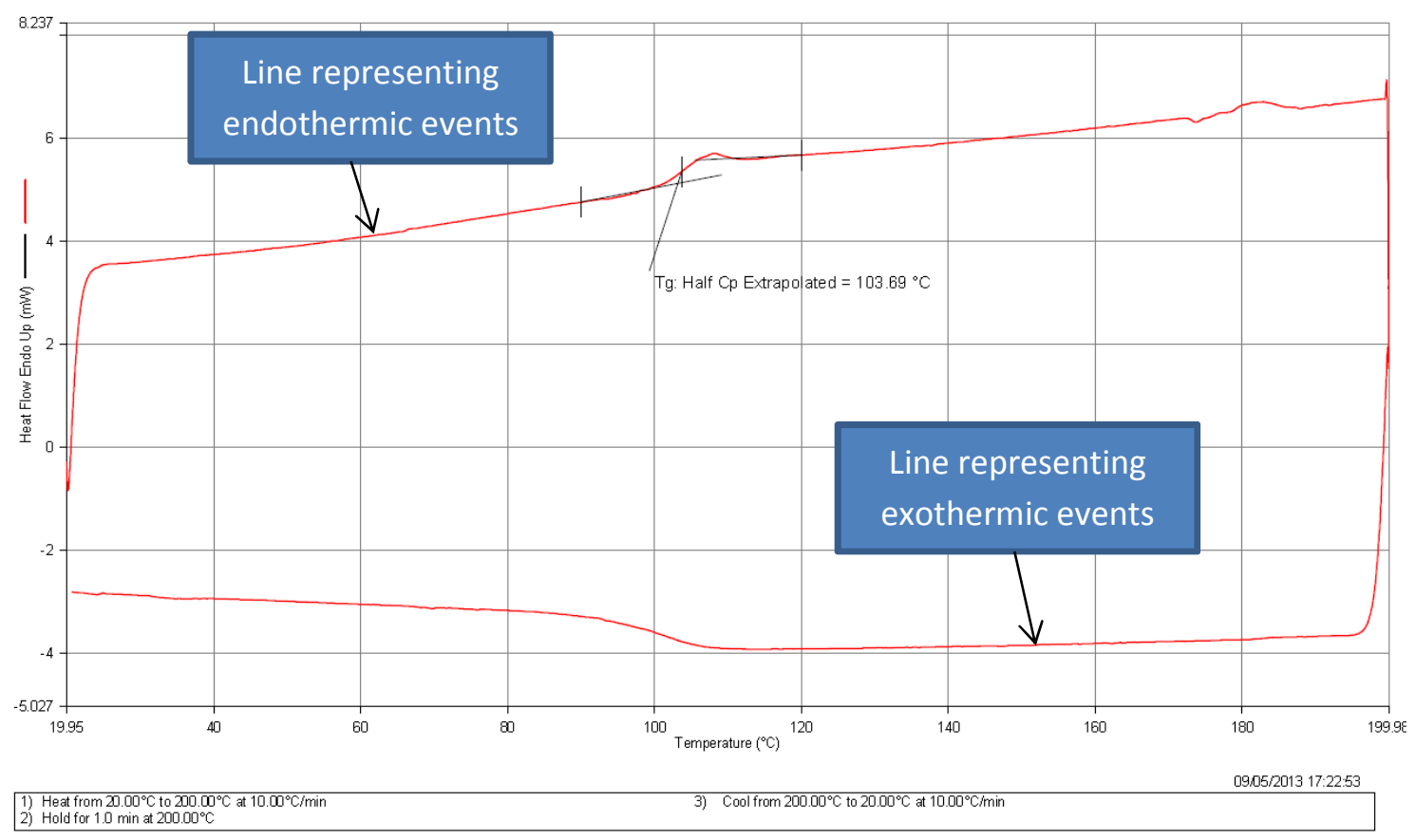

Figure 7.3 DSC result for ABS 
From Figure 7.4, the melting or softening temperature for PP was found to be $170.55{ }^{\circ} \mathrm{C}$ which was deemed essential to weld PP properly. There was found a sharp peak of endothermic process during DSC testing for PP which suggested its melting point. In this DSC analysis, a weak signal was observed during endothermic events between 0 and $20{ }^{\circ} \mathrm{C}$ for glass transition temperature representing a difficulty in identifying the value of $T_{\mathrm{g}}$ for PP. Even though lower limit of heating range was set at $-50^{\circ} \mathrm{C}, \mathrm{T}_{\mathrm{g}}$ could not be quantified for PP.

Hypotheses A, C\&G were tested afterward by employing this DSC result for PP.

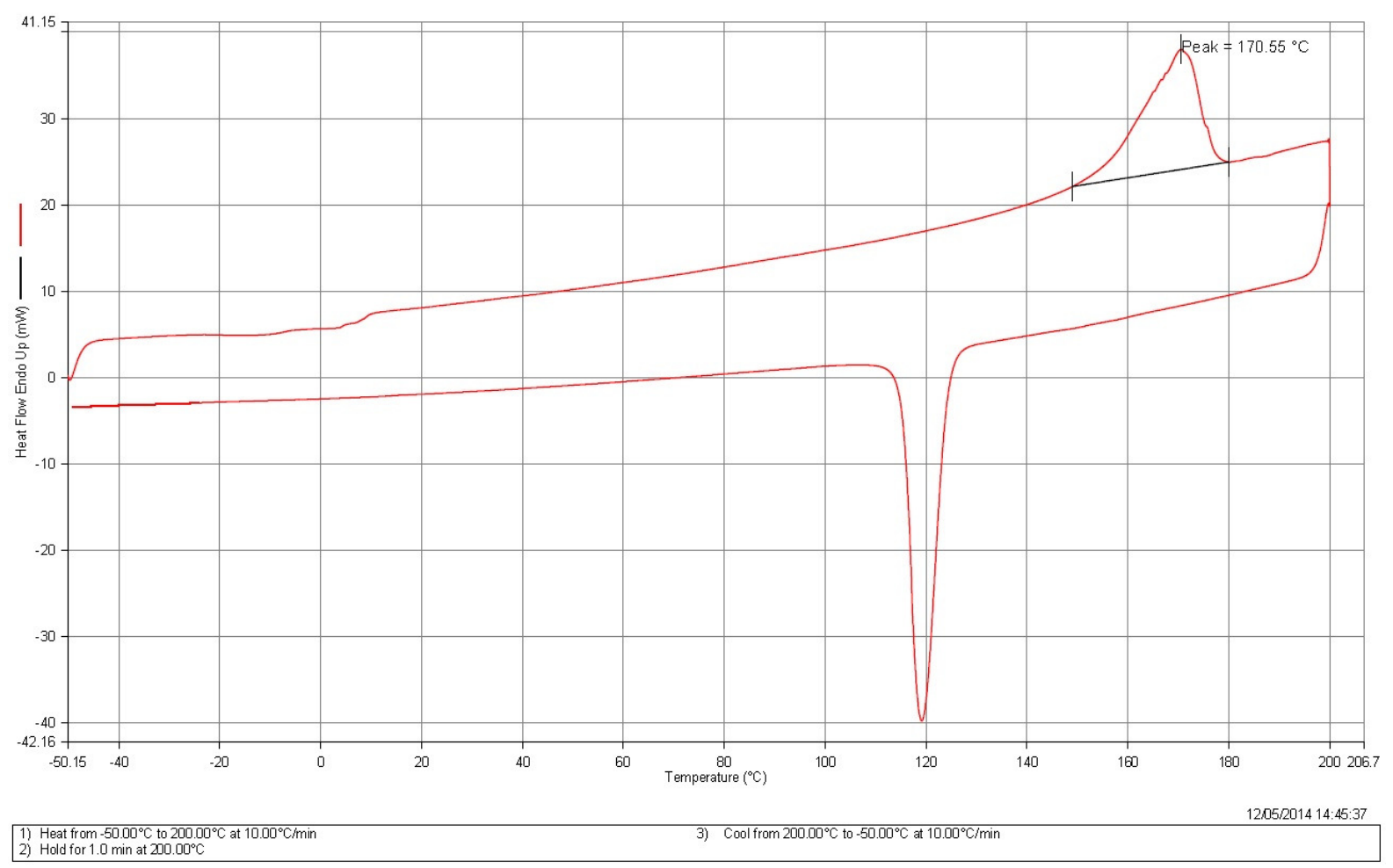

Figure 7.4 DSC result for PP

\subsubsection{Effect of softening temperatures on LSS}

\section{$\underline{A B S}$}

Hypothesis A

Hypothesis A has been stated as "ABS is an amorphous thermoplastic with higher tensile strength than that of PP"

Amorphous thermoplastic does not have a sharp melting point and can be melted over a range of temperatures. That is why; amorphous materials are well recognized by 
mentioning their glass transition temperatures. From DSC results, it was found that the glass transition or softening temperature of $A B S\left(103^{\circ} \mathrm{C}\right)$ was lower than that of PP $\left(170.55^{\circ} \mathrm{C}\right)$. In a welding process, the most important parameter is the welding temperature required to make bond. In other words, phase change was easily achievable during conduction of ultrasonic vibrations through $A B S$. Base material strength for $A B S(50 \mathrm{MPa}$ ) is also higher than that for PP (41 MPa). In contrast to PP, a better ultrasonic bond can be expected, therefore, for ABS. Hence hypothesis $A$ is partially proved hereby.

\section{Hypothesis C}

Hypothesis C states that "Welding temperature is more conveniently attainable for ABS owing to its higher loss modulus as compared to PP"

Lower softening temperature and higher loss modulus suggest keeping the weld factors lower for ABS in order to achieve better bonds. This was due to this reason that the weld factors were different for both $\mathrm{ABS}$ and PP in acquiring good ultrasonic bond. Temperature up to $103{ }^{\circ} \mathrm{C}$ is expected to be easily achieved owing to amorphous structure of $A B S$ as well. In this study, ABS took less than one second of weld time for welding completely and successfully in obtaining good weld ultimately. Value of softening temperature is certainly a valid reason for selecting proper level of weld factors for ABS and PP. Thus, hypothesis C seems to be somewhat proved.

\section{Hypothesis $G$}

Hypothesis G proposes that "Almost same resisting area is expected to be produced at weld interface by using higher amplitudes"

Having already been welded ultrasonically in this work, ABS was easier to weld due to its lower softening or glass transition temperature. Softening temperature is always important in making a good bond with proper resisting area. Also from FEA results (chapter 8), higher temperatures at weld zones for ABS helped back the idea of selecting proper welding factors. On setting higher amplitudes, ultrasonic weld resulted in higher LSS along with similar resisting area i.e. rectangular resisting area of almost equal dimensions. This resisting area also resulted in nearly similar shear force merely before fracture during tensile testing 
in shear. The LSS was ultimately evaluated to be stable. Hence hypothesis G appears to be partially proved here with the aid of DSC results.

\section{$\underline{P P}$}

Hypothesis A

Hypothesis A again specifies that "ABS is an amorphous thermoplastic with higher tensile strength than that of PP"

From the DSC results, PP was found to have a sharp melting point i.e. $170.55{ }^{\circ} \mathrm{C}$. In order to weld PP ultrasonically, this melting point must be attained and exceeded. Any temperature that was lower than this softening temperature of PP contributed towards collapse of ED without melting it properly resulting in low bond strength. During ultrasonic welding, PP took greater than one second of weld time i.e. high level of weld time for welding successfully. Comparatively smaller resisting area was also found by setting low levels of weld factors thereby resulting in lower LSS. Due to higher storage modulus and semicrystalline structure of PP, it is usually hard to weld PP. In present welding of PP, higher bond strength was only possible by setting high level of weld factors after breaking the orderly structure of PP. Hence hypothesis A seems to work partly here.

\section{Hypothesis C}

Hypothesis C states that "Welding temperature is more conveniently attainable for ABS owing to its higher loss modulus as compared to PP"

Softening temperature of PP was observed to be higher than that of ABS. On using low level of weld factors, there was a problem welding PP completely. This was due to the reason for not attaining the melting point of PP or the temperature more than the softening temperature for PP. Low weld factors either resulted in partial bonding or no weld at all. This difficulty in reaching the softening temperature of PP was due to its lower loss modulus and higher storage modulus causing a portion of applied ultrasonic energy to return back. Hence hypothesis $\mathrm{C}$ seems somehow good for its approval. 


\section{Hypothesis $G$}

Hypothesis G suggests that "Almost same resisting area is expected to be produced at weld interface by using higher amplitudes"

Only high levels of weld factors were successful in getting good bond achieved. FEA results (chapter 8) also show that low level of weld factors finds trouble in acquiring the melting point of PP at weld interface thereby resulting in low bond strength. $18.9 \& 21$ were considered as high levels of amplitude helping reach the softening temperature of PP. Adequate molten PP supply leading to similar resisting area is also guaranteed at weld interface in making better bond by using high level of weld factors. According to literature[189], bond strength is observed to be unstable for PP. In this work, the bond strength was found to be stable by using high level of amplitudes. This finding based on literature for PP is opposing the current hypothesis G. Hence hypothesis G is disapproved for PP.

\subsection{Measurement of Melt Flow Index (MFI)}

\subsubsection{AIM}

This section presents the measurement of viscosity for two thermoplastics under investigation by using melt flow indexer (MFI). Furthermore, this section was also presented to test certain hypotheses with the help of results from a melt flow indexer (MFI-10). In other words, hypotheses A\&F for ABS and C, G\&B for PP were checked. These hypotheses are; (A) ABS is an amorphous thermoplastic with higher tensile strength than that of $P P,(B)$ Volume of TRI ED is less than that of SEMI ED resulting in high concentration of ultrasonic energy, (C) Welding temperature is more conveniently attainable for ABS owing to its higher loss modulus as compared to PP, (F) Role of static force is different for ABS due to its higher viscosity and lower glass transition temperature and (G) Almost same resisting area is expected to be produced at weld interface by using higher amplitudes.

\subsubsection{Methodology}

Melt flow index determines the viscosity of the material with a numerical value showing the melt flow rate. The fluidity of a molten thermoplastic is known as its melt flow rate. Molten thermoplastic is extruded through a calibrated die by specifying its melt temperature and 
force with available weights. In this study, a melt flow indexer (MFI-10) was used encompassing a heated barrel with proper temperature controls, a calibrated die, a piston with graduated marks and different types of weights. MFI-10 set up is illustrated in Figure 7.5 .

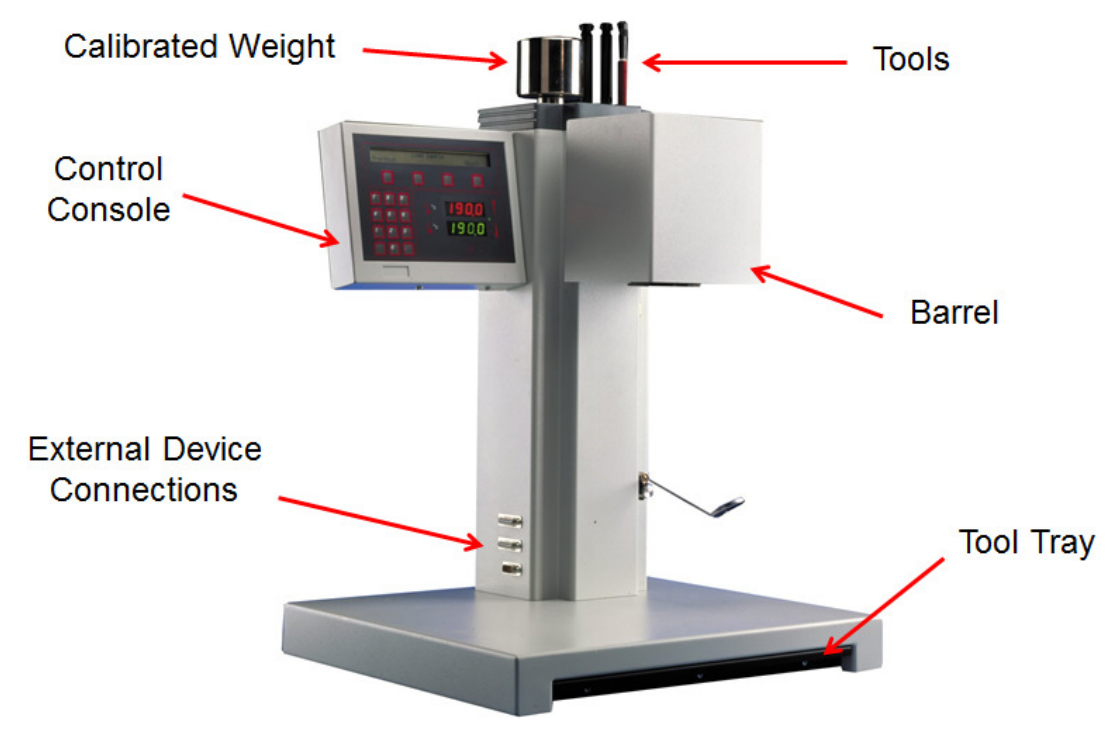

Figure 7.5 MFI-10 set up

Moreover, ultrasonic welding involves melting of thermoplastic material. This melted thermoplastic results in a definite spread at weld zone depending significantly on viscosity of the material. A proper molten material flow is essential in securing better bond strength. In this research, the difference in welding $A B S$ and PP was also thought of having relationship with fluidity of these two thermoplastics. If the thermoplastic was more fluid to overcome high viscosity issue then ultrasonic welding ensured a good spread of material at the weld interface which resulted in better bond. Hence results from MFI test were used to understand the bond development phenomenon during ultrasonic welding of thermoplastics. These results also help support or oppose the hypotheses.

\subsubsection{Variables used}

In an MFI test, various variables are used including cut off weight, cut off time intervals, testing temperature for material. Cut off weight is the weight of extruded and cut sample after a time interval that is called the cut off time interval. The testing temperature is the 
temperature of the extrusion barrel depending upon the typical thermoplastic under investigation. Melt flow index is eventually the dependent variable and the result desired.

\subsubsection{Description of procedure}

The Melt Flow Rate (MFR) is generally the mass in grams extruded in 10 minutes (grams per 10 minutes) and can be calculated by one of two methods which are defined as method $A$ and method B in the standards. In this research, method A was used with MFI-10. Method A is called the manual method and is suitable for polymers with an MFR below 25 grams per 10 minutes. Method A involves cutting the extruded polymer at accurate time intervals and testing temperature then weighing cut polymer to an accuracy of $0.1 \mathrm{~g}$ by using a balance.

Having used in injection moulding for producing samples (section 4.3 and Table 4.1), testing temperatures for ABS and PP (i.e. 200 and $200-230{ }^{\circ} \mathrm{C}$ respectively) were kept the same in MFI tester. Same temperature worked well for ABS but for PP it resulted in a molten material of lower viscosity generating difficulty in performing MFI test. Testing temperature was then reduced to $190{ }^{\circ} \mathrm{C}$ using control console, as shown in Figure 7.6, in order to get viscous molten PP necessary for MFI test. Hence thermal knowledge (i.e. melting temperature) of material was necessary before performing MFI Test as it might badly affect the barrel and piston.

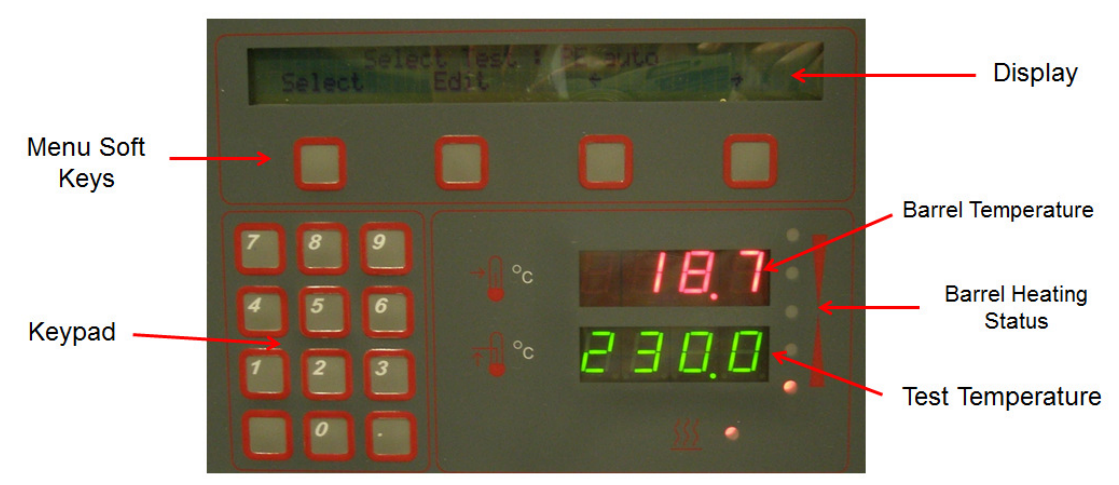

Figure 7.6 Control Console

In this work, Method A considered following important steps which were carefully observed to measure MFI for PP and ABS, as can be visualized with simple diagram in Figure 7.8:

1. Entering the cut-off time in sec via control console. For ABS it was $90 \mathrm{sec}$ and for PP it was $5 \mathrm{sec}$. 
2. Entering the testing temperature value in control console of MFI tester. $200{ }^{\circ} \mathrm{C} \& 190$ ${ }^{\circ} \mathrm{C}$ were the values of set temperatures for $A B S$ and $P P$ respectively.

3. Feeding the ABS/PP (test samples) into the heated barrel.

4. Putting the piston and piston support. Piston support is exhibited in Figure 7.7.

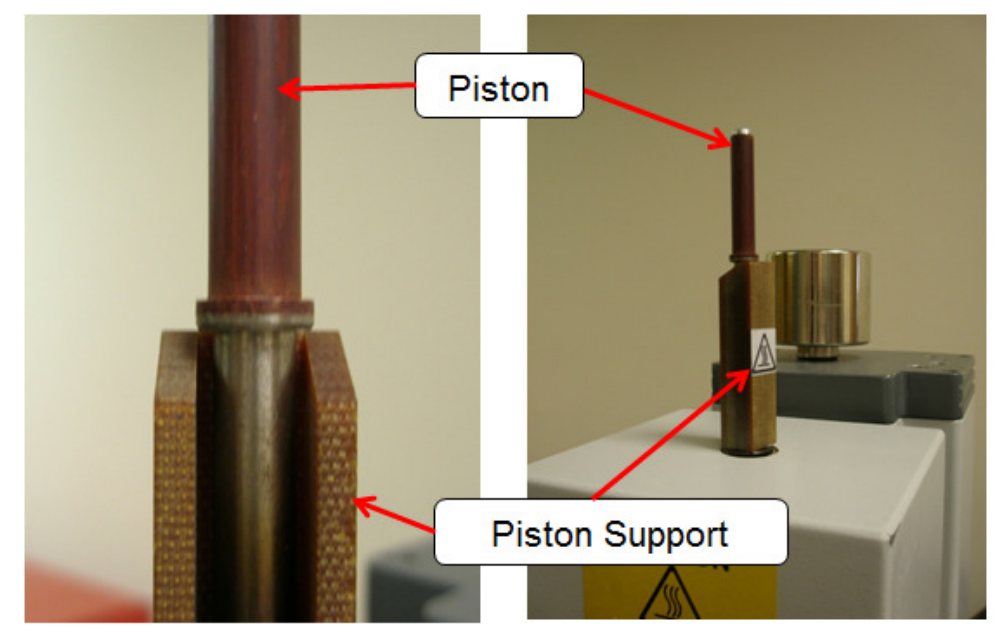

Figure 7.7 Piston and piston support

5. Placing the weight on the top of piston.

6. Removing the piston support.

7. Cutting off the waste material before starting the actual test according to above settings.

8. The measurements were taken between two scribe marks on the piston. 
Figure 7.8 Simple Diagram showing that how MFI test works[193]

9. Cutting off the samples that were extruded through a die after observing the cut off time. In this study, the polymer was manually cut by using a cut-off knife. Manual cutting is shown in Figure 7.9. The polymer was also cut at three equal time intervals during the test and each of the cut-off extrusions was weighed.

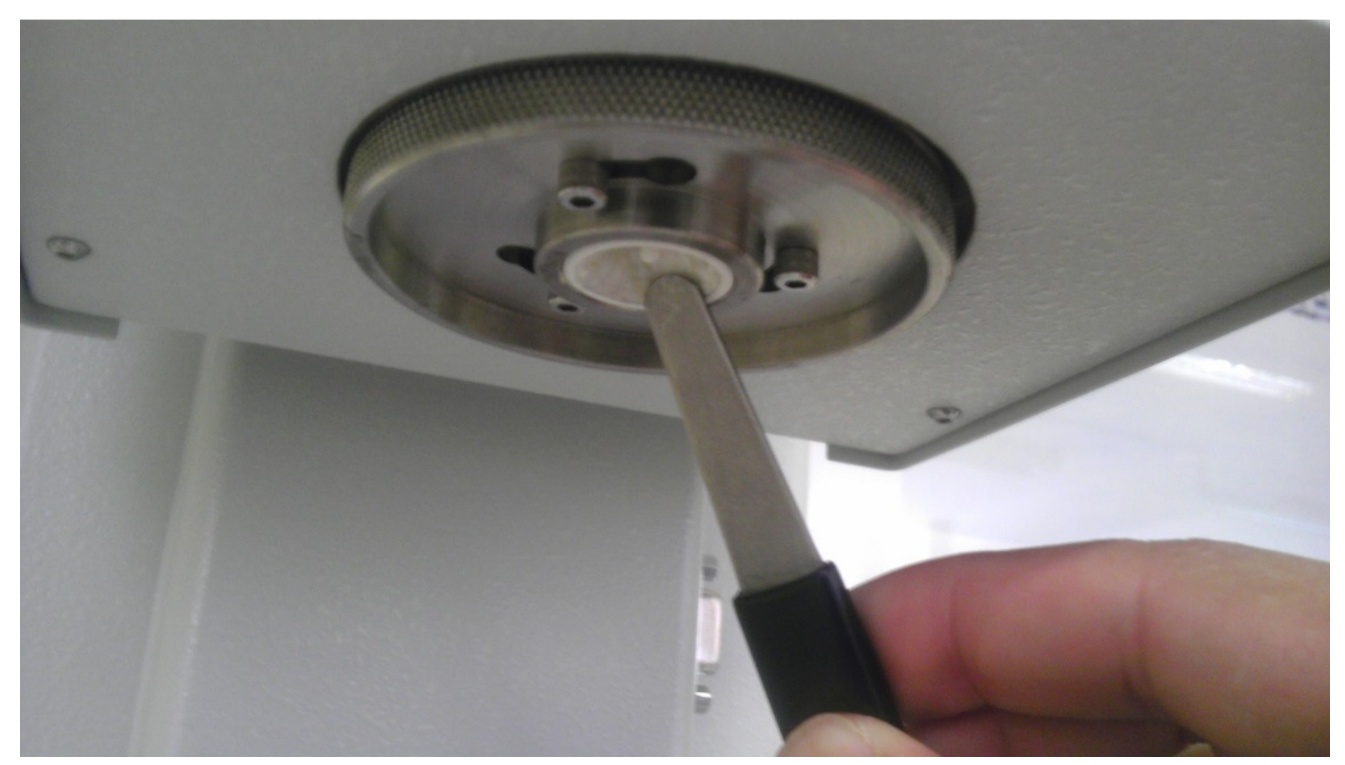

Figure 7.9 Manual Cutting with cut-off knife

10. Accepting or rejecting the sample due to immediate contamination in it e.g. air bubbles etc.

11. Labelling the cut off samples as 1,2 \& so on. 
12. Weighing the samples in grams.

13. Using the numerical keypad in control console for entering the sample weights.

14. Pressing finish key after entering the weights. Machine now showed the MFI for material under investigation in $\mathrm{g} / \mathrm{mins}$.

\subsubsection{Results}

Table 7.3 and Table 7.4 show the results from MFI tests for both ABS and PP respectively. Three samples were cut and weighed to determine the MFI for both ABS and PP. It is apparent from these tables that MFI of ABS is lower than that of PP. These results show that ABS has been found to be substantially more viscous than that of PP.

Table 7.3 MFI for ABS

\begin{tabular}{||c||c||c||}
\hline Sr. No. & $\begin{array}{c}\text { *Weight } \\
\text { (g) }\end{array}$ & $\begin{array}{c}\text { Mass Flow Index (g/10 } \\
\text { mins) }\end{array}$ \\
\hline \hline 1 & 0.141 & \\
\hline 2 & 0.139 & \multirow{2}{*}{0.92} \\
\hline 3 & 0.136 & \\
\hline
\end{tabular}

Table 7.4 MFI for PP

\begin{tabular}{||c||c||c||}
\hline \hline Sr. No. & $\begin{array}{c}* \text { Weight } \\
\text { (g) }\end{array}$ & $\begin{array}{c}\text { Mass Flow Index (g/10 } \\
\text { mins) }\end{array}$ \\
\hline \hline 1 & 0.1235 & \\
\hline 2 & 0.1232 & \multirow{2}{*}{14.81} \\
\hline 3 & 0.1237 & \\
\hline
\end{tabular}

Table 7.5 is a summary table showing the thermal properties of ABS and PP respectively that have been obtained by differential scanning calorimeter and melt flow indexer.

Table 7.5 Softening Temperatures and MFI for both materials

\begin{tabular}{|c|c||c|}
\hline Material Type & Softening Temperatures & Melt Flow Indices (g/10 min) \\
\hline \hline $\mathrm{ABS}$ & ${ }^{*} \mathrm{~T}_{\mathrm{g}}: 103{ }^{\circ} \mathrm{C}$ & $0.92 @ 200{ }^{\circ} \mathrm{C}$ \\
\hline $\mathrm{PP}$ & ${ }^{\alpha} \mathrm{T}_{\mathrm{m}}: 170.55^{\circ} \mathrm{C}$ & $14.81 @ 190{ }^{\circ} \mathrm{C}$ \\
\hline
\end{tabular}

* Glass Transition Temperature for ABS, ${ }^{\alpha}$ Melting Point of PP 


\subsubsection{Effect of viscosity on bond strength}

\section{$\underline{A B S}$}

Hypothesis A

Hypothesis A detects that "ABS is an amorphous thermoplastic with higher tensile strength than that of PP"

From MFI tests, ABS was found to be more viscous than that of PP. Higher loss modulus of $A B S$ is an important property to damp the ultrasonic vibrations without letting them return back. These damped vibrations are then dispersed within the samples in the form of viscoelastic heating. This internal heating is considered providing with an opportunity of dropping the high viscosity of ABS. After attaining sufficient fluidity of molten $A B S$, ultrasonic welding resulted in best bond formation. Hence hypothesis A appears to be approved partly again.

\section{Hypothesis F}

Hypothesis F notices that "Role of static force is different for ABS due to its higher viscosity and lower glass transition temperature"

In fact, viscosity drop for liquids is directly proportional to the supplied temperature. In this study, softening temperature for ABS was just $103{ }^{\circ} \mathrm{C}$ contributing a lot to reduce the viscosity of molten $A B S$ during welding. This softened $A B S$ with viscosity drop should not be allowed to be forced out of the weld zone until a higher static force was applied. Hence low level of static force is required to acquire higher LSS which proves somehow the hypothesis F here.

\section{$\underline{P P}$}

\section{Hypothesis B}

Hypothesis B proposes that "Volume of TRI ED is less than that of SEMI ED resulting in high concentration of ultrasonic energy"

From MFI results, PP was found to have lower viscosity. SEMI ED has already been identified for its higher volume $\left(140.13 \mathrm{~mm}^{3}\right)$ than that of TRI ED $\left(90 \mathrm{~mm}^{3}\right)$. Although TRI ED was found 
to be better in concentrating the ultrasonic energy at weld zone but the supply of molten material was poorer with TRI ED during welding PP. On welding PP with high level of weld factors, sufficient supply of molten PP along with proper fluidity was achieved with SEMI ED. Hence possibility of attaining higher LSS with SEMI ED was superior to that with TRI ED. Current ultrasonic welding of PP with SEMI ED was having near field welding scenario. Thus MFI test seems to approve this hypothesis B to some extent even in near field welding situation.

\section{Hypothesis C}

Hypothesis $C$ declares that "Welding temperature is more conveniently attainable for ABS owing to its higher loss modulus as compared to PP"

For PP, the viscosity was found to be lower than that of $A B S$ from MFI results. In this research, it was hard to melt PP due to its lower loss modulus by using low level of weld factors. On utilizing high levels of weld factors, a better bond was expected for PP which meant that chances of softening/melting were higher. This molten PP delivered higher spread owing to its lower viscosity over a larger weld zone thus resulting in higher LSS. Hypothesis $C$ seems to be appropriate hereby to some extent.

\section{Hypothesis $G$}

Hypothesis G suggests that "Almost same resisting area is expected to be produced at weld interface by using higher amplitudes"

Achieving uniform LSS at high levels of weld factors suggested us that there was a uniform outcome with both EDs. This stable outcome/LSS provided with comparatively bigger HAZ. Bigger HAZ was directly associated with lower viscosity of PP and higher weld factors with any ED. Also the higher storage modulus for PP needed higher static force in order to prevent the ultrasonic energy from returning back. This modulus always caused a portion of ultrasonic vibrations to return back on welding PP. But according to literature[189], bond strength for PP was found to be uneven disagreeing with the bond stability from current work. This hypothesis $\mathrm{G}$ is again partially disproved. 


\subsection{Summary}

Softening temperatures and melt flow indices were evaluated by DSC and MFI tests for both $A B S$ and PP. Softening temperature for ABS was found to be lower than that for PP. On the other hand, viscosity of PP was found to be higher than that of ABS. Hypotheses A, C, F\&G for $A B S$ and $A, B, C \& G$ for PP were also tested with the help of both DSC and MFI. These hypotheses were somewhat approved convincingly with the assistance of DSC and MFI results except hypothesis $\mathrm{G}$. 


\section{CHAPTER 8 FINITE ELEMENT ANALYSIS (FEA)}

\subsection{AIM}

In this chapter, temperature spread was registered with the help of FEA at the weld interface. Distribution of numerical/simulated temperatures obtained was also utilized to test hypotheses. Previous chapter 6 recognized the facts that several hypotheses (section 6.5.1 and Table 6.13) could be affected by the temperature distribution at the weld region. This chapter was also aimed at presenting FEA to test those hypotheses. These hypotheses were stated; $(A) A B S$ is an amorphous thermoplastic with higher tensile strength than that of PP, (B) Volume of TRI ED is less than that of SEMI ED resulting in high concentration of ultrasonic energy, (C) Welding temperature is more conveniently attainable for ABS owing to its higher loss modulus as compared to PP, (D) EDs made of ABS have higher heat distortion temperature (HDT) \& higher loss modulus than those for PP, (F) Role of static force is different for ABS due to its higher viscosity and lower glass transition temperature and $(G)$ Almost same resisting area is expected to be produced at weld interface by using higher amplitudes

\subsection{Methodology}

Prediction of temperature was also desired as a function of various weld factors at the weld zone in an attempt to obtain optimized bond strength. An FEA approach was developed to attain a thorough investigation of temperature at weld interface.

Finite element analysis is a great computational technique to evaluate the approximate solution of various tangible engineering world problems. These problems may have intricate domain with certain boundary conditions. FEA involves dividing the complex domain into subdomains for finding an approximate solution with the aid of variational or weighted residual methods. After getting the approximated solution for each subdomain, next and final step of FEA is to assemble solution of each subdomain into solution of whole domain. Based on discretizing the complicated domains into finite elements, FEA has been used in solving number of different problems e.g. eigenvalue, initial and boundary value problems which arise from various physical and natural processes. Several technical papers and books have been published so far after marvellous commencement of FEA. 
For ultrasonic welding process, FEA has covered various aspects so far:

1. Designing of optimal horn [122]

2. Studying the stress distributions in horn [121]

3. Studying the vibratory behaviour of large sized vibrator [119]

4. Studying the heating mechanism $[117,37]$

5. Finding out the temperature distribution during ultrasonic welding [37]

6. Analysing the thermo-viscoelastic phenomenon in plastics during welding [33].

In fact, FEA has been utilized as an effective computational technique in determining numerous facets of ultrasonic welding process before actually assembling the parts/specimens.

In this FEA, simulation was planned and implemented by selecting suitable analysis type, element type, material properties, approximations, mesh size, boundary and initial conditions. Mathematical relationship for viscoelastic heating was used to calculate the heat generation at the weld interface. Simulations were run in an effort to acquire maximum temperature at the weld zone. Simulation results were required to be validated by utilizing the ultrasonic welding equipment in an attempt to check the accuracy of simulation. Hence these simulated temperatures were validated by using data acquisition system, thermocouples, and labview software.

\subsection{Variables Used}

In this analysis, temperature is the dependent variable. In order to make this study more explicit, this temperature was desired throughout the analysis. The output temperature may result from the simulation/FEA and experiments/validation of simulation. The governing differential equation is:

$$
k \partial^{2} T / \partial x^{2}+k \partial^{2} T / \partial y^{2}+k \partial^{2} T / \partial z^{2}+Q-\rho c \partial T / \partial t=0[174]
$$

Where,

$T=$ Temperature, ${ }^{\circ} \mathrm{C}$

$K=$ Isotropic Thermal Conductivity, $\mathrm{W} / \mathrm{m}$. ${ }^{\circ} \mathrm{C}$

$\rho=$ Density of the material, $\mathrm{Kg} / \mathrm{m}^{3}$

$c=$ Specific Heat Capacity of the material, $\mathrm{J} / \mathrm{Kg} .{ }^{\circ} \mathrm{C}$ 
$Q=$ Volumetric Heat Generation Rate, $\mathrm{W} / \mathrm{m}^{3} .{ }^{\circ} \mathrm{C}$

$K, \rho$ and $c$ are the material properties that are shown in Table 8.1 for both ABS and PP. And $Q$ can be calculated by an equation:

$$
\mathrm{Q}_{\text {avg }}=\omega \epsilon_{\mathrm{A}}^{2} E^{\prime \prime} / 2[37]
$$

Where, $\omega=$ Angular velocity

$\epsilon_{\mathrm{A}}=$ Strain amplitude

$E^{\prime \prime}=$ Loss modulus

$\omega=2 \pi f$ and $f=20 \mathrm{KHz}$

Angular velocity and strain amplitude strongly depend on weld factors and geometry of specimens. Loss modulus is the material property, as shown in Table 8.1.

\subsection{Description of Procedure}

Description of procedure is presented for simulation and validation of simulation one by one respectively.

\subsubsection{Simulation}

\section{FEA Set Up}

In this research, simplified thermoviscoelastic analysis [33] was used. This analysis was chosen because it could be used for any thermoplastic which might be processed by standard methods (e.g. injection moulding). Any shape of ED could easily be accommodated by this thermoviscoelastic analysis. Moreover, this simplified analysis was suitable for lap type of weld joints. Temperature variations were modelled in FEA by considering the viscoelastic heating as a key heating mechanism. Transient thermal analysis was simulated via ANSYS software package leading to its validation later via empirical results. At the end, a good harmony between numerical and validation results was expected.

\section{Analysis Type}

Thermal (Full Transient)

A transient thermal analysis is defined as an analysis used to evaluating the temperature and various thermal quantities which fluctuate as a function of time. Also the imposed loads in transient analysis always vary with time. In this analysis, the localized viscoelastic heating 
was produced as a strong function of ultrasonic vibration i.e. dynamic load and static force i.e. static load. Hence transient thermal analysis seemed to be logical to cover these loads.

\section{Element Type}

Thermal Solid (Solid70)

Since 3D geometry was decided to be used throughout the thermal analyses, 3D thermal element was needed to perform these analyses. Solid70 has been utilized for its 3D thermal conduction capability. This element is applicable to a 3D transient thermal analysis. This element consists of eight nodes with single temperature for each node. This element also takes convection, heat flux and radiation as surface loads.

\section{Material Properties}

Material properties for ABS and PP are given in Table 8.1.

In this analysis, all the unreferenced material properties were used by going through the respective datasheets of both materials in Appendix $B$.

Table 8.1 Properties for both materials

\begin{tabular}{|c||c|c|c|c|c|c||}
\hline Material & $\begin{array}{c}\text { Density } \\
\left(\mathrm{Kg} / \mathbf{m}^{3}\right)\end{array}$ & $\begin{array}{c}\text { Thermal } \\
\text { Conductivity } \\
\left(\mathbf{W} / \mathbf{m}^{\circ}{ }^{\circ} \mathbf{C}\right)\end{array}$ & $\begin{array}{c}\text { Loss } \\
\text { Modulus } \\
(\mathbf{G P a})[\mathbf{1 8 9}]\end{array}$ & $\begin{array}{c}\text { Storage } \\
\text { Modulus } \\
(\mathbf{G P a})[189]\end{array}$ & $\begin{array}{c}\text { Specific } \\
\text { Heat }(\mathrm{J} / \mathrm{Kg} . \\
\left.{ }^{\circ} \mathrm{C}\right)\end{array}$ & $\begin{array}{c}\text { Softening } \\
\text { Temperature }\left({ }^{\circ} \mathrm{C}\right)\end{array}$ \\
\hline \hline $\mathrm{PP}$ & 905 & $0.2[194]$ & 0.32 & 3.87 & $1800[195]$ & 170.55 \\
\hline $\mathrm{ABS}$ & 1040 & $0.15[37]$ & 0.42 & 2.75 & $2000[37]$ & 103 \\
\hline
\end{tabular}

\section{Approximations used}

In this analysis, following approximations were set.

1. Each cross section (Triangular and Semi-circular) of EDs is approximated to be rectangular ED of equal cross sectional area [37]. For instance, SEMI ED_Area is equal to $3.5 * 4 \mathrm{~mm}^{2}$ and TRI ED_Area is equal to $2.5 * 3.5 \mathrm{~mm}^{2}$. Prakasan et al. [37] has performed a similar analysis with 2D geometry and element.

2. Amplitude of ultrasonic vibrations is given to the top part and the ED [37].

3. Heat loss due to convection is considered negligible for very short periods of welding. To be more precise, up to $1.5 \mathrm{Sec}$ of weld time, convection is neglected.

4. Loss modulus of material is not dependent on temperature [37]. 


\section{Mesh Size}

ANSYS Mechanical APDL uses a way of meshing the geometrical model that is called the smart sizing (10 to 1). Literature has suggested a mesh smart size of 2 for this type of analysis that is a finer mesh size [37]. In this analysis, smart sizes 10 (coarsest) to 1 (finest) were selected to verify the suitability of mesh size 2 for the present geometry, thermal simulations were run for these mesh sizes, and numerical results in terms of temperature distribution were compared between two consecutive mesh sizes e.g. 10 \& 9. An approximated geometry with mesh is exhibited in Figure 8.1.

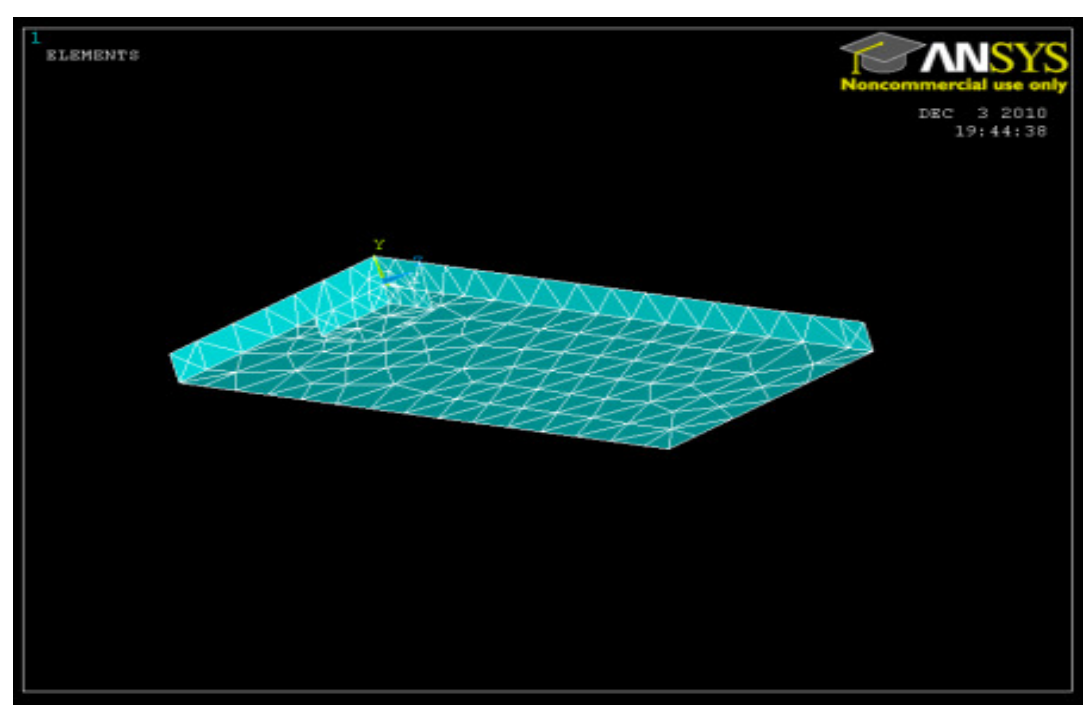

Figure 8.1 Mesh generated after approximating the symmetric geometry

An example of mesh verification is shown in Table 8.2 before deciding proper mesh size for simulation runs. From Table 8.2, after mesh size 6, maximum temperature values were highly fluctuated because these mesh sizes were coarser. Although temperature values were found to be almost same for finer mesh sizes before mesh size 6, mesh size 2 was frequently used to run simulations, as used in literature [37]. Due to this reason, finer meshes were considered more reliable than coarser meshes for current analysis. Therefore, mesh sizes $1 \& 2$ were finally decided to be used alternatively. 
Table 8.2 Mesh Verification

\begin{tabular}{|c||c||c||c||c||c||c|}
\hline $\begin{array}{c}\text { Mesh Smart } \\
\text { Sizes }\end{array}$ & $\begin{array}{c}\text { Material } \\
\text { Type }\end{array}$ & ED & $\begin{array}{c}\text { Static Force } \\
(\mathbf{N})\end{array}$ & $\begin{array}{c}\text { Weld Time } \\
(\mathbf{S e c})\end{array}$ & $\begin{array}{c}\text { Frequency } \\
(\mathbf{H z})\end{array}$ & $\begin{array}{c}\text { Max. Temp. value } \\
\left.\mathbf{}^{\mathbf{C}} \mathbf{C}\right)\end{array}$ \\
\hline \hline 1 & ABS & SEMI & 750 & 0.9 & 20000 & 27.64 \\
\hline 2 & ABS & SEMI & 750 & 0.9 & 20000 & 27.79 \\
\hline 3 & ABS & SEMI & 750 & 0.9 & 20000 & 27.79 \\
\hline 4 & ABS & SEMI & 750 & 0.9 & 20000 & 27.73 \\
\hline 5 & ABS & SEMI & 750 & 0.9 & 20000 & 27.73 \\
\hline 6 & ABS & SEMI & 750 & 0.9 & 20000 & 27.73 \\
\hline 7 & ABS & SEMI & 750 & 0.9 & 20000 & 27.68 \\
\hline 8 & ABS & SEMI & 750 & 0.9 & 20000 & 31.22 \\
\hline 9 & ABS & SEMI & 750 & 0.9 & 20000 & 27.07 \\
\hline 10 & ABS & SEMI & 750 & 0.9 & 20000 & 27.07 \\
\hline
\end{tabular}

\section{Boundary and Loading Conditions}

In this FEA, initial temperature was specified to be $21{ }^{\circ} \mathrm{C}$ as initial conditions. Initial conditions were indicated for the room temperature before and after welding the thermoplastic specimens ultrasonically.

Generation of heat comprises intermolecular friction and viscoelastic heating when thermoplastics are welded ultrasonically. Following equation is held responsible for calculation of heat generation at weld zone due to ultrasonic vibrations.

$$
\mathrm{Q}_{\mathrm{avg}}=\omega \epsilon_{\mathrm{A}}^{2} E^{\prime \prime} / 2[37]
$$

This relationship was used to calculate viscoelastic heating for current simulation at ED during ultrasonic welding. Temperatures were then obtained owing to viscoelastic heating. To simulate ultrasonic welding of thermoplastics, viscoelastic heating due to intermolecular friction was given as Heat Flux (thermal surface load).

\section{Crucial Weld Factors}

In this FEA, thermal simulations were run for various ranges of weld factors to investigate the spread of temperatures. Weld time ranging from 0.1 to $1.5 \mathrm{sec}$ and static force ranging from 250 to $2000 \mathrm{~N}$ were used. Specifically for ABS, weld time was varied from 0.1 to $1 \mathrm{sec}$ and static force from 250 to 2000 N. For PP, weld time (up to $1.5 \mathrm{sec}$ ) was higher than that (up to $1 \mathrm{sec}$ ) of ABS but static force's range was same. These ranges of weld time and static 
force were established to encompass the same range of these factors that was used in experimentation, as discussed earlier in chapter 4.

Nodal temperatures (temperatures at nodes of an element) were obtained after running simulations to study their effects on heat affected zone and shear strength. For each material e.g. $A B S$ and anyone $E D$, all static forces ( 8 in number) were used one by one at a constant weld time to acquire a numerical temperature. Then this was again repeated for different but constant weld time and so on, as shown in Figure 8.7. Hence a detailed FEA was achieved by using a comprehensive set of weld factors.

\subsubsection{Validation of simulation}

\section{Equipment Utilized}

In this analysis, ultrasonic welding rig was used to validate the simulation with the assistance of lab view software. An interface of lab view software is shown in Figure 8.2.

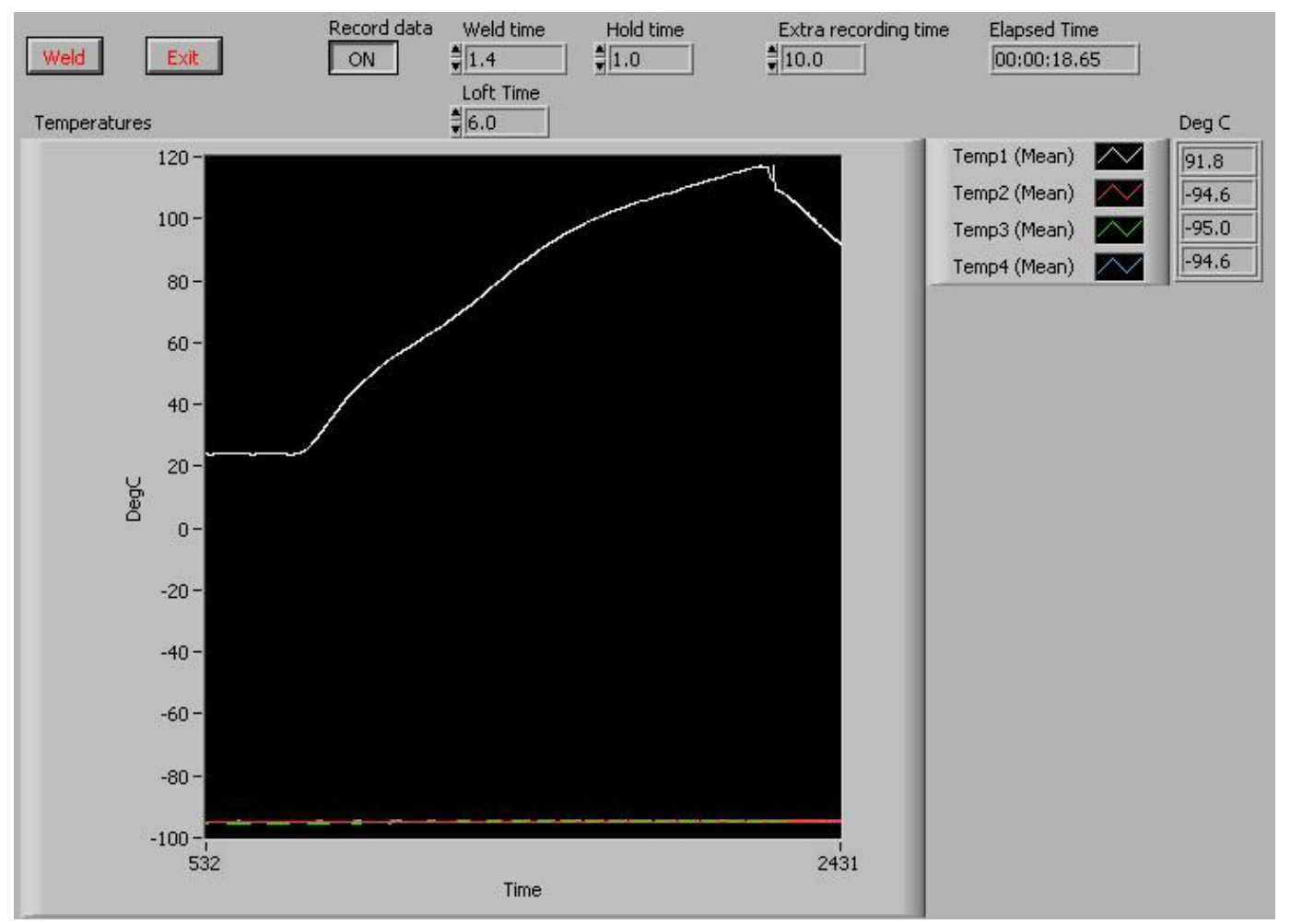

Figure 8.2 Lab view software screen shot for particular validation case 


\section{Thermocouples and Their Positioning}

To validate the simulated results, thermocouples (K-Type-0.005" Dia-12" Length-Bare Insulation) were inserted at the interface of energy director and flat surface of lower specimen, as illustrated in Figure 8.3. Likewise, thermocouples were repeatedly inserted, as shown in Figure 8.3 and Figure 8.3(A), to validate all simulation results. This thermocouple grade is able to measure temperature range between -270 to $1372{ }^{\circ} \mathrm{C}$.

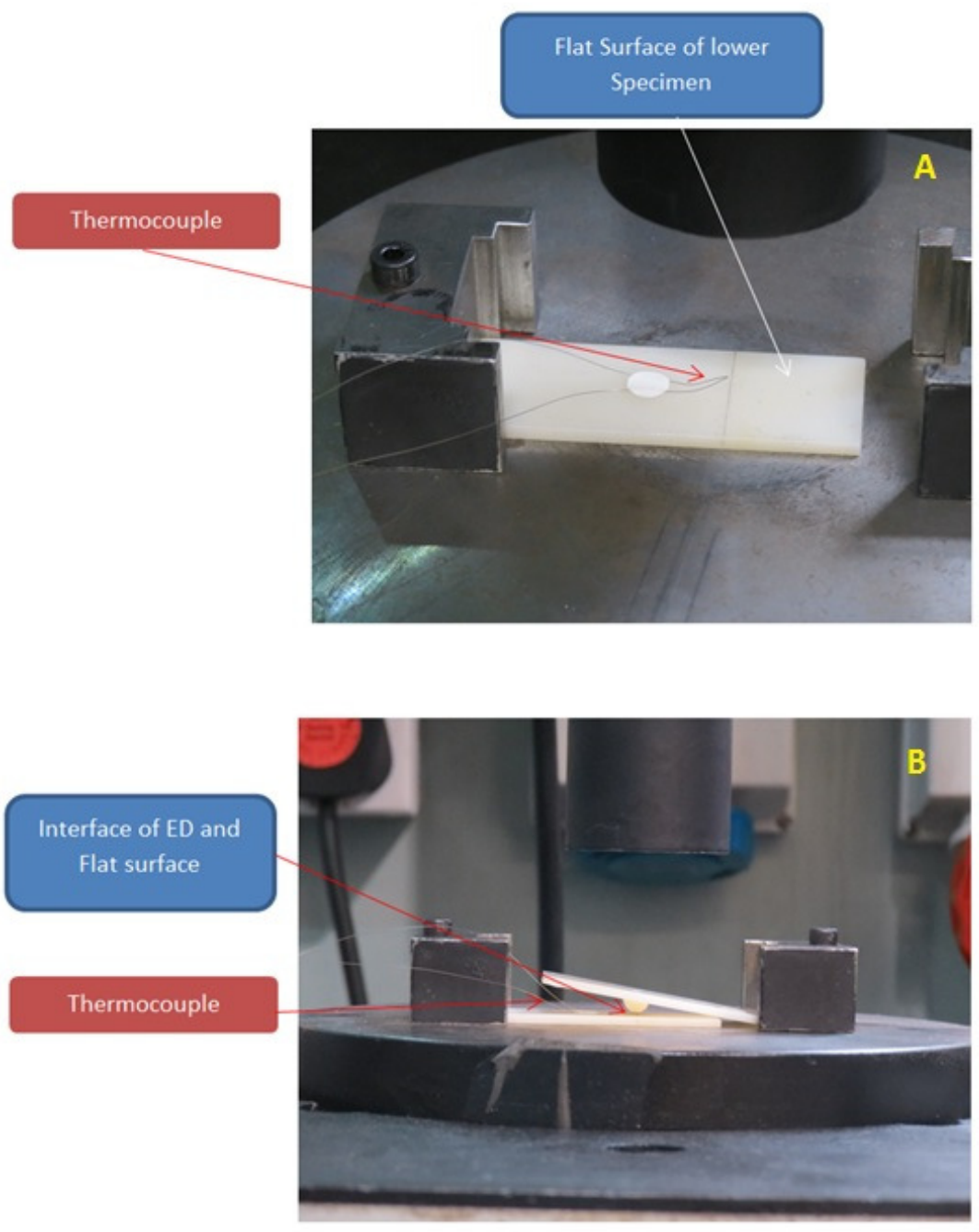

Figure 8.3 Insertion of thermocouple 


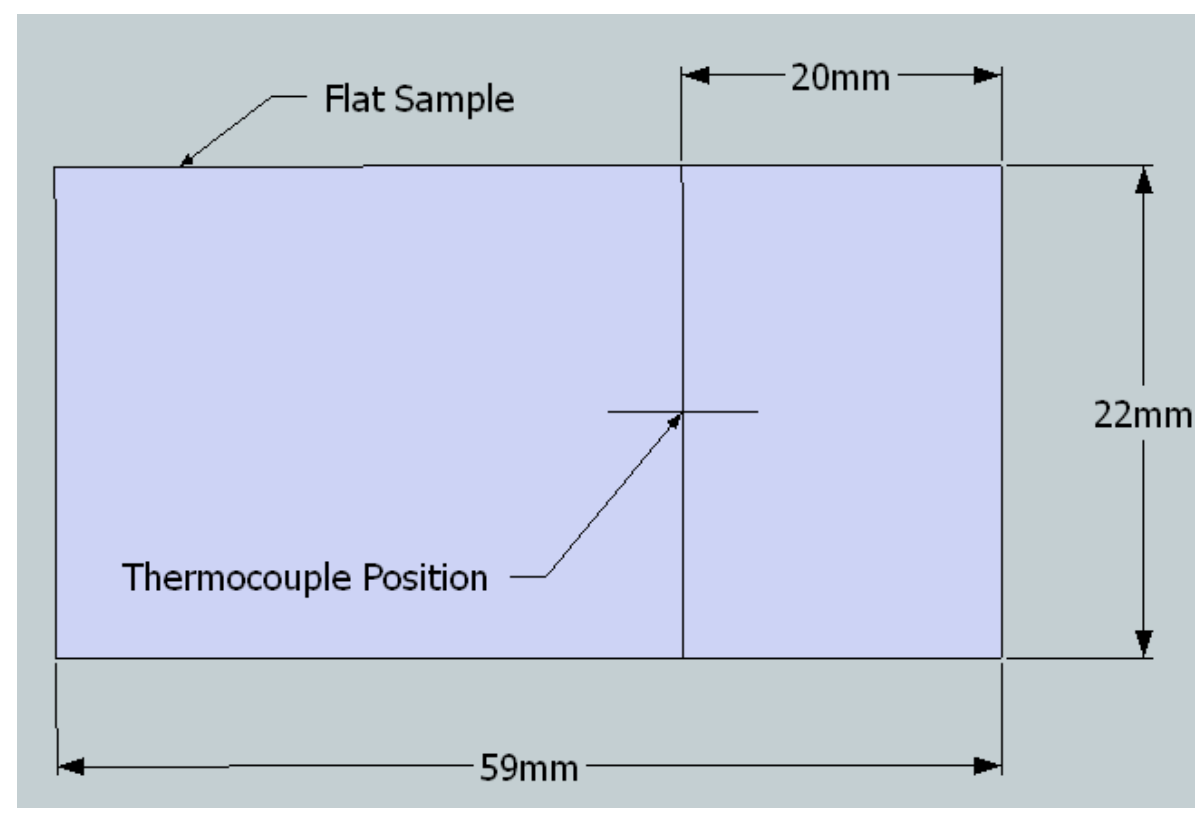

Figure 8.3(A) Thermocouple Position

A sticky tape was utilized in order to prevent the thermocouple from misplacing its desired position during up and down movement of anvil. Thermocouple's two terminals were fixed by a connecting electric cable with the help of small screws. Cable's other end was attached with the data acquisition box, as shown in Figure 8.6. Finally data acquisition black box was attached with the dedicated PC by using USB interface, as exhibited in Figure 8.5. Before welding $A B S$ and PP for validation, thermocouples were initially calibrated by measuring the room temperature from both thermocouple and room thermometer. A good agreement was found for a room temperature of $22{ }^{\circ} \mathrm{C}$, as shown in Figures (8.15 to 8.26).

\section{$\underline{\text { Temperature Measurement }}$}

In order to validate simulated results, a proper data acquisition system was made and put into a black box in an effort to register temperatures at the weld interface, as shown in Figure 8.5. Fine bead is a term representing small thermal mass for dynamic temperature measurement. Mini K-Type thermocouple was used to get an electrical signal owing to temperature development at HAZ. The value of this electrical signal was $2 \mu \mathrm{v}$ needing to be amplified. For this purpose, an amplifier AD595 was used to amplify the lowest value of signal up to a value of $5 \mu \mathrm{v}$. This signal was filtered by using low pass filter and sent to labview software via NI USB 6008 later. A detailed diagram of USB DAQ is shown in Figure 8.4. 


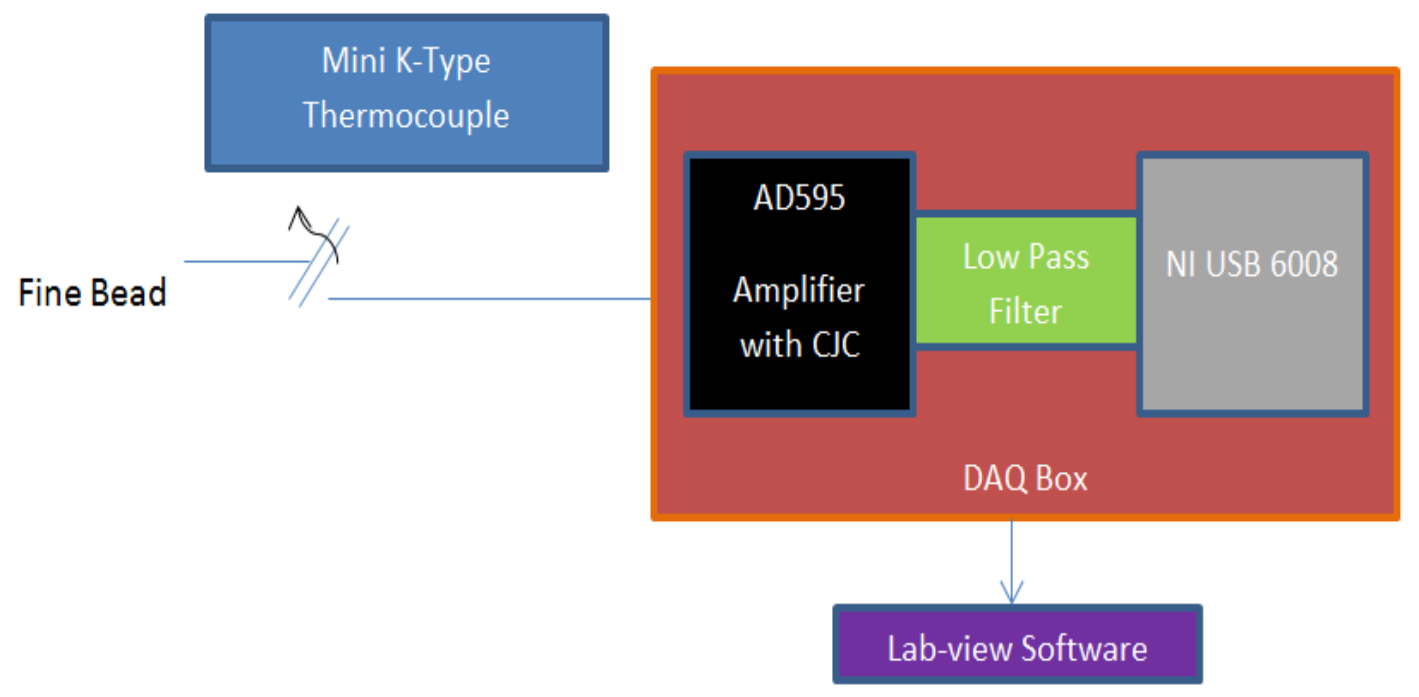

Figure 8.4 Details of USB DAQ
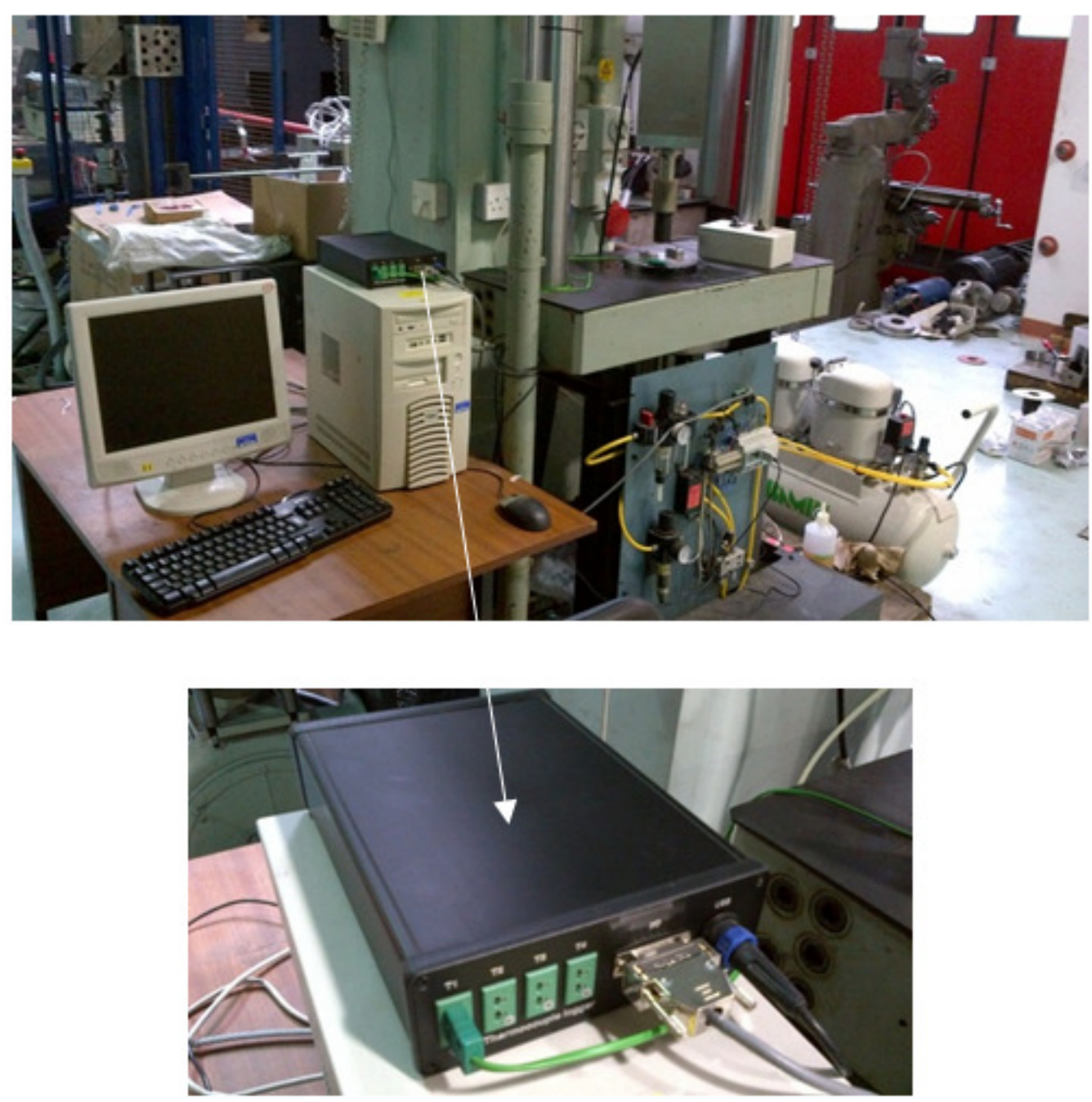

Figure 8.5 DAQ in black box as a part of UW rig 


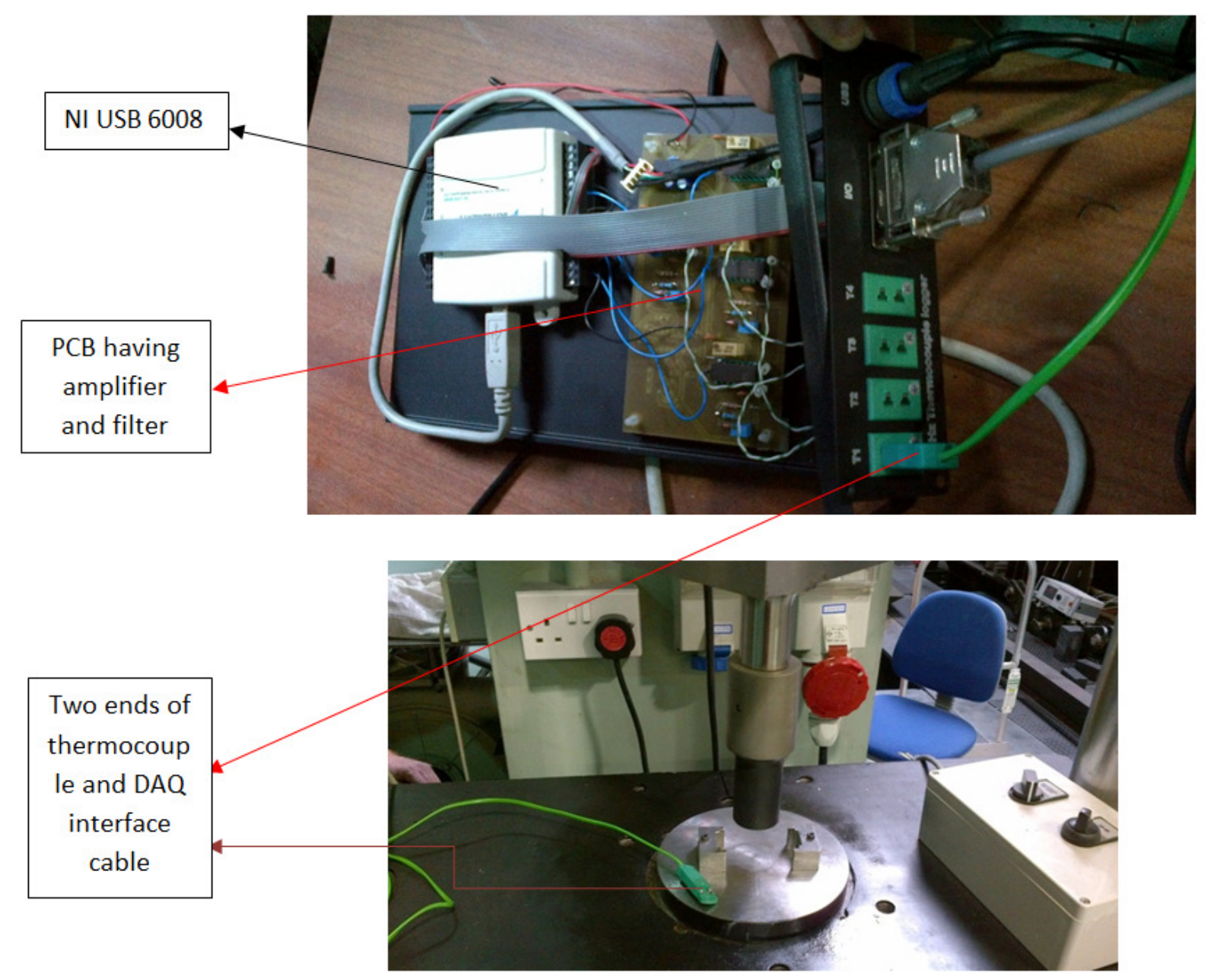

Figure 8.6 UWP with USB DAQ

\subsection{Results}

In this section, results from both simulation and validation of simulation are investigated for their useful interpretation.

\subsubsection{Simulation Results}

\section{Crucial Weld Factors}

In this FEA, thermal simulations were run for various ranges of weld factors to investigate the spread of temperatures. Weld time ranging from 0.1 to $1.5 \mathrm{sec}$ and static force ranging from 250 to $2000 \mathrm{~N}$ were used. Specifically for ABS, weld time was varied from 0.1 to $1 \mathrm{sec}$ and static force from 250 to 2000 N. For PP, weld time was little higher than that of ABS but static force's range was same. 
Nodal temperatures (temperatures at nodes of an element) were obtained after running simulations to study their effects on heat affected zone and shear strength. For each material e.g. ABS and anyone ED, all static forces ( 8 in number) were used one by one at a constant weld time to acquire a numerical temperature. Then this was again repeated for different but constant weld time and so on, as shown in Figure 8.7.

\section{Temperature Spread for TRI ED having PP and ABS respectively}

Relationship between temperature and static force is shown in Figure 8.7 and Figure 8.8 for a constant weld time with triangular ED by using PP and ABS respectively. Similarly, temperature variations as a function of weld time at constant static forces are illustrated in Figure 8.9 and Figure 8.10. Please note that these relationships are straight lines or linear. In fact, a critical temperature is required to get intended function achieved i.e. assembly of parts using right levels of weld factors.

From Figure 8.7 and Figure 8.8, desired critical temperatures can be obtained with the fact that some levels of weld factors may result in unnecessary higher temperature which is not desired. For instance, static force of $1500 \mathrm{~N}$ and higher weld time up to $1.2 \mathrm{sec}$ seem to be essential to attain or exceed sufficiently the softening temperature of PP, as shown in Figure 8.7 and Figure 8.9. Likewise, lower static force of $500 \mathrm{~N}$ with an appropriate weld time can easily reach the softening temperature of ABS, as shown in Figure 8.8 and Figure 8.10. Hence static force of $2000 \mathrm{~N}$ and weld time of $1 \mathrm{sec}$ may produce enormous heating at weld interface for ABS preventing us from using them in actual welding scenario. 


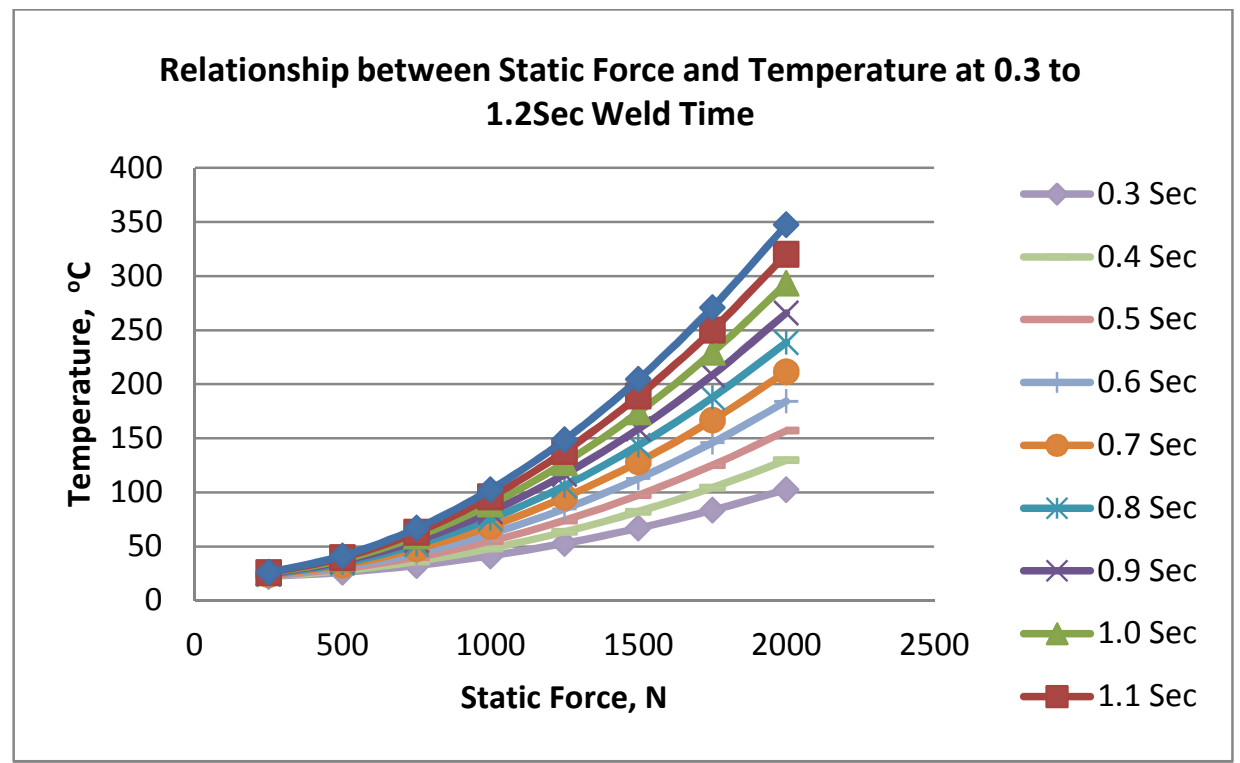

Figure 8.7 Temperature spread Vs Static Force for polypropylene with triangular ED

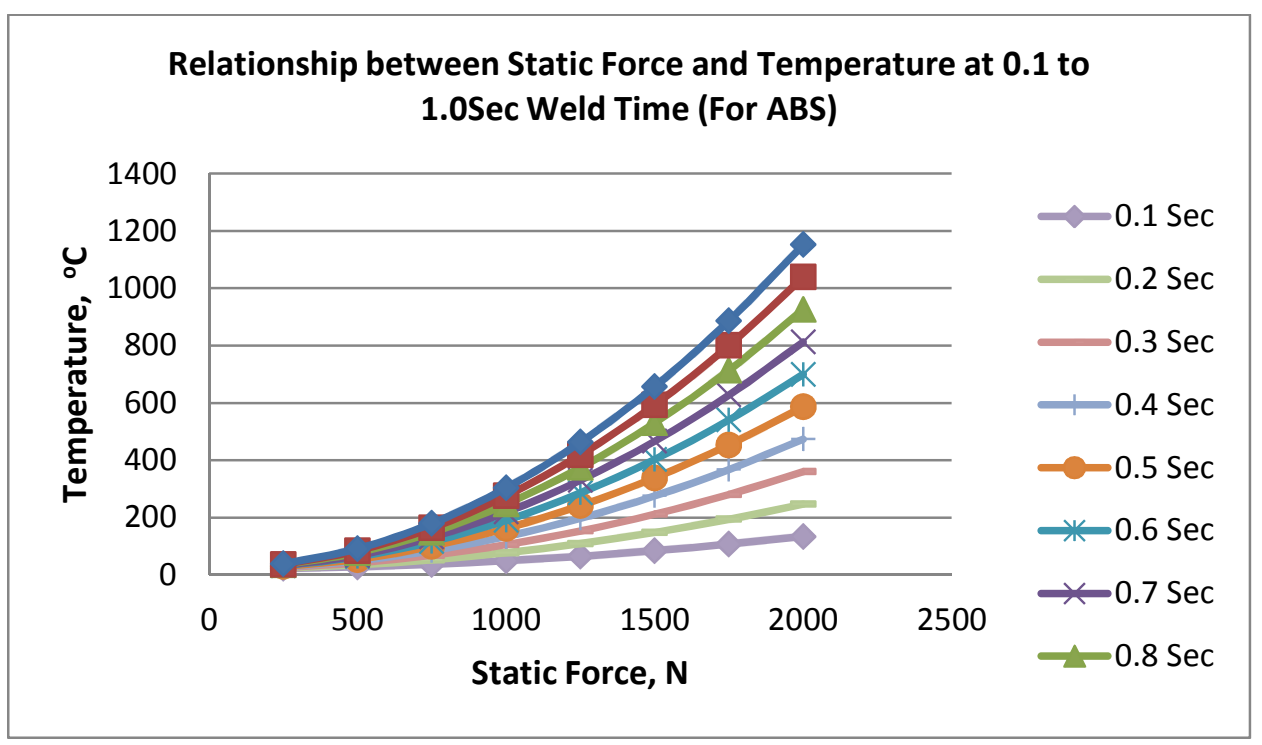

Figure 8.8 Temperature Distribution Vs Static Force for ABS with TRI ED 


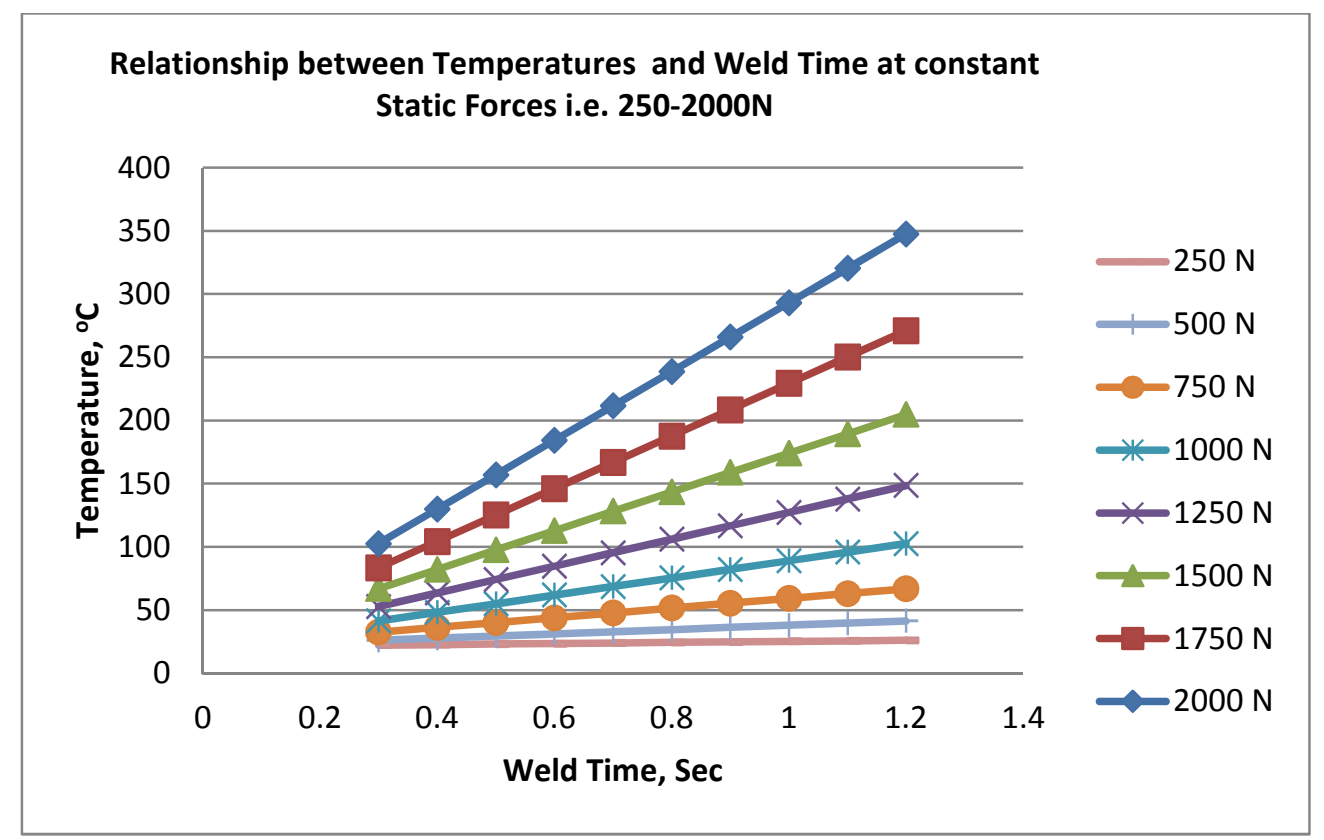

Figure 8.9 Relationship between weld time and Temperature for PP and TRI ED

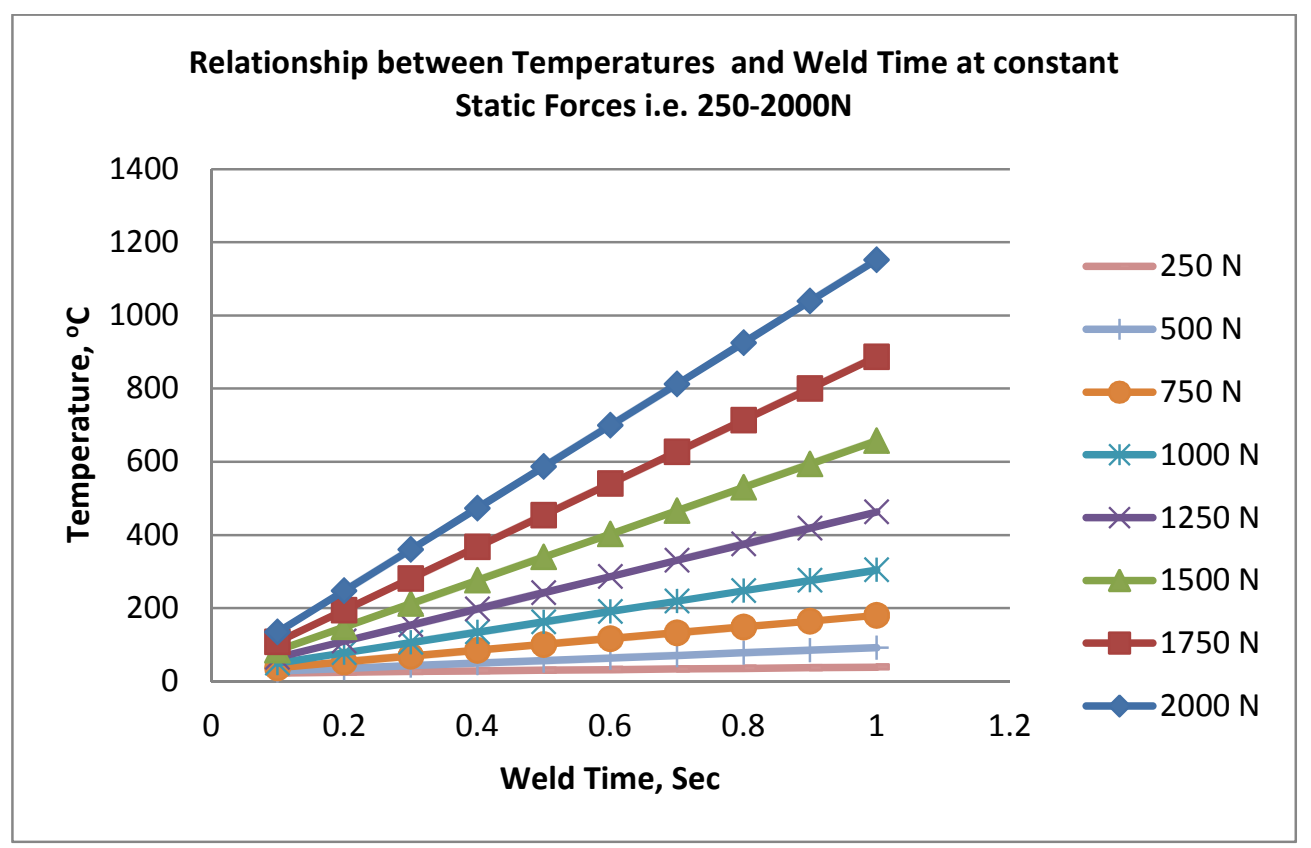

Figure 8.10 Relationship between weld time and Temperature for ABS and TRI ED

\section{$\underline{\text { Temperature Spread for SEMI ED having ABS and PP respectively }}$}

Relationship between temperature distributions and static forces with constant weld times is exhibited in Figure 8.11. From Figures 8.7, 8.8, 8.11 and 8.12, maximum values for temperatures developed at weld interface are greater for any plastic with TRI EDs than those with SEMI EDs. This is due to the reason that volume of TRI ED is less than that of SEMI ED pointing towards large concentration of ultrasonic energy with TRI ED. 
Relationships between temperature distribution and weld time at constant static forces are also illustrated in Figure 8.13 and Figure 8.14. Again, lower static force with higher weld time and higher static force with higher weld time may be needed to acquire sufficient temperature at weld interface for welding ABS and PP respectively.

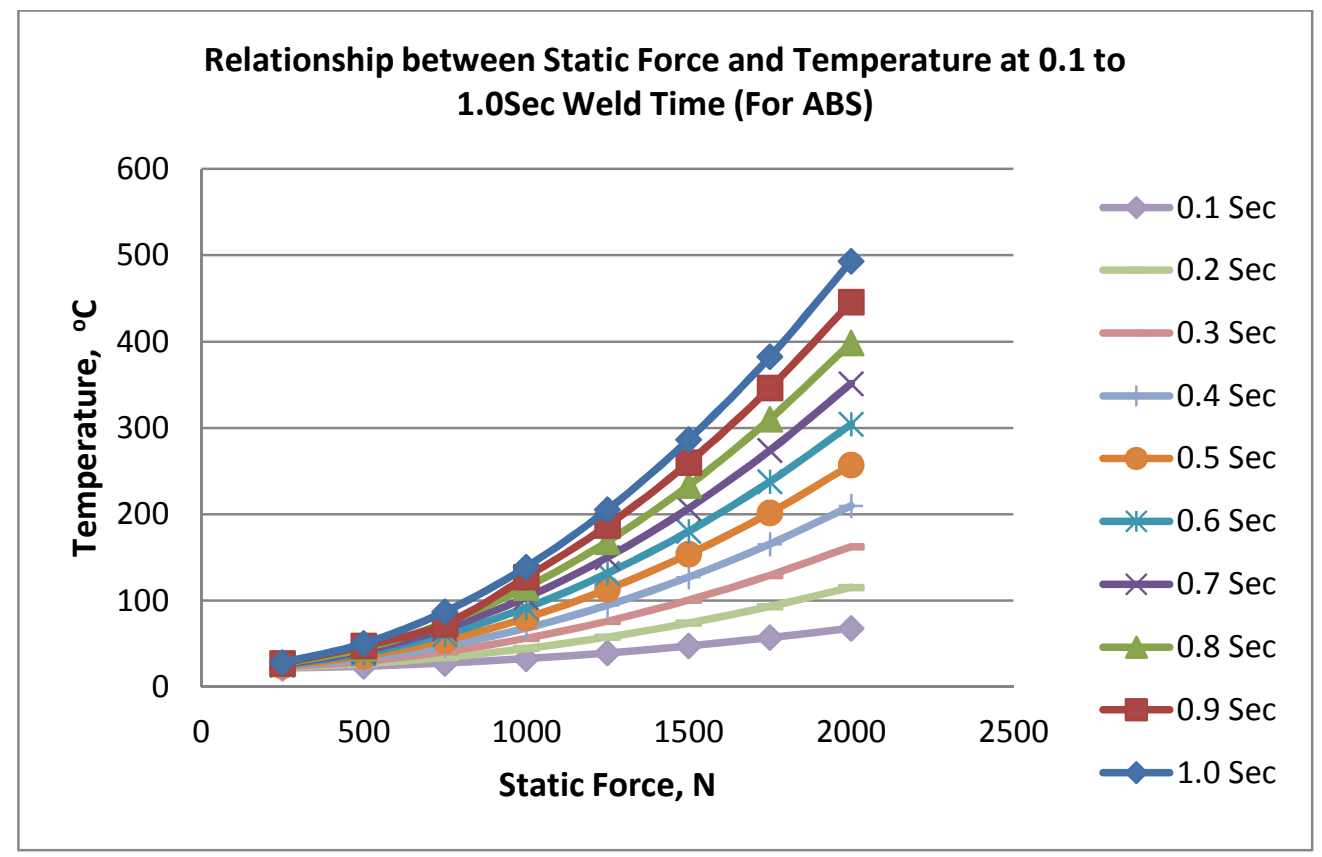

Figure 8.11 Temperature Distribution for ABS with SEMI ED Vs Static Force

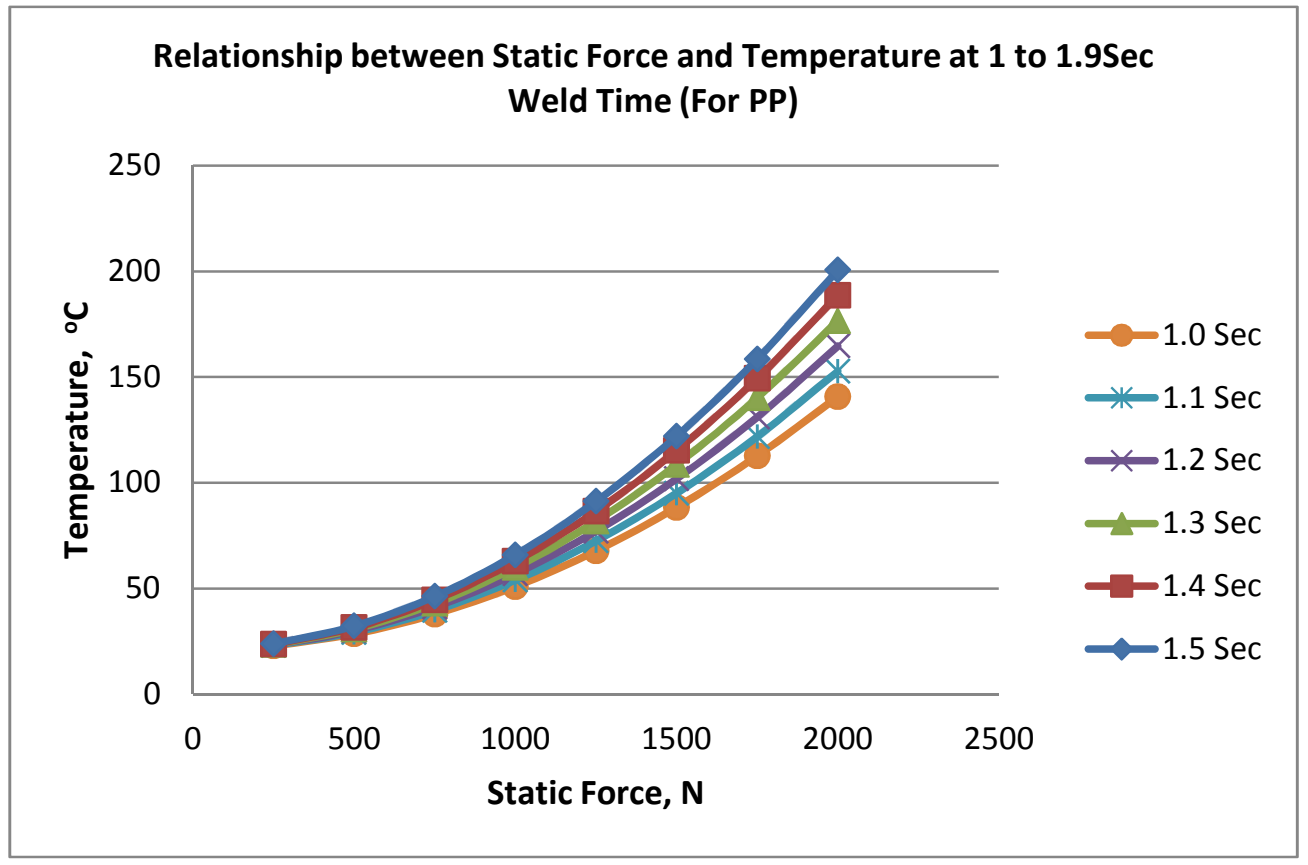

Figure 8.12 Temperature Distribution for PP with SEMI ED Vs Static Force 


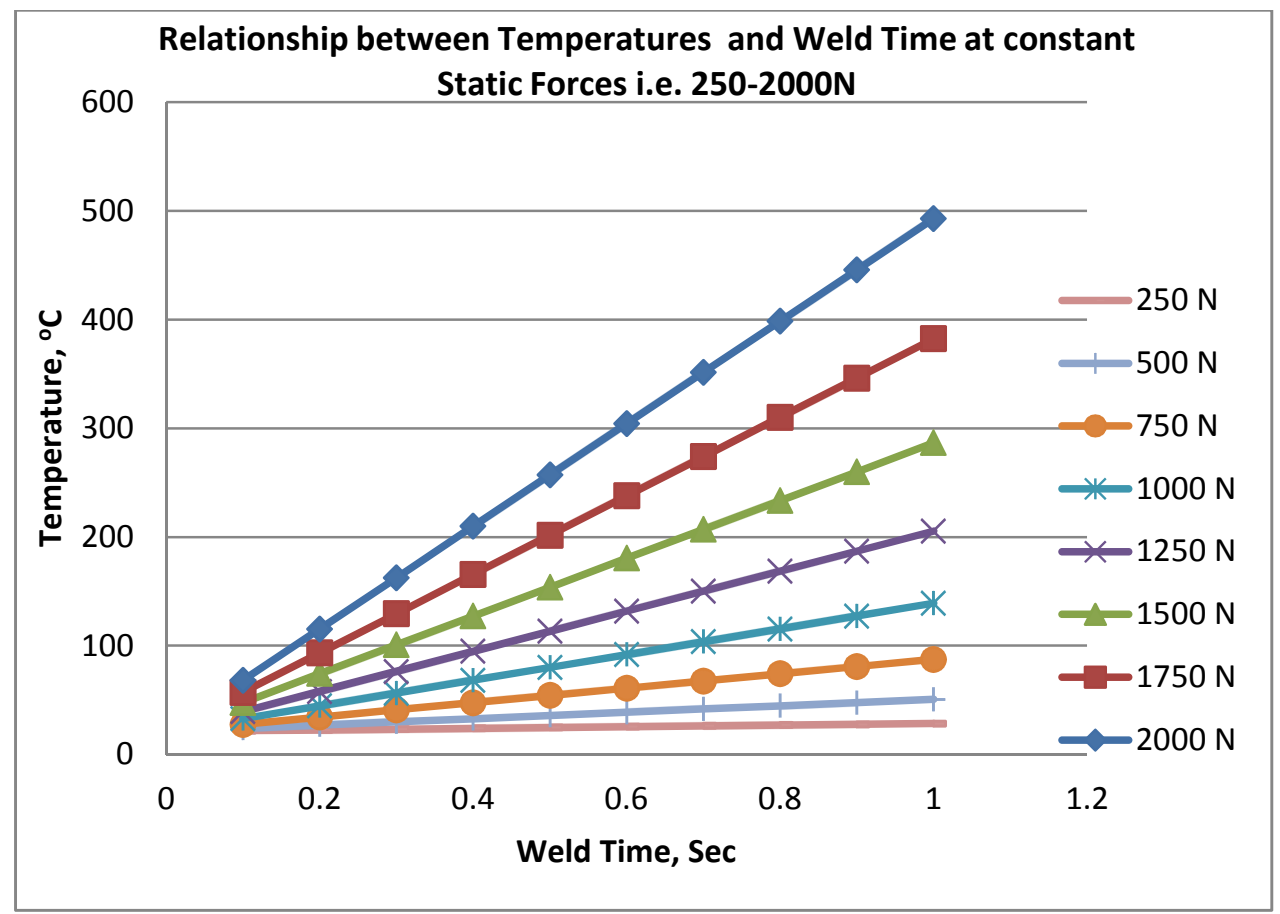

Figure 8.13 Relationship between weld time and Temperature for ABS and SEMI ED

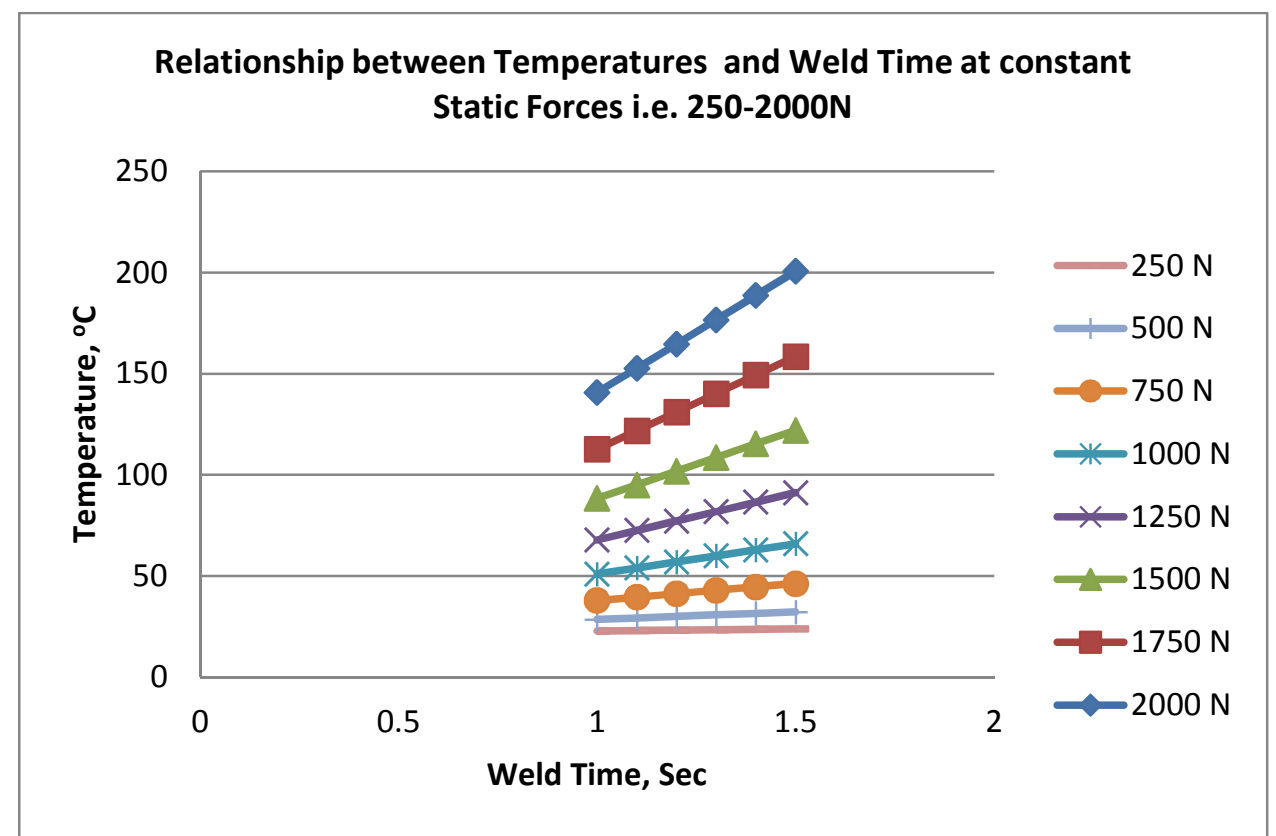

Figure 8.14 Relationship between weld time and Temperature for PP and SEMI ED

For $A B S$, temperature values are found to be higher than those for PP. This is due to difference in molecular structure for these materials. PP is a semi-crystalline thermoplastic having molecular chains in proper order. Higher energy is required to break these chains for PP than that for ABS which comprises random molecular chains. 
Neither of these materials is purely viscous nor purely elastic. For ABS, value of loss modulus is greater than that for PP resulting in highest temperature regions. As far as storage modulus is concerned, PP has slightly greater storage modulus value than that for ABS [189]. Hence first guidelines for selecting right weld factors may be provided by these simulation results in an effort to weld ABS and PP in real world of ultrasonic welding.

\subsubsection{Validation Results}

In this study, twelve validation attempts were made to check the accuracy of simulated results, as can be seen from Table 8.3 to Table 8.14 . Thermocouples and their positioning were kept same for each validation attempt, as described with the help of Figures 8.3 and 8.3(A) under section 8.4.2. Let us discuss these in detail.

\section{Selection of Weld Factors for validation}

In this study, six numerical results were endeavoured to match with experimental results for each ABS \& PP, as shown in Table $8.15^{1}$. Experimental conditions were selected in such a way that a good range of weld factors was covered which led to better temperature spread.

\section{$1^{\text {st }}$ Attempt}

First experimental trial was attempted to match the empirical result with numerical result, as illustrated in Figure 8.15. An account of weld factors resulting in experimental and numerical results with percentage error is shown in Table 8.3.

Table 8.3 First Attempt

\begin{tabular}{|c|c|c|c|c|c|c|c|}
\hline $\begin{array}{c}\text { Material } \\
\text { Type }\end{array}$ & $\begin{array}{l}\text { Static } \\
\text { Force } \\
\text { (N) }\end{array}$ & $\begin{array}{l}\text { Weld } \\
\text { Time } \\
\text { (sec) }\end{array}$ & $\begin{array}{l}\text { Hold } \\
\text { Time } \\
\text { (sec) }\end{array}$ & $\begin{array}{c}\text { ED } \\
\text { Type }\end{array}$ & $\begin{array}{l}\text { Experimenta } \\
\text { I Value }\left({ }^{\circ} \mathrm{C}\right)\end{array}$ & $\begin{array}{l}\text { Predicted/ } \\
\text { Numerical } \\
\text { Value }\left({ }^{\circ} \mathrm{C}\right)\end{array}$ & $\begin{array}{l}\% \text { age } \\
\text { Error }\end{array}$ \\
\hline ABS & 500 & 0.5 & 0.2 & $\begin{array}{c}\text { TRI } \\
\end{array}$ & 61.26 & 62.24 & 1.60 \\
\hline
\end{tabular}

\footnotetext{
${ }^{1}$ Table 8.15 is mentioned at page 212.
} 


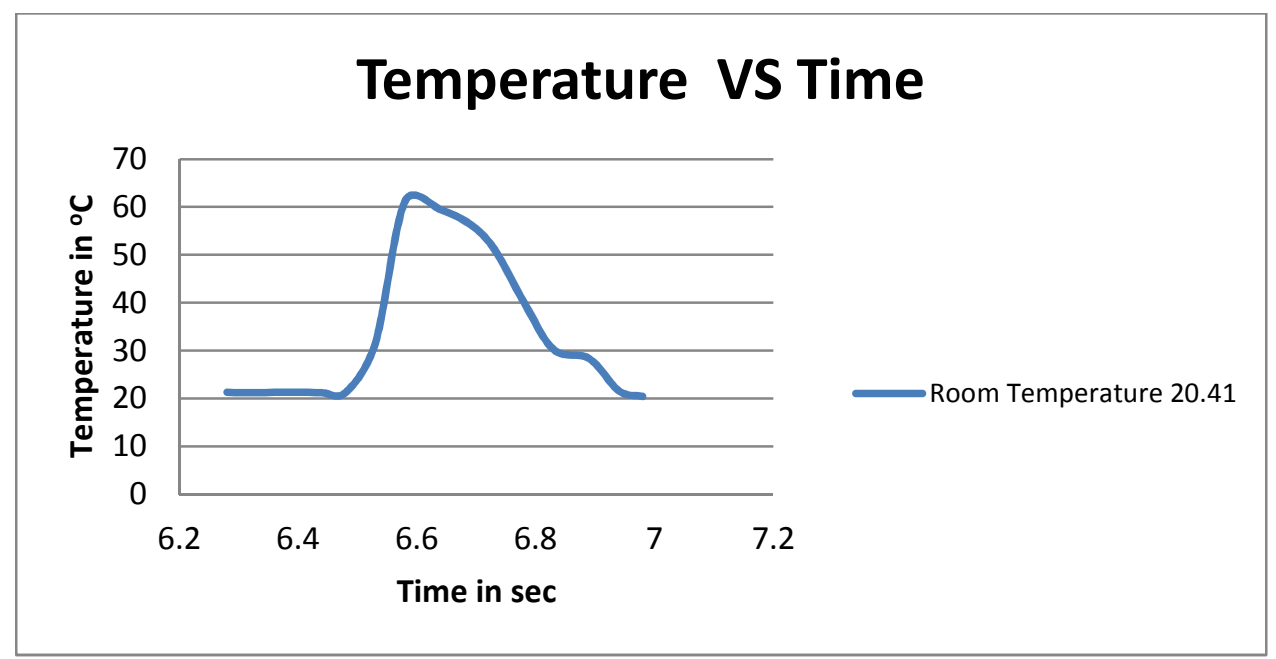

Figure 8.15 Experimental results for 1st attempt

\section{$2^{\text {nd }}$ Attempt}

On increasing the weld time from 0.5 to 0.7 , it was noted that the experimental value of temperature was increased abruptly which left behind the numerical value. Percentage error this time was found to be 33.47. Validation result for this attempt is shown in Table 8.4 and Figure 8.16. The reason for having this big error might be due to various factors e.g. untidy thermocouples at micro level with debris, discoloration, oxidization and corrosion. In order to clean the bare wires of thermocouples, various cleaning solutions can be used e.g. alcohol etc. In addition to this, thermocouple should not be contaminated with moisture which results in erratic reading at the end. Fine plastic gloves should be worn before setting the thermocouples at the desired place.

Another cause suspected for this big error was the temperature difference between room and thermocouple wires before welding. This temperature difference can develop temperature gradients resulting in troubles in sensing temperature. This problem needs to be solved by conducting the dummy checks with thermocouple for its room temperature measurement [196]. 
Table 8.4 Second Attempt

\begin{tabular}{|c||c|c|c|c||c||c|c||}
\hline $\begin{array}{c}\text { Material } \\
\text { Type }\end{array}$ & $\begin{array}{c}\text { Static } \\
\text { Force } \\
(\mathbf{N})\end{array}$ & $\begin{array}{c}\text { Weld } \\
\text { Time } \\
(\mathbf{s e c})\end{array}$ & $\begin{array}{c}\text { Hold } \\
\text { Time } \\
(\mathbf{s e c})\end{array}$ & $\begin{array}{c}\text { ED } \\
\text { Type }\end{array}$ & $\begin{array}{c}\text { Experimental } \\
\text { Value }\left({ }^{\circ} \mathrm{C}\right)\end{array}$ & $\begin{array}{c}\text { Predicted/Numerical } \\
\text { Value }\left({ }^{\circ} \mathrm{C}\right)\end{array}$ & $\begin{array}{c}\% \\
\text { age } \\
\text { Error }\end{array}$ \\
\hline \hline ABS & 500 & 0.7 & 0.2 & TRI & 122.81 & 81.70 & 33.47 \\
\hline
\end{tabular}

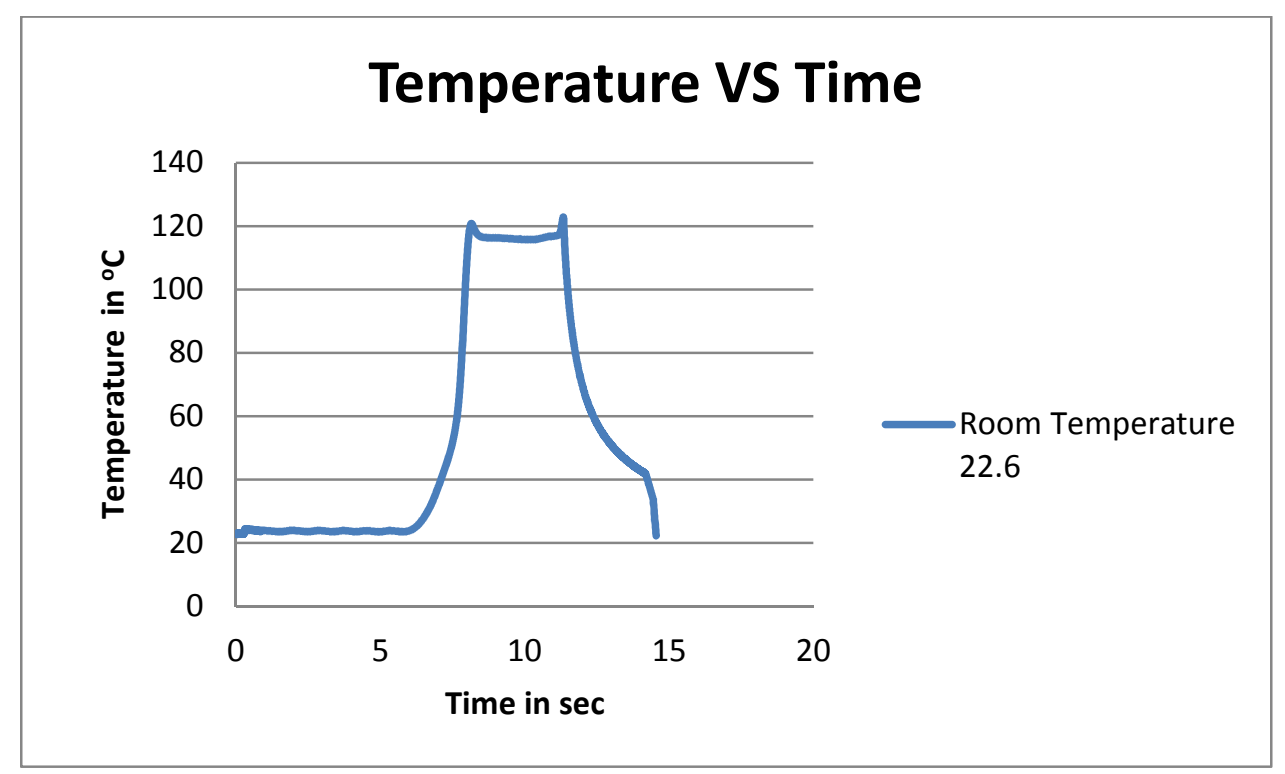

Figure 8.16 Experimental results for 2 nd attempt

\section{$3^{\text {rd }}$ Attempt}

In this case, again both experimental and numerical results remained matchless because of setting higher weld time for SEMI ED. Experimental temperature resulting from weld factors in Table 8.5 was initially predicted to match with 76.02 due to higher volume of SEMI ED than that of TRI ED. In fact, lower concentration of ultrasonic vibrations producing lower temperature was expected at weld interface due to higher volume of SEMI ED. But later it was found that the percentage error was noted to be 37.8. Maximum experimental value of temperature is shown in Figure 8.17. The possible reason allowing this error to occur was the excessive movement of upper specimen with SEMI ED on thermocouple that generates some extra heating due to friction. Such movement is expected to happen for a short period of time. But ultrasonic vibration is recognized to develop high temperature region even for such a tiny time on using $A B S$. 
Table 8.5 Third Attempt

\begin{tabular}{|c|c|c|c|c|c||c|c|}
\hline $\begin{array}{c}\text { Material } \\
\text { Type }\end{array}$ & $\begin{array}{c}\text { Static } \\
\text { Force } \\
(\mathbf{N})\end{array}$ & $\begin{array}{c}\text { Weld } \\
\text { Time } \\
(\mathbf{s e c})\end{array}$ & $\begin{array}{c}\text { Hold } \\
\text { Time } \\
(\mathbf{s e c})\end{array}$ & $\begin{array}{c}\text { ED } \\
\text { Type }\end{array}$ & $\begin{array}{c}\text { Experimental } \\
\text { Value }\left({ }^{\circ} \mathbf{C}\right)\end{array}$ & $\begin{array}{c}\text { Predicted/Numerical } \\
\text { Value }\left({ }^{\circ} \mathbf{C}\right)\end{array}$ & $\begin{array}{c}\% \\
\text { age } \\
\text { Error }\end{array}$ \\
\hline \hline ABS & 750 & 0.9 & 0.2 & SEMI & 122.23 & 76.02 & 37.80 \\
\hline
\end{tabular}

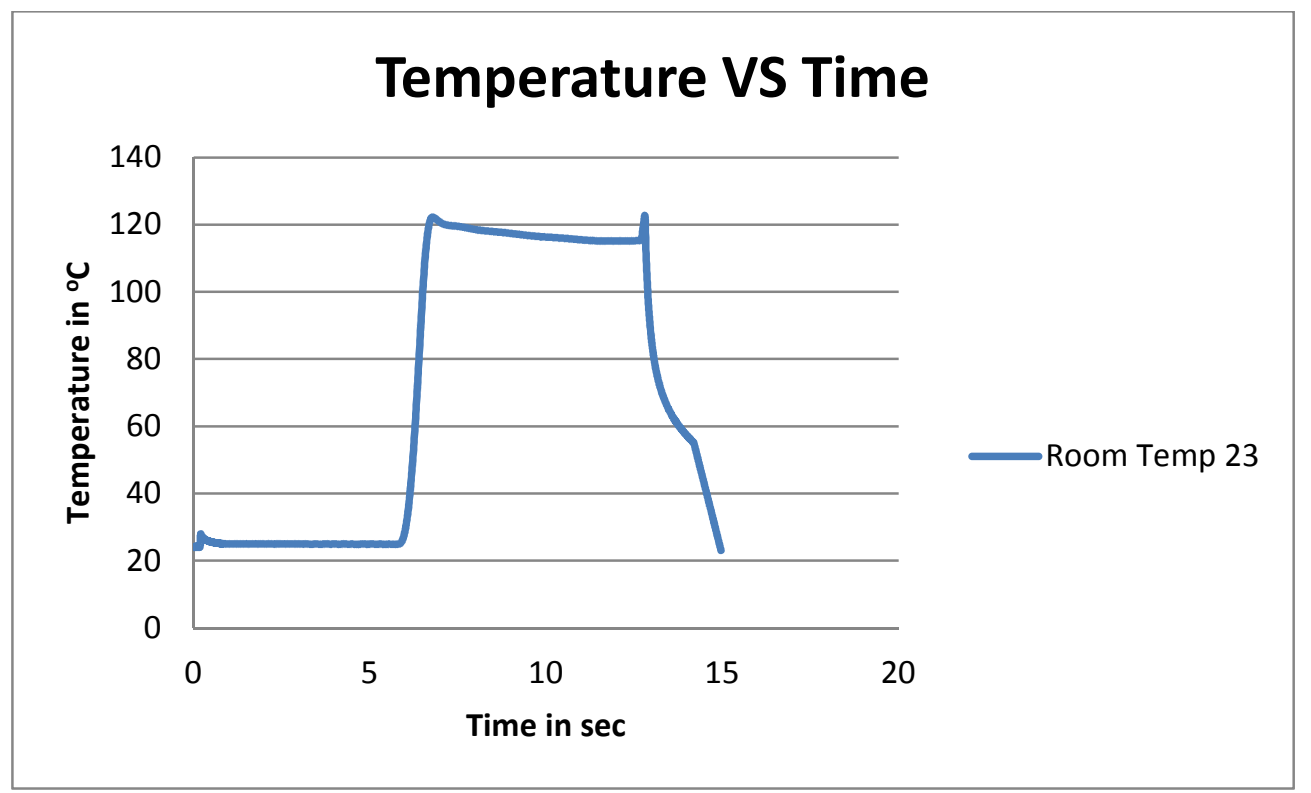

Figure 8.17 Experimental results for 3rd attempt

\section{$4^{\text {th }}$ Attempt}

In this trial, experimental result was found to be closer to numerical counterpart. Percentage error was found to be 1.07, as shown in Table 8.6. Notably, this time the weld time is $1 \mathrm{sec}$ which is considered large for welding ABS. It is also noted that static force has been increased this time from 750 to $1000 \mathrm{~N}$. Experimental result is also shown in Figure 8.18 .

Table 8.6 Fourth Attempt

\begin{tabular}{|c||c||c||c|c||c||c||c|}
\hline \hline $\begin{array}{c}\text { Material } \\
\text { Type }\end{array}$ & $\begin{array}{c}\text { Static } \\
\text { Force } \\
(\mathbf{N})\end{array}$ & $\begin{array}{c}\text { Weld } \\
\text { Time } \\
(\mathbf{s e c})\end{array}$ & $\begin{array}{c}\text { Hold } \\
\text { Time } \\
(\mathbf{s e c})\end{array}$ & $\begin{array}{c}\text { ED } \\
\text { Type }\end{array}$ & $\begin{array}{c}\text { Experimental } \\
\text { Value }\left({ }^{\circ} \mathrm{C}\right)\end{array}$ & $\begin{array}{c}\text { Predicted/Numerical } \\
\text { Value }\left({ }^{\circ} \mathrm{C}\right)\end{array}$ & $\begin{array}{c}\% \\
\text { age } \\
\text { Error }\end{array}$ \\
\hline \hline $\mathrm{ABS}$ & 1000 & 1 & 0.2 & SEMI & 122.44 & 123.75 & 1.07 \\
\hline
\end{tabular}




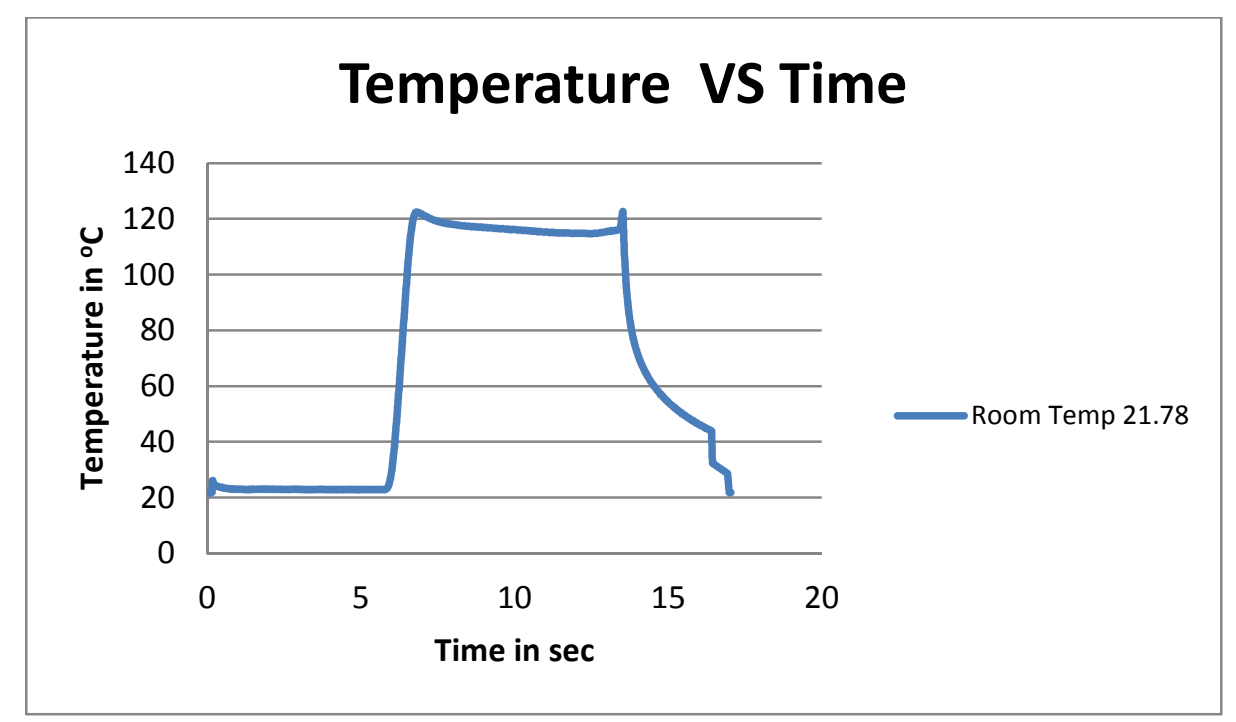

Figure 8.18 Experimental results for 4 th attempt

\section{$5^{\text {th }}$ Attempt}

In this trial, both results were again found to match with each other. From Table 8.7, percentage error remained 5.95. Again static force was kept same i.e. $1000 \mathrm{~N}$. This shows that each weld factor has its own contribution in delivering true temperature data during welding. Experimental temperature for this attempt is illustrated in Figure 8.19.

Table 8.7 Fifth Attempt

\begin{tabular}{|c||c||c||c|c||c||c||c|}
\hline $\begin{array}{c}\text { Material } \\
\text { Type }\end{array}$ & $\begin{array}{c}\text { Static } \\
\text { Force } \\
(\mathbf{N})\end{array}$ & $\begin{array}{c}\text { Weld } \\
\text { Time } \\
(\mathbf{s e c})\end{array}$ & $\begin{array}{c}\text { Hold } \\
\text { Time } \\
(\mathbf{s e c})\end{array}$ & $\begin{array}{c}\text { ED } \\
\text { Type }\end{array}$ & $\begin{array}{c}\text { Experimental } \\
\text { Value }\left({ }^{\circ} \mathrm{C}\right)\end{array}$ & $\begin{array}{c}\text { Predicted/Numerical } \\
\text { Value }\left({ }^{\circ} \mathrm{C}\right)\end{array}$ & $\begin{array}{c}\% \\
\text { age } \\
\text { Error }\end{array}$ \\
\hline \hline $\mathrm{ABS}$ & 1000 & 0.7 & 0.2 & SEMI & 99.60 & 93.67 & 5.95 \\
\hline
\end{tabular}




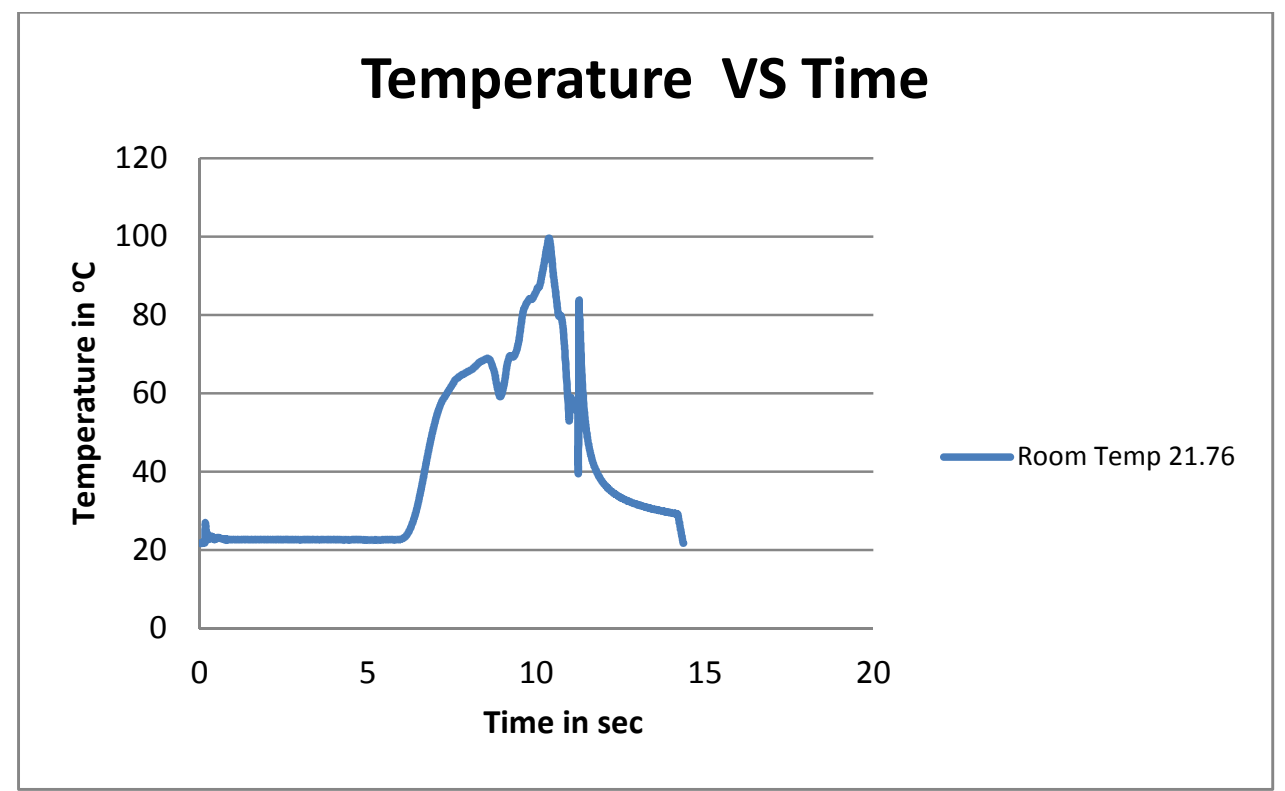

Figure 8.19 Experimental results for 5 th attempt

\section{$6^{\text {th }}$ Attempt}

In this trial, the numerical value was found to be higher than that of experimental. Please note that the static force is $750 \mathrm{~N}$, as can be seen in Table 8.8. At start, it was thought that the experimental temperature should go beyond $150.13{ }^{\circ} \mathrm{C}$ i.e. numerical/predicted value but later it was not observed on validating, as shown in Figure 8.20. The likely reason for getting this error is the poor placement of thermocouple.

Table 8.8 Sixth Attempt

\begin{tabular}{|c||c|c|c|c||c||c|c||}
\hline $\begin{array}{c}\text { Material } \\
\text { Type }\end{array}$ & $\begin{array}{c}\text { Static } \\
\text { Force } \\
(\mathbf{N})\end{array}$ & $\begin{array}{c}\text { Weld } \\
\text { Time } \\
(\mathbf{s e c})\end{array}$ & $\begin{array}{c}\text { Hold } \\
\text { Time } \\
(\mathbf{s e c})\end{array}$ & $\begin{array}{c}\text { ED } \\
\text { Type }\end{array}$ & $\begin{array}{c}\text { Experimental } \\
\text { Value }\left({ }^{\circ} \mathbf{C}\right)\end{array}$ & $\begin{array}{c}\text { Predicted/Numerical } \\
\text { Value }\left({ }^{\circ} \mathrm{C}\right)\end{array}$ & $\begin{array}{c}\% \\
\text { age } \\
\text { Error }\end{array}$ \\
\hline ABS & 750 & 0.9 & 0.2 & TRI & 121.25 & 150.13 & 23.82 \\
\hline
\end{tabular}




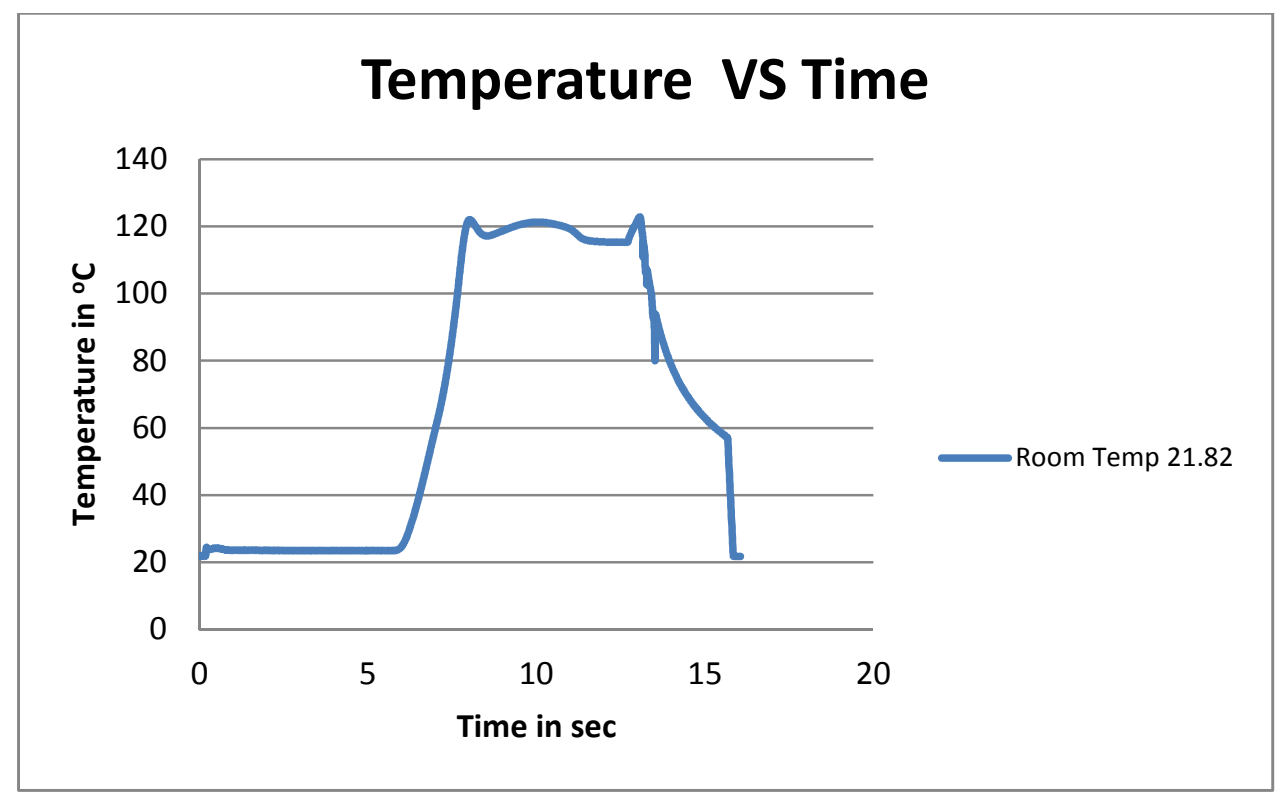

Figure 8.20 Experimental results for 6 th attempt

For PP, all the validation attempts resulted in close match between experimental and simulation results.

$7^{\text {th }}$ Attempt

In this case the experimental and numerical results were almost found to be similar with a percentage error of 2.94, as shown in Table 8.9. Experimental result for this attempt is shown in Figure 8.21.

Table 8.9 Seventh Attempt

\begin{tabular}{|c|c||c||c|c||c|c|c||}
\hline $\begin{array}{c}\text { Material } \\
\text { Type }\end{array}$ & $\begin{array}{c}\text { Static } \\
\text { Force } \\
(\mathbf{N})\end{array}$ & $\begin{array}{c}\text { Weld } \\
\text { Time } \\
(\mathbf{s e c})\end{array}$ & $\begin{array}{c}\text { Hold } \\
\text { Time } \\
(\mathbf{s e c})\end{array}$ & $\begin{array}{c}\text { ED } \\
\text { Type }\end{array}$ & $\begin{array}{c}\text { Experimental } \\
\text { Value }\left({ }^{\circ} \mathrm{C}\right)\end{array}$ & $\begin{array}{c}\text { Predicted/Numerical } \\
\text { Value }\left({ }^{\circ} \mathrm{C}\right)\end{array}$ & $\begin{array}{c}\% \\
\text { age } \\
\text { Error }\end{array}$ \\
\hline \hline PP & 1000 & 1.2 & 1 & TRI & 120.72 & 117.17 & 2.94 \\
\hline
\end{tabular}




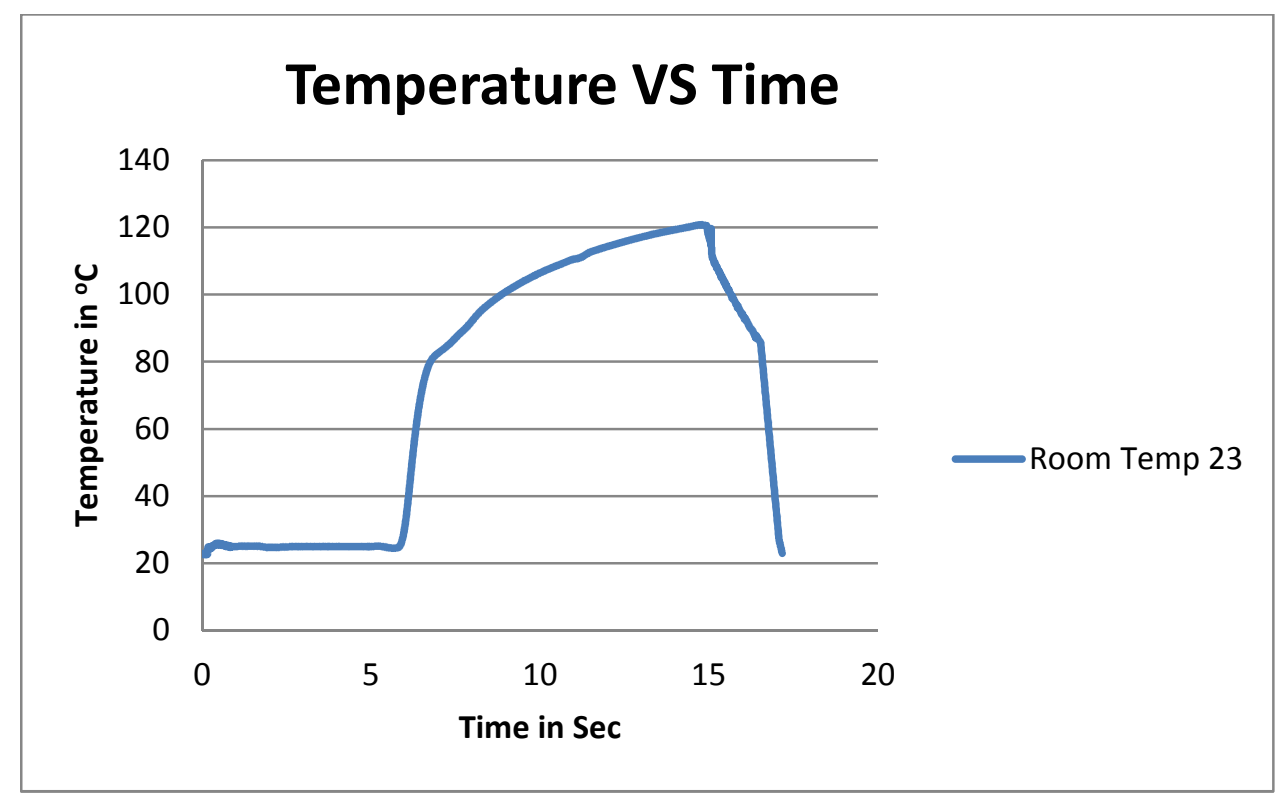

Figure 8.21 Experimental results for 7th attempt

\section{$8^{\text {th }}$ Attempt}

In current case, there was less difference between experimental and numerical results with a percentage error of 11.18 , as illustrated in Table 8.10 and Figure 8.22.

Table 8.10 Eighth Attempt

\begin{tabular}{|c||c|c|c|c||c||c|c||}
\hline $\begin{array}{c}\text { Material } \\
\text { Type }\end{array}$ & $\begin{array}{c}\text { Static } \\
\text { Force } \\
(\mathbf{N})\end{array}$ & $\begin{array}{c}\text { Weld } \\
\text { Time } \\
(\mathbf{s e c})\end{array}$ & $\begin{array}{c}\text { Hold } \\
\text { Time } \\
(\mathbf{s e c})\end{array}$ & $\begin{array}{c}\text { ED } \\
\text { Type }\end{array}$ & $\begin{array}{c}\text { Experimental } \\
\text { Value }\left({ }^{\circ} \mathrm{C}\right)\end{array}$ & $\begin{array}{c}\text { Predicted/Numerical } \\
\text { Value }\left({ }^{\circ} \mathrm{C}\right)\end{array}$ & $\begin{array}{c}\% \\
\text { age } \\
\text { Error }\end{array}$ \\
\hline \hline PP & 1000 & 1.1 & 1 & TRI & 122.79 & 109.06 & 11.18 \\
\hline
\end{tabular}




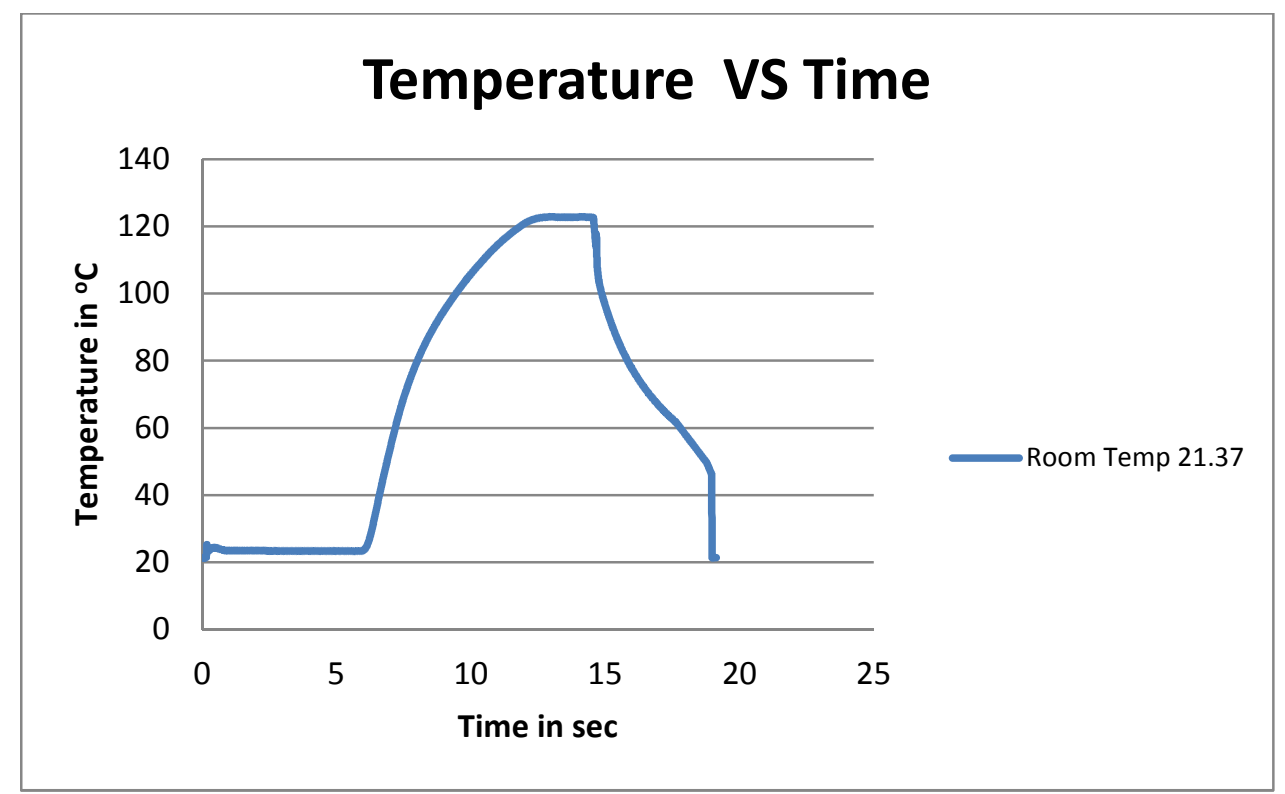

Figure 8.22 Experimental results for 8 th attempt

\section{$9^{\text {th }}$ Attempt}

Experimental result in this case nearly agreed with numerical one with a percentage error of 3.55, as exhibited in Table 8.11. Increasing static force and decreasing weld time resulted in increment in both experimental and numerical values of temperatures. Overall for TRI ED, experimental values of temperatures were found to be higher than those of their numerical counterparts for PP, as shown in Table 8.11 and previous Tables 8.9\&8.10. Sudden variation in temperature before reaching its maximum value of 122.81 degree $C$ is shown in Figure 8.23. It seems possible that the TRI ED with upper specimen has lost the contact several times with thermocouple for a while and then again made the contact owing to the presence of static force.

Table 8.11 Ninth Attempt

\begin{tabular}{|c|c||c||c||c||c||c||c|}
\hline $\begin{array}{c}\text { Material } \\
\text { Type }\end{array}$ & $\begin{array}{c}\text { Static } \\
\text { Force } \\
(\mathbf{N})\end{array}$ & $\begin{array}{c}\text { Weld } \\
\text { Time } \\
(\mathbf{s e c})\end{array}$ & $\begin{array}{c}\text { Hold } \\
\text { Time } \\
(\mathbf{s e c})\end{array}$ & $\begin{array}{c}\text { ED } \\
\text { Type }\end{array}$ & $\begin{array}{c}\text { Experimental } \\
\text { Value }\left({ }^{\circ} \mathrm{C}\right)\end{array}$ & $\begin{array}{c}\text { Predicted/Numerical } \\
\text { Value }\left({ }^{\circ} \mathrm{C}\right)\end{array}$ & $\begin{array}{c}\% \\
\text { age } \\
\text { Error }\end{array}$ \\
\hline PP & 1250 & 1 & 1 & TRI & 122.81 & 118.45 & 3.55 \\
\hline
\end{tabular}




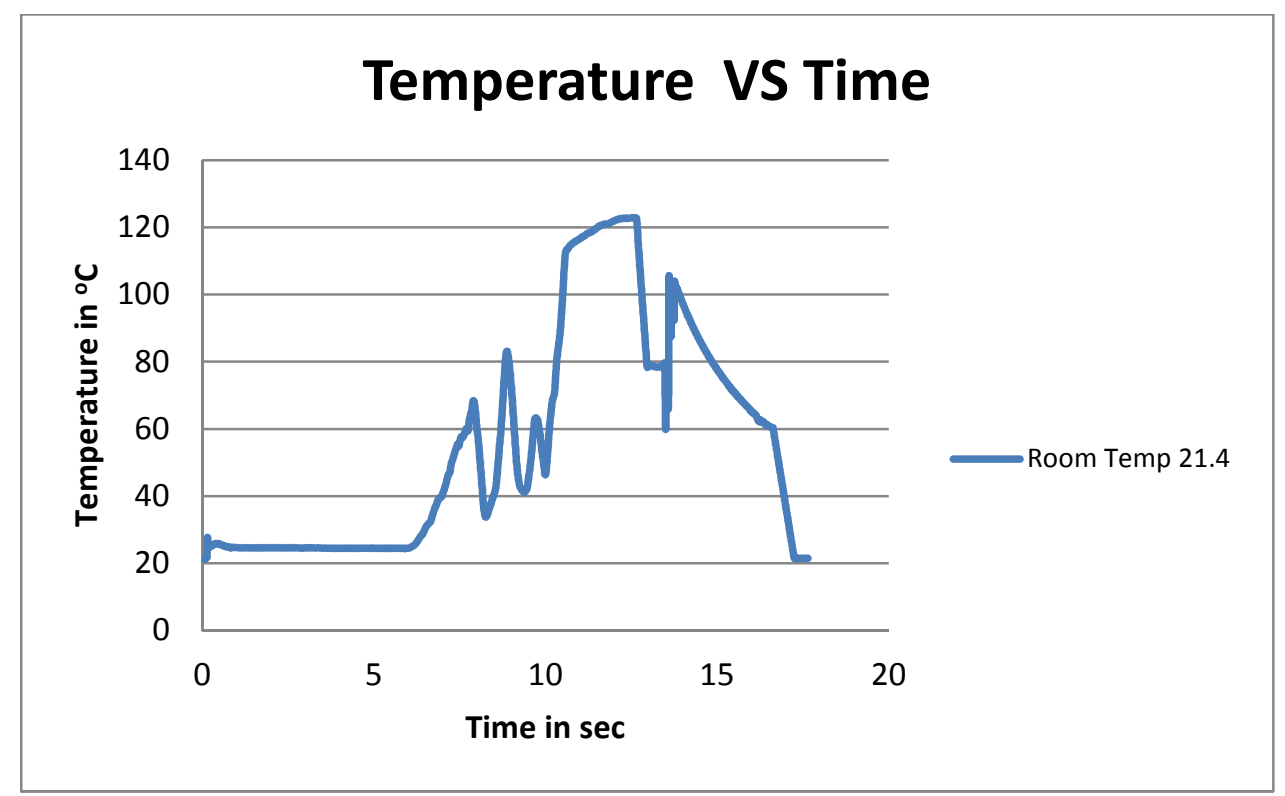

Figure 8.23 Experimental results for 9th attempt

$10^{\text {th }}$ Attempt

In this case, a minor difference was observed between experimental and numerical values with percentage error of 9.86, as can be seen in Table 8.12. Experimental result is illustrated in Figure 8.24.

Table 8.12 Tenth Attempt

\begin{tabular}{|c||c||c|c|c||c||c||c|}
\hline $\begin{array}{c}\text { Material } \\
\text { Type }\end{array}$ & $\begin{array}{c}\text { Static } \\
\text { Force } \\
(\mathbf{N})\end{array}$ & $\begin{array}{c}\text { Weld } \\
\text { Time } \\
(\mathbf{s e c})\end{array}$ & $\begin{array}{c}\text { Hold } \\
\text { Time } \\
(\mathbf{s e c})\end{array}$ & $\begin{array}{c}\text { ED } \\
\text { Type }\end{array}$ & $\begin{array}{c}\text { Experimental } \\
\text { Value }\left({ }^{\circ} \mathrm{C}\right)\end{array}$ & $\begin{array}{c}\text { Predicted/Numerical } \\
\text { Value }\left({ }^{\circ} \mathrm{C}\right)\end{array}$ & $\begin{array}{c}\% \\
\text { age } \\
\text { Error }\end{array}$ \\
\hline \hline PP & 1500 & 1.4 & 1 & SEMI & 117.16 & 105.61 & 9.86 \\
\hline
\end{tabular}




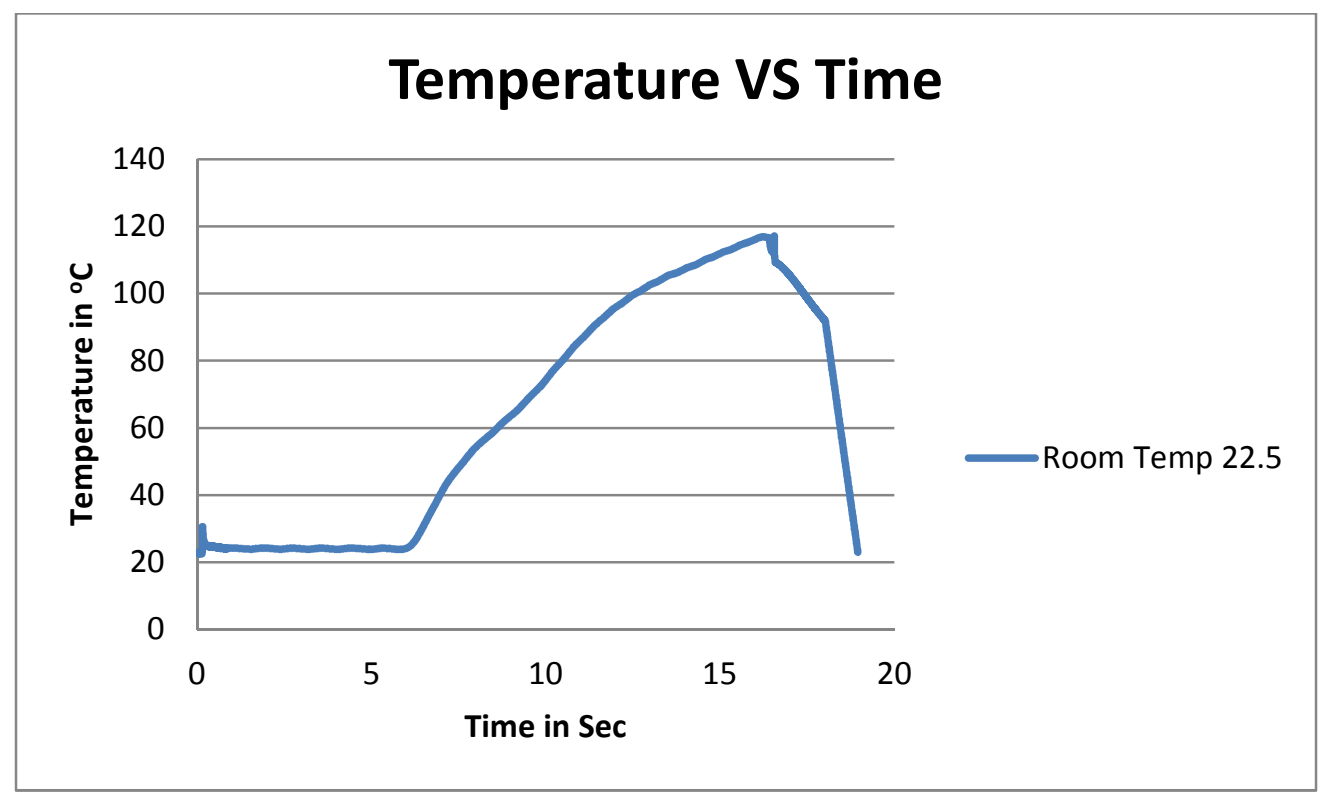

Figure 8.24 Experimental results for 10th attempt

\section{$11^{\text {th }}$ Attempt}

In this case, the experimental value of temperature was found to be lower than that of its numerical counterpart with a negligible percentage error of 1.4, as shown in Table 8.13. Experimental value of temperature is exhibited in Figure 8.25.

Table 8.13 Eleventh Attempt

\begin{tabular}{|c||c||c|c|c||c||c||c|}
\hline $\begin{array}{c}\text { Material } \\
\text { Type }\end{array}$ & $\begin{array}{c}\text { Static } \\
\text { Force } \\
(\mathbf{N})\end{array}$ & $\begin{array}{c}\text { Weld } \\
\text { Time } \\
(\mathbf{s e c})\end{array}$ & $\begin{array}{c}\text { Hold } \\
\text { Time } \\
(\mathbf{s e c})\end{array}$ & $\begin{array}{c}\text { ED } \\
\text { Type }\end{array}$ & $\begin{array}{c}\text { Experimental } \\
\text { Value }\left({ }^{\circ} \mathrm{C}\right)\end{array}$ & $\begin{array}{c}\text { Predicted/Numerical } \\
\text { Value }\left({ }^{\circ} \mathrm{C}\right)\end{array}$ & $\begin{array}{c}\% \\
\text { age } \\
\text { Error }\end{array}$ \\
\hline \hline PP & 1750 & 1.1 & 0.2 & SEMI & 109.75 & 111.28 & 1.4 \\
\hline
\end{tabular}




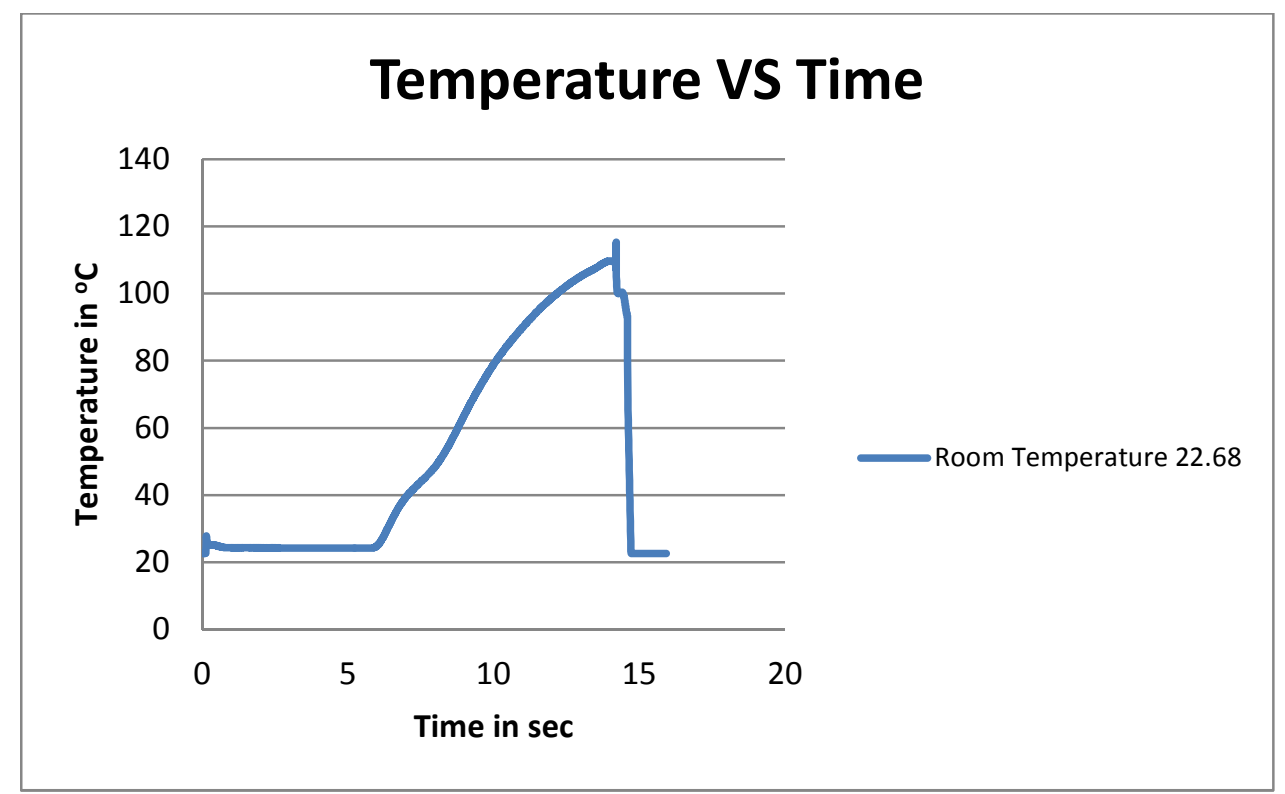

Figure 8.25 Experimental results for 11th attempt

\section{$12^{\text {th }}$ Attempt}

In this case, experimental value of temperature was again found matching with numerical value with percentage error of 2.32 , as shown in Table 8.14. From previous case, on increasing the weld time and hold time, a considerable increment in both results was noted. Experimental result is illustrated in Figure 8.26.

Table 8.14 Twelfth Attempt

\begin{tabular}{|c||c||c||c|c||c||c||c|}
\hline $\begin{array}{c}\text { Material } \\
\text { Type }\end{array}$ & $\begin{array}{c}\text { Static } \\
\text { Force } \\
(\mathbf{N})\end{array}$ & $\begin{array}{c}\text { Weld } \\
\text { Time } \\
(\mathbf{s e c})\end{array}$ & $\begin{array}{c}\text { Hold } \\
\text { Time } \\
(\mathbf{s e c})\end{array}$ & $\begin{array}{c}\text { ED } \\
\text { Type }\end{array}$ & $\begin{array}{c}\text { Experimental } \\
\text { Value }\left({ }^{\circ} \mathrm{C}\right)\end{array}$ & $\begin{array}{c}\text { Predicted/Numerical } \\
\text { Value }\left({ }^{\circ} \mathrm{C}\right)\end{array}$ & $\begin{array}{c}\% \\
\text { age } \\
\text { Error }\end{array}$ \\
\hline \hline PP & 1750 & 1.3 & 1 & SEMI & 122.81 & 125.67 & 2.32 \\
\hline
\end{tabular}




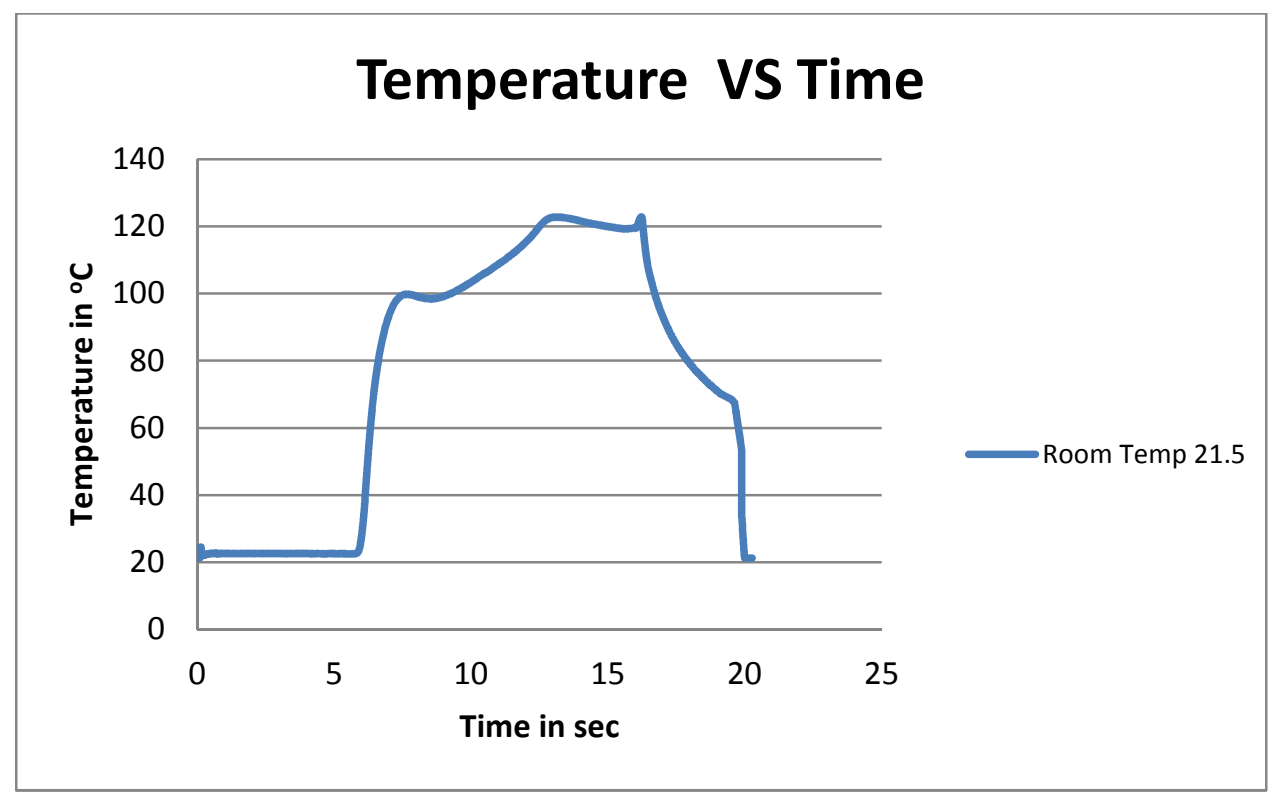

Figure 8.26 Experimental results for 12th attempt

Holistically, this simulation proves itself to be used for future in order to predict the temperature at weld zone and to make some quick comparisons of numerical temperature with the empirical temperature. From Table 8.15, there are few cases which present considerable percentage errors. These errors can be avoided by using good fixtures in preventing the specimens from displacing, by fixing the thermocouples in a better way and by carefully observing the shooting up nature of ultrasonic welding that depends upon various weld factors. By shooting up nature of ultrasonic welding process, it is meant that temperature jumps quickly in most of the cases with ABS, in general, and TRI ED of both materials. 
Table 8.15 Results from validation of simulation

\begin{tabular}{|c|c||c||c|c|c|c|c||c|}
\hline $\begin{array}{c}\text { Attempt } \\
\text { No. }\end{array}$ & $\begin{array}{c}\text { Static } \\
\text { Force } \\
(\mathbf{N})\end{array}$ & $\begin{array}{c}\text { Weld } \\
\text { Time } \\
(\mathbf{s e c})\end{array}$ & Material & $\begin{array}{c}\text { Hold } \\
\text { Time } \\
(\mathbf{s e c})\end{array}$ & $\begin{array}{c}\text { ED } \\
\text { Type }\end{array}$ & $\begin{array}{c}\text { Experimental } \\
\text { Value }{ }^{\circ} \mathbf{C} \text { ) }\end{array}$ & $\begin{array}{c}\text { Predicted/ } \\
\text { Numerical } \\
\left.\text { Value ( }{ }^{\circ} \mathbf{C}\right)\end{array}$ & $\begin{array}{c}\text { \% age } \\
\text { Error }\end{array}$ \\
\hline \hline 1 & 500 & 0.5 & ABS & 0.2 & TRI & 61.26 & 62.24 & 1.6 \\
\hline 2 & 500 & 0.7 & ABS & 0.2 & TRI & 122.81 & 81.70 & 33.47 \\
\hline 3 & 750 & 0.9 & ABS & 0.2 & SEMI & 122.23 & 76.02 & 37.8 \\
\hline 4 & 1000 & 1 & ABS & 0.2 & SEMI & 122.44 & 123.75 & 1.07 \\
\hline 5 & 1000 & 0.7 & ABS & 0.2 & SEMI & 99.6 & 93.67 & 5.95 \\
\hline 6 & 750 & 0.9 & ABS & 0.2 & TRI & 121.25 & 150.13 & 23.82 \\
\hline 7 & 1000 & 1.2 & PP & 1 & TRI & 120.72 & 117.17 & 2.94 \\
\hline 8 & 1000 & 1.1 & PP & 1 & TRI & 122.8 & 109.06 & 11.18 \\
\hline 9 & 1250 & 1 & PP & 1 & TRI & 122.81 & 118.45 & 3.55 \\
\hline 10 & 1500 & 1.4 & PP & 1 & SEMI & 117.16 & 105.61 & 9.86 \\
\hline 11 & 1750 & 1.1 & PP & 0.2 & SEMI & 109.75 & 111.28 & 1.4 \\
\hline 12 & 1750 & 1.3 & PP & 1 & SEMI & 122.81 & 125.67 & 2.32 \\
\hline
\end{tabular}

\subsection{Hypotheses Testing}

\subsection{1. $\underline{A B S}$}

\section{Hypothesis A}

Hypothesis A vocalizes as "ABS is an amorphous thermoplastic with higher tensile strength than that of PP"

In this research, higher LSS was observed for whole ranges of weld factors e.g. weld time $(0.4-0.9 \mathrm{sec})$ \& static force $(500-1500 \mathrm{~N})$. This was due to the reason that high temperature was guaranteed by FEA results for $A B S$. For instance, temperature up to 280 degree $C$ is achieved by using high levels of weld time and static force for SEMI ED. In the same manner, temperature up to 590 degree $C$ is achievable on using high levels of weld time and static force for TRI ED. These temperature values for both EDs are really sufficient in reaching the glass transition temperature of ABS thereby resulting in higher LSS, as shown in Figure 8.27. Hence hypothesis A seems to be again considered for its approval in this way after proving it partly from DSC \& MFI results. 

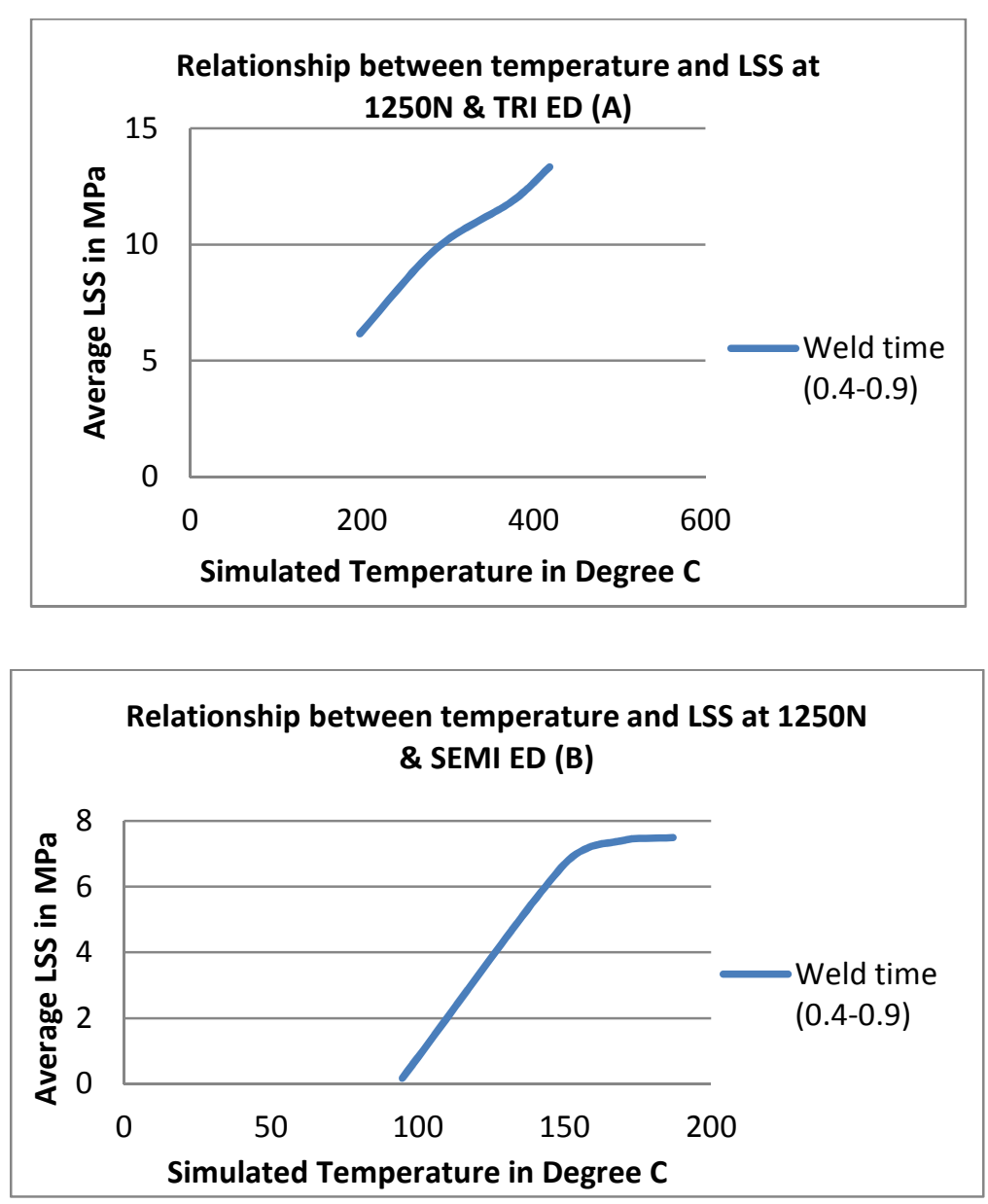

Figure 8.27(A\&B) Relationship between simulated temperature and LSS for TRI and SEMI EDs respectively

\section{Hypothesis B}

Hypothesis B expresses that "Volume of TRI ED is less than that of SEMI ED resulting in high concentration of ultrasonic energy"

Temperature value up to 590 degree $C$ is easily attainable by using high levels of weld time and static force for TRI ED from FEA results. Therefore, TRI ED appears to be a better heating source which contrasts with that of SEMI ED according to FEA results. So, in current ultrasonic welding, higher LSS was expected to be delivered conveniently. Since the chances of uniformly heating weld interface are possibly better for TRI ED due to its lower volume, hence hypothesis B also appears to be accepted again somehow hereby after having proved from MFI results.

\section{Hypothesis C}


Hypothesis $C$ discusses that "Welding temperature is more conveniently attainable for ABS owing to its higher loss modulus as compared to PP"

The relationship for viscoelastic heating [37] requires the value of loss modulus which is higher for ABS than that for PP. This high value of loss modulus always assures the consistent supply of heat at the weld interface. There is almost no chance of returning back for vibrations during welding $A B S$ owing to its higher loss modulus. Therefore, vibrations are dissipated as heat owing to damping. This higher loss modulus also makes ABS a better conductor of ultrasonic waves. Hence higher LSS was easily achieved for ABS by using even low level of weld factors. Abrupt drop in LSS can be possible with high level of weld factors because of heating too much above glass transition temperature of ABS. Therefore, FEA results cover the hypothesis $C$ for its sanction after having already checked it from DSC and MFI results.

\section{Hypothesis D}

Hypothesis D states that "EDs made of ABS have higher heat distortion temperature (HDT) \& higher loss modulus than those for PP"

Although FEA results suggest achieving higher temperature values for ABS with both EDs, molten $A B S$ has higher viscosity. Also high viscosity of $A B S$ can result in lower collapse of ED except for very few cases where the welded joint has shown complete ED collapse. High loss modulus does not ensure high collapse of ED for ABS as loss modulus is not dependent on the temperature and it is a material property. The heat distortion temperature (HDT) of ABS is also higher than that of PP implying that distortion rate of ED under ultrasonic vibration is not higher for ABS than that for PP. Hence higher HDT and higher viscosity can yield lower ED collapse finally for ABS unless high level of weld factors is used which further proves the hypothesis D from FEA results to some extent.

\section{Hypothesis F}

Hypothesis F states that "Role of static force is different for ABS due to its higher viscosity and lower glass transition temperature." 
MFI tests have evaluated a higher viscosity for ABS. From the FEA results it is clear that higher temperature zones are possible at the weld interface for ABS. Viscosity drop is, in fact, directly proportional to the temperature. On using the high level of static force, molten $A B S$ is expected to be pushed out of weld interface which is detrimental to bond strength. Hence the bond strength is controlled by lowering the static force to ensure bond improvement offering a better resisting area. Henceforth FEA proves partially the hypothesis F once again after having proved it from MFI results.

\section{Hypothesis G}

Hypothesis G states that "Almost same resisting area is expected to be produced at weld interface by using higher amplitudes"

In this study, high temperature regions were observed for ABS from FEA results. At high amplitudes, high temperature is confidently expected to exceed the softening temperature of ABS. Hence good bond strength is expected at higher amplitudes first of all. But this LSS seems to be flattened at high amplitudes. One of the possible reasons of this smoothness is development of nearly same temperature at weld interface by using high amplitudes. This temperature results in similar flow of molten $A B S$ and similar resisting area. Same shear force is also required to break bond area thereby resulting in almost similar LSS. Hence this hypothesis is again somehow approved after being partially sanctioned by DSC and MFI results.

\subsection{2. $P P$}

\section{Hypothesis A}

Hypothesis A puts forward that "ABS is an amorphous thermoplastic with higher tensile strength than that of PP"

From FEA results, it is easily observed that temperature is lower for PP than that for ABS by using both TRI and SEMI ED with high levels of weld time and static forces. For instance, with SEMI ED and high levels of weld factors (2000N and $1.5 \mathrm{sec}$ ), the possibility of acquiring the highest temperature is 200 degree C. But this is not true for lower levels of weld factors e.g. $1500 \mathrm{~N}$ and $1 \mathrm{sec}$ etc. Hence resulting temperature almost struggles to reach the sharp 
melting point of PP i.e. 165 degree $C$ which results in an overall lower LSS by using SEMI ED. This difficulty in reaching the melting point of PP suggests setting different weld factors for this semicrystalline material. In this work, SEMI ED was found to contribute its utmost empirically to obtain higher LSS for PP. But an overall lower LSS is also expected for lower level of weld factors from simulation's results which again proves the hypothesis $A$ to some extent after its partial approval from DSC and MFI results.

\section{Hypothesis C}

Hypothesis C declares that "Welding temperature is more conveniently attainable for ABS owing to its higher loss modulus as compared to PP"

In this research, an overall difficulty in welding PP was observed with low levels of weld factors. Low levels of weld factors for PP always struggle to reach the melting point of PP leading to no or little melting of PP. Even at high levels of weld factors, most of the ultrasonic energy is expected to return back owing to higher storage modulus of PP resulting in acquiring low bonding temperature. Hence this again proves somewhat the hypothesis $C$ from FEA results.

\section{Hypothesis D}

Hypothesis D states that "EDs made of ABS have higher heat distortion temperature (HDT) \& higher loss modulus than those for PP"

It is found that numerical/predicted temperatures are hardly touching the melting point of PP. When reaching near the melting temperature, numerical temperature contributes in sudden softening of material further flattening the ED without actually melting PP, therefore, resulting in lower LSS. Heat distortion temperature (HDT) is the distortion rate of a material at a particular temperature. It has already been known that the HDT is lower for PP. So, higher collapse of ED is achieved ultimately with lower LSS. Henceforth hypothesis D is also working well again for FEA results.

\section{Hypothesis G}

Hypothesis G suggests that "Almost same resisting area is expected to be produced at weld interface by using higher amplitudes" 
In this study, high levels of weld factors were necessary to obtain comparatively bigger welded area and higher temperature regions for PP. At higher amplitudes, same shear force for similar resisting area is found after testing bond strength acquiring ultimately the stability of LSS. This is against the finding from literature[189]. So, hypothesis G seems to be disproved here as well after having disproved from DSC and MFI results.

\subsection{Summary}

Thermal simulations were run in this chapter for registering temperatures at weld zone. These simulated temperatures were finally validated by utilizing the ultrasonic welding rig. Current FEA approach has a great potential to be used in future for ultrasonic welding of thermoplastics. FEA results were also utilized to test a set of hypotheses for both ABS and PP. Most of the hypotheses for both materials were partly proved with the aid of simulated results hereby. 


\section{CHAPTER 9 HIGH SPEED VIDEO CAMERA (HSVC)}

\subsection{AIM}

This chapter presents an application of high speed video camera for measuring collapse of energy director (ED). HSVC was also utilized to test few hypotheses relevant to the collapses of EDs from both ABS and PP. Hypothesis D for both ABS and PP was tested hereby stating that "EDs made of ABS have higher heat distortion temperature (HDT) \& higher loss modulus than those for PP".

\subsection{Methodology}

High speed video camera is extensively used to record fast/high-speed phenomena in order to analyse those events that occur too fast. A series of images are recorded at high frame rate and played back in slow motion to permit the researcher to find interesting results. When the images are played back in slow motion or stopped for investigation, amazing details can be seen that remain unnoticed at ordinary speed. In fact, high speed video cameras provide the researcher with motion analysis for various applications e.g. research facilities, automotive industry, machine tools, etc. Likewise, ultrasonic welding is also a fast welding process which takes less than a second to weld thermoplastic. In this work, HSVC was used to record ultrasonic welding of both ABS \& PP for different weld factors leading to the measurement of ED collapse. Accessories of HSVC are shown in Figure 9.1.

In this work, it was expected that weld strength could be largely influenced by collapse of ED. Various weld factors were employed in order to investigate the way ED collapses for both $\mathrm{ABS}$ and PP during UWP, as shown in Table $9.3^{2}$. Collapse of different EDs was measured with the help of Richmas software. Finally the relationship between LSS and ED collapse was observed for both materials.

\subsection{Variables used}

Quality of recorded images can be affected by various factors. These factors involve setting zoom, exposure time, depth of field, aperture, focus, window size and frame rate properly. Size of objects is maximized to increase accuracy by using zoom. Moreover, the amount of

\footnotetext{
${ }^{2}$ Table 9.3 is shown at page 223.
} 
light entering the camera is reduced by using zoom. Higher exposure time increases the brightness of images and produces better contrast of images. The amount of light entering the camera is controlled by the aperture. Depth of field is the distance at which objects do not lose focus. It is frequently convenient to zoom in an area and focus. Aperture setting is usually a compromise between depth of field and exposure time. Window size limits the number of images that may be stored. It is usually better to keep the window size as large as possible. Frame rate is the number of frames that is recorded per second. A proper frame rate strongly depends on the purpose of recording events. In this research, frame rate was set equal to 1000 frames per second (fps) with window size equal to $512 * 256$, as shown in Table 9.1. Finally, the ED collapse is the dependent variable defined as distance between specimens to be welded before and after welding. ED collapse will thoroughly be discussed in current chapter.

Table 9.1 Variables Setting

\begin{tabular}{|c|c|}
\hline Variable & $\begin{array}{c}\text { Numerical } \\
\text { Description }\end{array}$ \\
\hline \hline Frame Rate (fps) & 1000 \\
\hline Window Size & $512 * 256$ \\
\hline $\begin{array}{c}\text { Zoom, Focus, Depth of Field, Aperture, and Exposure Time } \\
\text { Settings }\end{array}$ & As appropriate \\
\hline
\end{tabular}

9.4. Description of procedure

In this research, Phantom $1 \& 2$ high speed cameras were employed to observe ultrasonic welding for thermoplastics and to measure ED collapse. Photographic images could be stored and played back for current analysis. It started working by fixing it on tripod and placing floodlights to get fine details of images. Equipment and accessories required for recording events are shown in Figure 9.1. An interface between physical camera and especially designed laptop was made via Ethernet connection to acquire quick interaction of ultrasonic vibrations on samples. Camera was fixed with lenses requiring aperture, zoom and focus setting. After having set the lens, interface software, between physical camera and laptop, was opened and rest of control was feasible through laptop. Window size was kept large to get proper frame rate and good storage of recorded frames. Exposure time was set to get considerable brightness of images and good contrast ultimately. Continuous 
recording was accomplished by triggering camera after the event happened. After capturing event, cine files were converted into AVI files and finally into MPEG files.

To measure ED collapse, ultrasonic welding factors were also chosen for both ABS and PP after setting variables for HSVC. For ABS, high level of weld factors was selected which resulted in higher LSS and higher collapse. With PP, both high and low level of weld factors were selected because of expecting higher collapse on utilizing them, as can be seen in Table 9.3.

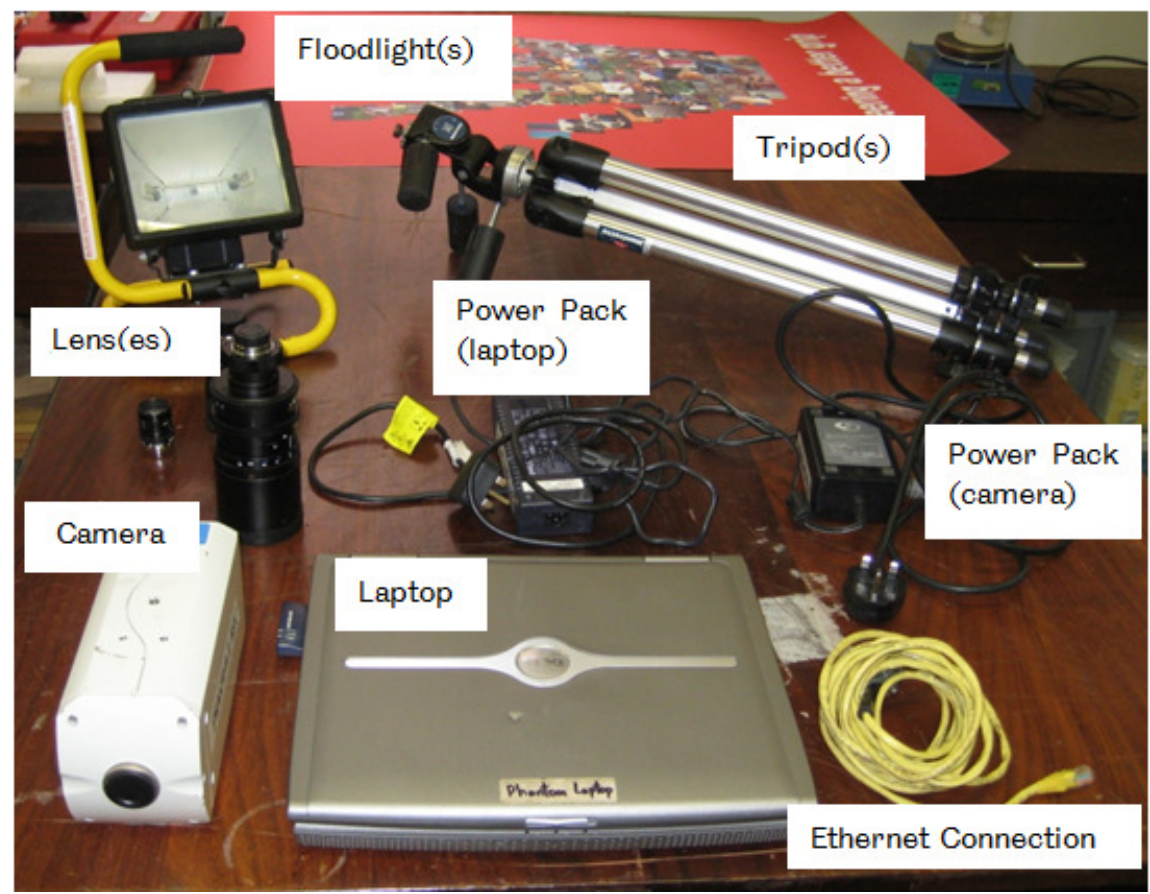

Figure 9.1 Equipment and accessories required for filming

The way of measuring ED collapse, as shown in Figure 9.2, requires a reference on any frame to convert pixels into millimetres $(\mathrm{mm})$ by using MPEG analyser i.e. Richmas software. 


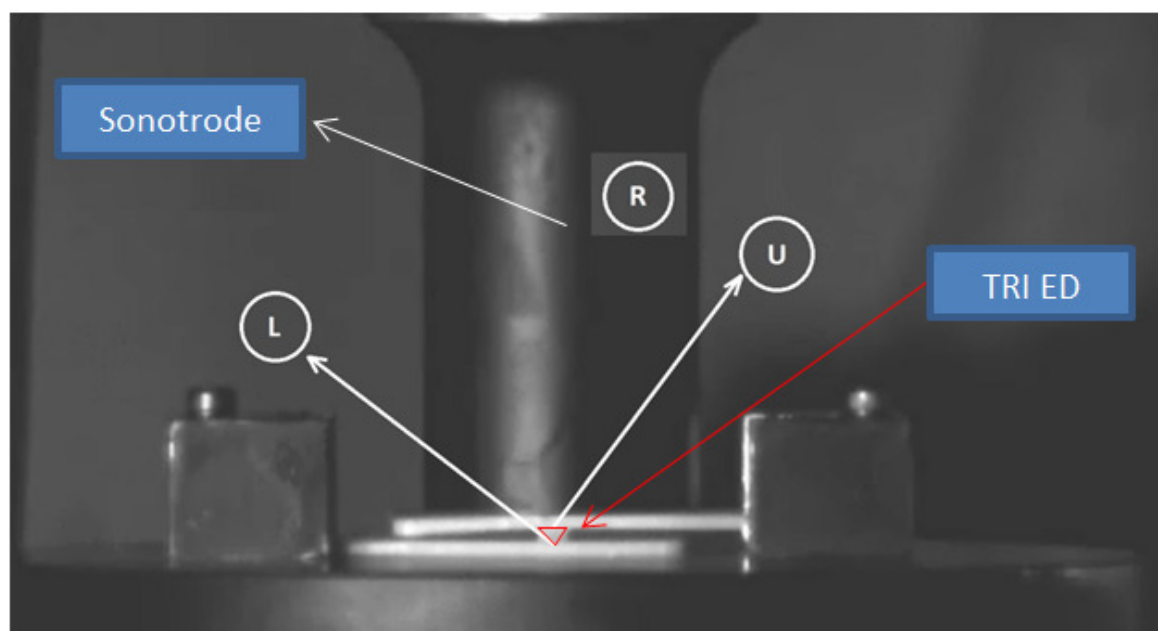

Figure 9.2 Method of calculating ED Collapse

The point $\mathrm{R}$ represents any random reference e.g. sonotrode diameter, on a particular frame. This gives the pixels' information in $X$ or $Y$ direction. For instance, it is known that the base of TRI or SEMI ED is equal to $6 \mathrm{~mm}$, as exhibited earlier in Figure 4.3. To convert pixels into $\mathrm{mm}$, it is needed to measure the pixels for two ends of base line of TRI ED in X axis. An energy director collapsed is separately shown in Figure 9.3. Suppose, the reference R is represented by a base line of TRI ED with vertices $X 1$ and $X 2$, as shown in Figure 9.3. From Table 9.2, it is found that length of base line in pixels is 7.14. Since the actual distance between $\mathrm{X} 1$ and $\mathrm{X} 2$ is $6 \mathrm{~mm}$, it can easily be evaluated that what value of pixel is equal to 1 $\mathrm{mm}$. Hence ED Collapse in $\mathrm{mm}$ is then calculated by evaluating the difference between upper (U) and lower (L) points of ED. From Figure 9.2 and Figure 9.3, $U$ is the half of the base line of TRI ED, whereas $L$ is in line with the apex of TRI ED. As far as $U$ and $L$ are concerned, measurements of ED collapse will now be in $Y$ axis.

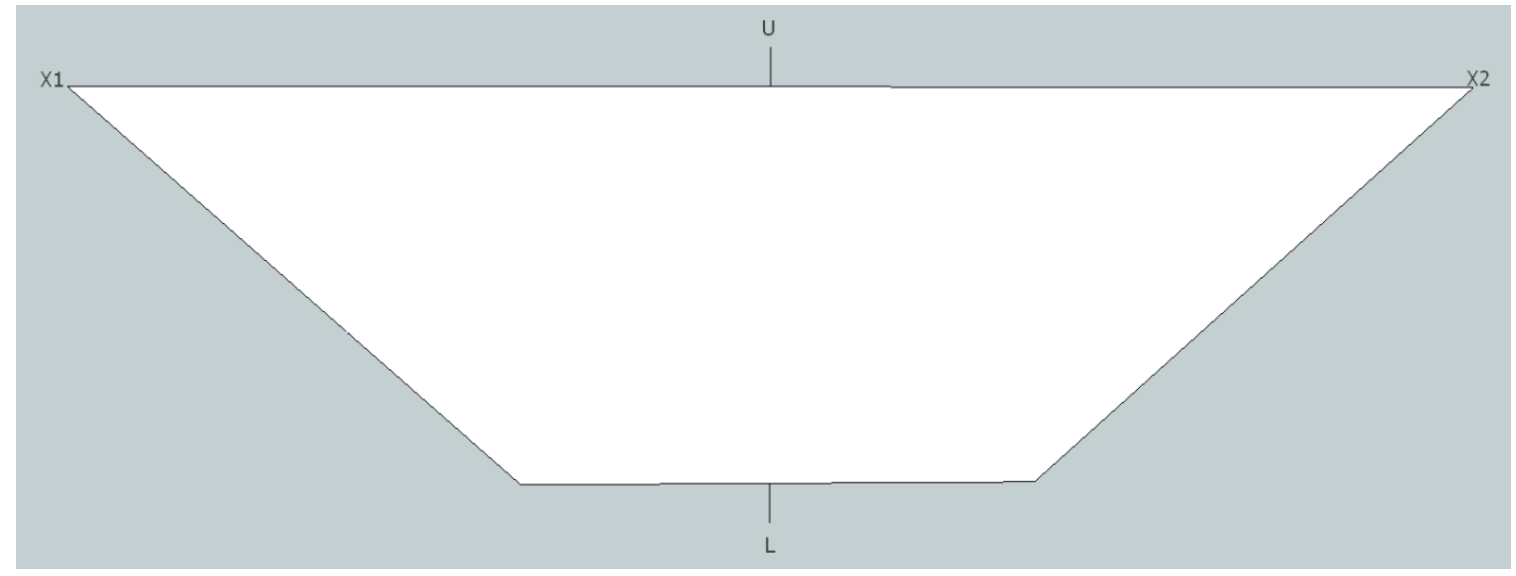

Figure 9.3 Base line of TRI ED with vertices $X 1$ and $X 2$ 
Table 9.2 Pixels to $\mathrm{mm}$ conversion

\begin{tabular}{||c||c||c||}
\hline Vertices & Pixels & $\begin{array}{c}\text { Length of base } \\
\text { line in Pixels }\end{array}$ \\
\hline $\mathrm{X} 1$ & 263.81 & \multirow{2}{*}{7.14} \\
\hline $\mathrm{X} 2$ & 270.95 & \\
\hline
\end{tabular}

Hence collapse is generally the arithmetic difference between $U$ and $L$ in $Y$ axis after welding. Therefore, a state of full collapse is expected to occur if difference between points $\mathrm{U}$ and $\mathrm{L}$ is nearly zero. A flow chart showing the steps for measuring ED collapse is shown in Figure 9.4.

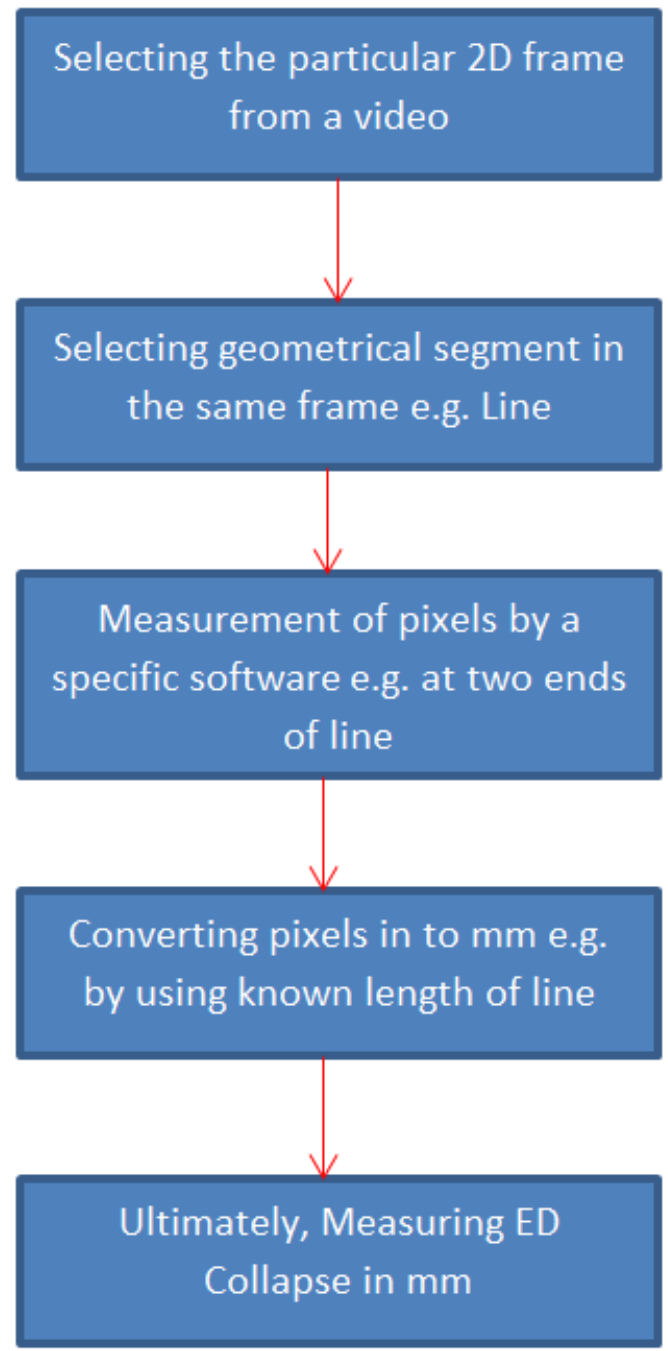

Figure 9.4 Flow chart for measuring ED Collapse 


\subsection{Results}

In this HSVC analysis, effect of amplitude of ultrasonic vibrations on ED collapse was studied due to its uniform impact on LSS for both ABS and PP, as discussed in sections 6.3.1 and 6.3.2. The relationship between amplitude and ED collapse is shown in Figure 9.5. The highest collapse is not consistent with the high level of amplitude for SEMI ED, as illustrated in Figure 9.5. From the data in Figure 9.5, consistently increasing trend of ED collapse is found for TRI ED on increasing the amplitude. Moreover, welding energy associated with each set of weld factors is also exhibited in Table 9.3. Relationship for calculating weld energy has been shown in Table 3.1 under section 3.11.

Table 9.3 Weld factors which were used for HSVC analysis

\begin{tabular}{|c|c|c|c|c|c|c|c|}
\hline Sr.No. & $\begin{array}{l}\text { Static } \\
\text { Force } \\
\text { (N) }\end{array}$ & $\begin{array}{l}\text { Weld } \\
\text { Time } \\
\text { (sec) }\end{array}$ & $\begin{array}{l}\text { Hold } \\
\text { Time } \\
\text { (sec) }\end{array}$ & $\begin{array}{c}\text { Material } \\
\text { Type }\end{array}$ & $\begin{array}{l}\text { ED } \\
\text { Type }\end{array}$ & $\begin{array}{c}\text { Amplitude } \\
(\mu \mathrm{m})\end{array}$ & $\begin{array}{l}\text { *Weld } \\
\text { Energy } \\
\text { (J) }\end{array}$ \\
\hline 1_a & 750 & 0.9 & 0.2 & ABS & SEMI & 21 & $\mathrm{a} a(283.5)$ \\
\hline 1_b & 750 & 0.9 & 0.2 & ABS & SEMI & 18.9 & $\mathrm{~b}(255.2)$ \\
\hline 1_c & 750 & 0.9 & 0.2 & ABS & SEMI & 14.7 & $c(198.5)$ \\
\hline 2_a & 1500 & 0.9 & 0.2 & ABS & TRI & 21 & $a(567)$ \\
\hline 2_b & 1500 & 0.9 & 0.2 & ABS & TRI & 18.9 & $b(510.3)$ \\
\hline 2_c & 1500 & 0.9 & 0.2 & ABS & TRI & 14.7 & $c(397)$ \\
\hline 3_a & 1250 & 1.4 & 1 & PP & TRI & 21 & $a(735)$, \\
\hline 3_b & 1250 & 1.4 & 1 & PP & TRI & 18.9 & $\mathrm{~b}(662)$ \\
\hline 3_c & 1250 & 1.4 & 1 & PP & TRI & 14.7 & $c(515)$ \\
\hline 4_a & 1500 & 1 & 1 & $\mathrm{PP}$ & SEMI & 21 & $a(630)$ \\
\hline 4_b & 1500 & 1 & 1 & PP & SEMI & 18.9 & $b(567)$ \\
\hline 4_c & 1500 & 1 & 1 & PP & SEMI & 14.7 & $c(441)$ \\
\hline
\end{tabular}

*weld energy was calculated for each of three amplitudes i.e. $a=21 \mu \mathrm{m}, b=18.9 \mu \mathrm{m}, \mathrm{c}=14.7 \mu \mathrm{m}$

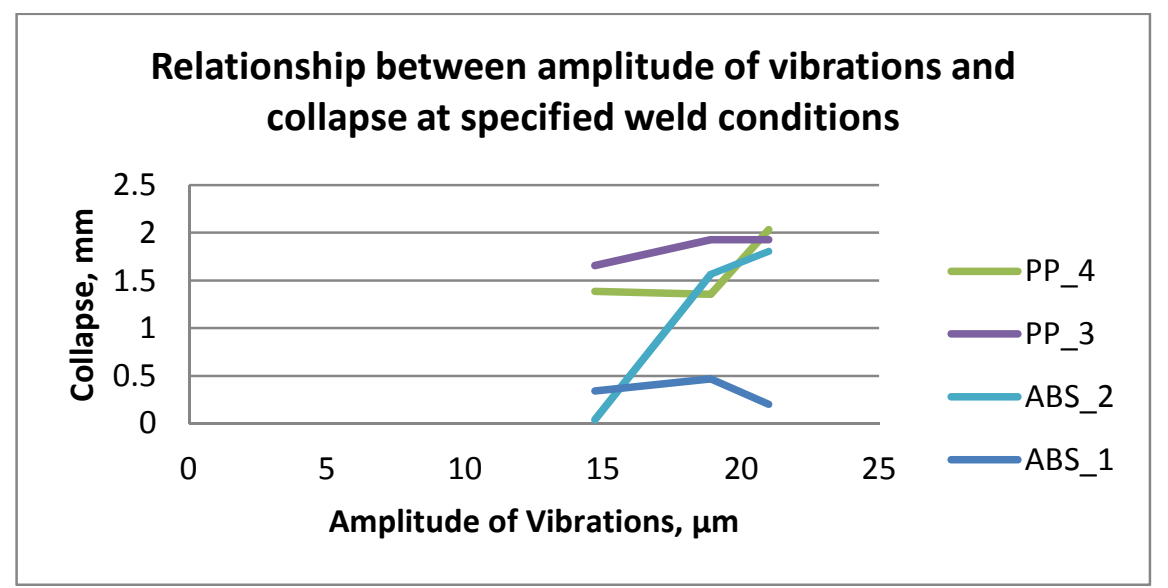

Figure 9.5 Relationship between amplitude and ED Collapse 


\subsection{Effect of ED collapse on LSS}

\subsection{1. $\underline{A B S}$}

\section{Hypothesis D}

Hypothesis D proposes that "EDs made of ABS have higher heat distortion temperature (HDT) \& higher loss modulus than those for PP"

From Figure 9.6, the relationship between ED collapse and LSS suggests that higher the collapse, the greater will be the LSS for both types of EDs. The possible reasons for achieving higher ED collapse are utilization of high level of weld factors and higher loss modulus for ABS. Hence hypothesis $D$ seems to be proved somehow here after having approved it by FEA results.

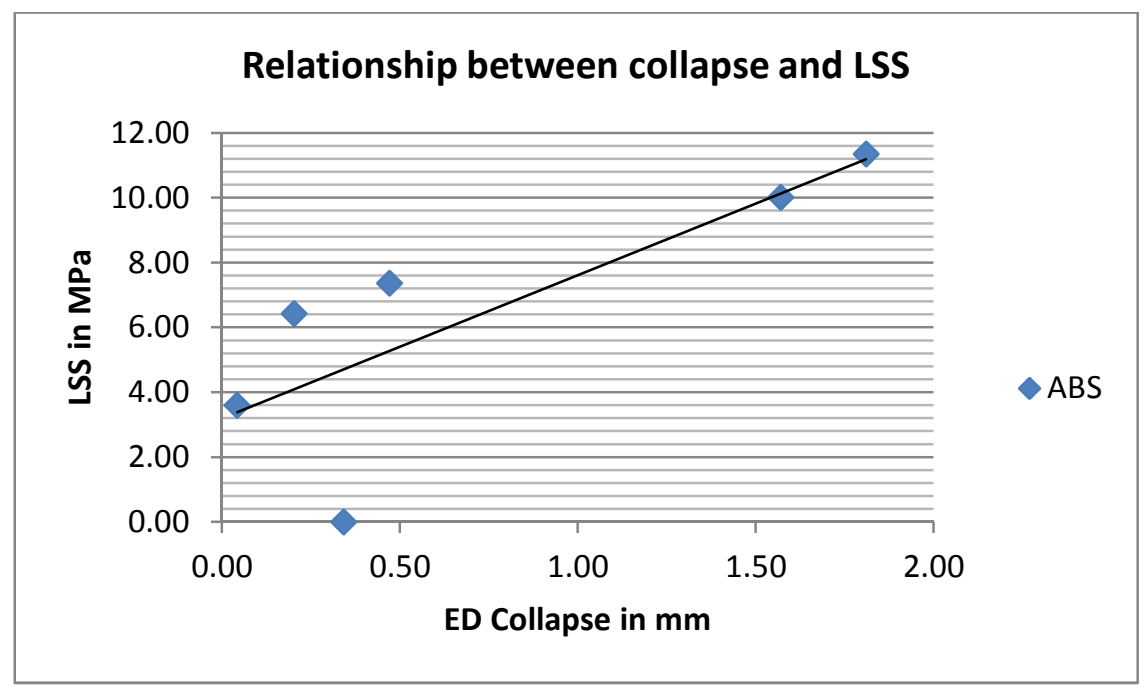

Figure 9.6 Relationship between ED Collapse and LSS for ABS

\subsection{2. $\mathrm{PP}$}

\section{Hypothesis D}

Hypothesis D specifies that "EDs made of ABS have higher heat distortion temperature (HDT) \& higher loss modulus than those for PP"

In this work, full collapse was frequently observed for PP by employing even low levels of weld factors. On increasing amplitude from low to high level, a uniformly increasing trend of collapse was obtained for both types of EDs. By plotting the relationship between LSS and 
ED collapse, it was again found rising consistently on increasing the collapse which was further a function of amplitude, as shown in Figure 9.7.

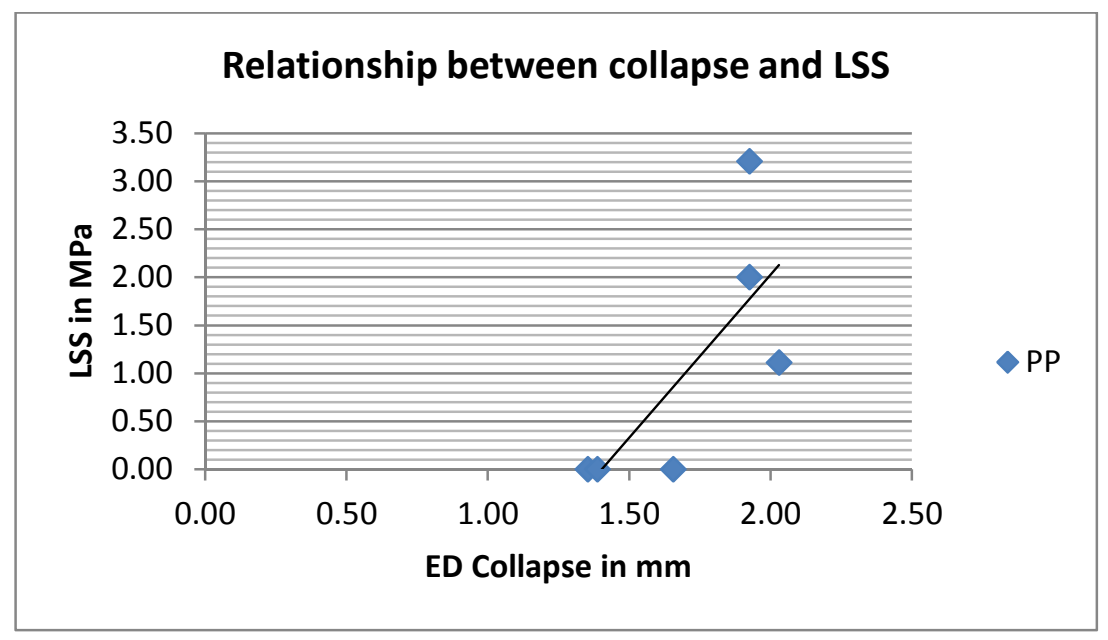

Figure 9.7 Relationship between ED Collapse and LSS for PP

The overall LSS was found to be lower because of setting low levels of weld factors. Various reasons needed to mention here for lower LSS e.g. lower heat distortion temperature for PP, lower loss modulus, orderly molecular structure of PP and under melt situation at weld interface by using low levels of weld factors. These findings do not only prove partially our hypothesis D for PP but also indirectly insist us to set high levels of factors in order to get higher LSS with higher collapse of ED. Hence hypothesis D is moderately validated by HSVC.

\subsection{Summary}

ED collapse was calculated by HSVC. Hypothesis D for ABS and PP was tested with the help of HSVC. This hypothesis was proved somehow for both ABS and PP. 


\section{CHAPTER 10 MICROSCOPY}

\subsection{AIM}

In this chapter, microscopic study of weld zone is presented before and after breaking the ultrasonic bond through tensile testing in shear. Chapter 6 also identified that various hypotheses could be affected by weld morphology and microscopic studies. Current microscopic study was performed to test those hypotheses i.e. D \& E for both ABS and PP. These hypotheses are presented as; (D) EDs made of ABS have higher heat distortion temperature (HDT) \& higher loss modulus than those for PP and (E) Crazing affects bond fracture without influencing fracture mode for ABS and PP.

\subsection{Methodology}

A general account of microscopy can be seen in section 3.10.3. Optical microscopy (OM) entails an object that is illuminated and the scattered light is collected by different lenses to produce an image. Fine details can be revealed in the samples at a range of magnifications $2 \mathrm{X}$ to $2000 \mathrm{X}$. The possibility of resolution is about $0.5 \mu \mathrm{m}$ depending upon the nature of sample, the objective lens and the light wavelength. Information based on size, shape and relative arrangement of visible features is obtained by optical microscope. A high quality digital camera is typically used for recording images connected to a computer system for image processing and analysis. A typical optical microscope is shown in Figure 10.1(B).

Moreover, a scanning probe and a focused electron beam are used by scanning electron microscope (SEM) to form an image of the sample under investigation. If a sample is impinged by an electron beam, the electrons are scattered through the sample within a well-defined area that is called interaction volume. When interaction between electron beam and sample is occurred, secondary electrons (of low energy) are often produced. Development of these secondary electrons is usually used to form a SEM image. SEM can result in higher resolution and much larger depth of field as compared to incident light optical microscope. The specimen chamber is larger in SEM and larger samples can be used. Conductive coating is required by non-conductive materials during sample preparation. SEM images are interpreted conveniently and qualitatively. A simple diagram of SEM is illustrated in Figure 10.1(A). 
Figure 10.1(A) Simple diagram of SEM[197]

In this research, the study of heat affected zone (HAZ) was done by optical microscope before getting bond fractured. And the microscopic study of fractured surface was accomplished by both optical microscope and SEM after the bond ruptured i.e. fractography.

Moreover, this chapter is divided into two sections i.e. study of HAZ and fractography owing to difference in description of procedure for these two sections.

\subsection{Variables used}

An optical microscope is utilized to study the small samples. Quality of image examined depends upon three main factors which include magnification, resolution and amount of light. Magnification is usually associated with lens making the object under investigation appear bigger. If the magnification is lower, the sample will look smaller and more features of it can easily be viewed. On the other hand, if the magnification is higher, the sample will appear larger and less features of it may be seen. Magnification can be varied by using a particular lens type. Resolution is a variable on which fine details of a sample depends. High magnification does not yield high resolution. Resolution can be improved by fine and coarse adjustments (4-5 in Figure 10.1(B)) that are used to bring the image into focus. Resolution can also be modified by adjusting the contrast which distinguishes the sample from the background. A diaphragm (8) is also employed by optical light microscopes in order to control the light which comes in. 
Figure 10.1(B) Optical Microscope[198]

As far as SEM is concerned, a better SEM image may often be attained by understanding the effect of various variables. Some crucial variables comprise condenser lens current (CLC), stigmatism, working distance and accelerating voltage. In practice, adjustments can be made in order to achieve a balance between these SEM variables. Condenser lens current varies the diameter of electrons beam or spot size which is used to scan the sample. When a CLC is increased, smaller spot size and better resolution are achieved. However, beam current will be reduced by increasing CLC. In other words, lower beam current means that fewer electrons interact with sample thereby resulting in fewer secondary electrons which are used to form an image. Therefore, a correct tradeoff between CLC and beam current is needed to obtain. Distortions in spot roundness are controlled by stigmator knob. In Latin, Stigme implies 'mark' or 'spot'. When electron beam is not round e.g. elliptical, unimportant information may be obtained which is called the astigmatism. Working distance is the distance between final condenser lens and the sample. Effect on spherical aberration and resolution of image is expected by changing the working distance. Spherical aberration is associated with the geometry of electromagnetic lenses. 
When working distance is reduced, effect of spherical aberration will be less and resolution will finally be improved. Accelerating voltage is a higher voltage applied to the filament. When this voltage is applied with a small current, the electrons will be caused to leave the filament. The accelerating voltage often set in SEM varies between 5KV and $20 \mathrm{KV}$. Holistically, if accelerating voltage is increased, the spherical aberration is reduced to give optimal resolution. However, interaction between electron beam and sample may badly be affected by this increase in accelerating voltage. This effect needs to be properly adjusted for samples with low atomic number e.g. biological samples. Hence an appropriate balance among SEM variables is needed to form the best image. Moreover, some of these SEM variables can also be generated on SEM images while SEM has been utilizing. From Figure $10.2,20 \mathrm{~mm}$ is the working distance, $20 \mathrm{KV}$ is the accelerating voltage, bar is $10 \mu \mathrm{m}$ and magnification is $\times 600$.

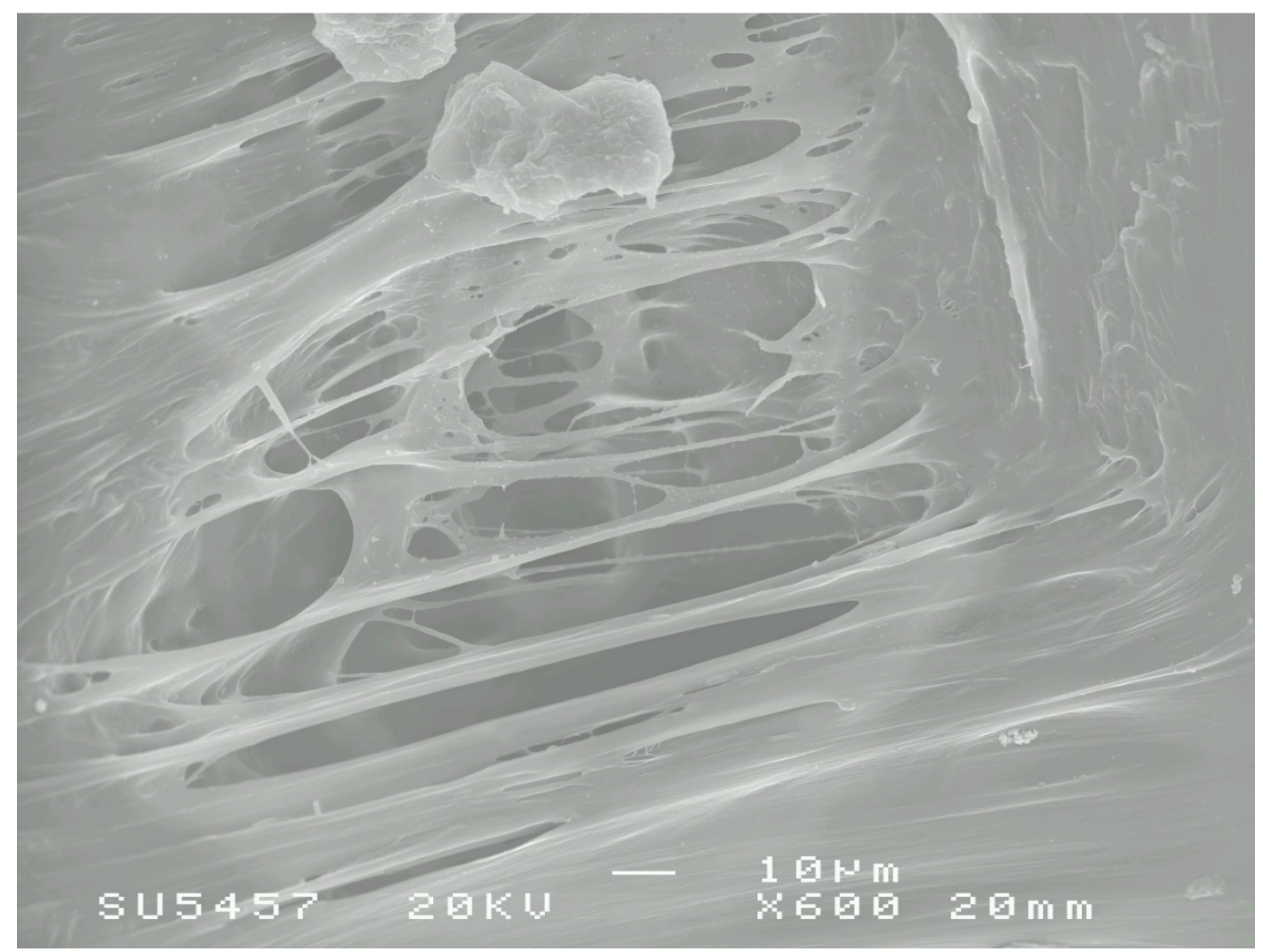

Figure 10.2 Variables used for a particular SEM image

Lap shear strength can be affected by several dependent variables e.g. HAZ thickness, weld fracture features and pit depth. Optical and SEM microscopes can be utilized to find the relationship between weld factors, dependent variables and LSS. Different welding 
conditions produce different properties of bonds having great impact on microstructure of welded joints. Therefore, microscopic study of welded joints is crucial to understand the links between weld factors and weld strength. Dissimilar weld factors generate different sizes of HAZ in thickness direction of specimens that are called the HAZ thicknesses, as shown in Figure 10.4(B). When testing in shear, the welded samples result in fracture surfaces. Crazes are the main source of weld fracture. Microscopic study of fractured joints is called fractography. Pit depth is the penetration of ED into the specimens during welding. These pits can be analysed after testing bond strength of welded specimens.

\subsection{HEAT AFFECTED ZONE (HAZ)}

\subsubsection{Description of procedure for studying $H A Z$}

In this study, bond features were analysed before fracture (i.e. HAZ) resulting from weld factors which produced high LSS. A microscopic investigation of weld zone was planned using extensively POLYVAR MET Reichert-Jung optical microscope, as exhibited in Figure 10.3 .

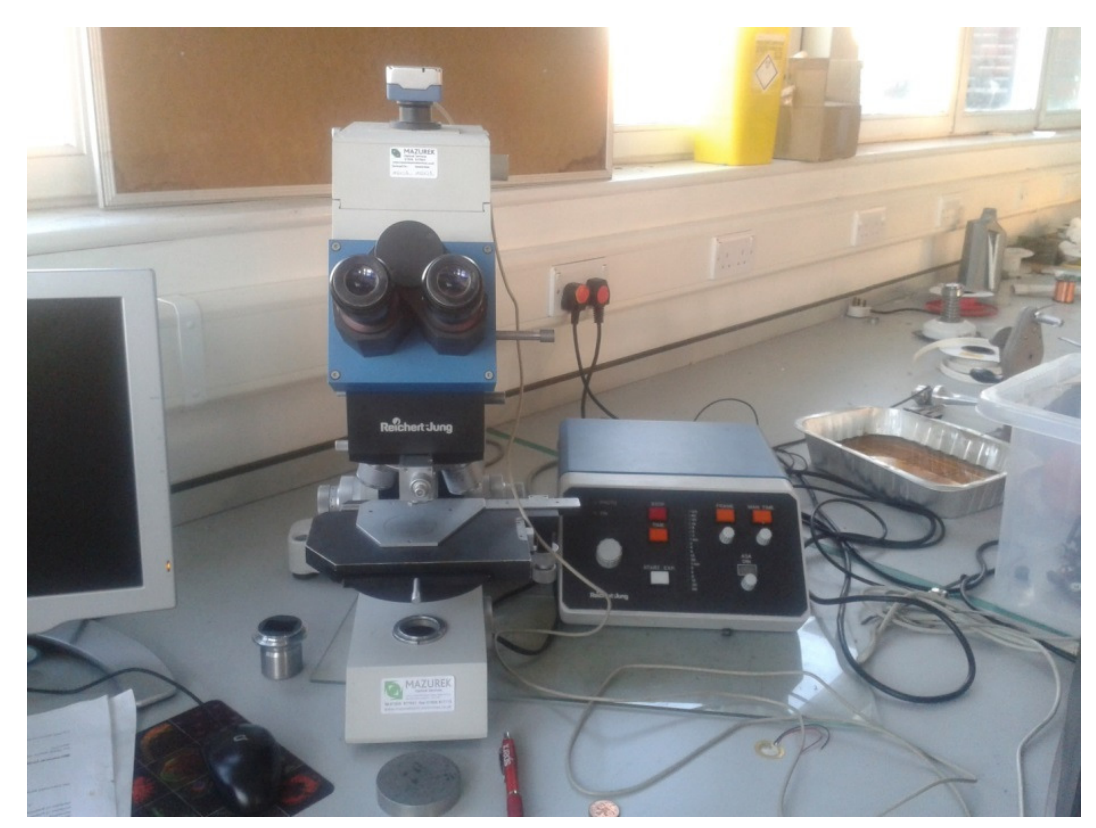

Figure 10.3 POLYVAR MET Reichert-Jung optical microscope

After welding PP and ABS ultrasonically, HAZ was identified either by examining the gap between lower and upper specimen or by finding the molten material which came out of the weld zone. In case of PP, HAZ was really obvious due to its transparent appearance. Welded specimens were cut from four sides via two cutters, as shown in Figure 10.4 and 
Figure 10.4(A). HAZ was clearly exposed after properly cutting the welded samples. These samples prepared were microscopically observed by using optical microscope.

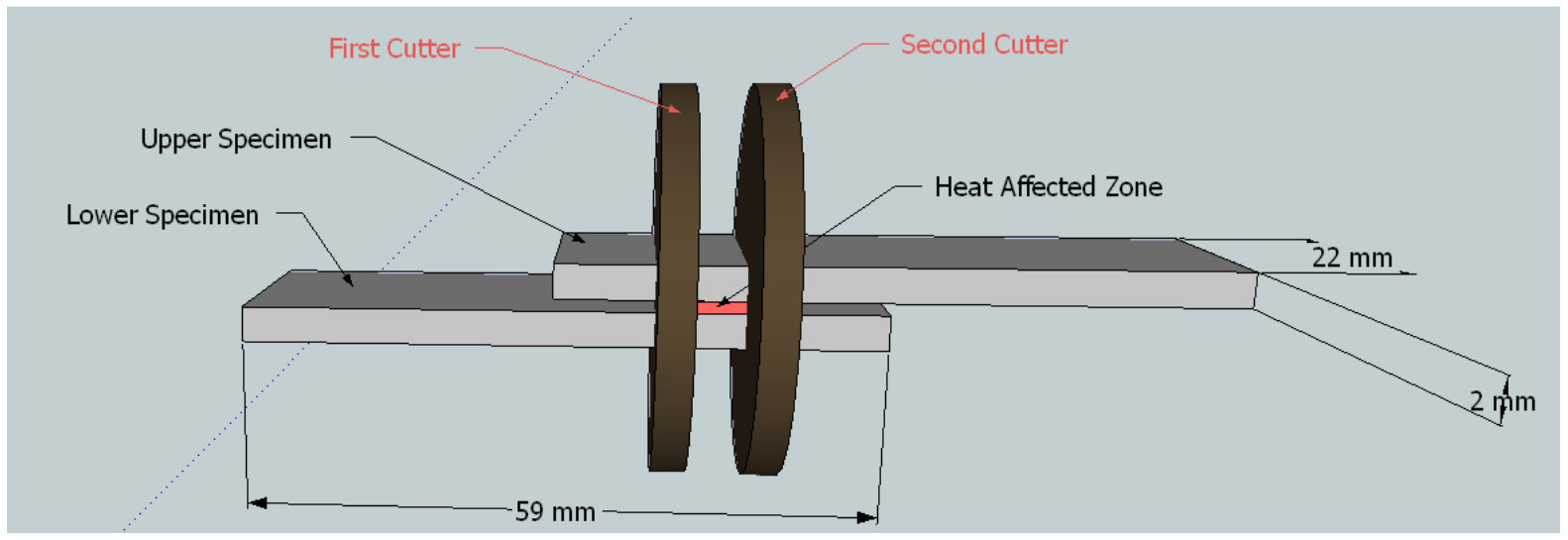

Figure 10.4 Cutting procedure of welded joint for ABS

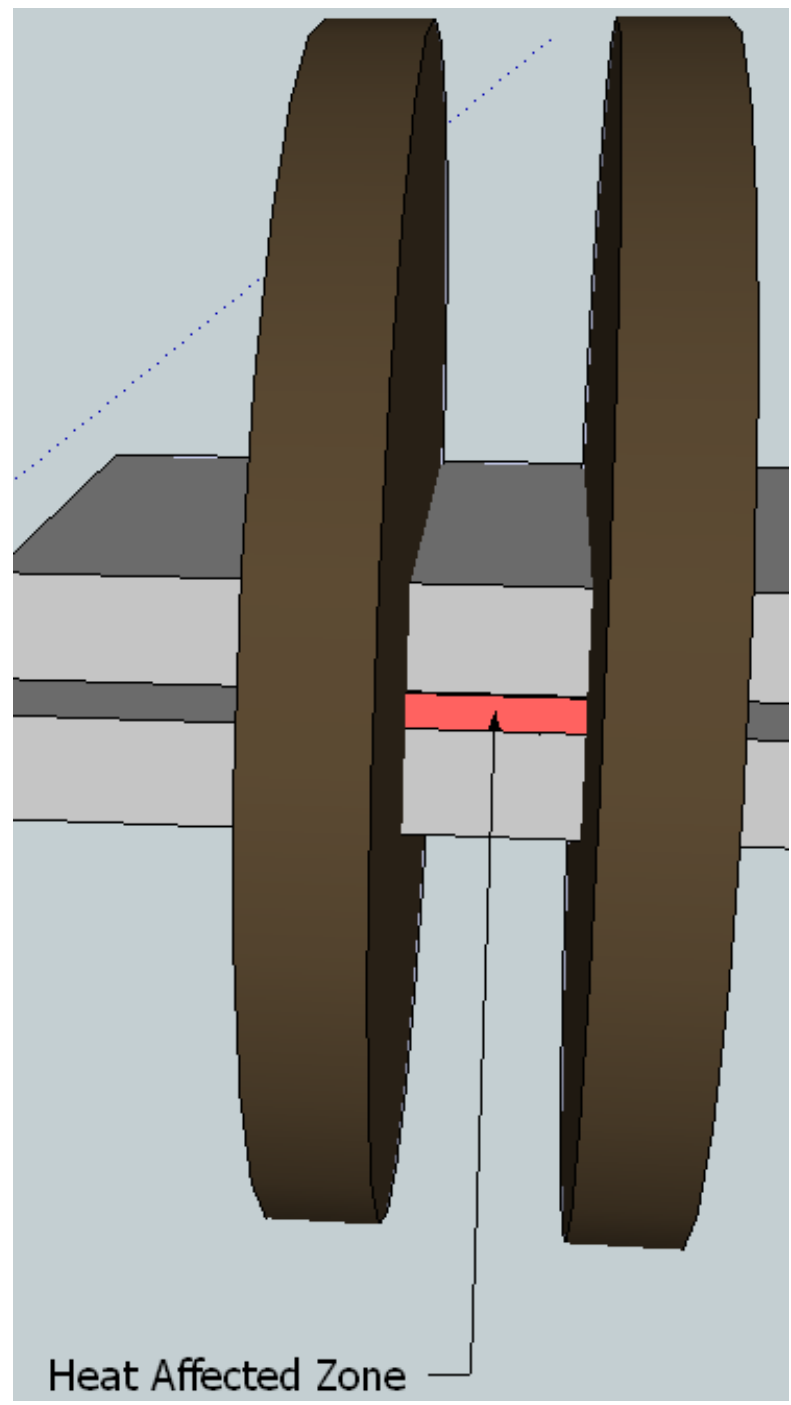

Figure 10.4(A) HAZ 


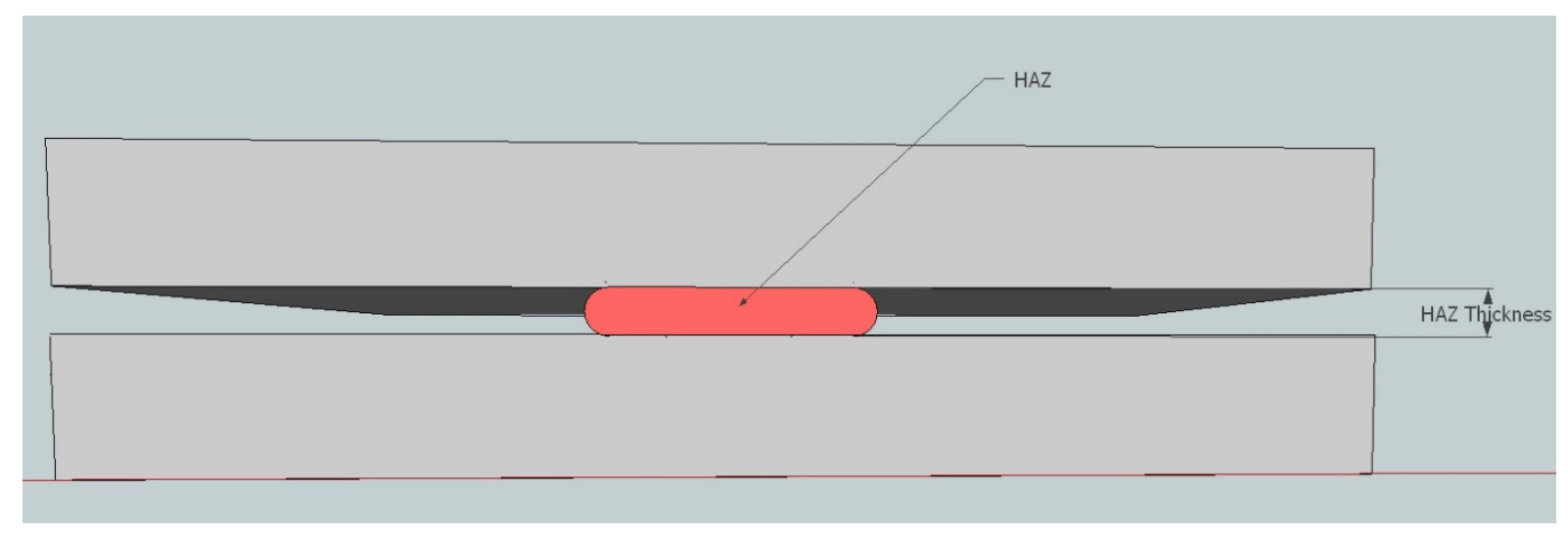

Figure 10.4(B) HAZ Thickness

A specimen is located on a stage (6 in Figure 10.1(B)), as shown in Figure 10.1(B), which often has metal clips to hold the sample in place. Two types of lens are frequently used in microscopes ocular lens (1) (that is looked through) and objective lens (3) that is usually attached with the rotating nose piece (2). In general, two magnifications of lenses were used throughout current optical microscopy i.e. 5 and 10 in an effort to attain better understanding of HAZ, as written in Table 10.1.

Table 10.1 Variables for optical microscope

\begin{tabular}{||c||c||}
\hline Variables & $\begin{array}{c}\text { Numerical } \\
\text { Description }\end{array}$ \\
\hline \hline Objective Lens & 5 \& 10 \\
\hline $\begin{array}{c}\text { Resolution } \\
\text { Setting }\end{array}$ & $\begin{array}{c}\text { As Appropriate in } \\
\text { accordance with } \\
\text { sample dimensions }\end{array}$ \\
\hline $\begin{array}{c}\text { Diaphragm } \\
\text { Setting }\end{array}$ & $\begin{array}{c}\text { As Appropriate in } \\
\text { accordance with } \\
\text { sample dimensions }\end{array}$ \\
\hline
\end{tabular}

Various measurements related to HAZ thickness were obtained by using bar scale on captured images and vernier callipers, as shown in Figure 10.5. Moreover, 5 and 10 magnifications of lenses are graphically presented by $200 \mu \mathrm{m}$ and $100 \mu \mathrm{m}$ bars respectively on each captured image. In this optical microscopy, resolution and diaphragm settings were made in accordance with the sample dimensions to acquire the best and detailed images. 


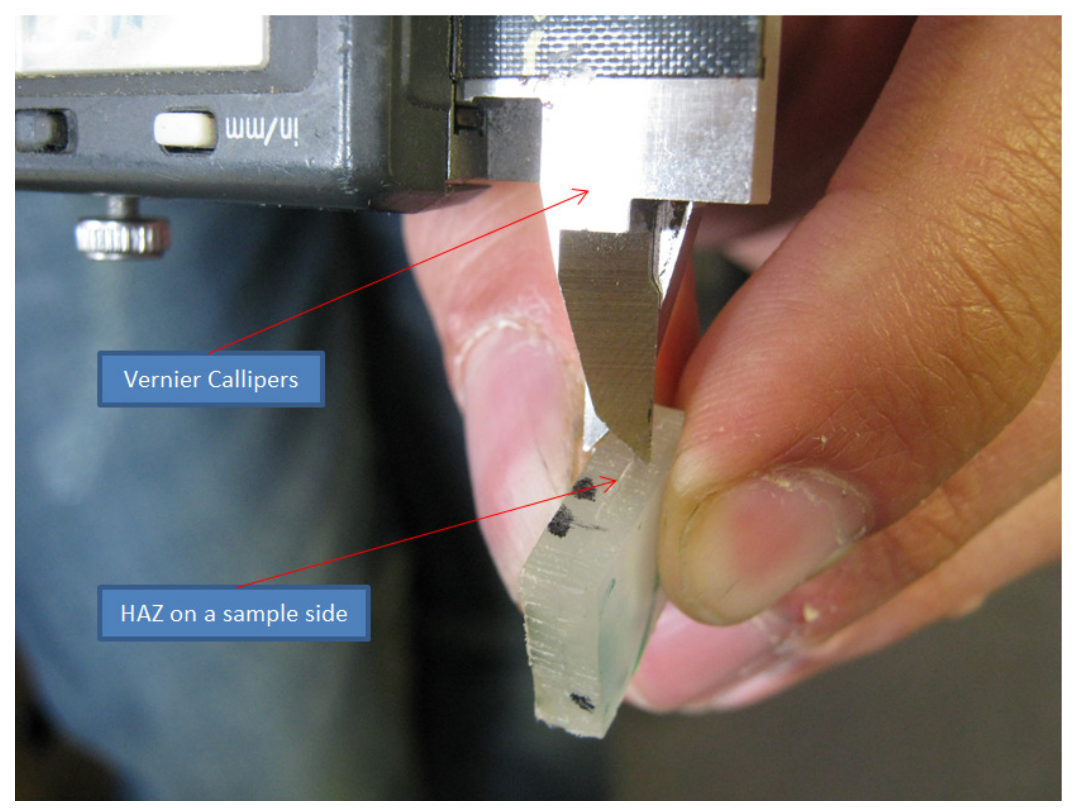

Figure 10.5 Measurement of HAZ thickness with vernier callipers

After being cut, the samples were examined microscopically at four available sides, as illustrated in Figure 10.6. Area of any parallel sides of prepared samples was almost equal whereas the area of any adjacent perpendicular sides was always different.
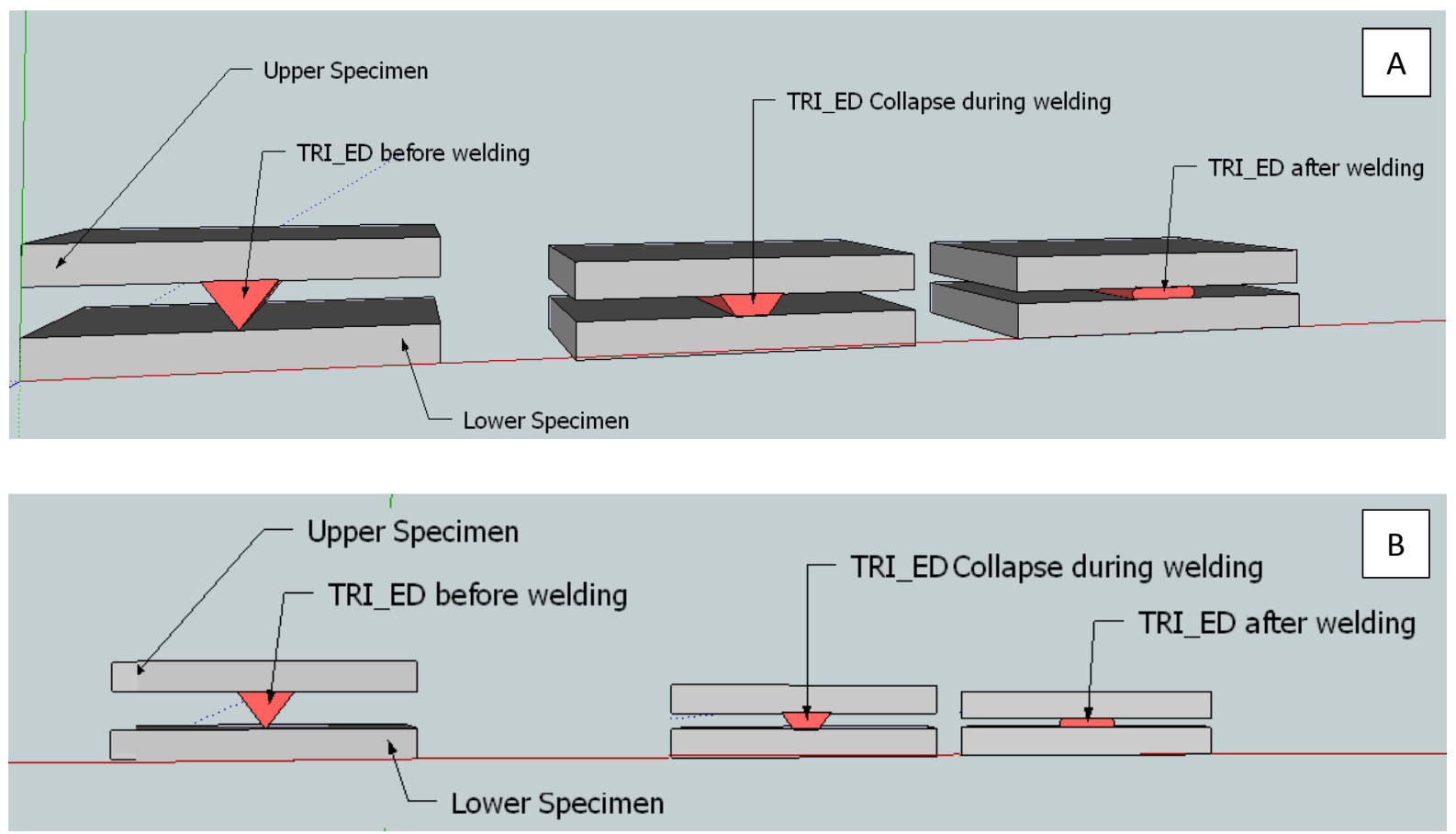


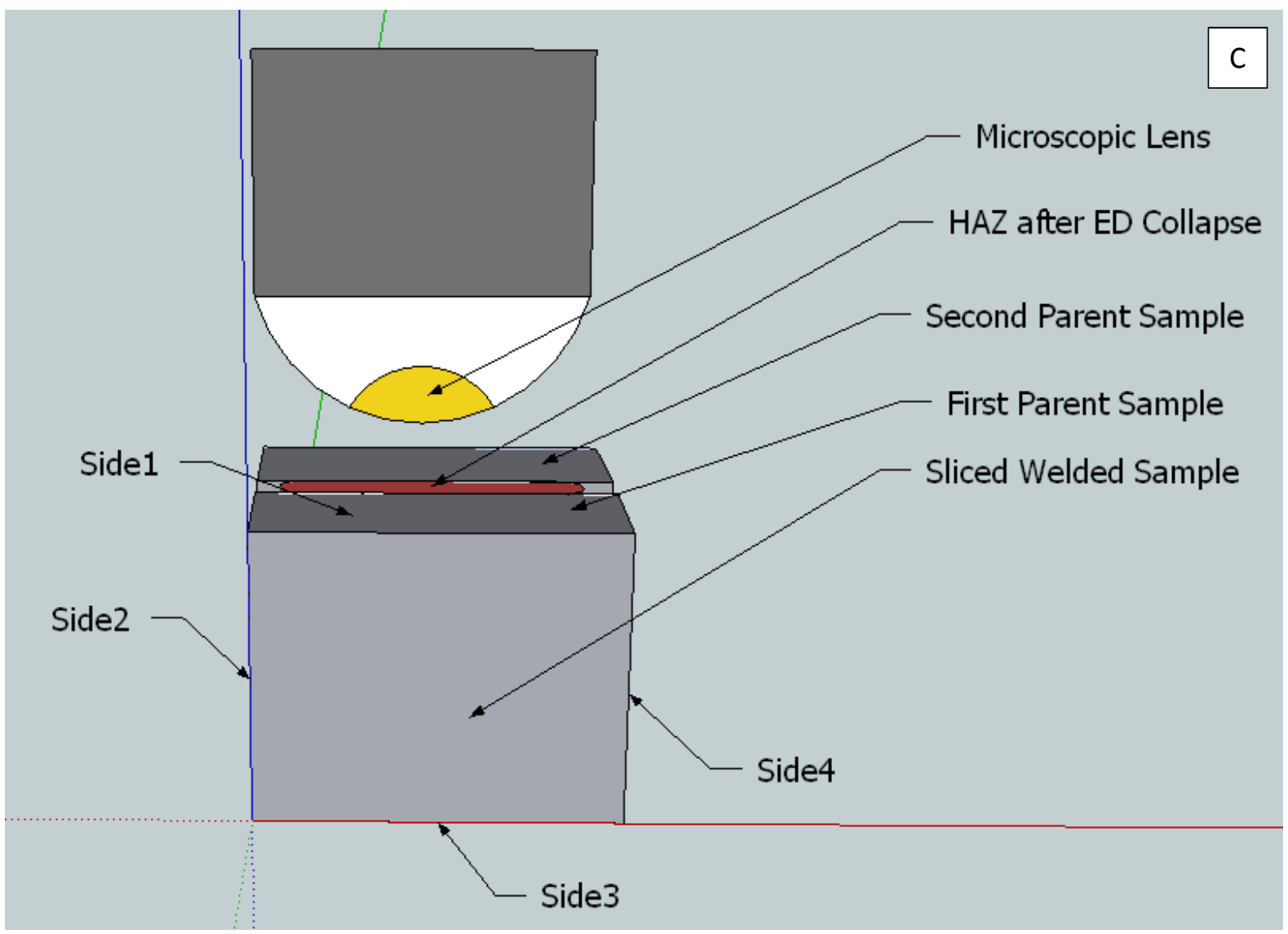

Figure 10.6(A, B\&C) Anatomy of Ultrasonic HAZ

\subsection{2. $\quad \underline{\text { Results }}$}

Table 10.2 shows effect of weld factors on HAZ thickness from four available sides for both thermoplastics.

\section{Effect of HAZ on LSS}

Hypothesis D states that "EDs made of ABS have higher heat distortion temperature (HDT) \& higher loss modulus than those for PP"

From Table 10.2, it is quite clear that the LSS for ABS is much higher than that for PP. Higher ED collapse implies the lower HAZ thickness. In this work, higher HAZ thickness was found for PP instead of ABS because of using low level of weld factors. In fact, it was expected that higher ED collapse would be obtained due to lower HDT and lower loss modulus for PP. Higher LSS was observed for ABS with lower HAZ thickness (i.e. higher ED collapse) but lower LSS was evaluated for PP with higher HAZ thickness (i.e. lower ED collapse) at same 
amplitude and ED, as exhibited in Table 10.2. Hence hypothesis D seems to be approved for $A B S$ and disproved for PP.

Table 10.2 Weld factors and HAZ Thickness

\begin{tabular}{|c|c|c|c|c|c|c|c|}
\hline $\begin{array}{c}\text { Serial } \\
\text { No. }\end{array}$ & $\begin{array}{c}\text { Material } \\
\text { Type }\end{array}$ & $\begin{array}{l}\text { ED } \\
\text { Type }\end{array}$ & $\begin{array}{c}\text { Static } \\
\text { Force } \\
(\mathrm{N})\end{array}$ & $\begin{array}{l}\text { Weld } \\
\text { Time } \\
\text { (Sec) }\end{array}$ & $\begin{array}{c}\text { Amplitude } \\
(\mu \mathrm{m})\end{array}$ & $\begin{array}{c}\text { HAZ } \\
\text { Thickness } \\
\text { (mm) }\end{array}$ & $\begin{array}{c}\text { Lap } \\
\text { Shear } \\
\text { Strength } \\
\text { (MPa) }\end{array}$ \\
\hline 1 & PP & SEMI & 1750 & 1 & 18.9 & 0.9 & 1.14 \\
\hline 2 & PP & SEMI & 1750 & 1 & 21 & 0.7 & 0.99 \\
\hline 3 & $A B S$ & TRI & 500 & 0.9 & 14.7 & 0.4 & 12.3 \\
\hline 4 & $A B S$ & TRI & 500 & 0.9 & 21 & 0.6 & 10.5 \\
\hline 5 & ABS & TRI & 500 & 0.9 & 18.9 & 0.61 & 8.85 \\
\hline 6 & $A B S$ & SEMI & 1500 & 0.8 & 21 & 0.20 & 8.63 \\
\hline 7 & $A B S$ & SEMI & 1500 & 0.8 & 18.9 & 0.25 & 7.97 \\
\hline
\end{tabular}

The relationship between LSS and HAZ thicknesses for both PP and ABS is also plotted, as depicted in Figures 10.7 to 10.9. From Figure 10.7, a direct relationship is found between LSS and HAZ thickness for PP. An inverse relationship is observed between LSS and HAZ thickness finally for ABS, as can be seen from Figure 10.8 and Figure 10.9.

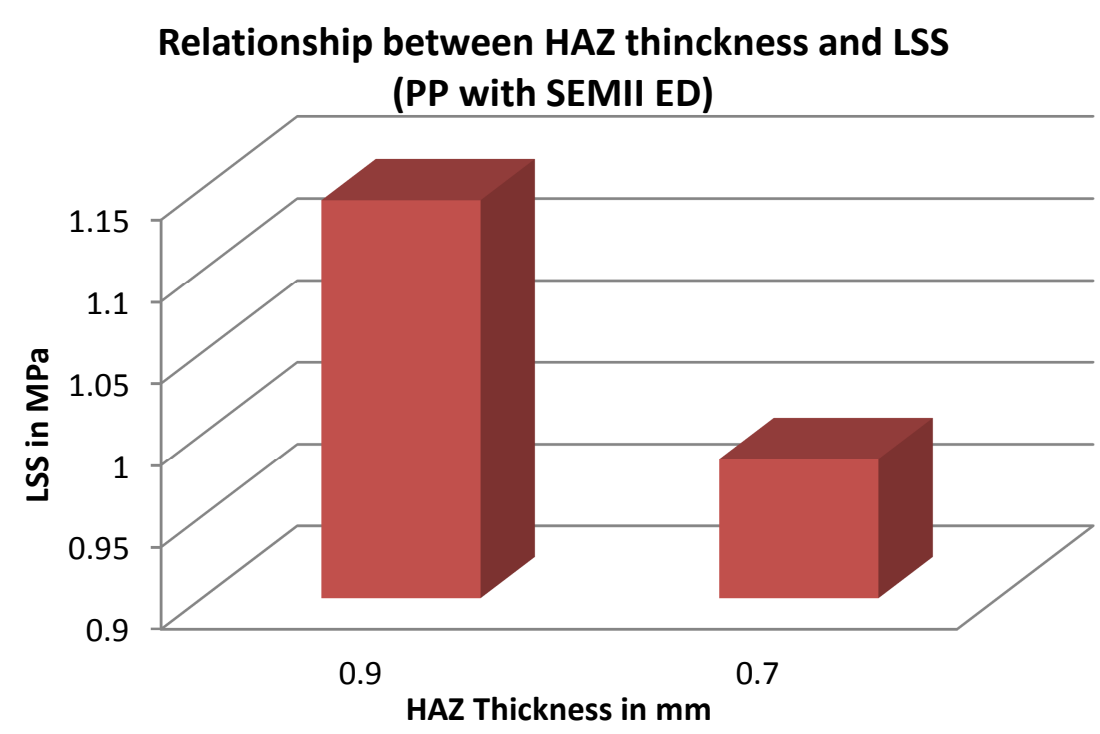

Figure 10.7 Relationship between LSS and HAZ thickness for PP (Sr. No. 1 \& 2 of Table 10.2) 


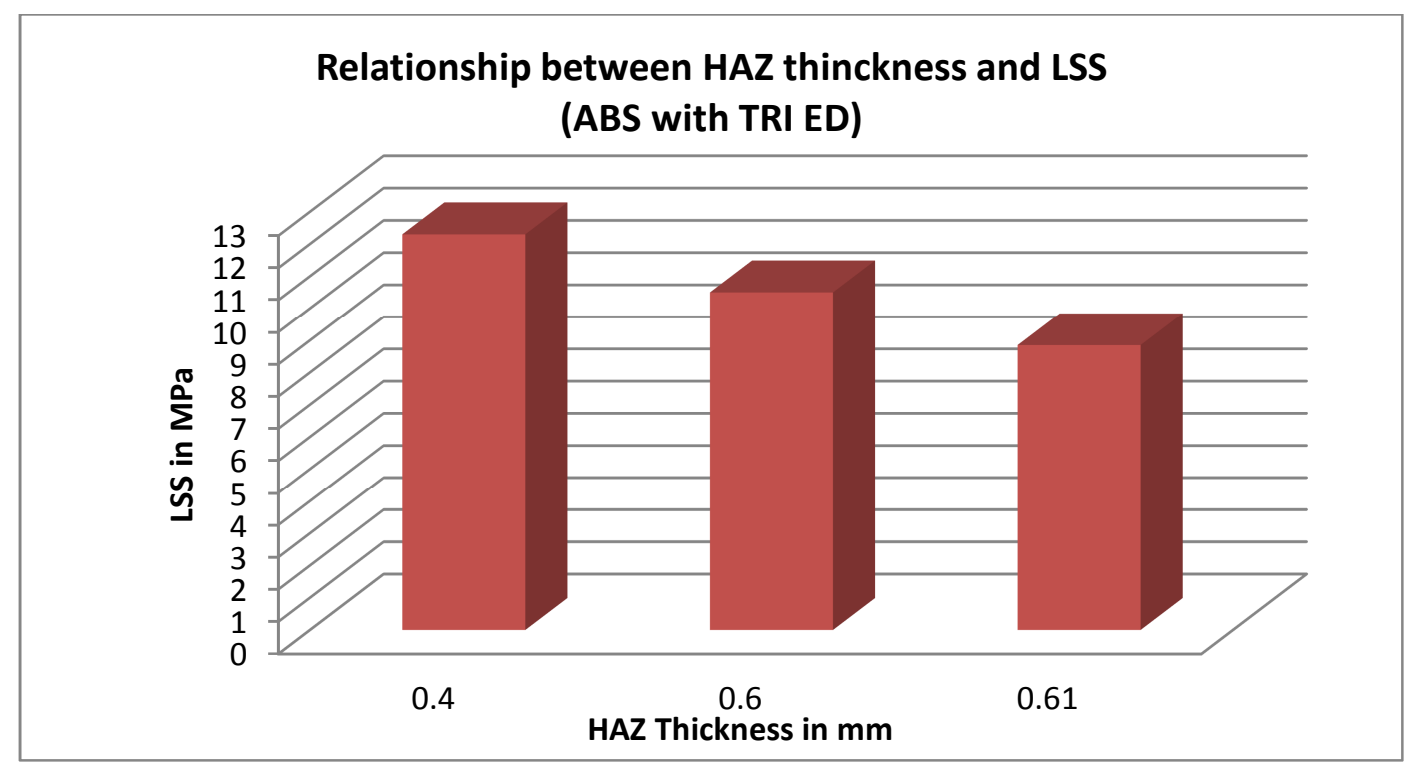

Figure 10.8 Relationship between LSS and HAZ thickness for ABS (Sr. No. 3, 4 \& 5)

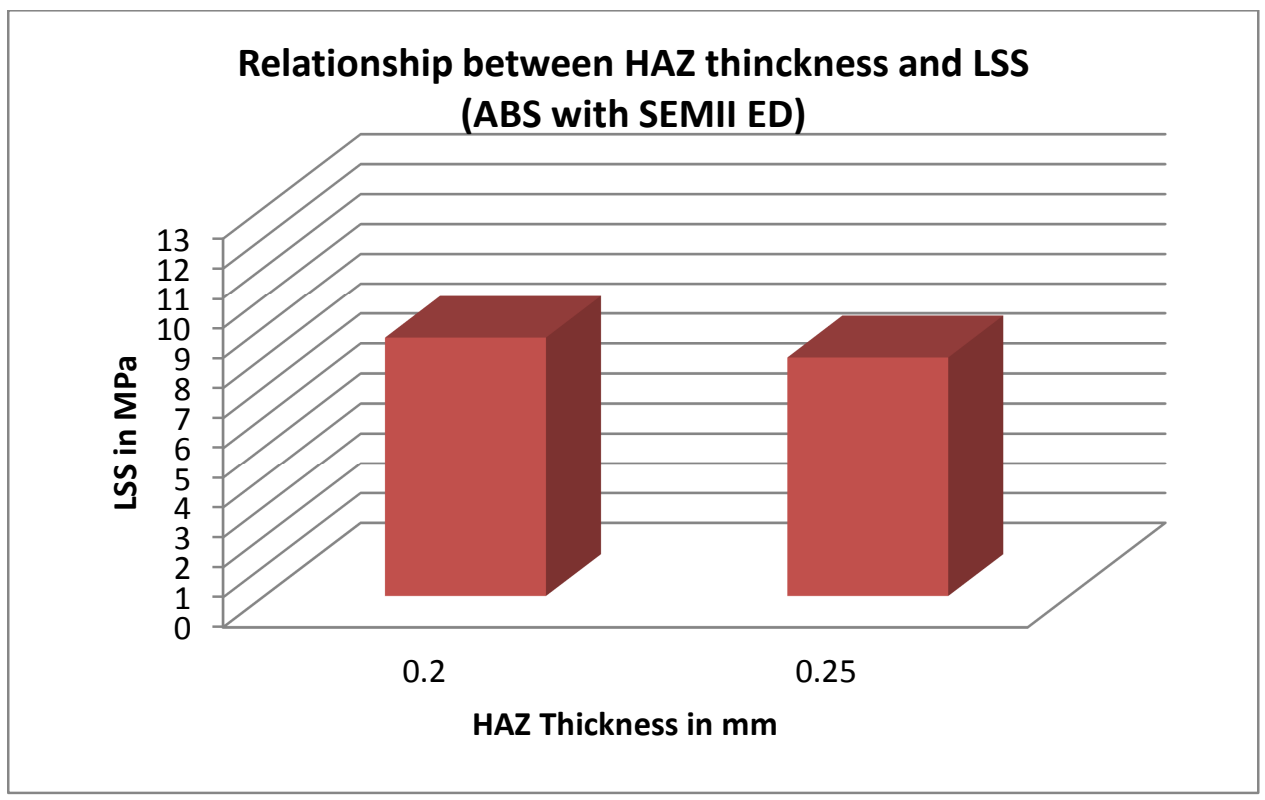

Figure 10.9 Relationship between LSS and HAZ thickness for ABS (Sr. No. 6 \& 7)

Heat affected zones (HAZES) for serials 1, 2, 3, 4, 5, 6 \& 7 respectively of Table 10.2 are shown in Figures 10.10 to 10.16 . 

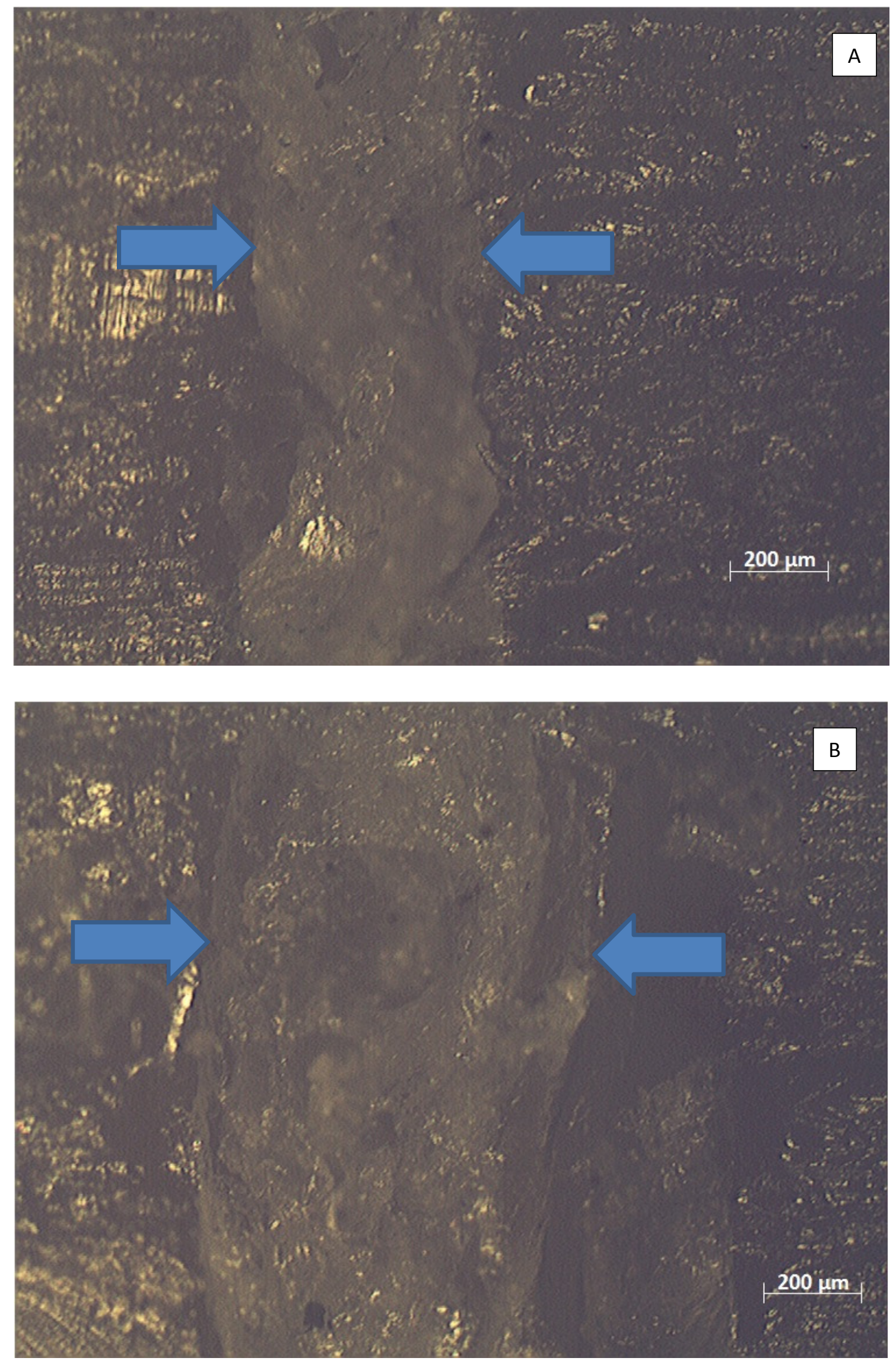

Figure 10.10(A\&B) HAZES viewed from side1 for Serial No.1 


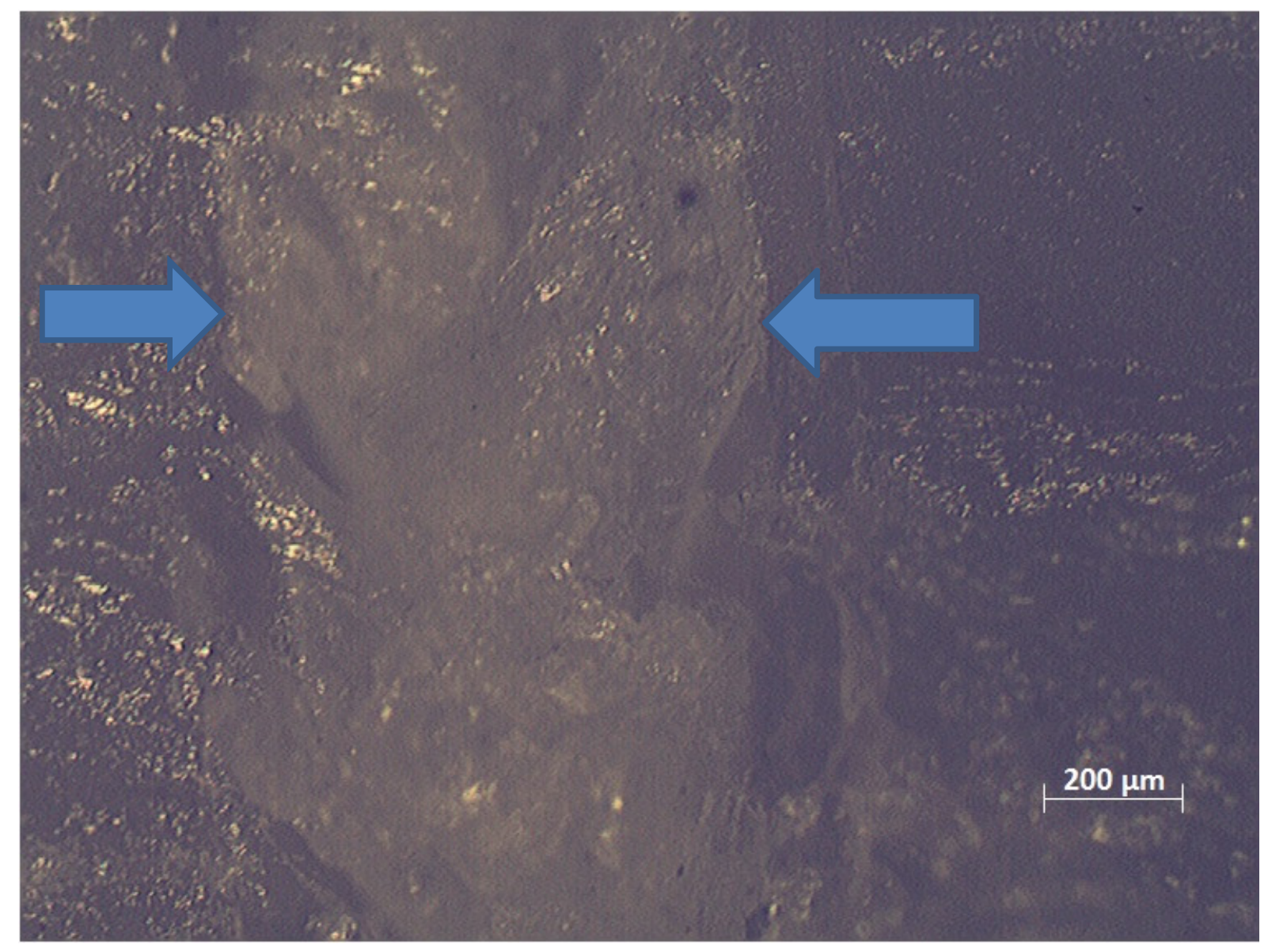

Figure 10.11 HAZ viewed from side1 for Serial No. 2 


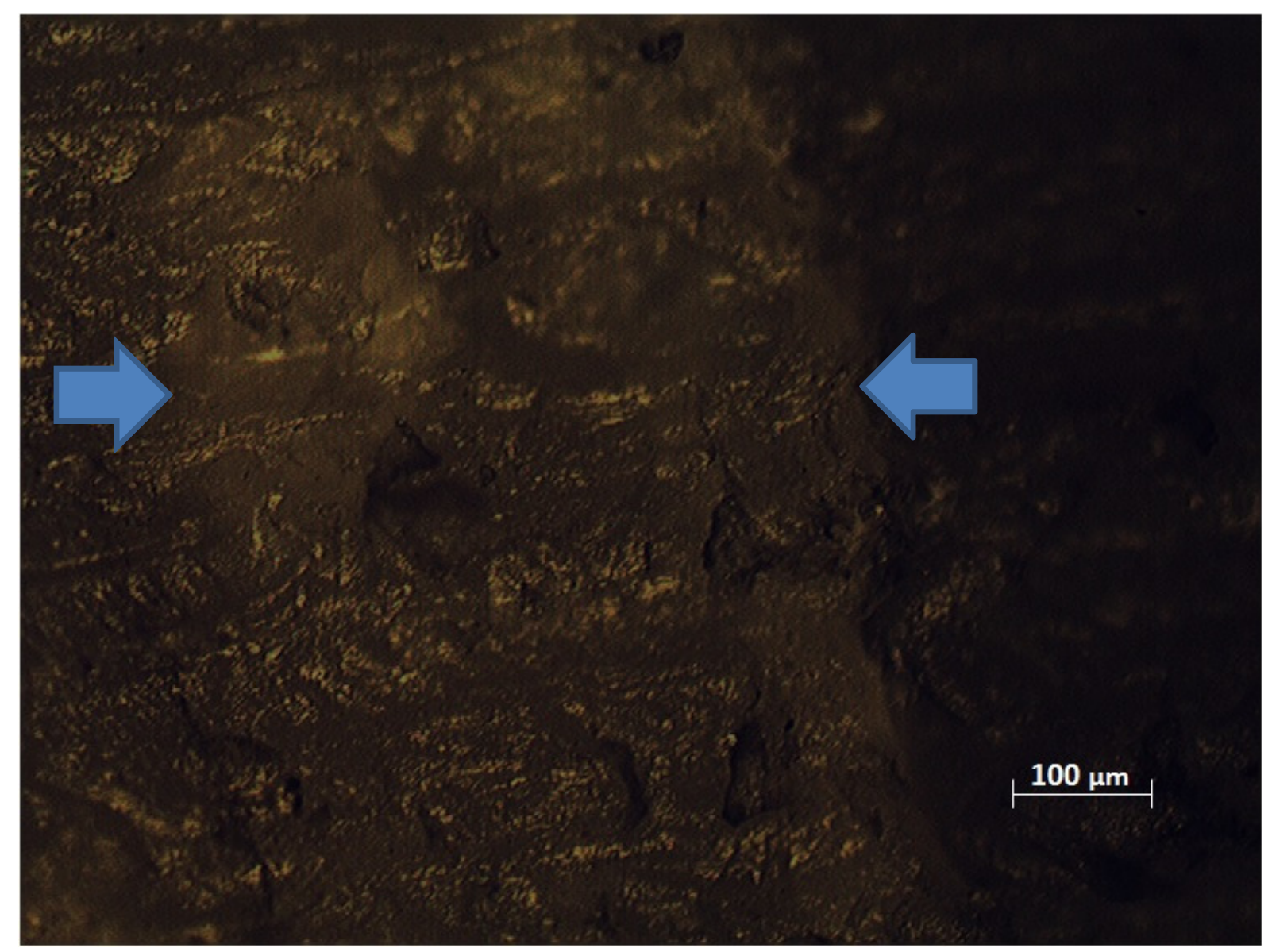

Figure 10.12 HAZES viewed from side3 for Serial No. 3 

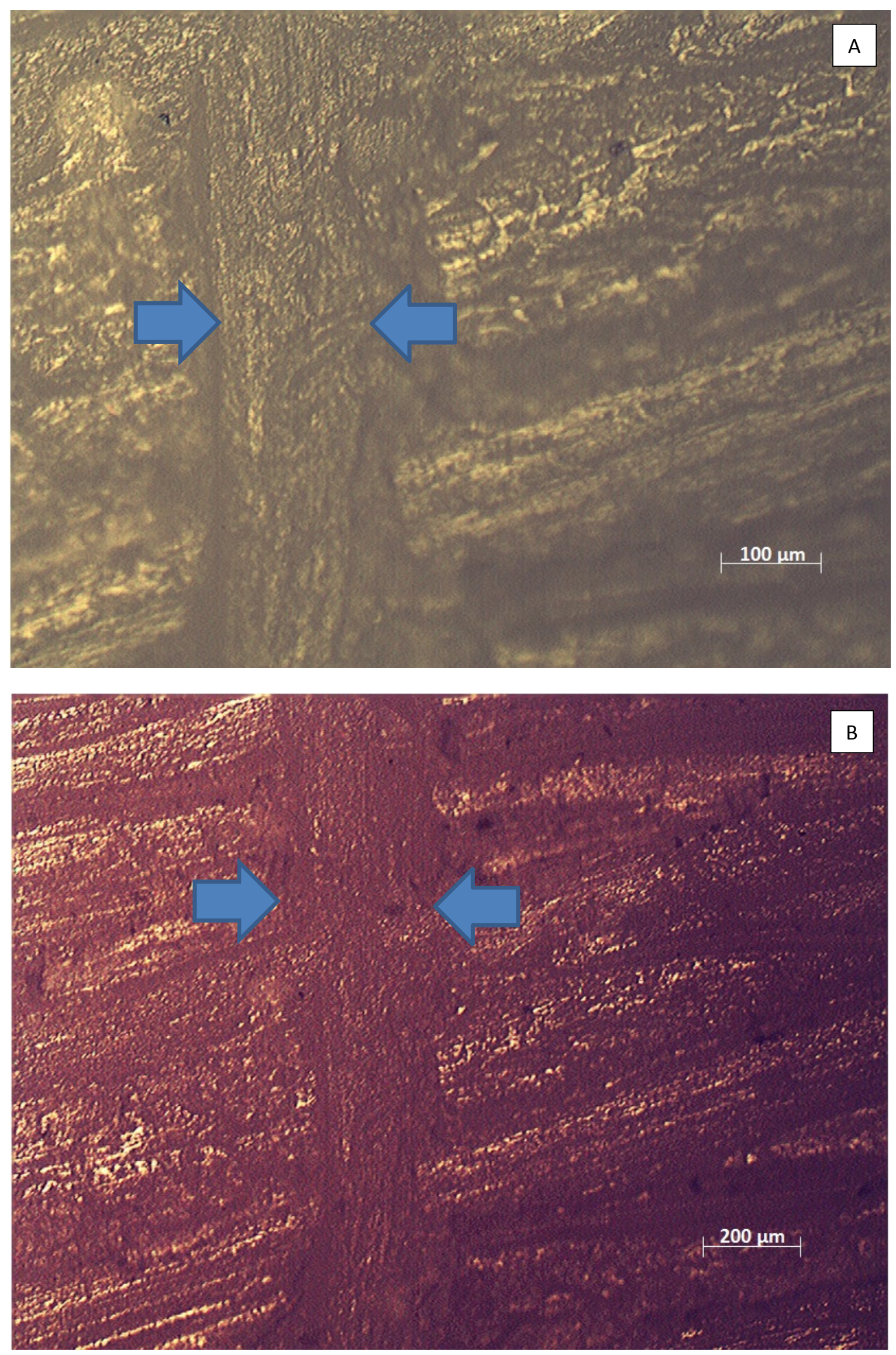

Figure 10.13(A\&B) HAZES viewed from side1 for Serial No. 4 


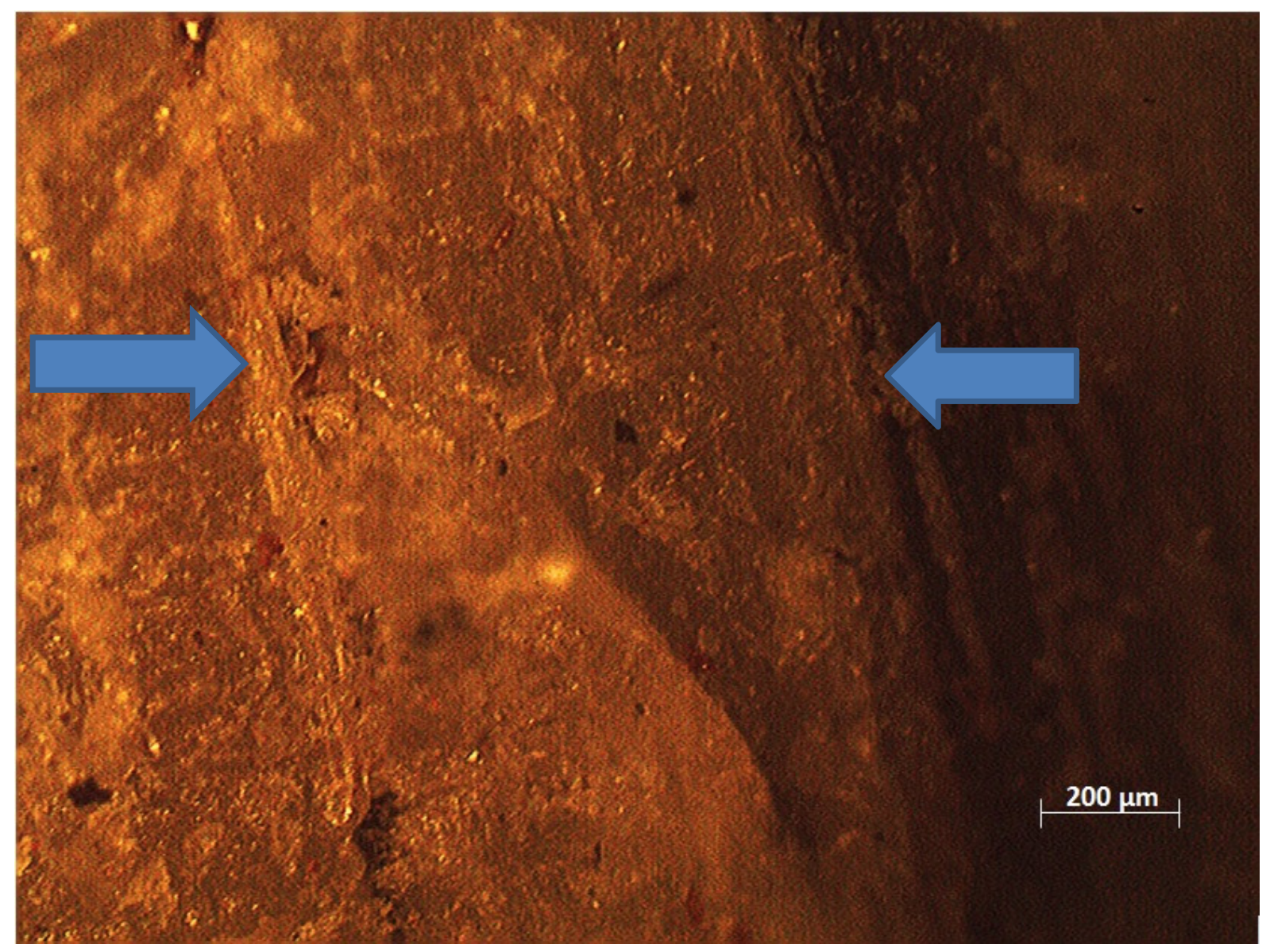

Figure 10.14 HAZ viewed from side4 for Serial No. 5 

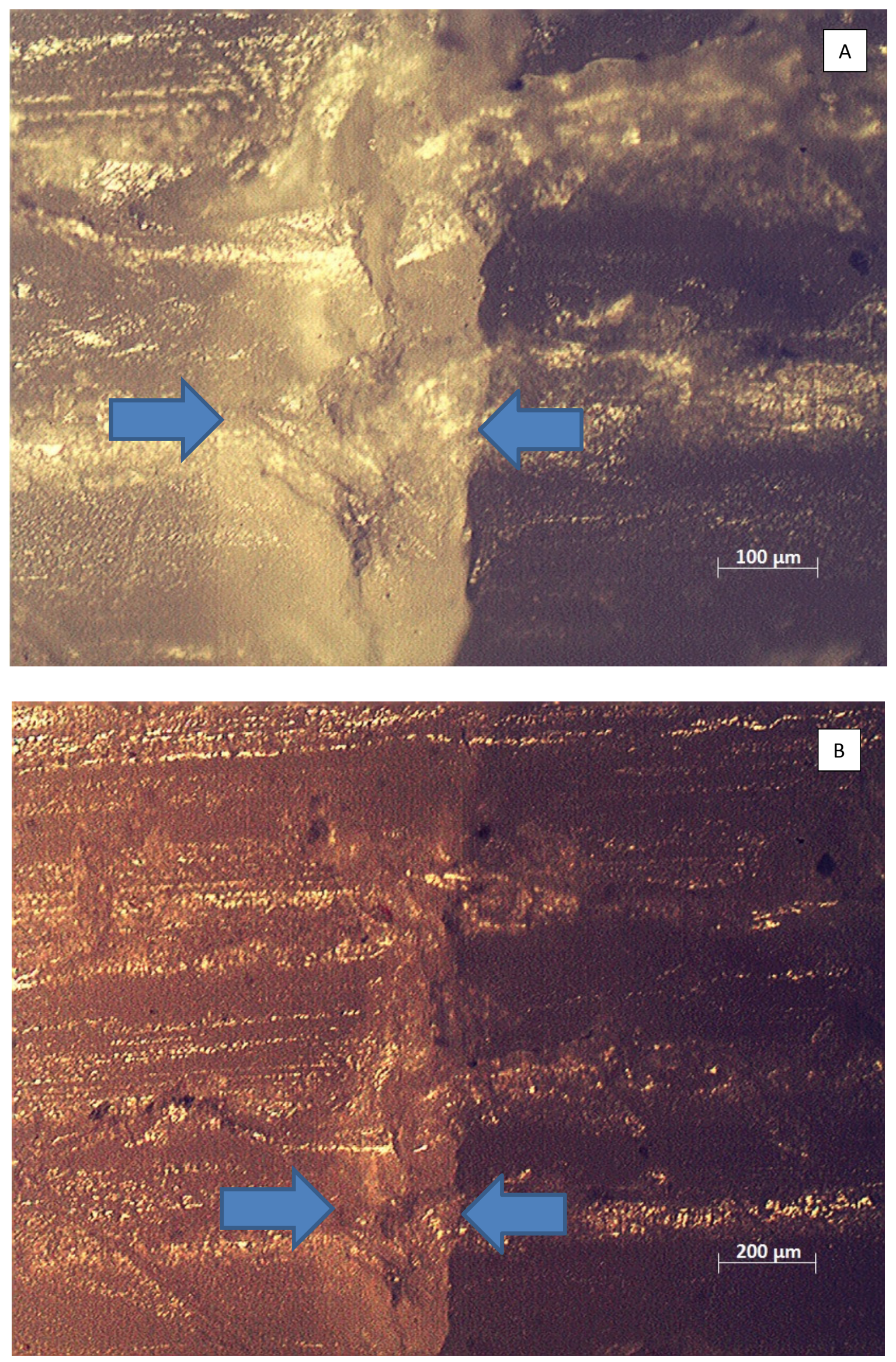

Figure 10.15(A\&B) HAZES viewed from side1 for Serial No. 6 

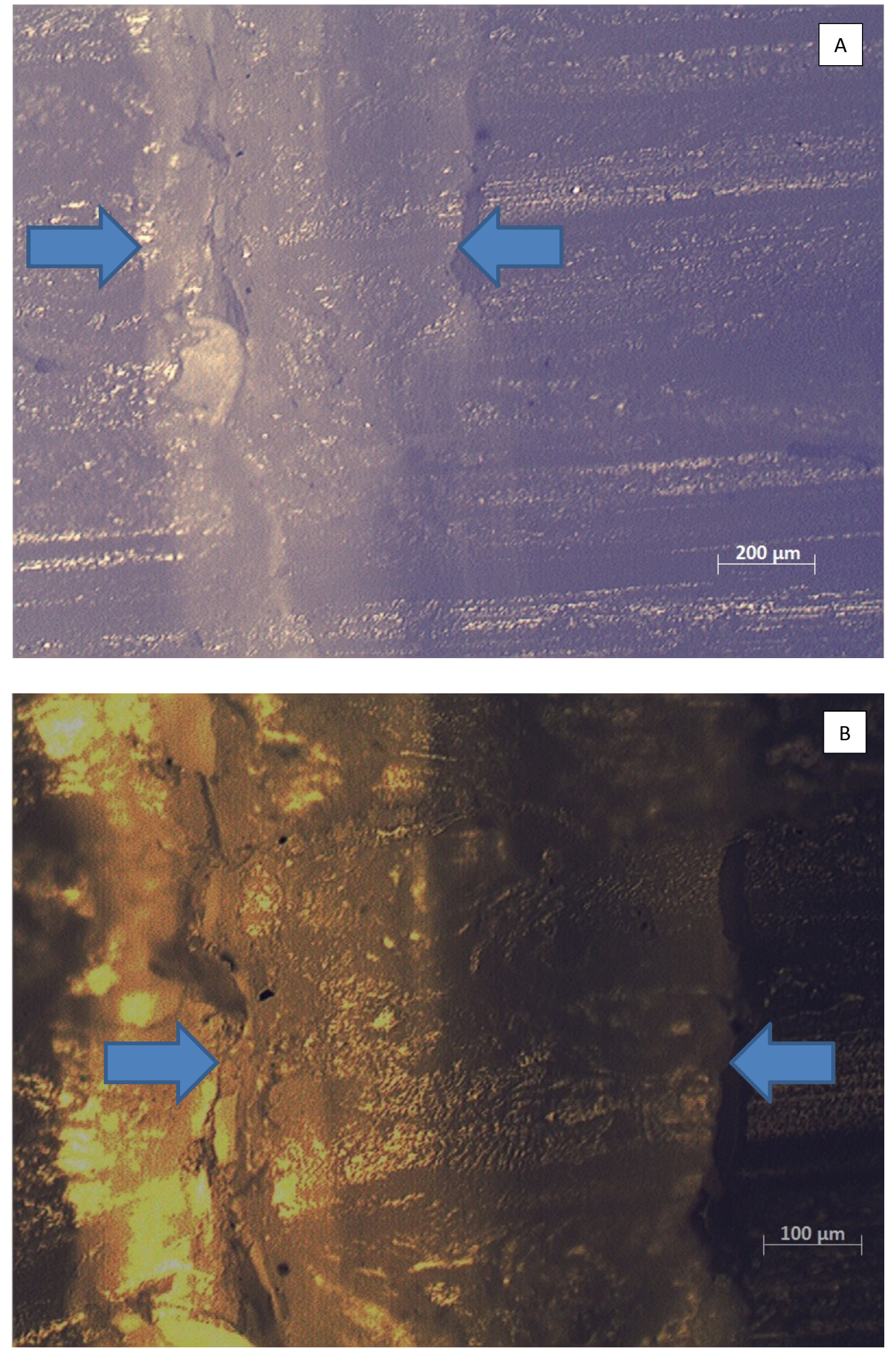

Figure 10.16(A\&B) HAZES viewed from side1 for Serial No. 7 


\subsection{FRACTOGRAPHY}

In this research, fracture analysis or fractography was achieved to test again a hypothesis for both ABS and PP. Hypothesis E was checked for ABS and PP.

\subsubsection{Brief Literature Review}

The fractography of polymers depends upon material macrostructure, microstructure, stress environment, time and temperature. Fracture of polymeric components is commonly a macroscopic term emerging from sequences of microscopic and sub microscopic procedures [166]. Fractography is studied to explore the welding, loading and environmental conditions which produce fracture by interpreting the visual appearance of fractured parts [166].

Previous studies have reported that stress crazing, stress cracking, and stress whitening have great impact on weld failure. Various features are observed on the fractured surfaces e.g. mirror zone, mist region, hackle region, wallner lines, parabolic markings and rib markings [166].

Fractography gives lot of hints about the causes of fracture. Fractography is not merely an examination of fractured surface but it has the ability to give crucial clues about the state of stress which causes crack initiation and growth to ultimate failure.

Structure, loading conditions and temperature affect the mechanisms of ductile fracture (fracture with plastic deformation) and brittle fracture (fracture with little or no plastic deformation) on loading. Deformation is generated by viscoplastic flow processes in thermoplastic polymers. Thermoset plastics are usually brittle. Viscoplastic deformation is a strong function of temperature and strain rate. Plastic flow in polymer leads to bond rupture, shear sliding, chain straightening and chain alignment in the direction of load. Strain rates may also affect plastic flow. Moreover, application of load on perpetual basis results in formation of crazes. Crazes are crack predecessors. Formation of cavities in polymers points to plastic deformation. Instead of coalescing into a crack, the micro voids are stabilized by fibrils which comprise polymer. This region of voids and fibrils is collectively called as a craze. Crazing is usually observed in polymers which have long chains. Crazing 
fibrils are shown in Figures 10.23 to $10.26^{3}$. In the absence of long chains, it is impossible to have fibrils which surround the craze and to stop the exchange of crazes into crackformation. Amorphous polymers are considered to have crazing on stressing which leads to their deformation. Semi-crystalline polymers have also been thought of getting crazing found and even in thermosetting resins [166]. Crake formation from a craze is shown in Figure 10.17. Resemblance to Figure 10.17 from current research is exhibited in Figure $10.22^{4}$.

Figure 10.17 Crack formation from a craze [166]

\subsubsection{Description of procedure for fractography}

After investigating the $H A Z$, fracture analysis was planned to be conducted for $A B S$ and Polypropylene. In this study, ABS and polypropylene were welded ultrasonically and tested for their lap shear strength (LSS) which resulted in a complete fracture. Selection of ultrasonic weld factors for fractography depended on their contribution to either make necessarily good bond or acquire better weld strength.

In this fracture analysis, cutting procedure and anatomy for fractured samples were almost similar to what were adopted in HAZ analysis except only one fracture side was under investigation. Cutting procedure for fractured surface is illustrated in Figure 10.18.

\footnotetext{
${ }^{3}$ Figures 10.23 to 10.26 are illustrated one by one from page 250 to page 252 .

${ }^{4}$ Figure 10.22 is available at page 249.
} 


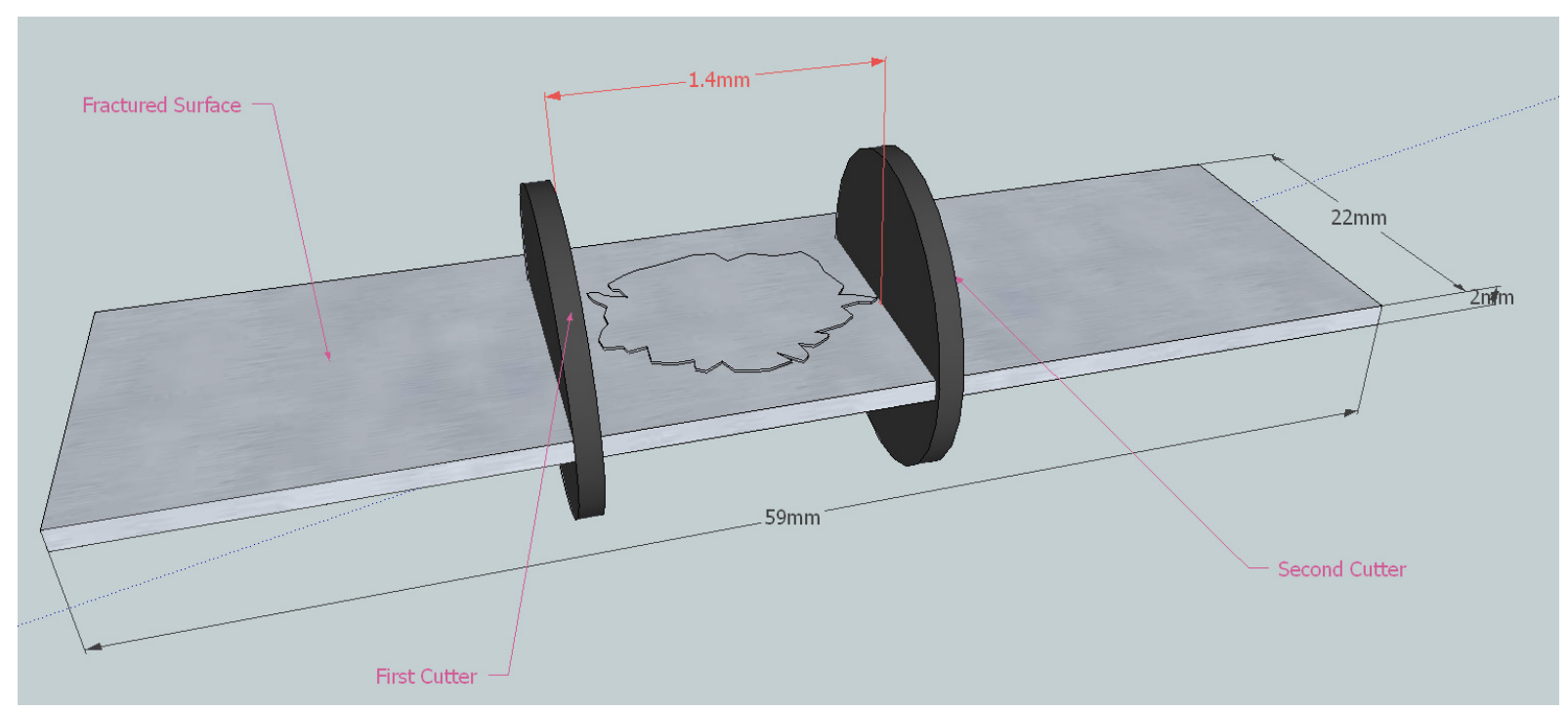

Figure 10.18 Cutting of fractured surface for PP

The fractured surfaces after cutting were observed by using optical microscope (OM) (POLYVAR MET Reichert-Jung) and scanning electron microscope (SEM) (JEOL JSM 6400), as shown in Figure 10.19.

Figure 10.19 SEM (JEOL JSM 6400) [199]

Cracking in thermoplastics can be addressed to some extent by inspecting their visual appearance. But micro crack may best be examined by using either optical microscope or scanning electron microscope. In this research, optical microscope was extensively used 
because it was most appropriate for our studies. SEM was also used to gain additional information where necessary.

In present fracture analysis, although procedure for optical microscopy of fractured surfaces was the same, as discussed earlier in section 10.4.1, description of procedure for fractography with SEM must be described here. For SEM, a necessary sample preparation was done for fractured surfaces of ABS and PP. Moreover, polymer samples prepared were coated by gold before utilizing SEM.

A brief account of coating gold on fractured sample has been presented here. In this SEM imaging, samples and sample platform were cleaned using acetone after cutting the samples. Any contamination on broken sample surface was removed by this cleaning activity. Sample and its platform were then dried in a dryer. The neat samples were pasted/stuck on the platforms by using sliver dag, as shown in Figure 10.20.

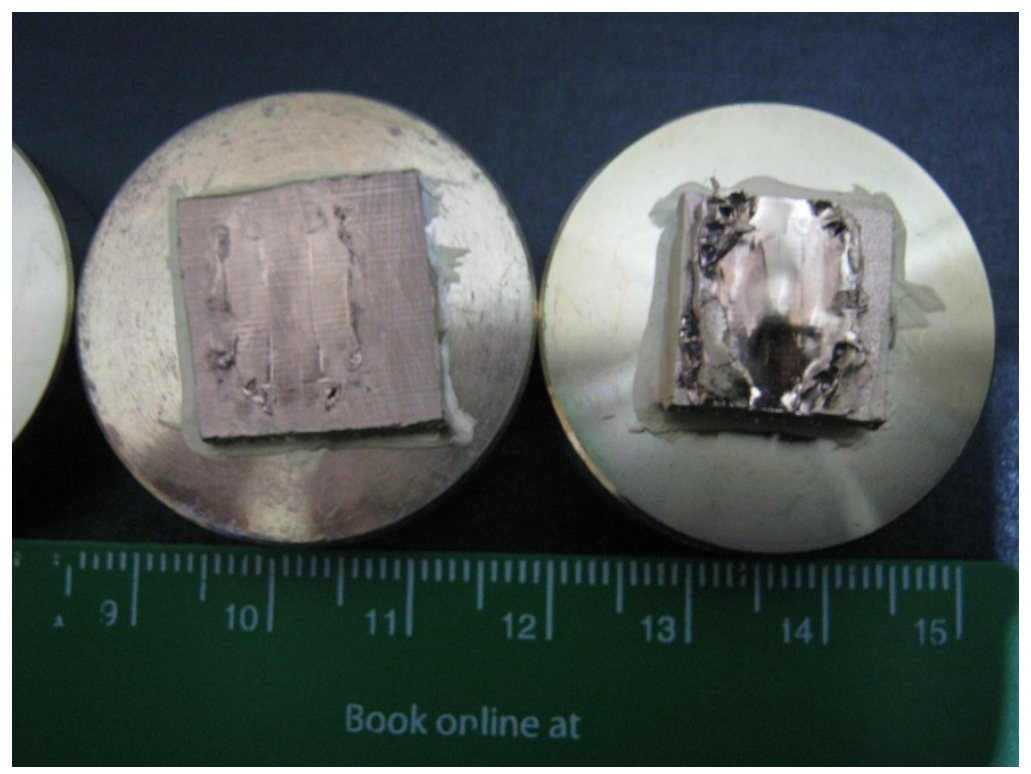

Figure 10.20 Gold Coated and Pasted Samples on SEM Sample Platform

Having been cleaned, stuck and placed on the platform, the sample was now ready to be gold coated. Sample stuck was taken to the gold sputtering machine, as shown in Figure 10.21. Sample was gently and safely placed in coater. Gold coater was turned ON for coating after switching $\mathrm{ON}$ the pump. 


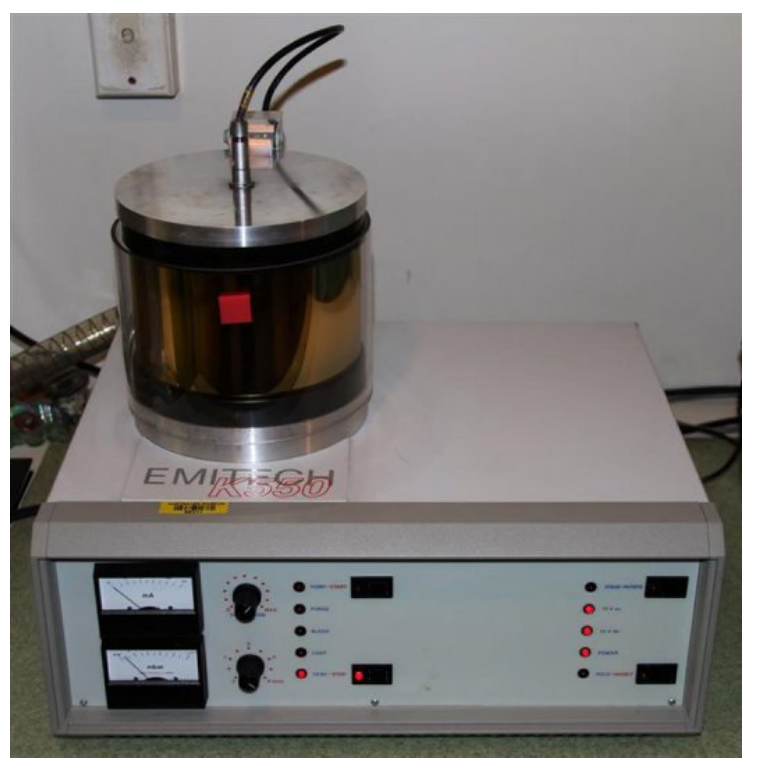

Figure 10.21 Gold Sputtering Machine

JEOL JSM 6400 (SEM) was firstly turned ON. Gold coated sample was then placed heedfully according to operating manual of SEM. After locating sample, a proper working distance (that involves switching ON the TV monitor) and accelerating voltage were set. Digital imaging was started after astigmatism correction as per instructions of manual. Sample was removed after capturing the SEM images.

\subsection{3. $\quad \underline{\text { Results }}$}

In this research, welded samples were acquired by utilizing ultrasonic weld factors, as shown in Table 10.3. Fractured surfaces were obtained after testing these welded samples for LSS. Fractured surfaces were finally analysed by microscopes.

Table 10.3 Weld factors for fractography of welded specimens

\begin{tabular}{|c|c||c||c||c||c|c||}
\hline Sr. No. & $\begin{array}{c}\text { Material } \\
\text { Type }\end{array}$ & $\begin{array}{c}\text { ED } \\
\text { Type }\end{array}$ & $\begin{array}{c}\text { Static } \\
\text { Force } \\
(\mathbf{N})\end{array}$ & $\begin{array}{c}\text { Weld } \\
\text { Time } \\
(\mathbf{S e c})\end{array}$ & $\begin{array}{c}\text { Amplitude } \\
(\boldsymbol{\mu m})\end{array}$ & $\begin{array}{c}\text { Lap Shear } \\
\text { Strength } \\
(\mathbf{M P a})\end{array}$ \\
\hline \hline 1 & ABS & TRI & 1500 & 0.9 & 21 & 11.4 \\
\hline 2 & ABS & SEMI & 1500 & 0.8 & 18.9 & 7.97 \\
\hline 3 & PP & TRI & 1000 & 1.5 & 21 & 1.23 \\
\hline 4 & PP & SEMI & 1750 & 1.0 & 18.9 & 1.14 \\
\hline
\end{tabular}




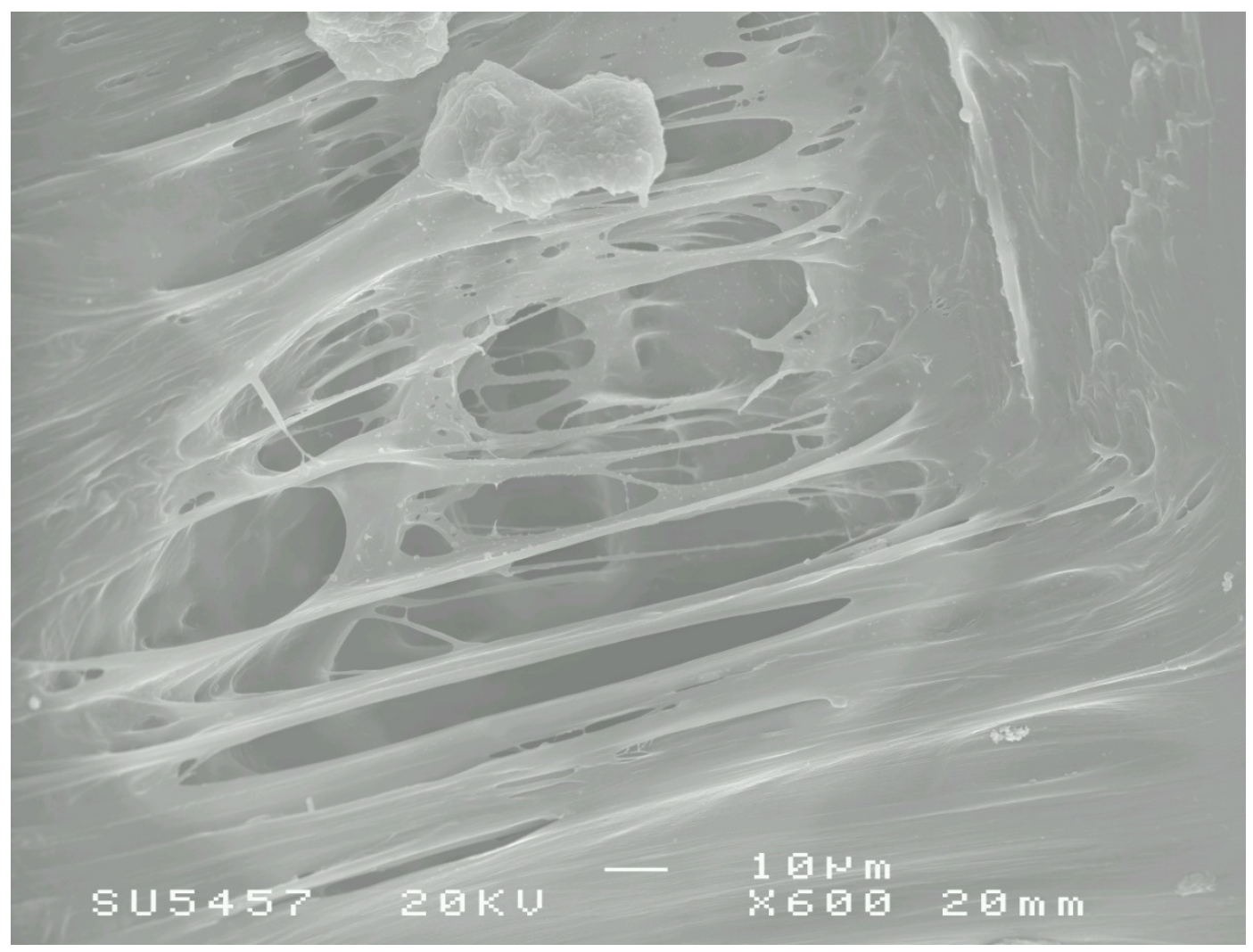

Figure 10.22 SEM image for Serial No.3 of Table 10.3

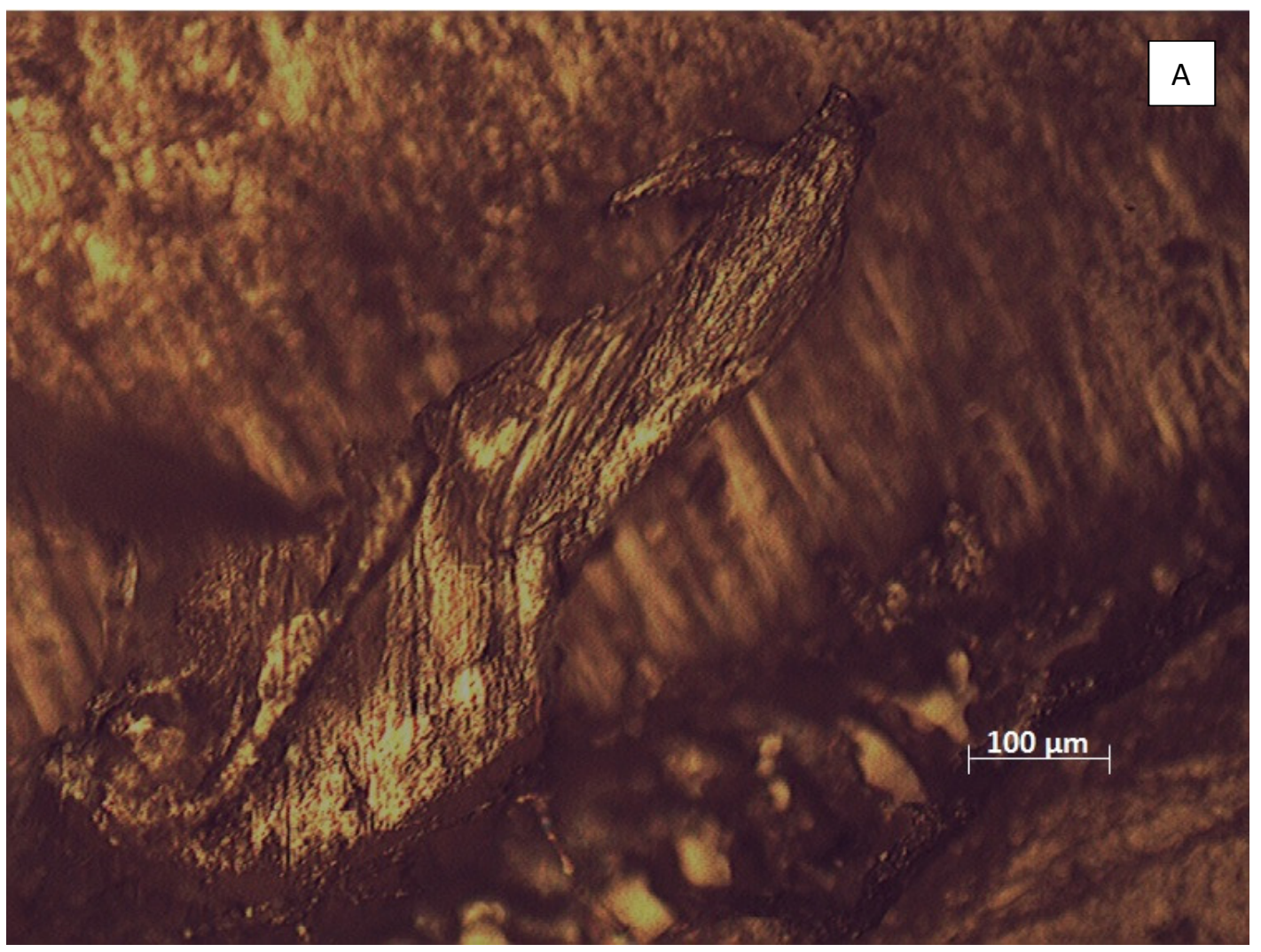




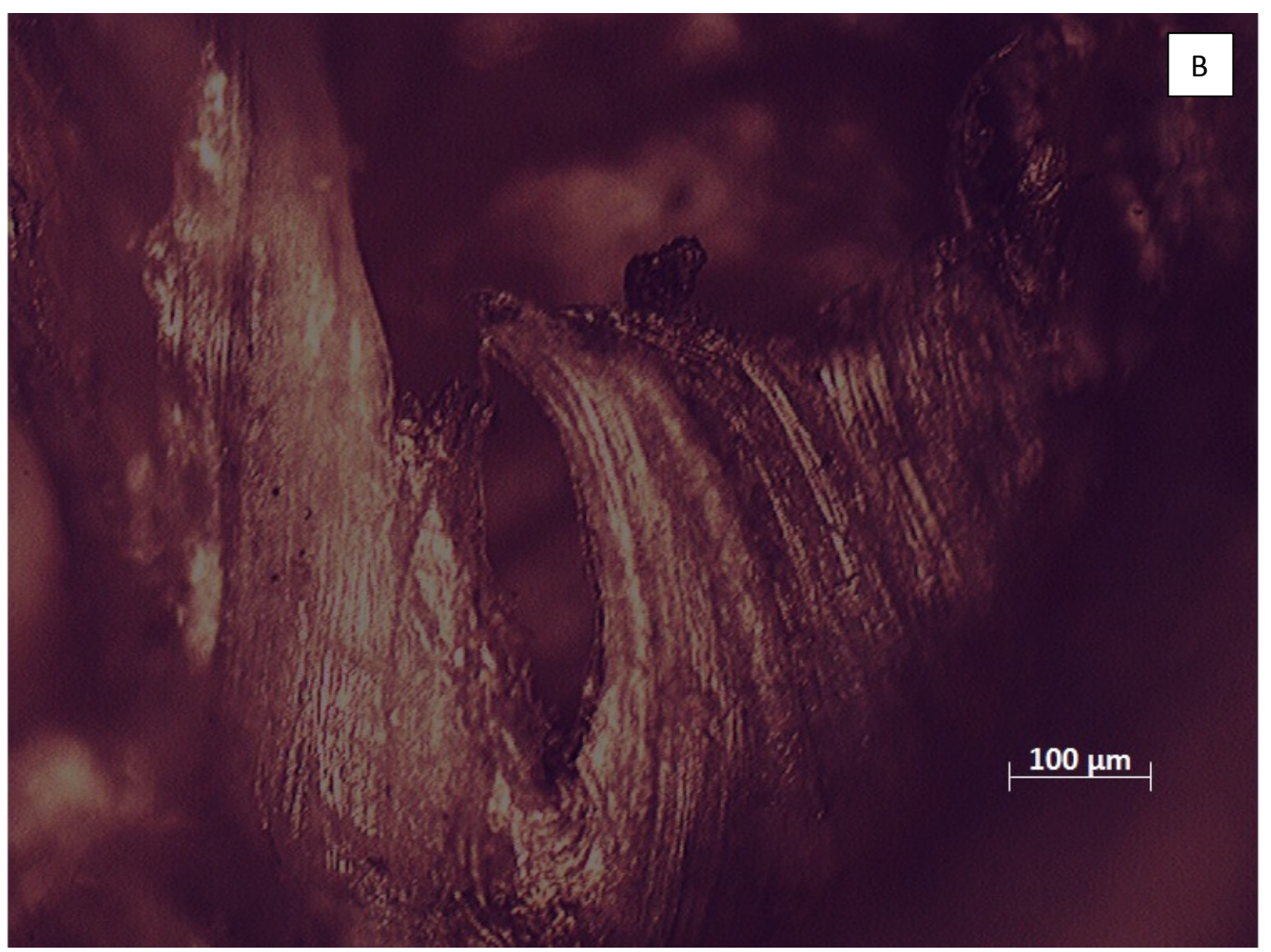

Figure 10.23(A\&B) Microscopic pictures of crazing fibrils for $A B S$

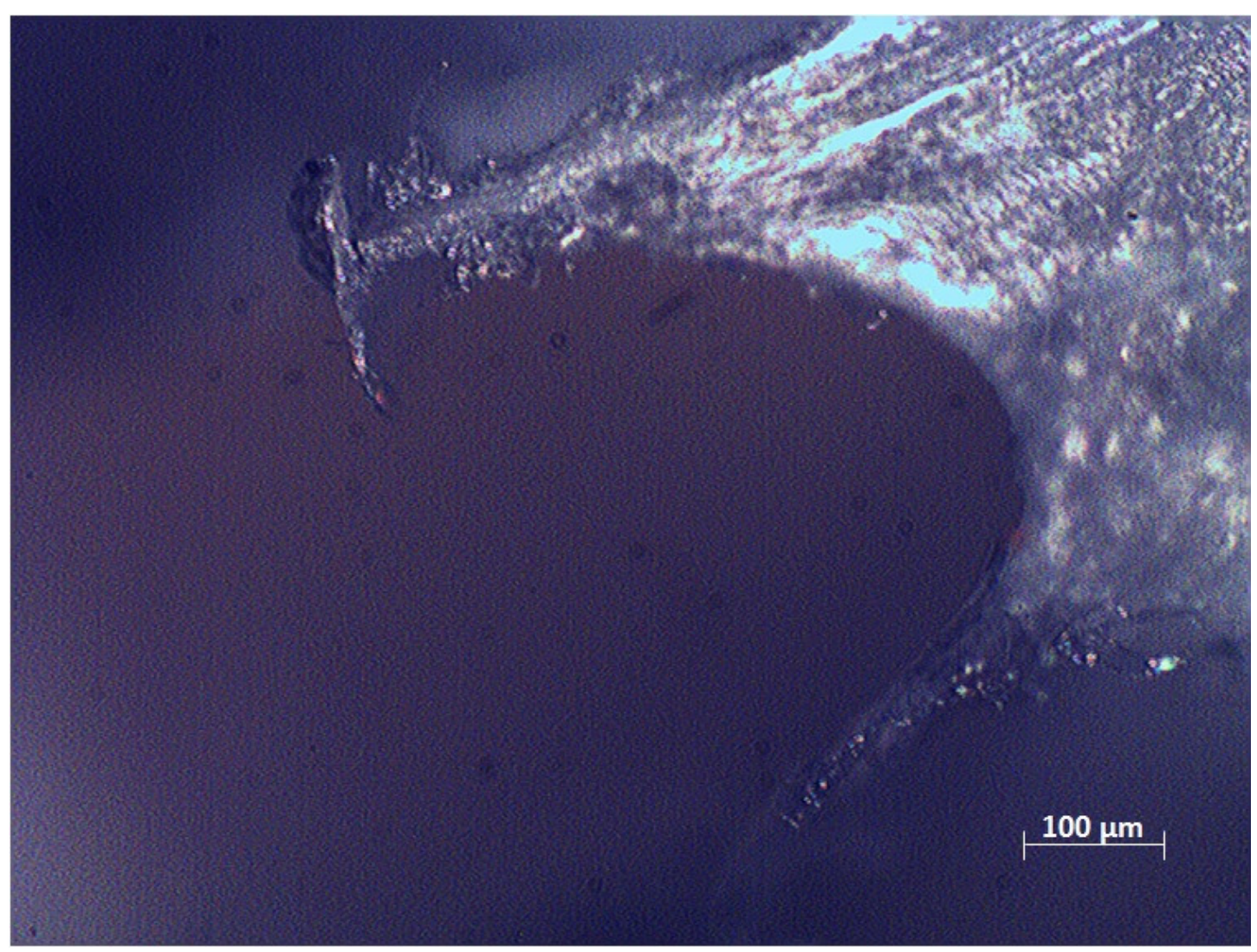

Figure 10.24 Microscopic pictures of crazing fibrils for PP 

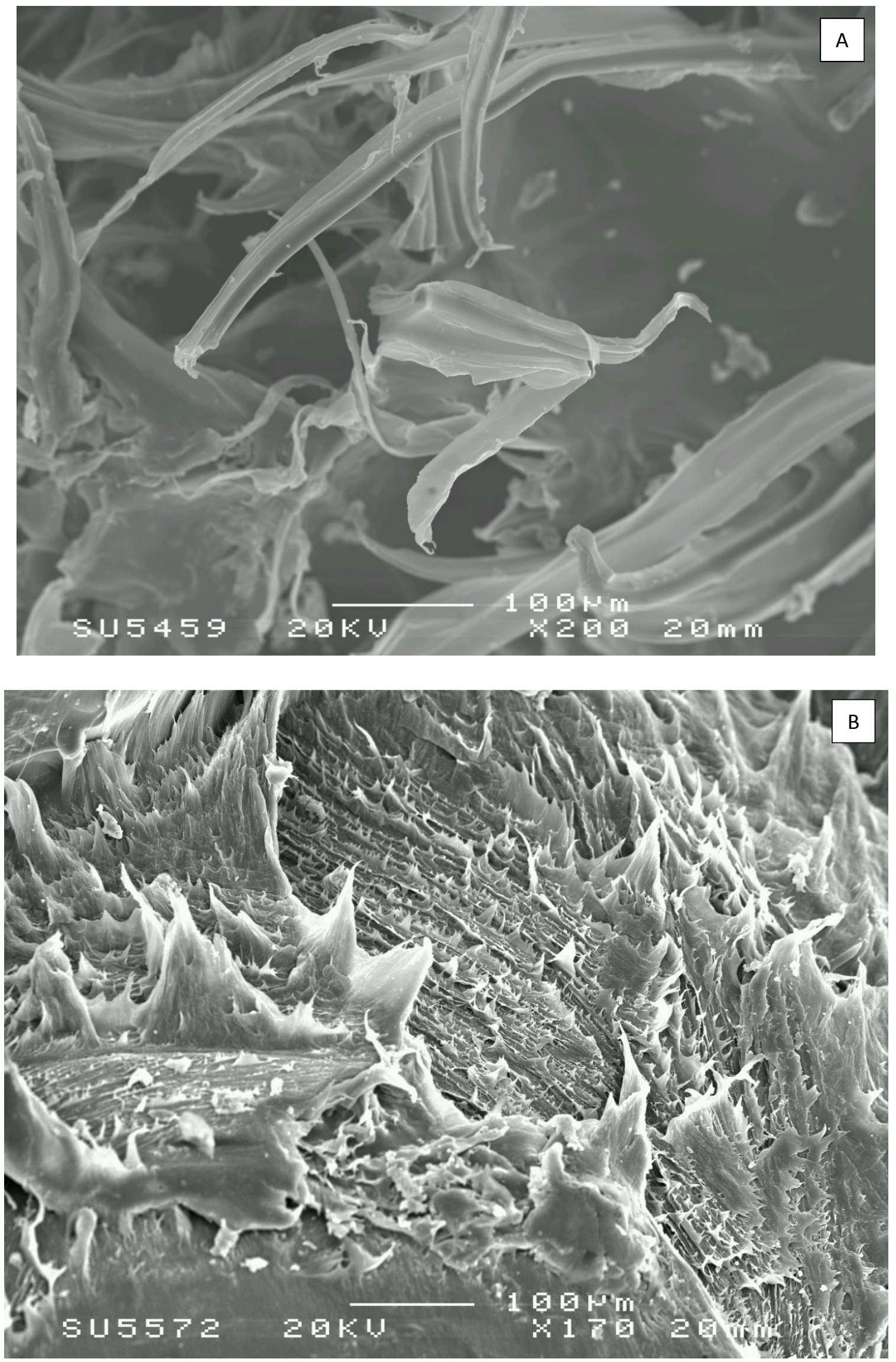

Figure 10.25(A\&B) SEM images showing crazing fibrils for Serial No.3 


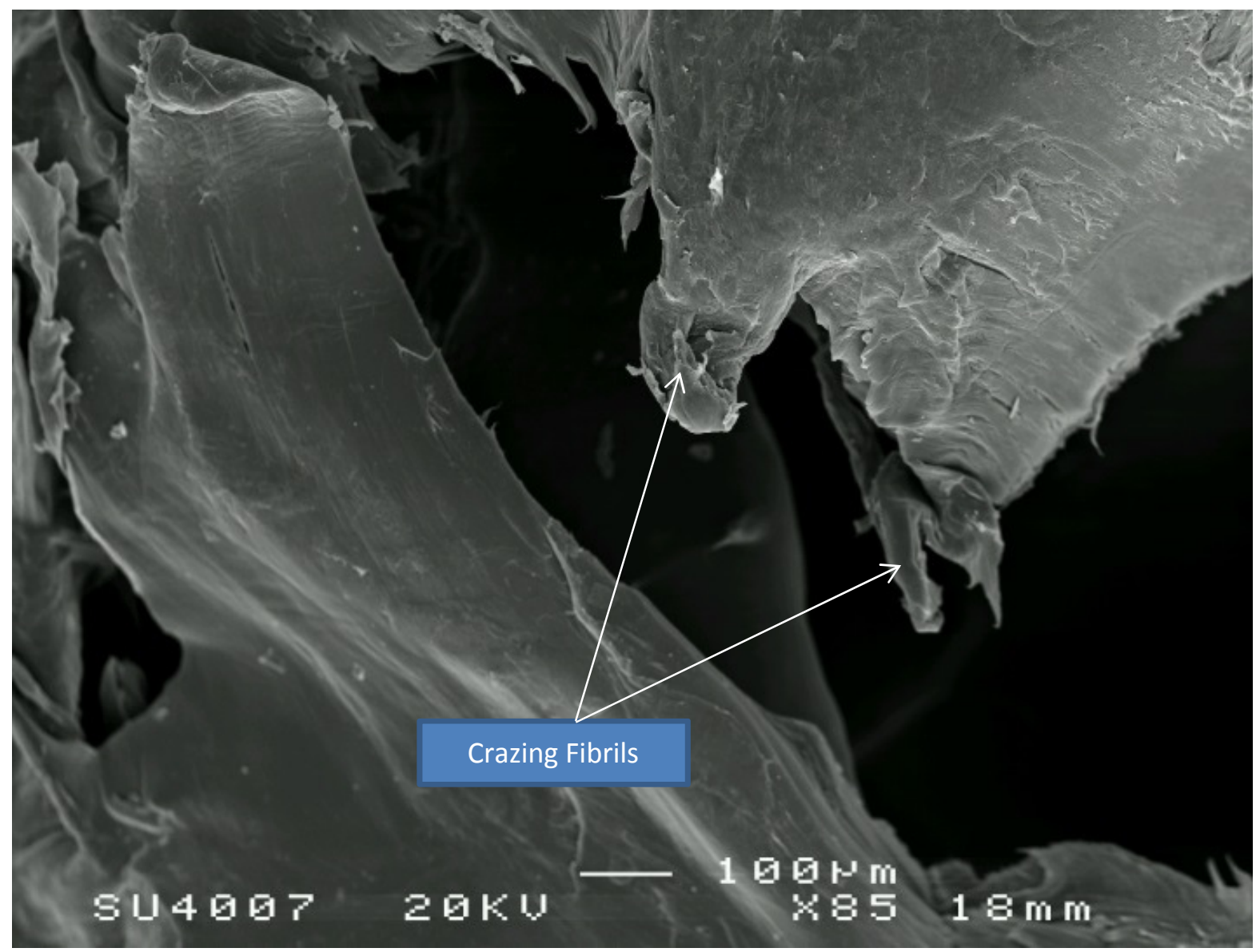

Figure 10.26 SEM image showing crazing fibrils for Serial No.4

\section{Effect of fracture mode on LSS}

Hypothesis E suggests that "Crazing affects bond fracture without influencing fracture mode for ABS and PP"

In this work, three modes of fractures were possible during testing of bond strength.

1. Ductile Fracture [166]

2. Brittle Fracture [166]

3. No Fracture

When the specimens were fractured in ductile manner, higher LSS was expected for both materials, whereas brittle fracture resulted in comparatively lower LSS. When achieving no fracture/failure of ultrasonic bond, the highest LSS was termed as high welding efficiency.

When fracture was investigated microscopically, crazing was definitely found there without having effect on the fracture mode. When delivering high welding efficiency, the crazing 
occurred at a region away from the weld zone. Because ultrasonic bond was unbreakable and fracture occurred near the clamping points of lap shear joint, as shown in Figure 10.27.

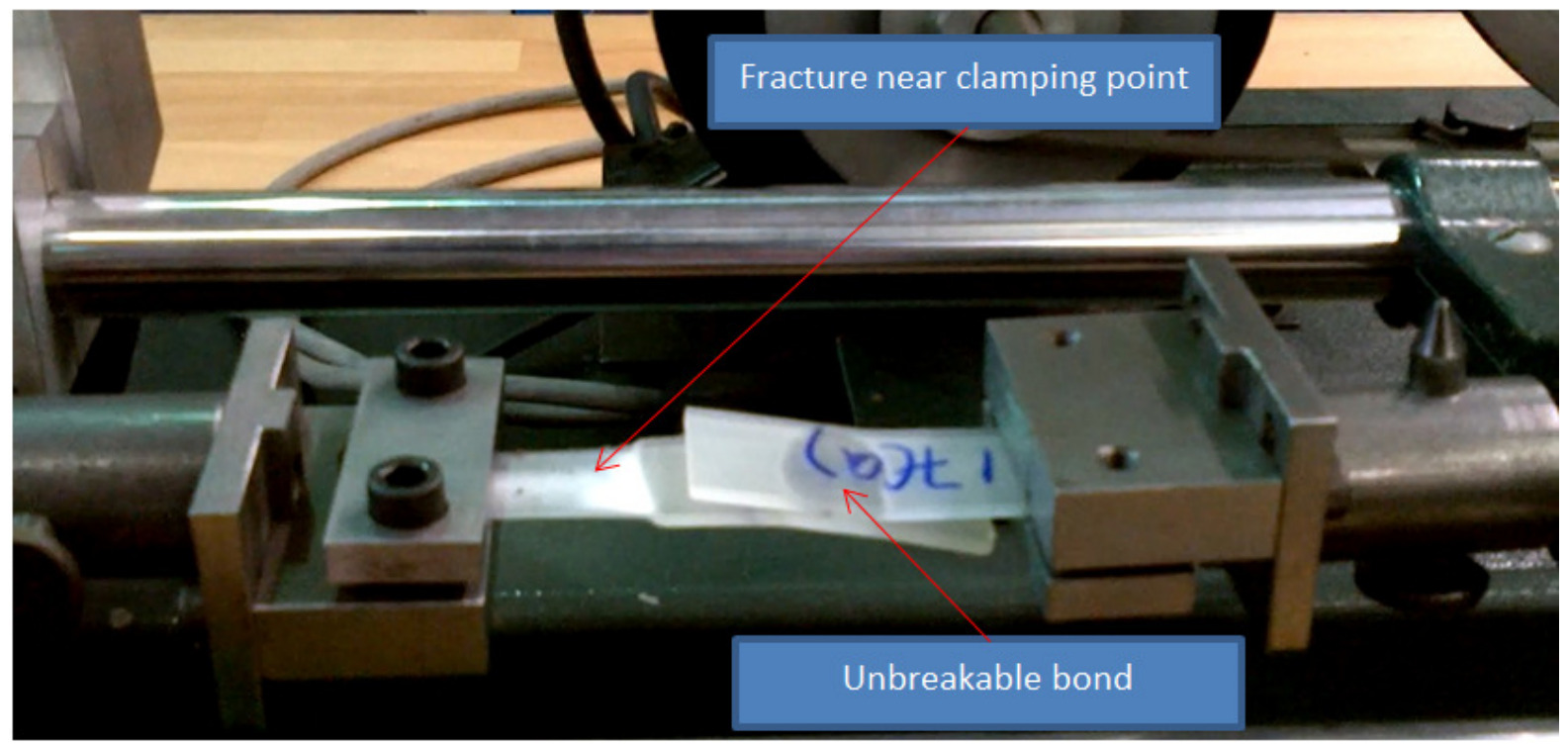

Figure 10.27 Fracture for unbreakable bond

In this fractography, ABS and PP expected to show ductile fracture [166] were utilized. It was also expected due to the shape of ultrasonic bond i.e. localized bonding at specific area that both modes of fracture were possible on shear testing. That was why; there were some cases relevant to both ABS and PP which showed sudden fracture i.e. brittle fracture. This was due to the reason that fracture of virgin polymer having dog-bone shape may be definitely different than that of welded joints.

Supportive findings to approve hypothesis $E$

In this work, following features on fractured surfaces were found helping support the hypothesis E for ABS and PP.

1. Presence of more than one HAZES (Heat Affected Zones)

2. Sharp Edges' Intervals

3. Blisters or bubbles in PP material

4. Beast mouth fractured site, rock like fractured location, and horn like shapes in PP

Presence of different HAZES was found owing to variation in temperature at weld zone as a result of different weld factors, as exhibited in Figure 10.28. 
These HAZES are considered to be major causes of ductile fracture for both ABS and PP because each HAZ contributes to shear when shear force is applied on a lap shear joint. After failure of any $\mathrm{HAZ}$, next $\mathrm{HAZ}$ comes in to resist facture which gives rise to a ductile fracture.

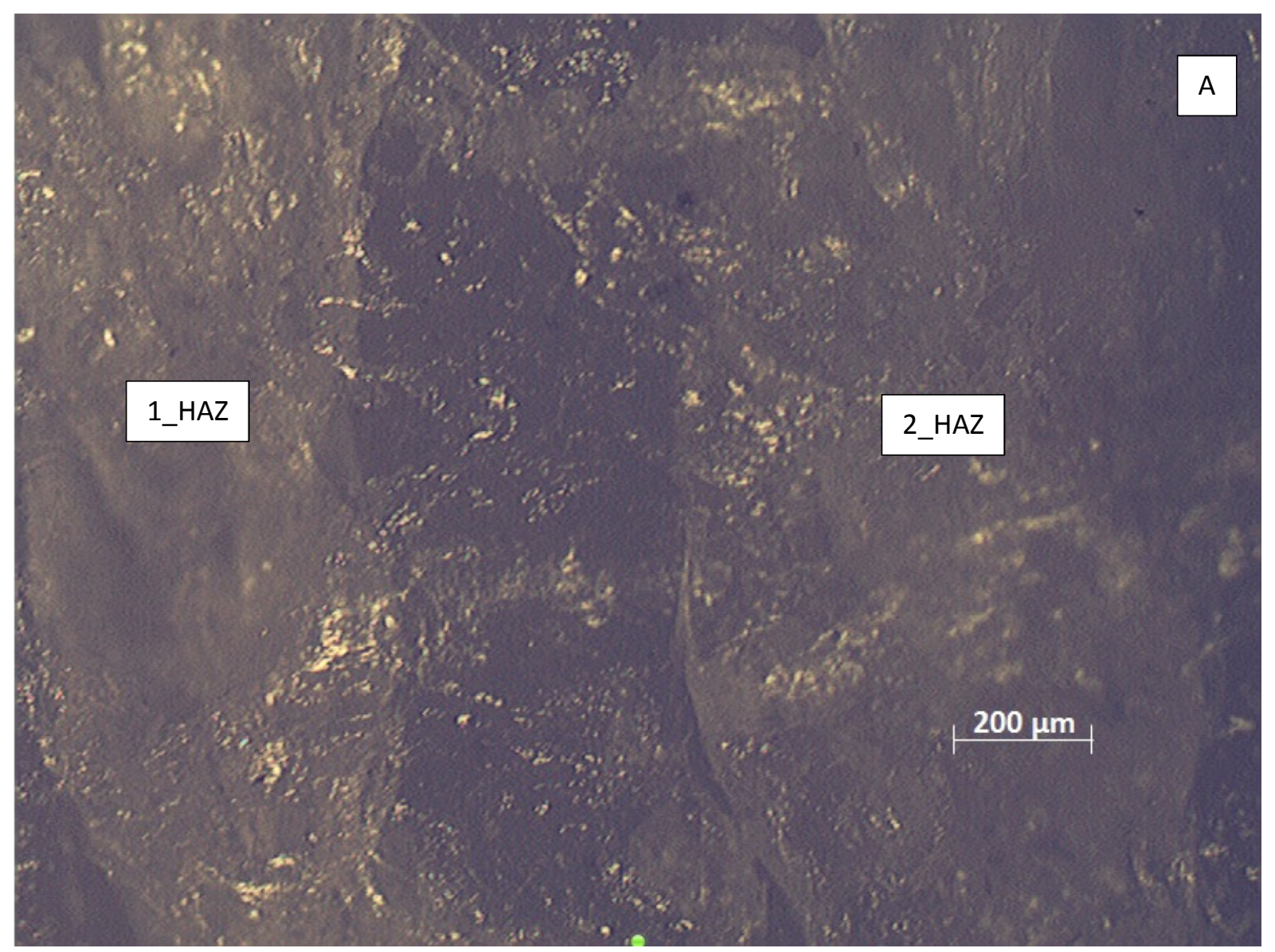




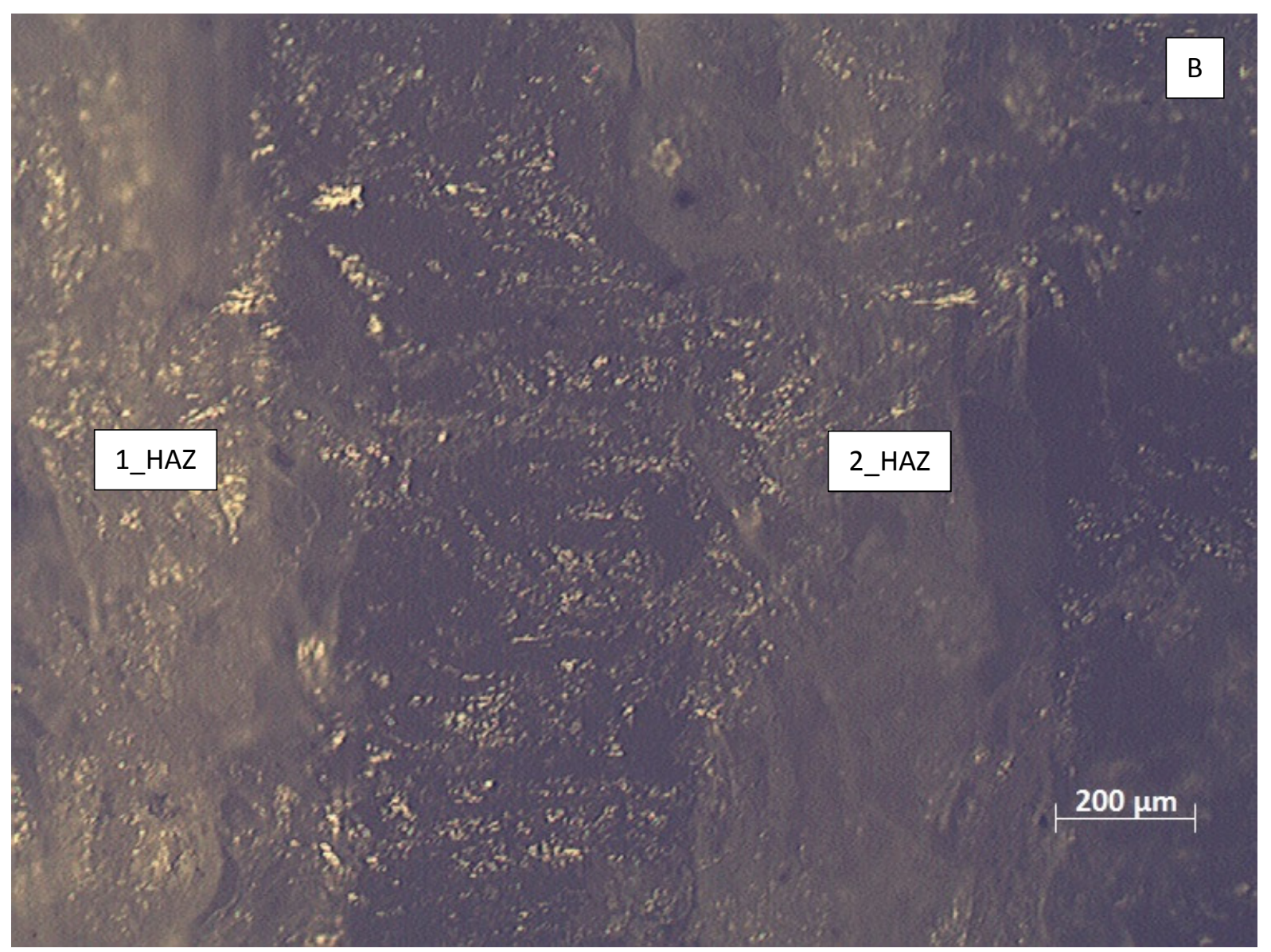

Figure 10.28(A\&B) Two HAZES viewed from side1 for PP

As illustrated in Figure 10.29, sharp edges' intervals were also observed microscopically which were present due to the sudden fracture/brittle fracture. 


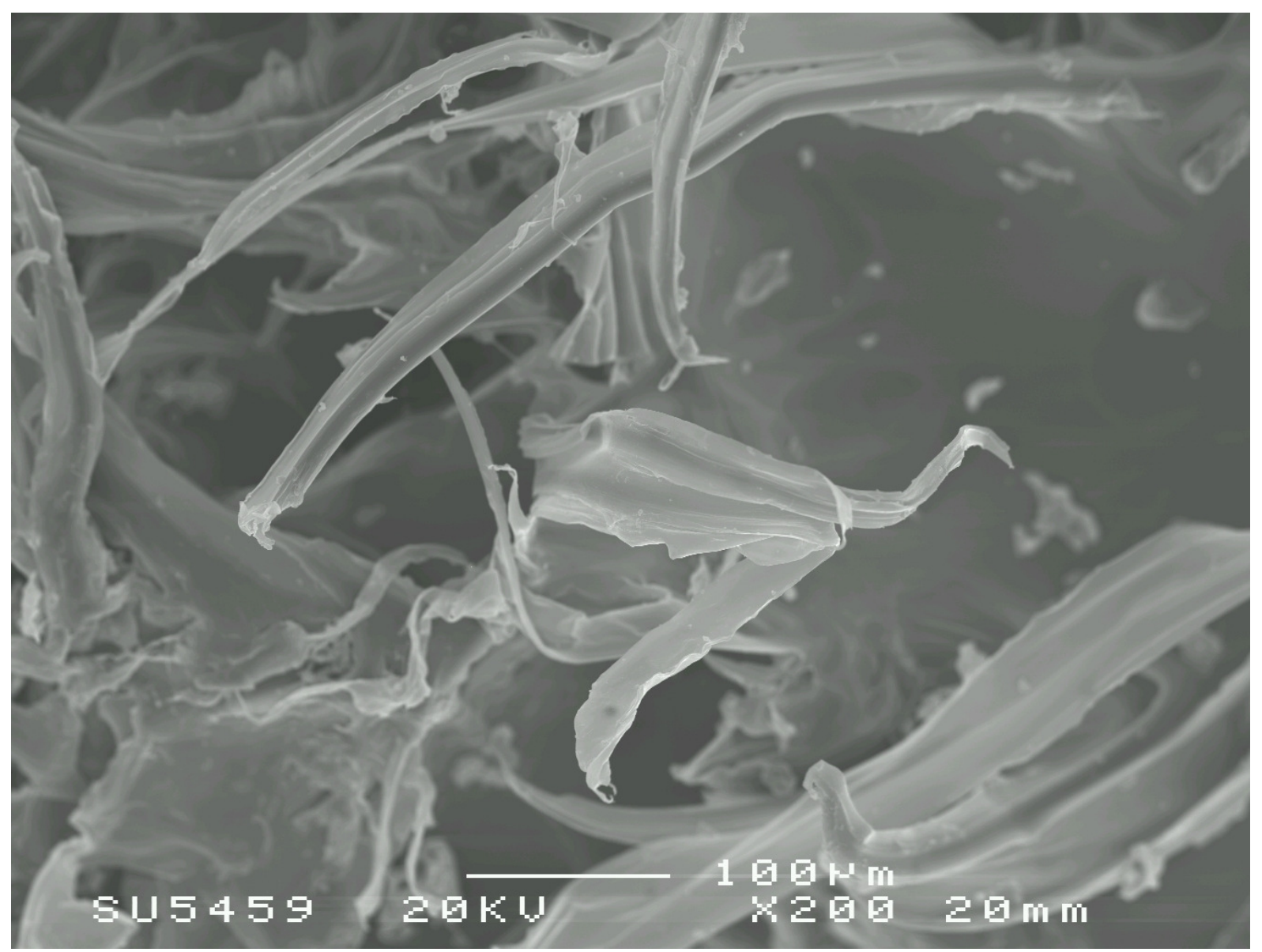

Figure 10.29 Sharp edges for PP

\section{Presence of bubbles/blisters}

Blisters and bubbles were observed in both ABS and PP owing to the interaction of molten plastic with atmospheric air, as exhibited in Figure 10.30. Air was entrapped by molten thermoplastic from atmosphere before and during welding. So, it is always good to properly take care of preventing the atmospheric air from entering molten polymers. These blisters and air bubbles can greatly turn the failure mode into brittle one. 


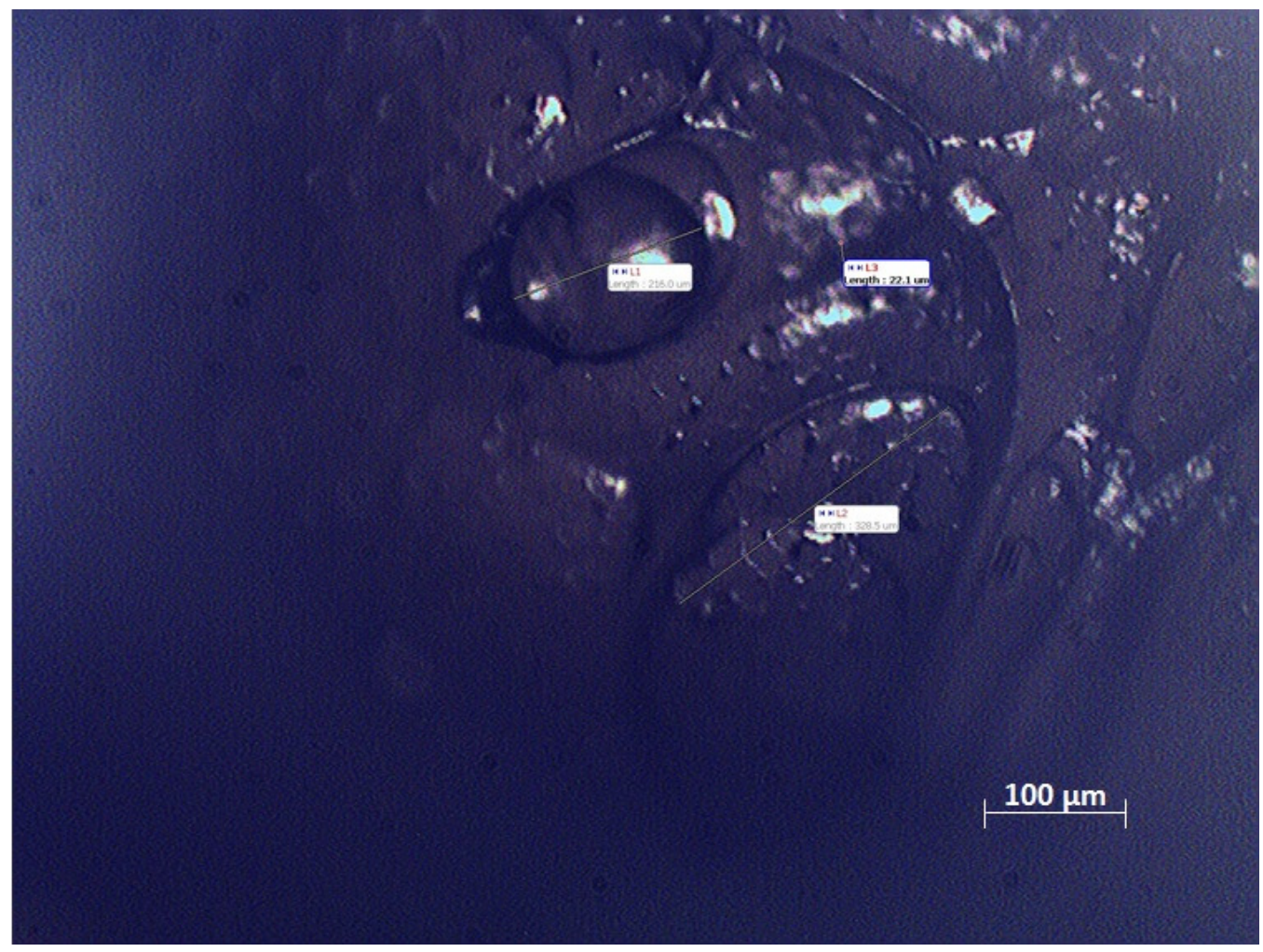

Figure 10.30 Bubbles and blisters

Presence of beast mouth fibril

Beast mouth micro fibril was the result of brittle fracture in case of PP, as depicted in Figure 10.31. 


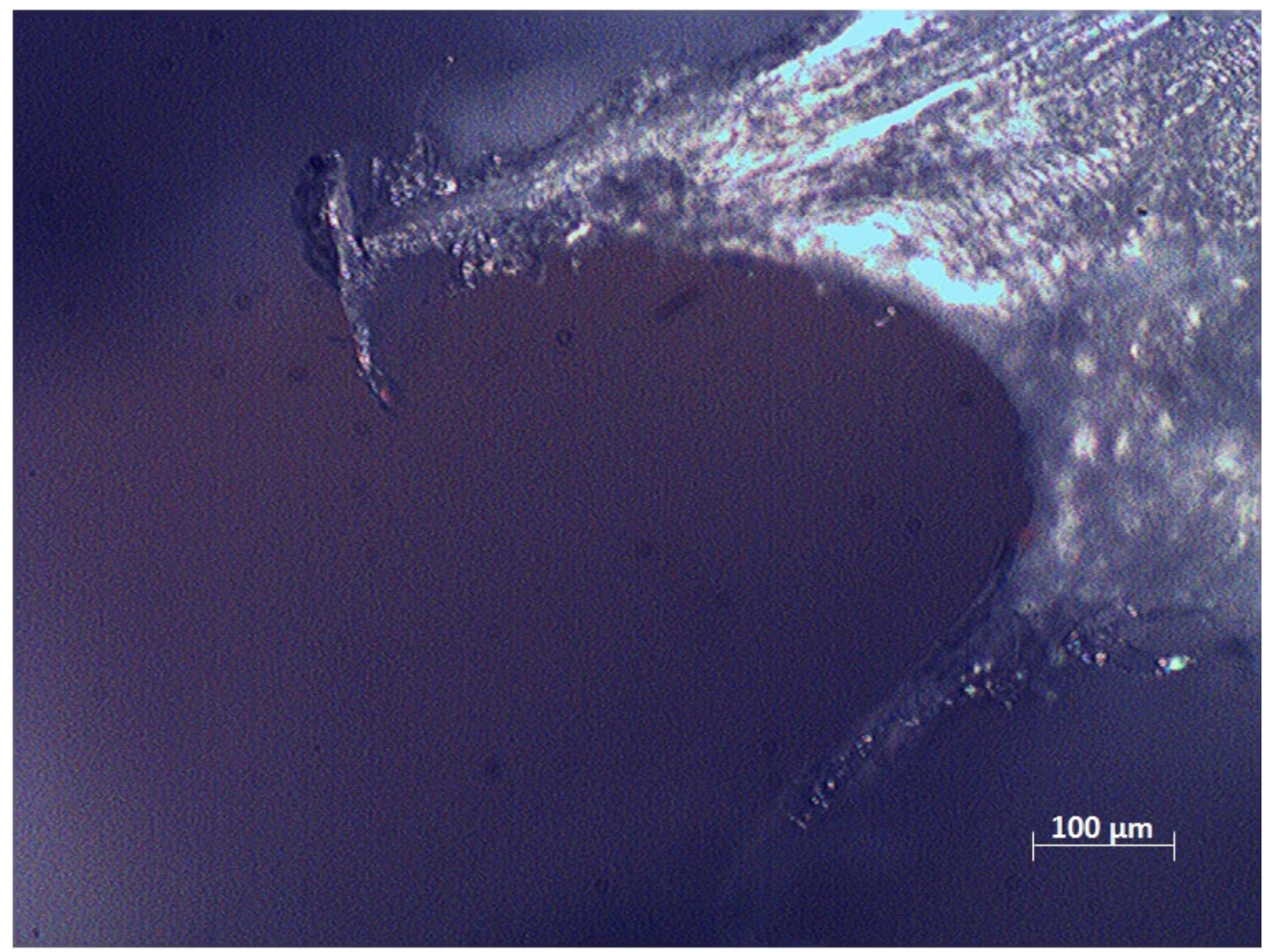

Figure 10.31 Beast mouth micro fibril

Presence of rock like micro fibril

Rock like fractured micro fibril was due to again brittle fracture, as represented in Figure 10.32. And this form of micro fibrils might be due to that part of weld zone that showed great resistance to shear forces in acquiring fracture. 

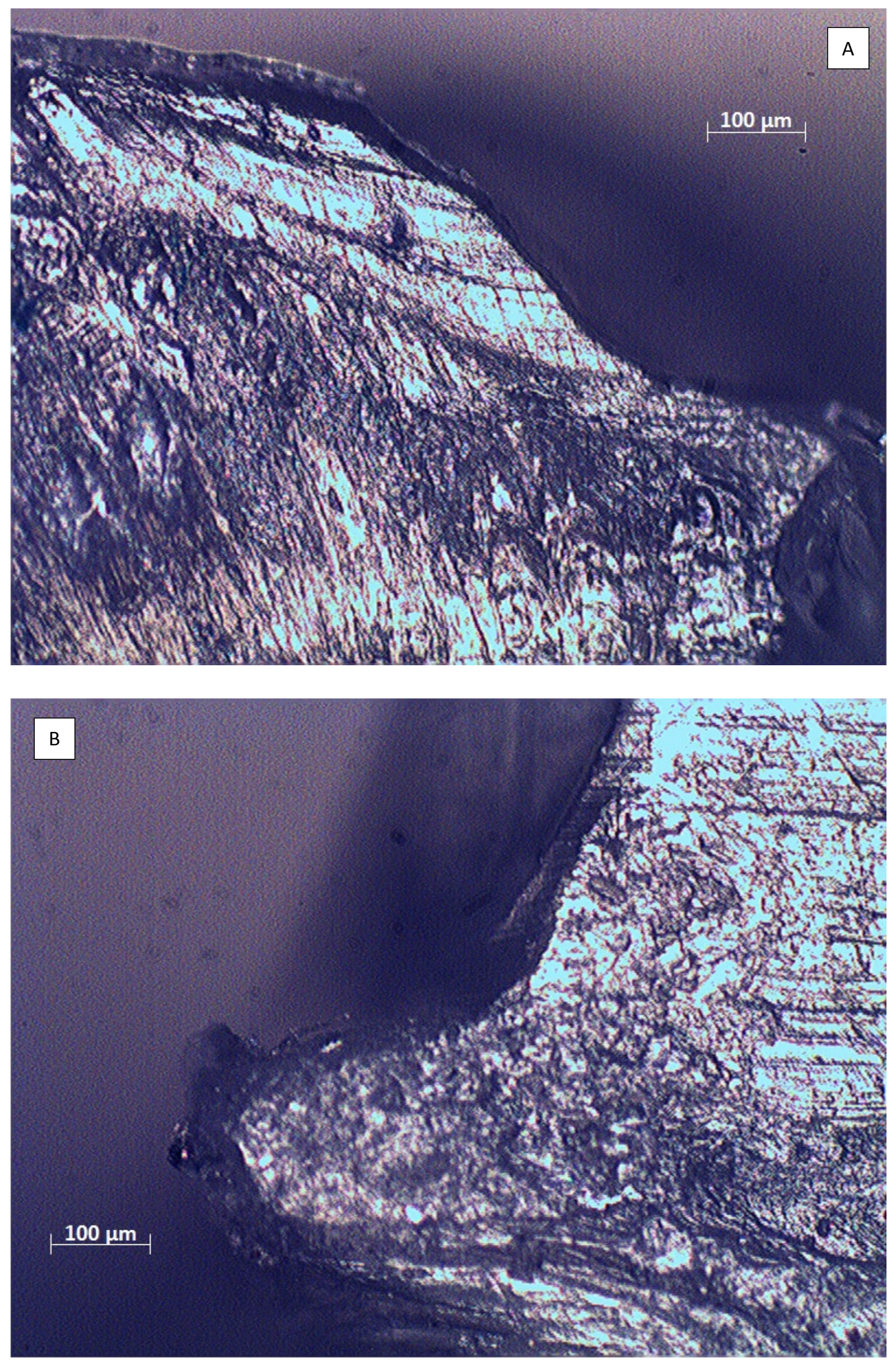


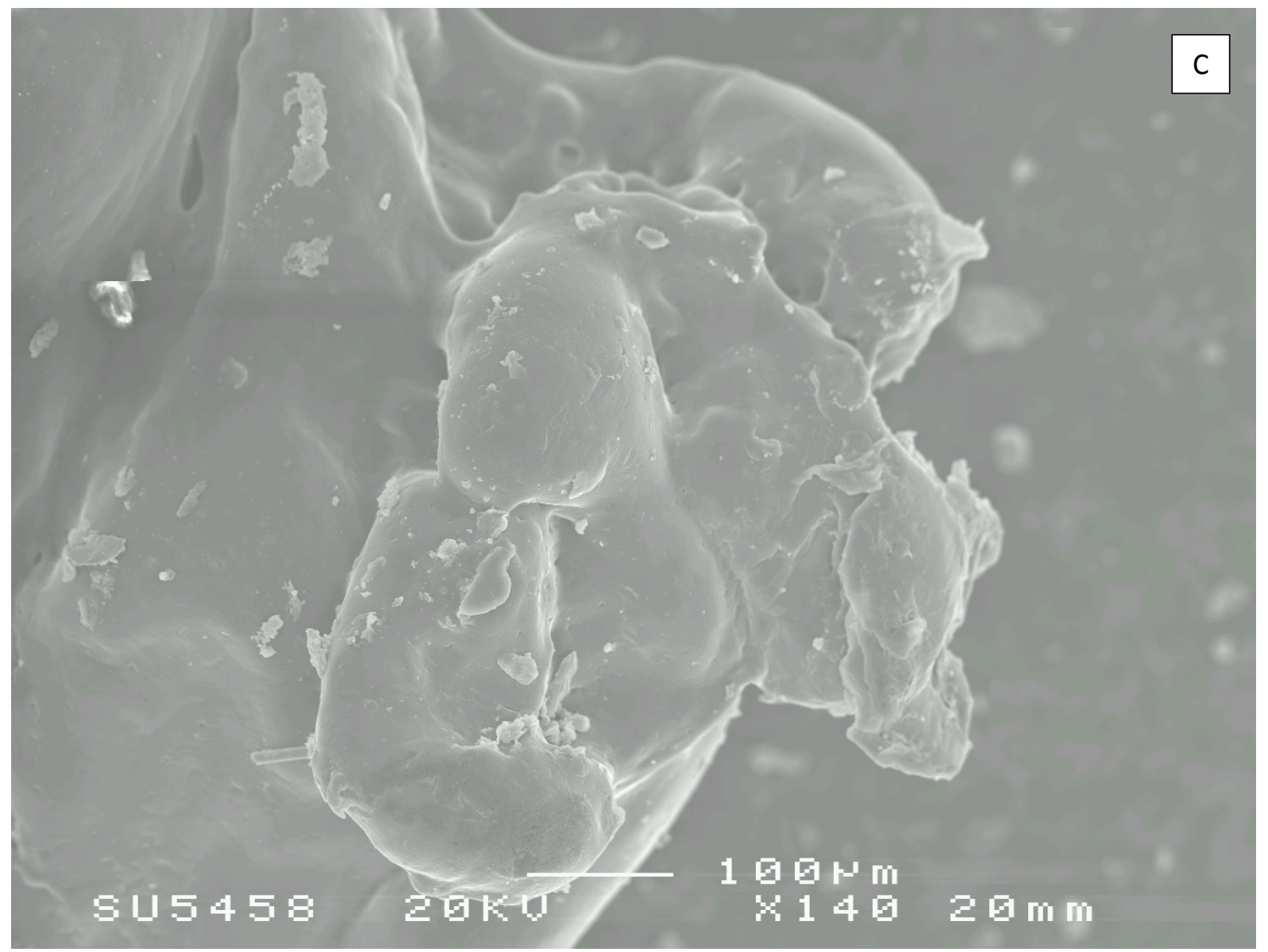

Figure 10.32(A,B\&C) Rock like fractured micro fibril

Presence of horn like fibrils

Horn like fibrils (another form of micro fibrils) was again originated by brittle fracture, as shown in Figure 10.33. 

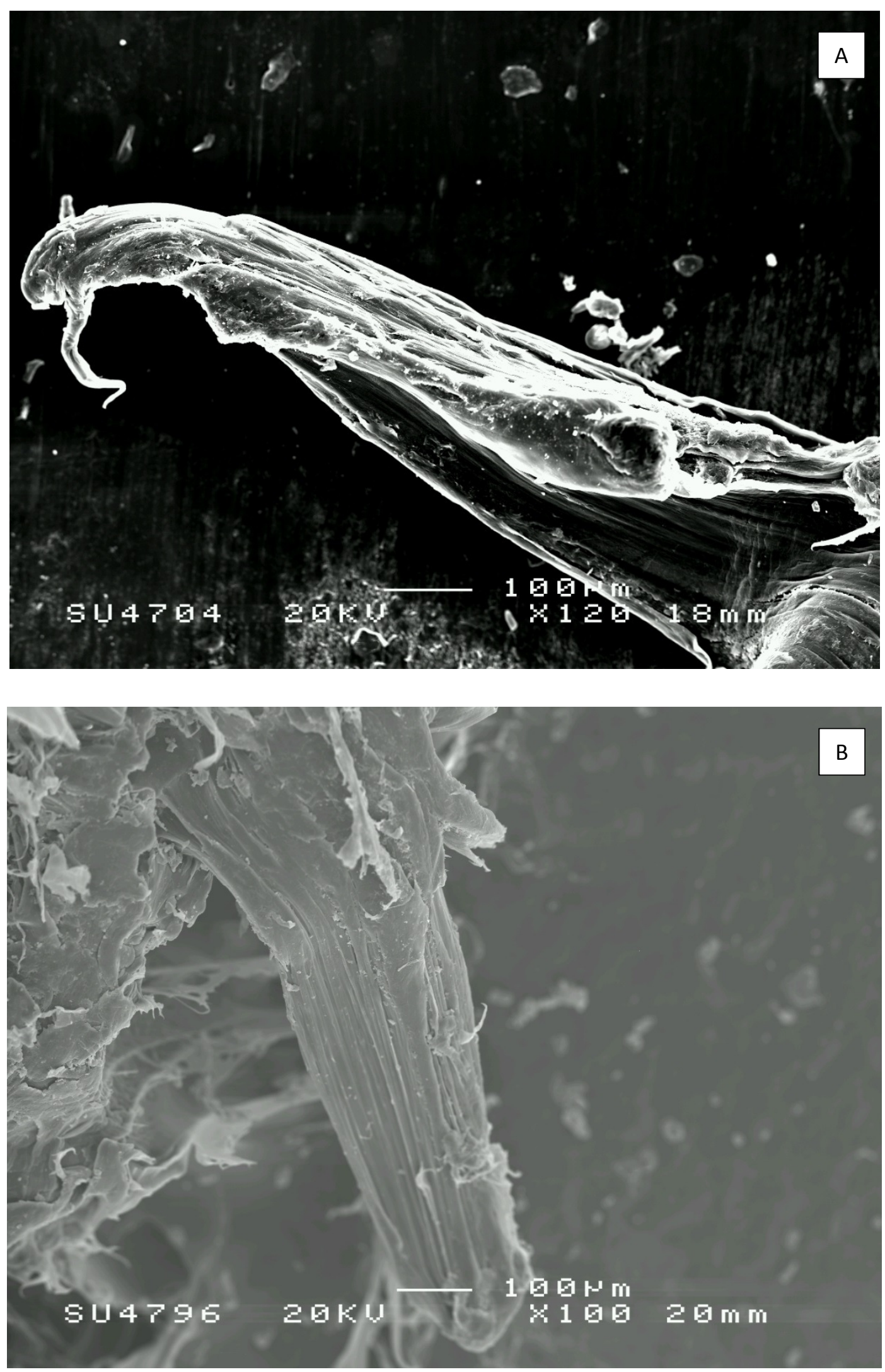

Figure 10.33(A\&B) Horn like fibrils 
Hence hypothesis E for ABS and PP is moderately accepted with the support of fractography.

\section{Effect of pit depth on LSS}

After testing the bond strength, another aspect of fractured surfaces was inspected with unaided eyes for both materials. This was called the pit depth. Pit depth is the penetration of ED into the specimen during welding.

In this study, pit depth at the weld zone was concluded to have direct relationship with the LSS. In order to understand this, let us assume that $1 \mathrm{~mm}$ thickness of parent material is equal to 1000 micro meter layers. Our each sample has $2 \mathrm{~mm}$ of overall thickness. So, $2 \mathrm{~mm}$ will contain 2000 layers of thickness 1 micro meter.

In this research, range of pit depth was found out with vernier calliper using more than 5 samples for both materials. Pit depth of fractured surfaces was fluctuated from 0.00 to 0.80 $\mathrm{mm}$ and from 0.00 to $0.40 \mathrm{~mm}$ mostly uneven for ABS and PP respectively, as shown in Figure 10.34 and Figure 10.35. In other words, maximum pith depth was acquired for $A B S$ after tearing away up to 800 micro layers by a set of weld factors. Maximum number for PP reached up to 400 layers. Weld factors resulting in no weld were not able some times to tear even a single micro layer properly. Hence for those factors, pit depth was assumed to be zero for both ABS and PP.
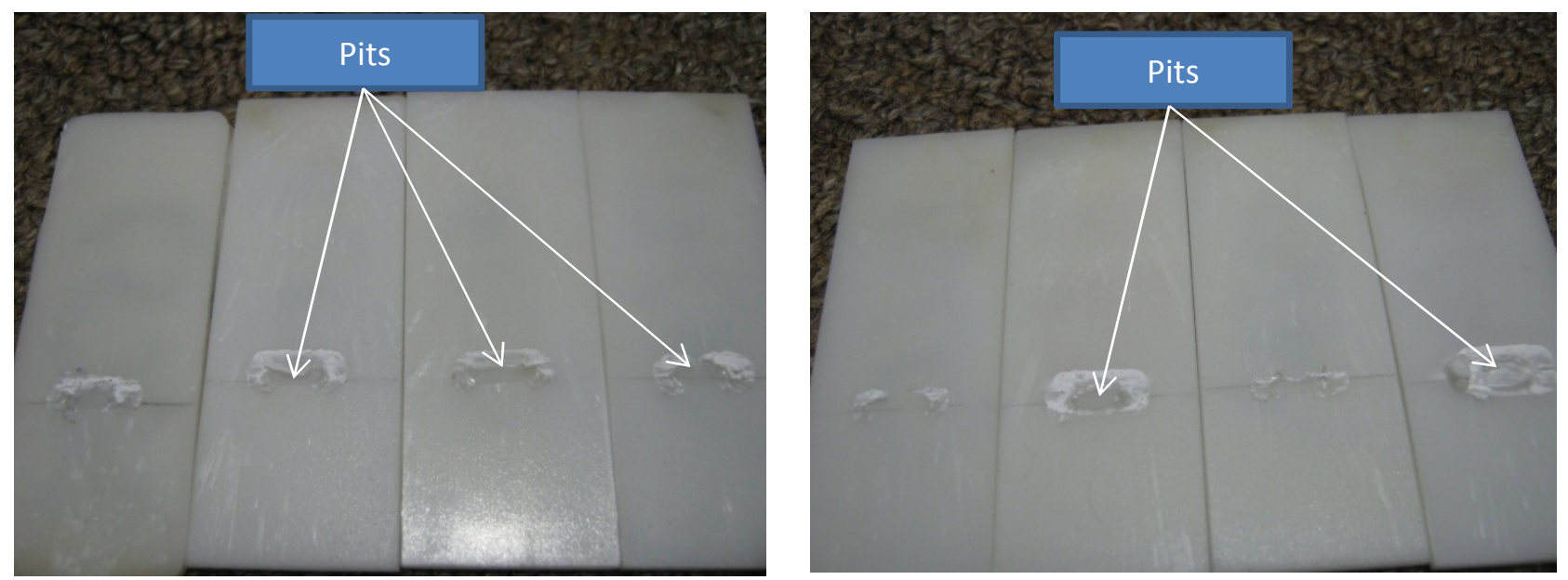

Figure 10.34 Pit depth for ABS 

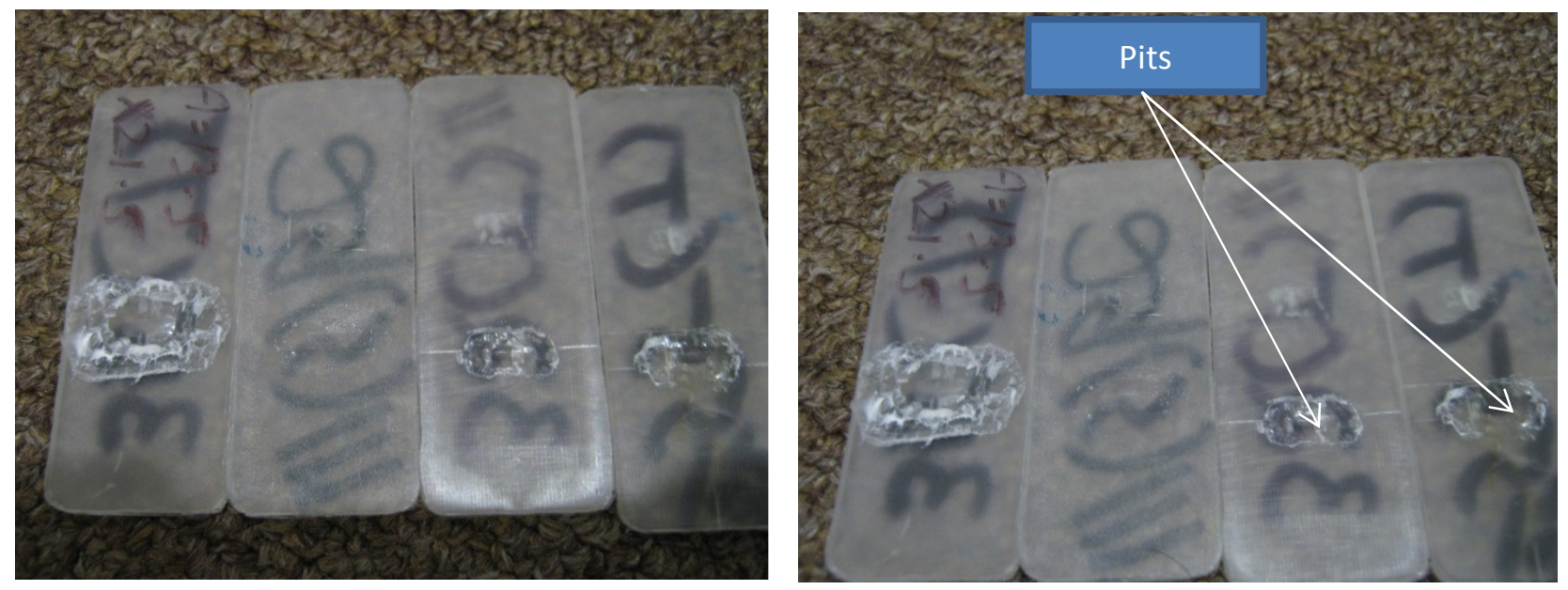

Figure 10.35 Pit depth for PP

\subsection{Summary}

HAZ and fracture analysis were performed to test the hypotheses D\&E respectively for both materials i.e. ABS and PP. Optical microscope (OM) and scanning electron microscope (SEM) were used to study the HAZ and fracture surfaces. Ductile fracture resulted in higher LSS than that of brittle fracture. Fractured surface features were also deliberately investigated for their contribution towards fracture mode. These features included presence of more than one HAZES, air bubbles, blisters, sharp edges, rock \& horn like fractured edges etc. Both hypotheses D\&E were tested by microscopic results. Hypothesis E was verified for both ABS and PP. Although hypothesis D was approved for ABS, it was disproved for PP. 


\section{CHAPTER 11 GENERAL SUMMARY OF HYPOTHESES TESTING}

\subsection{AIM}

Previous chapters involve hypothesizing various interesting results and testing these hypotheses. In this section, a general summary is intended to amalgamate the results from testing those hypotheses helping draw the inclusive picture of their approval or disapproval.

In this study, hypotheses A to $G$ were checked for both ABS \& PP.

\subsection{Hypotheses Testing Methods}

In current study, several state of the art tools were used in order to investigate the hypotheses which were set out at the end of Chapter 6. Differential scanning calorimeter (DSC) was used to obtain the thermal characteristics of ABS and PP and melt flow index (MFI) was measured by MFI tests. High speed video camera (HSVC) was used to determine the collapse of energy directors, finite element analyses were conducted to find out the temperature information at weld interface for both materials and microscopy was used to determine the thickness of heat affect zone (HAZ) and to study the various features on fractured surfaces.

\subsubsection{Hypothesis A}

Hypothesis A declares that "ABS is an amorphous thermoplastic with higher tensile strength than that of PP"

After welding ABS and PP ultrasonically, it was observed that the weld strength of ABS was higher than that of PP at identical factors. Literature has also suggested this because of following reasons:

Base material strengths for $\mathrm{ABS}$ and $\mathrm{PP}$ are $50 \mathrm{MPa}$ and $41 \mathrm{MPa}$ respectively. Literature shows that bond strength has been found up to $25 \%$ and $11 \%$ of base material strengths for both $A B S$ and PP respectively. In present study, these percentages were observed to be $34 \%$ of base ABS material and $14.63 \%$ for that of PP agreeing nearly with literature. Obtained with higher strength of base material, higher LSS was expected for ABS rather than for PP.

However, as well as that, DSC (utilized in this work) showed that the lower softening temperature of $A B S$ was $103{ }^{\circ} \mathrm{C}$ which meant that $A B S$ material started melting and flowing 
at a lower temperature during welding. From DSC results, a sharp and higher melting point of PP was found i.e. $170.55^{\circ} \mathrm{C}$.

FEA results explored that higher temperature regions were obtained for ABS. These high temperature zones achieved the glass transition temperature of $A B S$ more quickly which was necessary in acquiring sufficient bond strength. For PP, the results from FEA suggested us that temperature reached the melting temperature slowly at weld interface resulting in difficulty for PP to make a good joint.

As far as MFI was concerned, this test showed higher value of viscosity for ABS at start i.e. $0.92 \mathrm{~g} / 10$ mins. But thanks to the higher temperature regions discovered by FEA, the ABS fluidity was increased due to the viscosity drop at temperatures more than the softening temperature of $A B S$, thereby resulting in better bond strength. Viscosity of PP was found to be lower (i.e. $14.81 \mathrm{~g} / 10$ mins) differing from that of ABS and an advantage for PP until it exceeded the under melt situation.

So, possibility of gaining high temperature at weld interface explored by FEA, lower softening temperature of ABS which was determined by DSC and viscosity drop of higher $A B S$ viscosity from MFI proved that good bond strength could be obtained with $A B S$.

The danger of acquiring lower temperature states, sharp melting point and lower viscosity for PP justified collectively that chances of difficulty in welding PP were higher until high level of weld factors was utilized (this will be summarized in next paragraphs under hypothesis C). DSC and MFI results backed well the results of FEA. So, MFI, FEA and DSC altogether proved hypothesis A strongly for both ABS \& PP.

\subsection{2. $\quad$ Hypothesis B}

Hypothesis B was deduced in an attempt to state the TRI ED as the best ED for ABS and SEMI ED for PP in attaining higher LSS. It expresses "Volume of TRI ED is less than that of SEMI ED resulting in high concentration of ultrasonic energy"

It was found that higher LSS was further likely with TRI ED for ABS and SEMI ED for PP. Following possible reasons were found to use alternate ED from literature and current experimental work: 
On comparing the TRI and SEMI EDs geometrically, the volume of TRI ED $\left(90 \mathrm{~mm}^{3}\right)$ is less than that of SEMI ED $\left(140.13 \mathrm{~mm}^{3}\right)$. In ultrasonic welding of thermoplastics, heating phenomenon occurs internally predominantly due to molecular friction rather than the surface friction. An improvement in this heating source can increase the efficiency of ultrasonic welding with minimum power input. TRI ED, therefore, is expected to produce more concentrated heating at the weld zone due to its lower volume.

In this research, for $A B S$, this concentration of heat was good and beneficial in achieving higher LSS owing to its amorphous structure, higher loss modulus and lower softening temperature. Because heat concentration broke the random molecular bonds easily to attain the molten state of ABS at weld interface.

However, in the case of PP, this heat concentration did not have a pronounced effect because of its semi-crystalline structure, higher storage modulus and higher softening or melting temperature. Heat concentration could not break the orderly chains of PP. Also according to literature, storage modulus returns a portion of ultrasonic vibrations resulting in lower heat development at weld zone.

In this study, heat affected zone was also analysed and results showed that this was higher for SEMI ED than that for TRI ED using PP. HAZ was again higher for TRI ED than that for SEMI ED after joining ABS i.e. opposite to PP. In other words, by delivering higher heat concentration due to substantial supply of ultrasonic energy at EDs, higher HAZ was obtained; this further resulted in higher LSS.

These results supported this hypothesis. Differences in material structure, viscoelastic and thermal characteristics implied that highly concentrated energy produced a positive effect on $A B S$ and less influence on PP.

Concentrated energy also resulted in localized viscosity drop for ABS which was deemed to be crucial in making a better joint. Lower volume of TRI ED did not generate concentrated supply of heat for PP owing to different material properties and molecular structure of PP.

Based on FEA findings, higher temperature regions were further possible with TRI ED contrasting with those of SEMI ED for ABS. This was again owing to the reason of lower volume of TRI ED and concentrated supply of internal heating at a localized area. 
So, the flow requirements were fulfilled for molten ABS with the assistance of higher temperature regions with TRI ED to develop a weld with higher LSS. From FEA results, high temperature values up to $350{ }^{\circ} \mathrm{C}$ were possible for TRI ED than those for SEMI ED with PP but these values were lower than those of ABS.

Also temperature at weld interface was striving to reach the softening temperature/sharp melting point of PP at low level of weld factors. TRI ED was found to have good molten state by utilizing high level of weld factors. But the heat affected zone was better for SEMI ED than that for TRI ED thereby resulting in higher LSS for PP. Also TRI ED was achieving good molten state for ABS unlike TRI ED for PP.

From literature, SEMI ED has been found to be the best option for ultrasonically welding semi-crystalline thermoplastics in far field configurations [107]. In this research, the volume of SEMI ED was higher than that of TRI ED, hence at high level of weld factors, better supply of molten PP and better flow at weld interface were justifying the suitability of SEMI ED for PP.

Hence FEA and MFI helped approve this hypothesis B for both ABS and PP to some extent. Henceforth SEMI ED was declared the best ED option for PP even in near field ultrasonic welding configurations.

\subsection{3. $\quad$ Hypothesis C}

Hypothesis C proposes that "Welding temperature is more conveniently attainable for ABS owing to its higher loss modulus as compared to PP"

It was detected that welding temperature for ABS was more conveniently achievable than that for PP which suggested us utilizing different factor settings for both ABS and PP. This happened because of following reasons from literature and recent work:

FEA results for ABS displayed possibility of acquiring high temperature values at weld area. Due to availability of higher temperatures for ABS, ABS was easier to weld. Accessibility of right temperature was also guaranteed due to higher loss modulus of ABS. Hence FEA discovered superior heating sections for ABS. Gaining welding temperature for PP was not as easy as for ABS which was determined by FEA results. This was due to the crystalline structure of PP, lower loss modulus of PP, higher sharp melting point of PP, and higher 
storage modulus of PP. As a result, there was also observed a struggle to reach the melting temperature of PP where the phase change was expected to occur. This struggle was found at its peak by setting low level of weld factors. Also from literature, the storage modulus of $\mathrm{PP}$ is responsible for bouncing back a portion of ultrasonic vibrations without raising temperature during welding.

On the other hand, DSC evaluated lower softening temperature for ABS. So, it was also easier to change the phase for $A B S$ at ED from glassy to rubbery during welding ultrasonically. With low level of weld factors, it was difficult to weld PP because welding temperature was not reaching the sharp melting point of PP at weld interface. In other words, this was owing to the truth that softening temperature for PP was comparatively higher than that for ABS.

MFI test found the higher viscosity for $A B S$ than that for PP. Higher viscosity for $A B S$ also prevented the molten ABS from flowing properly on welding. But this problem was resolved with the availability of high temperature values at weld area from FEA results. So, a better bond strength was achieved finally. Fluidity of PP was better than that of ABS due to its lower viscosity which was verified by MFI test. But this fluidity was expected to be lower if PP was not melted properly on welding. After having attained the softening temperature of PP with high level of weld factors, advantage of better flow of PP was always there to get a better bond strength.

Henceforth by utilizing the high levels of weld factors for ABS, an over melt state was also encountered leading to its detrimental effects on ultimate bond strength. This was due to this reason that high level of weld factors for ABS was also considered to be low level of weld factors for PP. In other words, a difference in setting dissimilar level of weld factors was a distinguishing fact between ABS and PP.

This is the point where hypothesis $C$ is different from hypothesis $A$. In other words, hypothesis $\mathrm{C}$ justifies that why are different weld factors settings required for welding both $A B S$ and PP?, Whereas hypothesis $A$ justifies that why does higher bond strength achieve with $\mathrm{ABS}$ rather than with $\mathrm{PP}$ ? 
At the end, this hypothesis C was checked by FEA, DSC and MFI. All three testing methods in combination assisted somehow to approve this hypothesis $C$ for both ABS and PP.

\subsection{4. $\quad$ Hypothesis D}

Hypothesis D states that "EDs made of ABS have higher heat distortion temperature (HDT) \& higher loss modulus than those for PP"

It was noticed that ED from ABS resulted in higher LSS than that from PP at same level of weld factors. Following were the reasons of this difference in LSS values for both ABS and PP:

In this work, FEA, HSVC and microscopy tested this hypothesis.

High speed video camera was used to quantify collapse. For ABS with TRI ED, it was found that higher LSS of 11.34 MPa was achieved at high level of weld factors with collapse of 1.81 $\mathrm{mm}$. Lower LSS of 3.6 MPa was obtained at lower level of weld conditions which gave a collapse of $0.043 \mathrm{~mm}$. For ABS with SEMI ED, LSS and collapse were found to be $7.36 \mathrm{MPa}$ and $0.471 \mathrm{~mm}$ at high level of weld factors \& $6.41 \mathrm{MPa}$ and $0.203 \mathrm{~mm}$ at low level of weld factors respectively.

For PP with TRI ED, by using high level of weld conditions, a collapse of $1.93 \mathrm{~mm}$ was obtained which delivered an LSS of 3.2 MPa and a collapse of $1.66 \mathrm{~mm}$ with no weld at low level of weld factors. For PP with SEMI ED, collapse of $2.03 \mathrm{~mm}$ with $1.11 \mathrm{MPa}$ was achieved by using low level of welding factors.

Thus it could be noticed that at different factors, with TRI ED the collapse of PP was higher than that of $A B S$ by $6.6 \%$ and for almost same weld factors with SEMI ED, the collapse of PP was much higher than that of $A B S$ by $330 \%$. But the relationship between the degree of ED collapse and LSS was direct for ABS and vice versa for PP.

Microscopy showed the effect of HAZ thickness for ABS with both EDs. An inverse relationship between HAZ thickness and LSS was perfectly found. It implied that lower the HAZ thickness, higher would be the LSS. Furthermore, the lower HAZ thickness implied the higher ED collapse. Hence microscopic results seemed to be tied with HSVC results for ABS. 
As far as PP was concerned, with SEMI ED, higher HAZ thickness (lower ED Collapse) was resulted in lower LSS with moderate weld conditions.

Difference between values of collapse for both materials was due to the difference in their material properties e.g. loss modulus and heat distortion temperature. Heat distortion temperature (HDT) is associated with the distortion rate of a material at a particular temperature. And loss modulus is related to the energy dissipated as heat by applying ultrasonic vibrations to the specimens.

For $\mathrm{ABS}$ and $\mathrm{PP}, \mathrm{HDT}$ is $85^{\circ} \mathrm{C}$ and $56^{\circ} \mathrm{C}$ respectively. Similarly, the loss moduli for ABS and PP are $0.42 \mathrm{GPa}$ and $0.32 \mathrm{GPa}$ respectively. Hence these both properties are higher for ABS than those for PP. In other words, energy dissipation as heat is supposed to be higher for $A B S$ creating the molten state of $A B S$ and the viscosity drop simultaneously. Lower loss modulus means lower energy dissipation as heat which is totally insufficient for gaining the molten state of PP. But lower HDT means higher possibility of ED complete collapse for PP. In this research, owing to possibility of not reaching the melting point of PP, the temperature at weld interface was not sufficient to melt properly the ED for low level of weld factors. On the other hand, the temperature near melting point of PP resulted in flattening of ED without actually melting it.

Attainment of molten state of ABS was also guaranteed because of high temperature predictions from FEA results. Hence better bond strength was achieved. But due to higher HDT for ABS, ED needed more heat to acquire its complete collapse. As far as PP was concerned low levels of weld factors were expected to flatten ED without achieving melting point of PP.

Collapse for PP was found to be higher than that for ABS at same weld conditions. Hence higher collapse of ED was observed for PP without having substantial effect on LSS with the low level of weld factors. Thus, a lower LSS was obtained.

Hence the ED collapse was found to be lower for PP than that for ABS at same weld factors which resulted in disproving the hypothesis $D$ until high level of weld factors was used for both materials. 


\subsubsection{Hypothesis E}

Hypothesis E proposes that "Crazing affects bond fracture without influencing fracture mode for ABS and PP"

In this research, it was found that the failure mode (ductile or brittle) for several types of thermoplastics was not dependent on various micro level occurrences e.g. crazing. Crazing was the main reason for fracture in thermoplastics. The failure mode depended on how did the weld interface develop itself on welding? If the weld interface was achieving or approaching the original material properties then a ductile fracture was expected. But if this interface was lacking parent material characteristics then brittle fracture was resulted. LSS testing was required to get an idea of the failure mode.

Ductile type of fracture was deemed to be the failure mode contributing more to achieve higher LSS. In ductile fracture, a clear yield point was observed before rupturing weld for evaluating LSS.

On the other hand, brittle fracture did not result in any yielding and abrupt fracture was achieved rather. This type of failure mode delivered higher LSS but lower than that of ductile fracture.

Both ABS and PP are included in ductile type of thermoplastics. In this work, if a bond broke in ductile manner, it was expected that bond strength was approaching the original material strength. But as far as brittle fracture was concerned, weld looked quite strong apparently. But after testing for LSS, bond strength was found to be lower than that of parent material strength. In other words, brittle mode of fracture did not reveal characteristics of original material.

Crazing was identified microscopically by various other features on the fractured surfaces. These features resulted from either ductile or brittle fracture with crazing as main failure mechanism. For instance, fractography showed the presence of more than one HAZES at the weld interface which was one cause of ductile failure. The presence of sharp edge's intervals, blisters, bubbles, beast mouth micro fibrils, rock like micro fibrils and horn like micro fibrils was produced by brittle fracture. 
In this research, for ABS, it was expected that the ductile fracture achieved LSS up to its maximum value i.e. $17 \mathrm{MPa}$. Brittle fracture resulted in LSS of $10 \mathrm{MPa}$ or less than $10 \mathrm{MPa}$. Similarly for PP, $6 \mathrm{MPa}$ of LSS was achieved by ductile fracture and 3 or less than $3 \mathrm{MPa}$ resulted from brittle fracture.

Detailed discussion on crazing could be found in chapter 10 (section 10.5.1).

Current experimental study showed that three modes of fracture were found for both $A B S$ and PP which affected the bond strength. When the specimens were fractured in ductile manner, higher LSS was expected for both materials, whereas brittle fracture resulted in comparatively lower LSS. When achieving no fracture/failure of ultrasonic bond, the highest LSS was termed as high welding efficiency. When fracture was investigated microscopically, crazing was definitely found there without having effect on the fracture mode. When delivering high welding efficiency, the crazing occurred at a region away from the weld zone. Because ultrasonic bond was unbreakable and fracture occurred near the clamping points of lap shear joint.

So, the crazing was the main cause of fracture without affecting the fracture mode. Hence hypothesis E was approved to a reasonable degree for both ABS and PP.

\subsection{6. $\quad$ Hypothesis F}

Hypothesis F states that "Role of static force is different for ABS due to its higher viscosity and lower glass transition temperature."

As discussed in Hypothesis $A$, it was easier to obtain required weld temperature for ABS due to its amorphous molecular structure. But, once this temperature was acquired, the $A B S$ viscosity fell abruptly which implied that low static force was needed to squeeze it fairly to form a uniform HAZ. So, higher LSS was ultimately gained by low level of static force.

However, for PP, the viscosity was found to be lower but weld temperature was not obtained as easy as with ABS owing to its crystalline structure until high level of static force was utilized. So, once the welding temperature was attained, molten material started flowing. A comparatively bigger HAZ was obtained due to high level of static force resulting sometimes in high welding efficiency. 
Viscosity of both ABS and PP was observed to be dropped down which leaded to improved LSS because of obtaining higher temperature at weld interface. Hence the role of static force was different for $A B S$ due to its higher viscosity. Probable reasons behind this viscosity drop and role of static force were presented here with the support of both literature and current experimental work:

For $A B S$, softening temperature was found to be lower and viscosity was evaluated to be higher by using DSC and MFI respectively. The viscosity of PP was found to be lower which was beneficial in welding PP specimens. Softening temperature for PP was found to be higher from DSC results. Higher viscosity for ABS could originate flow issues in attaining a proper ultrasonic bond. In other words, after acquiring the softening temperature of $A B S$, a phase change was occurred from glassy to rubbery. But at this particular stage, ABS was not having proper flow necessary for good welding zone. Hence a decrement in viscosity was required by any way to attain the proper flow of ABS. This was done by ensuring high temperature at weld zone with high level of welding factors. Over melt condition must be properly taken into considerations when high level of weld factors was used. In other words, higher viscosity of ABS was expected to be reduced to a greater extent by setting high level of weld factors which generated higher temperature regions (from FEA results). For PP, FEA results predicted comparatively lower temperature values at weld interface. There was a problem welding PP ultrasonically in this context. Melting point of PP was either not reached or never exceeded using low level of weld factors. Hence high level of weld factors must be used in order to attain the phase change and melting state of PP. Once the proper molten state of PP was achieved, lower viscosity could result in making a reliable bond.

After achieving the viscosity drop for $A B S$, the next stage was to prevent the molten $A B S$ from leaving the weld zone. This molten ABS required low level of static force in order to protect it from being forced out of the welding area. Hence low level of static force was necessary for ABS. But for PP, high level of static force was, still, required in order to achieve the molten state of PP first.

MFI, FEA and DSC results tested this hypothesis $F$ for ABS and PP in the light of above explanation. These tests seemed to verify the hypothesis $F$ to an acceptable degree. 


\subsubsection{Hypothesis G}

Hypothesis G states that "Almost same resisting area is expected to be produced at weld interface by using higher amplitudes"

In current study, it was observed for both materials that LSS was going to achieve a constant value at higher amplitudes. In other words, both materials strength seemed to attain uniformity at higher amplitudes. For both materials, percent increase in LSS was also found to be lower at higher amplitudes meaning that further increase in LSS was stopped.

Based on literature, viscoelastic heating has a direct relationship with the square of the amplitude (please see the relationship for viscoelastic heating under section 8.3). Hence substantial amount of heat is gained at weld interface by using higher amplitudes. Also amplitude is found to significantly contribute in attaining higher LSS.

For ABS, softening temperature was easily achieved at higher amplitudes and viscosity was also expected to be dropped to make a good bond. Possibility of attaining necessary temperature was also higher at weld interface. Hence a better weld was obtained.

For PP, melting point was easily reached by utilizing higher amplitudes. Lower viscosity of PP was an advantage. And FEA results also backed the convenience of acquiring melting point of PP at higher amplitudes. Therefore, better bond strength was also achieved ultimately.

Applications of higher amplitudes suggested gaining the bonding temperature for ABS and $\mathrm{PP}$, therefore, causing to achieve almost similar resisting area and the shear force for breaking the bond. As a result, uniform bond strength appeared at high amplitude settings.

These results appeared to support this hypothesis $\mathrm{G}$ somehow. This hypothesis was tested by DSC, MFI and FEA. DSC, MFI and FEA proved this hypothesis for ABS to an acceptable degree but for PP, this hypothesis was disproved by finding instability of bond strength in literature. 


\section{CHAPTER 12 CONCLUSION}

Knowledge on welding thermoplastics ultrasonically is fairly limited needing to be improved. The present study involves a comprehensive understanding of the UWP for thermoplastics and the weld factors which affect the weld strength.

Ultrasonic welding of thermoplastics (ABS and PP) has been achieved with new specimen and rig design. A matrix of experimentation has been planned and implemented for UW with various weld conditions. Bond strength can be largely affected by the strain rate. Hence a constant strain rate has been applied to determine weld strength in an attempt to minimize the variation in bond strength's data as much as possible. SEMI and TRI EDs have been used in an effort to find their effects on LSS. There exists a clear difference in setting weld factors for both ABS and PP. ABS needs low level of weld factors differing from PP that requires high level of weld factors. Highest LSS has been possible for ABS with TRI ED and PP with SEMI ED. ABS is easier to weld contrasting with that of PP due to its amorphous type of molecular structure. Owing to higher base material strength (50 MPa) for ABS, bond strength has also been expected to be higher for this type of thermoplastic. Maximum LSS of $17 \mathrm{MPa}$ has been evaluated with ABS i.e. $34 \%$ of the parent material strength. On the other hand, $6 \mathrm{MPa}$ has been calculated for PP that is $14.63 \%$ of the original PP strength.

A GLM statistical analysis has been utilized to acquire meaningful information from the welding results. All weld factors and their possible interactions have been found to be significant after running the GLM analysis for both types of thermoplastics. The degree of significance, to achieve higher LSS, can be categorised by using the F-Ratio which is associated with each weld factor. Based on F-Ratio, weld time has been found to be the most significant factor then ED, amplitude and so on for ABS. Similarly for PP, the most significant factor has again been weld time and then the amplitude etc. That is why; an improved LSS has been achieved at higher weld times. Moreover, weld time has been that weld factor requiring different settings for each $A B S$ and PP. For ABS, this time is $0.9 \mathrm{sec}$ as the maximum owing to its higher effect on LSS and greater than one second for PP in an effort to have good weld strength. This is also owing to different material behaviour (softening temperature, fluidity of material, HDT and dynamic moduli), that different settings of weld factors have been essential in welding both types of thermoplastics. 
Present study has been completed to arrive at deeper analysis on ultrasonic welding of thermoplastics gathering various interesting results. Taken together, these interesting results suggest following vital conclusions:

1. Higher LSS is obtained for ABS than that of PP at same weld conditions.

2. TRI ED is better for getting higher LSS with ABS than that of SEMI ED with PP.

3. $A B S$ requires lower level of weld factors than that of PP resulting in higher LSS.

4. Higher ED collapse leads to higher LSS for ABS but opposite for PP at similar weld factors.

5. Brittle and ductile fractures are observed for welded joints from both ABS and PP.

6. Lower static force is required to achieve higher LSS for ABS.

7. LSS is found to be stable at higher amplitudes for both ABS and PP.

Literature on ultrasonic welding has extensively been reviewed in getting detailed idea of existing knowledge. Then, a range of hypotheses tested has been developed by experimentation.

These hypotheses have been tested by various state of the art experimental techniques e.g. DSC, MFI, HSVC, FEA \& microscopy. These techniques have been used to find the reasons for interesting effects. During a UWP, various technical considerations are needed at the joint interface. These considerations consist of attaining right weld temperature (FEA and DSC cover this), fluidity of molten specimen (MFI determines this), ED collapse (HSVC and microscopy address this), viscoelastic and thermal properties of specimens to be joined. Hence main causes of strong or weak joint comprising low or high level of weld factors, EDs, fracture mechanism and ED collapse etc. have actually been explained by using the above techniques.

Hypothesis A has been verified for the fact that higher LSS is achieved with ABS rather than with PP. Difference in the softening temperature and molecular structure have been the reasons for having difference in LSS for $A B S$ and PP. Lower softening temperature of $A B S$ provides with better chances of complete welding. Hypothesis A suggests that ultrasonic welding must be recommended perfectly for ABS more than that for PP. 
Energy director must be used to obtain desired weld strength. Moreover, geometry of ED has a great impact on bond strength which varies from one material to another material. Hypothesis B focuses on the role of ED geometry in achieving good weld strength. To weld PP, SEMI ED is found as the ideal ED configuration for both near (from current work) as well as far field (from literature) arrangements of ultrasonic welding. Near field and far field weldings must also be recommended with current rig settings. But according to literature, some problems may appear from far field welding of thermoplastics.

Hypothesis $C$ addresses different settings of weld factors for both ABS and PP owing to their dissimilar mechanical (viscoelastic) and thermal properties. In this context, ABS has again been preferred to the PP owing to lower glass transition temperature of ABS. By delivering ultrasonic vibrations to the specimens to be welded, role of complex moduli must be taken into account for its contribution to the bond strength. For instance, ultrasonic vibrations applied to a viscoelastic material result in two components of modulus to be there i.e. in phase and out of phase. In phase component is called as storage modulus and out of phase component is known as loss modulus. Evaluation of these dynamic moduli has been considered essential in a struggle to know the behaviour of thermoplastic material to be welded ultrasonically. In this study, ABS has higher loss modulus than that of PP, whereas ABS has lower storage modulus than that of PP. Joint strength depends largely on the weld temperature development at the weld interface. Due to higher loss modulus and lower softening temperature for ABS, low level of weld factors is set for ABS and opposite for PP. Hence knowledge of various material properties must be acquired before setting the right weld factors for welding the material.

Hypothesis D addresses the effect of ED collapse on LSS for both materials. A direct relationship has been found between ED collapse and LSS for ABS. But ED collapse does not affect LSS for PP when low level of weld factors is used until high level of weld factors is utilized. Also the ED collapse has been found to be lower for PP than that for ABS at the same weld conditions. The difference in the values of ED collapse is associated with the different HDT values for both ABS and PP. Hence this hypothesis appeared to be disproved at low level of weld factors. 
Hypothesis E covers the effect of fracture mode on LSS. Microscopic phenomena have resulted the bond fracture either in ductile or in brittle manner if welded joint is tested for its strength. For instance, crazing is the main cause of bond fracture in thermoplastics and it results in either ductile or brittle fracture which depends upon the weld morphology. Therefore, crazing does not have major impact on the occurrence of failure mode. Each fracture mode has a definite impact on the bond strength.

Hypothesis F suggests that sometimes low level of static force must be required to weld different thermoplastics. Fluidity of material must also be known well before welding in order to decide the levels of factors which are required to join specimens. For instance, if a thermoplastic material has lower viscosity at weld interface during welding then low level of static force is required in an effort to prevent the material from forcing out of the weld interface.

Hypothesis $\mathrm{G}$ covers the finding of previous studies with respect to stability of bond strength for ABS as contrary to PP. Current study proves this hypothesis for ABS but stability of bond strength has also been obtained for PP at higher amplitude. Hence this hypothesis has been disproved for PP.

All the hypotheses proposed seem to work well for both materials except hypothesis D \& G. These hypotheses have been tested by microscopic, DSC, MFI, HSVC \& FEA results.

Ultrasonic welding has been a better welding technique to join various thermoplastics. Ultrasonic welding appears to be flexible in accommodating new specimen design, rig design and range of materials. The mould making activity and welding new materials can be difficult and time consuming. Also using a new thermoplastic material leading to initial experimentation can take considerable time in acquiring desired weld strength. Furthermore, hypotheses testing have produced more confidence and reliance on GLM analysis for both materials. Overall this present work has made a substantial progress in getting more understanding about UWP for thermoplastics.

\subsection{FUTURE WORK AND RECOMMENDATIONS}

Whilst this research has made considerable improvements in our understanding of the UWP for thermoplastics, further investigation can also be recommended in following areas: 
Hypothesis D has been tested by HSVC, FEA and microscopy. These testing tools approve the hypothesis D for ABS and PP except for PP with low level of weld factors. Hence hypothesis D does not hold true at low level of weld factors for PP. Further work should investigate this. Other tools must also be used to test this hypothesis e.g. thermal imaging camera can be used to identify the temperature variation at weld zone.

In fact, ED region has been a vital part of specimen design. In this context, right factor settings, important material properties e.g. HDT, complex moduli, ED geometry, specimen design and shape of sonotrode have affected the ED collapse which leads to ultimately affect the LSS. Future work can examine various ED sizes and geometries.

Thickness of HAZ must be further researched in order to develop a certain relationship between HAZ thickness and LSS. In doing so, different weld factors with suitable sample size must be utilized for microscopic examination. This microscopic study must not be restricted to just optical and scanning electron microscopes. X-Ray analysis, atomic force microscope and other similar microscopic tools may be used because these give information of molecular orientation at HAZ and surface morphology.

Hypothesis $G$ has also been disproved contradicting the stable LSS for PP at higher amplitudes in present study. This hypothesis addresses an important characteristic of ultrasonic vibration i.e. amplitude. Hence sophisticated tools must be used to appropriately monitor the effect of higher amplitudes on LSS.

Also various GLM results have not been included in the main body of thesis and are presented in appendices C\&D at the end. These include the parameter estimates and prediction of LSS in the form of a general linear equation (slope-intercept form of linear equation) [200] by considering all the factors of ultrasonic welding, as presented in appendix D. Hence a researcher has also been allowed to predict an LSS with the support of main as well as interaction of main factors without actually doing ultrasonic welding. This linear model for both $A B S$ and PP may be validated in future. Henceforth a considerable contribution has been ensured to the existing knowledge of joining thermoplastics ultrasonically. 
Current work can be expanded to join wider range of polymers e.g. high performance thermoplastics i.e. amorphous (PEI, PSU, PPSU, PPE, etc.) and semi crystalline (PEEK, PPS, PA, PBT etc.). This will further help recognize the potentials of present welding scenario with proper industrial prospective.

Further research can also examine the potential of present UWP for high performance composites, thermoset composites and dissimilar materials. 


\section{REFERENCES}

[1] (Accessed 24 January 2015). Overview of Ultrasonic Welding - Ultrasonic welding for plastics. Available: http://www.weldmyworld.com/blog/overview-of-ultrasonic-welding.html

[2] (Accessed 24 January 2015). Ultrasonic FAQ's - Ultrasonic Technology. Available: http://www.sonobondultrasonics.com/more-info/ultrasonic-faqs

[3] (Accessed 23 January 2015). Ultrasonic Welding Machine utilizes rotary technology Ultrasonic fabric welder. Available: http://news.thomasnet.com/fullstory/UltrasonicWelding-Machine-utilizes-rotary-technology-543973

[4] (Accessed 24 January 2015). Ultrasonic Hand Welders. Available: http://www.xfurth.com/ultrasonic hand welders.html

[5] (Accessed 5th November 2014). Automatic Hand Held Ultrasonic Welder For Non-woven Fabric Sewing / Clothing - Good Quality Hand Held Ultrasonic Welder Products. Available: http://www.ultrasonic-weldingmachines.com/china-

automatic hand held ultrasonic welder for non woven fabric sewing clothing1827441.html

[6] J. W. Nicholson, The Chemistry of Polymers, 4th ed. Cambridge: The Royal Society of Chemistry, 2012.

[7] (Accessed 24 January 2015). TECHNICAL NEWSLETTER - Focus on: PEEK. Available: http://www.zeusinc.com/sites/default/files/files/Misc\%20Files/FocusPEEK Zeus.pdf

[8] D. T. G. a. G. F. M. Linda C. Sawyer, Polymer Microscopy, Third ed. New York: Springer, 2008.

[9] (Accessed 23 January 2015). GRAFUNET - Application of plastics in furniture manufacturing. Available: http://grafunkt.com/products/peach-chair/

[10] (Accessed 23 January 2015). THERMOPLASTICS VS THERMOSETTING PLASTICS - Basic structures of thermoplastics and thermosets. Available: http://www.recycledplastic.com/plastics/thermoplastics-vs-thermosetting-plastics/

[11] (Accessed 23 January 2015). Polymer Structure and Synthesis - Linear, branched and crosslinked plastics. Available: http://www.globalspec.com/reference/64225/203279/1-2polymer-structure-and-synthesis

[12] (Accessed 23 January 2015). Thermoplastic polymers - Basic chain structures of amorphous and semicrystalline thermoplastics. Available: http://www.whatischemistry.unina.it/en/thermoplastic.html

[13] (Accessed 24 January 2015). Primary Differences between Amorphous \& Semi-Crystalline Thermoplastics. Available: https://plasticsnetwork.wordpress.com/2007/12/26/primarydifferences-between-amorphous-semi-crystalline-thermoplastics/

[14] A. Yousefpour, M. Hojjati, and J. P. Immarigeon, "Fusion bonding/welding of thermoplastic composites," Journal of Thermoplastic Composite Materials, vol. 17, pp. 303-341, Jul 2004.

[15] (Accessed 23 January 2015). Fundamentals of adhesive bonding - Configuration of adhesive bond.

Available:

http://www.substech.com/dokuwiki/doku.php?id=fundamentals of adhesive bonding

[16] (Accessed 24 January 2015). ADHESIVES, GLUES AND SEALANTS - Disadvantages and drawbacks of the adhesives. Available: http://www.adhesiveandglue.com/adhesivedisadvantage.html

[17] (Accessed 23 January 2015). Heating of plastics with microwaves - Microwave heating effect. Available:

http://www.ict.fraunhofer.de/en/comp/pe/mp/heating plastics microwaves.html

[18] (Accessed 23 January 2015). Advanced Thermoplastic Composites - Resistance Welding. Available: http://www.composites.nl/innovation-technology/welding/

[19] (Accessed 24 January 2015). Hot Plate Plastic Welding Machine Available: http://www.diytrade.com/china/pd/3718083/Hot Plate Plastic Welding Machine.html 
[20] (Accessed 24 January 2015). Case Study: Plastics Heat Staking and Welding - Infrared welding for plastics. Available: http://noblelight.net/resources/infrared case studies/heat staking and welding.shtml

[21] (Accessed 23 January 2015). Plastic Welding - Hot Gas Welding. Available: http://www.greweco.com/fabrication.html

[22] (Accessed 24 January 2015). Impact of Lasers in Plastics Manufacturing - Laser welding. Available: $\quad$ http://www.laserstoday.com/2013/09/impact-of-lasers-in-plasticsmanufacturing/

[23] (Accessed 24 January 2015). Vibration Welders - Linear vibration welding. Available: http://www.dukane.com/us/PVI VibeWeld.htm

[24] (Accessed 24 January 2015 ). Choosing the Best Plastic Joining Process for the Application Spin welding of plastics. Available: http://www.plasticsdecorating.com/articlesdisplay.asp?ID=98

[25] (Accessed 24 January 2015). Finite Element Modeling of Friction Stir Welding - Stir welding. Available: http://anisotropy.mae.cornell.edu/dplab/projects/Projects.html

[26] (Accessed 24 January 2015). Ultrasonic welding machine. Available: http://www.usinenouvelle.com/industry/sirius-electric-8768/ultrasonic-welding-machinep38626.html

[27] (Accessed 24 January 2015). What are the benefits of microwave welding of thermoplastics FAQs. Available: http://www.twi-global.com/technical-knowledge/faqs/process-faqs/faqwhat-are-the-benefits-of-microwave-welding-of-thermoplastics/

[28] (Accessed 24 January 2015). Resistance welding benefits and limitations. Available: https://zhweijun.wordpress.com/2010/07/01/resistance-welding-benefits-and-limitations/

[29] (Accessed 24 January 2015). FRIMO Infrared welding. Available: http://www.frimo.com/en/products/frimo-technologies/joining/infrared-welding.html

[30] (Accessed 24 January 2015). Friction Stir Welding. Available: http://www.mtiwelding.com/friction-stir-welding.html

[31] (Accessed 24 January 2015). Manufacturing Processes - Friction Welding Metal \& Plastic. Available: http://technoman.hubpages.com/hub/Friction-Welding

[32] (Accessed 24 January 2015). Friction Stir Welding - Advantages of Friction Welding. Available: http://www.azom.com/article.aspx?ArticlelD=1170

[33] D. G. W. C.J. Aloisio, E.E. Whetsel, "A simplified thermoviscoelastic analysis of the ultrasonic bonding," ed. Brookfield, CT: SPE Technical Papers, 1972, pp. 445-451.

[34] D. E. a. L. J. Bond, Ultrasonics, Third ed. Boca Raton London New York: CRC Press (Taylor \& Francis Group), 2012.

[35] J. D. N. Cheeke, Fundamentals and Applications of Ultrasonic Waves. Boca Raton CRC Press LLC, 2002.

[36] (Accessed 24 January 2015). Ultrasonic welding of injection moulded components - Part 2. Component design and weld parameters - Near and far-field ultrasonic welding. Available: http://www.twi-global.com/technical-knowledge/job-knowledge/ultrasonic-welding-ofinjection-moulded-components-part-2-component-design-and-weld-parameters-062/

[37] K. S. Suresh, A. R. Rani, K. Prakasan, and R. Rudramoorthy, "Modeling of temperature distribution in ultrasonic welding of thermoplastics for various joint designs," Journal of Materials Processing Technology, vol. 186, pp. 138-146, May 2007.

[38] (Accessed 25 January 2015). Ultrasonic welding of injection moulded components - TWI UK. Available: http://www.twi-global.com/technical-knowledge/job-knowledge/ultrasonicwelding-of-injection-moulded-components-part-1-process-and-equipment-061/

[39] (Accessed 25 January 2015). 10 - Assembly Techniques - Category II Welding, Adhesive Bonding. Available: http://plastics.dupont.com/plastics/pdflit/europe/design/L12565 10.pdf

[40] J. Devine, "Ultrasonic Plastics Welding Basics," ed. New York: Welding Journals, 2001, pp. 2933. 
[41] (Accessed 24 January 2015). Choosing the Best Plastic Joining Process for the Application Ultrasonic welding equipment.

Available: http://www.plasticsdecorating.com/articlesdisplay.asp?ID $=98$

[42] C. J. Nonhof and G. A. Luiten, "Estimates for process conditions during the ultrasonic welding of thermoplastics," Polymer Engineering and Science, vol. 36, pp. 1177-1183, May 1996.

[43] J. Tsujino, M. Hongoh, and T. Ueoka, "Welding characteristics of $40 \mathrm{kHz}$ ultrasonic plastic welding system using fundamental and higher resonance frequency vibrations," 2002 leee Ultrasonics Symposium Proceedings, Vols 1 and 2, pp. 699-702, 20022002.

[44] V. N. Khmelev, A. N. Slivin, R. V. Barsukov, S. N. Tsyganok, Savin, II, A. V. Shalunov, et al., Development of the new principle of batching of energy at ultrasonic welding and creation of the equipment for connection of thermoplastic materials, 2006.

[45] I. F. Villegas and H. E. N. Bersee, "Ultrasonic welding of advanced thermoplastic composites: An investigation on energy-directing surfaces," Advances in Polymer Technology, vol. 29, pp. 112-121, 2010.

[46] H. Tsujino, M. Hongoh, M. Yoshikuni, H. Hashii, and T. Ueoka, "Welding characteristics of 27, 40 and $67 \mathrm{kHz}$ ultrasonic plastic welding systems using fundamental- and higher-resonance frequencies," Ultrasonics, vol. 42, pp. 131-137, Apr 2004.

[47] R. V. Barsukov, A. N. Slivin, V. N. Khmelev, S. N. Tsyganok, A. V. Shalunov, Savin, II, et al., "An equipment for continuous joint ultrasonic welding and cutting of various thermoplastic polymeric materials," 2004 International Siberian Workshops and Tutorials on Electron Devices and Materials, Edm 2004, Proceedings, pp. 197-201, 20042004.

[48] (Accessed 24 January 2015). KAER SONIC POWER - Geogrid Ultrasonic Welding Production Line. Available: http://www.kaer-welder.com/product/viewproduct4969ec6937567.html

[49] T. U. a. K. K. Jiromaru TSUJINO, "RECENT ADVANCE IN ULTRASONIC WELDING OF METAL AND PLASTICS," ed: IEEE ULTRASONICS SYMPOSIUM, 1985, pp. 557-562.

[50] V. N. Khmelev, A. N. Slivin, A. D. Abramov, and leee, "Model of process and calculation of energy for a heat generation of a welded joint at ultrasonic welding polymeric thermoplastic materials," Edm 2007: 8th International Workshop and Tutorials on Electron Devices and Materials, pp. 316-322, 20072007.

[51] J. T. a. T. UEOKA, "ULTRASONIC BUTT WELDING OF ALUMINUM, ANTICORROSIVE ALUMINUM AND COPPER PLATE SPECIMENS," ed: IEEE ULTRASONICS SYMPOSIUM, 1988, pp. 493-496.

[52] (Accessed 24 January 2015). Common joint types - Various welded joints. Available: http://www.conceptdraw.com/examples/sketches-of-various-welding-joints-in-engineering

[53] J. Tsujino, "Recent developments of ultrasonic welding," 1995 leee Ultrasonics Symposium Proceedings, Vols 1 and 2, pp. 1051-1060, 19951995.

[54] S. F. Ling, X. Li, Z. Sun, and Asme, "Process Analysis for Ultrasonic Welding of Thermoplastics," Imece2008: Proceedings of the Asme International Mechanical Engineering Congress and Exposition, Vol 4, pp. 251-256, 20092009.

[55] A. SHOH, "Welding of thermoplastics by ultrasound," ed, 1976, pp. 209-217.

[56] Y. Huang, "Study on Design of Welding-joint and Structure of Welding Based on Ultrasonic Welding Technology," 1-2, 2012.

[57] Y. Gao and C. Doumanidis, "Mechanical analysis of ultrasonic bonding for rapid prototyping," Journal of Manufacturing Science and Engineering-Transactions of the Asme, vol. 124, pp. 426-434, May 2002.

[58] Y. W. e. al., "Proposal of a new ultrasonic welding technique for thermoplastic polymer," vol. 83-86, ed, 2010, pp. 1129-1134.

[59] K. M. Shu, C. S. Chang, W. J. Chuang, S. I. Wang, and Y. Y. Jang, "Study on Application of Taguchi Method to Ultrasonic-Aided Spin Welding of Heterogeneous Plastic Materials," Advances in Abrasive Technology Xiii, vol. 126-128, pp. 381-387, 20102010. 
[60] C. Y. Wu, A. Benatar, and A. Mokhtarzadeh, "COMPARISON OF ULTRASONIC WELDING AND VIBRATION WELDING OF THERMOPLASTIC POLYOLEFIN," Welding in the World, vol. 56, pp. 69-75, Jan-Feb 2012.

[61] (Accessed 24 January 2015). VIBRATION WELDERS - Vibration welding process Available: http://www.rincoindia.com/main/page products vibration welders.html

[62] F. Haddadi, "Rapid intermetallic growth under high strain rate deformation during high power ultrasonic spot welding of aluminium to steel," Materials \& Design, vol. 66, pp. 459472, Feb 52015.

[63] M. Shakil, N. H. Tariq, M. Ahmad, M. A. Choudhary, J. I. Akhter, and S. S. Babu, "Effect of ultrasonic welding parameters on microstructure and mechanical properties of dissimilar joints," Materials \& Design, vol. 55, pp. 263-273, Mar 2014.

[64] C. Q. Zhang, J. D. Robson, O. Ciuca, and P. B. Prangnell, "Microstructural characterization and mechanical properties of high power ultrasonic spot welded aluminum alloy AA6111-TiAI6V4 dissimilar joints," Materials Characterization, vol. 97, pp. 83-91, Nov 2014.

[65] A. Levy, S. Le Corre, and I. F. Villegas, "Modeling of the heating phenomena in ultrasonic welding of thermoplastic composites with flat energy directors," Journal of Materials Processing Technology, vol. 214, pp. 1361-1371, Jul 2014.

[66] N. Stoehr, B. Baudrit, E. Haberstroh, M. Nase, P. Heidemeyer, and M. Bastian, "Ultrasonic Welding of Plasticized PLA Films," Journal of Applied Polymer Science, vol. 132, Jan 202015.

[67] (Accessed 24 January 2015). Viscoelasticity - Polymer viscoelasticity with an elastic spring and a viscous dashpot. Available: http://www.gfz-potsdam.de/en/research/organizationalunits/departments-of-the-gfz/department-1/earth-system-modelling/topics/ice-sheet-andsolid-earth-dynamics/solid-earth-dynamics/

[68] E. H. LEE, "Special Issue on Rheology of Polymers," vol. 27, ed, 1956, pp. 665-672.

[69] F. Bueche, "THE VISCOELASTIC PROPERTIES OF PLASTICS," Journal of Chemical Physics, vol. 22, pp. 603-609, 19541954.

[70] L. M. Nicholson, K. S. Whitley, and T. S. Gates, "The role of molecular weight and temperature on the elastic and viscoelastic properties of a glassy thermoplastic polyimide," International Journal of Fatigue, vol. 24, pp. 185-195, Feb-Apr 2002.

[71] V. S. Chevali, D. R. Dean, and G. M. Janowski, "Flexural creep behavior of discontinuous thermoplastic composites: Non-linear viscoelastic modeling and time-temperature-stress superposition," Composites Part a-Applied Science and Manufacturing, vol. 40, pp. 870-877, Jul 2009.

[72] W. G. F. Sengers, P. Sengupta, J. W. M. Noordermeer, S. J. Picken, and A. D. Gotsis, "Linear viscoelastic properties of olefinic thermoplastic elastomer blends: melt state properties," Polymer, vol. 45, pp. 8881-8891, Dec 92004.

[73] J. S. Lyons, "Linear viscoelastic analysis of the room-temperature creep behavior of glassreinforce aromatic and aliphatic thermoplastics," Polymer Testing, vol. 22, pp. 545-551, Aug 2003.

[74] P. Dasappa, P. Lee-Sullivan, X. Xiao, and P. H. Foss, "Tensile Creep of a Long-Fiber Glass Mat Thermoplastic Composite. I. Short-Term Tests," Polymer Composites, vol. 30, pp. 1146-1157, Aug 2009.

[75] P. Dasappa, P. Lee-Sullivan, X. Xiao, and P. H. Foss, "Tensile Creep of a Long-Fibre Glass Mat Thermoplastic (GMT) Composite. II. Viscoelastic-Viscoplastic Constitutive Modeling," Polymer Composites, vol. 30, pp. 1204-1211, Sep 2009.

[76] T. B. Van Erp, C. T. Reynolds, T. Peijs, J. A. W. Van Dommelen, and L. E. Govaert, "Prediction of Yield and Long-Term Failure of Oriented Polypropylene: Kinetics and Anisotropy," Journal of Polymer Science Part B-Polymer Physics, vol. 47, pp. 2026-2035, Oct 152009.

[77] (Accessed 24 January 2015). Polypropylene (PP) strapping tape. Available: http://www.somi.lt/en/Products/packing-materials/strapping-tapes/polypropylene-ppstrapping-tape/ 
[78] F. Lionetto and A. Maffezzoli, "Polymer Characterization by Ultrasonic Wave Propagation," Advances in Polymer Technology, vol. 27, pp. 63-73, Sum 2008.

[79] Y. C. Lu and D. M. Shinozaki, "Temperature Dependent Viscoelastic Properties of Polymers Investigated by Small-Scale Dynamic Mechanical Analysis," Experimental Mechanics, vol. 50, pp. 71-77, Jan 2010.

[80] J. Wang, G. Liang, W. Zhao, and Z. Zhang, "Viscoelastic, thermal and mechanical properties of dicyclopentadiene bisphenol diyanate ester/epoxy co-polymers," Polymer Bulletin, vol. 57, pp. 945-952, Oct 2006.

[81] W. Brostow and H. E. H. Lobland, "Predicting wear from mechanical properties of thermoplastic polymers," Polymer Engineering and Science, vol. 48, pp. 1982-1985, Oct 2008.

[82] (Accessed 24 January 2015). Mechanical Analysis - DMA. Available: http://www.perkinelmer.com/catalog/category/id/mechanical\%20analysis\%20tma\%20dma

[83] C. Gauthier, J. M. Pelletier, Q. Wang, and J. J. Blandin, "Viscoelastic and viscoplastic properties of bulk metallic glasses - Comparison with oxide glasses and amorphous polymers," Journal of Non-Crystalline Solids, vol. 345, pp. 469-472, Oct 152004.

[84] S. K. Yoshinobu ISONO, Toshio KASE, "Nonlinear viscoelastic properties and change in entanglement structure of linear polymers 2. Double step large shearing deformations at moderately long time-scale," vol. 31, ed, 2003, pp. 201-206.

[85] M. Horio, S. Onogi, C. Nakayama, and K. Yamamoto, "VISCOELASTIC PROPERTIES OF SEVERAL HIGH POLYMERS," Journal of Applied Physics, vol. 22, pp. 966-970, 19511951.

[86] R. R. Mocellini, O. A. Lambri, C. L. Matteo, and P. A. Sorichetti, "Dielectric properties and viscoelastic response in two-phase polymers," leee Transactions on Dielectrics and Electrical Insulation, vol. 15, pp. 982-993, Aug 2008.

[87] E. van Ruymbeke, R. Keunings, and C. B. Bailly, "Prediction of linear viscoelastic properties for polydisperse mixtures of entangled star and linear polymers: Modified tube-based model and comparison with experimental results," Journal of Non-Newtonian Fluid Mechanics, vol. 128, pp. 7-22, Jun 12005.

[88] V. K. Stokes, "Experiments on the hot-tool welding of three dissimilar thermoplastics," Polymer, vol. 39, pp. 2469-2477, Jun 1998.

[89] (Accessed 24 January 2015). Hot Plate Weld Tooling. Available: http://www.forwardtech.com/plastic-assembly/hot-plate-welders/hot-plate-weld-tooling

[90] P. Yarlagadda and T. C. Chai, "An investigation into welding of engineering thermoplastics using focused microwave energy," Journal of Materials Processing Technology, vol. 74, pp. 199-212, Feb 1998.

[91] X. Y. Dai and P. J. Bates, "Mechanical properties of vibration welded short- and long-glassfiber-reinforced polypropylene," Composites Part a-Applied Science and Manufacturing, vol. 39, pp. 1159-1166, 20082008.

[92] S. Strand, "JOINING PLASTICS - CAN FRICTION STIR WELDING COMPETE?," ed, 2003, pp. 321326.

[93] (Accessed 24 January 2015). Friction Stir Welding. Available: http://www.frictionstirlink.com/desc.html

[94] V. Kagan, "Optimized mechanical performance of welded and molded butt joints: Part I Similarities and differences," Journal of Reinforced Plastics and Composites, vol. 22, pp. 773784, 20032003.

[95] V. Kagan, "Optimized mechanical performance of welded and molded butt joints: Part II Weld and knit lines integrity," Journal of Reinforced Plastics and Composites, vol. 22, pp. 867-879, 20032003.

[96] H. T. Sanchez, P. Sanchez, and M. Estrems, "SCADA system improvement in PVC high frequency plastic welding," International Journal of Advanced Manufacturing Technology, vol. 40, pp. 84-94, Jan 2009. 
[97] (Accessed 24 January 2015). High Frequency Welding Machine - Arm Hanging type. Available:

hg.com.tw/front/bin/ptdetail.phtml?Part=Boomtype\&Category=715

[98] S. H. McKnight, S. T. Holmes, J. W. Gillespie, C. L. T. Lambing, and J. M. Marinelli, "Scaling issues in resistance-welded thermoplastic composite joints," Advances in Polymer Technology, vol. 16, pp. 279-295, Win 1997.

[99] S. J. Liu and I. T. Chang, "Optimizing the weld strength of ultrasonically welded nylon composites," Journal of Composite Materials, vol. 36, pp. 611-624, 20022002.

[100] V. N. Khmelev, A. N. Slivin, A. D. Abramov, S. V. Levin, and U. Novosibirsk State Tech, "Weld Strength Test of Thermoplastics Obtained by Ultrasonic Welding," Edm 2008: International Workshop and Tutorials on Electron Devices and Materials, Proceedings, pp. 227-230, 2008 2008.

[101] V. N. Khmelev, A. N. Slivin, A. D. Abramov, and U. Novosibirsk State Tech, "Research of Parameter Influence of Ultrasonic Welding Process on Conjuncture of Polymeric Thermoplastic Materials," Edm 2008: International Workshop and Tutorials on Electron Devices and Materials, Proceedings, pp. 272-278, 20082008.

[102] H. P. C. DANIELS, "ULTRASONIC WELDING," ed, 1965, pp. 190-196.

[103] J. N. Antonevich, "ULTRASONIC WELDING EQUIPMENT," Proceedings of the Institute of Radio Engineers, vol. 47, pp. 487-487, 19591959.

[104] J. Tsujino, T. Ueoka, I. Watanabe, Y. Kimura, T. Mori, K. Hasegawa, et al., "NEW METHODS OF ULTRASONIC METAL WELDING," leee 1993 Ultrasonics Symposium Proceedings, Vols 1 and 2, pp. 405-410, 19931993.

[105] G. Jandali and P. K. Mallick, "Vibration welding of continuous-fiber thermoplastic matrix composites," Journal of Thermoplastic Composite Materials, vol. 17, pp. 343-358, Jul 2004.

[106] F. Balle and D. Eifler, "Statistical test planning for ultrasonic welding of dissimilar materials using the example of aluminum-carbon fiber reinforced polymers (CFRP) joints," Materialwissenschaft Und Werkstofftechnik, vol. 43, pp. 286-292, Apr 2012.

[107] Y. K. Chuah, L. H. Chien, B. C. Chang, and S. J. Liu, "Effects of the shape of the energy director on far-field ultrasonic welding of thermoplastics," Polymer Engineering and Science, vol. 40, pp. 157-167, Jan 2000.

[108] S. J. Liu, I. T. Chang, and S. W. Hung, "Factors affecting the joint strength of ultrasonically welded polypropylene composites," Polymer Composites, vol. 22, pp. 132-141, Feb 2001.

[109] M. R. Rani, K. Prakasan, and R. Rudramoorthy, "STUDY OF DIFFERENT JOINTS FOR ULTRASONIC WELDING OF SEMICRYSTALLINE POLYMERS," Experimental Techniques, vol. 33, pp. 36-42, Jul-Aug 2009.

[110] S. J. Liu, W. F. Lin, B. C. Chang, G. M. Wu, and S. W. Hung, "Optimizing the joint strength of ultrasonically welded thermoplastics," Advances in Polymer Technology, vol. 18, pp. 125-135, Sum 1999.

[111] R. M. Rani, K. S. Suresh, K. Prakasan, and R. Rudramoorthy, "A statistical study of parameters in ultrasonic welding of plastics," Experimental Techniques, vol. 31, pp. 53-58, Sep-Oct 2007.

[112] I. F. Villegas, "Strength development versus process data in ultrasonic welding of thermoplastic composites with flat energy directors and its application to the definition of optimum processing parameters," Composites Part a-Applied Science and Manufacturing, vol. 65, pp. 27-37, Oct 2014.

[113] I. F. Villegas, L. Moser, A. Yousefpour, P. Mitschang, and H. E. N. Bersee, "Process and performance evaluation of ultrasonic, induction and resistance welding of advanced thermoplastic composites," Journal of Thermoplastic Composite Materials, vol. 26, pp. 10071024, Sep 2013.

[114] (Accessed 24 January 2015). Ultrasonic horn design using SonoAnalyzer - a basic guide. Available: $\quad$ http://www.sonoanalyzer.com/content/ultrasonic-horn-design-usingsonoanalyzer-basic-guide 
[115] J. Zhang, K. K. Chawla, and U. K. Vaidya, "FEA of Temperature Profile in the Ultrasonic Welded Joint," Advanced Materials and Processes li, Pts 1-3, vol. 557-559, pp. 1317-1321, 20122012.

[116] M. R. Rani and R. Rudramoorthy, "Computational modeling and experimental studies of the dynamic performance of ultrasonic horn profiles used in plastic welding," Ultrasonics, vol. 53, pp. 763-772, Mar 2013.

[117] X. Wang, "FEM Investigation of the Temperature Field of Energy Director During Ultrasonic Welding of PEEK Composites," Journal of Thermoplastic Composite Materials, vol. 19, pp. 593-607, 2006.

[118] D. A. Wang, W. Y. Chuang, K. Hsu, and H. T. Pham, "Design of a Bezier-profile horn for high displacement amplification," Ultrasonics, vol. 51, pp. 148-156, Feb 2011.

[119] E. A. a. Y. Kagawa, "Finite Element Simulation of a Ultrasonic Vibrator for Plastic Welding," pp. 563-566, 1985.

[120] (Accessed 24 January 2015). Ultrasonic vibrators - UCE Ultrasonic Co., LTD. Available: http://www.ultrasonic-transducers.cn/ultrasonic-welding-transducer/

[121] S. Elangovan, S. Semeer, and K. Prakasan, "Temperature and stress distribution in ultrasonic metal welding-An FEA-based study," Journal of Materials Processing Technology, vol. 209, pp. 1143-1150, Feb 2009.

[122] P. L. S. F. N. Dr Ying Ming, Dr Sun Zheng, "A New Optimization Method for Horn Designs in Ultrasonic Welding Systems," ed, 2002, pp. 1-6.

[123] (Accessed 24 January 2015). State-of-the art FEA technology and our abundant experiences create suitable ultrasonic tools. Available: http://www.cosmo-stm.com/e-ultrasonic/eustool.html

[124] C. Zhang and L. Li, "A Friction-Based Finite Element Analysis of Ultrasonic Consolidation," Welding Journal, vol. 87, pp. 187S-194S, Jul 2008.

[125] D. R. Wenguang Zhao, John Player, "Finite Element Analysis of Ultrasonic Processing of a Polymer- Matrix Composite," ed, pp. 1-15.

[126] E. Ando and Y. Kagawa, "FINITE-ELEMENT SIMULATION OF TRANSIENT HEAT RESPONSE IN ULTRASONIC TRANSDUCERS," leee Transactions on Ultrasonics Ferroelectrics and Frequency Control, vol. 39, pp. 432-440, May 1992.

[127] A. Siddiq and E. Ghassemieh, "Thermomechanical analyses of ultrasonic welding process using thermal and acoustic softening effects," Mechanics of Materials, vol. 40, pp. 982-1000, Dec 2008.

[128] H.-j. Yin, Y.-c. Su, H.-m. Jiang, L. Fang, and leee, "Numerical Simulation Study for Improvement of Polymer Flooding by Viscoelastic Effect," in Asia-Pacific Power and Energy Engineering Conference (APPEEC), Wuhan, PEOPLES R CHINA, 2009, pp. 2271-2276.

[129] R. M. Roopa, K. Prakasan, and R. Rudramoorthy, "Studies on high density polyethylene in the far-field region in ultrasonic welding of plastics," Polymer-Plastics Technology and Engineering, vol. 47, pp. 762-770, 20082008.

[130] M. Dube, P. Hubert, A. Yousefpour, and J. Denault, "Fatigue failure characterisation of resistance-welded thermoplastic composites skin/stringer joints," International Journal of Fatigue, vol. 31, pp. 719-725, Apr 2009.

[131] A. Levy, S. Le Corre, and A. Poitou, "Ultrasonic welding of thermoplastic composites: a numerical analysis at the mesoscopic scale relating processing parameters, flow of polymer and quality of adhesion," International Journal of Material Forming, vol. 7, pp. 39-51, Mar 2014.

[132] A. Levy, S. Le Corre, N. Chevaugeon, and A. Poitou, "A level set based approach for the finite element simulation of a forming process involving multiphysics coupling: Ultrasonic welding of thermoplastic composites," European Journal of Mechanics a-Solids, vol. 30, pp. 501-509, Jul-Aug 2011. 
[133] C. Gsell, A. Dahoun, V. Favier, J. M. Hiver, M. J. Philippe, and G. R. Canova, "Microstructure transformation and stress-strain behavior of isotactic polypropylene under large plastic deformation," Polymer Engineering and Science, vol. 37, pp. 1702-1711, Oct 1997.

[134] N. Brusselle-Dupend, D. Lai, X. Feaugas, M. Guigon, and M. Clavel, "Mechanical behavior of a semicrystalline polymer before necking. Part I: Characterization of uniaxial behavior," Polymer Engineering and Science, vol. 41, pp. 66-76, Jan 2001.

[135] (Accessed 24 January 2015). X-RAY DIFFRACTION BASICS - WELCOMETO CRAIN'S PETROPHYSICAL HANDBOOK. Available: https://www.spec2000.net/09-xrd.htm

[136] N. Brusselle-Dupend, D. Lai, X. Feaugas, M. Guigon, and M. Clavel, "Mechanical behavior of a semicrystalline polymer before necking. Part II: Modeling of uniaxial behavior," Polymer Engineering and Science, vol. 43, pp. 501-518, Feb 2003.

[137] G. D. Dean and W. Broughton, "A model for non-linear creep in polypropylene," Polymer Testing, vol. 26, pp. 1068-1081, Dec 2007.

[138] A. D. Drozdov and J. D. Christiansen, "Model for the viscoelastic and viscoplastic responses of semicrystalline polymers," Journal of Applied Polymer Science, vol. 88, pp. 1438-1450, May 92003.

[139] J. Takahashi, T. Yamamoto, and K. Shizawa, "Modeling and simulation for ductile fracture prediction of crystalline polymer based on craze behavior," International Journal of Mechanical Sciences, vol. 52, pp. 266-276, Feb 2010.

[140] H. Louche, F. Piette-Coudol, R. Arrieux, and J. Issartel, "An experimental and modeling study of the thermomechanical behavior of an ABS polymer structural component during an impact test," International Journal of Impact Engineering, vol. 36, pp. 847-861, Jun 2009.

[141] R. D. Sudduth, "New description of viscoelasticity that can be applied to mechanical properties such as constant strain rate, creep, and stress relaxation analysis," Polymer Engineering and Science, vol. 45, pp. 1600-1605, Dec 2005.

[142] S. R. Hummel and H. F. Nied, "A procedure for measuring biaxial viscoelastic behavior of thermoplastics," Experimental Mechanics, vol. 44, pp. 381-386, Aug 2004.

[143] R. D. Sudduth, "Comparison of the failure conditions for creep, stress relaxation, and constant strain rate measurements to predict pipe burst for two ABS materials using the universal viscoelastic model," Journal of Applied Polymer Science, vol. 93, pp. 247-260, Jul 5 2004.

[144] S. Wang, A. Makinouchi, M. Okamoto, T. Kotaka, and T. Nakagawa, "Viscoplastic modeling of ABS material under high-strain-rate uniaxial elongational deformation," Journal of Materials Science, vol. 34, pp. 5871-5878, Dec 1999.

[145] (Accessed 24 January 2015). Higher Institute For Plastics Fabrication - Thermoforming. Available: http://www.hipf.edu.sa/Courses-TF.html

[146] R. D. Sudduth, "Development of a simplified relationship between uniaxial creep, stress relaxation, and constant strain-rate results for viscoelastic polymeric materials," Journal of Applied Polymer Science, vol. 82, pp. 527-540, Oct 172001.

[147] T. Chen, "Determining a Prony Series for a Viscoelastic Material From Time Varying Strain Data," ed, 2000, pp. 1-21.

[148] (Accessed 24 January 2015). Chapter 12 - Thermal Analysis of Phase Transitions and Crystallization in Polymeric Fibers - Open Access. Available: http://www.intechopen.com/books/applications-of-calorimetry-in-a-wide-contextdifferential-scanning-calorimetry-isothermal-titration-calorimetry-andmicrocalorimetry/thermal-analysis-of-phase-transitions-and-crystallization-in-polymericfibers

[149] H. Blom, R. Yeh, R. Wojnarowski, and M. Ling, "Detection of degradation of ABS materials via DSC," Thermochimica Acta, vol. 442, pp. 64-66, Mar 152006.

[150] L. C. Bandeira, B. M. de Campos, E. H. de Faria, K. J. Ciuffi, P. S. Calefi, E. J. Nassar, et al., "TG/DTG/DTA/DSC as a tool for studying deposition by the sol-gel process on materials 
obtained by rapid prototyping," Journal of Thermal Analysis and Calorimetry, vol. 97, pp. 6770, Jul 2009.

[151] T. Streibel, R. Geissler, M. Saraji-Bozorgzad, M. Sklorz, E. Kaisersberger, T. Denner, et al., "Evolved gas analysis (EGA) in TG and DSC with single photon ionisation mass spectrometry (SPI-MS): molecular organic signatures from pyrolysis of soft and hard wood, coal, crude oil and ABS polymer," Journal of Thermal Analysis and Calorimetry, vol. 96, pp. 795-804, Jun 2009.

[152] (Accessed 24 January 2015). CAE - Thermosetting Plastic Injection Molding and Differential Scanning Calorimetry. Available: http://www.plastics-u.com/2013/08/28/thermosettingplastic-injection-molding-and-differential-scanning-calorimetry/

[153] M. M. Shapi, "TG AND DSC STUDIES OF SOME THERMAL-PROPERTIES AND STABILITY ASPECTS OF POLY(ACRYLONITRILE BUTADIENE STYRENE), POLYSTYRENE AND POLY(ACRYLONITRILE STYRENE) PLASTICS," Thermochimica Acta, vol. 175, pp. 25-34, Feb 28 1991.

[154] Y.-S. Duh, T.-C. Ho, J.-R. Chen, and C.-S. Kao, "Study on Exothermic Oxidation of Acrylonitrilebutadiene-styrene (ABS) Resin Powder with Application to ABS Processing Safety," Polymers, vol. 2, pp. 174-187, Sep 2010.

[155] S. S. Parikh and L. Zlatkevich, "Chemiluminescence in thermal oxidation of ABS," Angewandte Makromolekulare Chemie, vol. 247, pp. 255-268, May 1997.

[156] P. A. Tarantili, A. N. Mitsakaki, and M. A. Petoussi, "Processing and properties of engineering plastics recycled from waste electrical and electronic equipment (WEEE)," Polymer Degradation and Stability, vol. 95, pp. 405-410, Mar 2010.

[157] Z. Chen, B. Wang, J. Kang, H. Peng, J. Chen, F. Yang, et al., "Crystallization behavior and morphology of beta-nucleated isotactic polypropylene with different stereo-defect distribution," Polymers for Advanced Technologies, vol. 25, pp. 353-363, Apr 2014.

[158] Y. Pan, S. Shi, W. Xu, G. Zheng, K. Dai, C. Liu, et al., "Wide distribution of shish-kebab structure and tensile property of micro-injection-molded isotactic polypropylene microparts: a comparative study with injection-molded macroparts," Journal of Materials Science, vol. 49, pp. 1041-1048, Feb 2014.

[159] G. M. Shashidhara, S. H. K. Devi, and S. Preethi, "Isothermal crystallization behavior of PP and PP-g-GMA copolymer at high undercooling," Polymer Science Series A, vol. 55, pp. 393403, Jun 2013.

[160] B. Luijsterburg and H. Goossens, "Assessment of plastic packaging waste: Material origin, methods, properties," Resources Conservation and Recycling, vol. 85, pp. 88-97, Apr 2014.

[161] K. H. Ha, "Identification by thermal analysis for open-loop recycling of post-consumer waste polypropylene to apply refrigerator plastics," Materials \& Design, vol. 36, pp. 359-365, Apr 2012.

[162] "SYMPOSIUM ON LIGHT MICROSCOPY," in STP143-EB, ed: ASTM International, Nov. 1953.

[163] (Accessed 24 January 2015). Parts of a Compound Microscope - MicrobeHunter Microscopy Magazine. Available: http://www.microbehunter.com/parts-of-a-compound-microscope/

[164] (Accessed 24 January 2015). Upright \& Inverted Microscopes - Vision Engineering Available: http://www.visioneng.com/products/upright-and-inverted-microscopes

[165] (Accessed 24 January 2015). OPTICS \& PHOTONICS, TEST \& MEASURING INSTRUMENTS, VISION SYSTEMS - Video Microscope inspects textiles and fabrics. Available: http://news.thomasnet.com/fullstory/Video-Microscope-inspects-textiles-and-fabrics603817

[166] A. International, Characterization and Failure Analysis of Plastics: United States of America, 2003.

[167] Y.-M. Chung and M. R. Kamal, "Morphology of PA-6 vibration welded joints and its effects on weld strength," Polymer Engineering and Science, vol. 48, pp. 240-248, Feb 2008. 
[168] M. J. Oliveira, C. A. Bernardo, and D. A. Hemsley, "Morphology and mechanical behavior of polypropylene hot plate welds," Polymer Engineering and Science, vol. 41, pp. 1913-1922, Nov 2001.

[169] O. Balkan, H. Demirer, A. Ezdesir, and H. Yildirim, "Effects of welding procedures on mechanical and morphological properties of hot gas butt welded PE, PP, and PVC sheets," Polymer Engineering and Science, vol. 48, pp. 732-746, Apr 2008.

[170] M. Cakmak, J. Robinette, and S. Schaible, "Structure development and dynamics of vibration welding of poly(ethylene naphthalate) from amorphous and semicrystalline precursors," Journal of Applied Polymer Science, vol. 70, pp. 89-108, Oct 1998.

[171] M. Dube, P. Hubert, A. Yousefpour, and J. Denault, "Resistance welding of thermoplastic composites skin/stringer joints," Composites Part a-Applied Science and Manufacturing, vol. 38, pp. 2541-2552, 2007.

[172] M. Carboni, "Failure analysis of two aluminium alloy sonotrodes for ultrasonic plastic welding," International Journal of Fatigue, vol. 60, pp. 110-120, Mar 2014.

[173] F. Balle, S. Emrich, G. Wagner, D. Eifler, A. Brodyanski, and M. Kopnarski, "Improvement of Ultrasonically Welded Aluminum/Carbon Fiber Reinforced Polymer-Joints by Surface Technology and High Resolution Analysis," Advanced Engineering Materials, vol. 15, pp. 814820, Sep 2013.

[174] X. Wang, J. Yan, R. Li, and S. Yang, "FEM investigation of the temperature field of energy director during ultrasonic welding of PEEK composites," Journal of Thermoplastic Composite Materials, vol. 19, pp. 593-607, Sep 2006.

[175] D. G. W. C.J. Aliosio, E.E. Whetsel, "A simplified thermoviscoelastic analysis of the ultrasonic bonding," ed. Brookfield, CT: SPE Technical Papers, 1972, pp. 445-451.

[176] (Accessed 24 January 2015). Polypropylene (PP) - Examples of Use. Available: http://www.bpf.co.uk/Plastipedia/Polymers/PP.aspx

[177] G. Strobl, The Physics of Polymers, Third Revised and Expanded ed. Berlin, Heidelberg and New York: Springer, 2007.

[178] (Accessed 24 January 2015). Acrylic-Butadiene-Styrene-copolymers (ABS) - Formula. Available:

http://www.mecadi.com/en/literature tools/encyclopedia/categorial/Thermoplastic/Acrylic -Butadiene-Styrene-copolymers ABS/

[179] (Accessed 24 January 2015). Acrylonitrile Butadiene Styrene (ABS) and Other Specialist Styrenics - $\quad$ Applications. Available: http://www.bpf.co.uk/Plastipedia/Polymers/ABS and Other Specialist Styrenics.aspx

[180] (Accessed 25 January 2015). HAAKE MINIJET II (POLYMER) - HAAKE* MiniJet Piston Injection Molding System. Available: http://www.simtech.a-star.edu.sg/equipments/haake-minijet-ii(polymer).aspx

[181] (Accessed 24 January 2015). Transformations: an introduction. Available: http://fmwww.bc.edu/repec/bocode/t/transint.html

[182] (Accessed 24 January 2015). Generalized Linear Models - Transforming the Data. Available: http://data.princeton.edu/wws509/notes/c2s10.html

[183] ENCYCLOPEDIA OF MEASUREMENT AND STATISTICS vol. 1. Thousand Oaks, London and New Delhi: Rolf Janke, 2007.

[184] I. Software, "IBM SPSS Statistics Help," 20 ed.

[185] R. K. ROY, DESIGN OF EXPERIMENTS USING THE TAGUCHI APPROACH: JOHN WILEY \& SONS, INC., 2001.

[186] S. W. a. D. C. Shirley Dowdy, Statistics for Research, Third ed. Hoboken, New Jersey: John Wiley \& Sons, Inc., 2004.

[187] P. Larry J. Stephens, Beginning Statistics, Second ed.: McGraw-Hill, 2009. 
[188] (Accessed 24 January 2015). Chapter 7: Analysing the Data Part IV : Analysis of Variance One-Way ANOVA Post hoc tests. Available: http://webstat.une.edu.au/unit materials/c7 anova/oneway post hoc.htm

[189] R. V. E. a. S. K. N. A. BENATAR, "Ultrasonic Welding of Thermoplastics in the Near Field," POLYMER ENGINEERING AND SCIENCE., vol. 29, pp. 1689 - 1698, 1989.

[190] S. S. Carey, A Beginner's Guide to Scientific Method 4th ed. Boston: Wadsworth, Cengage Learning, 2011.

[191] M. M. W.J. Sichina. (Accessed 31 May 2015). Measurement of Tg of Polypropylene Using the PYRIS Power Compensation DSC. Available: http://www.perkinelmer.com/fr/PDFs/downloads/APP ThermalTgPolypropylenePYRISPowe rComp.pdf

[192] (Accessed 25 January 2015). NPL Image gallery - Placing a sample pan in DSC chamber. Available: http://www.npl.co.uk/

[193] (Accessed 31 May 2015). Adhesives Toolkit. Available: http://www.adhesivestoolkit.com/Docs/test/Physical\%20Analysis\%20\%20Capillary\%20Rheometers.xtp

[194] (Accessed 24 January 2015). Thermal Conductivity of some common Materials and Gases The Engineering Toolbox. Available: http://www.engineeringtoolbox.com/thermalconductivity-d 429.html

[195] (Accessed 24 January 2015). Thermal properties of Polypropylene, PP. Available: http://www.segerfrojd.com/thermal properties.htm

[196] (Accessed 24 January 2015). Thermocouple Trouble Shooting - TEMPERATURE MEASUREMENT SYSTEMS (TMS). Available: http://tempmeassystems.com/products/thermocouple-troubleshooting.htm

[197] (Accessed 11 June 2015). History of Imaging - Part 3. Available: http://blog.eyewire.org/history-of-imaging-part-3/

[198] (Accessed 19 April 2015). Variables (Learning the Parts of the Microscope). Available: http://microscopy4kids.org/Variables (Learning the Parts of the Microscope)\#VARIABLES

[199] (Accessed 26 January 2015). JEOL JSM 6400 Scanning Electron Microscope. Available: http://www.uh.edu/research/mcf/SEM.html

[200] (Accessed 14 August 2015). Calculus - GET IN LINE. Available: https://www.learner.org/courses/mathilluminated/units/12/textbook/03.php 


\section{$\underline{\text { APPENDICES }}$}

\section{Appendix A}

Matrices of experiments have been presented here for welding ABS and PP ultrasonically, as shown in Table A.1 and Table A.2;

Matrix of experiments for ABS

Table A.1

\begin{tabular}{|c||c||c||c|c|c|c|}
\hline Sr. No. & ED & Material & $\begin{array}{c}\text { Static Force } \\
(\mathbf{N})\end{array}$ & $\begin{array}{c}\text { Weld Time } \\
(\mathbf{s e c})\end{array}$ & $\begin{array}{c}\text { Hold Time } \\
(\mathbf{s e c})\end{array}$ & $\begin{array}{c}\text { Amplitude } \\
(\boldsymbol{\mu} \mathbf{m})\end{array}$ \\
\hline \hline 1 & SEMI & ABS & 500 & 0.9 & 0.2 & 21 \\
\hline 2 & SEMI & ABS & 500 & 0.8 & 0.2 & 21 \\
\hline 3 & SEMI & ABS & 500 & 0.7 & 0.2 & 21 \\
\hline 4 & SEMI & ABS & 500 & 0.6 & 0.2 & 21 \\
\hline 5 & SEMI & ABS & 500 & 0.4 & 0.2 & 21 \\
\hline 6 & SEMI & ABS & 750 & 0.9 & 0.2 & 21 \\
\hline 7 & SEMI & ABS & 750 & 0.8 & 0.2 & 21 \\
\hline 8 & SEMI & ABS & 750 & 0.7 & 0.2 & 21 \\
\hline 9 & SEMI & ABS & 750 & 0.5 & 0.2 & 21 \\
\hline 10 & SEMI & ABS & 750 & 0.4 & 0.2 & 21 \\
\hline 11 & SEMI & ABS & 1250 & 0.9 & 0.2 & 21 \\
\hline 12 & SEMI & ABS & 1250 & 0.8 & 0.2 & 21 \\
\hline 13 & SEMI & ABS & 1250 & 0.7 & 0.2 & 21 \\
\hline 14 & SEMI & ABS & 1250 & 0.4 & 0.2 & 21 \\
\hline 15 & SEMI & ABS & 1000 & 0.9 & 0.2 & 21 \\
\hline 16 & SEMI & ABS & 1000 & 0.8 & 0.2 & 21 \\
\hline 17 & SEMI & ABS & 1000 & 0.7 & 0.2 & 21 \\
\hline 18 & SEMI & ABS & 1000 & 0.5 & 0.2 & 21 \\
\hline 19 & SEMI & ABS & 1000 & 0.4 & 0.2 & 21 \\
\hline 20 & SEMI & ABS & 1500 & 0.9 & 0.2 & 21 \\
\hline 21 & SEMI & ABS & 1500 & 0.8 & 0.2 & 21 \\
\hline 22 & SEMI & ABS & 1500 & 0.7 & 0.2 & 21 \\
\hline 23 & SEMI & ABS & 1500 & 0.5 & 0.2 & 21 \\
\hline 24 & SEMI & ABS & 1500 & 0.4 & 0.2 & 21 \\
\hline 25 & TRI & ABS & 500 & 0.9 & 0.2 & 21 \\
\hline 26 & TRI & ABS & 500 & 0.8 & 0.2 & 21 \\
\hline 27 & TRI & ABS & 500 & 0.6 & 0.2 & 21 \\
\hline 28 & TRI & ABS & 500 & 0.4 & 0.2 & 21 \\
\hline 29 & TRI & ABS & 1000 & 0.9 & 0.2 & 21 \\
\hline 30 & TRI & ABS & 1000 & 0.8 & 0.2 & 21 \\
\hline
\end{tabular}




\begin{tabular}{|c|c|c|c|c|c|c|}
\hline Sr. No. & ED & Material & $\begin{array}{l}\text { Static Force } \\
\text { (N) }\end{array}$ & $\begin{array}{l}\text { Weld Time } \\
\text { (sec) }\end{array}$ & $\begin{array}{l}\text { Hold Time } \\
\text { (sec) }\end{array}$ & $\begin{array}{c}\text { Amplitude } \\
(\mu \mathrm{m})\end{array}$ \\
\hline 31 & TRI & ABS & 1000 & 0.6 & 0.2 & 21 \\
\hline 32 & TRI & ABS & 1000 & 0.4 & 0.2 & 21 \\
\hline 33 & TRI & $A B S$ & 1250 & 0.9 & 0.2 & 21 \\
\hline 34 & TRI & $\mathrm{ABS}$ & 1250 & 0.8 & 0.2 & 21 \\
\hline 35 & TRI & ABS & 1250 & 0.6 & 0.2 & 21 \\
\hline 36 & TRI & $A B S$ & 1250 & 0.4 & 0.2 & 21 \\
\hline 37 & TRI & ABS & 750 & 0.9 & 0.2 & 21 \\
\hline 38 & TRI & $A B S$ & 750 & 0.8 & 0.2 & 21 \\
\hline 39 & TRI & ABS & 750 & 0.6 & 0.2 & 21 \\
\hline 40 & TRI & $A B S$ & 750 & 0.4 & 0.2 & 21 \\
\hline 41 & TRI & ABS & 1500 & 0.9 & 0.2 & 21 \\
\hline 42 & TRI & ABS & 1500 & 0.8 & 0.2 & 21 \\
\hline 43 & TRI & $A B S$ & 1500 & 0.6 & 0.2 & 21 \\
\hline 44 & TRI & ABS & 1500 & 0.4 & 0.2 & 21 \\
\hline 45 & SEMI & $A B S$ & 500 & 0.9 & 0.2 & 18.9 \\
\hline 46 & SEMI & ABS & 500 & 0.8 & 0.2 & 18.9 \\
\hline 47 & SEMI & ABS & 500 & 0.7 & 0.2 & 18.9 \\
\hline 48 & SEMI & $\mathrm{ABS}$ & 500 & 0.6 & 0.2 & 18.9 \\
\hline 49 & SEMI & ABS & 500 & 0.4 & 0.2 & 18.9 \\
\hline 50 & SEMI & $A B S$ & 750 & 0.9 & 0.2 & 18.9 \\
\hline 51 & SEMI & ABS & 750 & 0.8 & 0.2 & 18.9 \\
\hline 52 & SEMI & $A B S$ & 750 & 0.7 & 0.2 & 18.9 \\
\hline 53 & SEMI & ABS & 750 & 0.5 & 0.2 & 18.9 \\
\hline 54 & SEMI & ABS & 1250 & 0.9 & 0.2 & 18.9 \\
\hline 55 & SEMI & $\mathrm{ABS}$ & 1250 & 0.8 & 0.2 & 18.9 \\
\hline 56 & SEMI & ABS & 1250 & 0.7 & 0.2 & 18.9 \\
\hline 57 & SEMI & $A B S$ & 1000 & 0.9 & 0.2 & 18.9 \\
\hline 58 & SEMI & ABS & 1000 & 0.8 & 0.2 & 18.9 \\
\hline 59 & SEMI & $A B S$ & 1000 & 0.7 & 0.2 & 18.9 \\
\hline 60 & SEMI & ABS & 1000 & 0.5 & 0.2 & 18.9 \\
\hline 61 & SEMI & ABS & 1500 & 0.9 & 0.2 & 18.9 \\
\hline 62 & SEMI & ABS & 1500 & 0.8 & 0.2 & 18.9 \\
\hline 63 & SEMI & ABS & 1500 & 0.7 & 0.2 & 18.9 \\
\hline 64 & SEMI & ABS & 1500 & 0.5 & 0.2 & 18.9 \\
\hline 65 & TRI & ABS & 500 & 0.9 & 0.2 & 18.9 \\
\hline 66 & TRI & ABS & 500 & 0.8 & 0.2 & 18.9 \\
\hline 67 & TRI & $\mathrm{ABS}$ & 500 & 0.6 & 0.2 & 18.9 \\
\hline 68 & TRI & ABS & 500 & 0.4 & 0.2 & 18.9 \\
\hline 69 & TRI & ABS & 1000 & 0.9 & 0.2 & 18.9 \\
\hline 70 & TRI & ABS & 1000 & 0.8 & 0.2 & 18.9 \\
\hline 71 & TRI & ABS & 1000 & 0.6 & 0.2 & 18.9 \\
\hline
\end{tabular}




\begin{tabular}{|c|c|c|c|c|c|c|}
\hline Sr. No. & ED & Material & $\begin{array}{l}\text { Static Force } \\
\text { (N) }\end{array}$ & $\begin{array}{l}\text { Weld Time } \\
\text { (sec) }\end{array}$ & $\begin{array}{l}\text { Hold Time } \\
\text { (sec) }\end{array}$ & $\begin{array}{c}\text { Amplitude } \\
(\mu \mathrm{m})\end{array}$ \\
\hline 72 & TRI & ABS & 1000 & 0.4 & 0.2 & 18.9 \\
\hline 73 & TRI & ABS & 1250 & 0.9 & 0.2 & 18.9 \\
\hline 74 & TRI & $A B S$ & 1250 & 0.8 & 0.2 & 18.9 \\
\hline 75 & TRI & $\mathrm{ABS}$ & 1250 & 0.6 & 0.2 & 18.9 \\
\hline 76 & TRI & ABS & 1250 & 0.4 & 0.2 & 18.9 \\
\hline 77 & TRI & $A B S$ & 750 & 0.9 & 0.2 & 18.9 \\
\hline 78 & TRI & ABS & 750 & 0.8 & 0.2 & 18.9 \\
\hline 79 & TRI & $A B S$ & 750 & 0.6 & 0.2 & 18.9 \\
\hline 80 & TRI & ABS & 750 & 0.4 & 0.2 & 18.9 \\
\hline 81 & TRI & $A B S$ & 1500 & 0.9 & 0.2 & 18.9 \\
\hline 82 & TRI & ABS & 1500 & 0.8 & 0.2 & 18.9 \\
\hline 83 & TRI & $A B S$ & 1500 & 0.6 & 0.2 & 18.9 \\
\hline 84 & TRI & $A B S$ & 1500 & 0.4 & 0.2 & 18.9 \\
\hline 85 & SEMI & ABS & 500 & 0.9 & 0.2 & 16.8 \\
\hline 86 & SEMI & $A B S$ & 500 & 0.8 & 0.2 & 16.8 \\
\hline 87 & SEMI & ABS & 500 & 0.7 & 0.2 & 16.8 \\
\hline 88 & SEMI & ABS & 750 & 0.9 & 0.2 & 16.8 \\
\hline 89 & SEMI & $A B S$ & 750 & 0.8 & 0.2 & 16.8 \\
\hline 90 & SEMI & ABS & 750 & 0.7 & 0.2 & 16.8 \\
\hline 91 & SEMI & $A B S$ & 750 & 0.5 & 0.2 & 16.8 \\
\hline 92 & SEMI & ABS & 1250 & 0.9 & 0.2 & 16.8 \\
\hline 93 & SEMI & $A B S$ & 1250 & 0.8 & 0.2 & 16.8 \\
\hline 94 & SEMI & ABS & 1250 & 0.7 & 0.2 & 16.8 \\
\hline 95 & SEMI & ABS & 1250 & 0.6 & 0.2 & 16.8 \\
\hline 96 & SEMI & $\mathrm{ABS}$ & 1000 & 0.9 & 0.2 & 16.8 \\
\hline 97 & SEMI & ABS & 1000 & 0.8 & 0.2 & 16.8 \\
\hline 98 & SEMI & $A B S$ & 1000 & 0.7 & 0.2 & 16.8 \\
\hline 99 & SEMI & ABS & 1000 & 0.5 & 0.2 & 16.8 \\
\hline 100 & SEMI & $A B S$ & 1500 & 0.9 & 0.2 & 16.8 \\
\hline 101 & SEMI & ABS & 1500 & 0.8 & 0.2 & 16.8 \\
\hline 102 & SEMI & ABS & 1500 & 0.7 & 0.2 & 16.8 \\
\hline 103 & SEMI & $A B S$ & 1500 & 0.5 & 0.2 & 16.8 \\
\hline 104 & TRI & ABS & 500 & 0.9 & 0.2 & 16.8 \\
\hline 105 & TRI & ABS & 500 & 0.8 & 0.2 & 16.8 \\
\hline 106 & TRI & ABS & 500 & 0.6 & 0.2 & 16.8 \\
\hline 107 & TRI & ABS & 500 & 0.4 & 0.2 & 16.8 \\
\hline 108 & TRI & $A B S$ & 1000 & 0.9 & 0.2 & 16.8 \\
\hline 109 & TRI & ABS & 1000 & 0.8 & 0.2 & 16.8 \\
\hline 110 & TRI & $A B S$ & 1000 & 0.6 & 0.2 & 16.8 \\
\hline 111 & TRI & ABS & 1000 & 0.4 & 0.2 & 16.8 \\
\hline 112 & TRI & $A B S$ & 1250 & 0.9 & 0.2 & 16.8 \\
\hline
\end{tabular}




\begin{tabular}{|c|c|c|c|c|c|c|}
\hline Sr. No. & ED & Material & $\begin{array}{l}\text { Static Force } \\
\text { (N) }\end{array}$ & $\begin{array}{l}\text { Weld Time } \\
\text { (sec) }\end{array}$ & $\begin{array}{l}\text { Hold Time } \\
\text { (sec) }\end{array}$ & $\begin{array}{c}\text { Amplitude } \\
(\mu \mathrm{m})\end{array}$ \\
\hline 113 & TRI & ABS & 1250 & 0.8 & 0.2 & 16.8 \\
\hline 114 & TRI & ABS & 1250 & 0.6 & 0.2 & 16.8 \\
\hline 115 & TRI & $A B S$ & 1250 & 0.4 & 0.2 & 16.8 \\
\hline 116 & TRI & $\mathrm{ABS}$ & 750 & 0.9 & 0.2 & 16.8 \\
\hline 117 & TRI & ABS & 750 & 0.8 & 0.2 & 16.8 \\
\hline 118 & TRI & $A B S$ & 750 & 0.6 & 0.2 & 16.8 \\
\hline 119 & TRI & ABS & 750 & 0.4 & 0.2 & 16.8 \\
\hline 120 & TRI & $A B S$ & 1500 & 0.9 & 0.2 & 16.8 \\
\hline 121 & TRI & $A B S$ & 1500 & 0.8 & 0.2 & 16.8 \\
\hline 122 & TRI & $A B S$ & 1500 & 0.6 & 0.2 & 16.8 \\
\hline 123 & TRI & ABS & 1500 & 0.4 & 0.2 & 16.8 \\
\hline 124 & SEMI & ABS & 500 & 0.9 & 0.2 & 14.7 \\
\hline 125 & SEMI & $A B S$ & 500 & 0.7 & 0.2 & 14.7 \\
\hline 126 & SEMI & ABS & 500 & 0.6 & 0.2 & 14.7 \\
\hline 127 & SEMI & $A B S$ & 500 & 0.4 & 0.2 & 14.7 \\
\hline 128 & SEMI & ABS & 750 & 0.9 & 0.2 & 14.7 \\
\hline 129 & SEMI & ABS & 750 & 0.8 & 0.2 & 14.7 \\
\hline 130 & SEMI & $A B S$ & 750 & 0.7 & 0.2 & 14.7 \\
\hline 131 & SEMI & ABS & 750 & 0.4 & 0.2 & 14.7 \\
\hline 132 & SEMI & $A B S$ & 750 & 0.5 & 0.2 & 14.7 \\
\hline 133 & SEMI & ABS & 1250 & 0.9 & 0.2 & 14.7 \\
\hline 134 & SEMI & $A B S$ & 1000 & 0.9 & 0.2 & 14.7 \\
\hline 135 & SEMI & $A B S$ & 1500 & 0.9 & 0.2 & 14.7 \\
\hline 136 & TRI & ABS & 500 & 0.9 & 0.2 & 14.7 \\
\hline 137 & TRI & $\mathrm{ABS}$ & 500 & 0.8 & 0.2 & 14.7 \\
\hline 138 & TRI & ABS & 500 & 0.6 & 0.2 & 14.7 \\
\hline 139 & TRI & $A B S$ & 500 & 0.4 & 0.2 & 14.7 \\
\hline 140 & TRI & ABS & 1000 & 0.9 & 0.2 & 14.7 \\
\hline 141 & TRI & $A B S$ & 1000 & 0.8 & 0.2 & 14.7 \\
\hline 142 & TRI & ABS & 1000 & 0.6 & 0.2 & 14.7 \\
\hline 143 & TRI & ABS & 1000 & 0.4 & 0.2 & 14.7 \\
\hline 144 & TRI & ABS & 1250 & 0.9 & 0.2 & 14.7 \\
\hline 145 & TRI & ABS & 1250 & 0.8 & 0.2 & 14.7 \\
\hline 146 & TRI & ABS & 1250 & 0.6 & 0.2 & 14.7 \\
\hline 147 & TRI & ABS & 1250 & 0.4 & 0.2 & 14.7 \\
\hline 148 & TRI & ABS & 750 & 0.9 & 0.2 & 14.7 \\
\hline 149 & TRI & $A B S$ & 750 & 0.8 & 0.2 & 14.7 \\
\hline 150 & TRI & ABS & 750 & 0.6 & 0.2 & 14.7 \\
\hline 151 & TRI & ABS & 750 & 0.4 & 0.2 & 14.7 \\
\hline 152 & TRI & ABS & 1500 & 0.9 & 0.2 & 14.7 \\
\hline 153 & TRI & $A B S$ & 1500 & 0.8 & 0.2 & 14.7 \\
\hline
\end{tabular}




\begin{tabular}{||c||c||c||c||c||c||c|}
\hline \hline Sr. No. & ED & Material & $\begin{array}{c}\text { Static Force } \\
\text { (N) }\end{array}$ & $\begin{array}{c}\text { Weld Time } \\
(\mathbf{s e c})\end{array}$ & $\begin{array}{c}\text { Hold Time } \\
(\mathbf{s e c})\end{array}$ & $\begin{array}{c}\text { Amplitude } \\
(\boldsymbol{\mu m})\end{array}$ \\
\hline \hline 154 & TRI & ABS & 1500 & 0.6 & 0.2 & 14.7 \\
\hline 155 & TRI & ABS & 1500 & 0.4 & 0.2 & 14.7 \\
\hline 156 & TRI & ABS & 1500 & 0.4 & 0.2 & 14.7 \\
\hline
\end{tabular}

\section{Matrix of experiments for PP}

Table A.2

\begin{tabular}{|c|c|c|c|c|c|c|}
\hline $\begin{array}{l}\text { Sr. } \\
\text { No. }\end{array}$ & ED & Material & $\begin{array}{l}\text { Static Force } \\
\text { (N) }\end{array}$ & $\begin{array}{c}\text { Weld Time } \\
\text { (sec) }\end{array}$ & $\begin{array}{l}\text { Hold Time } \\
\text { (sec) }\end{array}$ & $\begin{array}{c}\text { Amplitude } \\
(\mu \mathrm{m}) \\
\end{array}$ \\
\hline 1 & TRI & PP & 750 & 1.5 & 3 & 21 \\
\hline 2 & TRI & PP & 750 & 1.3 & 3 & 21 \\
\hline 3 & TRI & PP & 750 & 1.2 & 3 & 21 \\
\hline 4 & TRI & PP & 750 & 1.1 & 3 & 21 \\
\hline 5 & TRI & $\mathrm{PP}$ & 1000 & 1.5 & 3 & 21 \\
\hline 6 & TRI & PP & 1000 & 1.3 & 3 & 21 \\
\hline 7 & TRI & PP & 1000 & 1.2 & 3 & 21 \\
\hline 8 & TRI & $\mathrm{PP}$ & 1000 & 1.1 & 3 & 21 \\
\hline 9 & TRI & PP & 1250 & 1.4 & 3 & 21 \\
\hline 10 & TRI & $\mathrm{PP}$ & 1250 & 1.3 & 3 & 21 \\
\hline 11 & TRI & $\mathrm{PP}$ & 1250 & 1.2 & 3 & 21 \\
\hline 12 & TRI & PP & 1250 & 1.1 & 3 & 21 \\
\hline 13 & TRI & $\mathrm{PP}$ & 1500 & 1.5 & 3 & 21 \\
\hline 14 & TRI & PP & 1500 & 1.4 & 3 & 21 \\
\hline 15 & TRI & $\mathrm{PP}$ & 1500 & 1.2 & 3 & 21 \\
\hline 16 & TRI & $\mathrm{PP}$ & 1500 & 1.1 & 3 & 21 \\
\hline 17 & SEMI & $\mathrm{PP}$ & 750 & 1.4 & 3 & 21 \\
\hline 18 & SEMI & $\mathrm{PP}$ & 750 & 1.2 & 3 & 21 \\
\hline 19 & SEMI & $\mathrm{PP}$ & 750 & 1 & 3 & 21 \\
\hline 20 & SEMI & PP & 750 & 0.8 & 3 & 21 \\
\hline 21 & SEMI & PP & 1250 & 1.5 & 3 & 21 \\
\hline 22 & SEMI & $\mathrm{PP}$ & 1250 & 1.4 & 3 & 21 \\
\hline 23 & SEMI & PP & 1250 & 1.1 & 3 & 21 \\
\hline 24 & SEMI & PP & 1250 & 0.8 & 3 & 21 \\
\hline 25 & SEMI & $\mathrm{PP}$ & 1000 & 1.5 & 3 & 21 \\
\hline 26 & SEMI & $\mathrm{PP}$ & 1000 & 1.4 & 3 & 21 \\
\hline 27 & SEMI & PP & 1000 & 1 & 3 & 21 \\
\hline 28 & SEMI & $\mathrm{PP}$ & 1000 & 0.8 & 3 & 21 \\
\hline 29 & SEMI & PP & 1500 & 1.4 & 3 & 21 \\
\hline 30 & SEMI & $\mathrm{PP}$ & 1500 & 1.3 & 3 & 21 \\
\hline 31 & SEMI & $\mathrm{PP}$ & 1500 & 1 & 3 & 21 \\
\hline 32 & SEMI & $\mathrm{PP}$ & 1500 & 0.8 & 3 & 21 \\
\hline
\end{tabular}




\begin{tabular}{|c|c|c|c|c|c|c|}
\hline $\begin{array}{l}\text { Sr. } \\
\text { No. }\end{array}$ & ED & Material & $\begin{array}{l}\text { Static Force } \\
\text { (N) }\end{array}$ & $\begin{array}{l}\text { Weld Time } \\
\text { (sec) }\end{array}$ & $\begin{array}{l}\text { Hold Time } \\
\text { (sec) }\end{array}$ & $\begin{array}{c}\text { Amplitude } \\
(\mu \mathrm{m})\end{array}$ \\
\hline 40 & TRI & PP & 750 & 1.5 & 3 & 18.9 \\
\hline 41 & TRI & $\mathrm{PP}$ & 750 & 1.3 & 3 & 18.9 \\
\hline 42 & TRI & PP & 750 & 1.2 & 3 & 18.9 \\
\hline 43 & TRI & PP & 750 & 1.1 & 3 & 18.9 \\
\hline 44 & TRI & PP & 1000 & 1.5 & 3 & 18.9 \\
\hline 45 & TRI & PP & 1000 & 1.3 & 3 & 18.9 \\
\hline 46 & TRI & PP & 1000 & 1.2 & 3 & 18.9 \\
\hline 47 & TRI & $\mathrm{PP}$ & 1000 & 1.1 & 3 & 18.9 \\
\hline 48 & TRI & PP & 1250 & 1.4 & 3 & 18.9 \\
\hline 49 & TRI & PP & 1250 & 1.3 & 3 & 18.9 \\
\hline 50 & TRI & $\mathrm{PP}$ & 1250 & 1.2 & 3 & 18.9 \\
\hline 51 & TRI & $\mathrm{PP}$ & 1250 & 1.1 & 3 & 18.9 \\
\hline 52 & TRI & PP & 1500 & 1.5 & 3 & 18.9 \\
\hline 53 & TRI & PP & 1500 & 1.4 & 3 & 18.9 \\
\hline 54 & TRI & $\mathrm{PP}$ & 1500 & 1.2 & 3 & 18.9 \\
\hline 55 & TRI & PP & 1500 & 1.1 & 3 & 18.9 \\
\hline 56 & TRI & PP & 1500 & 0.6 & 3 & 18.9 \\
\hline 57 & SEMI & $\mathrm{PP}$ & 750 & 1.4 & 3 & 18.9 \\
\hline 58 & SEMI & PP & 750 & 1.2 & 3 & 18.9 \\
\hline 59 & SEMI & PP & 750 & 1 & 3 & 18.9 \\
\hline 60 & SEMI & $\mathrm{PP}$ & 750 & 0.8 & 3 & 18.9 \\
\hline 61 & SEMI & PP & 1000 & 1.5 & 3 & 18.9 \\
\hline 62 & SEMI & PP & 1000 & 1.4 & 3 & 18.9 \\
\hline 63 & SEMI & $\mathrm{PP}$ & 1000 & 1 & 3 & 18.9 \\
\hline 64 & SEMI & PP & 1000 & 0.8 & 3 & 18.9 \\
\hline 65 & SEMI & $\mathrm{PP}$ & 1250 & 1.5 & 3 & 18.9 \\
\hline 66 & SEMI & PP & 1250 & 1.4 & 3 & 18.9 \\
\hline 67 & SEMI & PP & 1250 & 1.1 & 3 & 18.9 \\
\hline 68 & SEMI & PP & 1250 & 0.8 & 3 & 18.9 \\
\hline 69 & SEMI & $\mathrm{PP}$ & 1500 & 1.4 & 3 & 18.9 \\
\hline 70 & SEMI & PP & 1500 & 1.3 & 3 & 18.9 \\
\hline 71 & SEMI & PP & 1500 & 1 & 3 & 18.9 \\
\hline 72 & SEMI & $\mathrm{PP}$ & 1750 & 1 & 3 & 18.9 \\
\hline 73 & SEMI & PP & 1750 & 0.7 & 3 & 18.9 \\
\hline 81 & TRI & PP & 1000 & 1.7 & 3 & 16.8 \\
\hline 82 & TRI & PP & 1000 & 1.5 & 3 & 16.8 \\
\hline 83 & TRI & PP & 1000 & 1.3 & 3 & 16.8 \\
\hline 84 & TRI & PP & 1000 & 1.2 & 3 & 16.8 \\
\hline 85 & TRI & $\mathrm{PP}$ & 1000 & 1.1 & 3 & 16.8 \\
\hline 86 & TRI & PP & 1250 & 1.5 & 3 & 16.8 \\
\hline 87 & TRI & PP & 1250 & 1.4 & 3 & 16.8 \\
\hline 88 & TRI & PP & 1250 & 1.2 & 3 & 16.8 \\
\hline 89 & TRI & PP & 1250 & 1.1 & 3 & 16.8 \\
\hline
\end{tabular}




\begin{tabular}{|c|c|c|c|c|c|c|}
\hline $\begin{array}{l}\text { Sr. } \\
\text { No. }\end{array}$ & ED & Material & $\begin{array}{l}\text { Static Force } \\
\text { (N) }\end{array}$ & $\begin{array}{c}\text { Weld Time } \\
\text { (sec) }\end{array}$ & $\begin{array}{l}\text { Hold Time } \\
\text { (sec) }\end{array}$ & $\begin{array}{c}\text { Amplitude } \\
(\mu \mathrm{m})\end{array}$ \\
\hline 90 & TRI & $\mathrm{PP}$ & 1500 & 1.5 & 3 & 16.8 \\
\hline 91 & TRI & PP & 1500 & 1.4 & 3 & 16.8 \\
\hline 92 & TRI & PP & 1500 & 1.2 & 3 & 16.8 \\
\hline 93 & TRI & PP & 1500 & 1.1 & 3 & 16.8 \\
\hline 94 & SEMI & PP & 750 & 1.4 & 3 & 16.8 \\
\hline 95 & SEMI & $\mathrm{PP}$ & 750 & 1.2 & 3 & 16.8 \\
\hline 96 & SEMI & PP & 750 & 1 & 3 & 16.8 \\
\hline 97 & SEMI & PP & 750 & 0.8 & 3 & 16.8 \\
\hline 98 & SEMI & $\mathrm{PP}$ & 1000 & 1.5 & 3 & 16.8 \\
\hline 99 & SEMI & $\mathrm{PP}$ & 1000 & 1.4 & 3 & 16.8 \\
\hline 100 & SEMI & $\mathrm{PP}$ & 1000 & 1.4 & 3 & 16.8 \\
\hline 101 & SEMI & PP & 1000 & 1 & 3 & 16.8 \\
\hline 102 & SEMI & PP & 1000 & 0.8 & 3 & 16.8 \\
\hline 103 & SEMI & PP & 1250 & 1.5 & 3 & 16.8 \\
\hline 104 & SEMI & PP & 1250 & 1.4 & 3 & 16.8 \\
\hline 105 & SEMI & PP & 1250 & 1.2 & 3 & 16.8 \\
\hline 106 & SEMI & $\mathrm{PP}$ & 1250 & 1.1 & 3 & 16.8 \\
\hline 107 & SEMI & $\mathrm{PP}$ & 1500 & 1.4 & 3 & 16.8 \\
\hline 108 & SEMI & PP & 1500 & 1.3 & 3 & 16.8 \\
\hline 109 & SEMI & $\mathrm{PP}$ & 1500 & 1.1 & 3 & 16.8 \\
\hline 110 & SEMI & $\mathrm{PP}$ & 1500 & 1 & 3 & 16.8 \\
\hline 111 & SEMI & PP & 1500 & 0.8 & 3 & 16.8 \\
\hline 120 & TRI & PP & 500 & 1.3 & 3 & 14.7 \\
\hline 121 & TRI & PP & 500 & 1.5 & 3 & 14.7 \\
\hline 122 & TRI & PP & 500 & 1.7 & 3 & 14.7 \\
\hline 123 & TRI & $\mathrm{PP}$ & 750 & 1.1 & 3 & 14.7 \\
\hline 124 & TRI & PP & 750 & 1.3 & 3 & 14.7 \\
\hline 125 & TRI & PP & 750 & 1.5 & 3 & 14.7 \\
\hline 126 & TRI & $\mathrm{PP}$ & 750 & 1.7 & 3 & 14.7 \\
\hline 127 & TRI & PP & 1000 & 1.7 & 3 & 14.7 \\
\hline 128 & TRI & PP & 1000 & 1.5 & 3 & 14.7 \\
\hline 129 & TRI & $\mathrm{PP}$ & 1000 & 1.3 & 3 & 14.7 \\
\hline 130 & TRI & $\mathrm{PP}$ & 1000 & 1.1 & 3 & 14.7 \\
\hline 131 & TRI & PP & 1250 & 1.1 & 3 & 14.7 \\
\hline 132 & TRI & $\mathrm{PP}$ & 1250 & 1.3 & 3 & 14.7 \\
\hline 133 & TRI & PP & 1250 & 1.5 & 3 & 14.7 \\
\hline 134 & TRI & PP & 1250 & 1.7 & 3 & 14.7 \\
\hline 135 & TRI & $\mathrm{PP}$ & 1500 & 0.6 & 3 & 14.7 \\
\hline 136 & TRI & $\mathrm{PP}$ & 1250 & 1.4 & 3 & 14.7 \\
\hline 137 & TRI & PP & 1500 & 1.4 & 3 & 14.7 \\
\hline 138 & TRI & $\mathrm{PP}$ & 1500 & 1.5 & 3 & 14.7 \\
\hline 139 & TRI & $\mathrm{PP}$ & 1500 & 1.2 & 3 & 14.7 \\
\hline 140 & TRI & PP & 1250 & 1.2 & 3 & 14.7 \\
\hline
\end{tabular}




\begin{tabular}{|c||c||c|c||c||c||c|}
\hline $\begin{array}{c}\text { Sr. } \\
\text { No. }\end{array}$ & ED & Material & $\begin{array}{c}\text { Static Force } \\
(\mathbf{N})\end{array}$ & $\begin{array}{c}\text { Weld Time } \\
(\mathbf{s e c})\end{array}$ & $\begin{array}{c}\text { Hold Time } \\
(\mathbf{s e c})\end{array}$ & $\begin{array}{c}\text { Amplitude } \\
(\boldsymbol{\mu m})\end{array}$ \\
\hline \hline 141 & TRI & PP & 1000 & 1.2 & 3 & 14.7 \\
\hline 142 & TRI & PP & 1500 & 1.1 & 3 & 14.7 \\
\hline 143 & SEMI & PP & 1250 & 1.5 & 3 & 14.7 \\
\hline 144 & SEMI & PP & 1250 & 1.4 & 3 & 14.7 \\
\hline 145 & SEMI & PP & 1250 & 1.4 & 3 & 14.7 \\
\hline 146 & SEMI & PP & 1250 & 1.1 & 3 & 14.7 \\
\hline 147 & SEMI & PP & 1250 & 0.8 & 3 & 14.7 \\
\hline 148 & SEMI & PP & 1500 & 1.3 & 3 & 14.7 \\
\hline 149 & SEMI & PP & 1500 & 1 & 3 & 14.7 \\
\hline 150 & SEMI & PP & 1500 & 0.8 & 3 & 14.7 \\
\hline 151 & SEMI & PP & 1500 & 0.6 & 3 & 14.7 \\
\hline 152 & SEMI & PP & 1000 & 1.4 & 3 & 14.7 \\
\hline 153 & SEMI & PP & 750 & 1.4 & 3 & 14.7 \\
\hline 154 & SEMI & PP & 750 & 1.2 & 3 & 14.7 \\
\hline 155 & SEMI & PP & 750 & 1 & 3 & 14.7 \\
\hline 156 & SEMI & PP & 750 & 0.8 & 3 & 14.7 \\
\hline 157 & SEMI & PP & 1500 & 1.4 & 3 & 14.7 \\
\hline 158 & SEMI & PP & 1750 & 1 & 3 & 14.7 \\
\hline 159 & SEMI & PP & 1000 & 0.8 & 3 & 14.7 \\
\hline 160 & SEMI & PP & 1000 & 1 & 3 & 14.7 \\
\hline 161 & SEMI & PP & 1000 & 1.4 & 3 & 14.7 \\
\hline 162 & SEMI & PP & 1000 & 1.5 & 3 & 14.7 \\
\hline 163 & SEMI & PP & 1250 & 1.2 & 3 & 14.7 \\
\hline 164 & SEMI & PP & 1750 & 0.7 & 3 & 14.7 \\
\hline 165 & SEMI & PP & 1500 & 0.6 & 3 & 14.7 \\
\hline & & & & & 3 & \\
\hline
\end{tabular}




\section{Appendix B}

Datasheets have been presented for both ABS and PP, as can be seen in Table B.1 and Table B.2;

\section{Datasheet for ABS}

Table B.1

\begin{tabular}{|c|c|c|c|}
\hline Test Item Unit & $\begin{array}{c}\text { Test } \\
\text { Method } \\
\text { (ASTM) }\end{array}$ & Test Condition & Properties \\
\hline $\begin{array}{l}\text { Tensile Strength }\left\{\mathrm{Kg} / \mathrm{cm}^{\wedge} 2\right. \\
(\mathrm{psi}\}\}\end{array}$ & D638 & $\begin{array}{c}23 \text { Degree C, } \\
50 \mathrm{~mm} / \mathrm{min}\end{array}$ & $510(7240)$ \\
\hline Elongation (\%) & D638 & $50 \mathrm{~mm} / \mathrm{min}$ & 30 \\
\hline $\begin{array}{l}\text { Flexural Strength }\{ \\
\left.\mathrm{Kg} / \mathrm{cm}^{\wedge} 2(\mathrm{psi}\}\right\}\end{array}$ & D790 & 23 Degree C & $720(10,220)$ \\
\hline $\begin{array}{l}\text { Flexural Modulus }\{ \\
\mathrm{Kg} / \mathrm{cm}^{\wedge} 2 \text { (psi\}\} }\end{array}$ & D790 & 23 Degree C & $24000(340,800)$ \\
\hline Rckwell Hardness & D785 & R Scale & 110 \\
\hline \multirow{2}{*}{ Izod Impad Strength } & \multirow{2}{*}{ D256 } & $\begin{array}{c}\text { 3.2mm, Notched, } 23 \\
\text { Degree C (73 Degree F) }\end{array}$ & $25(4.5)$ \\
\hline & & $\begin{array}{c}6.4 \mathrm{~mm}, \text { Notched, } 23 \\
\text { Degree C (73 Degree F) }\end{array}$ & $22(4.1)$ \\
\hline $\begin{array}{c}\text { Heat Distortion } \\
\text { Temperature }\{\text { Degree C, } \\
\text { Degree F }\end{array}$ & D648 & $\begin{array}{c}\text { 18.6. } \mathrm{kgf} / \mathrm{cm}^{\wedge} 2,(264 \mathrm{psi}), \\
\text { unannealed }\end{array}$ & $\begin{array}{c}85 \\
(185)\end{array}$ \\
\hline $\begin{array}{l}\text { Vicat Softening Temp } \\
\{\text { Degree C, Degree F)\} }\end{array}$ & D1525 & - & - \\
\hline \multirow{3}{*}{ Melt Flow Index (g/10 min) } & \multirow{3}{*}{ D1238 } & 200 Degree C, 5.0kg & 4.2 \\
\hline & & 220 Degree C,10.0kg & 35 \\
\hline & & 200Degree C, 21.6kg & 48 \\
\hline Moulding Shrinkage (\%) & D955 & - & $0.4-0.7$ \\
\hline Specific Gravity & D792 & 23 Degree C & 1.04 \\
\hline Flammability (Rating) & UL94 & Class & $\mathrm{HB}$ \\
\hline
\end{tabular}




\section{Datasheet for PP}

Table B.2

\begin{tabular}{|c|c|c|c|}
\hline Properties & Unit (SI) & Values & $\begin{array}{c}\text { Test } \\
\text { methods }\end{array}$ \\
\hline \multicolumn{4}{|l|}{ Polymer properties } \\
\hline Melt flow rate (MFR) & & & ISO 1133 \\
\hline at $230^{\circ} \mathrm{C}$ and $2.16 \mathrm{~kg}$ & $\begin{array}{l}\mathrm{g} / 10 \\
\mathrm{~min}\end{array}$ & 47 & \\
\hline at $230^{\circ} \mathrm{C}$ and $5 \mathrm{~kg}$ & $\begin{array}{l}\mathrm{g} / 10 \\
\mathrm{~min}\end{array}$ & 158 & \\
\hline Density & $\mathrm{kg} / \mathrm{m}^{3}$ & 905 & ISO 1183 \\
\hline \multicolumn{4}{|l|}{ Mechanical properties } \\
\hline Tensile test & & & ISO 527 \\
\hline stress at yield & $\mathrm{MPa}$ & 41 & \\
\hline stress at break & $\mathrm{MPa}$ & 22 & \\
\hline strain at break & $\%$ & 500 & \\
\hline Flexural test & & & $\begin{array}{c}\text { ASTM D } \\
790\end{array}$ \\
\hline Flexural modulus & $\mathrm{MPa}$ & 1900 & \\
\hline Izod impact notched & & & ISO 180/4A \\
\hline at $23^{\circ} \mathrm{C}$ & $\mathrm{kJ} / \mathrm{m}^{2}$ & 2 & \\
\hline Charpy impact notched & & & ISO 179 \\
\hline at $23^{\circ} \mathrm{C}$ & $\mathrm{kJ} / \mathrm{m}^{2}$ & 2 & \\
\hline Hardness Shore D & - & 72 & ISO 868 \\
\hline \multicolumn{4}{|l|}{ Thermal properties } \\
\hline \multicolumn{4}{|l|}{ Heat deflection temperature } \\
\hline at $1.80 \mathrm{MPa}(\mathrm{HDT} / \mathrm{A})$ & ${ }^{\circ} \mathrm{C}$ & 56 & ISO 75/A \\
\hline at $0.45 \mathrm{MPa}$ (HDT/B) & ${ }^{\circ} \mathrm{C}$ & 108 & ISO 75/B \\
\hline \multicolumn{4}{|l|}{ Vicat softening temperature } \\
\hline at $10 \mathrm{~N}(\mathrm{VST} / \mathrm{A})$ & ${ }^{\circ} \mathrm{C}$ & 152 & ISO 306/A \\
\hline at $50 \mathrm{~N}$ (VST/B) & ${ }^{\circ} \mathrm{C}$ & 90 & ISO 306/B \\
\hline
\end{tabular}




\section{Appendix C}

\section{GLM Results}

Results from ANOVA and post hoc tests have been shown here in form of tables for both $A B S$ and $P P$ respectively;

\section{$\underline{A B S}$}

Table C.1 ANOVA Results

Dependent Variable: LSS in MPa

\begin{tabular}{|c|c|c|c|c|c|}
\hline Source & $\begin{array}{l}\text { Type III } \\
\text { Sum of } \\
\text { Squares }\end{array}$ & df & $\begin{array}{l}\text { Mean } \\
\text { Square }\end{array}$ & $\mathbf{F}$ & $\begin{array}{l}\text { P- } \\
\text { Value }\end{array}$ \\
\hline Corrected Model & $25727.911^{a}$ & 31.00 & 829.93 & 133.46 & 0.00 \\
\hline Intercept & 5811.96 & 1.00 & 5811.96 & 934.58 & 0.00 \\
\hline ED & 2970.80 & 1.00 & 2970.80 & 477.72 & 0.00 \\
\hline Static_Force_Categories & 679.23 & 2.00 & 339.62 & 54.61 & 0.00 \\
\hline Amplitude_of_Vibrations & 3064.81 & 3.00 & 1021.60 & 164.28 & 0.00 \\
\hline Weld_Categories & 7768.73 & 2.00 & 3884.37 & 624.62 & 0.00 \\
\hline $\begin{array}{c}E D^{*} \\
\text { Amplitude_of_Vibrations }\end{array}$ & 417.11 & 3.00 & 139.04 & 22.36 & 0.00 \\
\hline $\begin{array}{l}\text { Static_Force_Categories } \\
\text { Amplitude_of_Vibrations }\end{array}$ & 190.07 & 6.00 & 31.68 & 5.09 & 0.00 \\
\hline $\begin{array}{l}\text { Amplitude_of_Vibrations } \\
\text { *Weld_Categories }\end{array}$ & 1138.76 & 6.00 & 189.79 & 30.52 & 0.00 \\
\hline $\begin{array}{c}\text { ED * } \\
\text { Static_Force_Categories }\end{array}$ & 1625.74 & 2.00 & 812.87 & 130.71 & 0.00 \\
\hline ED * Weld_Categories & 148.76 & 2.00 & 74.38 & 11.96 & 0.00 \\
\hline $\begin{array}{l}\text { Static_Force_Categories } \\
\text { *Weld_Categories }\end{array}$ & 492.75 & 4.00 & 123.19 & 19.81 & 0.00 \\
\hline Error & 8171.48 & 1314.00 & 6.22 & & \\
\hline Total & 105573.11 & 1346.00 & & & \\
\hline Corrected Total & 33899.39 & 1345.00 & & & \\
\hline
\end{tabular}

Table C.3 is the extension of Table C.2 for categories of static forces. It shows that all means of LSS are different from each other. 
Table C.2 Results from post hoc tests

\begin{tabular}{|c|c|c|c|c|}
\hline \multirow{2}{*}{$\begin{array}{c}(I) \\
\text { Categories } \\
\text { of Static } \\
\text { Force } \\
\end{array}$} & \multirow{2}{*}{$\begin{array}{c}(\mathrm{J}) \\
\text { Categories } \\
\text { of Static } \\
\text { Force } \\
\end{array}$} & \multirow{2}{*}{$\begin{array}{c}\text { Mean } \\
\text { Difference } \\
(\mathrm{I}-\mathrm{J})\end{array}$} & \multicolumn{2}{|c|}{ 95\% Confidence Interval } \\
\hline & & & $\begin{array}{l}\text { Lower } \\
\text { Bound }\end{array}$ & $\begin{array}{l}\text { Upper } \\
\text { Bound }\end{array}$ \\
\hline \multirow{2}{*}{$\begin{array}{l}500 \text { thru } \\
750\end{array}$} & $\begin{array}{c}1000 \text { thru } \\
1250\end{array}$ & 1.09 & 0.73 & 1.45 \\
\hline & $\begin{array}{c}1500 \text { thru } \\
1750\end{array}$ & 2.39 & 1.96 & 2.82 \\
\hline \multirow{2}{*}{$\begin{array}{c}1000 \text { thru } \\
1250\end{array}$} & $\begin{array}{c}500 \text { thru } \\
750\end{array}$ & -1.09 & -1.45 & -0.73 \\
\hline & $\begin{array}{c}1500 \text { thru } \\
1750\end{array}$ & 1.30 & 0.87 & 1.73 \\
\hline \multirow{2}{*}{$\begin{array}{c}1500 \text { thru } \\
1750\end{array}$} & $\begin{array}{c}500 \text { thru } \\
750 \\
\end{array}$ & -2.39 & -2.82 & -1.96 \\
\hline & $\begin{array}{c}1000 \text { thru } \\
1250\end{array}$ & -1.30 & -1.73 & -0.87 \\
\hline
\end{tabular}

Table C.3 LSS in MPa

\begin{tabular}{|c|c|c|c|c|}
\hline \multirow{2}{*}{$\begin{array}{l}\text { Categories } \\
\text { of Static } \\
\text { Force }\end{array}$} & \multirow{2}{*}{$\mathbf{N}$} & \multicolumn{3}{|c|}{ Subset } \\
\hline & & 1 & 2 & 3 \\
\hline $\begin{array}{c}1500 \text { thru } \\
1750\end{array}$ & 281.00 & 5.84 & & \\
\hline $\begin{array}{c}1000 \text { thru } \\
1250\end{array}$ & 536.00 & & 7.14 & \\
\hline $\begin{array}{c}500 \text { thru } \\
750\end{array}$ & 529.00 & & & 8.23 \\
\hline
\end{tabular}

Table C.5 is the extension of Table C.4 for amplitude of vibrations showing that subset 3 of mean LSS is related to 21 and 18.9 amplitudes and their means are almost similar. But 14.7 and 16.8 have different mean LSS. Likewise, Table C.6 and Table C.7 can be easily interpreted now.

Table C.4 Results from post hoc tests

\begin{tabular}{|c|c|c|c|c|}
\hline \multirow{2}{*}{$\begin{array}{c}\text { (I) } \\
\text { Amplitude }\end{array}$} & \multirow{2}{*}{$\begin{array}{c}(\mathrm{J}) \\
\text { Amplitude }\end{array}$} & \multirow{2}{*}{$\begin{array}{c}\text { Mean } \\
\text { Difference } \\
(\mathrm{I}-\mathrm{J})\end{array}$} & \multicolumn{2}{|c|}{ 95\% Confidence Interval } \\
\hline & & & $\begin{array}{l}\text { Lower } \\
\text { Bound }\end{array}$ & $\begin{array}{l}\text { Upper } \\
\text { Bound }\end{array}$ \\
\hline \multirow{3}{*}{14.7} & 16.8 & -1.15 & -1.74 & -0.55 \\
\hline & 18.9 & -3.13 & -3.72 & -2.53 \\
\hline & 21 & -2.85 & -3.44 & -2.26 \\
\hline \multirow{3}{*}{16.8} & 14.7 & 1.15 & 0.55 & 1.74 \\
\hline & 18.9 & -1.98 & -2.44 & -1.52 \\
\hline & 21 & -1.7 & -2.16 & -1.25 \\
\hline \multirow{3}{*}{18.9} & 14.7 & 3.13 & 2.53 & 3.72 \\
\hline & 16.8 & 1.98 & 1.52 & 2.44 \\
\hline & 21 & 0.28 & -0.18 & 0.73 \\
\hline \multirow{3}{*}{21} & 14.7 & 2.85 & 2.26 & 3.44 \\
\hline & 16.8 & 1.7 & 1.25 & 2.16 \\
\hline & 18.9 & -0.28 & -0.73 & 0.18 \\
\hline
\end{tabular}


Table C.5 LSS in MPa

Tukey HSD

\begin{tabular}{|c|c|c|c|c|}
\hline \multirow{2}{*}{ Amplitude } & \multirow{2}{*}{$\mathbf{N}$} & \multicolumn{3}{|c|}{ Subset } \\
\hline & & 1 & 2 & 3 \\
\hline 14.7 & 166 & 5.19 & & \\
\hline 16.8 & 376 & & 6.34 & \\
\hline 21 & 413 & & & 8.04 \\
\hline 18.9 & 391 & & & 8.32 \\
\hline
\end{tabular}

Table C.6 Results from post hoc tests

Dependent Variable: LSS in MPa

Tukey HSD

\begin{tabular}{|c|c|c|c|c|}
\hline \multirow{2}{*}{ (I) Weld Time } & \multirow{2}{*}{ (J) Weld Time } & \multirow{2}{*}{$\begin{array}{c}\text { Mean } \\
\text { Difference (I-J) }\end{array}$} & \multicolumn{2}{|c|}{ 95\% Confidence Interval } \\
\hline & & & Lower Bound & Upper Bound \\
\hline \multirow{2}{*}{0.4 thru 0.5} & 0.6 thru 0.7 & -4.16 & -4.63 & -3.69 \\
\hline & 0.8 thru 0.9 & -6.37 & -6.78 & -5.95 \\
\hline \multirow{2}{*}{0.6 thru 0.7} & 0.4 thru 0.5 & 4.16 & 3.69 & 4.63 \\
\hline & 0.8 thru 0.9 & -2.21 & -2.59 & -1.83 \\
\hline \multirow{2}{*}{0.8 thru 0.9} & 0.4 thru 0.5 & 6.37 & 5.95 & 6.78 \\
\hline & 0.6 thru 0.7 & 2.21 & 1.83 & 2.59 \\
\hline
\end{tabular}

Table C.7 LSS in MPa

Tukey HSD

\begin{tabular}{||c|c|c|c|c||}
\hline \multirow{2}{*}{ Weld Time } & \multirow{2}{*}{$\mathbf{N}$} & \multicolumn{3}{|c||}{ Subset } \\
\cline { 3 - 5 } & & $\mathbf{1}$ & $\mathbf{2}$ & $\mathbf{3}$ \\
\hline \hline 0.4 thru 0.5 & 274 & 2.82 & & \\
\hline 0.6 thru 0.7 & 360 & & 6.98 & \\
\hline 0.8 thru 0.9 & 712 & & & 9.18 \\
\hline
\end{tabular}

Mean LSS for TRI ED is much higher than that for SEMI ED, as can be seen in Table C.8. 
Table C.8 Energy Director

Dependent Variable: LSS in MPa

\begin{tabular}{||c||c||c|c||}
\hline \multirow{2}{*}{$\begin{array}{c}\text { Energy } \\
\text { Director }\end{array}$} & \multirow{2}{*}{ Mean } & \multicolumn{2}{|c|}{$95 \%$ Confidence Interval } \\
\cline { 3 - 4 } & & $\begin{array}{c}\text { Lower } \\
\text { Bound }\end{array}$ & $\begin{array}{c}\text { Upper } \\
\text { Bound }\end{array}$ \\
\hline \hline SEMI & 1.44 & 0.93 & 1.94 \\
\hline TRI & 7.34 & 7.13 & 7.55 \\
\hline
\end{tabular}

Mean LSS for category of static force 500 thru 750 is the highest one and 1000 thru 1250 is having the second highest mean LSS, as presented in Table C.9.

Table C.9 Categories of Static Force

Dependent Variable: LSS in MPa

\begin{tabular}{|c|c|c|c|}
\hline \multirow{2}{*}{$\begin{array}{c}\text { Categories } \\
\text { of Static } \\
\text { Force }\end{array}$} & \multirow{2}{*}{ Mean } & \multicolumn{2}{|c|}{ 95\% Confidence Interval } \\
\hline & & $\begin{array}{l}\text { Lower } \\
\text { Bound }\end{array}$ & $\begin{array}{l}\text { Upper } \\
\text { Bound }\end{array}$ \\
\hline $\begin{array}{c}500 \text { thru } \\
750\end{array}$ & 5.6 & 5.24 & 5.95 \\
\hline $\begin{array}{c}1000 \text { thru } \\
1250\end{array}$ & 4.29 & 3.97 & 4.6 \\
\hline $\begin{array}{c}1500 \text { thru } \\
1750\end{array}$ & 3.28 & 2.83 & 3.72 \\
\hline
\end{tabular}

Mean LSS for amplitude 21 is the highest and then 18.9, $16.8 \& 14.7$ respectively, as exhibited in Table C.10.

Table C.10 Amplitude

Dependent Variable: LSS in MPa

\begin{tabular}{|c||c||c|c|}
\hline \multirow{2}{*}{ Amplitude } & \multirow{2}{*}{ Mean } & \multicolumn{2}{|c|}{$95 \%$ Confidence Interval } \\
\cline { 3 - 4 } & \multicolumn{2}{|c|}{$\begin{array}{c}\text { Lower } \\
\text { Bound }\end{array}$} & $\begin{array}{c}\text { Upper } \\
\text { Bound }\end{array}$ \\
\hline \hline 14.7 & -1.34 & -2.35 & -0.32 \\
\hline 16.8 & 4.35 & 4.06 & 4.64 \\
\hline 18.9 & 7.11 & 6.84 & 7.39 \\
\hline 21 & 7.42 & 7.16 & 7.68 \\
\hline
\end{tabular}

Mean LSS for category of weld time 0.8 thru 0.9 is the highest and then for 0.6 thru 0.7 is the second highest. And the lowest is for category 0.4 thru 0.5 , as can be seen in Table C.11. 
Table C.11 Weld Time

Dependent Variable: LSS in MPa

\begin{tabular}{||c||c||c|c||}
\hline \multirow{2}{*}{ Weld Time } & \multirow{2}{*}{ Mean } & \multicolumn{2}{|c|}{ 95\% Confidence Interval } \\
\cline { 3 - 4 } & & $\begin{array}{c}\text { Lower } \\
\text { Bound }\end{array}$ & $\begin{array}{c}\text { Upper } \\
\text { Bound }\end{array}$ \\
\hline \hline 0.4 thru 0.5 & 0.29 & -0.17 & 0.75 \\
\hline 0.6 thru 0.7 & 4.8 & 4.42 & 5.19 \\
\hline 0.8 thru 0.9 & 8.07 & 7.79 & 8.35 \\
\hline
\end{tabular}

Post hoc tests have not been performed for Energy Directors because there are fewer than three levels of ED.

\section{$\underline{\text { PP }}$}

Table C.12 ANOVA Results

Dependent Variable: Sqrt_LSS

\begin{tabular}{|c|c|c|c|c|c|}
\hline Source & $\begin{array}{l}\text { Type III } \\
\text { Sum of } \\
\text { Squares }\end{array}$ & df & $\begin{array}{l}\text { Mean } \\
\text { Square }\end{array}$ & $\mathbf{F}$ & P-Value \\
\hline Corrected Model & 265.41 & 25.00 & 10.62 & 37.73 & 0.00 \\
\hline Intercept & 121.43 & 1.00 & 121.43 & 431.57 & 0.00 \\
\hline ED & 4.01 & 1.00 & 4.01 & 14.24 & 0.00 \\
\hline Static_Force_Categories & 30.60 & 2.00 & 15.30 & 54.37 & 0.00 \\
\hline Amplitude_of_Vibrations & 19.88 & 2.00 & 9.94 & 35.32 & 0.00 \\
\hline Weld_Categories & 58.12 & 2.00 & 29.06 & 103.27 & 0.00 \\
\hline $\begin{array}{c}\text { ED * } \\
\text { Amplitude_of_Vibrations }\end{array}$ & 3.88 & 2.00 & 1.94 & 6.89 & 0.00 \\
\hline $\begin{array}{l}\text { Static_Force_Categories } \\
\text { Amplitude_of_Vibrations }\end{array}$ & 10.62 & 4.00 & 2.66 & 9.44 & 0.00 \\
\hline $\begin{array}{c}\text { Amplitude_of_Vibrations } \\
{ }^{*} \text { Weld_Categories }\end{array}$ & 3.43 & 4.00 & 0.86 & 3.04 & 0.02 \\
\hline $\begin{array}{c}\text { ED * } \\
\text { Static_Force_Categories }\end{array}$ & 5.07 & 2.00 & 2.54 & 9.02 & 0.00 \\
\hline ED *Weld_Categories & 3.46 & 2.00 & 1.73 & 6.14 & 0.00 \\
\hline $\begin{array}{l}\text { Static_Force_Categories } \\
{ }^{*} \text { Weld_Categories }\end{array}$ & 15.39 & 4.00 & 3.85 & 13.68 & 0.00 \\
\hline Error & 211.31 & 751.00 & 0.28 & & \\
\hline Total & 1541.32 & 777.00 & & & \\
\hline Corrected Total & 476.72 & 776.00 & & & \\
\hline
\end{tabular}


Now, again all main and interaction effects are significant.

Table C.14 is the extension of Table C.13. Now means of LSS for amplitudes 21 and 18.9 are close to each other and higher than that for 16.8 .

Table C.13 Results from post hoc tests

\begin{tabular}{|c|c||c||c|c|}
\hline \multirow{2}{*}{$\begin{array}{c}\text { (I) } \\
\text { Amplitude }\end{array}$} & \multirow{2}{*}{$\begin{array}{c}(\mathbf{J}) \\
\text { Amplitude }\end{array}$} & $\begin{array}{c}\text { Mean } \\
\text { Difference (I-J) }\end{array}$ & \multicolumn{2}{|c|}{ 95\% Confidence Interval } \\
\cline { 4 - 5 } & & Lower Bound & Upper Bound \\
\hline \hline \multirow{2}{*}{16.8} & 18.9 & -0.40 & -0.51 & -0.29 \\
\cline { 2 - 5 } & 21 & -0.42 & -0.53 & -0.31 \\
\hline \multirow{2}{*}{18.9} & 16.8 & 0.40 & 0.29 & 0.51 \\
\cline { 2 - 5 } & 21 & -0.03 & -0.13 & 0.08 \\
\hline \multirow{2}{*}{21} & 16.8 & 0.42 & 0.31 & 0.53 \\
\cline { 2 - 5 } & 18.9 & 0.03 & -0.08 & 0.13 \\
\hline
\end{tabular}

Table C.14 Sqrt_LSS

\begin{tabular}{|c|c|c|c|}
\hline \multirow{2}{*}{ Amplitude } & \multirow{2}{*}{$\mathbf{N}$} & \multicolumn{2}{|c|}{ Subset } \\
\hline & & 1 & 2 \\
\hline 16.8 & 240 & 0.89 & \\
\hline 18.9 & 275 & & 1.28 \\
\hline 21 & 262 & & 1.31 \\
\hline
\end{tabular}

Table C.16 is the extension of Table C.15 for categories of static forces. It shows that means of LSS for categories 500 thru 750 \& 1500 thru 1750 are close to each other and higher than that for remaining category i.e. 0.97 .

Table C.15 Results from post hoc tests

\begin{tabular}{|c|c|c|c|c|}
\hline \multirow{2}{*}{$\begin{array}{c}\text { (I) } \\
\text { Categories } \\
\text { of Static } \\
\text { Force }\end{array}$} & \multirow{2}{*}{$\begin{array}{c}(\mathrm{J}) \\
\text { Categories } \\
\text { of Static } \\
\text { Force }\end{array}$} & \multirow{2}{*}{$\begin{array}{c}\text { Mean } \\
\text { Difference } \\
(\mathrm{I}-\mathrm{J})\end{array}$} & \multicolumn{2}{|c|}{$95 \%$ Confidence Interval } \\
\hline & & & $\begin{array}{l}\text { Lower } \\
\text { Bound }\end{array}$ & $\begin{array}{l}\text { Upper } \\
\text { Bound }\end{array}$ \\
\hline \multirow{2}{*}{500 thru 750} & $\begin{array}{c}1000 \text { thru } \\
1250\end{array}$ & 0.35 & 0.23 & 0.47 \\
\hline & $\begin{array}{c}1500 \text { thru } \\
1750\end{array}$ & -0.08 & -0.20 & 0.05 \\
\hline \multirow{2}{*}{$\begin{array}{c}1000 \text { thru } \\
1250\end{array}$} & 500 thru 750 & -0.35 & -0.47 & -0.23 \\
\hline & $\begin{array}{c}1500 \text { thru } \\
1750\end{array}$ & -0.43 & -0.53 & -0.33 \\
\hline \multirow{2}{*}{$\begin{array}{c}1500 \text { thru } \\
1750\end{array}$} & 500 thru 750 & 0.08 & -0.05 & 0.20 \\
\hline & $\begin{array}{c}1000 \text { thru } \\
1250\end{array}$ & 0.43 & 0.33 & 0.53 \\
\hline
\end{tabular}


Table C.16 Sqrt_LSS

Tukey HSD

\begin{tabular}{|c|c||c|c||}
\hline \hline \multirow{2}{*}{$\begin{array}{c}\text { Categories } \\
\text { of Static } \\
\text { Force }\end{array}$} & $\mathbf{N}$ & $\mathbf{2}$ & $\mathbf{2}$ \\
\cline { 2 - 4 } & & $\mathbf{1}$ & \\
\hline $\begin{array}{c}1000 \text { thru } \\
1250\end{array}$ & 385 & 0.97 & 1.32 \\
\hline $\begin{array}{c}500 \text { thru } \\
750\end{array}$ & 152 & & 1.40 \\
\hline $\begin{array}{c}1500 \text { thru } \\
1750\end{array}$ & 240 & & \\
\hline
\end{tabular}

Table C.18 is the extension of Table C.17 showing that the means of LSS for categories of weld time i.e. 1.1 thru $1.3 \& 0.8$ thru 1.0 are smaller and closer to each other as compared to that for weld category 1.4 thru 1.7.

Table C.17 Results from post hoc tests

\begin{tabular}{|c|c|c|c|c|c|}
\hline \multirow{2}{*}{$\begin{array}{l}\text { (I) Weld } \\
\text { Time }\end{array}$} & \multirow{2}{*}{$\begin{array}{l}\text { (J) Weld } \\
\text { Time }\end{array}$} & \multirow{2}{*}{$\begin{array}{c}\text { Mean } \\
\text { Difference } \\
(\mathrm{I}-\mathrm{J})\end{array}$} & \multirow{2}{*}{ Std. Error } & \multicolumn{2}{|c|}{$95 \%$ Confidence Interval } \\
\hline & & & & $\begin{array}{l}\text { Lower } \\
\text { Bound }\end{array}$ & $\begin{array}{l}\text { Upper } \\
\text { Bound }\end{array}$ \\
\hline \multirow{2}{*}{0.8 thru 1.0} & 1.1 thru 1.3 & 0.06 & 0.05 & -0.06 & 0.18 \\
\hline & 1.4 thru 1.7 & -0.68 & 0.05 & -0.80 & -0.57 \\
\hline \multirow{2}{*}{1.1 thru 1.3} & 0.8 thru 1.0 & -0.06 & 0.05 & -0.18 & 0.06 \\
\hline & 1.4 thru 1.7 & -0.75 & 0.04 & -0.85 & -0.64 \\
\hline \multirow{2}{*}{1.4 thru 1.7} & 0.8 thru 1.0 & 0.68 & 0.05 & 0.57 & 0.80 \\
\hline & 1.1 thru 1.3 & 0.75 & 0.04 & 0.64 & 0.85 \\
\hline
\end{tabular}

Table C.18 Sqrt_LSS

\begin{tabular}{|c|c|c|c|}
\hline \multirow{2}{*}{ Weld Time } & \multirow{2}{*}{$\mathbf{N}$} & \multicolumn{2}{|c|}{ Subset } \\
\hline & & 1 & 2 \\
\hline 1.1 thru 1.3 & 269 & 0.84 & \\
\hline 0.8 thru 1.0 & 180 & 0.90 & \\
\hline 1.4 thru 1.7 & 328 & & 1.59 \\
\hline
\end{tabular}

Means of LSS for the EDs can be ordered as: SEMI>>TRI, as shown in Table C.19. 
Table C.19 Energy Director

Dependent Variable: Sqrt_LSS

\begin{tabular}{|c|c|c||c|c||}
\hline \multirow{2}{*}{$\begin{array}{c}\text { Energy } \\
\text { Director }\end{array}$} & Mean & \multirow{2}{*}{ Std. Error } & \multicolumn{2}{|c|}{$95 \%$ Confidence Interval } \\
\cline { 4 - 5 } & & $\begin{array}{c}\text { Lower } \\
\text { Bound }\end{array}$ & $\begin{array}{c}\text { Upper } \\
\text { Bound }\end{array}$ \\
\hline \hline SEMI & 1.22 & 0.03 & 1.15 & 1.28 \\
\hline TRI & 0.83 & 0.10 & 0.64 & 1.01 \\
\hline
\end{tabular}

Means of LSS for the categories of static force can be ordered as: 1500 thru $1750 \gg>500$ thru $750 \gg>1000$ thru 1250, as can be seen in Table C.20.

Table C.20 Categories of Static Force

Dependent Variable: Sqrt_LSS

\begin{tabular}{|c|c|c|c|c|}
\hline \multirow{2}{*}{$\begin{array}{l}\text { Categories } \\
\text { of Static } \\
\text { Force }\end{array}$} & \multirow{2}{*}{ Mean } & \multirow{2}{*}{ Std. Error } & \multicolumn{2}{|c|}{ 95\% Confidence Interva } \\
\hline & & & $\begin{array}{l}\text { Lower } \\
\text { Bound }\end{array}$ & $\begin{array}{l}\text { Upper } \\
\text { Bound } \\
\end{array}$ \\
\hline $\begin{array}{c}500 \text { thru } \\
750 \\
\end{array}$ & 1.17 & 0.07 & 1.04 & 1.31 \\
\hline $\begin{array}{c}1000 \text { thru } \\
1250\end{array}$ & 0.72 & 0.06 & 0.61 & 0.83 \\
\hline $\begin{array}{c}1500 \text { thru } \\
1750\end{array}$ & 1.18 & 0.05 & 1.07 & 1.28 \\
\hline
\end{tabular}

Means of LSS for the amplitudes can be ordered as: $21>>18.9>>16.8$, as shown in Table C.21.

Table C.21 Amplitude

Dependent Variable: Sqrt_LSS

\begin{tabular}{|c||c|c||c|c||}
\hline \multirow{2}{*}{ Amplitude } & \multirow{2}{*}{ Mean } & \multirow{2}{*}{ Std. Error } & \multicolumn{2}{|c|}{$95 \%$ Confidence Interval } \\
\cline { 4 - 5 } & & $\begin{array}{r}\text { Lower } \\
\text { Bound }\end{array}$ & $\begin{array}{c}\text { Upper } \\
\text { Bound }\end{array}$ \\
\hline \hline 16.8 & 0.73 & 0.07 & 0.60 & 0.85 \\
\hline 18.9 & 1.16 & 0.05 & 1.06 & 1.26 \\
\hline 21 & 1.18 & 0.06 & 1.07 & 1.30 \\
\hline
\end{tabular}

Means of LSS for the categories of weld time may be ordered as: 1.4 thru 1.7 $\gg>1.1$ thru $1.3>>0.8$ thru 1.0, as exhibited in Table C.22. 
Table C.22 Weld Time

Dependent Variable: Sqrt_LSS

\begin{tabular}{|c|c|c|c|c|}
\hline \multirow{2}{*}{ Weld Time } & \multirow{2}{*}{ Mean } & \multirow{2}{*}{ Std. Error } & \multicolumn{2}{|c|}{$95 \%$ Confidence Interval } \\
\hline & & & $\begin{array}{l}\text { Lower } \\
\text { Bound }\end{array}$ & $\begin{array}{l}\text { Upper } \\
\text { Bound }\end{array}$ \\
\hline 0.8 thru 1.0 & 0.42 & 0.14 & 0.15 & 0.68 \\
\hline 1.1 thru 1.3 & 0.99 & 0.04 & 0.91 & 1.07 \\
\hline 1.4 thru 1.7 & 1.66 & 0.04 & 1.59 & 1.73 \\
\hline
\end{tabular}




\section{Appendix D}

A general linear model results in an equation using the LSS data. This equation may help the analyser predict the parameters (e.g. LSS or Sqrt_LSS) which are linearly dependent on weld factors and their interaction. This may be understood by presenting the concept of slopeintercept form of linear equation, as shown in Figure D.1.

Figure D.1 Slope-intercept form of linear equation [200]

Where, LSS or Sqrt_LSS is represented by Y-axis. Weld factors and their interactions are represented by X-axis. Although slope is usually represented by ' $\mathrm{m}$ ', slope relevant to each level of weld factor is being represented by B particularly for appendix $D$, as mentioned in second column of Table D.1 and Table D.2, except intercept which is shown in second row of Table D.1 and Table D.2. Hence the slope-intercept equation resulting from GLM may be given as;

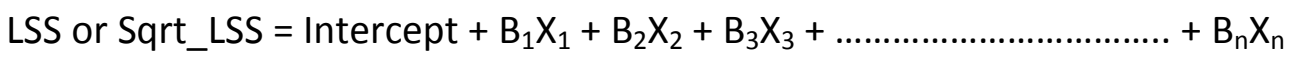

Where, $B_{1}$ is the slope for a particular level of a weld factor $\left(X_{1}\right)$ and so on. Therefore, equations D1 and D2 can be developed by using Table D.1 and Table D.2 respectively. Equations D1 and D2 for $A B S$ and PP respectively show how different parameters are predicted, estimated and associated with weld factors. The numbers (1/0) imply that whether a level of weld factor or an interaction between factors has been (1)/has not been 
(0) used for welding a thermoplastic. These numbers (1/0) have frequently been used in both equations D1 and D2.

\section{$\underline{A B S}$}

By looking at the parameter estimates' Table D.1, the predicted model for ABS may be written as;

Predicted LSS $=9.78-5.01 *(14.7 *(1 / 0))+1.24 *(16.8 *(1 / 0))+0.32 *(18.9 *(1 / 0))-$ $1.36 *(\operatorname{SEMI} *(1 / 0))+3.64 *(500$ thru $750 *(1 / 0))+2.07 *(1000$ thru $1250 *(1 / 0))-6.69 *(0.8$ thru $1.0 *(1 / 0))-1.05 *(1.1 \quad$ thru $1.3 *(1 / 0))-2.66 *((14.7 *$ SEMI $) *(1 / 0))-2.42 *((16.8 *$ SEMI $) *(1 / 0))+$ $0.20 *((18.9 *$ SEMI $) *(1 / 0))))+1.53 *((14.7 * 500$ thru 750$) *(1 / 0))-0.15 *((14.7 * 1000$ thru $1250 *(1 / 0))-$ $1.10 *((16.8 * 500$ thru 750$) *(1 / 0))-0.55 *((16.8 * 1000$ thru 1250$) *(1 / 0))-0.27 *((18.9 * 500$ thru $750 *(1 / 0))+0.54 *((18.9 * 1000$ thru 1250$) *(1 / 0))-2.82 *\left((14.7 * 0.8\right.$ thru $1.0 *(1 / 0))-5.81 *\left(\left(14.7^{*} 1.1\right.\right.$ thru 1.3$\left.)^{*}(1 / 0)\right)-4.30 *((16.8 * 0.8$ thru 1.0$) *(1 / 0))-3.36 *((16.8 * 1.1$ thru 1.3$) *(1 / 0))-1.67 *((18.9 * 0.8$ thru 1.0$\left.)^{*}(1 / 0)\right)-0.79 *((18.9 * 1.1$ thru 1.3$) *(1 / 0))-6.16 *((\operatorname{SEMI} * 500$ thru 750$) *(1 / 0))-$ $2.60 *(($ SEMI*1000 thru 1250) $) *(1 / 0))+0.31 *(($ SEMI $) *(0.8$ thru 1.0$)) *(1 / 0))-1.51 *(($ SEMI)*(1.1 thru $1.3)) *(1 / 0))+3.06 *(((500$ thru 750$) *(0.8$ thru 1.0$)) *(1 / 0))+2.09 *(((500$ thru 750$) *(1.1$ thru $1.3)) *(1 / 0))-0.18 *(((1000$ thru 1250)*( 0.8 thru 1.0) $) *(1 / 0))+0.99 *(((1000$ thru 1250)*( 1.1 thru 1.3))

Parameter Estimate's Table D.1 for ABS;

Table D.1 Parameter Estimates

Dependent Variable: LSS in MPa

\begin{tabular}{|c|c|c|c|}
\hline \multirow{2}{*}{ Parameter } & \multirow{2}{*}{ B } & \multicolumn{2}{|c|}{$\begin{array}{c}95 \% \text { Confidence } \\
\text { Interval }\end{array}$} \\
\hline & & $\begin{array}{l}\text { Lower } \\
\text { Bound } \\
\end{array}$ & $\begin{array}{l}\text { Upper } \\
\text { Bound }\end{array}$ \\
\hline Intercept & 9.78 & 9.02 & 10.53 \\
\hline [Amplitude_of_Vibrations=14.7] & -5.01 & -6.23 & -3.79 \\
\hline [Amplitude_of_Vibrations=16.8] & 1.24 & 0.33 & 2.16 \\
\hline [Amplitude_of_Vibrations=18.9] & 0.32 & -0.58 & 1.23 \\
\hline [Amplitude_of_Vibrations=21.0] & $0^{\mathrm{a}}$ & . & . \\
\hline$[E D=1]$ & -1.36 & -2.16 & -0.57 \\
\hline [ED=2] & $0^{\mathrm{a}}$ & . & . \\
\hline [Static_Force_Categories=1.00] & 3.64 & 2.79 & 4.49 \\
\hline [Static_Force_Categories=2.00] & 2.07 & 1.22 & 2.92 \\
\hline [Static_Force_Categories=3.00] & $0^{\mathrm{a}}$ & . & . \\
\hline [Weld_Categories=1.00] & -6.69 & -7.66 & -5.72 \\
\hline [Weld_Categories=2.00] & -1.05 & -1.99 & -0.11 \\
\hline [Weld_Categories=3.00] & $0^{\mathrm{a}}$ & . & . \\
\hline [Amplitude_of_Vibrations $=14.7]^{*}[\mathrm{ED}=1]$ & -2.66 & -4.49 & -0.84 \\
\hline
\end{tabular}


Table D.1 Parameter Estimates

Dependent Variable: LSS in MPa

\begin{tabular}{|c|c|c|c|}
\hline \multirow{2}{*}{ Parameter } & \multirow{2}{*}{ B } & \multicolumn{2}{|c|}{$\begin{array}{c}95 \% \text { Confidence } \\
\text { Interval }\end{array}$} \\
\hline & & $\begin{array}{l}\text { Lower } \\
\text { Bound } \\
\end{array}$ & $\begin{array}{l}\text { Upper } \\
\text { Bound } \\
\end{array}$ \\
\hline [Amplitude_of_Vibrations=14.7] * [ED=2] & $0^{\mathrm{a}}$ & . & . \\
\hline [Amplitude_of_Vibrations $=16.8]^{*}[E D=1]$ & -2.42 & -3.13 & -1.71 \\
\hline [Amplitude_of_Vibrations $=16.8]^{*}[\mathrm{ED}=2]$ & $0^{\mathrm{a}}$ & . & . \\
\hline [Amplitude_of_Vibrations=18.9] * [ED=1] & 0.20 & -0.50 & 0.89 \\
\hline [Amplitude_of_Vibrations=18.9] * [ED=2] & $0^{\mathrm{a}}$ & . & . \\
\hline [Amplitude_of_Vibrations $=21.0] *[E D=1]$ & $0^{\mathrm{a}}$ & . & . \\
\hline [Amplitude_of_Vibrations=21.0] * [ED=2] & $0^{\mathrm{a}}$ & . & . \\
\hline $\begin{array}{l}\text { [Amplitude_of_Vibrations }=14.7]^{*} \\
{[\text { Static_Force_Categories }=1.00]}\end{array}$ & 1.53 & 0.14 & 2.91 \\
\hline $\begin{array}{c}\text { [Amplitude_of_Vibrations }=14.7]{ }^{*} \\
{[\text { Static_Force_Categories }=2.00]}\end{array}$ & -0.15 & -1.55 & 1.25 \\
\hline $\begin{array}{l}\text { [Amplitude_of_Vibrations }=14.7]^{*} \\
{[\text { Static_Force_Categories }=3.00]}\end{array}$ & $0^{\mathrm{a}}$ & . & . \\
\hline $\begin{array}{l}\text { [Amplitude_of_Vibrations }=16.8]^{*} \\
{[\text { Static_Force_Categories }=1.00]}\end{array}$ & -1.10 & -2.04 & -0.15 \\
\hline $\begin{array}{l}\text { [Amplitude_of_Vibrations }=16.8]^{*} \\
\text { [Static_Force_Categories }=2.00]\end{array}$ & -0.55 & -1.48 & 0.39 \\
\hline $\begin{array}{l}\text { [Amplitude_of_Vibrations }=16.8]^{*} \\
\text { [Static_Force_Categories }=3.00]\end{array}$ & $0^{\mathrm{a}}$ & . & . \\
\hline $\begin{array}{c}\text { [Amplitude_of_Vibrations }=18.9]^{*} \\
{[\text { Static_Force_Categories }=1.00]}\end{array}$ & -0.27 & -1.20 & 0.66 \\
\hline $\begin{array}{c}\text { [Amplitude_of_Vibrations }=18.9]^{*} \\
{[\text { Static_Force_Categories }=2.00]}\end{array}$ & 0.54 & -0.39 & 1.47 \\
\hline $\begin{array}{c}\text { [Amplitude_of_Vibrations }=18.9]^{*} \\
{[\text { Static_Force_Categories }=3.00]}\end{array}$ & $0^{\mathrm{a}}$ & . & . \\
\hline $\begin{array}{c}\text { [Amplitude_of_Vibrations }=21.0]^{*} \\
\text { [Static_Force_Categories }=1.00]\end{array}$ & $0^{\mathrm{a}}$ & . & . \\
\hline $\begin{array}{c}{[\text { Amplitude_of_Vibrations }=21.0]} \\
{[\text { Static_Force_Categories }=2.00]}\end{array}$ & $0^{\mathrm{a}}$ & . & . \\
\hline $\begin{array}{l}\text { [Amplitude_of_Vibrations }=21.0]^{*} \\
{[\text { Static_Force_Categories }=3.00]}\end{array}$ & $0^{\mathrm{a}}$ & . & . \\
\hline [Amplitude_of_Vibrations=14.7] * [Weld_Categories=1.00] & -2.82 & -4.21 & -1.44 \\
\hline [Amplitude_of_Vibrations $=14.7]^{*}$ [Weld_Categories=2.00] & -5.81 & -7.00 & -4.62 \\
\hline [Amplitude_of_Vibrations=14.7] * [Weld_Categories=3.00] & $0^{\mathrm{a}}$ & . & . \\
\hline [Amplitude_of_Vibrations=16.8] * [Weld_Categories=1.00] & -4.30 & -5.23 & -3.38 \\
\hline [Amplitude_of_Vibrations $=16.8]^{*}$ [Weld_Categories=2.00] & -3.36 & -4.20 & -2.53 \\
\hline [Amplitude_of_Vibrations=16.8] * [Weld_Categories=3.00] & $0^{\mathrm{a}}$ & . & . \\
\hline [Amplitude_of_Vibrations=18.9] * [Weld_Categories=1.00] & -1.67 & -2.55 & -0.78 \\
\hline [Amplitude_of_Vibrations=18.9] * [Weld_Categories=2.00] & -0.79 & -1.61 & 0.04 \\
\hline [Amplitude_of_Vibrations=18.9] * [Weld_Categories=3.00] & $0^{\mathrm{a}}$ & . & . \\
\hline [Amplitude_of_Vibrations=21.0] * [Weld_Categories=1.00] & $0^{\mathrm{a}}$ & . & . \\
\hline [Amplitude_of_Vibrations=21.0] * [Weld_Categories=2.00] & $0^{a}$ & . & . \\
\hline [Amplitude_of_Vibrations=21.0] * [Weld_Categories=3.00] & $0^{\mathrm{a}}$ & . & . \\
\hline$[E D=1]^{*}[$ Static_Force_Categories $=1.00]$ & -6.16 & -6.94 & -5.38 \\
\hline$[E D=1]^{*}[$ Static_Force_Categories $=2.00]$ & -2.60 & -3.36 & -1.83 \\
\hline$[E D=1]^{*}[$ Static_Force_Categories $=3.00]$ & $0^{\mathrm{a}}$ & . & . \\
\hline$[E D=2]{ }^{*}[$ Static_Force_Categories $=1.00]$ & $0^{\mathrm{a}}$ & . & . \\
\hline$[E D=2] *[$ Static_Force_Categories $=2.00]$ & $0^{\mathrm{a}}$ & . & . \\
\hline$[E D=2]{ }^{*}[$ Static_Force_Categories $=3.00]$ & $0^{\mathrm{a}}$ & . & . \\
\hline
\end{tabular}


Table D.1 Parameter Estimates

Dependent Variable: LSS in MPa

\begin{tabular}{|c|c|c|c|}
\hline \multirow{2}{*}{ Parameter } & \multirow{2}{*}{ B } & \multicolumn{2}{|c|}{$\begin{array}{c}95 \% \text { Confidence } \\
\text { Interval }\end{array}$} \\
\hline & & $\begin{array}{l}\text { Lower } \\
\text { Bound } \\
\end{array}$ & $\begin{array}{l}\text { Upper } \\
\text { Bound }\end{array}$ \\
\hline$[E D=1]^{*}[$ Weld_Categories $=1.00]$ & 0.31 & -0.47 & 1.08 \\
\hline$[E D=1]^{*}[$ Weld_Categories $=2.00]$ & -1.51 & -2.18 & -0.83 \\
\hline$[E D=1]^{*}[$ Weld_Categories=3.00] & $0^{a}$ & . & . \\
\hline$[E D=2] *[$ Weld_Categories $=1.00]$ & $0^{\mathrm{a}}$ & . & . \\
\hline$[E D=2] *[$ Weld_Categories=2.00] & $0^{\mathrm{a}}$ & . & . \\
\hline$[E D=2] *[$ Weld_Categories=3.00] & $0^{\mathrm{a}}$ & . & . \\
\hline [Static_Force_Categories $=1.00]^{*}[$ Weld_Categories $=1.00]$ & 3.06 & 2.12 & 4.01 \\
\hline [Static_Force_Categories $=1.00]^{*}[$ Weld_Categories $=2.00$ ] & 2.09 & 1.20 & 2.98 \\
\hline [Static_Force_Categories $=1.00]^{*}[$ Weld_Categories $=3.00]$ & $0^{\mathrm{a}}$ & . & . \\
\hline [Static_Force_Categories $=2.00]^{*}[$ Weld_Categories $=1.00]$ & -0.18 & -1.11 & 0.74 \\
\hline [Static_Force_Categories $=2.00]^{*}[$ Weld_Categories $=2.00]$ & 0.99 & 0.10 & 1.89 \\
\hline$[$ Static_Force_Categories $=2.00]{ }^{*}[$ Weld_Categories $=3.00]$ & $0^{\mathrm{a}}$ & . & . \\
\hline [Static_Force_Categories $=3.00]^{*}[$ Weld_Categories $=1.00]$ & $0^{\mathrm{a}}$ & . & . \\
\hline [Static_Force_Categories $=3.00]^{*}[$ Weld_Categories $=2.00]$ & $0^{\mathrm{a}}$ & . & . \\
\hline [Static_Force_Categories $=3.00]$ * [Weld_Categories $=3.00]$ & $0^{\mathrm{a}}$ & . & . \\
\hline
\end{tabular}

a. This parameter is set to zero because it is redundant.

$\underline{P P}$

By looking at the parameter estimates' Table D.2, the predicted model for PP can be written as;

Predicted Square Root (LSS) $=2.34-0.31 *(16.8 *(1 / 0))-0.09 *(18.9 *(1 / 0))-0.16 *($ SEMI*(1/0))$1.08 *(500$ thru $750 *(1 / 0))-0.71^{*}(1000$ thru $1250 *(1 / 0))-2.03^{*}(0.8$ thru $1.0 *(1 / 0))-1.15^{*}(1.1$ thru $1.3 *(1 / 0))-0.33 *((16.8 *$ SEMI $) *(1 / 0))+0.08 *((18.9 *$ SEMI $) *(1 / 0))+0.39 *((16.8 * 500$ thru 750$) *(1 / 0))-$ $0.33 *((16.8 * 1000$ thru 1250$) *(1 / 0))-0.07 *((18.9 * 500$ thru 750$) *(1 / 0))+0.14 *((18.9 * 1000$ thru $\left.1250)^{*}(1 / 0)\right)-0.27 *((16.8 * 0.8$ thru 1.0$) *(1 / 0))+0.26 *((16.8 * 1.1$ thru 1.3$) *(1 / 0))-0.21 *((18.9 * 0.8$ thru $1.0) *(1 / 0))+0.08 *((18.9 * 1.1$ thru 1.3$) *(1 / 0))+0.71 *(($ SEMI $* 500$ thru 750$) *(1 / 0))+0.15 *(($ SEMI*1000 thru 1250$)) *(1 / 0))+0.74 *(($ SEMI $) *(0.8$ thru 1.0$)) *(1 / 0))+0.27 *(($ SEMI $) *(1.1 \quad$ thru $1.3)) *(1 / 0))+1.13 *(((500$ thru 750$) *(0.8$ thru 1.0$)) *(1 / 0))+0.55 *(((500$ thru 750$) *(1.1$ thru $1.3)) *(1 / 0))+0.60 *(((1000$ thru 1250$) *(0.8$ thru 1.0$)) *(1 / 0))+0.12 *(((1000$ thru 1250)*( 1.1 thru 1.3))

After predicting square root LSS value, it must be squared immediately e.g. if;

Predicted Square Root (LSS) $=$ X MPa 
Then

Predicted LSS $=\mathrm{X}^{2} \mathrm{MPa}$

Parameter Estimates' Table D.2 for PP;

Table D.2 Parameter Estimates

Dependent Variable: Sqrt LSS

\begin{tabular}{|c|c|c|c|}
\hline \multirow{2}{*}{ Parameter } & \multirow[b]{2}{*}{ B } & \multicolumn{2}{|c|}{ 95\% Confidence Interval } \\
\hline & & $\begin{array}{l}\text { Lower } \\
\text { Bound }\end{array}$ & $\begin{array}{l}\text { Upper } \\
\text { Bound }\end{array}$ \\
\hline Intercept & 2.34 & 2.15 & 2.52 \\
\hline [Amplitude_of_Vibrations=16.8] & -0.31 & -0.54 & -0.07 \\
\hline [Amplitude_of_Vibrations=18.9] & -0.09 & -0.32 & 0.15 \\
\hline [Amplitude_of_Vibrations=21.0] & $0^{\mathrm{a}}$ & & \\
\hline$[E D=1]$ & -0.16 & -0.39 & 0.08 \\
\hline$[E D=2]$ & $0^{\mathrm{a}}$ & & \\
\hline [Static_Force_Categories=1.00] & -1.08 & -1.35 & -0.80 \\
\hline [Static_Force_Categories=2.00] & -0.71 & -0.92 & -0.50 \\
\hline [Static_Force_Categories=3.00] & $0^{\mathrm{a}}$ & & \\
\hline [Weld_Categories=1.00] & -2.03 & -2.59 & -1.48 \\
\hline [Weld_Categories=2.00] & -1.15 & -1.36 & -0.94 \\
\hline [Weld_Categories=3.00] & $0^{\mathrm{a}}$ & & \\
\hline [Amplitude_of_Vibrations $=16.8]^{*}[E D=1]$ & -0.33 & -0.55 & -0.10 \\
\hline [Amplitude_of_Vibrations=16.8] * [ED=2] & $0^{\mathrm{a}}$ & & \\
\hline [Amplitude_of_Vibrations $=18.9]^{*}[E D=1]$ & 0.08 & -0.15 & 0.31 \\
\hline [Amplitude_of_Vibrations=18.9] * [ED=2] & $0^{\mathrm{a}}$ & & \\
\hline [Amplitude_of_Vibrations $=21.0]^{*}[E D=1]$ & $0^{\mathrm{a}}$ & & \\
\hline [Amplitude_of_Vibrations=21.0] * [ED=2] & $0^{\mathrm{a}}$ & & \\
\hline [Amplitude_of_Vibrations $=16.8]^{*}$ [Static_Force_Categories $=1.00$ ] & 0.39 & 0.06 & 0.71 \\
\hline
\end{tabular}


Table D.2 Parameter Estimates

Dependent Variable: Sqrt_LSS

\begin{tabular}{|c|c|c|c|}
\hline \multirow[b]{2}{*}{ Parameter } & \multirow[b]{2}{*}{ B } & \multicolumn{2}{|c|}{ 95\% Confidence Interval } \\
\hline & & $\begin{array}{l}\text { Lower } \\
\text { Bound }\end{array}$ & $\begin{array}{l}\text { Upper } \\
\text { Bound }\end{array}$ \\
\hline [Amplitude_of_Vibrations $=16.8]^{*}$ [Static_Force_Categories $=2.00$ ] & -0.33 & -0.55 & -0.12 \\
\hline [Amplitude_of_Vibrations $=16.8]^{*}$ [Static_Force_Categories $=3.00$ ] & $0^{\mathrm{a}}$ & & \\
\hline [Amplitude_of_Vibrations $=18.9$ ] * [Static_Force_Categories $=1.00$ ] & 0.07 & -0.19 & 0.33 \\
\hline [Amplitude_of_Vibrations $=18.9]^{*}$ [Static_Force_Categories $=2.00$ ] & 0.14 & -0.07 & 0.35 \\
\hline [Amplitude_of_Vibrations $=18.9]^{*}$ [Static_Force_Categories $=3.00$ ] & $0^{\mathrm{a}}$ & & \\
\hline [Amplitude_of_Vibrations $=21.0]^{*}$ [Static_Force_Categories $=1.00$ ] & $0^{\mathrm{a}}$ & & \\
\hline [Amplitude_of_Vibrations $=21.0]$ * [Static_Force_Categories $=2.00$ ] & $0^{\mathrm{a}}$ & & \\
\hline [Amplitude_of_Vibrations $=21.0]^{*}$ [Static_Force_Categories $=3.00$ ] & $0^{\mathrm{a}}$ & & \\
\hline [Amplitude_of_Vibrations=16.8] * [Weld_Categories=1.00] & -0.27 & -0.56 & 0.02 \\
\hline [Amplitude_of_Vibrations=16.8] * [Weld_Categories=2.00] & 0.26 & 0.04 & 0.48 \\
\hline [Amplitude_of_Vibrations=16.8] * [Weld_Categories=3.00] & $0^{\mathrm{a}}$ & & \\
\hline [Amplitude_of_Vibrations=18.9] * [Weld_Categories=1.00] & -0.21 & -0.46 & 0.04 \\
\hline
\end{tabular}


Table D.2 Parameter Estimates

Dependent Variable: Sqrt_LSS

\begin{tabular}{|c|c|c|c|}
\hline \multirow[b]{2}{*}{ Parameter } & \multirow[b]{2}{*}{ B } & \multicolumn{2}{|c|}{ 95\% Confidence Interval } \\
\hline & & $\begin{array}{l}\text { Lower } \\
\text { Bound }\end{array}$ & $\begin{array}{l}\text { Upper } \\
\text { Bound }\end{array}$ \\
\hline [Amplitude_of_Vibrations=18.9] * [Weld_Categories=2.00] & 0.08 & -0.14 & 0.30 \\
\hline [Amplitude_of_Vibrations=18.9] * [Weld_Categories=3.00] & $0^{\mathrm{a}}$ & & \\
\hline [Amplitude_of_Vibrations=21.0] * [Weld_Categories=1.00] & $0^{\mathrm{a}}$ & & \\
\hline [Amplitude_of_Vibrations=21.0] * [Weld_Categories=2.00] & $0^{\mathrm{a}}$ & & \\
\hline [Amplitude_of_Vibrations=21.0] * [Weld_Categories=3.00] & $0^{\mathrm{a}}$ & & \\
\hline$[E D=1]{ }^{*}[$ Static_Force_Categories $=1.00]$ & 0.71 & 0.38 & 1.04 \\
\hline$[E D=1]^{*}[$ Static_Force_Categories $=2.00]$ & 0.15 & -0.05 & 0.36 \\
\hline$[E D=1]^{*}[$ Static_Force_Categories $=3.00]$ & $0^{\mathrm{a}}$ & & \\
\hline$[E D=2] *[$ Static_Force_Categories $=1.00]$ & $0^{\mathrm{a}}$ & & \\
\hline$[E D=2]{ }^{*}[$ Static_Force_Categories $=2.00]$ & $0^{\mathrm{a}}$ & & \\
\hline$[E D=2] *[$ Static_Force_Categories $=3.00]$ & $0^{\mathrm{a}}$ & & \\
\hline$[E D=1]^{*}[$ Weld_Categories $=1.00]$ & 0.74 & 0.19 & 1.29 \\
\hline$[E D=1] *[$ Weld_Categories $=2.00]$ & 0.27 & 0.08 & 0.46 \\
\hline$[E D=1]{ }^{*}[$ Weld_Categories $=3.00]$ & $0^{\mathrm{a}}$ & & \\
\hline$[E D=2] *[$ Weld_Categories $=1.00]$ & $0^{a}$ & & \\
\hline$[E D=2] *[$ Weld_Categories=2.00] & $0^{a}$ & & \\
\hline$[E D=2]$ * [Weld_Categories=3.00] & $0^{\mathrm{a}}$ & & \\
\hline
\end{tabular}


Table D.2 Parameter Estimates

Dependent Variable: Sqrt_LSS

\begin{tabular}{|c|c|c|c|}
\hline \multirow{2}{*}{ Parameter } & \multirow{2}{*}{ B } & \multicolumn{2}{|c|}{ 95\% Confidence Interval } \\
\hline & & $\begin{array}{l}\text { Lower } \\
\text { Bound }\end{array}$ & $\begin{array}{l}\text { Upper } \\
\text { Bound }\end{array}$ \\
\hline [Static_Force_Categories $=1.00]^{*}[$ Weld_Categories $=1.00]$ & 1.13 & 0.82 & 1.45 \\
\hline [Static_Force_Categories $=1.00]^{*}[$ Weld_Categories $=2.00]$ & 0.55 & 0.26 & 0.84 \\
\hline [Static_Force_Categories $=1.00]^{*}[$ Weld_Categories $=3.00]$ & $0^{\mathrm{a}}$ & & \\
\hline [Static_Force_Categories $=2.00]^{*}[$ Weld_Categories $=1.00]$ & 0.60 & 0.32 & 0.87 \\
\hline [Static_Force_Categories $=2.00]^{*}[$ Weld_Categories $=2.00]$ & 0.12 & -0.09 & 0.32 \\
\hline [Static_Force_Categories $=2.00$ ] * [Weld_Categories $=3.00]$ & $0^{\mathrm{a}}$ & & \\
\hline [Static_Force_Categories $=3.00$ ] * [Weld_Categories $=1.00]$ & $0^{\mathrm{a}}$ & & \\
\hline [Static_Force_Categories $=3.00]^{*}[$ Weld_Categories $=2.00]$ & $0^{\mathrm{a}}$ & & \\
\hline [Static_Force_Categories $=3.00]^{*}$ [Weld_Categories=3.00] & $0^{\mathrm{a}}$ & & \\
\hline
\end{tabular}

a. This parameter is set to zero because it is redundant. 


\section{Appendix E}

Various drawings and a pneumatic circuit used for manufacturing and assembling ultrasonic welding rig have been presented in this appendix;

\section{Drawings of Clamps}

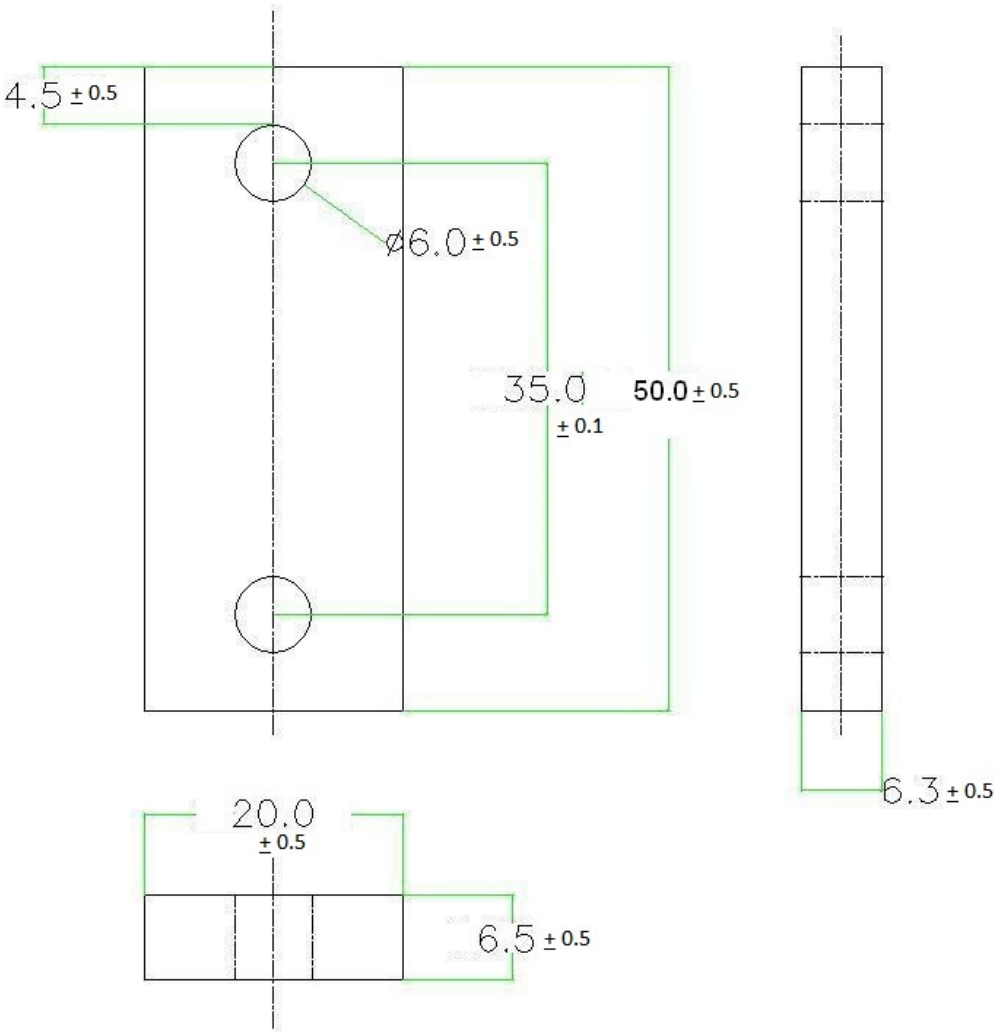

Figure E.1 Upper/stationary Jaw of Shear Clamp 


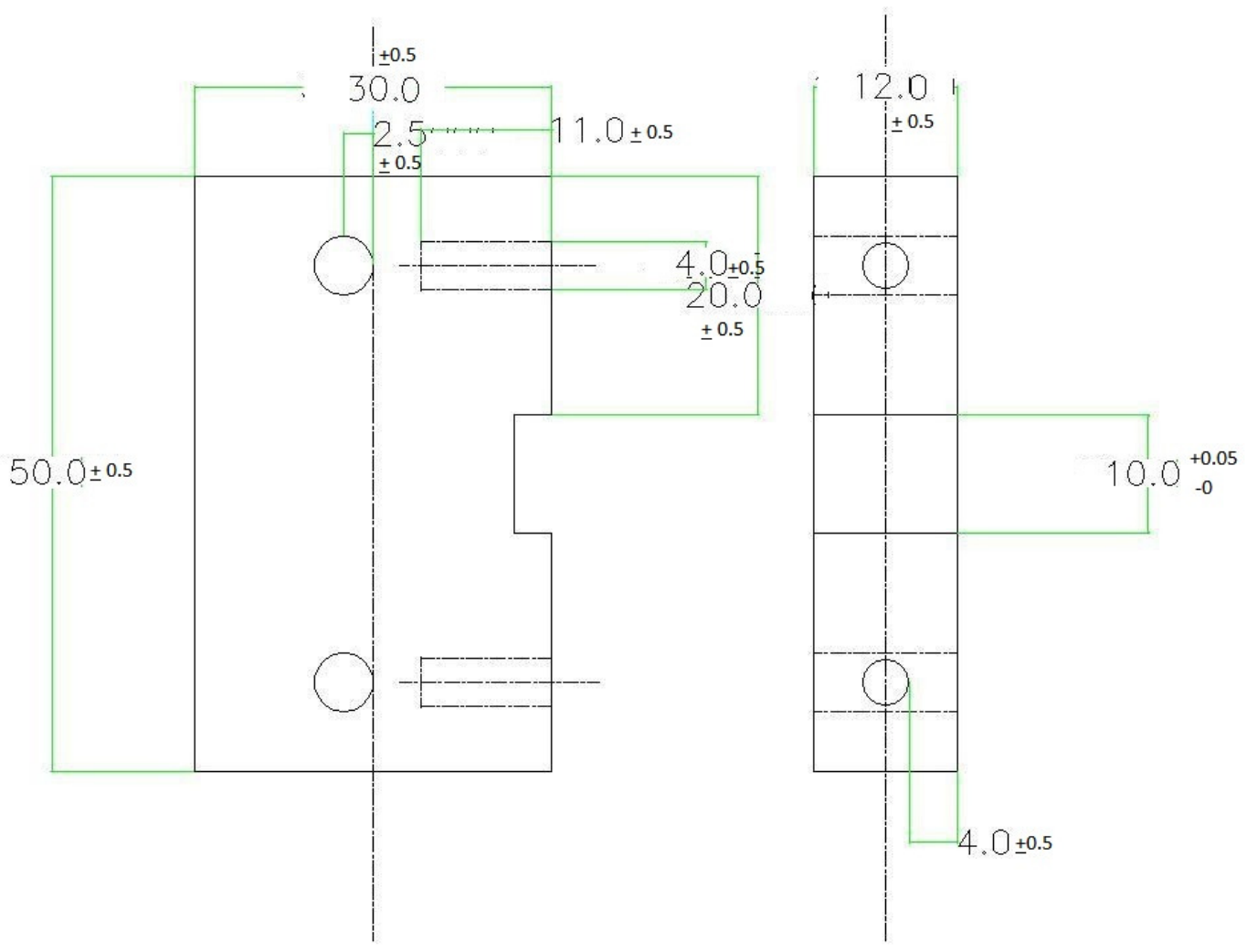

Figure E.2 Lower/moveable Jaw of Shear Clamp 
Drawing of Anvil

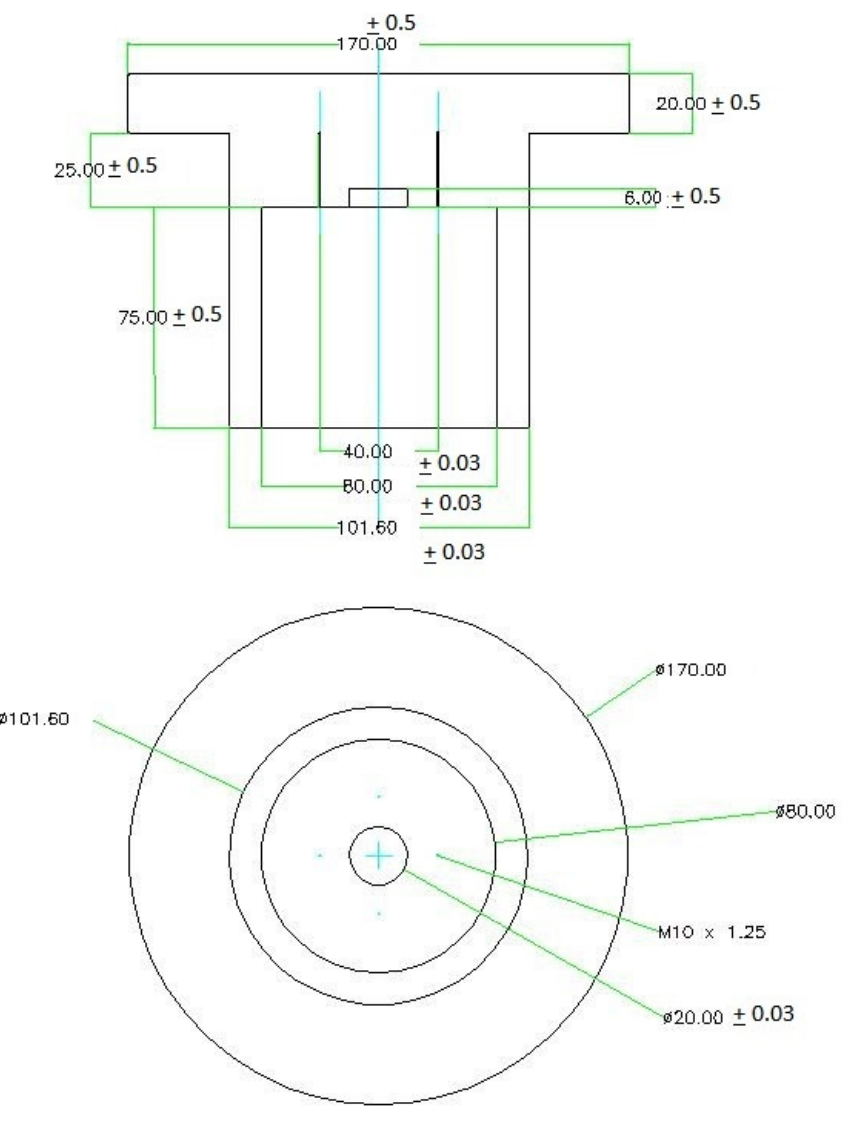

Figure E.3 Anvil for ultrasonic welding rig 


\section{Drawing of Fixtures}

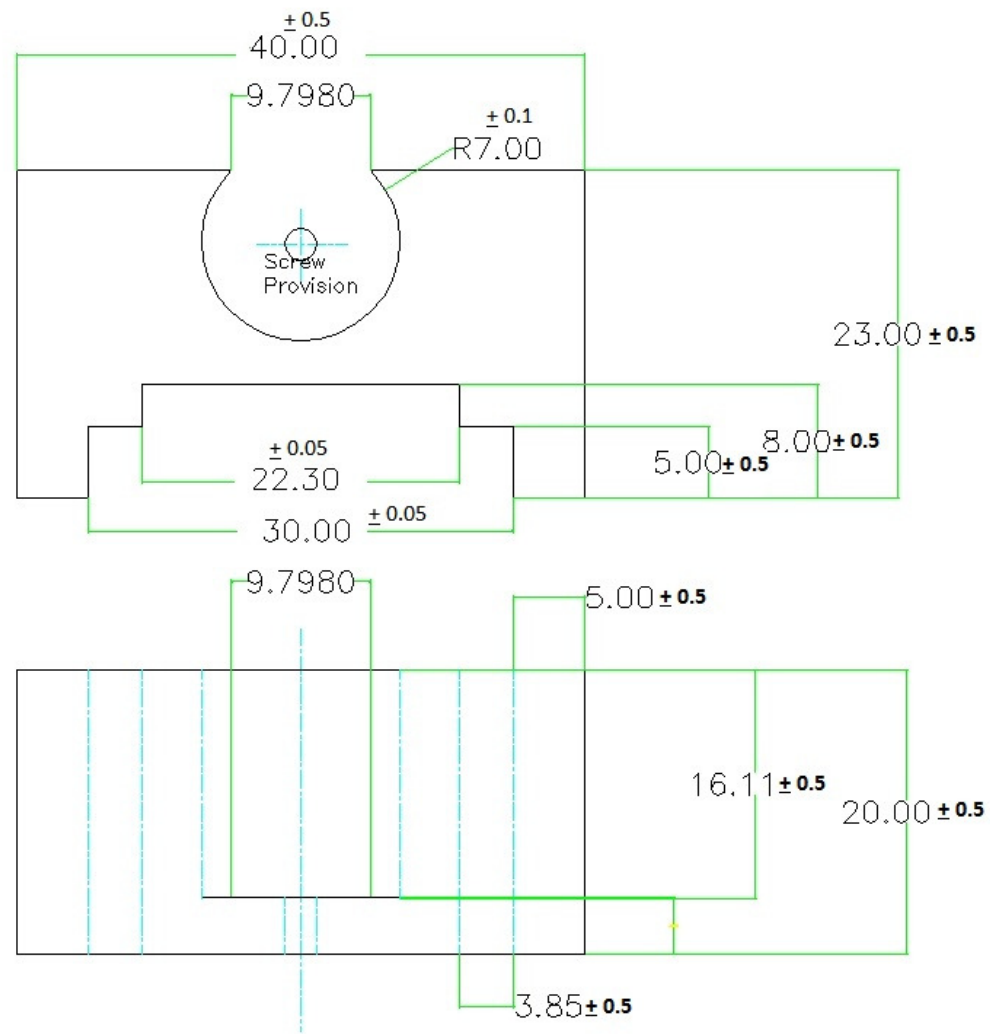

Figure E.4 Fixture to hold samples to be welded

\section{Pneumatic Circuit used in Ultrasonic Welding Rig}

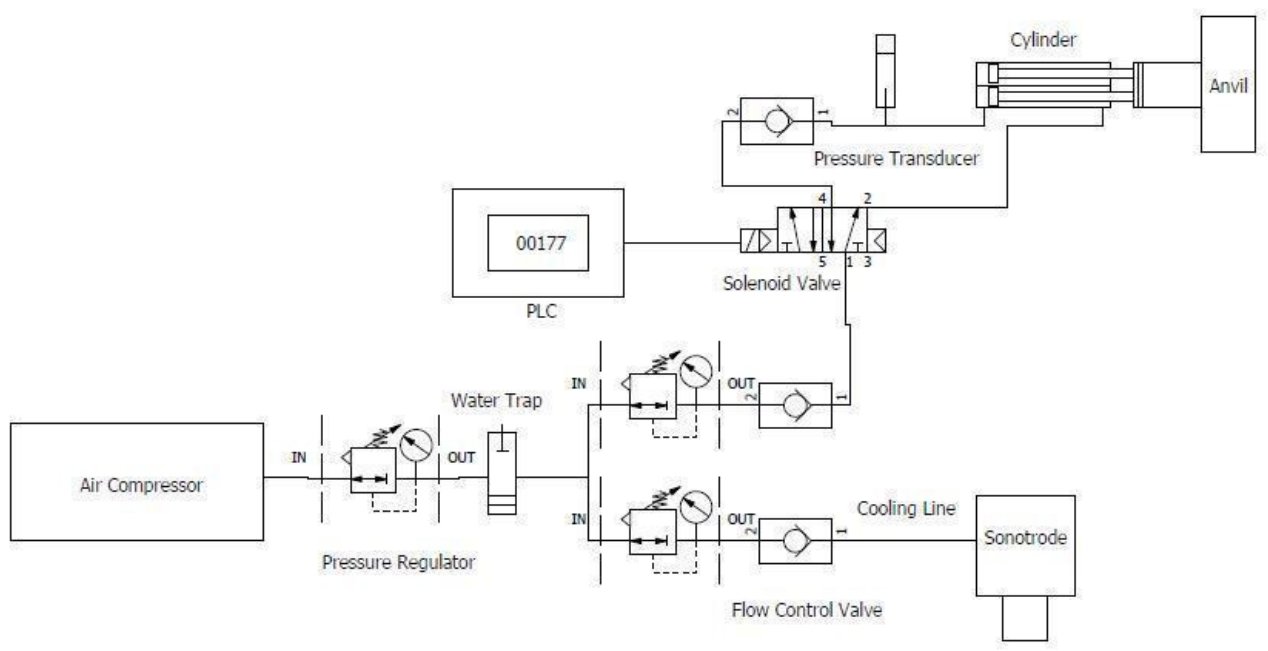

Pneumatic Circuit for Ultrasonic Welding Rig

Figure E.5 Pneumatic Circuit 